- An Introduction to the Passage of Energetic Particles

\title{
through Matter
}

N) Carron 


\section{An Introduction to the Passage of Energetic Particles through Matter}





\title{
An Introduction to the Passage of Energetic Particles through Matter
}

\author{
NJ Carron \\ ATK Mission Research Corporation \\ Santa Barbara, California, USA
}

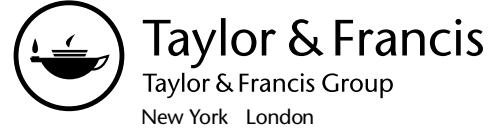


Sponsoring Consortium for

Open Access Publishing in Particle Physics

Open Access funded by SCOAP3

\title{
Copyright 2021 N J Carron
}

This eBook was converted to open access in 2021 through the sponsorship of SCOAP3 licensed under the terms of the creative commons Attribution-NonCommercial 4.0 International License (https://creativecommons.org/licenses/by-nc/4.0/) which permits use, sharing, adaptation distribution and reproduction in any medium or format, as long as you give appropriate credit to the author(s) and the source, provide a link to the creative commons license and indicate if changes were made, this license does not permit the Contribution to be used commercially.

\section{CRC Press}

Taylor \& Francis Group

6000 Broken Sound Parkway NW, Suite 300

Boca Raton, FL 33487-2742

(c) 2007 by Taylor \& Francis Group, LLC

CRC Press is an imprint of Taylor \& Francis Group, an Informa business

No claim to original U.S. Government works

Printed in the United States of America on acid-free paper

Version Date: 20141210

International Standard Book Number-10: 0-7503-0935-0 (Hardcover)

International Standard Book Number-13: 978-0-7503-0935-6 (Hardcover)

DOI: $10.1201 / 9781420012378$

This book contains information obtained from authentic and highly regarded sources. Reprinted material is quoted with permission, and sources are indicated. A wide variety of references are listed. Reasonable efforts have been made to publish reliable data and information, but the author and the publisher cannot assume responsibility for the validity of all materials or for the consequences of their use.

The Open Access version of this book, available at www.taylorfrancis.com, has been made available under a Creative Commons Attribution-Non Commercial 4.0 International.

Trademark Notice: Product or corporate names may be trademarks or registered trademarks, and are used only for identification and explanation without intent to infringe.

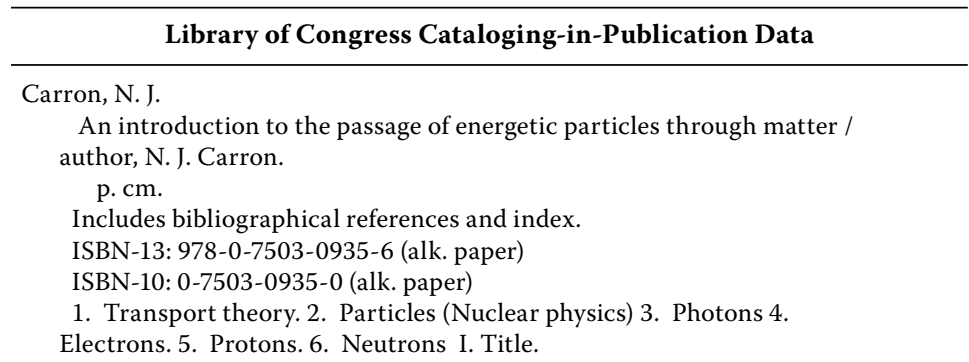

QC793.3.T7C37 2006

530.13 ' $8--\mathrm{dc} 22$

2006018871

\author{
Visit the Taylor \& Francis Web site at \\ http://www.taylorandfrancis.com \\ and the CRC Press Web site at \\ http://www.crcpress.com
}




\section{Preface}

The subject of the passage of neutral and charged particles through matter has been studied for a century. After decades of much early experimental work and nonquantum mechanical calculation, the basic physics of the passage of photons, electrons, protons, and heavier charged particles through matter was worked out theoretically in the decades following the completion of quantum mechanics, largely in the 1930's, and neutron diffusion shortly thereafter. There is excellent summary documentation, from the 1950's and later. The early, basic theoretical treatments, now textbook material, were compared with data available at the time. Today, one has available large data libraries with cross sections compiled from decades of measurements and sophisticated calculations.

The accuracy of data in current libraries, based on modern computations and continually accumulating experimental measurements, far surpasses that of the early, relatively elementary, calculations. As one example, below 100 $\mathrm{keV}$ the standard, original Bethe mean stopping power formula for electrons is less than $85 \%$ of the actual stopping power, and below $10 \mathrm{keV}$ may be less than $60 \%$ of the correct value in some materials. At still lower energies it is effectively inapplicable. Today's computing capabilities have allowed accurate calculations (although electron stopping power below a few $\mathrm{keV}$ is still somewhat problematical).

Given the long history of work on the passage of energetic particles through matter, there is little new that can be said of a fundamental nature. And almost all the data collected here are available elsewhere, often on the Web, or in journal articles. However, workers in need of certain parameters (cross sections, stopping powers, etc.) may not be aware of the availability of the needed data, which can be well-known to other disciplines. While nuclear physicists may be very familiar with the cross section compilation in the evaluated nuclear data files (ENDF), solid-state physicists and electronic engineers studying the effects of cosmic rays on satellites or micro-electronics may be less so. It is not widely known that photo-atomic and electro-atomic cross sections are also tabulated in ENDF, or that models for electron multiple scattering are available on the Web. Knowing where to access those and other data, extracting a needed subset from all that is available, knowing how to interpret the format in which it is presented, can be time-consuming tasks. That has often been the experience of the author and, according to a short, informal survey, the author's colleagues as well. Graphs of parameters as a function of the relevant independent variable (cross sections vs. energy or vs. scattering angle, etc.) are sometimes what is wanted, and occasionally are sufficient for the purpose at hand. 
It seemed worthwhile to try to collect in one place as much of these often needed data as possible, together with enough background physics so the reader can feel comfortable applying them, having some understanding of where they come from and why they have the order of magnitude they have. The idea is to make up-to-date data available and understandable to nonspecialists. The book and its accompanying data $\mathrm{CD}$ and contour plots are intended to be a working reference for scientists and engineers in industry, educational institutions, and laboratories, providing ready access to useful data. We have also tried to digest the data in the form of useful graphs, showing dependencies over a wide range of the independent variable(s), allowing quick approximations of a quantity. And it was decided to include much of the numerical data on a CD-ROM included with the book. Throughout, we include references to where the data came from, and where updates to them, and related information, can be found. While there are many articles and treatises on individual projectiles, there are fewer if any introductions providing an overview of the entire subject for photons, electrons, ions, and neutrons; we attempt to provide such an introduction here. It will be useful to practitioners of radiation physics, but the level of analysis is not intended to satisfy the expert. It is intermediate between a text book and usual reference. It is not intended to be a comprehensive treatise on the subject; that would be too vast a task. References that together may be taken to constitute such a treatise are given throughout. And the book may serve as an introduction to the massive, invaluable ENDF data library.

In addition, certain features of particles interacting with matter are not so well known, and worth bringing to a wider audience. It is widely appreciated, for example, that when a photon Compton scatters from a free electron, its angular distribution peaks in the forward direction. But it is less widely recognized that when a photon Compton scatters from an atom, its angular distribution vanishes in the forward direction.

For electrons, it is common knowledge that the Coulomb cross section for scattering from an isolated charge (nucleus) diverges. Screening of atomic electrons makes the electron-atom elastic scattering cross section finite. But its sharp forward peaking, even on a screened atom, is remarkable. The elastic differential cross section in exactly the forward direction $(\theta=0)$ increases in proportion to the square of the incident electron energy, while the total elastic cross section saturates to a constant as energy increases. On Fe, the forward differential cross section rises to a value of $10^{13} \mathrm{barn} / \mathrm{sr}$ at $100 \mathrm{MeV}$. At an angle of only $1^{\circ}$ it has fallen 9 orders of magnitude to $10^{4} \mathrm{~b} / \mathrm{sr}$. At that energy $99 \%$ of the scattering occurs at angles less than $0.1^{\circ}$, in a solid angle of only $10^{-8}$ of a sphere.

Any cross section, say the photon-atom Compton cross section $\sigma(E)$, is a function of incident photon energy $E$ for each target material. It is therefore clear that the cross section on all elements, $\sigma(Z, E)$, is a function of atomic number $Z$ and $E$, and so forms a surface over the $Z-E$ plane. That surface can 
be represented by a contour plot in the Z-E plane. One thereby displays an interaction for all elements over all energies of interest on a single graph. Numerical values of cross sections and/or stopping powers can be read quickly, often to quite useful accuracy, from such a graph, especially from the large, color $11^{\prime \prime} \times 17^{\prime \prime}$ plots. These plots are considered an integral part of this book. They are included as high resolution Portable Document Format (pdf) files on the accompanying CD-ROM, appropriate for printing on $11^{\prime \prime} \times 17^{\prime \prime}$ paper.

Such a contour plot assists in understanding the overall process, enables global trends to be discerned, and helps one choose a material with desired characteristics. In addition, there is satisfaction in knowing more than just the immediate number one needs; one develops more confidence in each number when it is viewed along side its neighbors.*

For a photon cross section such a plot brings to light the difference between the atomic cross section $\sigma$ (barn/atom) as a function of $Z$ and $E$, and the bulk matter cross section $\sigma\left(\mathrm{cm}^{2} / \mathrm{gram}\right)$ as a function of $Z$ and $E$. In the conversion between the two the atomic weight $A(Z)$ of elements in their natural isotopic composition enters the conversion factor. Since $A(Z)$ has irregular behavior as a function of $Z$, contours of $\sigma\left(\mathrm{cm}^{2} / \mathrm{gm}\right)$ exhibit an irregular pattern that does not occur in the smooth $\sigma$ (barn/atom). Similar graphs can be constructed for any cross section or for stopping powers or ranges of charged particles.

Cross section or stopping power contour plots are more than of academic interest. Not only does one see the full span of physics on a single page for, say, the total photon cross section, as the dominant process passes from photoelectric absorption to Compton scattering to pair production as energy increases, but in addition one can read the numerical value of the cross section often to better than a few percent. The author uses them routinely. It seems worthwhile to bring these and other features to the attention of a wider audience, and to provide comments clarifying and emphasizing important points.

Further, some published data are based on quite sophisticated calculations (for example self-consistent relativistic Dirac-Hartree-Fock models) that generally give a very believable result for cross sections, but may produce other unrealistic features. The self-consistent DHF model of elastic photon-atom scattering produces detailed form factors, but can be inaccurate for absorption edges. Such published data are most meaningful to other specialists in the field. The general user may wonder why a seemingly elementary quantity like

\footnotetext{
*We may appeal to a phrase by Oppenheimer, "Every part of the theory may be understood from more than one point of view; to see it always and only from one point of view, even if that be the most general, is to understand it only partially", taken, rather out of context, from his review of Dirac's new book The Principles of Quantum Mechanics; Phys. Rev. 31:97 (1931). The same may be said of data.
} 
the photo-ionization edge in $\mathrm{Fe}$, which is given as $7.9024 \mathrm{eV}$ in most tabulations, is given as 7.530 eV in LLNL's Evaluated Photon Data Library (EPDL) data base, and appears as $3.60 \mathrm{eV}$ in calculations of elastic form factors [C.T. Chantler J. Phys. Chem. Ref. Data 24:71 (1995)]. Likewise the separation of edges, such as $\mathrm{L}_{\mathrm{II}}$ to $\mathrm{L}_{\mathrm{III}}$, may not be calculated accurately. The reason is that detailed models accurate for their intended purpose (calculation of scattering cross sections) are not necessarily accurate for bound energies; they tend to break down at the $3-5 \mathrm{eV}$ level. The resulting photo-ionization edge may be off by more than $1 \mathrm{eV}$. In particular, Livermore's EPDL photo-atomic library was constructed together with its EEDL electro-atomic counterpart for the purpose of having a consistent set of cross sections for electron-photon transport calculations. Here consistency between data sets is more important than absolute accuracy. The library documentation makes that purpose clear, and cautions against using the cross sections for other purposes without checking other sources. Merely bringing these and other related facts to the attention of a wider audience seemed sensible.

Fortunately for the author the U.S. Air Force Office of Scientific Research agreed that this was a worthwhile undertaking, it hopefully being helpful to those unfamiliar with the data and saving workers much time. AFOSR has funded its writing for some time. The author is grateful for that support; there is no way the book could have been written without it.

Sources for data and their updates are given as Web addresses. Unfortunately, internet URLs change over time. We have found no simple way around this dilemma except to note that each address is usually associated with a particular organization which survives longer than the specific address for the data in question. Creative hunting for the new address within that organization may be necessary. The address for photo-nuclear cross sections at the International Atomic Energy Agency may change, but the IAEA will still be there.

Time and support limitations have resulted in photons and electrons being discussed more thoroughly than ions and neutrons. The former are important in their wide occurrence, are more penetrating than ions, and are a common source of effects. There are already a number of good reviews of heavy ion transport, and excellent, readily available calculational tools. Neutron interactions are too varied and numerous to attempt a full compilation. And the author is more familiar with photon and electron effects.

A broad discussion is given of the effects of multiple scattering on electron trajectories. We include a derivation and discussion of the conversion of a photon flux to an electron flux, via photoelectric, Compton, and pairproduction interactions (the " $1 \%$ Rule"), relevant, for example, to noise in pixellated detectors. Its opposite, the conversion of electrons to photons via Bremsstrahlung, is also discussed, with emphasis on the photon number flux in addition to the common energy flux, relevant, for example, to the problem of electrons penetrating material thicker than their range. 
In addition to the cross sections, stopping powers, angular distributions, etc., presented here, there are, of course, numerous other quantities involving radiation interacting with matter that are of interest in various applications. Among them are:

- The photon build-up factor, the increase in photon and electron flux with depth due to cascade showering as energetic gamma rays penetrate thick materials. A build-up factor may be defined for essentially any quantity. The transmission build-up factor is the transmission of all photons (or, if one wishes to define, all photons and electrons) through a thick absorber relative to the transmission of only unscattered photons; the flux build-up factor is the local omni-directional or directed flux of all photons (or all photons and electrons) relative to the flux of only unscattered photons; etc. But the concept is usually applied to the dose build-up factor, the dose of all particles at a given depth relative to the dose of unscattered photons; when a $\gamma$-ray Compton scatters, the scattered photon and the Compton electron now deliver more dose than the original photon alone.

- Differences between electron and positron cross sections, stopping powers, and ranges. These are of interest in the spatial distribution of ionization and dose following pair production by energetic photons, and in medical applications of positron emission tomography.

- Electron (and ion) restricted stopping power, the stopping power due to individual energy losses less than a specified amount. Or a related quantity, especially important in radiation effects in electronics, the radial profile of energy deposited along the trajectory of a primary electron or ion. This accounts in large measure for the principal difference between stopping power and Linear Energy Transfer (LET) in micro-electronic applications.

- The stopping power and ranges in compounds, as well as elements. Compound semi-conductors and particle detectors, plastics, metal alloys, dielectrics, biological tissue and other organic compounds, are some of the materials of interest. This is especially a problem for heavy ions; to be precise, unlike electrons, their stopping power is not simply the sum of the weighted elemental stopping powers. Fortunately, other readily available tools address this problem for ions.

- Stopping power and range of the mu meson, an important component of sea-level cosmic rays. Only stable particles are discussed.

- Photo-electron and photo-photon yield; the number of backscattered electrons and photons created when a photon strikes a solid surface.

- Backscattered and secondary electrons; the number and energy spectra of back-ejected electrons when an incident electron strikes a solid surface. 
- Ionization caused by secondary nuclear particles following energetic proton and heavier ion nuclear collisions.

- The small, but not always negligible, non-ionizing energy loss of electrons and photons.

Time limitations have prevented including these and many other topics of interest.

The author may be reached by e-mail at ncarron@alum.mit.edu for comments or to point out errors. Any necessary errata will be available at the website http://www.crcpress.com. 


\section{Acknowledgments}

It is a pleasure to thank a number of people, and an organization, who helped make this book materialize. The program director, Dr. Robert J. Barker, of The U.S. Air Force Office of Scientific Research (AFOSR), is one of the more level headed, sensible people the author has had the pleasure of knowing. In addition to exhibiting the patience of Job, he knows the value of long term research and how to foster it. " "If someone is enjoying the shade today it is because someone else planted a tree 40 years ago"-Warren Buffett, a level headed nonphysicist). I am grateful to him and the AFOSR for daring to fund the project leading to this book, and in the process tolerating intolerable delays.

I must thank Dr. Dermott (Red) Cullen who maintains and updates the current ENDF data library, now from LLNL, formerly from the IAEA. He tolerated and actually answered a nearly infinite email stream in my attempts to understand that compilation. Also my thanks to Dr. Robert MacFarlane of LANL, who similarly maintained the ENDF neutron part, who also increased my understanding of the subject.

Dr. Patrick J. Griffin of Sandia National Laboratory was kind enough to hasten his calculation of neutron kerma in silicon in time for his data to be included in the accompanying CD-ROM, and I thank him for a number of conceptual clarifications.

Drs. Carl Ekdahl (LANL), Victor A.J. van Lint (VvL, Inc.), John Hubbell (NIST), and John Baker (MRC) shared their considerable physical insight, some of which I hope has been passed on to the reader here. Other colleagues offered their written support early on. As not all revealed, I hesitate to mention those that did for fear of slighting those who did not but still labored as hard. I thank them all. 



\section{The Author}

Dr. N.J. Carron received his B.S. in physics from the Massachusetts Institute of Technology, and Ph.D. from the University of Illinois at ChampaignUrbana. After a post-doctoral appointment at Rice University Department of Physics, he joined the staff of Mission Research Corporation in Santa Barbara, California, where he has remained for more than 30 years.

During that time he has contributed to many programs, largely in electromagnetics and applied nuclear and plasma physics, including spacecraft radiation shielding, neutron and gamma ray detectors, relativistic electron beam propagation in air, intense laser propagation in air and laser-target interaction physics, air hydrodynamics, and others. He is an EMP fellow of the Summa Foundation, for his contributions to understanding systemgenerated EMP environments. The present book grew out of frustration at not being able to find readily available, digestible information and reliable data on the penetration of particles through matter. It is the book he wishes he had on his shelves 30 years ago. 



\section{Table of Contents}

\section{Chapter 1 \\ Introduction}

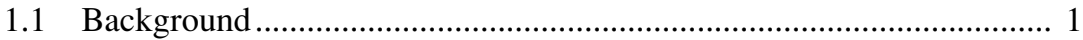

1.2 Charged vs. Neutral Particles ................................................................ 6

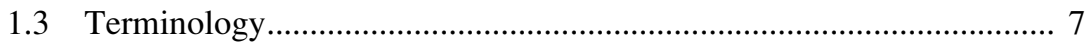

1.3.1 Field, Radiation, and Flux ................................................... 8

1.4 Distribution Functions and Flux........................................................ 9

1.4.1 Distribution Function and Flux .............................................. 10

1.4.2 Flux and Three-Dimensional Geometry................................... 11

1.4.2.1 Planar Fluxes .......................................................... 12

1.4.2.2 Omnidirectional Flux .............................................. 15

1.5 Reaction Rates in Planar and Omnidirectional Fluxes ........................ 17

1.6 Energy Spectrum and Spectral Fluence ............................................. 21

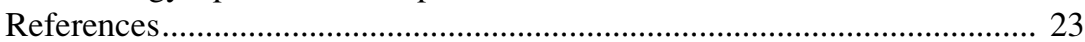

\section{Chapter 2}

\section{Photons}

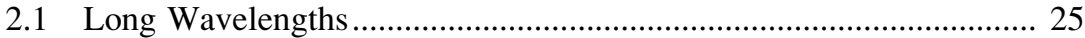

2.1.1 Limits of Applicability of Free Atom Cross

Sections in Condensed Media at Long Wavelengths ........... 26

2.1.2 Long-Wavelength Photon Attenuation in Metallic Aluminum ......................................................... 28

2.1.3 Long-Wavelength Photon Attenuation in Water ................... 30

2.2 Short Wavelengths............................................................................ 32

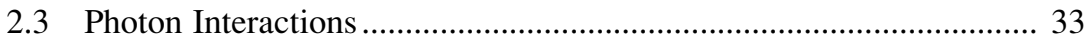

2.4 Photoelectric Absorption .................................................................... 37

2.4.1 Cross-Section Contour Plots................................................. 40

2.4.2 Contours in Barn/Atom ..................................................... 40

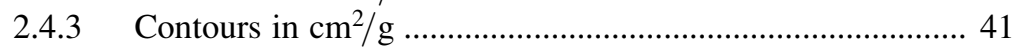

2.4.4 X-Ray Edge Energies ........................................................... 42

2.4.5 Angular Distribution of Photoelectrons ................................. 43

2.4.6 Fluorescence and Auger Electrons......................................... 46

2.4.7 Coster-Kronig Transitions .................................................. 47

2.4.8 Energy Transfer by the Photoelectric Effect ........................ 50

2.4.9 Energy-Transfer Coefficient.................................................. 51

2.4.10 Time Scale for Fluorescence .................................................. 53

2.4.11 Fluorescence and Auger Cascades ....................................... 53

2.4.12 Photoelectron and Auger-Electron Energies ......................... 54 
2.5 Coherent Rayleigh Scattering.......................................................... 54

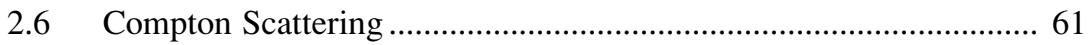

2.6.1 Compton Scattering from a Free Electron .......................... 64

2.6.1.1 Kinematics ............................................................ 64

2.6.1.2 Total Compton Cross Section .............................. 67

2.6.1.3 Particle Characteristic Energies ........................... 68

2.6.1.4 Compton Electron Energy Distribution .............. 69

2.6.1.5 Scattered Photon Energy Distribution................ 70

2.6.1.6 Photon Energy-Angle Relation........................... 71

2.6.1.7 Photon-Electron Angle Relations ....................... 71

2.6.1.8 Electron Angular Distribution.............................. 73

2.6.1.9 Scattered Photon Angular Distribution ............... 73

2.6.1.10 Cumulative Angular Distributions ...................... 73

2.6.1.11 Comment on Angular Distribution ..................... 79

2.6.1.12 Application to a Gamma Spectrometer.............. 80

2.6.1.13 Application to Penetration of Scattered Photons through a Shield ................................... 84

2.6.2 Compton Scattering from Atoms …………….................... 86

2.6.2.1 Angular Distribution......................................... 87

2.6.3 Compton Scattering in the Elements................................... 90

2.6.3.1 Total Compton Cross Section ............................. 90

$2.7 \quad$ Pair Production .......................................................................... 92

2.7.1 Threshold Energies for Pair Production.............................. 94

2.7.2 Z Dependence and Approximate Value of Cross

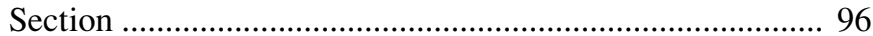

2.7.3 Graphs of Pair Production Cross Sections .......................... 98

$2.8 \quad$ Total Photon Cross Sections …………………………………........ 98

2.8.1 Use of Contour Plots in Shielding Applications................ 110

2.9 Attenuation and Energy Deposited in Target Material .................. 113

2.9.1 Attenuation of a Beam ..................................................... 113

2.9.2 Energy Transferred to the Target Material: Kerma........... 114

2.9.3 Photon Fluence-to-Kerma Conversion Factors.................. 118

2.9.4 Energy Deposited in the Target Material: Dose ................ 120

2.9.5 Kerma and Dose at a Material Interface............................ 123

2.9.6 Attenuation, Energy Transfer, and Energy Absorption ....... 125

2.10 Electron Flux from Photon Flux-The 1\% Rule............................. 126

2.10.1 The 1\% Rule .............................................................. 129

2.11 Dosimetric Quantities for Gammas and X-Rays ........................... 134

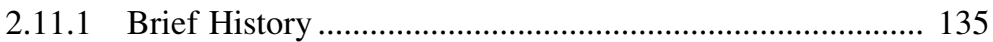

2.11.2 Nonbiological Measures .................................................. 136

2.11.2.1 Exposure, Dose, and Fluence ............................ 136

2.11.2.2 Fluence-to-Dose Conversion ............................. 137

2.11.2.3 Exposure-to-Fluence Conversion ...................... 138 
2.11.2.4 Exposure-to-Dose Conversion.......................... 140

2.11.2.5 Dose in Hydrogenous Material ....................... 141

2.11.3 Biological Measures ...................................................... 142

2.11.3.1 Relative Biological Effectiveness ................... 142

2.11.3.2 Quality Factor and Dose Equivalent.............. 143

2.11.3.3 Shallow vs. Deep Dose Equivalent................. 145

2.11.3.4 Dose Equivalent Averaged over an Organ .... 145

2.11.3.5 Dose Equivalent for Photons........................... 147

2.12 Sources of Cross-Section Data .......................................................... 148

2.12.1 Sources of Photon Cross-Section Data ............................ 148

2.12.2 Sources of General Nuclear and Particle Data ................ 149

2.12.3 The ENDF Data Library and Its Format.......................... 152

2.12.4 Structure of the ENDF Format........................................ 153

2.12.5 ENDF Preprocessing and Postprocessing Codes ............ 156

2.12.6 Extracting or Plotting a Cross Section from an ENDF File........................................................ 157

2.12.7 The Evaluated Photon Data Library in ENDF Format...... 157

2.12.8 The ENDL Data Library and Its Format ......................... 158

2.12.9 Comparison of NIST and LLNL Photon Data................ 159

2.12.10 Other Data Sources........................................................... 160

2.13 Large Contour Plots.......................................................................... 162

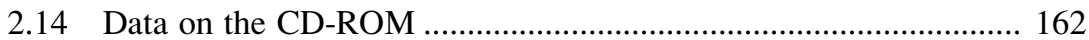

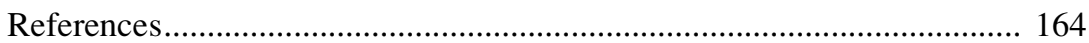

\section{Chapter 3}

\section{Electrons}

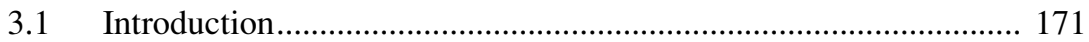

3.2 Elastic Scattering of an Electron from an Atom ............................ 172

3.2.1 Coulomb Scattering from a Bare Nucleus ....................... 172

3.2.2 Scattering from a Neutral Atom...................................... 174

3.2.3 Total Cross Section....................................................... 175

3.2.4 Differential Cross Section .............................................. 176

3.2.5 Differential Cross Section at $\theta=0$................................. 177

3.2.6 Differential Cross Section at Large

3.2.7 Angular Distribution........................................................ 180

3.3 Inelastic Scattering of an Electron from an Atom.......................... 181

3.4 Electron Energy Loss .............................................................. 185

3.5 Collisional Energy Loss ............................................................... 188

3.5.1 Accuracy of the Bethe Mean Stopping Power Formula.... 188

3.5.2 Density Effect .............................................................. 194

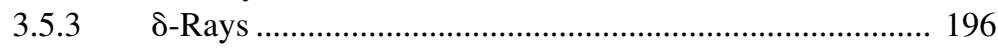


3.5.4 Energy and Number Distribution of $\delta$-Rays ..................... 196

3.5.5 Angular Distribution of 8-Rays....................................... 199

3.6 Radiative Energy Loss................................................................... 200

3.6.1 Bremsstrahlung Cross Section........................................... 200

3.6.2 Radiative Stopping Power .................................................. 202

3.6.3 Energy and Number Distribution

3.6.4 Bremsstrahlung Energy Spectrum Data ........................... 209

3.6.5 Angular Distribution of Bremsstrahlung Photons............. 209

3.6.6 Radiative Yield ................................................................ 209

3.6.7 Photon Number Yield........................................................ 210

3.6.8 Example of the Utility of Photon Number Yield:
Penetration of an Aluminum Slab .................................... 215

3.6.9 Aluminum Parameters ........................................................ 215

3.6.10 First Approximation ........................................................ 217

3.6.11 Improved Approximation ................................................. 218

3.7 Total Energy Loss and Mean Range against Total

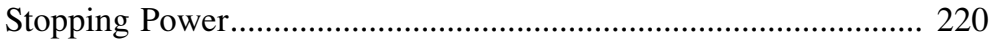

3.8 Multiple Angular Scattering ....................................................... 222

3.8.1 Williams, Molière, and Goudsmit-Saunderson Scattering ……................................................................. 227

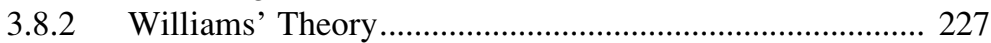

3.8.3 Angular Spread vs. Energy Loss ...................................... 232

3.8.4 Parameters Characterizing the Scattered Electron Distribution in a Thick Material ....................................... 234

3.8.5 Empirical Fit to the CSDA Range ..................................... 240

3.8.6 Empirical Fit to the Mean Forward Range ....................... 242

3.8.7 Longmire Treatment of Multiple Scattering

3.8.8 Range to a Specified Angular Divergence......................... 245

3.9 Cross Sections in Selected Elements ............................................ 247

3.10 Electrons in Low-Pressure Air ...................................................... 251

3.10.1 Energy Loss Rate........................................................... 251

3.10.2 Range against Energy Loss .............................................. 253

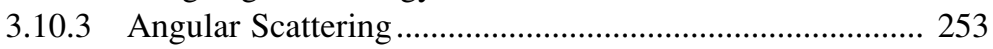

3.10.4 Scattering Range ............................................................. 257

3.10.5 Momentum-Transfer Mean Free Path .............................. 257

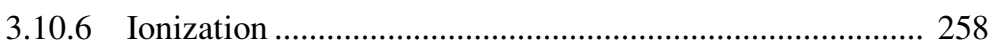

3.11 Large Contour Plots............................................................... 258

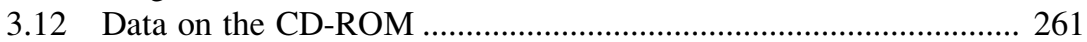

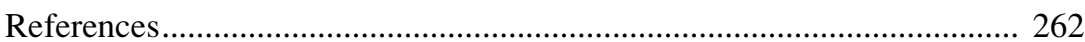




\section{Chapter 4}

\section{Protons and Heavier Ions}

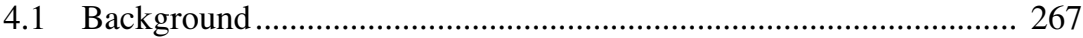

4.2 Principal Results of Ion Stopping Power Theory ………………….. 269

4.2.1 Maximum Energy Transfer in Elastic Scattering ................. 272

4.2.2 Shell Corrections ……………............................................ 273

4.2.3 The Density Effect............................................................. 274

4.2.4 Two Further Corrections to the Bethe Formula.................... 274

4.2.5 Charge State of the Ion...................................................... 275

4.2.6 The Bragg Peak ................................................................ 276

4.3 Ion Stopping Power in Compounds ................................................ 280

4.4 Proton and Alpha Particle Stopping Powers in Selected

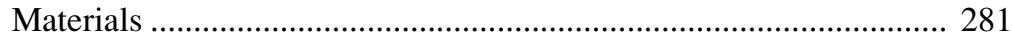

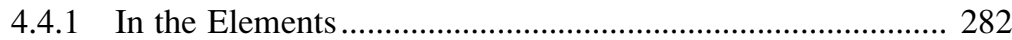

4.4.1.1 Protons ................................................................ 282

4.4.1.2 Alpha Particles...................................................... 286

4.5 Proton-Nucleus Elastic Scattering ................................................ 288

4.5.1 Nonionizing Energy Loss ..................................................... 300

4.6 Large Contour Plots................................................................... 300

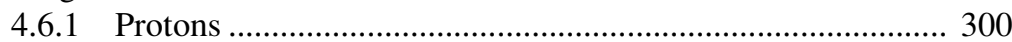

4.6.2 Alpha Particles...................................................................... 301

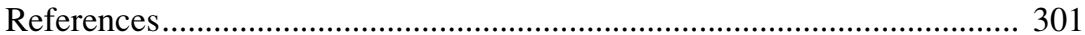

\section{Chapter 5}

\section{Selected Topics on Neutron Interactions}

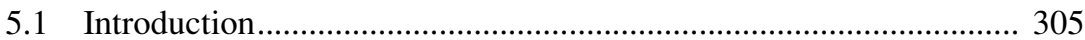

5.2 Neutron Energy and Reaction Nomenclature ……………………..... 307

5.2.1 Neutron Energy Ranges......................................................... 307

5.2.2 Neutron Reaction Nomenclature ........................................ 310

5.3 Good Neutron Absorbers.............................................................. 313

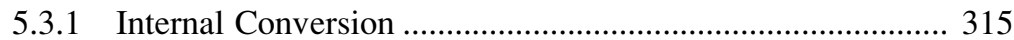

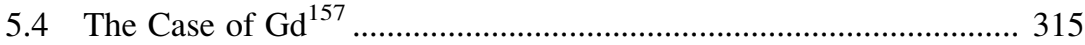

5.4.1 General Observations ………………………................. 316

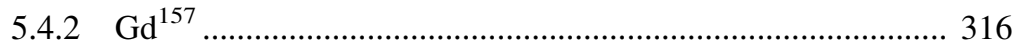

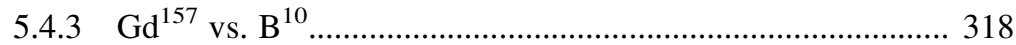

5.5 Cross-Section Dependence on Target Temperature ……………...... 320

5.5.1 Temperature Dependence of a Constant Cross Section ....... 321

5.5.2 Temperature Dependence of a $1 / v$ Cross Section .................. 322

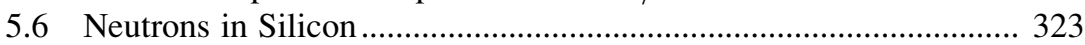

5.6.1 Basic Cross Sections in Silicon............................................ 324

5.6.2 Energy Transfer during Collision.......................................... 325

5.6.3 Kerma Due to Elastic Scattering ……………....................... 325 
5.6.4 Kerma Due to $n$ Capture and Gamma Emission 329

5.6.5 Energy Partitioning and Nonionizing Dose

5.6.6 1 MeV Displacement Equivalent Fluence

5.7 Neutron Lifetime in Air ........................................................... 332

5.7.1 Radiative Capture in Nitrogen ........................................... 334

5.7.2 The $(n, p)$ Reaction ........................................................ 334

5.7.3 Neutron Mean Free Path in Air........................................... 334

5.7.4 Neutron Mean Lifetime in Air ........................................... 334

5.8 Neutron Cross Sections in Selected Isotopes................................. 335

References............................................................................................. 344

Appendix A: Some Fundamental Constants and Defined Units ............. 347

Appendix B: Useful Web Sites ............................................................. 349

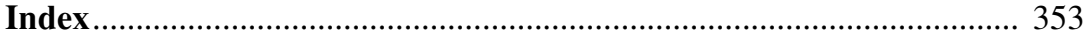




\section{Introduction}

DOI: $10.1201 / 9781420012378-1$

\subsection{BACKGROUND}

The study of the passage of photons, electrons, protons, $\alpha$ particles, and neutrons through matter is essentially as old as the discovery of these radiations themselves. Indeed, it could hardly be otherwise. Original production of energetic photons (x-rays), late in the nineteenth and early in the twentieth centuries, well before the name "photon" was invented, took place in vacuum chambers, and the radiations had to penetrate the walls of the chamber to propagate to detectors. Radiation detectors themselves were enclosed in a housing, and the radiation had also to penetrate its walls or entrance windows. Radiation names were also drawn from the notion of the passage of particles through matter. Before their underlying nature was understood, the names given to three forms of radiation, $\alpha, \beta$, and $\gamma$, were selected in the order of the penetrating power of the radiations, with $\alpha$ particles readily stopped by thin foils, $\beta$ rays more penetrating, and $\gamma$ rays capable of passing through relatively thick materials. Further, it was immediately realized that in penetrating foils (or scattering from foils), these radiations provide a probe of the interior matter, that is, a tool to investigate the atomic nature of matter. Understanding the energy loss and scattering processes thus became important endeavors. The historically minded reader may follow the chronology in, for example, the beautiful account by Pais [Pa86].

Over the decades, many fields of research have emerged in which energetic, neutral, and charged particles play a role. Their number seems to be increasing with time.

- Naturally trapped particles in the Earth's inner and outer radiation belts routinely bathe satellites in beams of energetic electrons and protons, and internal electronics must be designed to withstand their effects.

- Energetic cosmic rays, and their secondary showers, unavoidably strike satellites and aircraft (and their passengers).

- Nuclear weapon bursts put out intense $\gamma$-rays, x-rays, $\beta$ 's, and neutrons, and an entire industry has grown to study their effects and shielding against them.

- Reactor laboratories need to understand the penetration of neutrons and $\gamma$ 's, and the secondary particles generated by them. 
- Some laboratories routinely have pulsed or continuous x-ray sources, neutron guns, $\gamma$ and $\beta$ emitters, and other particle sources to study their effects on materials.

- The astronomy community is relying more and more on digital cameras in satellites, using CCDs in place of photographic emulsions. Performance of these electronic cameras is degraded over time as energetic particles, such as cosmic rays and trapped radiation, impinge on them, and needs to be taken into account in the original design.

- The health physics community employs radiation to destroy cancerous cells and is concerned with the effects of these radiations on biological tissue.

- The science and art of radiation detection require knowledge of the interaction of radiation with matter.

- Plasma physicists need to know the ionizing effects of charged particle beams passing through gases.

- Auroral and upper atmospheric studies require knowledge of the effects of energetic electrons and protons on atmospheric gases.

All these endeavors require basic cross sections and stopping powers of particles in matter.

When a particle passes through a material, it changes the matter in a number of ways. A charged projectile will knock out atomic electrons, causing ionization (ion pairs in a gas, electron-hole pairs in a semiconductor); it can electronically excite atoms, which may de-excite by delayed photon emission (fluorescence); it may collide directly with a nucleus, causing it to recoil and displace in a solid; or it may produce Bremsstrahlung photons, which travel some relatively large distance to interact elsewhere in the material or which escape the material target. An energetic neutron or proton may cause a nuclear reaction ejecting other nuclear particles. The study of the effects of a particle or particles on the target material is generally known as radiation effects.

Likewise, the matter affects the particle by scattering, slowing down, or absorbing it. The study of the effects of matter on the passage of particles is generally known as radiation transport. Both aspects of the process, the particle's effect on matter and the material's effect on the particle, are of interest. Radiation effects are of interest in radiation damage studies, as in cosmic ray effects in semiconductor electronics or in radiation cancer therapy, and in particle detection, where the change in the target is used to register the passage of a particle. Radiation transport is of interest, for example, in radiation shielding studies, or in particle stopping power such as that needed to compute the effect of a thin attenuating layer of solid purposely intended to slow down a proton beam.

As needed for these studies, it would clearly be useful to have at the ready a collection of the basic data on the relevant parameters concerning the 
passage of energetic particles through matter: A single place where one can find cross sections and mean free paths (mfps) of photons; stopping powers, ranges, and scattering of electrons, protons, $\alpha$ particles, and possibly other ions; kerma and dose; fluence-to-dose conversions, and so on, in the elements. It need not be a textbook, for most users would already be at least somewhat familiar with most concepts. However, in addition to data, it should have enough of a discussion of the physics to convince the users that they understand the data, what is behind them, and how to use them properly. The present book hopes to provide that source of information, at least partially. It is intended to complement the more fundamental references and provide a useful tool for researchers; to help workers use the mass of knowledge and data on the passage of energetic particles through matter, and to make it easier for the reader to find needed quantities. Some digital databases, while thorough, present numerical data in a compressed format, making them inconvenient to use readily. We have digested the most commonly used data (cross sections, stopping powers, fluence-to-kerma conversion factors, etc.), and provided them in straightforward format on an accompanying CDROM. Web sites are cited for updates.

In this well-studied subject, most of the just mentioned parameters are found scattered in books, articles, or on the Internet in a wide variety of sources. In particular, the three oft-referenced works have become classics:

Bethe, H.A. and Ashkin, J., Passage of radiations through matter, in E. Segre, Ed., Experimental Nuclear Physics, Vol. 1, J. Wiley, 1953. Evans, R.D., The Atomic Nucleus, McGraw-Hill, 1955.

Heitler, W., The Quantum Theory of Radiation, 3rd edn., Oxford University Press, 1954.

The series of reports by the Lawrence Livermore National Laboratory (LLNL), carrying basic number UCRL-50400, has provided a wide community with cross-section data for years. Particularly useful as data sources are certain web sites of the National Institute of Standards and Technology (NIST), the Los Alamos National Laboratory (LANL), the National Nuclear Data Center at Brookhaven National Laboratory (BNL), LLNL, and the ion transport tools of Ziegler and coworkers, originally at IBM. However, formulas and the discussion of the physics behind them are not so readily available on the Web, and it is no mean feat to get to the data from a laboratory's home page. And often enough, some extension of the raw data is desired. Having most of this in one place would save time.

Since the advent of powerful computers, and the growing need for particle transport calculations for shielding and for radiation effects studies, a number of equally powerful codes have been developed to solve the transport through matter of photons, neutrons, and charged particles. One of the most widely used is MCNP (Monte Carlo $\mathrm{n}$ Particle, formerly Monte Carlo 
Neutron Photon). Others are COG, TIGER (now ITS), TART, EGS, CASINO, PENELOPE, MC-SET, GEANT, and so forth. These are all very successful, and in common use. Many excel at handling complicated three-dimensional geometries, and are well developed. The December 2000 MCNP Version 4C manual states that there have been some 450 man-years (person-years, actually) in its development up to that time, and its conceptual origins date back to von Neuman and Fermi.

Partially as a result of the availability of these codes and computers, there is a growing tendency among scientists to run big, existing codes to solve almost every problem, rather than to ponder first with much effort about the physics involved. As a result, many studies in the disciplines listed earlier have been (and are) carried out with the well-established codes. The work involved in solving a problem then becomes the work involved in setting up the code input and deciding on useful forms of output from all those available; for complicated geometries, this is not simple. The trend toward numerical work is driven by current needs. Calculations involving complicated geometries using well-established codes are absolutely necessary; the geometries in most real problems are so involved that there is really no other way to get the right answer.

For the most part, the individuals who make use of the large codes are not those who wrote them, and often are not intimately familiar with them. For the uninitiated, the documentation occasionally does not quite achieve the epitome of ultimate clarity; it is impossible for code developers to write documentation in a way that anticipates every user's point of view. To check one's work, to make estimates, to scope the problem before setting up a large code computation, to convince oneself that numerical results are correct, to understand their meaning, one needs to see the raw data, their graphs, and perhaps the scaling laws (with particle energy, or with atomic number) from basic equations and data.

Further, one often wishes to write one's own (smaller) code rather than use the standard large ones. It gives one better control over the entire problem and an assurance of understanding. For this purpose, one needs the actual cross sections in numerical form.

The present book is intended primarily to present needed parameters, data, and concepts to satisfy these purposes. It makes good use of the huge ENDF cross-section database, and may serve as an introduction to that data library.

One might expect most atomic cross sections and particle stopping powers to be quite well known and well documented by now. While that is true in a general sense, practical problems remain. Uncorrected errors in published journal articles, differences among different data compilations, with little guidance as to which is more reliable, and errors in the documentation for the use of those compilations still exist. Some of those differences merely reflect the current state of knowledge of those parameters. Although the situation seems to be improving with time, it can be a chore to get reliable 
numbers for actual application. In documentations it can be nontrivial for experts to write in words understandable to the nonexpert, to try to anticipate questions by those not as familiar with the material as the authors. We provide here much of the data, together with sufficient discussion to provide the reader with a guide.

The standard data sources, some mentioned earlier, generally present neutral particle cross sections and charged particle stopping powers for the elements. For each element, these quantities are a function of projectile energy $E$. If we work with, say, 98 elements, there are then 98 tables and 98 graphs of, say, the total photon cross section as a function of incident photon energy $E$.

However, each quantity (a cross section or a stopping power) can be considered a function of $Z$ and $E$. As such, it forms a surface over the $Z, E$ plane. On a single page, this surface can be represented by a contour plot in the $Z, E$ plane, for all values of $Z$ and all desired values of $E$. In this way, one can condense these usual 98 plots of a particular cross section in the elements into a single graph. For example, the total photon cross section on all elements over the entire energy range of interest appears on one graph. Then, at a glance, one can grasp the entire behavior of any photon cross section over all elements for all energies. Trends are readily discerned. For instance, when plotted in units of $\mathrm{cm}^{2} / \mathrm{g}$, one can readily see that at $1 \mathrm{MeV}$ the total photon cross section is nearly $0.06 \mathrm{~cm}^{2} / \mathrm{g}$ in all elements; but when plotted in units of barn/atom, the cross section varies from 0.3 at $Z=2$ to more than 30 at $Z>90$. Similarly, the electron (or proton or alpha particle) stopping power at all energies in all elements can be represented by its own contour plot, and the full span of $Z$ and $E$ can be seen on a single page. Small versions of these contour plots are presented in later chapters. These contour plots are provided separately in color as Adobe Acrobat PDF files on the accompanying CD-ROM, intended to be printed out on $11^{\prime \prime} \times 17^{\prime \prime}$ paper. The plots are meant for this large size; in that size, they are much easier to read and are very useful. For some processes, the graphs can be read by the eye to better than a few percent accuracy, often sufficient for rough work.

This book is in no way intended to be a comprehensive treatise on the subject. The topic is too vast. Its primary purpose is to make modern data readily accessible, and to provide enough discussion of the underlying physics to make those data understandable. There has been something of a disconnect between expositions of the fundamental theory and complete presentations of modern, best, compiled data. This book is intended to fill that gap and to be a working reference.

Although the book touches on the subject of the effects of energetic particles on semiconductor electronic devices, it does not dwell on it. Rather, it presents the basic physical processes underlying all such effects. For a discussion of effects on semiconductor electronics, the reader is referred to standard works. Three of them are: 
Holmes-Siedle, A. and Adams, L., Handbook of Radiation Effects, 2nd edn., Oxford University Press, 2002.

Messenger, G.C. and Ash, M.S., The Effects of Radiation on Electronic Systems, 2nd edn., Van Nostrand Reinhold, 1992.

van Lint, V.A.J., Flanagan, T.M., Leadon, R.E., Naber, J.A., and Rogers, V.C., Mechanisms of Radiation Effects in Electronic Materials, Vol. 1, Wiley, 1980.

Likewise, radiation shielding is a major subject in its own right, and good texts are available. Among them are:

Goldstein, H., Fundamental Aspects of Reactor Shielding, AddisonWesley, 1959.

Profio, A.E., Radiation Shielding and Dosimetry, J. Wiley, 1979.

Shultis, J.K. and Faw, R.E., Radiation Shielding, American Nuclear Society, 2000.

The high-energy physics community, often requiring energies higher than those considered here, maintains its own summary of the passage of particles through matter, in the regularly updated review of particle properties; for example, Groom and Klein in [PDG04] or http://pdg.lbl.gov. The X-ray physics community maintains a site [http://xdb.lbl.gov] and a handy reference booklet [XRD01].

We first make note of some basic differences among the radiations.

\subsection{CHARGED VS. NEUTRAL PARTICLES}

It is worth making a quick observation about the difference between a charged particle passing through matter and a neutral particle. As will be seen, the cross section for a charged particle (electron, proton, or heavier ion) to interact with an atom is of the order $\sigma \sim 10^{-16} \mathrm{~cm}^{2}$, whereas that for a neutral particle (photon, neutron) is only of the order $10^{-24} \mathrm{~cm}^{2}$ to $10^{-20} \mathrm{~cm}^{2}$. Thus, in matter of number density $N \sim 10^{23} \mathrm{~cm}^{-3}$, the mfp between collisions, $1 / N \sigma$, is only a few Angstroms (1Angstrom $\equiv 1 \AA=10^{-8} \mathrm{~cm}$ ) for a charged particle, but is microns to centimeters for a neutral one.

As a result, a neutral particle (photon, neutron) interacts relatively infrequently with target atoms, and at each collision is either absorbed or scattered out of the original beam. The unscattered beam intensity is attenuated by a factor $e$ in one $\mathrm{mfp}, 1 / N \sigma$, the quantity which then characterizes its passage, and the intensity decreases exponentially with the distance traversed. Particles scattered out of the beam may or may not still be of interest. The mfp of a $1 \mathrm{MeV} \gamma$ ray is approximately $16 \mathrm{~g} / \mathrm{cm}^{2}$ in most elements (about $6.8 \mathrm{~cm}$ in Si, and about $125 \mathrm{~m}$ in STP air). 
However, a charged particle (electron, proton, alpha particle, heavier ion) interacts with nearly every atom along its path, and loses some energy each time to atomic excitation or ionization; but it continues on, perhaps scattered in direction. Heavy ions are scattered in direction only slightly, as are relativistic electrons; then the loss of particles from the original, unscattered beam is not of concern. The relevant parameter for a beam of charged particles is its rate of energy loss. Eventually, it loses all energy and stops after a fairly well-defined track length. A $1 \mathrm{MeV}$ electron has a track length of about $0.6 \mathrm{~g} / \mathrm{cm}^{2}$ in most elements (about $0.25 \mathrm{~cm}$ in $\mathrm{Si}$ and about $4.5 \mathrm{~m}$ in STP air). Lower-energy electrons, however, elastically scatter much, and their mean forward progress is considerably less than their track length.

Thus, beams of photons or neutrons are characterized by their mfps, but beams of charged particles are characterized by their instantaneous energy loss rate (the material's stopping power for that projectile) and a full path length or range.

Charged particles (electrons, protons, heavier nuclei, and charged mesons) are referred to as directly ionizing radiation. By their Coulomb collisions with atoms, they directly eject atomic electrons into the material. Many such ejected electrons, usually of low energy and small residual range, result from the passage of a single energetic charged particle.

Uncharged particles (photons, neutrons, and neutral mesons) are referred to as indirectly ionizing radiation. The basic interaction of a neutral particle with matter may cause one or more energetic charged particles to emerge from the interaction (a Compton electron; a photo-electron; a recoil target nucleus following nonelastic neutron scattering; protons, alpha particles, or nuclear fragments following nonelastic neutron scattering; etc.). Those energetic charged particles then ionize the matter by direct collisions with atomic electrons. The original neutral particle ionizes only indirectly via the ionization by the energetic particle(s) kicked out by the interaction of the neutral projectile. Some disciplines use the term ionizing radiation to apply to all particles, because all particles do, ultimately, produce ionization.

\subsection{TERMINOLOGY}

Over many decades, each of the disciplines that has had occasion to study or make use of the passage of energetic particles through matter has historically developed its own traditional terminology that may be inconsistent with, or at least confusing to, another. If a nuclear engineer (reactor designer), a basic nuclear physicist, a cosmic ray specialist, an electrical engineer studying gamma and neutron effects on electronics, and an infrared physicist were to enter a discussion to describe particle fluxes, the clash of nomenclature would bring useful information transfer nearly to a halt, even though all would be talking about the same phenomenon. Even the simple terms field, 
radiation, and flux, have different meanings in different specialties. Here we try to explain them or at least state unambiguously what we mean by the terminology we use.

\subsubsection{Field, Radiation, and Flux}

The classical electromagnetic fields $\overrightarrow{\mathbf{E}}$ and $\overrightarrow{\mathbf{B}}$ are indeed classical fields, because they are continuously distributed quantities and continuous point functions of position and time. No particles are involved.

However, and somewhat surprisingly for electromagneticists, the same word, field, is used, for example, in the neutron measurement community to mean the collection of all neutrons that may be bouncing around in a room. In this community, the neutron field is specified by the usual parameters describing a gas: either the number density of neutrons and their Boltzmann distribution function to specify their distribution in space and velocity, or, what is the same thing, their energy spectrum and angular distribution. A neutron field is the gas of neutrons in the vicinity of the observer, however one describes it, such as might occur near a reactor.

Once the particle nature of light and therefore of electromagnetic fields was realized in the 1920s, the term field in the context of electromagnetics has come to refer both to its original historical meaning of a classical, continuous point function, or, in the case of extremely high-frequency fields whose particle nature is important, to the neutron-community-like specification of the distribution of numbers of photons. A gamma field is the gas of photons in the vicinity of the observer, also such as might occur near a reactor.

Even the elementary term radiation has evolved to have a dual meaning. Since Maxwell's era in the latter half of the nineteenth century, in electromagnetics the term radiation has meant classical electromagnetic fields, arising from a time-dependent current distribution, which at large distances $r$ fall off as $1 / r$. The energy flux in the radiation field then falls off as $1 / r^{2}$. However, the discoverers of $\alpha$ rays and $\beta$ rays applied the term radiation to the emission of these particles from matter; the only similarity with the electromagnetic case, it turned out, was that an effect was observed some distance from a source. Even the distance dependence of their intensity did not fall off as $1 / r^{2}$ because of the additional exponential attenuation in passing through air or other materials. Therefore, the term as used had little quantitative value in common with its electromagnetic origins. Today, the term radiation has the general meaning of the emission and transport of classical electromagnetic fields, of photons, $\alpha$ or $\beta$ particles, neutrons, or anything else. A radioactive material is one that emits any of these particles.

In most areas of physics, the term flux means the rate at which some quantity passes through unit area. The number flux of particles is the number of particles passing per $\mathrm{cm}^{2}$ per second. In other disciplines, however, the term flux is used to mean the rate at which some quantity passes an observer, 
generally through a specified area, but not per unit area. In radiometry the word is used to mean the rate at which photonic energy passes a specified area, and is measured in Watts [Wo85]. Under this usage, a number flux would be the number of particles passing through a specified area per unit time, particles per second. The number passing through unit area would be called flux density, the number of particles per $\mathrm{cm}^{2}$ per second.* Older neutron literature (e.g., ICRU Report 13 [ICRU69]) used just such terminology (although the term fluence, rather than fluence density, is used to mean particles per $\mathrm{cm}^{2}$ ). Still other communities use the term flux to mean particles per $\mathrm{cm}^{2}$ (which we call fluence).

In this book, we adopt the more common usage. The flux of a quantity is the amount passing through unit area per unit time. An electron beam of 10 Amperes and cross-sectional area of $5 \mathrm{~cm}^{2}$ passes $2 \mathrm{C} / \mathrm{cm}^{2}$ per second. Its number flux is $2 /\left(1.602 \times 10^{-19}\right)=1.248 \times 10^{19}$ electrons $/ \mathrm{cm}^{2} / \mathrm{sec}$. Its charge flux is $2 \mathrm{C} / \mathrm{cm}^{2} / \mathrm{sec}=2 \mathrm{~A} / \mathrm{cm}^{2}$, also called its current density. A flux of $f$ (particles $/ \mathrm{cm}^{2} / \mathrm{sec}$ ) passes $f A$ (particles $/ \mathrm{sec}$ ) through an area $A$. There is no separate name for the quantity $f A$.

The fluence of a flux of particles is simply the time integral of flux, the number of particles passing through a unit area over a specified time, say particles $/ \mathrm{cm}^{2}$. Energy fluence is the energy of those particles that pass through unit area over a specified time, e.g., $\mathrm{MeV} / \mathrm{cm}^{2}$. As discussed presently, it is further necessary to specify the orientation of the unit area relative to the incident particles to fully specify the flux. Various definitions of this orientation result in different kinds of flux, such as planar or omnidirectional.

\subsection{DISTRIBUTION FUNCTIONS AND FLUX}

Although the focus of this book is on individual particles, one must eventually consider collections of particles. The dose in matter and the fluenceto-dose conversion factors make sense only for many particles. Here we discuss concepts and nomenclature of distribution functions and fluxes of particles.

From the previous discussion, once one defines flux as particles per unit area per unit time, the orientation of the unit area relative to the moving particles remains to be specified. Various orientations result in various definitions of flux: the omnidirectional flux; the planar, directed (current) flux; or the scalar directed flux, each useful in certain applications. All these follow from the Boltzmann distribution function $f(\overrightarrow{\boldsymbol{r}}, \overrightarrow{\boldsymbol{v}}, t)$, which contains more detailed information. Different disciplines define different distribution functions and fluxes for convenience.

\footnotetext{
* Magnetic flux (Wb) is the surface integral of the magnetic field $\mathbf{B}$ over a given area. $\mathbf{B}$ itself is called the magnetic flux density $\left(\mathrm{Wb} / \mathrm{m}^{2}\right)$. This use of the terms may be the origin of their similar use in other disciplines.
} 


\subsubsection{Distribution Function and Flux}

The basic Boltzmann distribution function, $f(\overrightarrow{\boldsymbol{r}}, \overrightarrow{\boldsymbol{v}}, t)$ particles per $\mathrm{cm}^{3}$ per $(\mathrm{cm} / \mathrm{sec})^{3}$, contains the most detailed description of a field of particles. $f(\overrightarrow{\boldsymbol{r}}, \overrightarrow{\boldsymbol{v}}, t) d^{3} \boldsymbol{v}$ is the number of particles per $\mathrm{cm}^{3}$ with velocity in $d^{3} v$ about $\overrightarrow{\boldsymbol{v}}$. The particle number density is $n(\overrightarrow{\boldsymbol{r}}, t)=\int f(\overrightarrow{\boldsymbol{r}}, \overrightarrow{\boldsymbol{v}}, t) d^{3} \boldsymbol{v}$. The energy spectrum $g$ is obtained by writing $d^{3} v=v^{2} d v d \Omega$ and integrating over velocity angles $\Omega$ and using $E=(1 / 2) m v^{2}$ (nonrelativistic) as the independent variable instead of $v: g(\overrightarrow{\boldsymbol{r}}, E, t)=g(E)=\int d \Omega f(\overrightarrow{\boldsymbol{r}}, \overrightarrow{\boldsymbol{v}}, t) \boldsymbol{v} / m$, where $m$ is the particle mass and $v^{2} d v=(v / m) d E . g(E) d E$ is the number of particles per $\mathrm{cm}^{3}$ with energy in $d E$ about $E$. The number density can also be written $n(\overrightarrow{\boldsymbol{r}}, t)=\int g(\overrightarrow{\boldsymbol{r}}, E, t) d E$.

$f$ itself is fundamental in kinetic theory, but it is often not the most useful direct description in applications. Rather, one is often interested not in the instantaneous velocity distribution, but in the number of particles that pass an area in a specified time. The quantity

$$
v f(\overrightarrow{\boldsymbol{r}}, \overrightarrow{\boldsymbol{v}}, t) d^{3} v
$$

is the number of particles $\mathrm{cm}^{-2} \mathrm{sec}^{-1}$ with velocity in $d^{3} v$ about $\vec{v}$, which cross a square centimeter with normal parallel to $\vec{v}$. It contains as much information as $f$ itself but addresses the commonly needed quantity more directly. Different disciplines define different differential fluxes. Seeing their connection to $f$ and their relation among one another lends some unified understanding to the subject. Even within a single discipline (notably neutron physics), nomenclature can differ.

As mentioned, in $f$ the three independent variables $\vec{v}$ can be replaced by $E$ and $\Omega$, using $d^{3} \boldsymbol{v}=(\boldsymbol{v} / m) d E d \Omega$. Then $\boldsymbol{v} f(\overrightarrow{\boldsymbol{r}}, \overrightarrow{\boldsymbol{v}}, t) d^{3} \boldsymbol{v}=$ $v f(\overrightarrow{\boldsymbol{r}}, E, \Omega, t)(\boldsymbol{v} / m) d E d \Omega$ is the number of particles $\mathrm{cm}^{-2} \mathrm{sec}^{-1}$ with energy in $d E$ about $E$ with direction $d \Omega$ about $\Omega$ that passes through the unit area perpendicular to the direction of $\Omega$. As indicated by their arguments, one takes $f(\overrightarrow{\boldsymbol{r}}, \vec{v}, t)$ to have dimensions of, for example, particles $/ \mathrm{cm}^{3} /(\mathrm{cm} / \mathrm{sec})^{3}$, but $f(\overrightarrow{\boldsymbol{r}}, E, \Omega, t)$ to be particles $/ \mathrm{cm}^{3} / \mathrm{MeV} / \mathrm{sr}$. Thus, the quantity

$$
J(\overrightarrow{\boldsymbol{r}}, E, \Omega, t)=\boldsymbol{v} f(\overrightarrow{\boldsymbol{r}}, E, \Omega, t) \quad\left(\text { particles } / \mathrm{cm}^{2} / \mathrm{sec} / \mathrm{MeV} / \mathrm{sr}\right)
$$

is a differential (in energy) flux with as much information as $f$ itself. In neutron transport theory, it is called the angular flux and is the fundamental quantity in that theory.

Nuclear reactor theory [Be70, He75] uses the notation $N(\overrightarrow{\boldsymbol{r}}, E, \Omega, t)$ for our $f(\overrightarrow{\boldsymbol{r}}, E, \Omega, t)$, and there $N$ is called the neutron angular density, emphasizing its dependence on $\Omega$, or the neutron density in phase space. It is simply the neutron Boltzmann distribution function with independent 
variables $E$ and $\Omega$ rather than $v_{x}, v_{y}, v_{z}$. The angular flux $J=v f=v N$ is denoted by $\Phi(\overrightarrow{\boldsymbol{r}}, E, \Omega, t)$ in Bell and Glasstone [Be70].* When integrated over the neutron velocity angles $\Omega$, the angular density becomes $\int N(\overrightarrow{\boldsymbol{r}}, E, \Omega, t) d \Omega=n(\overrightarrow{\boldsymbol{r}}, E, t)$, which Bell and Glasstone call the neutron density. Perhaps it should more properly be termed the neutron spectral density, for it is the number of neutrons per unit volume per unit energy interval, for example, neutrons $/ \mathrm{cm}^{3} / \mathrm{MeV}$. The full number of neutrons per unit volume is $\int n(\overrightarrow{\boldsymbol{r}}, E, t) d E$. The angular flux $\Phi$ may be integrated over velocity directions $\Omega$ to obtain what is called the total flux $\varphi(\overrightarrow{\boldsymbol{r}}, E, t)=\int \Phi(\overrightarrow{\boldsymbol{r}}, E, \Omega, t) d \Omega$ (particles $\left./ \mathrm{cm}^{2} / \mathrm{sec} / \mathrm{MeV}\right)$. This too might more properly be called the spectral flux, since it is differential in energy, or even the omnidirectional spectral flux, as it tabulates particles coming from all directions.

The same quantity $J(\vec{r}, E, \Omega, t)=v f$ is commonly the quantity specified in cosmic ray data. In that context, it is called the unidirectional differential intensity $[\mathrm{Sm} 85]$.

In problems of radiative transfer (i.e., photon transport), the photons are characterized by their frequency $\nu$ and velocity direction $\overrightarrow{\boldsymbol{n}}$, rather than their three-dimensional velocity vector (the magnitude of their velocity is, of course, always $c$, and is not a variable). The photon Boltzmann distribution function is then written $f_{R}(\overrightarrow{\boldsymbol{r}}, \overrightarrow{\boldsymbol{n}}, \nu, t)$ photons $\mathrm{cm}^{-3} \mathrm{~Hz}^{-1} \mathrm{sr}^{-1}$, and $f_{R}(\overrightarrow{\boldsymbol{r}}, \overrightarrow{\boldsymbol{n}}, \nu, t) d \Omega d \nu$ photons $/ \mathrm{cm}^{3}$ is the number density of photons with frequency in $d \nu$ about $\nu$, moving in direction $d \Omega$ about $\vec{n}$. The photon number flux is $c f_{R}(\overrightarrow{\boldsymbol{r}}, \overrightarrow{\boldsymbol{n}}, \vec{\nu}, t) d \Omega d \nu$ photons $\mathrm{cm}^{-2} \mathrm{sec}^{-1}$. The energy flux is often more useful than the number flux. As each photon has energy $h \nu$, the differential energy flux is

$$
I(\overrightarrow{\boldsymbol{r}}, \overrightarrow{\boldsymbol{n}}, \nu, t)=h v c f_{R}(\overrightarrow{\boldsymbol{r}}, \overrightarrow{\boldsymbol{n}}, \nu, t) \quad\left(\mathrm{erg} \mathrm{cm}^{-2} \mathrm{sec}^{-1} \mathrm{~Hz}^{-1} \mathrm{sr}^{-1}\right)
$$

and is known as the specific intensity [Ch60, Mi78]. It also contains the same information as $f$, but is more useful for formulating the equation of radiative transfer.

\subsubsection{Flux and Three-Dimensional Geometry}

The distribution function $f(\vec{r}, \vec{v}, t)$ or the differential flux $v f$ (or $J$ or $I$ ) specifies the most detailed information about a field of particles. Often such detail is not needed. Reduced fluxes, with less differential information, are defined for these purposes. They are usually obtained by integrating over velocity angles and/or magnitude.

In any application involving flux one must still specify the orientation of the unit area relative to the moving particles. Under various circumstances one

\footnotetext{
*Note that Weinberg and Wigner [We58] denote our $J=v f$ by $f$.
} 
has need for the omnidirectional flux, or the planar, directed(current)flux, or the scalar flux, according to the following considerations. Unfortunately, some articles report a particle flux without specifying whether it is omnidirectional or planar (they can differ by a factor of 2 or 4). Lack of specifying which flux is intended is often a source of confusion.

Fluxes of interest are broadly classified as (1) those particles crossing a fixed plane area or (2) omnidirectional. In type (1) the plane area is fixed, but the particles incident on it need not strike it normally. The particle field itself may, and usually does, contain particles moving in all directions.

Planar fluxes of type (1) may address particles coming from all directions but crossing a fixed plane area in only one Cartesian direction, say from $-z$ toward $+z$ across a unit area with normal parallel to the $z$ axis, counting only those particles whose $z$ component of velocity is positive. This is the flux of interest for studying, say, the flux of electrons striking a satellite surface and leading to spacecraft charging.

Alternatively in a planar flux, one may wish the net number of particles crossing a fixed area from $-z$ toward $+z$, counting as negative those crossing from $+z$ toward $-z$. This is the current of particles in the $+z$ direction.* This current is negative if more particles cross from $+z$ toward $-z$ than the other way. This flux is needed for electromagnetic applications. It is, for example, the electromagnetic current of an electron flow that has components flowing both toward $+z$ and toward $-z$.

Yet again, one may wish the total number of particles crossing the area in both directions, the scalar bidirectional flux, to be the absolute sum of those crossing from $-z$ toward $+z$ and those crossing from $+z$ toward $-z$. This flux is needed for the reaction rate in a thin foil immersed in a particle field, and is always a positive quantity.

\subsubsection{Planar Fluxes}

\subsection{Directed Planar Flux}

In a gas of particles with Boltzmann distribution function $f(\vec{v})$ and number density $n=\int f(\overrightarrow{\boldsymbol{v}}) d^{3} v$ particles $/ \mathrm{cm}^{3}$ (suppressing the $\overrightarrow{\boldsymbol{r}}, t$ variables), consider a plane circular disk of area $d A=1 \mathrm{~cm}^{2}$ at the origin, with normal parallel to $z$. Gas particles stream through $d A$. Figure 1.1 shows those coming from angle $\vartheta$.

The number of particles per $\mathrm{cm}^{2}$ per second that pass through $d A$ from one side, say from the left hemisphere $(z<0)$, toward the other side, is the directed, planar flux $F_{p}$ through $d A$. As the orientation of $d \vec{A}$ stays fixed during the integration over velocity directions, particles coming from angle $\vartheta$ face the

\footnotetext{
*Documentation of the code MCNP uses the term current to mean the absolute sum of particles crossing a surface in either direction, what we call the scalar bidirectional flux. This flux is never negative. The user may, however, request a tally of particles crossing only in one direction.
} 


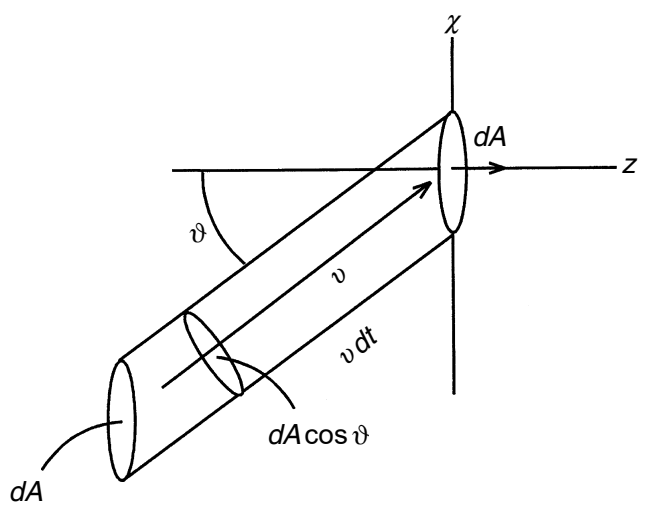

FIGURE 1.1 Particles streaming through fixed area element $d A$ from angle $\vartheta$.

smaller area $d A \cos \vartheta$ and contribute less to $F_{p}$ than those at $\vartheta=0$. (The $\cos \vartheta$ factor can as well be considered as arising from $v_{z}=v \cos \vartheta$, where $v=|\vec{v}|$ ). As usual, $F_{p}$ can be expressed as

$$
\begin{aligned}
F_{p} & =\frac{1}{d A} d \vec{A} \cdot \int f(\overrightarrow{\boldsymbol{v}}) \overrightarrow{\boldsymbol{v}} d^{3} \boldsymbol{v}=\int f(\overrightarrow{\boldsymbol{v}}) \boldsymbol{v}_{z} d^{3} \boldsymbol{v}=\int f(\overrightarrow{\boldsymbol{v}}) \boldsymbol{v} \cos \vartheta d^{3} \boldsymbol{v} \\
& =n(>0) \overline{\boldsymbol{v}_{z}(>0)}
\end{aligned}
$$

with the integral taken over only $v_{z}>0(\theta<\pi / 2)$. In Equation 1.1, $n(>0)$ is the density of particles with $v_{z}>0\left(=n / 2\right.$ in an isotropic gas), and $\overline{v_{z}(>0)}$ is the average $v_{z}$ of all particles with $v_{z}>0$. For example, if $d A$ is a fixed, small hole in the wall of a box containing a gas, $F_{p} d A$ is the rate at which molecules escape.

\subsection{Bidirectional Planar Flux and Current}

$F_{p}$ in Equation 1.1 was computed for particles passing from left to right, $F_{p}=F_{p}\left(v_{z}>0\right)$. One can as well compute it for those particles passing from right to left, obtaining $F_{p}\left(v_{z}<0\right)$. Their sum, $F_{\mathrm{bi}}^{\mathrm{scal}}=F_{p}\left(v_{z}>0\right)+F_{p}\left(v_{z}<0\right)$, is the scalar bidirectional flux, the total number of particles traversing a unit area per second from both directions. In a uniform, isotropic gas, $F_{p}\left(v_{z}>0\right)=F_{p}\left(v_{z}<0\right)$, and the scalar bidirectional flux is twice the planar flux in either direction.

The algebraic sum, $F_{\mathrm{bi}}^{\mathrm{net}}=F_{p}\left(v_{z}>0\right)-F_{p}\left(v_{z}<0\right)$, is the net number of particles crossing a unit area in one direction. It is simply the (net) current density of particles in the $z$ direction. In an isotropic gas, $F_{\mathrm{bi}}^{\text {net }}=0$.

$F_{\mathrm{bi}}^{\text {net }}$ is, for example, the flux determining the net charge transport across a surface in an electron gas. An example occurs when a planar beam of electrons is externally incident on the surface of the solid $(z=0) ; F_{\mathrm{bi}}^{\text {net }}(z)$ is the current passing through a surface at some fixed depth $z=d$ in the solid. 
Here, $F_{p}\left(z, v_{z}>0\right)$ is the flux of original electrons still moving forward at $z=d$, and $F_{p}\left(z, v_{z}<0\right)$ is the flux of electrons that have passed $z$ and been backscattered through the plane at $z=d$.

In neutron transport theory, for neutrons of a given energy $E$, the algebraic net $F_{\mathrm{bi}}^{\text {net }}$ is also called the neutron current and is commonly denoted [Be70] by $J(\vec{r}, E, t)$. Using $E$ and $\Omega$ instead of $\vec{v}$ for the independent variables,

$$
J_{z}(\overrightarrow{\boldsymbol{r}}, E, t)=\int f(\overrightarrow{\boldsymbol{r}}, E, \Omega, t) \boldsymbol{v}_{z} d \Omega=\int f(\overrightarrow{\boldsymbol{r}}, E, \Omega, t) v \cos \vartheta d \Omega=n(\overrightarrow{\boldsymbol{r}}, E, t) \overline{\boldsymbol{v}}_{z}
$$

where now the integral is taken over all $\vartheta(0<\vartheta<\pi) . n(\vec{r}, E, t)$ is the number density per unit energy interval, and $\overline{\boldsymbol{v}}_{z}$ is the average $z$ component of velocity, over all directions, of neutrons with energy $E$. The terminology neutron current is entrenched by usage, but it is actually the neutron spectral current density, because it is differential in energy (spectral), and is per unit area (density).* $J$ has units of, for example, particles/MeV/ $\mathrm{cm}^{2} / \mathrm{sec}$.

\subsection{Planar Flux for an Isotropic Gas}

In an isotropic gas, $f(\vec{v})=f(v)$, and with $v_{z}=v \cos \vartheta$, Equation 1.1 for the directed planar flux $F_{p}$ becomes

$$
\begin{aligned}
F_{p} & =\int_{v_{z}>0} f(v) v_{z} d^{3} v=\int f(v) v \cos \vartheta v^{2} d v d \Omega \\
& =\int_{0}^{\infty} d v f(v) v^{3} \int_{2 \pi} \cos \vartheta d \Omega \\
& =\frac{n}{2} \frac{\bar{v}}{2}
\end{aligned}
$$

where

$$
\overline{\boldsymbol{v}}=\frac{1}{n} \int f(\boldsymbol{v}) \boldsymbol{v} d^{3} \overrightarrow{\boldsymbol{v}}
$$

is the mean magnitude of the velocity over all particles (of density $n$ ), and $n=\int f(v) d^{3} v$ is the total number of particles per unit volume. In Equation 1.3 , one factor of $1 / 2$ arises because the density of particles with $v_{z}>0$ (i.e., $\cos \vartheta>0)$ is $n(>0)=n / 2$. The other is because of the factor $\cos \vartheta$ from $v_{z}$; the average $v_{z}$ over all particles with $v_{z}>0$ is $\bar{v} / 2$ :

\footnotetext{
*We note that the widely used Monte Carlo transport code MCNP, having its distant origins in nuclear reactor computations, has adopted the flux and current definitions and terminology of Bell and Glasstone [Be70].
} 


$$
\overline{v_{z}(>0)}=\frac{1}{n / 2} \int d v v^{3} f(v) \int_{0}^{2 \pi} d \varphi \int_{0}^{1} \mu d \mu=\frac{\bar{v}}{2}
$$

where $\mu \equiv \cos \vartheta$. Loosely stated, on $1 \mathrm{~cm}^{2}$ of a solid flat surface exposed to an isotropic gas on only one side, only $1 / 2$ of the particles are directed toward the surface, and of those the projected area, $\left(1 \mathrm{~cm}^{2}\right) \cdot \cos \theta$, reduces the number that hit the unit area by another factor $1 / 2$.

Reverting to a mathematical element of area $d A$ floating in an isotropic gas, the net directed flux through $d A$ vanishes. The total scalar, bidirectional flux is $F_{\mathrm{bi}}^{\mathrm{scal}}=2 F_{p}=n \overline{\boldsymbol{v}} / 2$, which is the total number of particles that pass through a fixed $d A$ in both directions.

\subsection{Planar Flux for a Plane Beam}

For a parallel, plane beam of particles of density $n$, velocity $v_{\mathrm{o}}$, the distribution function is

$$
f(\overrightarrow{\boldsymbol{v}})=n \delta\left(v_{x}\right) \delta\left(v_{y}\right) \delta\left(\boldsymbol{v}_{z}-\boldsymbol{v}_{0}\right) .
$$

When inserted in Equation 1.1 for the directed planar flux, this evaluates to the usual flux $n v_{\mathrm{o}}$ (particles $/ \mathrm{cm}^{2} / \mathrm{sec}$ ). If the beam has a spread in $\boldsymbol{v}_{z}, f(\overrightarrow{\boldsymbol{v}})=n \delta\left(\boldsymbol{v}_{x}\right) \delta\left(\boldsymbol{v}_{y}\right) g\left(\boldsymbol{v}_{z}\right)$, then the planar flux is $n \overline{\boldsymbol{v}}_{z}$.

In a gas of charged particles (usually electrons), the directed planar flux $F_{p}$ is the relevant flux for the electromagnetic charge transported across a fixed surface with particles incident from only one side. Hence, its alternate name current density. $F_{p}$ is, for example, the rate at which a unit surface on the exterior of a spacecraft is struck by ambient electrons (causing spacecraft charging). The bidirectional planar flux $F_{\text {bi }}^{\text {scal }}$ would be needed if particles are incident from both sides, as for example, would be the case for the charging of a large, thin solar panel.

\subsubsection{Omnidirectional Flux}

The omnidirectional flux $F_{\mathrm{om}}$ is the number of particles passing through $1 \mathrm{~cm}^{2}$ at the origin when the square $\mathrm{cm}$ is always directed toward the incoming particles. Thus, $\overrightarrow{d A}$ changes direction during the integration always to be parallel to the instantaneous $\vec{v}$. It is integrated over all velocity directions ( $4 \pi$ ster). Instead of Figure 1.1 we have Figure 1.2.

The number of particles passing through $d \vec{A}$ per second is $\int d^{3} v f(\vec{v}) \vec{v} \cdot d \vec{A}$. However, since $d \vec{A}$ is always parallel to $\vec{v}, \vec{v} \cdot d \vec{A}=v d A$, the number is $d A \int f(\vec{v}) v d^{3} v$. Thus, the expression for $F_{\text {om }}$ is

$$
F_{\mathrm{om}}=\int f(\overrightarrow{\boldsymbol{v}}) \boldsymbol{v} d^{3} \overrightarrow{\boldsymbol{v}}
$$



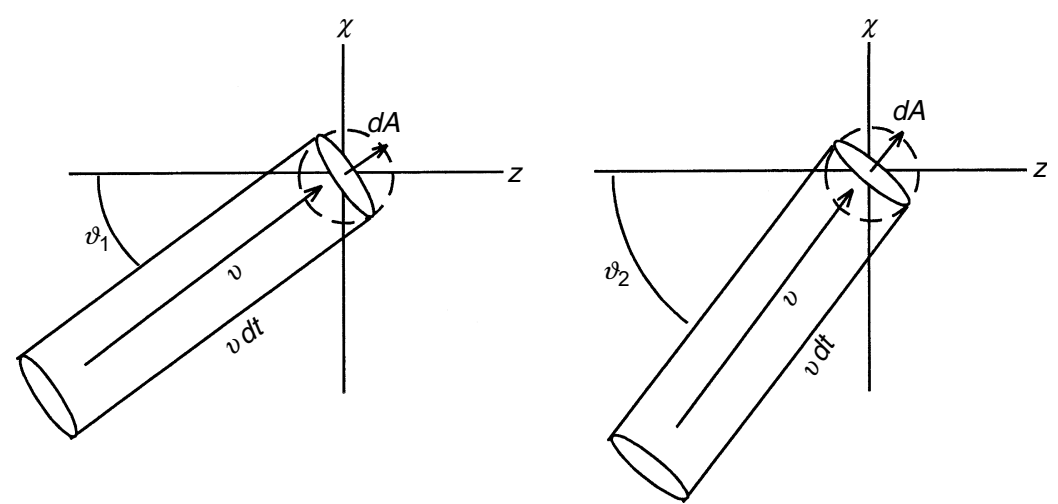

FIGURE 1.2 Particles streaming through area element always normal to velocity vector.

with the solid angle integral taken over $4 \pi$. The magnitude of velocity $v$ occurs in the integrand rather than $v_{z}=v \cos \theta$ as in Equation 1.1.

As $\vec{v}$ and $\overrightarrow{d A}$ sweep through all directions, the rim of $\overrightarrow{d A}$ marks out a sphere. As indicated in Figure 1.2, the omnidirectional flux is the number of particles passing into a sphere of unit projected area $\left(\pi r^{2}=1 \mathrm{~cm}^{2}\right)$.

The omnidirectional flux is important, and is the usually desired quantity in discussions of neutral particles (photons, neutrons) because it is the flux that addresses the common question: Suppose you have a small volume of material containing $N$ atoms $/ \mathrm{cm}^{3}$ buried in a gas of neutrons (or photons). How many collisions with $N$ do the neutrons undergo? The answer is $N \sigma F_{\text {om }}$ collisions per $\mathrm{cm}^{3} / \mathrm{sec}$, where $\sigma$ is the collision cross section.

The omnidirectional flux $F_{\text {om }}$ determines the reaction rate of the neutral particles with the material through which the particles traverse. By default, the flux computed by the Monte Carlo code MCNP, for example, is the omnidirectional flux. If material geometry blocks some particles from reaching the volume, the number of collisions will, of course, be less (see Section 1.5).

In neutron physics, the omnidirectional flux is sometimes expressed as a function of particle energy

$$
F_{\mathrm{om}}(\overrightarrow{\boldsymbol{r}}, E, t)=\int f(\overrightarrow{\boldsymbol{r}}, E, \Omega, t) v d \Omega
$$

and is the number of particles at $(\vec{r}, t)$ per unit energy at energy $E$ crossing a unit area per second. In neutron physics, it is called simply the total flux (Bell and Glasstone [Be70], p. 5), even though it is differential in energy; the total signifying all directions. Nomenclature is not universal, and the dimensions of some quantities of the same name differ according to displayed arguments; they may even use the same symbol. Weinberg and 
Wigner [We58], for example, use the term total flux, denoted by them as $\Phi$, to mean both $\Phi(\vec{r}, E, t)=F_{\mathrm{om}}(\overrightarrow{\boldsymbol{r}}, E, t)$ and also the energy integral of $F_{\mathrm{om}}(\overrightarrow{\boldsymbol{r}}, E, t), \Phi(\overrightarrow{\boldsymbol{r}}, t)=\int F_{\mathrm{om}}(\overrightarrow{\boldsymbol{r}}, E, t) d E=\int \Phi(\overrightarrow{\boldsymbol{r}}, E, t) d E$. In this case, the meaning and units of $\Phi$ are determined by the displayed arguments.

Words mean different things to different people. In discussions involving particle flux, current, and so on, it is prudent for writers who wish to be understood by those not already familiar with the field to specify at once their terminology for a quantity (e.g., total flux), its dimensions (e.g., particles per unit energy per unit area per unit time), and whether it is omnidirectional or planar; if planar, whether it is in only one direction across the plane, or both directions; if both, whether it is the absolute sum of both directions (called the bidirectional scalar flux here) or the algebraic sum (called current here). Failure to spell out these different possibilities has been the source of much confusion.

\subsection{Omnidirectional Flux for an Isotropic Gas}

In the case of an isotropic gas, $f(\vec{v})=f(v)$, and Equation 1.7 for the omnidirectional flux becomes

$$
F_{\mathrm{om}}=\int f(\overrightarrow{\boldsymbol{v}}) \boldsymbol{v} d^{3} \boldsymbol{v}=\int f(\boldsymbol{v}) \boldsymbol{v} d^{3} \boldsymbol{v}=n \overline{\boldsymbol{v}}
$$

with $\vec{v}$ given by Equation 1.4. As stated, it is the total number of particles passing per second through a unit area always normal to the particle velocity (i.e., through a sphere of radius $r$ with $\pi r^{2}=1 \mathrm{~cm}^{2}$ ). It is four times the oneway directed planar flux $F_{p}$ (Equation 1.3). And $F_{\text {om }}$ is twice the bidirectional scalar planar flux $F_{\mathrm{bi}}^{\text {scal }}$.

\subsection{Omnidirectional Flux for a Plane Beam}

The particle flux in a plane, parallel beam is elementary and hardly needs discussion; one need never introduce its omnidirectional flux. However, the omnidirectional flux of such a beam is still a meaningful concept. With the planar beam distribution function $f$ from Equation 1.6 inserted in Equation 1.7, the integrals are trivial, and one finds that the omnidirectional flux for a parallel beam is $F_{\text {om }}=n v_{0}$, the same as the directed planar flux $F_{p}$ and the common expression. All the particles $/ \mathrm{cm}^{2}$ in the beam enter a sphere of radius $r$ with $\pi r^{2}=1 \mathrm{~cm}^{2}$. The omnidirectional flux of a plane beam is the same as its ordinary planar flux.

\subsection{REACTION RATES IN PLANAR AND OMNIDIRECTIONAL FLUXES}

Without belaboring the issue, we discuss a point that can lead to confusion. That is the relation between the particle flux and the reaction rate or dose rate of particles striking a target. 


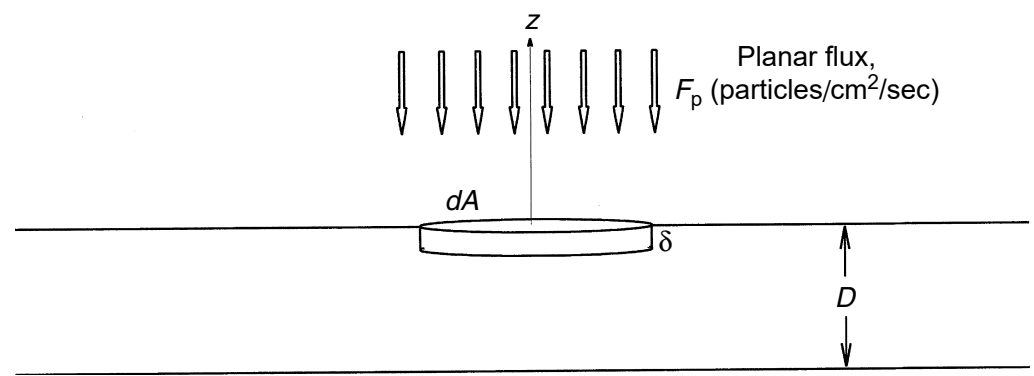

FIGURE 1.3 Planar flux $F_{\mathrm{p}}$ (particles $/ \mathrm{cm}^{2} / \mathrm{sec}$ ) of a parallel beam of particles impinging on one side of a surface. $F_{\mathrm{p}} d A$ particles/sec pass through area element $d A$ and volume element $d V=\delta d A$.

If a parallel beam of particles with flux $F_{\mathrm{p}}$ (particles $/ \mathrm{cm}^{2} / \mathrm{sec}$ ) is normally incident on a thick target, the number that strikes area $d A$ is $F_{\mathrm{p}} d A$ per second. Each particle can penetrate at least a small depth $\delta$, and so pass through the small volume $d V=\delta d A$ (Figure 1.3). Such a parallel beam may be a beam of gammas from a distant point source, or an electron beam impinging on the target.

Let $N$ be the atom number density in the target, and let $\sigma$ be the cross section for a reaction with the incident particles. The number of reactions per second per unit volume in the target is $R=N \sigma F_{\mathrm{p}}$, and the number of reactions per second in the volume is $R d V=N \sigma F_{\mathrm{p}} \delta d A$.

However, if the particle field surrounding the target is not a parallel beam directed normally at the surface but is an essentially isotropic gas of flux $F_{\text {om }}$ (particles $/ \mathrm{cm}^{2} / \mathrm{sec}$ ), then particles strike $d A$ from all directions, as

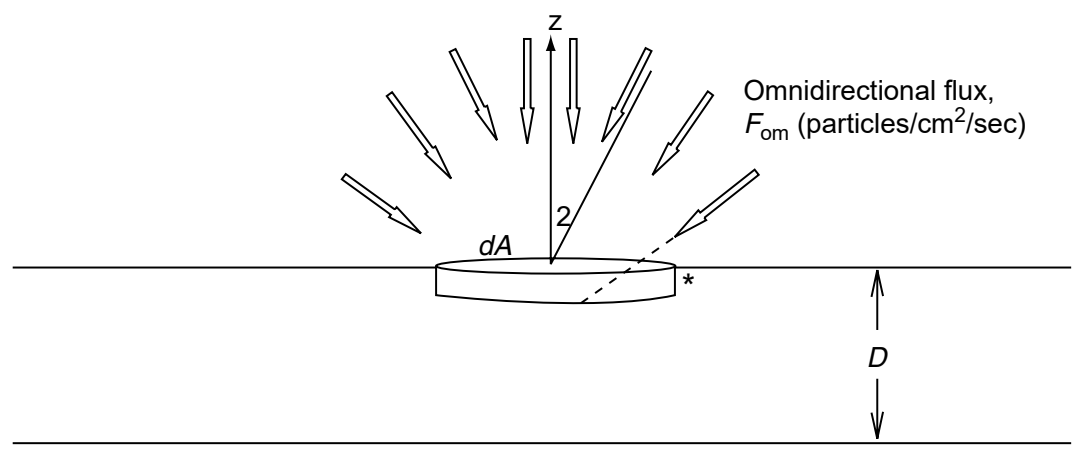

FIGURE 1.4 Omnidirectional flux $F_{\text {om }}$ (particles $/ \mathrm{cm}^{2} / \mathrm{sec}$ ) of a gas of particles impinging on one side of a material slab of thickness $D$. The flux is imagined nonzero in $4 \pi$ steradians in the space surrounding the slab, but for $D$ thick compared with a particle mean free path only those incident from above can reach volume element $d V=\delta d A$. 
in Figure 1.4. Such an omnidirectional flux may be that of a neutron field near a reactor, or environmental background neutrons or photons, or the flux of gammas in an experimental chamber in which there is much scattering.

Then, if target thickness $D$ is large compared with the particle mfp or range, only particles in the upper hemisphere with a downward velocity can strike $d A$; their flux is $F_{\mathrm{om}} / 2$. Field particles below the target are blocked by the target and cannot reach the surface of interest. The number of reactions per second in unit volume within $d V$ is $N \sigma\left(F_{\text {om }} / 2\right)$. Particles arriving from $\vartheta$ off axis see a smaller area $d A \cos \vartheta$, but experience a longer path $\delta / \cos \vartheta$ and so $\vartheta$ drops out.

On the other hand, if $D$ is thin compared with an mfp, particles may strike $d V$ from both hemispheres, and the number of reactions in one second in unit volume within $d V$ is $N \sigma F_{\text {om }}$. Recall that $F_{\text {om }}$ is defined with a $4 \pi$ solid angle integral (Equation 1.7 or Equation 1.8). That is, the nature of the particle field (parallel or omnidirectional) and the target properties (thickness $D$ relative to particle mfp or range) introduce a factor of two ambiguity in the relation between the particle flux and the reaction rate.

It is not uncommon for one to specify a desired reaction rate $R$, or a desired total number of reactions $R \Delta t$ occurring during a time interval $\Delta t$ and ask for the particle flux or fluence that will produce that number. If the target is thick relative to the particle $\mathrm{mfp}$, the flux sought is $F_{\mathrm{p}}=R / N \sigma$ if the particles are in a parallel beam, but is $F_{\text {om }}=2 R / N \sigma$ if the particles are in an isotropic gas. The flux or fluence needed to produce the specified number of reactions will differ by a factor of two according as the particles are in a parallel beam or an omnidirectional gas.

On the other hand, if the material thickness $D$ is thin compared with an mfp, all particles in the gas will strike volume element $d V$, and the flux needed in either an omnidirectional gas $\left(F_{\mathrm{om}}\right)$ or a parallel beam $\left(F_{\mathrm{p}}\right)$ is $F_{\mathrm{p}}=F_{\mathrm{om}}=R / N \sigma$. In this case, there is no ambiguity.

A common instance of this potential confusion arises when one is given a specified dose or dose rate and one wishes to realize it with a flux of particles. The dose (or kerma) deposited by a particle arises from the energy it deposits per centimeter of track length. On an average, each particle will deposit some energy $w$ (in, say, $\mathrm{MeV}$ ) per collision, or energy $w N \sigma$ $(\mathrm{MeV} / \mathrm{cm})$ per unit path length, or $W=w N \sigma / \rho\left(\mathrm{MeV} \mathrm{cm}^{2} / \mathrm{g}\right)$ per unit mass traversed, where $\rho$ is material mass density. For a photon or neutron, $w$ is the mean energy lost per collision; for a charged particle, $W$ is the mean stopping power. In terms of the collision rate $R, R \Delta t / \rho$ collisions occur per unit mass, and a dose $q=w R \Delta t / \rho(\mathrm{MeV} / \mathrm{g})$ is deposited.

Given a dose $q$ what fluence is required to produce it? If one works with a parallel beam of particles, one seeks their flux $F_{\mathrm{p}}$ and exposure duration $\Delta t$. Recall that in $F_{\mathrm{p}}$ the number of reactions $/ \mathrm{cm}^{3} / \mathrm{sec}$ is $R=N \sigma F_{\mathrm{p}}$. Then, the dose $q=w R \Delta t / \rho=w N \sigma F_{\mathrm{p}} \Delta t / \rho$, and the required fluence is 


$$
F_{\mathrm{p}} \Delta t=q \rho / w N \sigma=q / W \quad\left(\text { particles } / \mathrm{cm}^{2}, \text { parallel beam }\right) .
$$

If the available particle field, however, is a gas, one has an omnidirectional flux. Following the arguments given here, the required (assumed isotropic) fluence is

$F_{\mathrm{om}} \Delta t=2 q \rho / w N \sigma=2 q / W$ (particles $/ \mathrm{cm}^{2}$, omnidirectional flux, thick target)

if the exposed surface on which the dose desired is accessible to particles only from the upper hemisphere. If the surface mass element is accessible to all particles (as would be the case, for example, for neutrons or energetic gammas on a thin metal slab), the omnidirectional fluence needed to deposit a dose $q$ is

$$
F_{\text {om }} \Delta t=q \rho / w N \sigma=q / W \text { (particles } / \mathrm{cm}^{2}, \text { omnidirectional flux, thin target) }
$$

the same as the planar fluence. If the dose is to be realized by a photon gas with a distribution of energies, one must distinguish between those photons that are too soft to penetrate to the dosed surface, and those photons that are energetic enough to do so.

A common example illustrates the former case, that in which the exposed mass element is accessible only to particles (assumed isotropic) coming from one hemisphere. That geometry occurs for a thick body exposed to background gamma rays, which are supposed to be roughly isotropic. Such a background may be that in a reactor room, or that of the natural outdoor background. One may specify that the body surface must receive no more than a certain dose $q$ in a certain time span (e.g., the human body with radiological health exposure limits, or an electronic component with a maximum dose before performance failure). One can ask for the corresponding background photon fluence that delivers the dose $q$. Similarly, for laboratory photon sources, one can ask for the fluence of monoenergetic photons that produce the dose $q$.

Since there is a spectrum in the natural background, and as $w$ is a function of photon energy, it is easier to discuss the monoenergetic laboratory photon source. If that source is a plane parallel beam when it impinges on the target mass element, the planar fluence required to produce dose $q$ is, as before, $F_{\mathrm{p}} \Delta t=q \rho / w N \sigma$. Here, $w$ is the energy deposited per collision of cross section $\sigma$. If the source is isotropic, the fluence required to produce dose $q$ is $F_{\mathrm{om}} \Delta t=2 q \rho / w N \sigma$.

If one chooses a standard $\mathrm{Cs}^{137}$ source $(662 \mathrm{keV})$, the dominant collision with low $Z$ material is Compton scattering, and $w \approx 300 \mathrm{keV}$. The Compton cross section is $N \sigma / \rho \approx 0.07 \mathrm{~cm}^{2} / \mathrm{g}$. A dose of $q=1 \mathrm{rad}(=100 \mathrm{erg} /$ $g=6.24 \times 10^{7} \mathrm{MeV} / \mathrm{g}$ ) then requires a normally incident planar fluence of $F_{\mathrm{p}} \Delta t=q \rho / w N \sigma=3 \times 10^{9}$ photons $/ \mathrm{cm}^{2}$. However, if the photons were nearly isotropic when striking the surface, a $4 \pi$ omnidirectional fluence of 
$F_{\text {om }}=6 \times 10^{9}$ photons $/ \mathrm{cm}^{2}$ would be needed when photons cannot penetrate from the back of the body to the mass element in question. The same is true of the natural background or reactor room photons when weighted with their energy spectrum. This difference of a factor of two between the required flux of a parallel beam and that of a nearly isotropic particle field for thick targets is a common source of confusion. One must specify whether the particles comprising the fluence are in a parallel beam or a (nearly isotropic) gas, and if a gas, whether all particles can reach the volume in question.

\subsection{ENERGY SPECTRUM AND SPECTRAL FLUENCE}

As stated, one is often interested not in the instantaneous velocity distribution, but in the number of particles that pass an area in a specified time. Therefore, a flux or fluence differential in energy raises a distinction between the energy spectrum of particles and the energy spectrum of those that pass through an area in a specified time. The former is the energy spectrum per se of the field of particles, the latter is the energy spectrum of the fluence. The two are different, and their difference is another source of confusion, especially in neutron physics, owing partly to imprecise language. Some clarifying remarks are in order.

A particle field can be thought of as a gas or a beam of particles, albeit perhaps with a highly non-Maxwellian energy spectrum. The energy spectrum, $g(E)$, of a collection of particles in space is a point function of space and time, $g(E, \overrightarrow{\boldsymbol{r}}, t)$. At time $t$ the number of particles in an infinitesimal volume $d^{3} r$ with energy in interval $(E, E+d E)$ is $d n=g(E, \vec{r}, t) d^{3} r d E . g$ has dimensions of, say, particles per $\mathrm{cm}^{3}$ per $\mathrm{MeV}$.

Often, however, one is more interested in the energy spectrum $G(E)$ of all those particles that pass through a small area $d A$ in a specified time, say $d t$, or $1 \mathrm{sec}$. This quantity, with dimensions of particles per $\mathrm{cm}^{2}$ per $\mathrm{MeV}$, is the energy spectrum of the fluence.

The number of particles per unit volume in the energy interval $(E, E+d E)$ is $g d E$. Of these, the number that pass through an area element $d A$, chosen always to be normal to the particle (nonrelativistic) velocity $v=\sqrt{ }(2 E / m)$, in time $d t$ is $g d E v d t d A$. Per unit area, this is

$$
g v d E d t\left(\text { particles } / \mathrm{cm}^{2}\right) \text {, }
$$

a fluence. Per second it is

$$
\left.g v d E \text { (particles } / \mathrm{cm}^{2} / \mathrm{sec}\right) \text {, }
$$

a flux. Whereas the simple number of particles at one instant of time at a point in space in the energy interval $(E, E+d E)$ is proportional to $g$, the flux or fluence of particles that pass through a unit area is proportional to $v g$. 
Now, expressing this fluence or flux as per unit energy, as a function of energy, one has

$$
G=g v d t\left(\text { particles } / \mathrm{cm}^{2} / \mathrm{MeV}\right)
$$

with $g$ evaluated at the point where one accumulates the flux to obtain a fluence. $G$ is a spectral fluence, or a differential (in energy) fluence, and

$$
g v\left(\text { particles } / \mathrm{cm}^{2} / \mathrm{MeV} / \mathrm{sec}\right),
$$

a spectral flux, or a differential flux. Although the energy spectrum per se is $g(E, \vec{r}, t)$, the energy spectrum of particles that pass through a given area in time interval $\Delta t$ is $G=v g \Delta t$. The energy spectrum of the gas is not the same as the energy spectrum of the fluence accumulated over a finite time, simply because in that time, more high-energy particles are collected.

As an illustration of this distinction, chosen to clarify a common point of confusion, consider the energy spectrum $g$ of a gas of particles, with the Boltzmann distribution function $f(\overrightarrow{\boldsymbol{r}}, \overrightarrow{\boldsymbol{v}}, t)$. The particle distribution in velocity magnitude is proportional to $v^{2} f$. Then, using $E=\frac{1}{2} m v^{2}$ and $d E=m v d v$, the particle energy distribution $g$ is proportional to $v f$ or $\sqrt{ } E f$.

The Maxwellian distribution is $f \propto \exp (-E / T)$, with temperature $T$ in energy units. For energies smaller than $T, f$ is independent of $E$, and the low-energy Maxwellian energy spectrum $g \sim \sqrt{E} \cdot f$ is proportional to $\sqrt{E}$. Accordingly, the low-energy spectral fluence $G(E)$ would be proportional to $v \sqrt{E}$, which is simply proportional to $E$.

Unfortunately, nomenclature in common use is not so precise. Once energetic neutrons have scattered many times through shielding material, they have been downscattered enough to become largely thermal. Near and below thermal energies, they are Maxwellian. Figure 1.5 shows a neutron energy spectrum after moderation and thermalization, computed by MCNP. On this log-log plot, one sees that below the thermal peak the spectrum is proportional to $E$. One may suspect an error, as the spectrum should be Maxwellian and proportional to $\sqrt{E}$. In fact the plotted quantity, referred to as the energy spectrum, is actually the spectral fluence $G$. The spectral fluence is the more useful quantity, and is the one commonly computed. Documents often present what is called the energy spectrum, but which is in fact the spectral fluence $G=v g \Delta t$. The time span $\Delta t$ merely sets the normalization of $G$. As $G$ is often separately normalized (say, to unit integral), it is not necessary to specify $\Delta t$. 


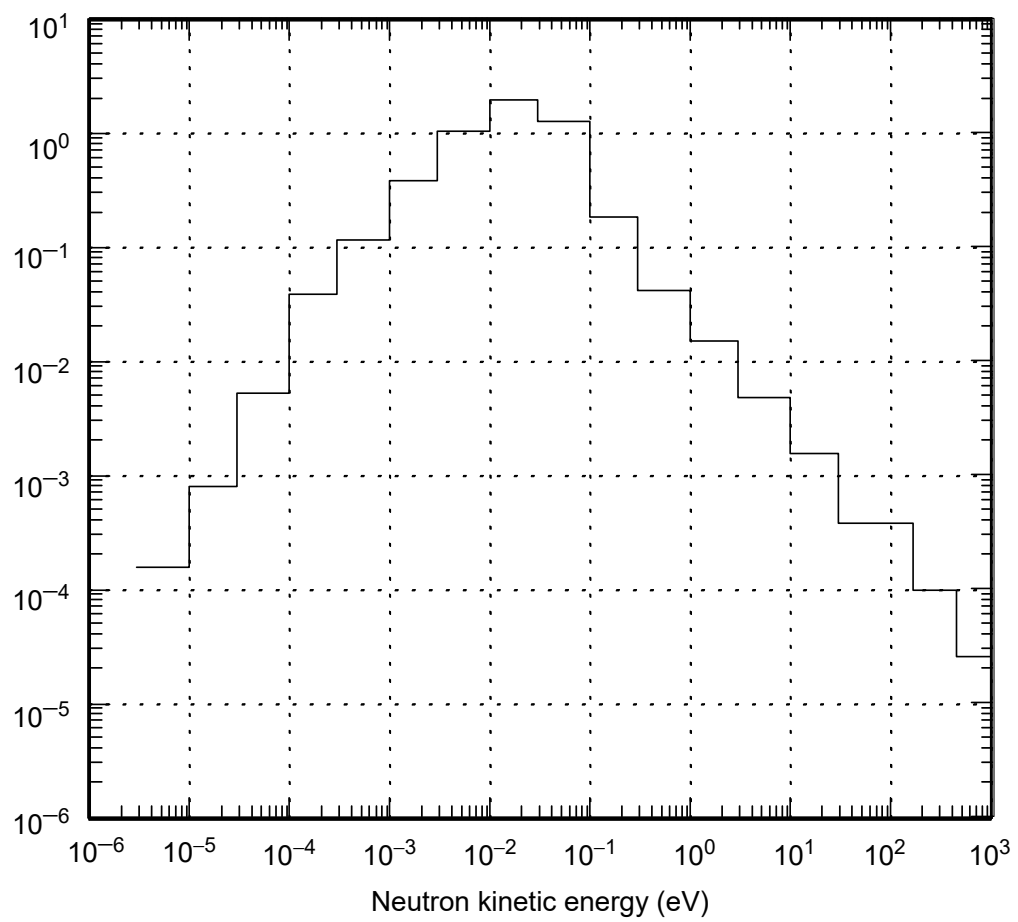

FIGURE 1.5 Low-energy part of neutron spectrum computed by MCNP at the center of a $50-\mathrm{kg}$ mass when energetic $(\sim \mathrm{MeV})$ neurons are externally incident. Below the thermal peak at $0.02 \mathrm{eV}$, the spectrum is more nearly proportional to $E$ than to $v \sqrt{E}$. The quantity computed is actually the spectral fluence.

\section{REFERENCES}

[Be70] Bell, G.I. and Glasstone, S., Nuclear Reactor Theory, Van Nostrand Reinhold, 1970.

[Ch60] Chandrasekhar, S., Radiative Transfer, Dover Publications, 1960.

[He75] Henry, A.F., Nuclear Reactor Analysis, MIT Press, 1975.

[ICRU69] International Commission on Radiation Units and Measurements, Neutron Fluence, Neutron Spectra and Kerma, ICRU Report 13, September 15, 1969.

[Mi78] Mihalas, D., Stellar Atmospheres, W.H. Freeman, 1978.

[Pa86] Pais, A., Inward Bound, Oxford University Press, 1986.

[PDG04] Bichsel, H., Groom, D.E., and Klein, S.R., Passage of particles through matter, in "Review of Particle Physics," Particle Data Group, Phys. Lett. B. 592, 242, 2004 [http://pdg.lbl.gov]. 
[Sm85] Smart, D.F., and Shea, M.A., Galactic cosmic radiation and solar energetic particles, Chapter 6 in Jursa, A.S., Ed., Handbook of Geophysics and the Space Environment, Air Force Geophysics Laboratory, 1985.

[We58] Weinberg, A.M., and Wigner, E.P., The Physical Theory of Neutron Chain Reactors, University of Chicago Press, 1958.

[Wo85] Wolfe, W.L. and Zissis, G.J., Eds., The Infrared Handbook, Revised Edition, Infrared Information Analysis Center, Environmental Research Institute of Michigan, 1985.

[XRD01] X-Ray Data Booklet, 15 authors, Center for X-Ray Optics Advanced Light Source, Lawrence Berkeley National Laboratory. 2nd edn., January 2001. [http://xdb.lbl.gov]. 


\section{Photons}

DOI: $10.1201 / 9781420012378-2$

Circumstances arise in which one has need to study the passage through matter of photons with wavelength $\lambda$ from as long as hundreds of kilometers or more (radio waves, photon energies $\lesssim 10^{-11} \mathrm{eV}$ ) to as short as the size of a nucleus ( $\gamma$ rays, energies $\gtrsim 100 \mathrm{MeV}$ ). Not surprisingly, over this 19 or more orders of magnitude in photon energy, the physics of the interactions between photon and matter varies widely. Somewhat surprisingly, only about four basic processes control the entire span of energies. At long wavelengths, photons interact with bulk matter as a whole; a classical description parameterizing the matter with a conductivity, dielectric constant, and magnetic permeability applies. As the wavelength decreases, individual photon-atom interactions dominate and photoelectric absorption, Compton scattering, and pair production become successively the dominant processes. Pair production remains the dominant process for all photon energies above about $100 \mathrm{MeV}$.

For interactions with matter in bulk, the primary focus of the subject matter in this book, a first distinction can be made based on the photon wavelength compared with the size of an atom. All atoms are of the order one Angstrom $\left(1 \AA=10^{-8} \mathrm{~cm}\right)$ in radius, and the distance $d$ between atomic planes in (ordered) solids is of the order of a few Angstroms. In the interaction with condensed matter, this leads to a basic difference between the cases in which photon wavelength is much less than or much greater than about one Angstrom. The distinction is important, particularly at long wavelengths, because most atomic cross-section databases, such as those used to construct graphs in this book, are the cross sections for photons interacting with free, isolated atoms at a very low temperature, that is, neglecting thermal effects and changes in atomic structure due to interaction with neighboring atoms.

\subsection{LONG WAVELENGTHS}

When $\lambda$ is longer than $d$ (photon energies roughly less than a few $\mathrm{keV}$ ), the photon interacts coherently with many atoms and with many atomic planes. In ordered solids, such as crystals, phase coherence is preserved, and gives rise to interference, which reflects the lattice structure. However, in liquids or amorphous solids phase coherence is lost because of the random positions of the scattering atoms. With wavelengths that are longer than the target (atom) size, the photon is not fruitfully thought of as a particle, so far as its 
interaction with the material target is concerned. Instead, the wave nature of the photon dominates its behavior, and the long-wavelength photon appears to be propagating through a continuous medium. Classical electromagnetics then allows the target medium to be characterized with electromagnetic parameters different from vacuum (dielectric constant $\varepsilon$, magnetic permeability $\mu$, and conductivity $\sigma$ ). The transmission can be treated by a conventional (complex) index of refraction $n=n_{r}+i n_{i}$ as a function of photon energy $E=h v$ or of wavelength $\lambda=c / v$. The real and imaginary parts of the index of refraction can vary widely with the wavelength, because of the resonances arising from atomic or molecular levels which may be comparable with the photon energy.

In some plasma or astrophysical applications, and when the gas is monoatomic rather than molecular, the material density can be far below that of conventional solids. In such cases, a photon interacts independently with each atom as it traverses the medium, and individual photon-atom cross sections are the appropriate parameters for describing the passage of photons through this gaseous matter.

Likewise, in diatomic or polyatomic gases, a restriction similar to that applying to condensed matter holds. At a relatively long wavelength $(\lambda \gtrsim \mathrm{a}$ few Angstroms, $E \lesssim$ a few $\mathrm{keV}$ ), the photon interacts with the molecule as a whole, not with its individual constituent atoms. The photon-molecule scattering cross section is not the incoherent sum of free atom cross sections. Molecular rotational and vibrational modes, and molecular electronic levels, are completely absent in free atom cross sections, and absorption edges or ionization potentials are changed in the molecule. For example, the ionization potential of the $\mathrm{H}$ atom is $13.598 \mathrm{eV}$, and that of atomic $\mathrm{O}$ is $13.618 \mathrm{eV}$, whereas that of an isolated $\mathrm{H}_{2} \mathrm{O}$ molecule is only $12.61 \mathrm{eV}$. For understanding the propagation of long-wavelength photons through polyatomic gases, one needs the correct photon-molecule cross sections. Although these can be computed approximately, they are best obtained from experimental measurements.

The fact that low-energy photons do not interact with solids, liquids, and polyatomic gases in the same way as they do with free, isolated atoms is no surprise and is well appreciated. A quantitative comparison to demonstrate the actual differences as a function of energy is worthwhile. One can see at what energies the use of free atom cross sections becomes inaccurate. Below these energies, more accurate photon propagation models or, better yet, actual data on photon attenuation, must be used.

\subsubsection{Limits of Applicability of Free Atom Cross Sections in Condensed Media at Long Wavelengths}

In condensed matter, atoms are closely packed so that their atomic orbitals and energy states are distorted from their free atom configurations by the effects of neighboring atoms. In metals, the distortion is so great that the outer 
electrons normally bound in an isolated atom become unbound and free to move as conduction electrons throughout the solid. In $\mathrm{Al}$, for example, the three $M$-shell electrons of the isolated atom become the free conduction electrons in the solid.

The actual scattering cross section from each atom therefore differs from that when the atom is isolated. In addition, photons may interact with solids and be scattered or absorbed by nonatomic processes altogether, such as phonon excitation or plasmon excitation [Ly85, Mi85]. These facts limit the applicability of photon-free atom cross sections to condensed media. These limits can be demonstrated by comparing calculations based on free atom cross sections with experimental data on the passage of relatively longwavelength photons through solids and liquids.

A point of comparison is usefully made via the measured total photon attenuation through solids or liquids at room temperature. Experimental attenuation is obtained from tabulated indices of refraction. With this, one can compare the attenuation that would be predicted using photon-free atom scattering.

Experimental real and imaginary parts of the index of refraction, $n_{r}$ and $n_{i}$ respectively, in many materials as a function of wavelength, are tabulated in [Pa98]. Energy and wavelength are related, of course, by

$$
E(\mathrm{eV})=h \nu=h c / \lambda=12398 / \lambda(\AA)
$$

The attenuation is obtained from the index of refraction as follows.

The electric field of a plane electromagnetic wave of vacuum wavelength $\lambda$ and wavenumber $k=2 \pi / \lambda$ behaves as

$$
E=E_{\mathrm{o}} e^{i n k x}=E_{\mathrm{o}} e^{i n_{r} k x} e^{-n_{i} k x}
$$

as it propagates through the material with index of refraction $n=n_{r}+i n_{i}$ in the $+x$ direction. Its amplitude decays exponentially with depth $x$ as $\exp \left(-n_{i} k x\right)$, and its intensity, proportional to $E^{2}$, decays as $\exp \left(-2 n_{i} k x\right)$. These are the amplitude and intensity of the remaining propagating wave. Its energy has been lost to nonconservative processes, parameterized by $n_{i}$, which represents a possible electrical conductivity or imaginary part of the dielectric constant $\varepsilon=n^{2}$.

It is common to introduce the parameter

$$
\alpha=2 n_{i} k=4 \pi n_{i} / \lambda
$$

so that the intensity-depth dependence may be written $\exp (-\alpha x) . \alpha$ has the dimensions of an inverse length, and is the attenuation coefficient. Its inverse, $1 / \alpha$, is the attenuation length, the distance at which the intensity drops to $1 / e$ of its initial value. From experimental data on $n_{i}$, one constructs $\alpha$ via Equation 2.2. 
On the other hand, in the free atom picture, we consider a photon flux of intensity $I$ (say, ergs $/ \mathrm{cm}^{2} / \mathrm{sec}$ ) passing through a tenuous material where photon-free atom cross sections are applicable. Let $N$ be the density of scattering atoms, and $\sigma$ the total scattering cross section. $N \sigma\left(\mathrm{cm}^{-1}\right)$ is the scattering cross section per unit volume. In a distance $\mathrm{d} x$, the unscattered intensity of this photon beam, $I$, is reduced by

$$
\mathrm{d} I=-I N \sigma \mathrm{d} x
$$

and so the intensity at depth $x$ is

$$
I(x)=I_{0} \exp (-N \sigma x)
$$

where $I_{0}$ is the original intensity. Then $1 / N \sigma$ is the distance at which the intensity drops to $1 / e$ of its initial value. This $N \sigma$, where $\sigma$ is the photon-free atom total cross section, may be compared with the $\alpha$ (Equation 2.2), determined from the experimental index of refraction. They will agree to the extent that photon-free atom cross sections are applicable.

We show two examples of this comparison, in aluminum and in water. First, we consider Al.

\subsubsection{Long-Wavelength Photon Attenuation in Metallic Aluminum}

The upper graph in Figure 2.1 shows $n_{i}$ vs. $E=h \nu=h c / \lambda$, constructed from measurements of $n_{i}$ vs. $\lambda$ or the imaginary part of $\varepsilon=n^{2}$ vs. $\lambda$ presented by Smith et al. [Sm85]. The Al $K$ edge at $1.56 \mathrm{keV}$ is evident. From $n_{i}$ we construct $\alpha$ (Equation 2.2), which is shown in the lower graph (solid line).

This $\alpha$ is to be compared with $N \sigma . \sigma$ is the photon-free Al atom total cross section. For it, we use the value tabulated by the Lawrence Livermore National Laboratory (LLNL) in the Evaluated Photon Data Library (EPDL97) database (which extends from $1 \mathrm{eV}$ to $100 \mathrm{GeV}$ ). The quantity $N \sigma$ is plotted as the dashed line in the same graph. If the photon-free atom cross sections were applicable to solid aluminum, the two curves should agree. In fact, they agree only above about $500 \mathrm{eV}$. Below this energy, and especially below about $50 \mathrm{eV}$, they differ by up to an order of magnitude. (Cross sections below $100 \mathrm{eV}$ compiled in EPDL97 are not to be believed with great accuracy. See discussion in Section 2.12. Nevertheless, the point to be made here is clear.)

The free atom $\mathrm{Al} L$ edges $\left(L_{1}=119.05 \mathrm{eV}, L_{2}=81.2 \mathrm{eV}, L_{3}=80.7 \mathrm{eV}\right)$ are seen in the dashed line. In the data (solid line) they are distorted, and the lowest $L$ edge has been downshifted about $8 \mathrm{eV}$ to approximately $72.6 \mathrm{eV}$. (The $\mathrm{Al} M_{1}$ edge is at about $10.16 \mathrm{eV}$.)

Below about $15 \mathrm{eV}$, the actual absorption is nearly an order of magnitude greater than that implied by free atom cross sections. The reason has to do with $\mathrm{Al}$ valence electrons. 

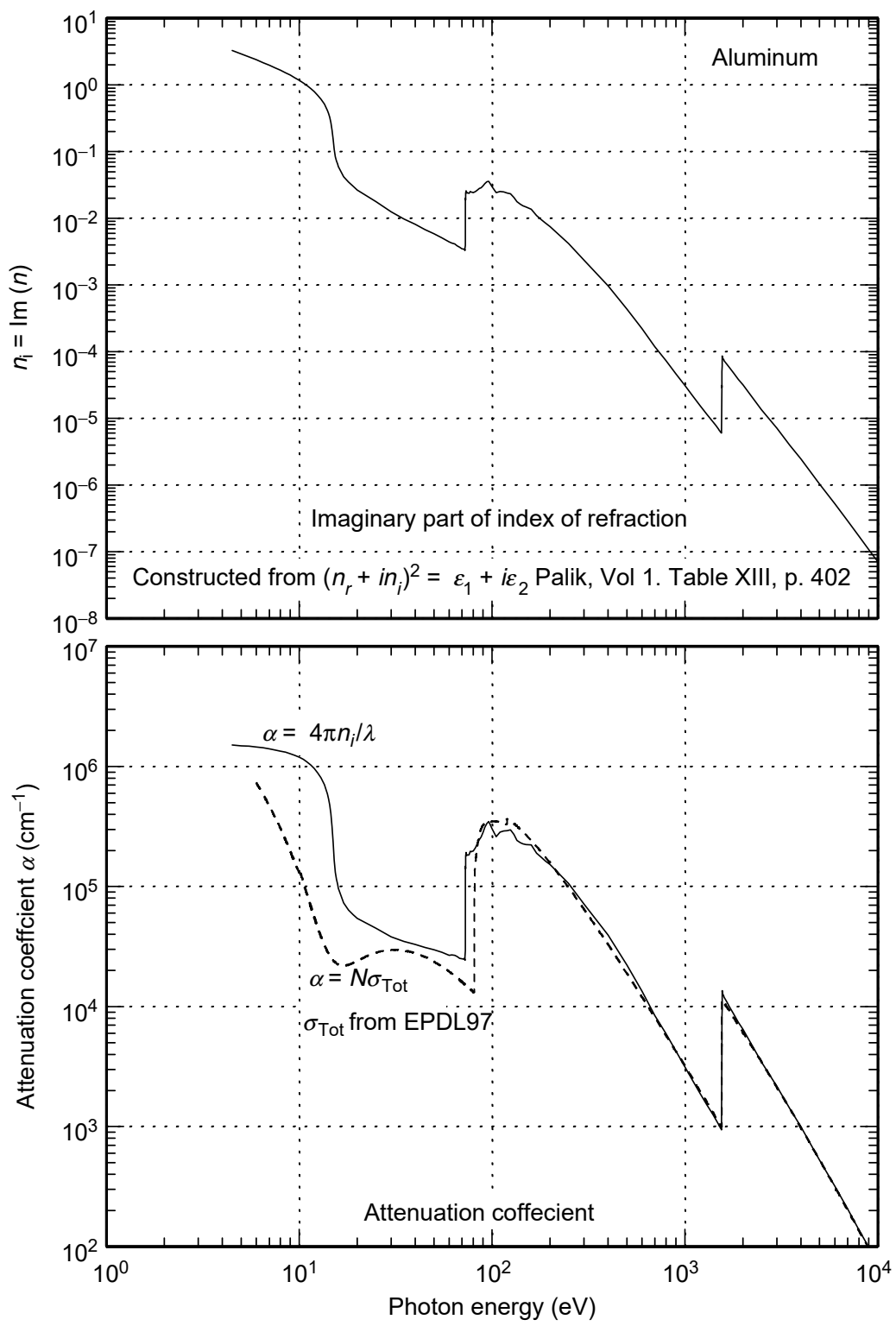

FIGURE 2.1 Comparison of $\mathrm{Al}$ attenuation data [Pa98] with that expected from free atom cross sections.

In metallic Al of mass density $\rho=2.698 \mathrm{~g} / \mathrm{cm}^{3}$, the atomic number density is

$$
N=N_{\mathrm{A}} \rho / A=6.022 \times 10^{22} \text { atoms } / \mathrm{cm}^{3}
$$


where

$$
N_{\mathrm{A}}=6.022142 \times 10^{23} \text { per mol }
$$

is Avogadro's number, and $A=26.982$ is the atomic weight. Al releases three valence electrons per atom, which are the free electrons that are responsible for its electrical conductivity. Thus, the free electron density in metallic $\mathrm{Al}$ is $N_{e}=1.807 \times 10^{23} \mathrm{elec} / \mathrm{cm}^{3}$. The plasma frequency of these electrons is $\omega_{p}=\left(4 \pi e^{2} N_{e} / m\right)^{1 / 2}=2.398 \times 10^{16} \mathrm{rad} / \mathrm{sec}$, or $f_{p}=\omega_{p} / 2 \pi=3.817 \times 10^{15} \mathrm{~Hz}$.

A collisionless plasma is opaque to electromagnetic radiation of frequency less than the plasma frequency. For $\mathrm{Al}$, the corresponding photon energy is $E=h f_{p}=15.78 \mathrm{eV}$. Photons of lesser energy cannot propagate in metallic Al. The actual attenuation is not infinite because the electrons are not truly free; they suffer collisions with the background ions, which permit some propagation of the incident wave. The collisions result in only finite attenuation, as shown in the data, in which the solid line rises from about $10^{5}$ to $10^{6} \mathrm{~cm}^{-1}$ suddenly as $E$ drops below $15.78 \mathrm{eV}$. No comparable sudden rise is seen in the free atom cross section calculation, although the photoelectric cross section rises as $E$ decreases. This attenuation is due to a bulk plasma effect, which is entirely nonatomic and is completely unaccounted for by ordinary photon-atom scattering. Similar plasma attenuation is seen in all metals at their plasma frequencies.

Thus, free atom cross sections reliably account for photon propagation through metallic $\mathrm{Al}$ only for photon energies above about $500 \mathrm{eV}$.

\subsubsection{Long-Wavelength Photon Attenuation in Water}

A similar comparison can be made in a dielectric, such as water. In Figure 2.2, the solid line shows $\alpha$ for liquid water, constructed as in Equation 2.2 with $n_{i}$ taken from the data presented by Querry et al. [Qu91]. The dashed line is the attenuation coefficient calculated from free atom cross sections from EPDL97, as $N(\mathrm{H}) \sigma(\mathrm{H})+N(\mathrm{O}) \sigma(\mathrm{O})$, where $N(x)$ is the density of species $x$, and $\sigma(x)$ is the free atom total cross section. These authors present data only up to $120 \mathrm{eV}$. Again, agreement is poor below at least $120 \mathrm{eV}$. As the atomic cross sections go virtually to zero below the $\mathrm{H} K$ edge $(13.56 \mathrm{eV})$, the free atom cross sections predict essentially no attenuation below that energy, whereas actual attenuation is substantial. In liquid water, free atom cross sections reliably account for photon propagation only for photon energies above a few hundred $\mathrm{eV}$.

Comparisons in other materials could easily be made. Although many cross-section graphs in this book go down to $10 \mathrm{eV}$, the reader must use judgment when applying them to condensed matter below a few hundred $\mathrm{eV}$. As discussed in Section 2.12, data on the CD-ROM is presented only above $100 \mathrm{eV}$. 


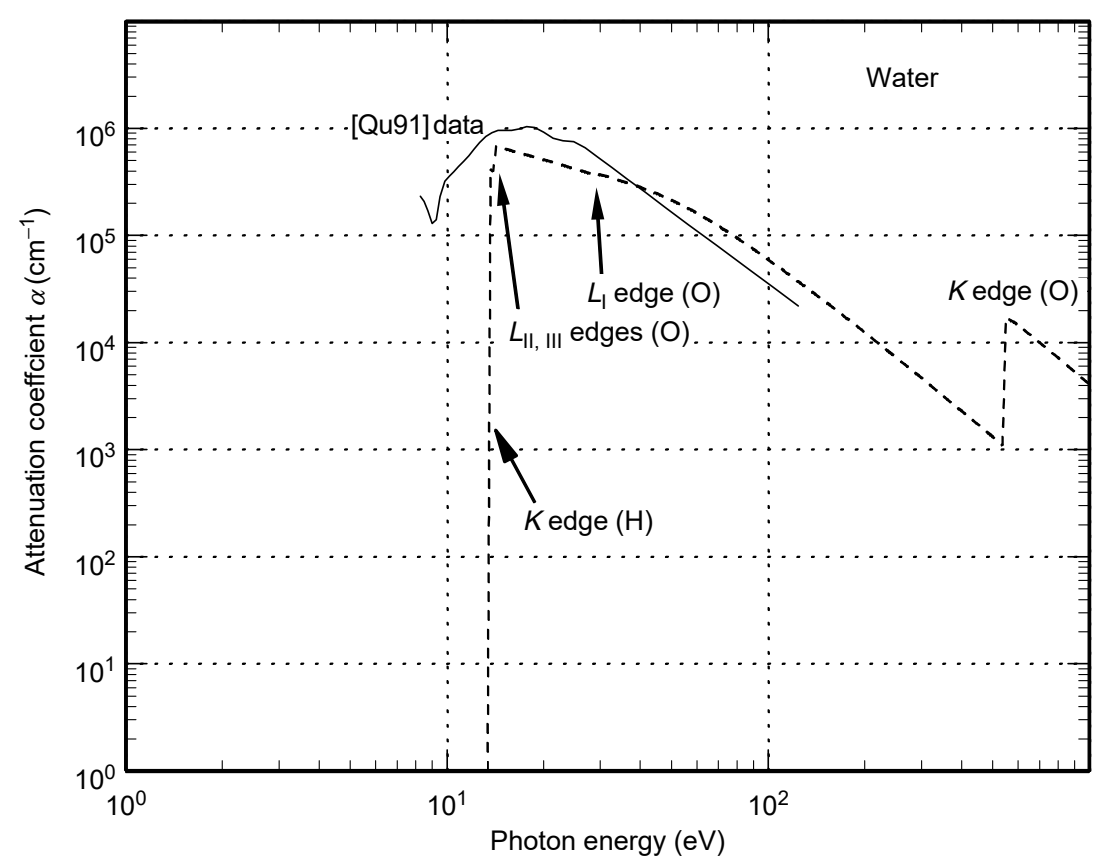

FIGURE 2.2 Comparison of water attenuation data [Qu91] with that expected from free atom cross sections.

In this long-wavelength region, several references are useful:

- The treatment by Ward [Wa94] is a good introduction to the theory of propagation of light through matter (the term "light" is used generically, and includes IR, visible, UV, etc.).

- The three-volume set edited by Palik [Pa98] contains thorough data on the real and imaginary parts of the index of refraction of many solids for wavelengths $10^{-4} \mu \mathrm{m} \lesssim \lambda \lesssim 1000 \mu \mathrm{m}\left(10^{-3} \mathrm{eV} \lesssim h \nu \lesssim 10 \mathrm{keV}\right)$.

- The IR Handbook [Wo85] contains theory and data on the transmission through materials for wavelengths in the IR (roughly 1-100 $\mu \mathrm{m}$ ).

- The compilation edited by Klocek [K191] includes IR properties of other materials than those given in the IR Handbook.

- J.H. Weaver, C. Krafka, D.W. Lynch, and E.E. Koch, Physics Data: Optical Properties of Metals, Volumes I and II, No. 18-1 and 18-2. Fachinformationzentrum, Karlsruhe, Germany (1981).

- S.S. Ballard, J.S. Browder, and J.F. Ebersole, Chapter $6 \mathrm{~b}$ and Chapter $6 \mathrm{c}$ in D.E. Gray, Ed., American Institute of Physics Handbook, 3rd Edition, McGraw-Hill, New York, 1972. 
- A short table of the index of refraction and absorption coefficients of metals is in the CRC Handbook of Chemistry and Physics, e.g., 75th Edition (1994) CRC Press, Boca Raton, Florida.

- The two-volume Handbook of Optics, Michael Bass, Ed., 2nd Edition, McGraw-Hill, New York (1995) contains a wealth of information concerning theory and data on the transmission of optical wavelengths through materials.

\subsection{SHORT WAVELENGTHS}

When wavelengths are comparable with or are lesser than atomic dimensions (i.e., photon energies greater than a few $\mathrm{keV}$ ), the particle nature of the photon dominates in the interaction with each atom (although, of course, when computing the interaction, its wave nature is used in quantum mechanics)* Then one can think of a particle of light passing through matter, undergoing more or less independent interactions with atoms on the way. Under these circumstances, the index of refraction is a less useful concept, because the photon does not merely elastically scatter, although an index of refraction can always be defined in terms of the complex scattering amplitude [Ne66].

Rather, each photon in the beam has a certain probability of interacting with an atom, measured by the cross section. For a given atom density, this translates into a certain probability per unit path length of interacting with the bulk material. It can interact with an atom in one of several distinct ways. It may be absorbed by photoelectric absorption, ejecting an electron. It may also elastically scatter from the atom, being a coherent scattering from all atomic electrons, leaving the atom undisturbed in its original state (called coherent scattering, or Rayleigh scattering). It may Compton scatter from a bound atomic electron, ejecting the electron and scattering off at longer wavelength. (This may be off any electron, leaving the atom in different states, and so is called incoherent scattering). Alternatively, in the electric field of the nucleus or the atomic electrons, it may disappear in the production of an electron-positron pair.

In any of these four ways (and other minor ways mentioned later), the original photon is removed from the incident beam. This higher-energy range is the subject of this book.

Photons of energy greater than some tens of $\mathrm{keV}$ are sometimes referred to as penetrating radiation. This historical term derives from the particle's

\footnotetext{
*The concept of a photon (but not the name) as a "quantum of electromagnetic radiation" was introduced early in the twentieth century. Physicists struggled with its meaning, and it was only in 1923 when A.H. Compton did the critical experiment on scattering of light off particles at high energy, and the kinematics were seen to be consistent with those of a (zero mass) particle, that physicists took the concept of a particle of light seriously. The name photon was assigned in 1926 by American chemist Gilbert N. Lewis. The story is beautifully told in Pais, Inward Bound [Pa86].
} 
ability to penetrate thin sheets of material, such as aluminum, and refers in general to radiations that penetrate to some macroscopic distance. Dental and medical x-rays fall in this class, as do nuclear $\gamma$ rays. Sometimes very energetic charged particles also fall in the class of penetrating radiation. However, charged ions (protons, $\alpha$ particles, etc.) must be at higher energies, for the range of a $10 \mathrm{MeV}$ proton in, say, water or polyethylene, is only about $0.1 \mathrm{~cm}$, and the range of higher mass ions is even less. A $10 \mathrm{MeV}$ electron has a range of the order $5 \mathrm{~cm}$ in polyethylene, and so may qualify as penetrating.

\subsection{PHOTON INTERACTIONS}

As mentioned, in general a photon interacts with an atom primarily in one of four ways:

- Elastic scattering in which the atom is left in its original state and a photon of the original energy continues on at a different angle (Rayleigh or coherent scattering). The photon loses no energy.

- Inelastic scattering in which an electron is ejected and a lower-energy photon is emitted (Compton or incoherent scattering). The photon loses some of its energy.

- Photoelectric absorption in which the photon is absorbed, kicking out an electron. The electron shell vacancy is then filled by an outer electron dropping down; the transition may emit a photon (fluorescence) or emit a different outer electron (Auger effect). Alternatively, its energy may be taken up by the medium by collisional quenching or phonon excitation. (Because fluorescence and Auger emission are so fast $\left(\lesssim 10^{-11} \mathrm{sec}\right)$, collisional quenching, although possible, is seldom the dominant process.) The original photon loses all energy.

- The destruction of the photon and the creation of an electron-positron pair. This occurs in the Coulomb field of the nucleus (nuclear pair production) or of an atomic electron (electronic pair production, also called triplet production). The photon loses all its energy.

These are the four dominant processes. Other possible processes in which the photon interacts with the atomic electrons, always small compared with the four given here, are:

First-order photoexcitation. In this process, the photon is absorbed by an electron in a low shell, say the $K$ shell, and excited to a high, vacant, bound state of the atom. The recoiling atom provides for momentum conservation. Subsequent de-excitation of the atom may be by emission of another photon (the electronic Raman effect, a form of fluorescence), Auger emission, phonon excitation (in solids), or by collisional quenching. As vacant, high atomic states are relatively closely spaced, often by only a few eV, this process is energetically possible only when the photon energy is just a few 
$\mathrm{eV}$ below the $K$ edge (or $L$ edge,...). It is manifested in small oscillations in the total absorption cross section just below the $K$ edge [Sa88]. Otherwise, it is seldom important, partly because such finely defined incident $\mathrm{x}$-rays are seldom encountered in practice. It is, however, more important for absorption of visible and UV photons (from the $L, M, \ldots$ shells). At those energies, electronic Raman scattering can be a useful diagnostic. It is a fairly new subject of research; it is employed, for example, in studying the electronic structure of superconductors [De00]. A review is given by Ter-Mikaelyan [Te97].

Second-order photoexcitation. In this process, the photon is scattered by an electron in a low shell, raising the electron to a high, vacant, bound state of the atom, while the reduced energy photon exits the interaction region (which also defines this as an electronic Raman effect). This is similar to Compton scattering, except that the electron is given insufficient energy to be ejected. Moreover, unlike first-order photoexcitation, the incident photon may have any energy. A review of this inelastic x-ray scattering without ionization is given by Åberg and Tulkki [Åb85].

Still other processes, in which the photon interacts with the atomic nucleus, also small compared with the primary four processes, are:

Nuclear Thomson scattering. This is elastic scattering from the nuclear charge. As it leaves the atom unchanged, it is coherent with Rayleigh scattering. The scattering amplitude for Thomson scattering from a single electron is, to order of magnitude, $r_{\mathrm{o}}=e^{2} / m c^{2}$ (see Equation 2.14). The amplitude for coherent, Rayleigh scattering from the $Z$ electrons in an atom is, to order of magnitude, $e^{2} F / m c^{2}$, where $F(q)$ is the elastic scattering form factor, a function of the momentum transferred to the atom, $q$ (see Equation 2.16). For small $q, F \sim Z$, but for large $q, F$ is much smaller. However, the amplitude for Thomson scattering from the nucleus is of the order $(Z e)^{2} / M c^{2}$, where $M$ is the mass of the nucleus. The nuclear Thomson amplitude is smaller than the electronic contribution by the factor $Z^{2} m / M F$. This ratio is about $\left(Z^{2} / A\right) / 1837 F$, where $A$ is the nuclear atomic weight. At low energies and small momentum transfer, this is of the order $3 \times 10^{-4}$ for all elements, and nuclear Thomson scattering is almost always negligible compared with scattering from atomic electrons. However, at high energies $(\sim 1 \mathrm{MeV})$, the momentum transfer is also large, $F$ can be less than or of the order 0.001, and the ratio can be several percent or more, especially for high $Z$. At energies greater than or of the order $1 \mathrm{MeV}$ in heavy elements, nuclear Thompson scattering can interfere with atomic Rayleigh scattering.

Nonelastic nuclear scattering. This includes the giant dipole resonance, photodisintegration, photopion production, and all nonelastic events. Figure 2.3 shows the nonelastic photonuclear cross section on four major isotopes. It is largest at 15-20 MeV (giant dipole resonance), and is usually less than about $5 \%$ of the atomic cross section. In $\mathrm{Pb}^{208}$, for example, the photonuclear cross 


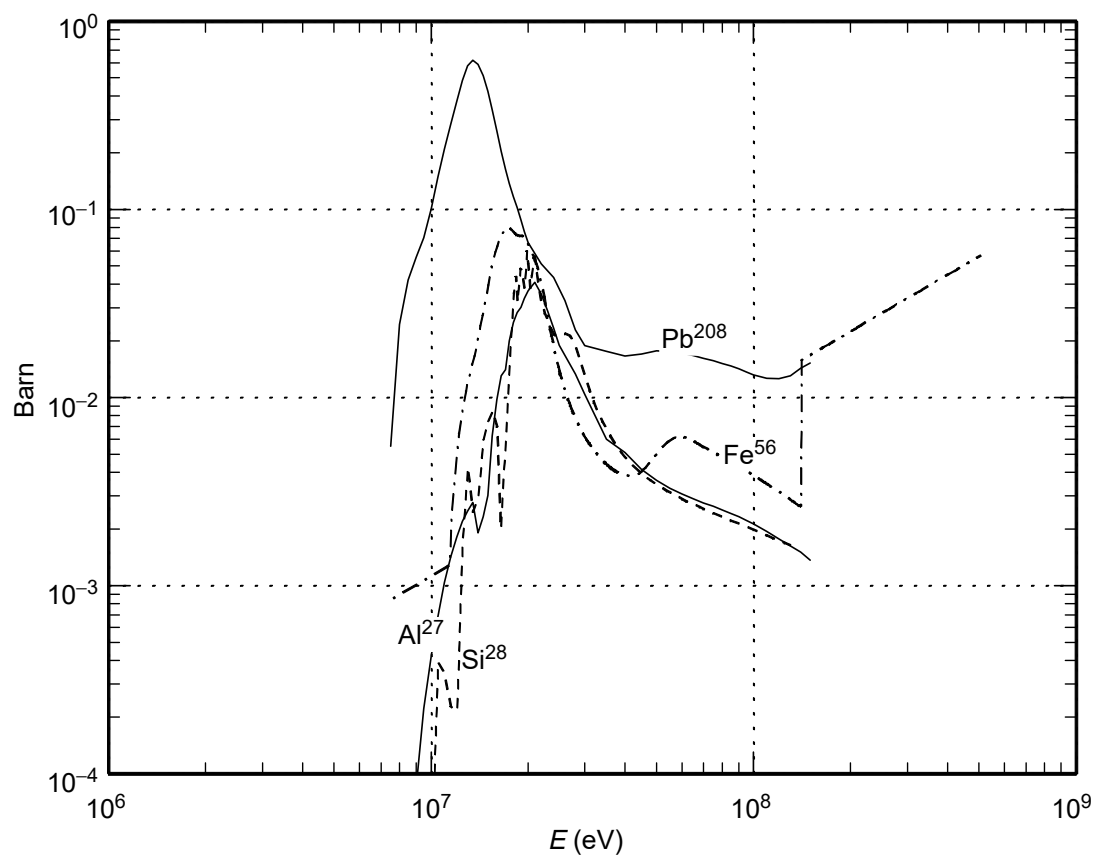

FIGURE 2.3 Total nonelastic photonuclear cross sections on four isotopes. (Data from http://www-nds.iaea.org/photonuclear/recommended/.)

section peaks near $14 \mathrm{MeV}$ at about 0.6 barn. At the same energy, the total atomic cross section is about 19 barn (primarily due to pair production). In this example, the photonuclear cross section is only some $3 \%$ of the atomic cross section.

Several countries have constructed photonuclear cross-section libraries (U.S., S. Korea, China, Japan, and Russia). All of these are available at the International Atomic Energy Agency [IAEApn].

At energies near the peak of the giant resonance, they agree quite well, but at other energies they can differ substantially from one another. The cross sections plotted in Figure 2.3 are the recommended ones. In the figure, only $\mathrm{Fe}^{56}$ is shown beyond $150 \mathrm{MeV}$. The sudden rise at $140 \mathrm{MeV}$ is due to the onset of pion production.

Delbrück scattering. This is elastic photon scattering from the Coulomb electric field surrounding the nucleus. The incident photon scatters from virtual electronpositron pairs and is effectively a radiative correction to Compton scattering off the nucleus. Enormous electric fields are necessary for it to occur with any appreciable probability; the unscreened electric field at $r=10^{-11} \mathrm{~cm}$ from a $\mathrm{Pb}$ nucleus is $1.2 \times 10^{19} \mathrm{~V} / \mathrm{m}$. Like the preceding two nuclear effects, it is never the dominant 
photon-atom interaction process. However, it can be important if attention is restricted to photon elastic scattering from atoms. In high $Z$ atoms at a few $\mathrm{MeV}$, there can be strong interference among atomic Rayleigh, nuclear Thomson, and Delbrück scattering [Ki85]. Its relative importance has been summarized by Hubbell and Bergstrom [Hu04].

Below 10 to $100 \mathrm{keV}$, depending on $Z$, photons are primarily absorbed by the photoelectric effect. From tens of $\mathrm{keV}$ to $\sim 10 \mathrm{MeV}$, or even lower energies in very low- $Z$ materials, the dominant process is Compton scattering. Above this energy, pair production is largest. Photon-atom cross sections in the standard databases almost always are for isolated, cold atoms. They do not include energy level shifts (and so cross-section changes) due to the neighboring atoms in condensed media, which may be particularly important near absorption edges. In addition, the smaller cross sections mentioned earlier, the photonuclear cross section, Delbrück scattering, nuclear Thomson scattering, radiative corrections to Compton scattering, and so on, are also not included in the databases. The largest of these omitted effects are the nonelastic photonuclear effects near the giant dipole resonance, from about 5 to $30 \mathrm{MeV}$; in that energy region, the ignored photonuclear processes could contribute a few percent to the total cross section.

The scope of magnitude is illustrated in Figure 2.4, which shows individual and total cross sections in aluminum as a function of incident photon

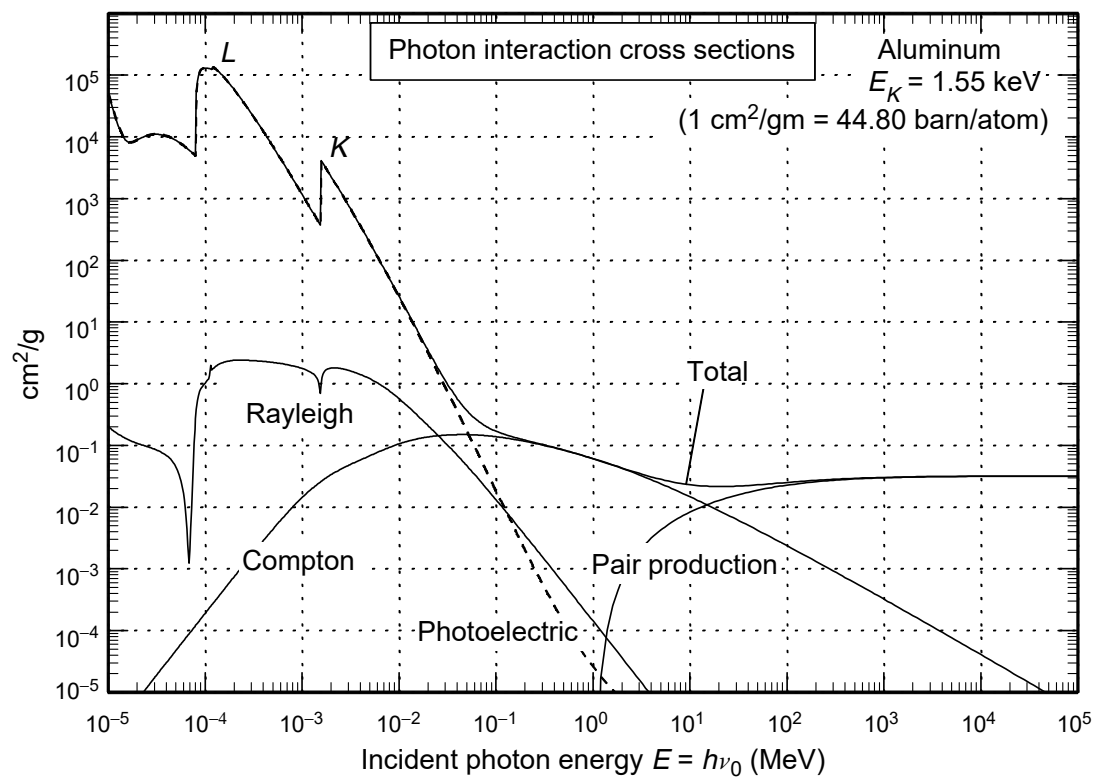

FIGURE 2.4 Photon interaction cross sections on an isolated, cold aluminum atom. (Data from LLNL EPDL97.) This database extends down to $1 \mathrm{eV}$. 
energy from $10 \mathrm{eV}$ to $10^{5} \mathrm{MeV}$. Data are taken from LLNL's Evaluated Photon Data Library, EPDL97. They are plotted in units of $\mathrm{cm}^{2} / \mathrm{g}$. This cross section is related to the individual atom cross section $\sigma$ (barn/atom) by

$$
\sigma\left(\mathrm{cm}^{2} / \mathrm{g}\right)=10^{-24} \frac{N_{\mathrm{A}}}{A} \sigma(\text { barn } / \text { atom })
$$

where $N_{\mathrm{A}}$ is Avogadro's number defined in Equation 2.5, and $A$ is the atomic weight (26.98 for $\mathrm{Al}$ ). For $\mathrm{Al}, 1 \mathrm{~cm}^{2} / \mathrm{g}=44.80$ barn/atom.

Similar cross-section plots for other elements are given in Section 2.8.

Standard tables of photon cross sections and related material parameters are widely available and listed later in Section 2.12.

We discuss photoelectric absorption first, in Section 2.4, then coherent Rayleigh scattering in Section 2.5. Section 2.6 presents a fairly thorough set of graphs for Compton scattering. Section 2.7 briefly discusses pair production, and Section 2.8 summarizes all processes for selected elements, and presents total cross-section and mean-free-path (mfp) contour plots for all elements at all energies.

A popular pocket book, the X-Ray Data Booklet is available free of charge from http://xdb.lbl.gov. It contains useful information on atomic x-ray properties, synchrotron radiation, electron binding energies, and so on.

\subsection{PHOTOELECTRIC ABSORPTION}

Photoelectric absorption is the most probable interaction for photon energies $E \lesssim 10 \mathrm{keV}$ (for $Z \lesssim 4$ ), or $E \lesssim 100 \mathrm{keV}$ (for $Z \lesssim 30$ ), or $E \lesssim 700 \mathrm{keV}$ (for $Z \lesssim 90)$. It is discussed by Heitler [He54], Evans [Ev55], and Bethe [Be53]; thorough plots are in [P175].

Remembering that

$$
1 \text { barn } \equiv 1 b=10^{-24}\left(\mathrm{~cm}^{2}\right)
$$

a convenient cross-section unit is the Thomson cross section

$$
\sigma_{\mathrm{T}}=\frac{8 \pi}{3} r_{\mathrm{o}}^{2}=0.665246 \text { barn }
$$

where $r_{\mathrm{o}} \equiv e^{2} / m c^{2}=2.818 \times 10^{-13} \mathrm{~cm}$ is the classical electron radius.

Kinematically, a free electron cannot absorb a photon, but an electron bound in an atom can. The less tightly bound it is, the less likely it is to absorb. Therefore, the atomic photoelectric cross section is largest when photon absorption takes place from the $K$ shell. The $K$ shell binding energy $E_{K}$ is approximately proportional to $Z^{2}$, and the cross section behaves roughly as a power of $E_{K}$, making the cross section per atom approximately proportional to a power of $Z$, roughly between $Z^{4}$ and $Z^{5}$. 
Likewise, the more energetic the photon the more nearly free does the electron appear, causing the cross section to decrease with increasing photon energy.

The calculation of photoelectric absorption is simple only in the Born approximation. In this case, and when the ejected electron is nonrelativistic $\left(h \nu \ll m c^{2}\right)$, the photoelectric cross section per atom from the $K$ shell is calculated to be [He54]

$$
\sigma_{K}=2 \sqrt{8} \alpha^{4} Z^{5} \sigma_{\mathrm{T}}\left(\frac{m c^{2}}{h \nu}\right)^{7 / 2}
$$

when the photon energy is not too close to the $K$ edge, where $\alpha=e^{2} / \hbar c=1 / 137$ is the fine structure constant. The factor 2 is because there are two electrons in the $K$ shell. Some distance from the $K$ edge is required for the validity of this equation so that the outgoing electron is energetic enough for the Born approximation to be valid.

Because it is simple absorption, one might expect the cross section for the photoelectric effect to be proportional to the square of the coupling constant $e$. However, as Thomson scattering is a second-order process, $\sigma_{\mathrm{T}} \propto e^{4}$, and $\alpha \propto e^{2}, \sigma_{K}$ is seen to be proportional to $e^{12}$. The interaction Hamiltonian is, of course, of first order in $e$. However, the initial state $(K$ shell) wave function contains $e$ in its radial dependence $\exp \left(-Z r / a_{0}\right)$, where $a_{0}=\hbar^{2} / m e^{2}=$ $0.529 \AA$ is the Bohr radius. The other powers of $e$ beyond 2 in Equation 2.9 for $\sigma_{K}$ arise from initial-state normalization and from evaluation of the matrix element integral itself, which brings $a_{\mathrm{o}}$ down from the exponent into an overall factor, making $\sigma_{K}$ proportional to a high power of $e$.

For very relativistic energies, when $h \nu \gg m c^{2}$,

$$
\sigma_{K}=\frac{3}{2} \alpha^{4} Z^{5} \sigma_{\mathrm{T}}\left(\frac{m c^{2}}{h \nu}\right)
$$

and the cross section drops off only as $1 / h \nu$. A more general formula holds in the moderately relativistic case $h \nu \sim m c^{2}$ [He54]. These expressions also hold only for sufficiently small $Z$ (again for the Born approximation to be valid).

The strong dependence on $Z$ means the photoelectric effect can still be important in heavy elements even at energies $(\sim 1 \mathrm{MeV})$ where the Compton effect is expected to dominate.

Calculations are simple, and very approximate, only for the elementary cases just mentioned. $L$ and $M$ shells should be added, the Born approximation must be circumvented, and all energies, including near edges and relativistic 
energies, must be computed, to obtain the actual photoelectric cross section per atom, $\sigma_{\mathrm{pe}}(h \nu)$. These calculations are done numerically using Dirac-HartreeFock and related sophisticated wave function models.

Absorption in the $K$ shell contributes the dominant amount to the total atom cross section. As a convenient rule of thumb, $L, M$, and higher shells contribute about one-fourth that of the $K$ shell, so that $\sigma_{\mathrm{pe}}(h \nu) \approx(5 / 4) \sigma_{K}$. However, over all $Z$ and photon energies $h \nu$, the atom cross section $\sigma_{\text {pe }}(h \nu)$ does not behave as a single power of $Z$ or of $h \nu$. Davisson and Evans [Da52] have reviewed theory and experiment for the photoelectric cross section.

Experimental atomic cross sections and theoretical models have been evaluated by the National Institute of Standards and Technology (NIST), and by the LLNL (see Section 2.12). Their compilations are widely used. We present some of these data here.

Figure 2.5 shows the photoelectric cross section $\left(\mathrm{cm}^{2} / \mathrm{g}\right)$ for several elements as a function of $E$, while Figure 2.6 shows it for selected energies as a function of $Z$.

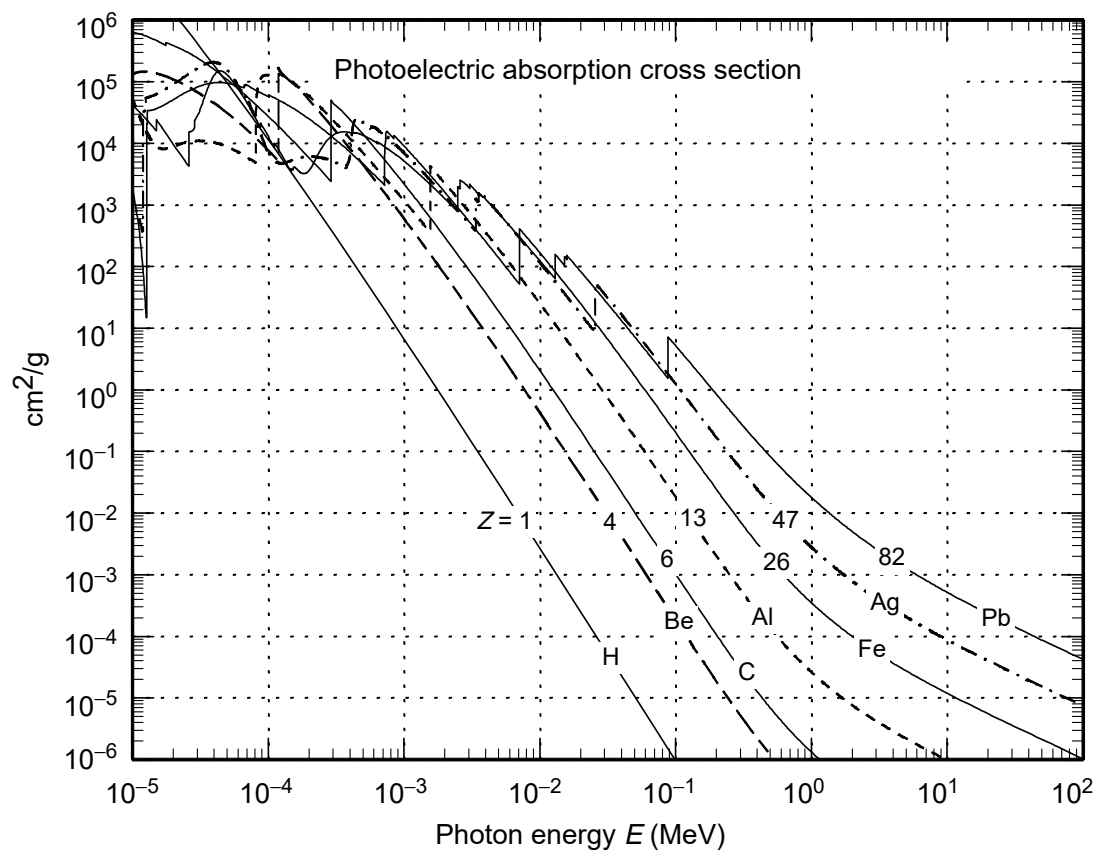

FIGURE 2.5 Photoelectric absorption cross section on several elements as a function of incident photon energy $E$. (Data from LLNL EPDL97.) 


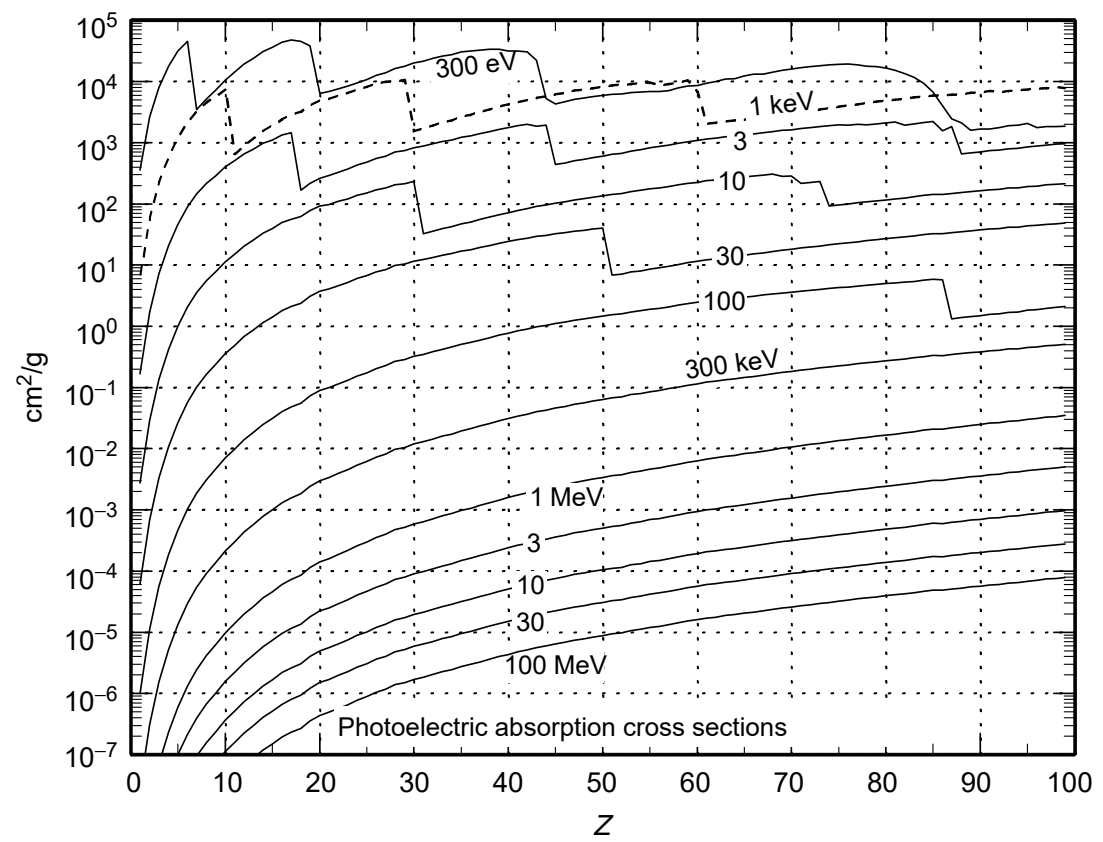

FIGURE 2.6 Photoelectric absorption cross section at several energies as a function of atomic number $Z$. (Data from LLNL EPDL97.)

\subsubsection{Cross-Section Contour Plots}

For each element the photoelectric cross section $\sigma_{\mathrm{pe}}(E)$ or the cross section for any other process is a function of $E=h \nu$. That is, the cross section is a function of $Z$ and $E$, and so forms a surface in $Z, E$ space. The cross section for all elements at all energies can then be represented by contours in that space, and one can grasp at a glance the behavior with both variables, over all energies and all elements.

\subsubsection{Contours in Barn/Atom}

Contours of the photoelectric cross section on a free, isolated atom at rest, $\sigma_{\mathrm{pe}}(Z, E)$, in barn/atom, are shown in Figure 2.7. The figure extends down to $100 \mathrm{eV}$. The user must observe the caveat in Section 2.1 when employing them in condensed media below several hundred $\mathrm{eV}$.

In contour plots, and in graphs of cross sections vs. $Z$ such as Figure 2.7, data exist only at integer $Z$. Between integral values of $Z$, the curves are eye guides only.

The complete photoelectric cross section on an atom, $\sigma_{\mathrm{pe}}$, has about the same dependence on $Z$ as $\sigma_{K}$. Although $\sigma_{\mathrm{pe}}$ (barn/atom) $\propto Z^{4}$ to $Z^{5}$, if one expresses it as $\mathrm{cm}^{2} / \mathrm{g}$ it scales more nearly between $Z^{3}$ and $Z^{4}$. 


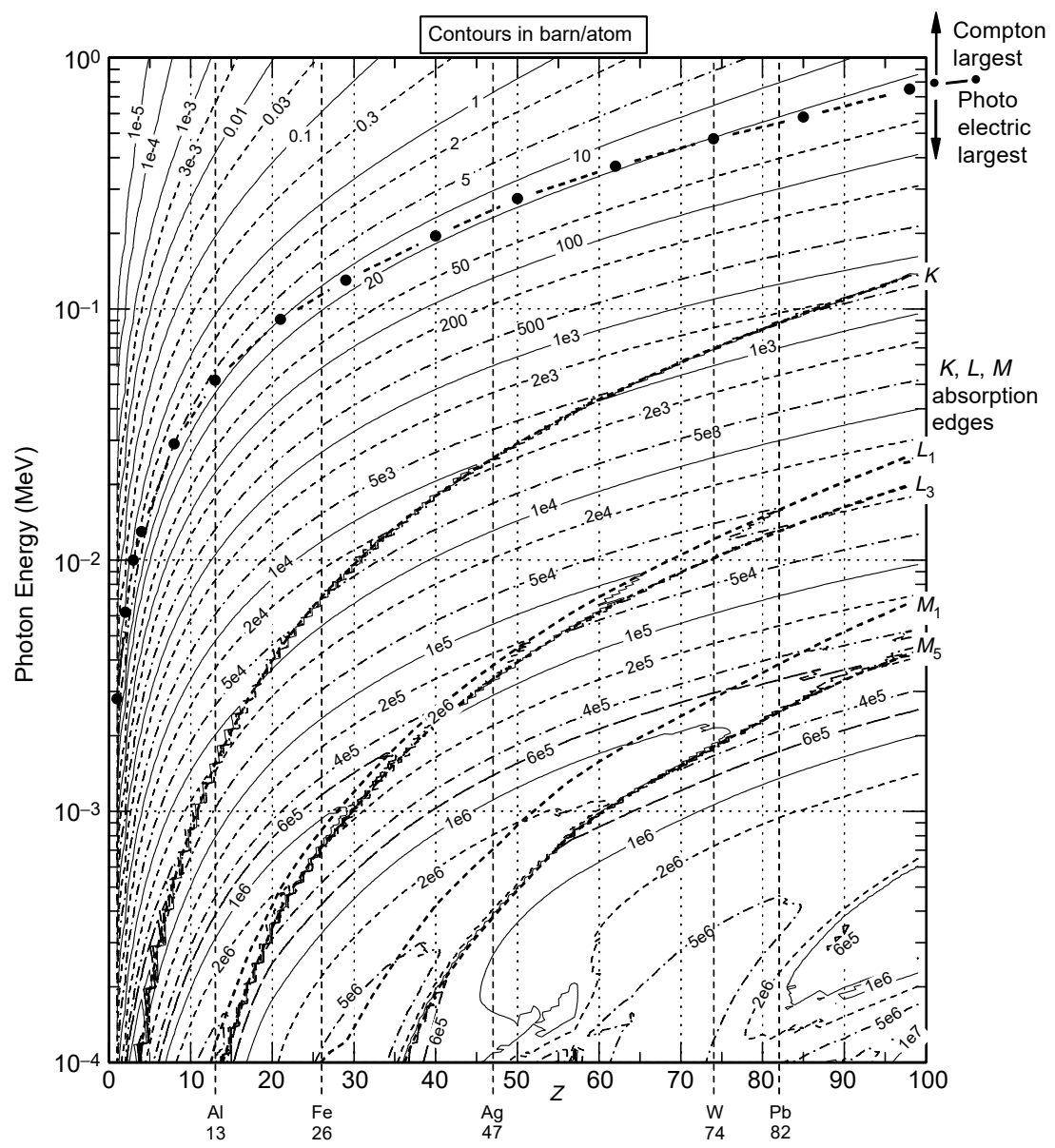

FIGURE 2.7 Contours of photoelectric absorption cross section in all elements, from $100 \mathrm{eV}$ to $1 \mathrm{MeV}$, in barn/atom. (Data from LLNL EPDL97.)

\subsubsection{Contours in $\mathrm{cm}^{2} / \mathrm{g}$}

In switching from $\sigma_{\mathrm{pe}}$ (barn/atom) to

$$
\sigma_{\mathrm{pe}}\left(\mathrm{cm}^{2} / \mathrm{g}\right)=10^{-24} \frac{N_{\mathrm{A}}}{A} \sigma_{\mathrm{pe}}(\text { barn } / \text { atom }),
$$

the $Z$ dependence shifts because of the dependence of $A$ on $Z$. First, the $Z$ dependence shifts down one power of $Z$ as just mentioned. Second, $A$ is not exactly proportional to $Z$; there are small, local deviations. The atomic weight $A(Z)$ of the elements in their natural isotopic composition is shown in Figure 2.8. 


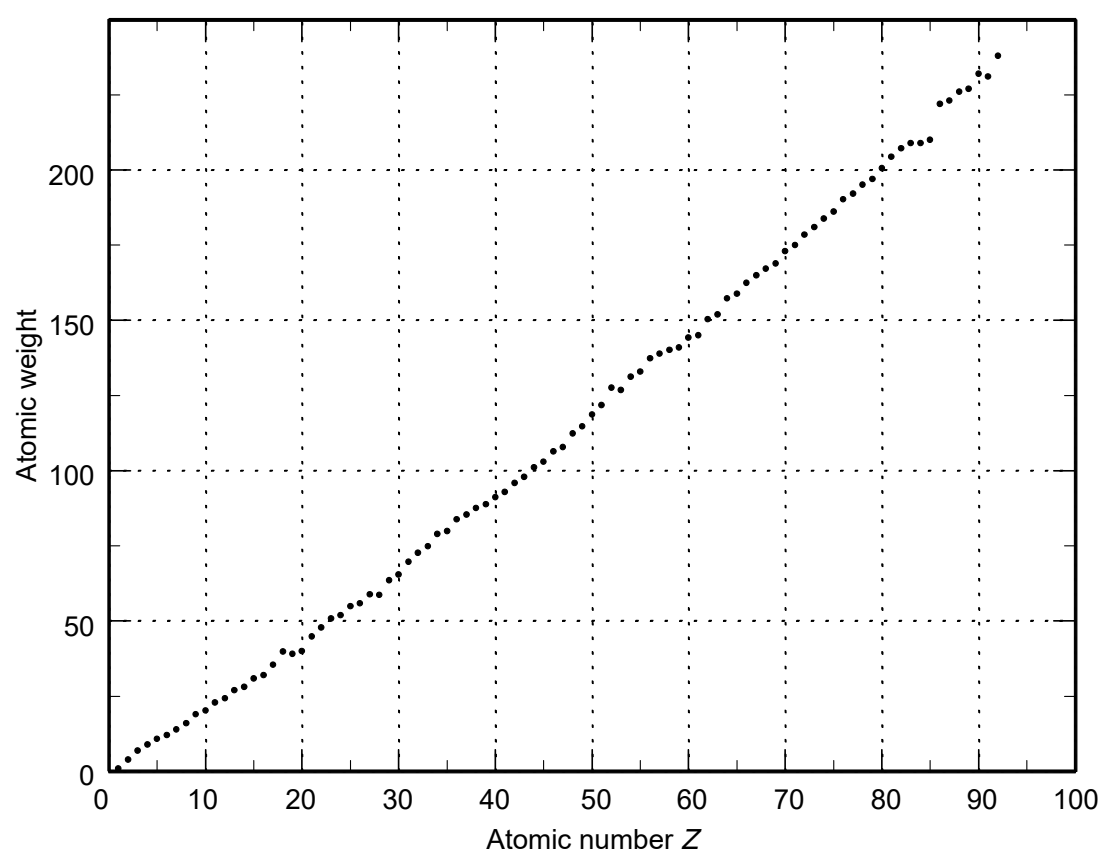

FIGURE 2.8 Atomic weight of the elements, naturally occurring isotopic abundances. (Data from http://physics.nist.gov/PhysRefData/Compositions/.)

The smooth, monotonically increasing dependence on $Z$ is interrupted at $Z=$ 18-19, 27-28, and 52-53, where $A$ momentarily decreases with increasing $Z$. Other irregular behaviors can be seen at different $Z$. This irregular dependence causes the irregularities in the contour plot of $\sigma_{\mathrm{pe}}\left(\mathrm{cm}^{2} / \mathrm{g}\right)$, Figure 2.9, at those values of $Z$. The irregularities are not very significant in this figure because they are small compared with the difference between adjacent contour lines. They are more pronounced when contour values are more closely spaced, as in the Compton cross section discussed in Figure 2.43 in Section 2.7.

\subsubsection{X-Ray Edge Energies}

Photoabsorption in the $K$ (or $L$, or $M, \ldots$ ) shell can occur only for photon energies greater than that shell's absorption edge. Some edges for the elements are shown in Figure 2.10. The $K$ edge in $\mathrm{Al}$ is $1.56 \mathrm{keV}$, that in $\mathrm{Pb}$ is $88 \mathrm{keV}$. The $L_{1}$ edge in $\mathrm{Al}$ is $119 \mathrm{eV}$, that in $\mathrm{Pb}$ is $15.85 \mathrm{keV}$. When less than a few hundred $\mathrm{eV}$, edge energies are known less accurately; they may be uncertain by perhaps $10 \%$, and at all energies can be uncertain to an absolute energy of 1 to $3 \mathrm{eV}$ [Ch95c]. A classic compilation of edge energies is that of Bearden and Burr [Be67]. More recent tabulations are at NIST's reference site [NIST], Biémont et al. [Bi99], and in Deslattes et al. [De03]. 


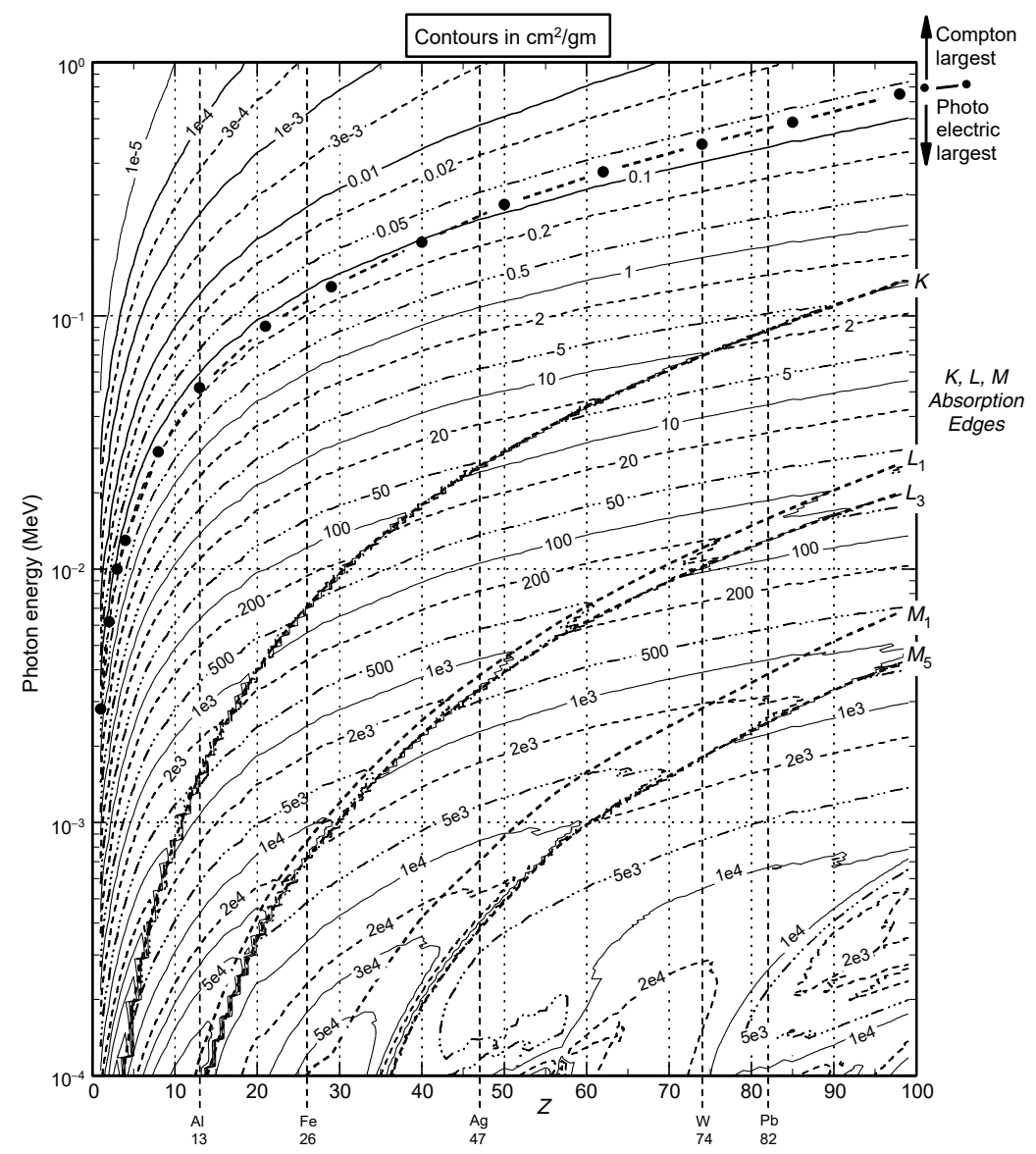

FIGURE 2.9 Contours of photoelectric absorption cross section in all elements, from $100 \mathrm{eV}$ to $1 \mathrm{MeV}$, in $\mathrm{cm}^{2} / \mathrm{g}$. (Data from LLNL EPDL97.)

\subsubsection{Angular Distribution of Photoelectrons}

The first-order interaction Hamiltonian between the electromagnetic field and an electron is $-(e / m c) \vec{p} \cdot \vec{A}$, where $\vec{A}=\hat{e} A$ is the vector potential of the incident photon, $\hat{e}$ the unit vector, and $\vec{p}$ the electron momentum. Thus, the matrix element contains the factor $\hat{e} \cdot \vec{p}$. As a result, for very low-energy photons $(\lesssim 10 \mathrm{keV})$, photoelectrons tend to be ejected parallel to the electric field vector $\vec{E}$ of the incident photon, and so perpendicular to the photon direction, in a general $\sin ^{2} \theta$ pattern (unpolarized photons), where $\theta$ is the angle between the original photon direction and the outgoing electron momentum. At all energies, the electron never exits in exactly the forward direction, $\theta=0$, where $\hat{e} \cdot \vec{p}$ vanishes. 


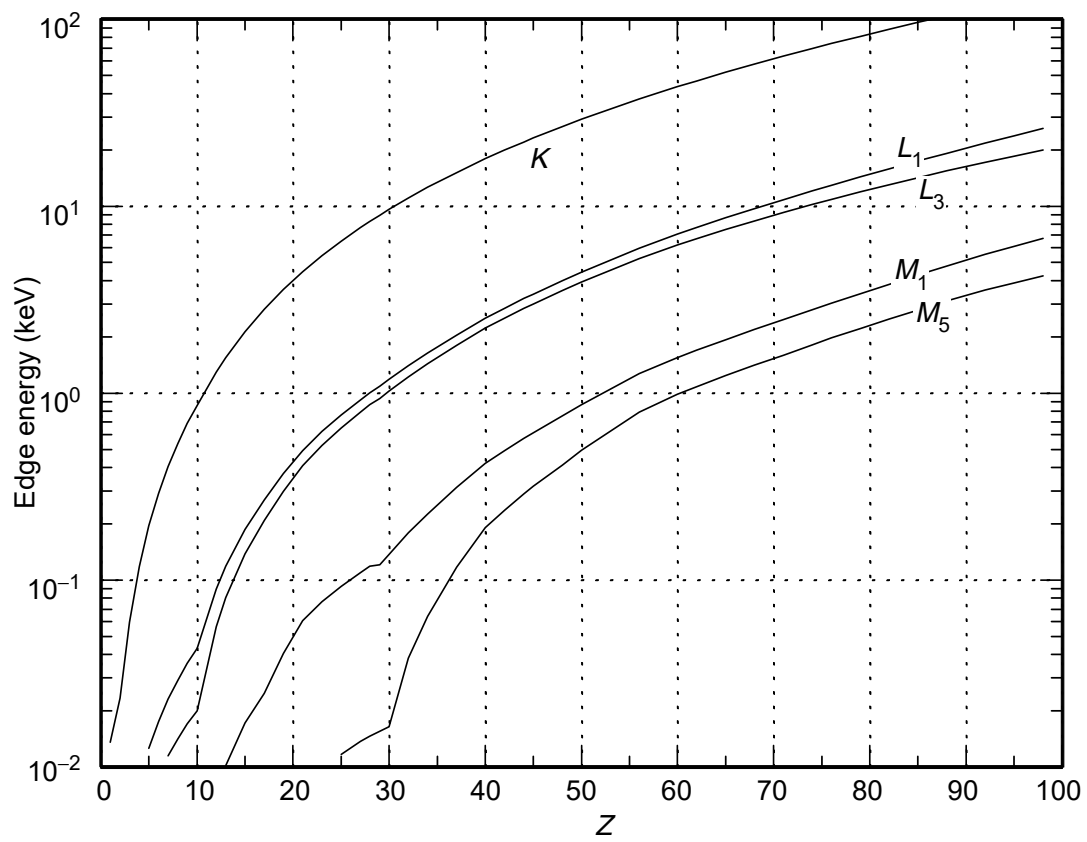

FIGURE 2.10 X-ray edge energies in the elements. (Data from EPDL97.)

At higher energies, the distribution is shifted forward, significantly so for $E \gtrsim 100 \mathrm{keV}$, while remaining zero at $\theta=0$. Nonrelativistically, the angular distribution, per unit solid angle, is approximately proportional to $\sin ^{2} \theta /(1-\beta \cos \theta)^{4}$, where $\beta$ is $v / c$ of the electron [He54]. A better, relativistic expression, valid for $Z / 137 \ll 1$, and neglecting electron binding (i.e., photon energy = electron energy) was given by Sauter [Sa31] and summarized by Davisson and Evans [Da52]

$$
\frac{\mathrm{d} \sigma}{\mathrm{d} \Omega} \sim \frac{\sin ^{2} \theta}{(1-\beta \cos \theta)^{4}}\left\{\frac{1}{\gamma}+\frac{1}{2}(\gamma-1)(\gamma-2)(1-\beta \cos \theta)\right\}
$$

where $\gamma=\left(1-\beta^{2}\right)^{-1 / 2}$ is the usual electron relativistic factor. A more recent survey of theory and experiments is given by Berkowitz [Be79]. These distributions are plotted in Figure 2.11 as functions of incident photon energy. There is little interest in photoabsorption above about $1 \mathrm{MeV}$, since in all materials the cross there is dominated by Compton scattering.

Figure 2.11a shows this angular distribution for several photon energies, and Figure 2.11b shows the cumulative angular distribution.

The polar angle (relative to the photon direction) inside of which half the electrons are ejected is shown in Figure 2.12. By $E=1 \mathrm{MeV}$, half the 


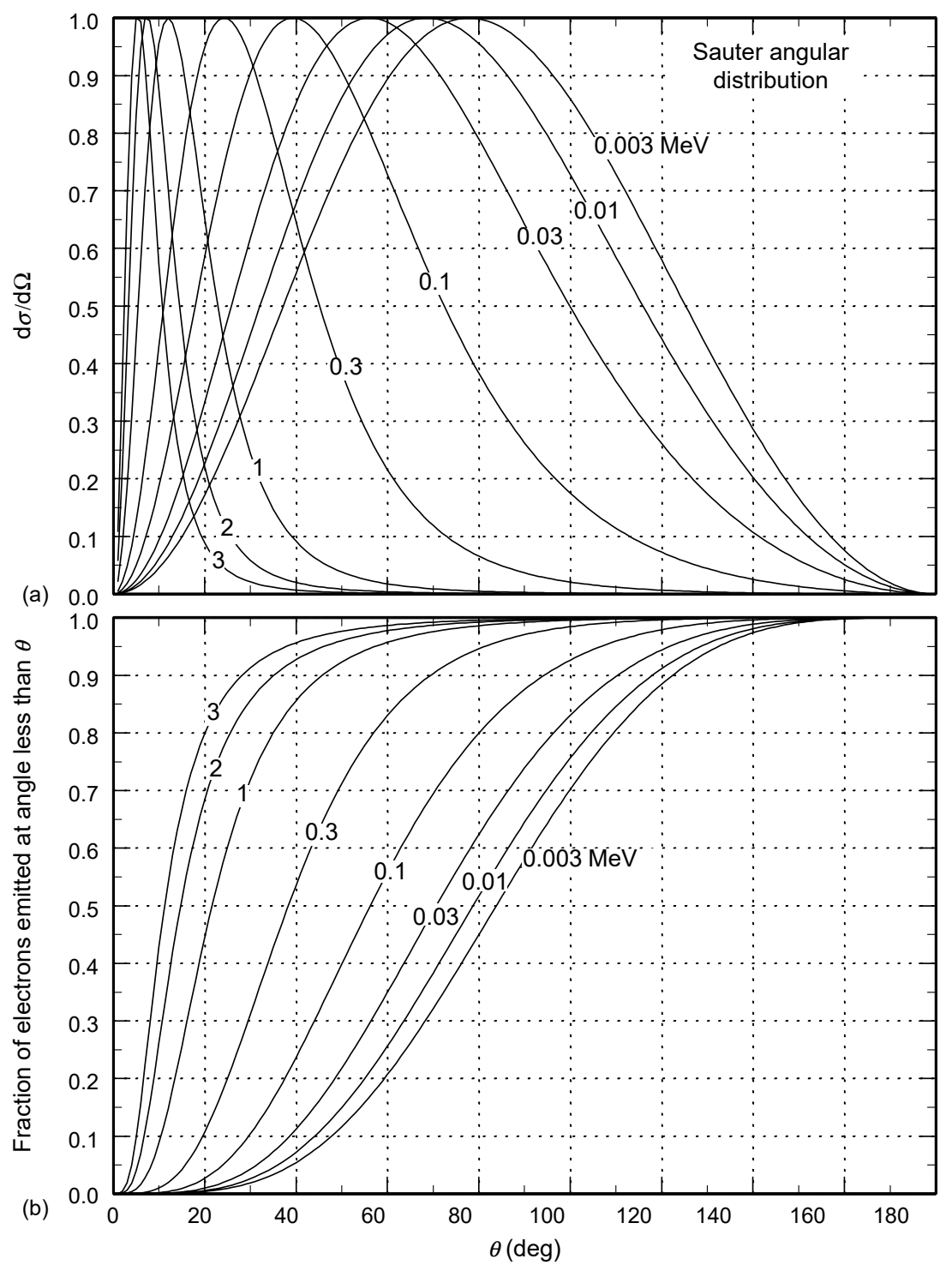

FIGURE 2.11 Angular distribution of photoelectrons, according to the calculations of Sauter [Sa31] (see also [Da52]). Above a few tens of $\mathrm{keV}$ the angular distribution is significantly shifted forward. (a) the solid angle distribution $\mathrm{d} \sigma / \mathrm{d} \Omega$, normalized to unit maximum. (b) fraction of photoelectrons ejected at less than polar angle $\theta$.

electrons come out within about $22^{\circ}$ of the original photon direction. Ignoring binding energies, the electron kinetic energy $T=E=h \nu$. Then, the electron momentum $p=\left[2 m T+T^{2}\right]^{1 / 2} / c=\left[1+2 m c^{2} / E\right]^{1 / 2} E / c$ always exceeds the 


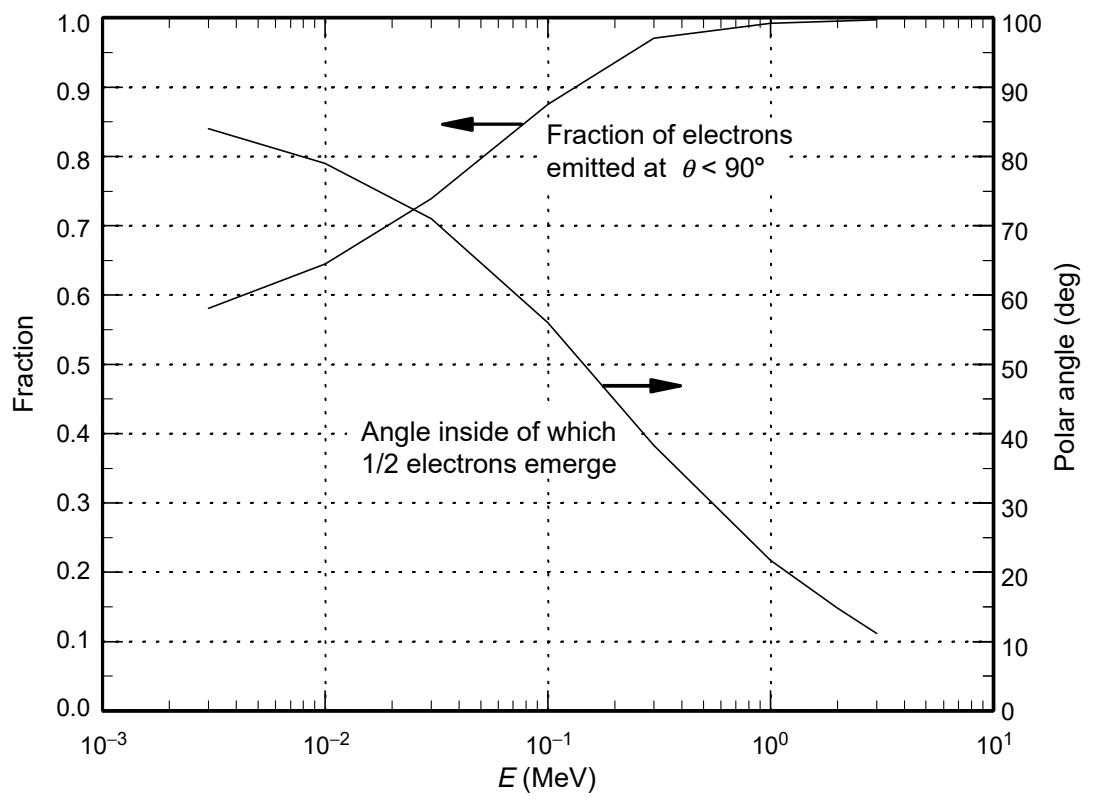

FIGURE 2.12 Summary angles for electron emission in photoelectric absorption. Binding energy is ignored; photon energy = electron energy, so that the abscissa represents either one.

photon momentum $E / c$; the difference is taken up by the atom recoiling backward. Also plotted is the fraction of all electrons that emerge in the forward hemisphere, $\theta<\pi / 2$. By $100 \mathrm{keV}$, some $87 \%$ of all ejected electrons exit in the forward hemisphere. An early discussion of the angular distribution was given by Kim et al. [Ki80]. Recent compilations of photoelectron angular distributions for elements are presented in [Tr01, Tr02]. Deviations from pure dipole behavior are discussed in [De99].

\subsubsection{Fluorescence and Auger Electrons}

After photoelectric absorption has occurred, a vacancy exists in the shell from which the electron was ejected. That vacancy may be filled by a higher shell electron jumping down, while a photon of energy equal to the difference of the two shells is emitted. Such photons are called characteristic x-rays, because their energy is characteristic of that atom's energy levels. This process of characteristic photon emission after photoabsorption is called fluorescence.

Alternatively, instead of a photon emission, while one electron jumps down to fill the vacancy a second electron from an outer shell may be emitted. For a vacancy in the $K$ shell, this Auger process is the only alternative to 
fluorescence. The probability that fluorescence will occur is called the Fluorescence Yield $\omega$, and the probability that Auger emission will occur is the Auger Yield $a$. For a $K$ shell vacancy, then, $\omega_{K}+a_{K}=1$. Radiative transitions are more probable $\left(\omega_{K}>1 / 2\right)$ for elements of $Z>30$, whereas Auger emission is more probable $\left(\omega_{K}<1 / 2\right)$ for $Z<30$.

Fluorescence is important for it affects the locality of incident photon energy deposition. Without it, the entire photon energy would be deposited locally (within the range of the emitted Auger electron). With it, the characteristic x-ray carries off a fraction of the original photon's energy and deposits it some distance away where that x-ray itself is absorbed. The mfp against absorption of a fluorescence $\mathrm{x}$-ray is many times the range of an electron of the same or lower energy.

The fluorescence photon has an energy just below the x-ray edge of the shell from which the original electron was ejected, for example, an energy $E_{K}-E_{L}$ if a photon was absorbed by the $K$ shell and fluorescence occurred when an $L$ shell electron dropped down to the $K$ shell. This is a meaningful example because $K$ shell absorption is about $80 \%$ of total photoabsorption. At the energies of these fluorescence photons, the photoabsorption cross section is several times smaller than just above the edge. Thus, these fluorescence photons have an mfp in the material several times greater than the original incident photons. Therefore, they are more penetrating in the target material than the incident photons, and will deposit energy to much greater depths. Photon transport codes therefore account for fluorescence.

Photoabsorption cross sections are large at low to moderate x-ray energies. In Fe, for example, just above the $K$ edge $(7.08 \mathrm{keV})$, the absorption cross section is about $3.8 \times 10^{4} \mathrm{barn} /$ atom, or $408 \mathrm{~cm}^{2} / \mathrm{g}$. Photons of this energy have an mfp against photoelectric absorption of only $2.45 \times$ $10^{-3} \mathrm{~g} / \mathrm{cm}^{2}$, or $3.1 \mu \mathrm{m}$. However, fluorescent photons with energy just below the $K$ edge experience a photoabsorption cross section of only $4.8 \times 10^{3}$ barn, and an mfp of about $25 \mu \mathrm{m}$. Thus, when Fe or other materials are exposed to $\mathrm{x}$-rays just above their $K$ edge, the photons tend to be absorbed very close to the surface. However, (half of) the fluorescent x-rays produced easily escape back out of the material. Consequently, fluorescence is a useful tool for studying surface composition, contamination, and surface structure. Puri et al. [Pu95] present a careful analysis of $K$ and $L$ shell cross sections for fluorescence applications.

\subsubsection{Coster-Kronig Transitions}

In addition to emitting fluorescence x-rays or Auger electrons, when photoabsorption occurs in a shell with subshells, that is, $L$ and higher, a third possibility exists. After photoabsorption by an electron in one subshell, the vacancy may be filled by an electron in the same shell but different subshell (of higher energy). These intra-shell transitions are known as Coster-Kronig 
(C-K) transitions. For photoabsorption in the $L$ shell, the Coster-Kronig yields $f_{1,2}$ and $f_{1,3}$ are the probabilities that a primary vacancy in the $L_{1}$ subshell is filled by a transition from subshell 2 or 3 , respectively; and $f_{2,3}$ is the probability that a primary vacancy in the $L_{2}$ subshell is filled by a transition from subshell 3. $L_{1}$ is always the deepest subshell, $L_{3}$ the shallowest. C-K transitions are radiationless and are accompanied by the emission of another electron either from another subshell within the same shell or from a higher shell.

Thus, by definition, the Auger yield $a_{\mathrm{S}}$ is the probability that a vacancy in shell $S(S=K, L, M, \ldots)$ is filled by a nonradiative transition by an electron from a higher shell. The Coster-Kronig yield is the probability that a vacancy in a subshell is filled by an electron from another subshell in the same shell, while the emitted electron may come from either the same shell or a higher shell. After the $\mathrm{C}-\mathrm{K}$ transition to a different subshell, the atom decays by a fluorescence or Auger process from that subshell.

The electron configuration of $L$ subshells and their approximate levels $(\mathrm{eV})$ in a few atoms are

\begin{tabular}{lccccc}
\hline Subshell & Configuration & ${ }_{13} \mathbf{A l}$ & ${ }_{26} \mathbf{F e}$ & ${ }_{50} \mathbf{S n}$ & ${ }_{\mathbf{8 2}} \mathbf{P b}$ \\
$L_{1}$ & $2 S_{1 / 2}$ & 119.05 & 843 & 4,440 & 15,847 \\
$L_{2}$ & $2 P_{1 / 2}$ & 81.2 & 734 & 4,161 & 15,251 \\
$L_{3}$ & $2 P_{3 / 2}$ & 80.73 & 721 & 3,927 & 13,040 \\
\hline
\end{tabular}

In the $L$ shell, it is also possible for an $L_{1}$ vacancy to be filled by a radiative transition from $L_{3}$. The symbol $f_{1,3}^{\prime}$ is commonly used for this probability. Radiative transitions within shells higher than $L$ are also possible. For known cases, these radiative probabilities are always much less than nonradiative $\mathrm{C}-\mathrm{K}$ transitions between the same subshells [Ba72]. For example, $f_{1,3}^{\prime} \ll f_{1,3}$.

In the absence of $\mathrm{C}-\mathrm{K}$ transitions, the radiative fluorescence yield for the $L$ and higher shells is defined to be an average of its subshell yields. In the presence of $\mathrm{C}-\mathrm{K}$ transitions, the average shell fluorescence yield must be defined more carefully. At least two methods have been proposed for this averaging process [Ba72]. $K$ shell yields, and $L, M$, and higher shell average yields are periodically tabulated as new data and calculations become available [Ba72, Kr79, Hu89, Hu94, Ca03]. The average radiative yield of shell $S$ is denoted by $\bar{\omega}_{S}$.

For photoabsorption in the $L_{1}$ subshell, the $\mathrm{C}-\mathrm{K}$ transition probability is $f_{1}=f_{1,2}+f_{1,3}$. It is larger than the sum of the $L_{1}$ subshell fluorescence yield and Auger yield combined for all elements except near $Z=50-60$, where it is almost equal to the $L_{1}$ Auger yield [Kr79, $\mathrm{Ca} 03$ ]

As $K$ shell absorption is about $80 \%$ of all photoabsorptions, that shell's fluorescence and Auger yields are the most important. Figure 2.13 shows 

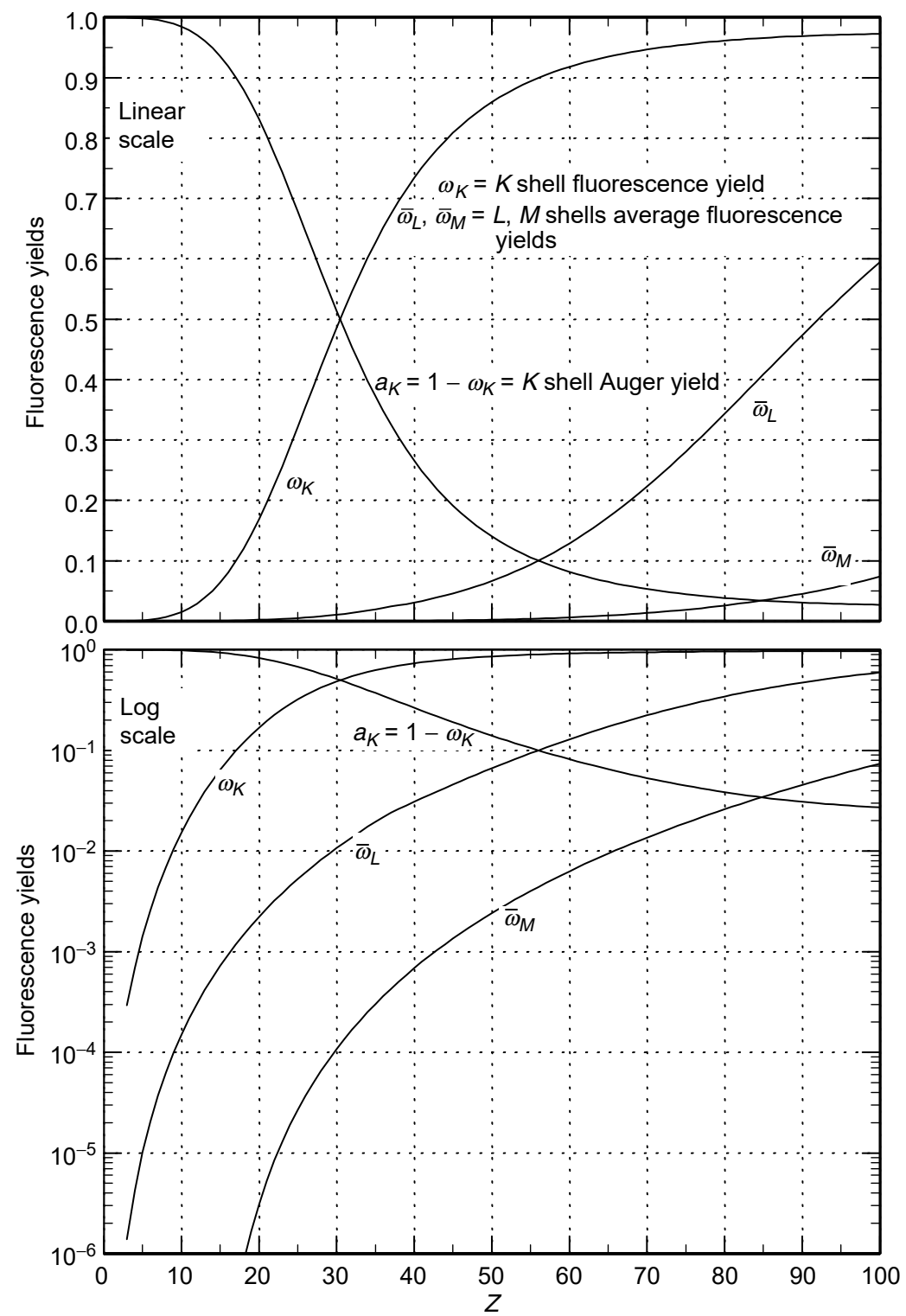

FIGURE 2.13 $K, L, M$ shell fluorescence and Auger yields. Linear and log scales. (Data from [Hu89].) 
$K$ shell fluorescence yield $\omega_{K}$, and the Auger yield, $a_{K}=1-\omega_{K}$, as a function of $Z$. What is actually plotted here is the convenient fit [Hu89]:

$$
\begin{array}{rlrl}
\omega_{\mathrm{K}}(Z) & =\left(\frac{S}{1-S}\right)^{4} & & \\
S & =\sum_{i=0}^{3} c_{i} Z^{i} & & \\
c_{0} & =0.037, & c_{1}=0.03112, \\
c_{2} & =5.44 \times 10^{-5}, & c_{3}=-1.25 \times 10^{-6}
\end{array}
$$

that represents the data very well [Hu89, Hu94]. Average $L$ shell and $M$ shell fluorescence yields, $\bar{\omega}_{L}$ and $\bar{\omega}_{M}$, are also shown.

\subsubsection{Energy Transfer by the Photoelectric Effect}

In addition to the fluorescence yields $\omega$ and $\bar{\omega}$, another parameter that is often tabulated, useful in determining energy transfer to charged particles in the photoelectric effect, is the fraction $f_{\text {pe }}$ of the incident photon energy that ends up in kinetic energy of ejected electrons, per absorbed photon. This includes the kinetic energy of the primary electron and those of subsequent Auger electrons emitted throughout the entire fluorescence cascade. Conversely, one can speak of the fraction of the incident photon energy that goes to fluorescence photons. Let $X$ be the average energy of fluorescence radiation emitted per absorbed photon of energy $E$. Then, the fraction of the incident photon energy that goes to fluorescence photons is $X / E$, and by conservation of energy, $E=X+f_{\mathrm{pe}} E$ or

$$
f_{\mathrm{pe}}=1-(X / E)
$$

$X$ includes the energies of all fluorescence $\mathrm{x}$-rays emitted throughout the entire cascade. Then, the energy put into the material in the form of kinetic energy of electrons is $E-X=f_{\mathrm{pe}} E$ per absorbed photon.

The energy $X$ is tabulated in EPDL97. In aluminum, for example, for an incident photon of any energy $E$ above the $K$ edge $(1.56 \mathrm{keV})$, the average fluorescence photon energy $X$ is approximately $49 \mathrm{eV}$ per photon absorbed, almost independent of $E$, and so $f_{\mathrm{pe}} \approx 1$. The reason for the small value of $X$ when a photon of energy close to $1.5 \mathrm{keV}$ can be emitted is that the fluorescence yield in $\mathrm{Al}$ is only about 0.04 , and it is more likely that no photon is emitted; the most probable photon energy is zero.

In $\mathrm{Au}$, the fluorescence yield is about 0.96, and the $K$ edge is $E_{K}=80.7 \mathrm{keV}$. There, for a photon of any energy $E$ above $E_{K}, X$ is about $59 \mathrm{keV}$, a substantial fraction of $E_{K}$, almost independent of $E$.

A quantitative example at high $Z$, where the fluorescence yield is large, is shown for $\mathrm{W}$ in Figure 2.14, which compares the incident photon energy 


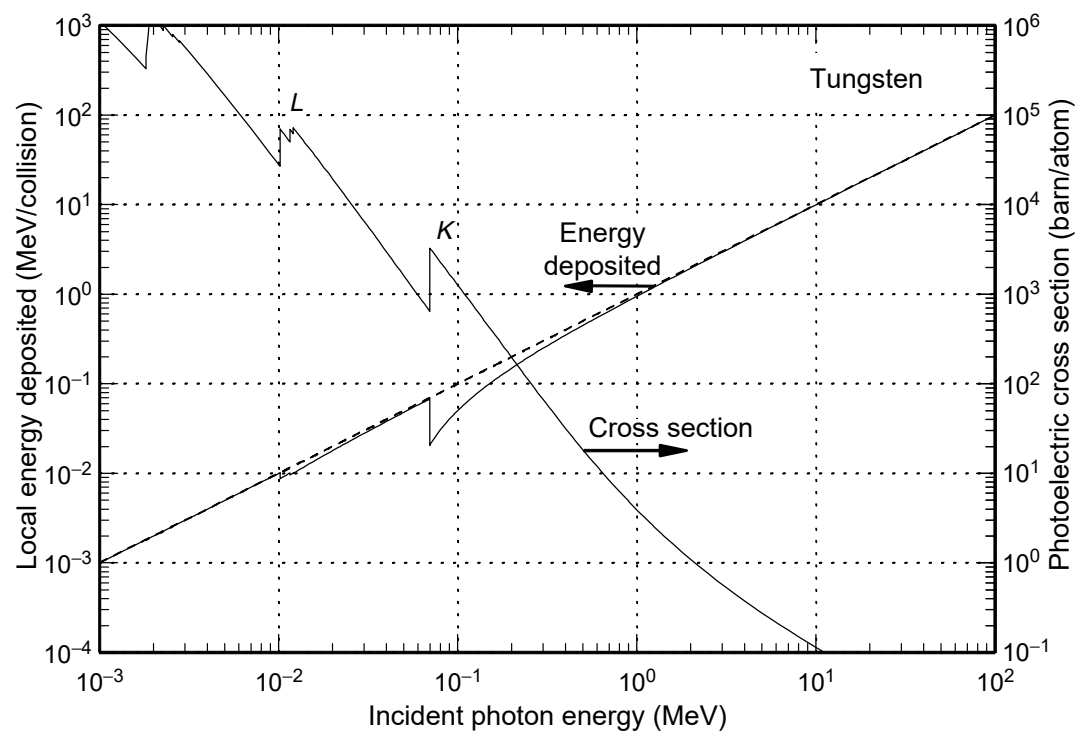

FIGURE 2.14 Energy deposition in Tungsten by photoelectric absorption as a function of incident photon energy. The right-hand scale shows the photoelectric absorption cross section in barn/atom. $K$ and $L$ edges are indicated. The dashed line is the incident photon energy; it would be the energy deposited were it not for fluorescence. Accounting for fluorescence, the solid line is the energy left in the material in the form of kinetic energy of electrons, and is the actual energy deposited locally. The difference from the incident energy is released as characteristic x-rays. With incident energy just above the $K$ edge the fluorescence x-rays have energy just below the $K$ edge, and a mean-free-path in the material some 4.2 times as long as the incident photons. (Data from EPDL97.)

(dashed curve) with the energy transferred to the material in the form of electron kinetic energy. Just above the $K$ edge, the energy deposited drops from the photon energy $E$ to about $0.3 E$, that is, to about that due to photoabsorption by the $L$ edge. As $\omega_{K}$ is large $(\sim 0.95)$, most of the energy due to $K$ shell absorption is returned to fluorescence photons and is not deposited locally. In high- $Z$ materials, fluorescence makes a big difference in energy deposited for photons above an absorption edge, especially the $K$ edge.

\subsubsection{Energy-Transfer Coefficient}

Consider a beam of photons, each of energy $E$, with number fluence $\varphi=\varphi(E)$ (photons $/ \mathrm{cm}^{2}$ ) incident on a material with atom number density $N\left(\mathrm{~cm}^{-3}\right)$. The photon energy fluence is $E \varphi\left(\mathrm{MeV} / \mathrm{cm}^{2}\right)$. The photoelectric cross section is $\sigma_{\mathrm{pe}}\left(\mathrm{cm}^{2} /\right.$ atom). There are $N \sigma_{\mathrm{pe}} \varphi$ photoelectric interactions 
per $\mathrm{cm}^{3}$. Each interaction absorbs a photon, so the energy removed from the beam, per unit volume, is $N \sigma_{\mathrm{pe}} E \varphi\left(\mathrm{MeV} / \mathrm{cm}^{3}\right)$.

Each interaction removes an energy $E$ and deposits an energy $f_{\text {pe }} E$ near the struck atom in the form of electron kinetic energy. The remaining energy $E-f_{\mathrm{pe}} E=X$ exits the atom as other photons. The kinetic energy in charged particles released per unit volume is therefore $f_{\mathrm{pe}} E N \sigma_{\mathrm{pe}} \varphi\left(\mathrm{MeV} / \mathrm{cm}^{3}\right)$. Relative to the incident energy fluence $E \varphi$, the energy transferred to the material (as electron kinetic energy) is $f_{\mathrm{pe}} N \sigma_{\mathrm{pe}}\left(\mathrm{cm}^{-1}\right)$.

The coefficient

$$
\mu_{\mathrm{tr}}=f_{\mathrm{pe}} \sigma_{\mathrm{pe}} N\left(\mathrm{~cm}^{-1}\right)
$$

is called the Linear energy-transfer coefficient. If the atom number density $N$ is expressed instead as per gram, $N_{\mathrm{g}}=N / \rho=N_{A} / A, \mu_{\text {tr }}$ can be written as

$$
\mu_{\mathrm{tr}} / \rho=f_{\mathrm{pe}} \sigma_{\mathrm{pe}} N_{\mathrm{g}}\left(\mathrm{cm}^{2} / \mathrm{g}\right)
$$

and then it is the mass energy-transfer coefficient. When multiplied by the incident photon energy fluence $E \varphi$ it results in the kinetic energy released per gram of material, known as the kerma

$$
\operatorname{kerma}=\left(\mu_{\mathrm{tr}} / \rho\right) E \varphi=f_{\mathrm{pe}} \sigma_{\mathrm{pe}} N_{\mathrm{g}} E \varphi(\mathrm{MeV} / \mathrm{g})
$$

Thus, the significance of $X$ or $f_{\mathrm{pe}}$ lies in its relation to the kerma delivered to the material, and the mass energy-transfer coefficient connects the incident energy fluence to the kerma. The kerma per photon $/ \mathrm{cm}^{2}$ (the specific kerma) is therefore

$$
\text { kerma-cm }{ }^{2}=\left(\mu_{\mathrm{tr}} / \rho\right) E=f_{\mathrm{pe}} \sigma_{\mathrm{pe}} N_{\mathrm{g}} E\left(\mathrm{MeV}-\mathrm{cm}^{2} / \mathrm{g}\right)
$$

This quantity itself, with units often given as $\mathrm{MeV}-\mathrm{cm}^{2} / \mathrm{g}$, is sometimes referred to just as the kerma. As the released electrons with average energy $f_{\mathrm{pe}} E$ then travel through the material, they may lose their own energy to ionization, excitation, or to Bremsstrahlung. The energy lost to ionization and excitation is considered local energy deposition by the original photon. The part lost to Bremsstrahlung is in the form of other photons, which generally travel a long distance compared with the electron range, and is considered removed from the volume of interest. It deposits some distance away only after the Bremsstrahlung photons are themselves absorbed.

The energy-transfer coefficient $\mu_{\mathrm{tr}}$ and kerma are to be distinguished from the energy-absorption coefficient and dose. $\mu_{\mathrm{tr}}$ and kerma measure energy immediately created in the form of charged particle kinetic energy out of the energy of neutral particles (here photons). However, the energy left in the target material, measured by the energy-absorption coefficient or dose, is the energy of the charged particles that is transferred to ionization and excitation. 
It is less than the kerma by the amount that is subsequently lost to Bremsstrahlung as the charged particles pass through the medium. It includes only that part of the energy in charged particles that ends up in the form of ionization or excitation. The energy-absorption coefficient and dose connect the incident energy fluence to the energy that is actually deposited by charged particles.

Further discussion of energy deposition, with proper definition of kerma, is given in Section 2.9.

\subsubsection{Time Scale for Fluorescence}

The term luminescence is a general term for emission of radiation. Fluorescence is a special instance of luminescence. Fluorescence is generally understood to mean the stimulated emission of radiation that occurs over times less than or in the order of $10^{-8} \mathrm{sec}$ [Wi58].

Phosphorescence is another special case of luminescence. It is the stimulated emission of radiation that persists much longer than fluorescence. Its time scale may be microseconds, seconds, days, or months or longer, and occurs primarily in molecular compounds rather than atoms.

Phosphorescence over the longer times may be affected by material temperature, and is the principle of operation of the thermoluminescent diode. Here, after exposure to x-rays, absorbed energy is trapped in a metastable molecular state. Upon heating, thermal excitation occurs to a higher, nearby state from which transitions to a much lower state are allowed, thus giving rise to luminescence when the sample is warmed. Its intensity as a function of temperature then reveals how much original x-ray exposure there was. Modern TLDs trap absorbed energy in trapping centers in semiconductors instead of metastable molecular states, but the principle is the same [Kn00].

The fluorescence yield $\omega_{S}$ by itself carries no information about the mean life $\tau$, or width $\Gamma=\hbar / \tau$, against radiative or Auger emission (although it does fix the radiative to Auger mean life ratio $\left.\tau_{\mathrm{R}} / \tau_{\mathrm{A}}\right)$. $K$ shell radiative mean lives $\tau_{\mathrm{R}}$ are less than $10^{-11} \mathrm{sec}\left(\Gamma_{\mathrm{R}}>10^{-4} \mathrm{eV}\right)$ and Auger mean lives $\tau_{\mathrm{A}}$ are less than $10^{-15} \sec \left(\Gamma_{\mathrm{A}} \gtrsim 10^{-1} \mathrm{eV}\right)$ for all elements with $Z>5$. At $Z=30$, where $\omega_{K} \approx a_{K}, \tau_{\mathrm{R}} \approx \tau_{\mathrm{A}} \approx 6 \times 10^{-16} \sec \left(\Gamma_{\mathrm{R}} \approx \Gamma_{\mathrm{A}} \approx 1 \mathrm{eV}\right)$. Both $\tau_{\mathrm{R}}$ and $\tau_{\mathrm{A}}$ are shorter for $Z>30$, and longer for $Z<30$ [Kr79].

\subsubsection{Fluorescence and Auger Cascades}

A vacancy in an atomic shell may be created by a number of processes: photoabsorption, Compton scattering, nuclear electron capture or internal conversion, or, in the case of an incident electron, electron scattering. No matter how the vacancy (hole) is created, the atom subsequently relaxes by having that vacancy filled either by fluorescence or by an Auger process (including possible $\mathrm{C}-\mathrm{K}$ transitions).

If the primary vacancy is in the $K$ shell, an $L_{2}$ or $L_{3}$ electron may drop down to fill it, and an Auger electron or a photon may then be emitted. If the 
process is fluorescence, there is now a vacancy in the $L$ shell. If by Auger, there may be two vacancies in the $L$ shell or one in the $L$ shell and one in a higher shell. These secondary holes are now filled again either by fluorescence or by an Auger process. The cascade can continue many times for high$Z$ elements. For a primary $K$ shell vacancy in uranium, for example, (U has $K$, $L, M, N, O, P$, and $Q$ shells consisting of 29 subshells) 154 different x-ray energies and 2772 different electron energies can be emitted [Cu95]. As mentioned, for simplicity fluorescence yields and Auger yields are usually computed and tabulated for each shell as averages over the subshells.

\subsubsection{Photoelectron and Auger-Electron Energies}

Energies of photoelectrons in many elements and selected compounds are available at the NIST x-ray Photoelectron Spectroscopy (XPS) Database, (NIST Standard Reference Database 20) [NIST20]. This database was built from an evaluation of the published literature, and contains over 19,000 photoelectron and Auger-electron lines, together with chemical shifts, doublet splittings, and so on. A version is available online.

\subsection{COHERENT RAYLEIGH SCATTERING}

A photon can elastically scatter from the atom as a whole, leaving the atom in its original, unexcited state. As the photon scatters coherently from all the electrons, the process is called coherent scattering (whereas Compton scattering from any one atomic electron is often referred to as incoherent scattering).* Evans [Ev55] has a brief but useful treatment. See also the discussion in [Ha58].

The differential atomic scattering cross section is conveniently written in terms of the differential cross section for elastic scattering from a single, isolated electron (Thomson scattering). The Thomson cross section for unpolarized light is

\footnotetext{
*In general, one should distinguish between elastic scattering and coherent scattering. The term elastic generally means that the scattered photon has the same energy as the incident photon. The term coherent means that the amplitudes for all contributing processes are summed, preserving their relative phases, before squaring to obtain a cross section. An elastic process is not necessarily coherent. The term incoherent means that the amplitudes for all contributing processes are squared before being summed to obtain a cross section. One could have, for example, a coherent scattering process that is not elastic. For present purposes, the terms coherent, elastic, and Rayleigh scattering are taken to be synonymous; the incident and scattered photons have the same energy and the atom is left in its original state. In addition, incoherent means Compton scattering, in which the scattered photon has less energy than the incident photon, and an electron is ejected from the atom.
} 


$$
\frac{\mathrm{d} \sigma_{\mathrm{T}}}{\mathrm{d} \Omega}=\frac{1+\cos ^{2} \theta}{2} r_{\mathrm{o}}^{2}
$$

with total cross section

$$
\int \mathrm{d} \Omega \frac{\mathrm{d} \sigma_{\mathrm{T}}}{\mathrm{d} \Omega}=\frac{8 \pi}{3} r_{\mathrm{o}}^{2} \equiv \sigma_{\mathrm{T}}
$$

whose numerical value was given in Equation 2.8.

To account for the coherent scattering from $Z$ electrons, the cross section for elastic, Rayleigh scattering* from a single, isolated atom with (spherically symmetric) electronic charge density $e \rho(r)$ ( $\rho$ is the electron number density) is written, as a first approximation,

$$
\frac{\mathrm{d} \sigma}{\mathrm{d} \Omega}=\frac{\mathrm{d} \sigma_{\mathrm{T}}}{\mathrm{d} \Omega} F(q)^{2}
$$

where

$$
F(q)=\int \mathrm{d}^{3} r e^{i \vec{q} \cdot \vec{r}} \rho(r)=4 \pi \int \mathrm{d} r r^{2} \frac{\sin (q r)}{q r} \rho(r)
$$

is the usual atomic form factor for scattering through angle $\theta$ while transferring momentum $\hbar \vec{q}$,

$$
\vec{q}=\vec{k}_{\mathrm{o}}-\vec{k}^{\prime}
$$

to the atom. Here, $\hbar \overrightarrow{k_{\mathrm{o}}}$ is the incident photon momentum, and $\hbar \vec{k}^{\prime}$ is the scattered photon momentum.

For elastic scattering, $k^{\prime}=k_{\mathrm{o}}$ and

$$
q=k_{\mathrm{o}} \sqrt{2[1-\cos \theta]}=2 k_{\mathrm{o}} \sin (\theta / 2)=4 \pi \sin (\theta / 2) / \lambda
$$

where $\lambda=2 \pi / k_{\mathrm{o}}$ is the incident wavelength. $F(q)$ is computed in various models for $\rho(r)$. From Equation 2.17, clearly $F(0)=Z$, and $F$ is monotonically decreasing with increasing $q$. Were it not for further corrections, in the forward direction $(q=0)$ the coherent sum of scattering from each electron would make the atomic differential cross section $Z^{2}$ times larger than the Thomson cross section $r_{\mathrm{o}}^{2}$.

\footnotetext{
*The term Rayleigh scattering means different things to different people. See A.T. Young, Phys. Today 35: 42 (January 1982).
} 
Equation 2.16 and Equation 2.17 describe scattering from a distributed charge density, and Equation 2.17 is the usual Born approximation for the form factor. This formulation is deficient in two respects. First, no account is taken of the internal energy level structure of the atom, and thus no resonances are treated, a serious problem at incident energies comparable to atomic levels and edge energies. The Rayleigh cross section has rapid variation and sharp dips near absorption edges; the simple form factor approximation does not account for this anomalous behavior. Second, the form factor is real, meaning the scattering amplitude has no imaginary part and thus violates unitarity. When these effects are accounted for, the differential cross section in the forward direction is no longer $Z^{2} r_{\mathrm{o}}^{2}$.

Anomalous scattering and an imaginary part of the scattering amplitude are usually taken into account by augmenting the form factor $F$ with the anomalous scattering factors $f_{1}$ and $f_{2}$

$$
F \Rightarrow F+f_{1}+i f_{2}
$$

so that the cross section for Rayleigh scattering from an atom becomes

$$
\begin{aligned}
\frac{\mathrm{d} \sigma}{\mathrm{d} \Omega} & =\frac{1+\cos ^{2} \theta}{2} r_{\mathrm{o}}^{2}\left|F(q)+f_{1}+i f_{2}\right|^{2} \\
& =\frac{1+\cos ^{2} \theta}{2} r_{\mathrm{o}}^{2}\left[\left(F(q)+f_{1}\left(k_{\mathrm{o}}\right)\right)^{2}+f_{2}\left(k_{\mathrm{o}}\right)^{2}\right]
\end{aligned}
$$

The anomalous factors are approximately functions of only the incident photon energy, not of $q$. Relativistic effects and nuclear Thomson scattering may or may not be included in the anomalous factors [Ch95a]. A good review of the theory is by Kissel and Pratt [Ki85].

Let $f_{\text {coh }}$ be the scattering amplitude for elastic scattering so that the differential coherent cross section is $\mathrm{d} \sigma / \mathrm{d} \Omega=\left|f_{\text {coh }}\right|^{2}$. According to the optical theorem, the imaginary part of $f_{\text {coh }}$ in the forward direction is related to the total cross section by

$$
\operatorname{Im} f_{\text {coh }}\left(k_{\mathrm{o}}, \theta=0\right)=\frac{k_{\mathrm{o}}}{4 \pi} \sigma_{\mathrm{Tot}}\left(k_{\mathrm{o}}\right)
$$

This means that $f_{2}$ is given by the total cross section according to

$$
f_{2}\left(k_{\mathrm{o}}\right)=\frac{E}{2 h c r_{\mathrm{o}}} \sigma_{\mathrm{Tot}}(E)
$$

where $E=\hbar k_{\mathrm{o}} c$ is the photon energy. A common alternative form of this last equation is $\sigma_{\mathrm{Tot}}(E)=2 \lambda r_{\mathrm{o}} f_{2}\left(k_{\mathrm{o}}\right)$. Except at higher energies where the 
Compton cross section contributes, $\sigma_{\text {Tot }}$ may be replaced in this equation by the total photoelectric cross section $\sigma_{\mathrm{pe}}$.

Recent compilations of form factors and anomalous scattering factors are given by Wang et al. [Wa93] and Chantler [Ch95a]; available in digitized tabular form on the NIST web sites [Ch95b, Ch95c, Ch00]. Henke et al. [He93] also provides a tabulation. In addition, $F, f_{1}$, and $f_{2}$ are given in the Evaluated Photon Data Library (EPDL) from LLNL (see Section 2.12). The form factor $F(q)$ is fairly well understood and agreed upon, and is not given in [He93] or [Ch95a]. However, recent compilations of $f_{1}$ and $f_{2}$ vary considerably below a few keV. Figure 2.15 compares three sets of anomalous form factors $f_{1}$ and $f_{2}$ in $\mathrm{Fe}$, while Figure 2.16 shows the resulting angular distribution and total Rayleigh cross section computed from the same three compilations of the anomalous scattering factors at $300 \mathrm{eV} . F(q)$ from EPDL97 was used in all four. Computations improve with time, and seem to be converging in the most recent tabulations, but there has been considerable change since 1989. Agreement is better at higher energies. A fifth compilation, from $50 \mathrm{keV}$ to $1.5 \mathrm{MeV}$, can be found in [Ch98]. The earlier 1989 compilation, EPDL89, is included in the figure to demonstrate progress toward convergence. However, in all cases, the 1997 compilation, EPDL97, is to be preferred over EPDL89.

The cross section for coherent scattering is almost never more than $10 \%$ of competing cross sections (photoelectric and Compton) at any energy. It is shown as a function of energy in the plots in Section 2.8 of all cross sections for many elements. It is largest (i.e., near $10 \%$ of the total) at energies of $10 \mathrm{keV}-100 \mathrm{keV}$, depending on $Z$. However, the differential cross section is strongly peaked in the forward direction. Figure 2.17 shows the differential Rayleigh cross section for atomic aluminum as a function of scattering angle $\theta$ for several energies, and Figure 2.18 shows the angular distribution in polar coordinates. Figure 2.19 and Figure 2.20 show the same for lead. Figure 2.21 shows the polar angle $\theta$ inside of which $50 \%$ or $90 \%$ of scattered photons emerge, in four elements. In $\mathrm{Al}$, for example, at $100 \mathrm{keV}$, one half of scattered photons emerge at less than about $9^{\circ}$. Scattered photons emerge more and more nearly in the forward direction as energy increases and $Z$ decreases.

Thus, Rayleigh scattering peaks generally in the forward direction. It will be seen later that Compton scattering from a free electron also peaks generally in the forward direction (although for scattering from atoms it vanishes in a narrow cone immediately around $\theta=0$ ). Thus, although both coherent and incoherent scattering do (may) scatter photons out of the original beam direction, if one needs accurate calculations of an incident photon beam attenuation, one must consider the angle from incidence inside of which one may consider a scattered photon still to be in the (attenuated) beam. 

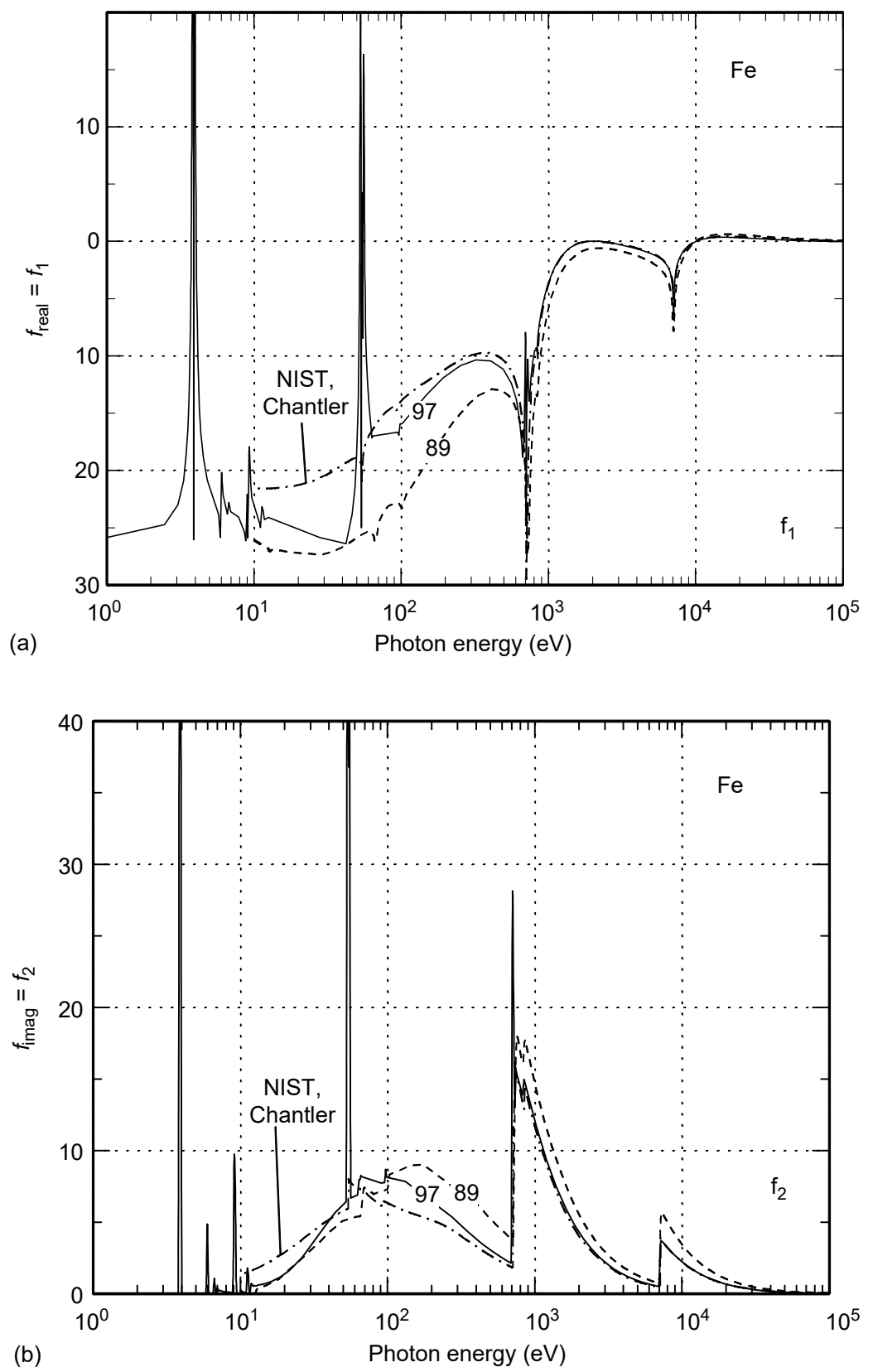

FIGURE 2.15 (a) Comparison of three recent compilations of the real part of the anomalous scattering factor $f_{1}$ in Fe. See text. (b) Comparison of three recent compilations of the imaginary part of the anomalous scattering factor $f_{2}$ in Fe. (Chantler's data are on the NIST web site, "89" is from EPDL89, "97" is from EPDL97. See text.) 


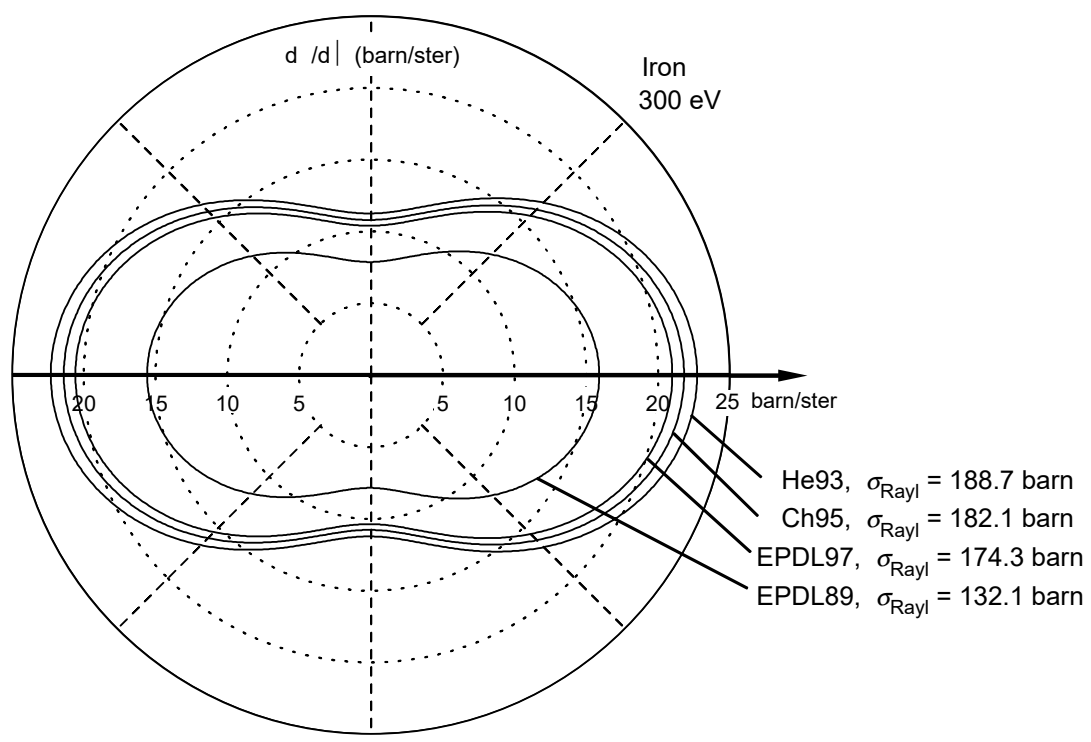

FIGURE 2.16 Illustrating variations in recent compilations of Rayleigh scattering angular distribution and total cross section, on $\mathrm{Fe}$ at incident photon energy of $300 \mathrm{eV}$. This plot was constructed from the form factors $F(q), f_{1}$, and $f_{2}$ compiled in the cited references, using Equation 2.21. Ch95 is from [Ch95a] and [Ch95b].

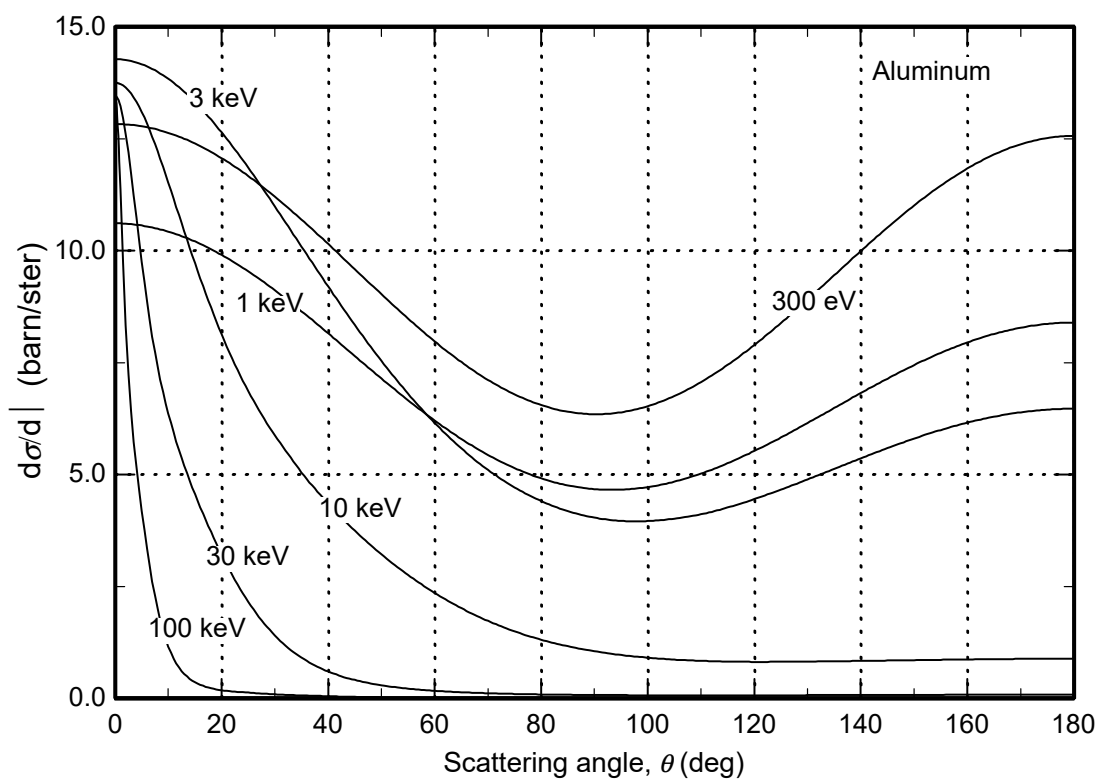

FIGURE 2.17 Coherent (Rayleigh) scattering angular distribution on atomic aluminum. (Data from EPDL97.) 

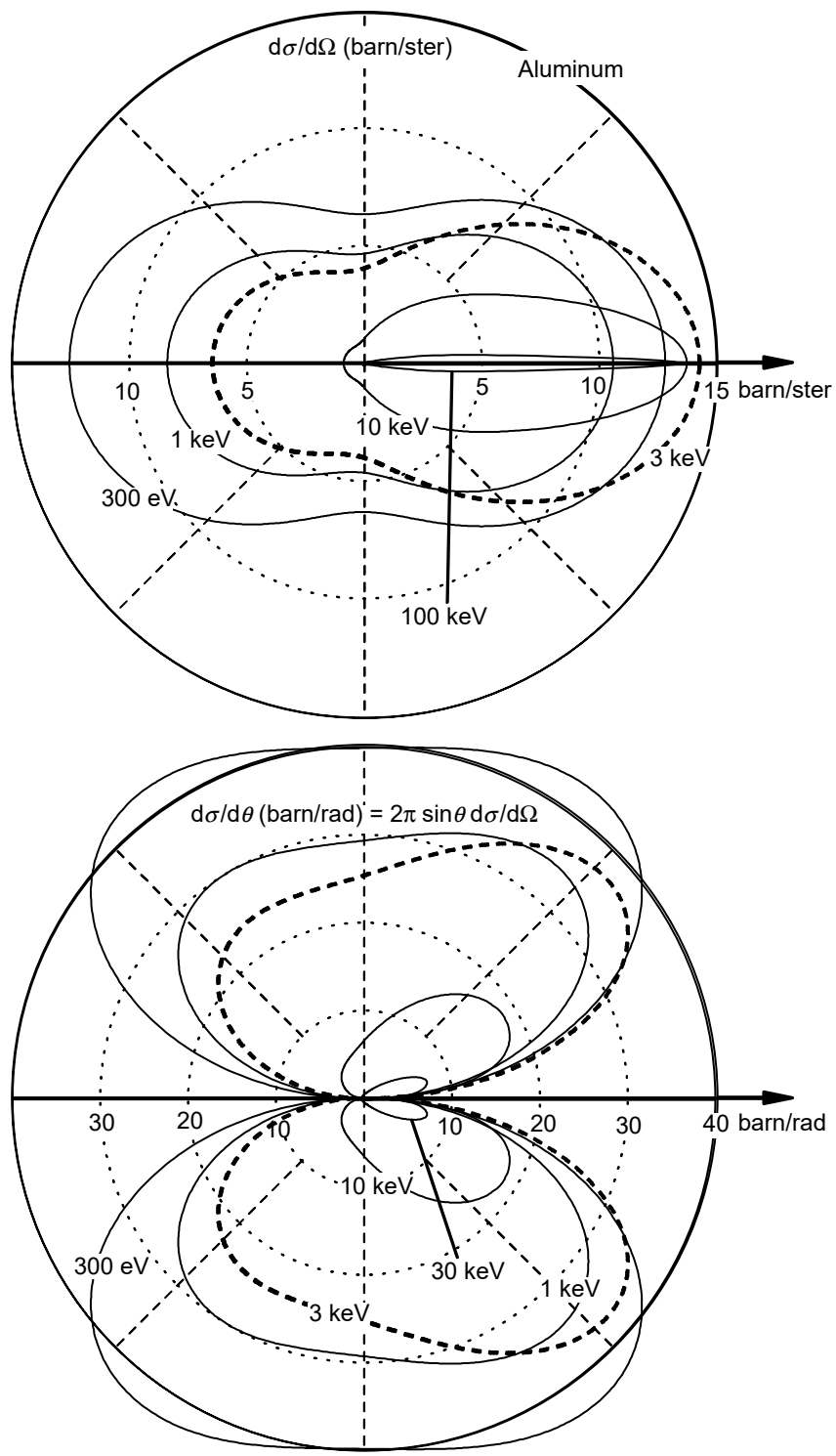

FIGURE 2.18 Angular distribution of coherent (Rayleigh) scattering on atomic aluminum. Upper panel: $\mathrm{d} \sigma / \mathrm{d} \Omega$ (barn/ster). Lower panel: $\mathrm{d} \sigma / \mathrm{d} \theta($ barn $/ \mathrm{rad})=2 \pi \times$ $\sin \theta \mathrm{d} \sigma / \mathrm{d} \Omega$. (Data from EPDL97.)

The latest developments in photon elastic scattering from atoms may be found on the LLNL web site: http://www-phys.llnl.gov/Research/scattering/. 


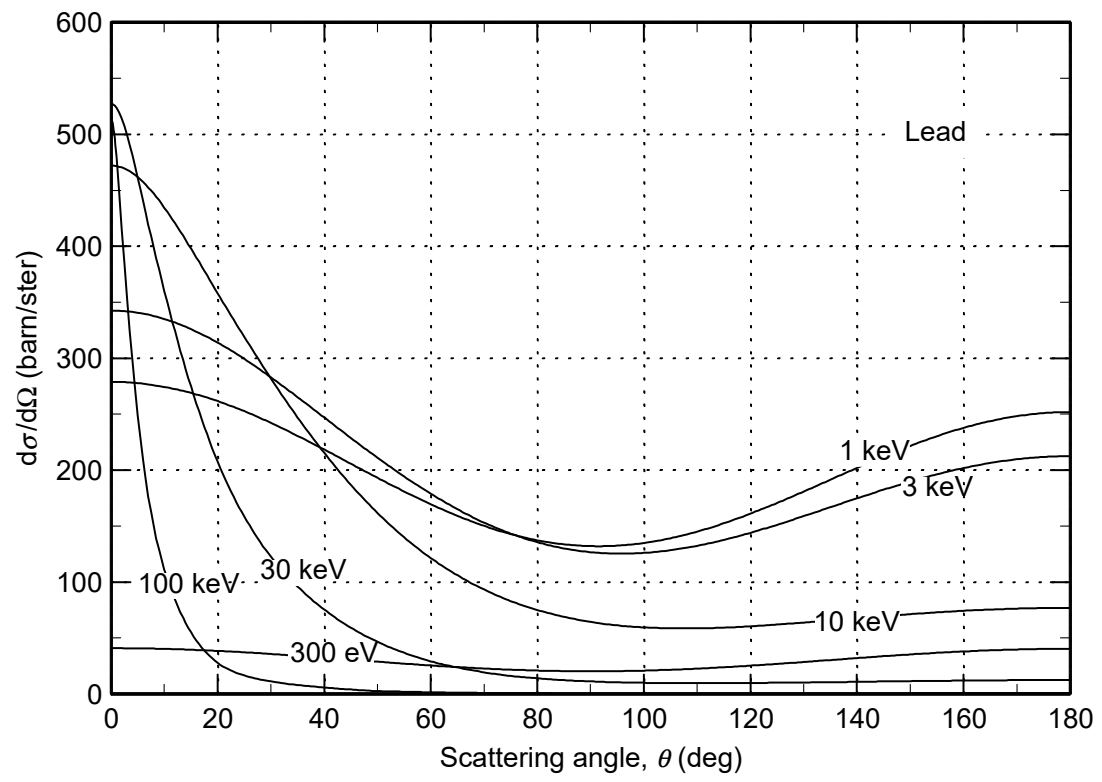

FIGURE 2.19 Coherent (Rayleigh) scattering angular distribution on atomic lead. (Data from EPDL97.)

\subsection{COMPTON SCATTERING}

The Compton effect is the inelastic scattering of a photon from an electron. The electron recoils and is ejected from the atom, the scattered photon emerges with reduced energy, and the atom is left ionized, with a vacancy in the shell that had been occupied by the ejected electron. It is the dominant process for typical energies of nuclear $\gamma$ rays, hundreds of $\mathrm{keV}$ to several $\mathrm{MeV}$.

Bethe and Ashkin [Be53], Evans [Ev55], and Heitler [He54] have excellent discussions of Compton scattering, and useful formulas. Work that is more recent is summarized by Williams [Wi77].

Compton scattering of a photon from electrons bound in an atom differs from scattering from a free electron. The differential cross section for Compton scattering from a single, free electron is the Klein-Nishina formula $\mathrm{d} \sigma_{\mathrm{KN}} / \mathrm{d} \Omega$, Equation 2.33. The differential (in outgoing photon angle) Compton scattering cross section from an atom is sometimes expressed in terms of the Compton scattering from a single electron as

$$
\frac{\mathrm{d} \sigma_{\mathrm{atom}}}{\mathrm{d} \Omega}=\frac{\mathrm{d} \sigma_{\mathrm{KN}}}{\mathrm{d} \Omega} S(q, Z)
$$

where $S(q, Z)$ is the incoherent scattering function for that atom. $S$ describes the effects of the different binding energies of electrons in 

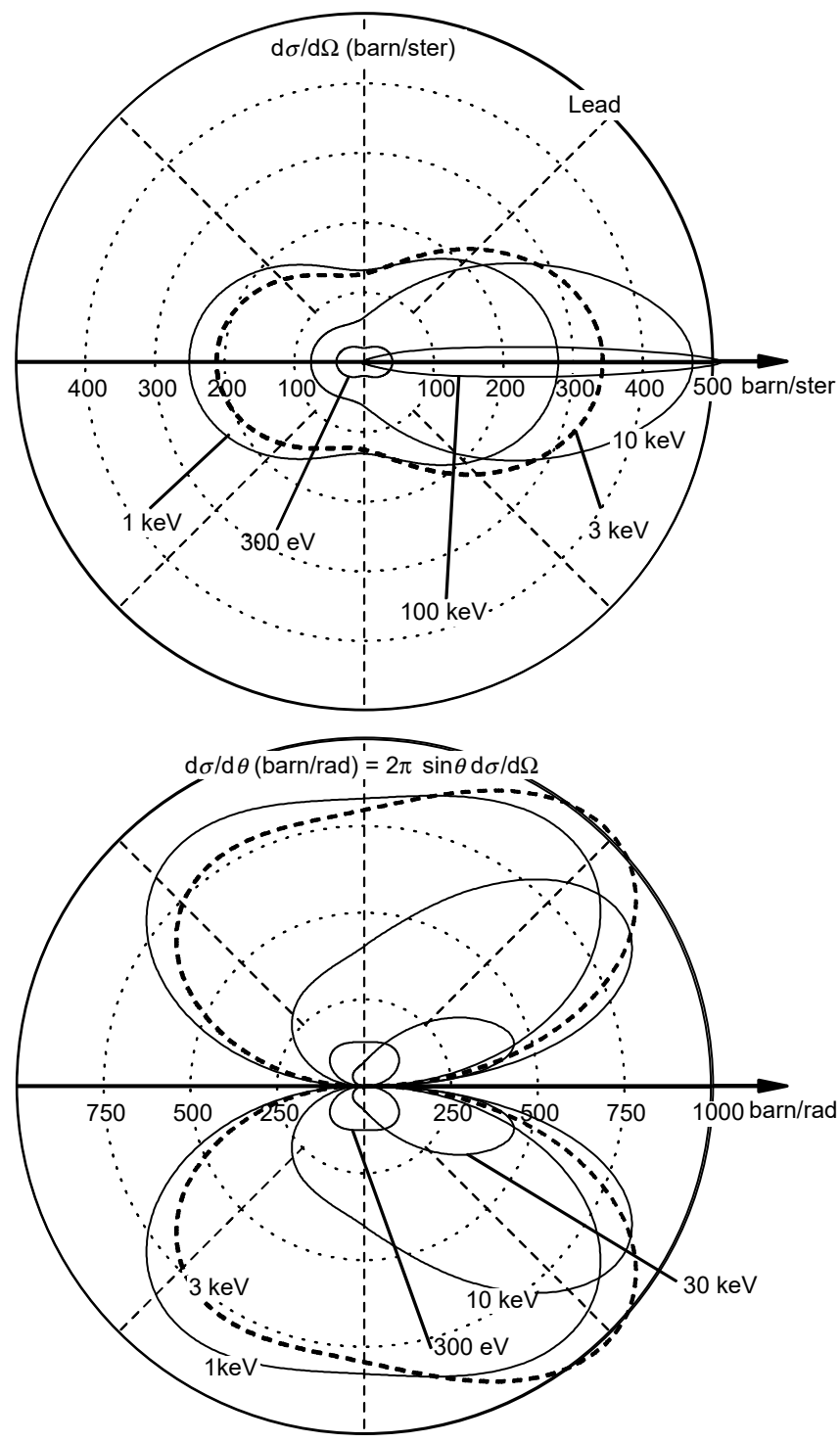

FIGURE 2.20 Angular distribution of coherent (Rayleigh) scattering on atomic lead. Upper panel: $\mathrm{d} \sigma / \mathrm{d} \Omega$ (barn/ster). Lower panel: $\mathrm{d} \sigma / \mathrm{d} \theta$ (barn/rad) $=2 \pi \sin \theta \mathrm{d} \sigma / \mathrm{d} \Omega$. (Data from EPDL97.) 


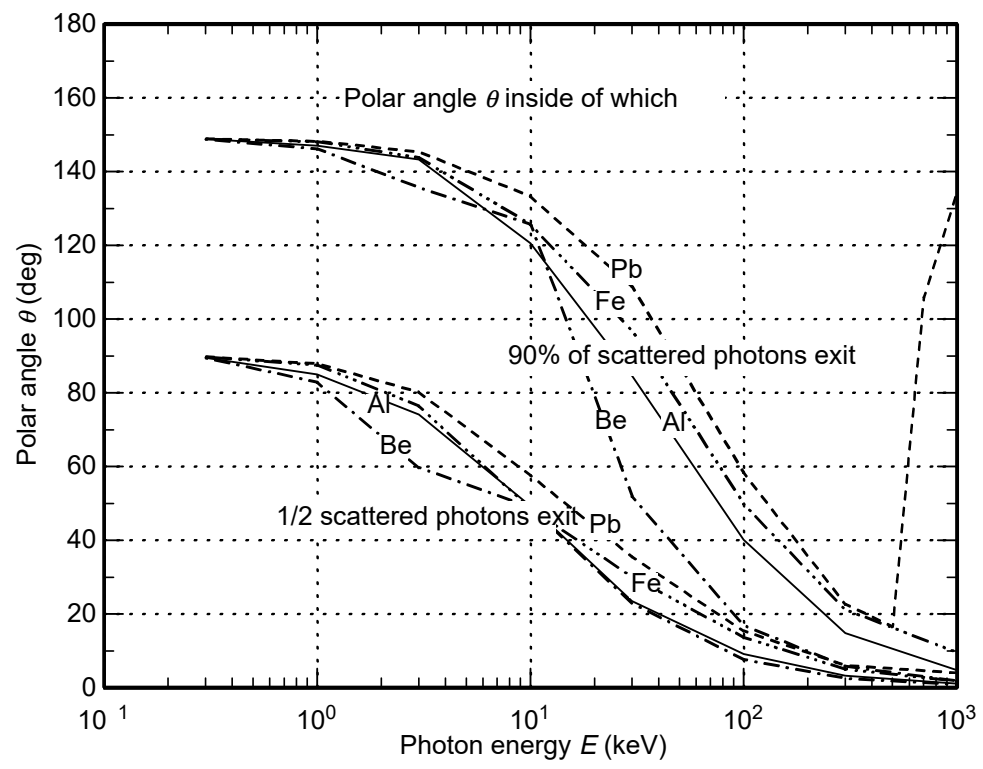

FIGURE 2.21 Concentration of coherent Rayleigh scattering in the forward direction at high energy, in $\mathrm{Be}, \mathrm{Al}, \mathrm{Fe}$, and $\mathrm{Pb}$. In $\mathrm{Be}$ at $100 \mathrm{keV} 50 \%$ of photons scatter at less than $7.5^{\circ}$, and $90 \%$ scatter at less than $17^{\circ}$. (Data from EPDL97). (In Pb above 500 $\mathrm{keV}$, in spite of sharp forward peaking, there is small scattering at all angles, and $90 \%$ does not accumulate until more than $100^{\circ}$.)

different atomic shells. Here, $\hbar q$ is the magnitude of the momentum transferred to the recoil electron,

$$
\begin{aligned}
& \vec{q}=\overrightarrow{k_{\mathrm{o}}}-\vec{k}^{\prime} \\
& q=\frac{E}{\hbar c}\left[1-2 \frac{\nu^{\prime}}{\nu_{0}} \cos \theta_{\gamma}+\left(\frac{\nu^{\prime}}{\nu_{0}}\right)^{2}\right]^{1 / 2}
\end{aligned}
$$

where $E=\hbar k_{\mathrm{o}} c=h \nu_{0}$ is the incident photon energy, $E^{\prime}=\hbar k^{\prime} c=h \nu^{\prime}$ is the scattered photon energy, and $\theta_{\gamma}$ is the outgoing angle of the scattered photon relative to the incident photon direction. At high-energy or high-momentum transfer $q$, binding energies make no difference, and $S(q \rightarrow \infty, Z) \rightarrow Z$; the cross section is simply the sum of the Compton scatterings for each electron. Cross sections add (rather than amplitudes) because the atom is left in a different state according to which electron is ejected. At very low energies, $\sigma_{\mathrm{KN}} \rightarrow \sigma_{\mathrm{T}}$, but $S$, $\mathrm{d} \sigma_{\text {atom }} / \mathrm{d} \Omega$, and the total cross section $\sigma_{\text {atom }}=$ $\int\left(\mathrm{d} \sigma_{\text {atom }} / \mathrm{d} \Omega\right) d \Omega$ approach 0 , because of binding energies. The incoherent scattering function on many elements has recently been reviewed by Wang et al. [Wa93] and Kahane [Ka98]. 
For a free electron, kinematics determines $E^{\prime}$ in terms of $\theta_{\gamma}$, so $q$ is a function of only $\theta_{\gamma}$ or $E^{\prime}$, but not both. The Compton electron takes the remaining energy. However, for an atom, the kinetic energy of the ejected electron is smaller than the free electron kinematic expression by the binding energy. If less than the binding energy is transferred to the bound electron, Compton scattering (defined on an atom as ejecting an electron) cannot occur. For incident photon energies well above absorption edges, the binding energy makes little difference, and the angular distribution from an atom will be close to that from a free electron (except very near $\theta_{\gamma}=0$; see Section 2.6.2 on Compton scattering from atoms).

When one wishes to investigate the detailed energy and angle distribution of the outgoing photon from atomic Compton scattering, one works with the doubly differential cross section $\mathrm{d}^{2} \sigma / \mathrm{d} E^{\prime} \mathrm{d} \Omega$ [Be93]. These considerations are usually of interest when one measures the scattered photon and wishes to infer information about the structure of the struck atom. In addition, the orbital velocity of the struck electron in the atom produces a Doppler broadening of the incident photon apparent frequency, resulting in a broadening of outgoing photon energy at a given angle. The shape of this broadened line is the Compton profile, and is a current topic of research ([Hu99a] and references therein). The current state of calculations of incoherent scattering has been summarized by Bergstrom and Pratt [Be97].

\subsubsection{Compton Scattering from a Free Electron}

Properties such as angular distributions are easier to understand in the case of scattering from a free electron, and the differences from atomic scattering are generally small. The major difference is in photon scattering near the forward direction, discussed separately in a later section.

Here we present formulas and graphs for Compton scattering from a single, free electron. Atomic (barn/atom) and bulk cross sections $\left(\mathrm{cm}^{2} / \mathrm{g}\right)$ and mfps in matter are presented in a later section.

Our discussion and graphs are for an unpolarized photon. Formulas for polarized photons, which are needed much less frequently, can be found in Heitler [He54], Evans [Ev55], and Evans [Ev58].

Figure 2.22 defines scattering angles in the scattering plane. The incident photon energy is $E=h \nu_{0}$, and the scattered photon energy is $E^{\prime}=h \nu^{\prime}$. The photon scattering angle relative to incident photon direction is $\theta_{\gamma}$; the electron scattering angle is $\theta_{\mathrm{e}}$. (Referring to Evans [Ev55], our photon scattering angle $\theta_{\gamma}$ is Evans' $\vartheta$; our electron scattering angle $\theta_{\mathrm{e}}$ is Evans' $\varphi$ ). $T$ is the outgoing electron kinetic energy.

\subsubsection{Kinematics}

The energy and momentum conservation laws determine all final momenta in terms of one final parameter, often taken as the scattered photon outgoing 


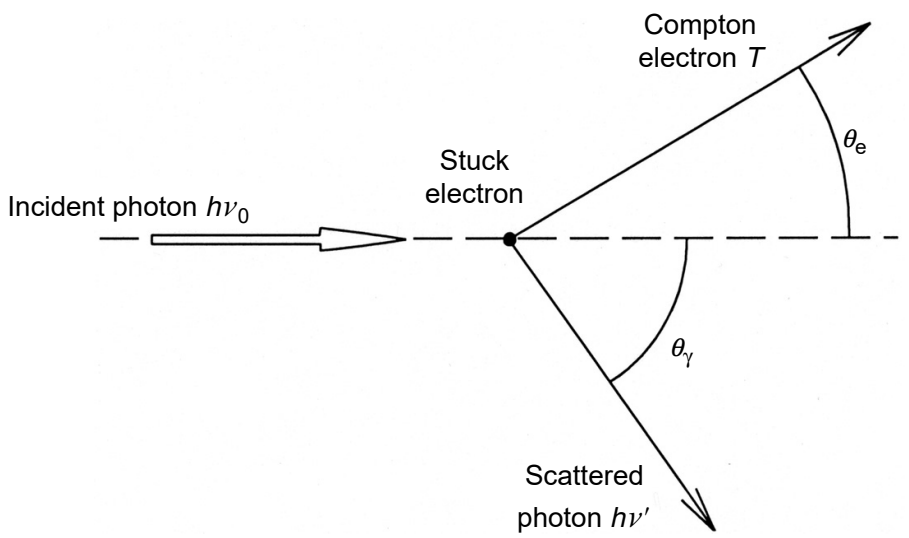

FIGURE 2.22 Angle and energy definitions for Compton scattering.

angle $\theta_{\gamma}$. The relations were first worked out by Compton in 1923. Following Davisson and Evans [Da52] and Evans [Ev55], the scattered photon energy is

$$
E^{\prime}=h \nu^{\prime}=\frac{h \nu_{0}}{1+\alpha\left(1-\cos \theta_{\gamma}\right)}
$$

which determines the frequency shift $\nu^{\prime}-\nu_{0}$ of the scattered radiation. Here, $\alpha$ is the commonly defined ratio of the incident photon energy to the electron rest energy

$$
\alpha \equiv \frac{h \nu_{0}}{m c^{2}}
$$

The photon may scatter through any angle from 0 to $\pi$. For incident photon energies much lesser than $m c^{2} / 2=255 \mathrm{keV}, \alpha \ll 1$, the scattered energy is independent of scattering angle, and the scattered energy is the incident energy; this low energy case is simply Thomson scattering. Equation 2.26 for scattered photon energy $h \nu^{\prime}$ as a function of $\theta_{\gamma}$ is graphed later in Figure 2.28.

When the photon backscatters, its energy is

$$
h \nu^{\prime}\left(\theta_{\gamma}=\pi\right)=\frac{h \nu_{0}}{1+2 \alpha}=\frac{1}{2} \frac{m c^{2}}{1+m c^{2} / 2 h \nu_{0}} \quad \text { (backscatter) }
$$

This is also the minimum energy the scattered photon may have, $h \nu^{\prime}\left(\theta_{\gamma}=\pi\right)<h \nu^{\prime}<h \nu_{0}$. At low energies (Thomson scattering), the backscattered photon may have its original energy. For incident photon energies much greater than $m c^{2} / 2$, the backscattered photon energy saturates to a maximum of $m c^{2} / 2$. 
The shift in photon wavelength, from the incident $\lambda_{0}=c / \nu_{0}$ to the scattered photon $\lambda^{\prime}=c / \nu^{\prime}$, is

$$
\lambda^{\prime}-\lambda_{0}=\lambda_{\mathrm{C}}\left(1-\cos \theta_{\gamma}\right)
$$

where $\lambda_{\mathrm{C}} \equiv h / m c=2.426 \times 10^{-10} \mathrm{~cm}$ is the Compton wavelength of the electron ( $m$ is the electron rest mass). In Compton scattering, the wavelength shift depends only on the scattered photon angle, not on the incident photon energy.

The outgoing electron has kinetic energy

$$
\begin{aligned}
T & =h \nu_{0}-h \nu^{\prime} \\
& =h \nu_{0} \frac{\alpha\left(1-\cos \theta_{\gamma}\right)}{1+\alpha\left(1-\cos \theta_{\gamma}\right)} \\
& =h \nu_{0} \frac{2 \alpha \cos ^{2} \theta_{\mathrm{e}}}{(1+\alpha)^{2}-\alpha^{2} \cos ^{2} \theta_{\mathrm{e}}}
\end{aligned}
$$

and its outgoing angle $\theta_{\mathrm{e}}$ is related to that of the scattered photon $\theta_{\gamma}$ by

$$
\cot \theta_{\mathrm{e}}=(1+\alpha) \frac{1-\cos \theta_{\gamma}}{\sin \theta_{\gamma}}=(1+\alpha) \tan \frac{\theta_{\gamma}}{2}
$$

or conversely,

$$
\cos \theta_{\gamma}=1-\frac{2}{(1+\alpha)^{2} \tan ^{2} \theta_{\mathrm{e}}+1}
$$

For scattering from a free electron $T=h \nu_{0}-h \nu^{\prime}$ as earlier, but in atomic scattering the outgoing electron kinetic energy is smaller than $h \nu_{0}-h \nu^{\prime}$ by its binding energy.

The cross section for scattering numbers of photons through angle $\theta_{\gamma}$ is the well-known Klein-Nishina cross section (for unpolarized photons)

$$
\frac{\mathrm{d} \sigma_{\mathrm{KN}}}{\mathrm{d} \Omega}=\frac{r_{\mathrm{o}}^{2}}{2} \frac{1}{\left[1+\alpha\left(1-\cos \theta_{\gamma}\right)\right]^{2}}\left[1+\cos ^{2} \theta_{\gamma}+\frac{\alpha^{2}\left(1-\cos \theta_{\gamma}\right)^{2}}{1+\alpha\left(1-\cos \theta_{\gamma}\right)}\right]
$$

where $\mathrm{d} \Omega=\sin \theta_{\gamma} \mathrm{d} \theta_{\gamma} \mathrm{d} \varphi_{\gamma}$ is the solid angle in the direction of the scattered photon. $\mathrm{d} \sigma_{\mathrm{KN}} / \mathrm{d} \Omega$ peaks in the forward direction, $\theta_{\gamma}=0$, but $\mathrm{d} \sigma_{\mathrm{KN}} / \mathrm{d} \theta_{\gamma}=2 \pi \sin \theta_{\gamma} \mathrm{d} \sigma_{\mathrm{KN}} / \mathrm{d} \Omega$ peaks at $\theta_{\gamma}>0$.

Only the energy $h \nu^{\prime}$ has been scattered, whereas the energy $T=h \nu_{0}-h \nu^{\prime}$ has been transferred to the electron. Since $h \nu^{\prime}$ has been scattered, while $h \nu_{0}$ was incident, the cross section for scattering photon energy through angle $\theta_{\gamma}$ is $\mathrm{d} \sigma_{\mathrm{s}} / \mathrm{d} \Omega=\left(h \nu^{\prime} / h \nu_{0}\right) \mathrm{d} \sigma_{\mathrm{KN}} / \mathrm{d} \Omega$. Because of the distinction between scattering numbers of photons and energy of photons, references [Da52] and [Ev55] 
refer to $\mathrm{d} \sigma_{\mathrm{KN}} / \mathrm{d} \Omega$ as the collision cross section and to $\mathrm{d} \sigma_{\mathrm{s}} / \mathrm{d} \Omega$ as the scattering cross section. However, this terminology does not seem to have gained wide acceptance; rather the terms collision cross section and scattering cross section are regarded as synonymous, both referring to the collision cross section for the scattering of numbers of particles (in this case $\mathrm{d} \sigma_{\mathrm{KN}} / \mathrm{d} \Omega$ ). If the scattering of energy is intended, it is stated explicitly.

For the given incident photon energy, $h \nu_{0}, \mathrm{~d} \sigma_{\mathrm{KN}} / \mathrm{d} \Omega$ is a function of only $\theta_{\gamma}$, but can also be written as

$$
\begin{aligned}
\frac{\mathrm{d} \sigma_{\mathrm{KN}}}{\mathrm{d} \Omega} & =\frac{r_{\mathrm{o}}^{2}}{2} \frac{\nu^{\prime 2}}{v_{0}^{2}}\left[\frac{\nu_{0}}{\nu^{\prime}}+\frac{\nu^{\prime}}{\nu_{0}}-\sin ^{2} \theta_{\gamma}\right] \\
& =r_{\mathrm{o}}^{2} \frac{\nu^{\prime 2}}{\nu_{0}^{2}}\left[\frac{1+\cos ^{2} \theta_{\gamma}}{2}+\frac{\left(\nu_{0}-\nu^{\prime}\right)^{2}}{2 \nu_{0} \nu^{\prime}}\right]
\end{aligned}
$$

showing that it differs from the Thomson differential cross section $\mathrm{d} \sigma_{\mathrm{T}} / \mathrm{d} \Omega$, Equation 2.14, in two ways. First, to the angular dependence $(1 / 2)\left(1+\cos ^{2} \theta_{\gamma}\right)$ is added the term $\left(\nu_{0}-\nu^{\prime}\right)^{2} / 2 \nu_{0} \nu^{\prime}$ which arises from the matrix element. This term vanishes as $h \nu_{0} \rightarrow 0$, since there $\nu^{\prime} \rightarrow \nu_{0}$. And second, it differs by the overall factor $\left(\nu^{\prime} / \nu_{0}\right)^{2}$ whose origin is purely due to phase space, not the matrix element. In concert with the first term, this second factor reduces scattering at large angles, where $h \nu^{\prime}$ can be much lesser than $h \nu_{0}$ (the electron carries off most of the energy). In the forward direction, $\theta_{\gamma}=0, \nu^{\prime}=\nu_{0}$, and the two corrections leave the Thomson differential cross section, $r_{\mathrm{o}}^{2}$; at all energies, the Compton differential cross section on a free electron in the forward direction is equal to the Thomson differential cross section, $\mathrm{d} \sigma_{\mathrm{KN}} / \mathrm{d} \Omega\left(\theta_{\gamma}=0\right)=\mathrm{d} \sigma_{\mathrm{T}} / \mathrm{d} \Omega\left(\theta_{\gamma}=0\right)=r_{\mathrm{o}}^{2}$.

At low energy, $\nu^{\prime} \rightarrow \nu_{0}$, and free electron Compton scattering approaches Thomson scattering at all angles, $\mathrm{d} \sigma_{\mathrm{KN}} / \mathrm{d} \Omega \rightarrow \mathrm{d} \sigma_{\mathrm{T}} / \mathrm{d} \Omega$.

\subsubsection{Total Compton Cross Section}

Integrating $\mathrm{d} \sigma_{\mathrm{KN}} / \mathrm{d} \Omega$ over solid angles, one obtains the Klein-Nishina total cross section for Compton scattering from a single, free electron (and for unpolarized incident and outgoing photons)

$$
\sigma_{\mathrm{KN}}=2 \pi r_{\mathrm{o}}^{2}\left\{\frac{1+\alpha}{\alpha^{2}}\left[\frac{2(1+\alpha)}{1+2 \alpha}-\frac{1}{\alpha} \ln (1+2 \alpha)\right]+\frac{1}{2 \alpha} \ln (1+2 \alpha)-\frac{1+3 \alpha}{(1+2 \alpha)^{2}}\right\}
$$

It is shown as the solid line in Figure 2.23.

The Compton cross section for photons scattering from atoms is approximately $Z \sigma_{\mathrm{KN}}$. However, at low energies it is less than $Z \sigma_{\mathrm{KN}}$ for two reasons. First, at energies below an element's $K$ edge, the $K$ electrons cannot 


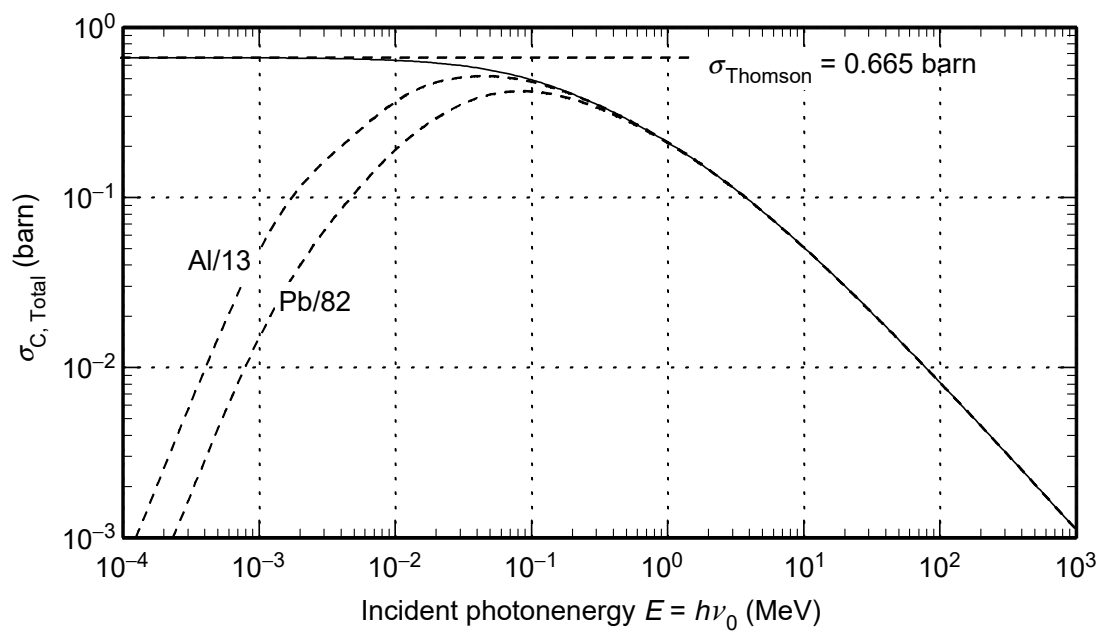

FIGURE 2.23 Klein-Nishina Compton cross section on a free electron (solid). Compton cross section on $\mathrm{Al}$ and $\mathrm{Pb}$ (dashed) divided by their atomic number.

be ejected, and so the effective atomic number for Compton scattering is $Z-2$. Below higher edges, even fewer electrons can contribute, and so the cross section is reduced again. Of course, this occurs only at energies below x-ray edges, where the Compton effect is much smaller than photoabsorption. Second, as will be seen later, at all energies the Compton differential scattering from an atom is smaller than $Z \mathrm{~d} \sigma_{\mathrm{KN}} / \mathrm{d} \Omega$ inside some fairly small angle near the forward direction (and actually vanishes inside a very small angle). When integrated over all solid angles, this forward dip makes the atomic Compton cross section smaller than $Z \sigma_{\mathrm{KN}}$, even at energies well above an atom's $K$ edge. Figure 2.23 shows $1 / Z$ times the total Compton cross section on $\mathrm{Al}$ and $\mathrm{Pb}$. The difference is dramatic below $100 \mathrm{keV}$.

\subsubsection{Particle Characteristic Energies}

Given an outgoing scattering angle for either the electron or scattered photon, kinematics fixes the energies. For the given incident photon energy $h \nu_{0}$, Figure 2.24 shows the maximum and the average Compton electron kinetic energies (averaged over all outgoing angles), and the average energy of the scattered photon, as a fraction of $h \nu_{0}$. From Equation 2.30 at $\theta_{\gamma}=\pi$, the electron maximum energy $T_{\max }$ is

$$
T_{\max }=\frac{h \nu_{0}}{1+1 /(2 \alpha)}
$$




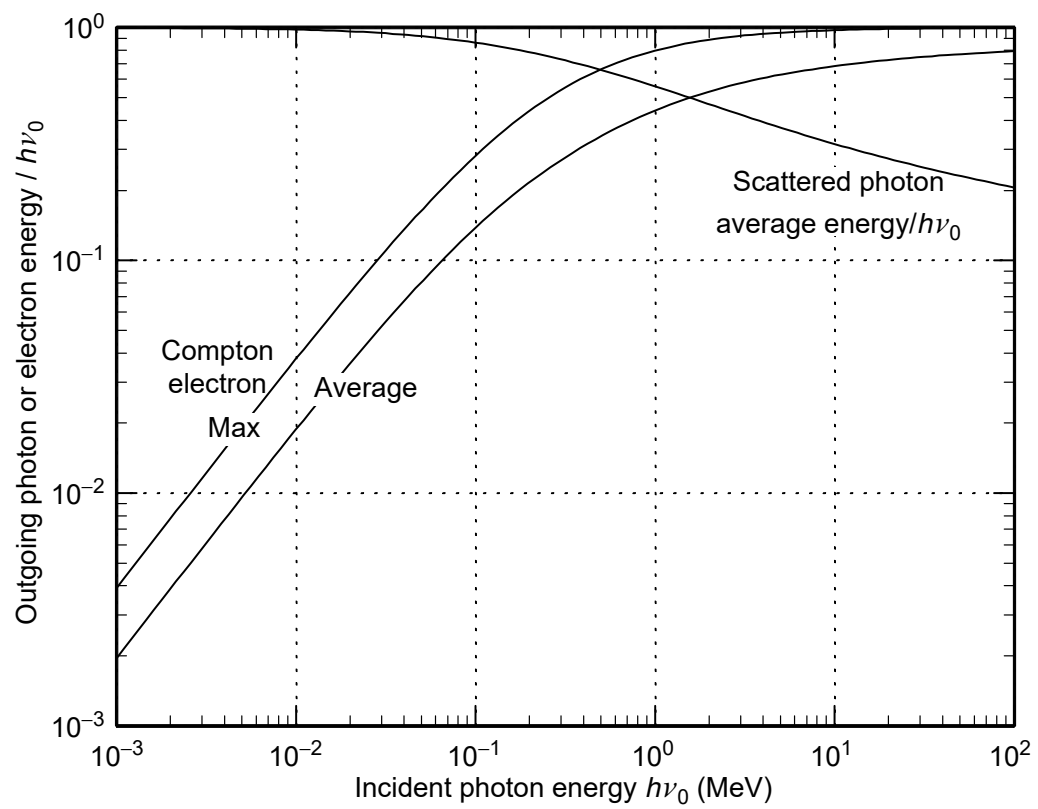

FIGURE 2.24 Average and maximum energies of Compton electron, and average energy of scattered photon, normalized to incident photon energy $h \nu_{0}$.

$\alpha$ was defined in Equation 2.27. The maximum electron energy $T_{\max }$ occurs when the electron exits in the forward direction, $\theta_{\mathrm{e}}=0$, and the photon backscatters, $\theta_{\gamma}=\pi$.

\subsubsection{Compton Electron Energy Distribution}

The distribution $\mathrm{d} \sigma / \mathrm{d} T$ of outgoing electron energy $T$ can be obtained by combining the photon angular distribution $\mathrm{d} \sigma_{\mathrm{KN}} / \mathrm{d} \mu=-2 \pi \mathrm{d} \sigma_{\mathrm{KN}} / \mathrm{d} \Omega$, where $\mu \equiv \cos \theta_{\gamma}$, with the relation between $T$ and $\mu$, the second of Equations 2.30

$$
\begin{aligned}
\frac{\mathrm{d} \sigma}{\mathrm{d} T} & =\frac{\mathrm{d} \sigma_{\mathrm{KN}}}{\mathrm{d} \mu} \frac{1}{\mathrm{~d} T / \mathrm{d} \mu}=-2 \pi \frac{\mathrm{d} \sigma_{\mathrm{KN}}}{\mathrm{d} \Omega} \frac{1}{\mathrm{~d} T / \mathrm{d} \mu} \\
& =\frac{\pi r_{\mathrm{o}}^{2}}{\alpha h \nu_{0}}\left[1+\left(1-\frac{1}{\alpha} \frac{T}{h \nu_{0}-T}\right)^{2}+\frac{T^{2}}{\left(h \nu_{0}-T\right) h \nu_{0}}\right]
\end{aligned}
$$

where $\mathrm{d} T / \mathrm{d} \mu$ was obtained by differentiating the second of Equations 2.27. Then, $(\mathrm{d} \sigma / \mathrm{d} T) \mathrm{d} T$ is the cross section for producing a Compton electron with energy in $(T, T+\mathrm{d} T)$.

The maximum energy the Compton recoil electron can have is $T_{\max }$ given by Equation 2.36. Equation 2.37 gives the distribution in energy. The electron energy distribution peaks at its end point, $T=T_{\max }$. 


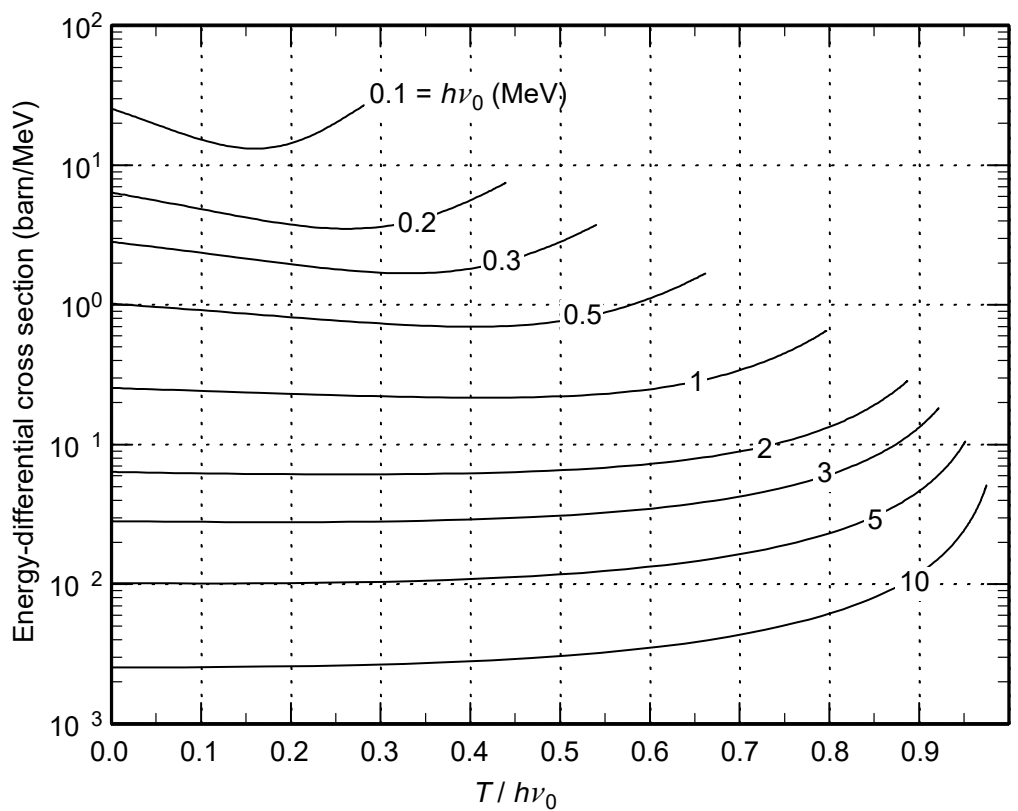

FIGURE 2.25 Compton electron energy distribution for Compton scattering from a free electron.

The energy-differential cross section given in Equation 2.37 is shown in Figure 2.25 (barn/MeV) for the Compton electron, for several incident photon energies. The electron maximum energy $T_{\max }$ is also its most probable energy, but its average energy is $\sim 0.5$ to 1 times its maximum. The distribution is rather flat at low energies, but rises to a sharp peak at $T_{\max }$.

Figure 2.26 is the Compton electron energy as a function of its outgoing polar angle $\theta_{\mathrm{e}}$ (relative to the incident photon direction), for several incident energies $h \nu_{0}$. It has its maximum energy in the forward direction $\left(\theta_{\mathrm{e}}=0\right)$ and zero energy as its angle of ejection approaches $\pi / 2$. Kinematically, the electron cannot exit in the backward hemisphere.

In the photoelectric effect, when the electron is ejected in the forward direction, its momentum can exceed that of the incident photon. Momentum conservation is assured because the atom recoils backward, and its momentum balances the total. In Compton scattering, the momentum of the forward-directed electron can also exceed that of the incident photon. However, in the Compton case, the photon can scatter in the backward hemisphere, carrying sufficient momentum to balance that of the electron.

\subsubsection{Scattered Photon Energy Distribution}

The scattered photon energy distribution $\mathrm{d} \sigma / \mathrm{d} h \nu^{\prime}$ is shown in Figure 2.27. Because the electron energy $T=h \nu_{0}-h \nu^{\prime}$, the photon energy spectrum is 


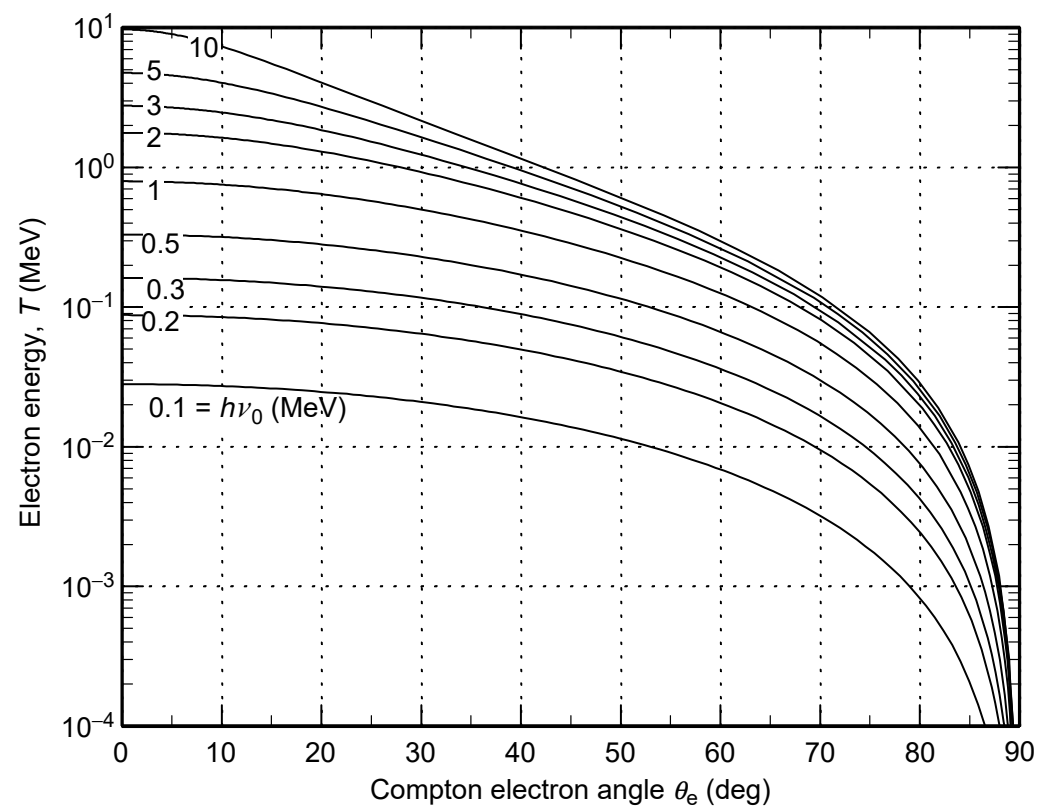

FIGURE 2.26 Compton electron energy vs. outgoing angle $\theta_{\mathrm{e}}$ for Compton scattering on a free electron.

given by the electron energy distribution, $\mathrm{d} \sigma / \mathrm{d} T$ (Equation 2.37), with $T$ replaced by $h \nu_{0}-h \nu^{\prime}$,

$$
\frac{\mathrm{d} \sigma}{\mathrm{d} h \nu^{\prime}}=\frac{\mathrm{d} \sigma}{\mathrm{d} T}\left(T=h \nu_{0}-h \nu^{\prime}\right)
$$

and thus its shape mirrors that of the electron distribution.

\subsubsection{Photon Energy-Angle Relation}

Figure 2.28 shows the outgoing photon energy $h \nu^{\prime}$ as a function of scattered photon angle $\theta_{\gamma}$. The photon always prefers the forward hemisphere, but the preference for small angles is pronounced above $h \nu_{0} \approx 2 \mathrm{MeV}$.

\subsubsection{Photon-Electron Angle Relations}

Figure 2.29 shows the outgoing electron angle $\theta_{\mathrm{e}}$ for given scattered photon angle $\theta_{\gamma}$.

For $h \nu_{0} \gtrsim 2 \mathrm{MeV}$, the electron strongly prefers to exit near the forward direction; the electron will exit at $\theta_{\mathrm{e}} \lesssim 30^{\circ}$ so long as the photon scatters at $\theta_{\gamma} \gtrsim 40^{\circ}$. 


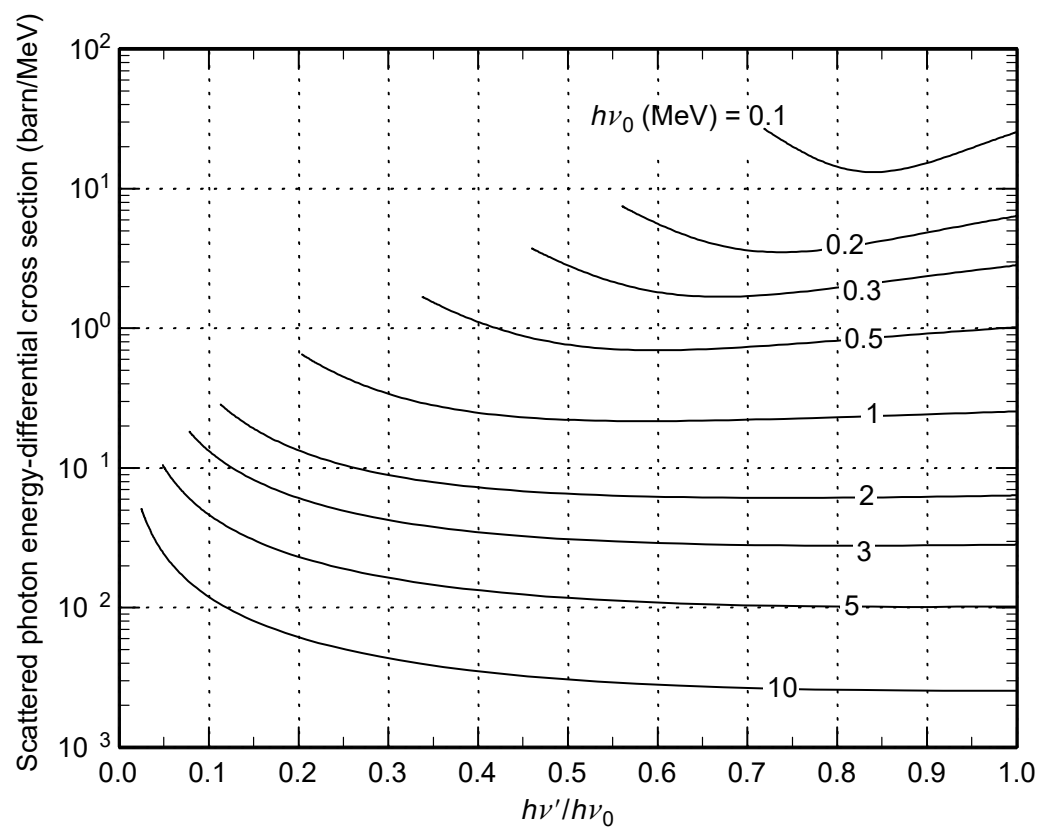

FIGURE 2.27 Scattered photon energy distribution for Compton scattering on a free electron.

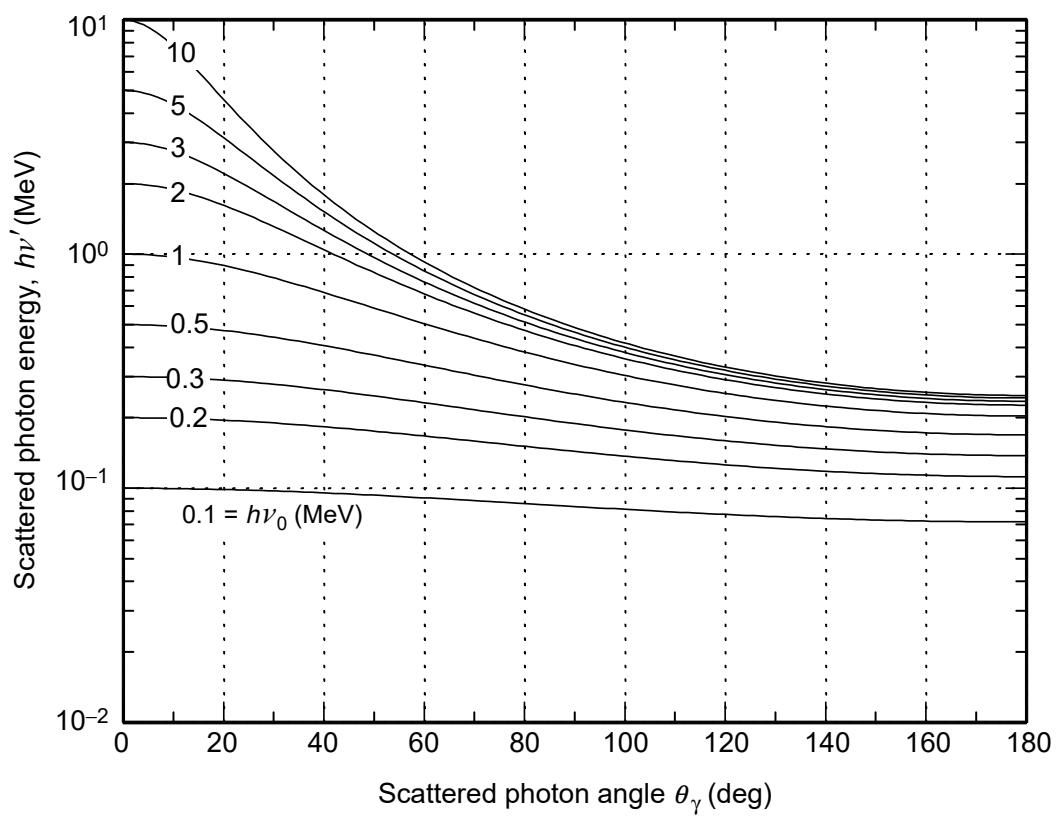

FIGURE 2.28 Scattered photon energy vs. outgoing angle $\theta_{\gamma}$. Compton scattering on a free electron. 


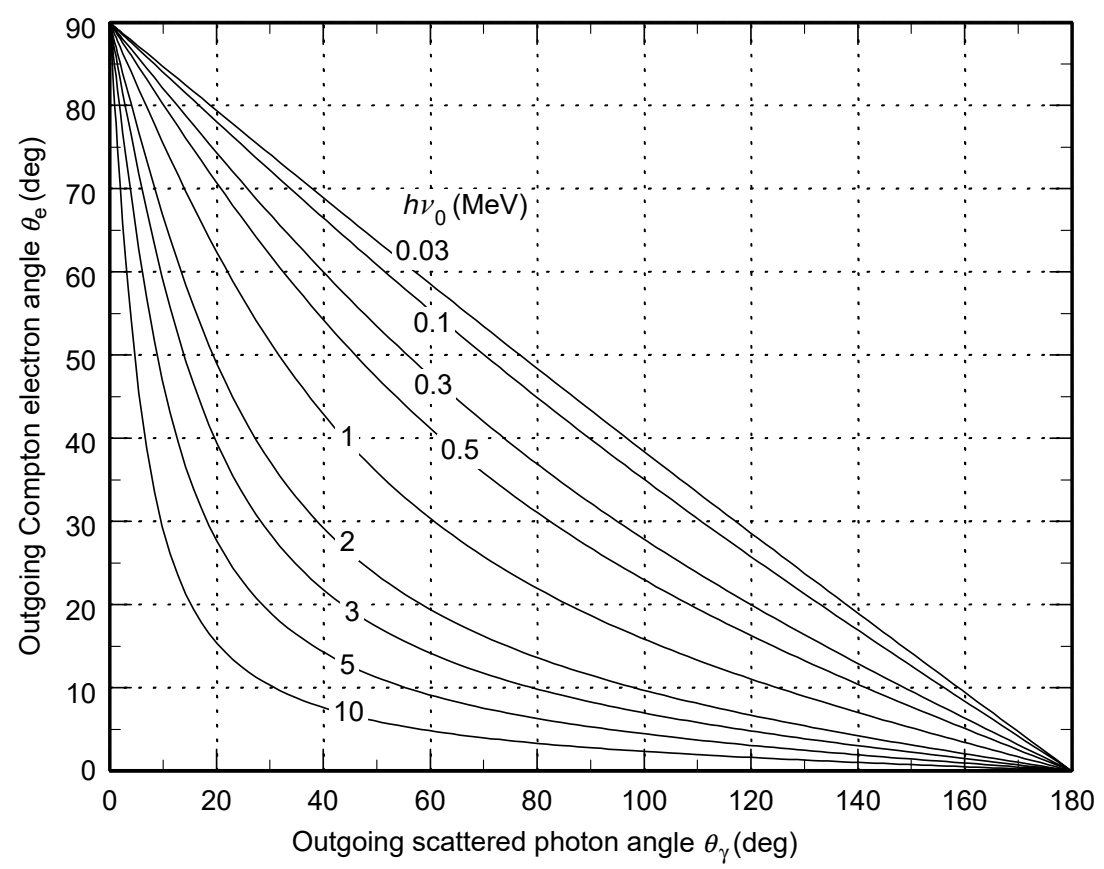

FIGURE 2.29 Compton electron angle of emission vs. scattered photon angle, for Compton scattering on a free electron.

\subsubsection{Electron Angular Distribution}

The electron differential scattering cross section $\mathrm{d} \sigma / \mathrm{d} \Omega_{\mathrm{e}}$ (barn/ster) is shown in Figure 2.30a, and the distribution in polar angle $\theta_{\mathrm{e}}, \mathrm{d} \sigma / \mathrm{d} \theta_{\mathrm{e}}=$ $2 \pi \sin \theta_{\mathrm{e}} \mathrm{d} \sigma / \mathrm{d} \Omega_{\mathrm{e}}(\mathrm{barn} / \mathrm{rad})$ in Figure 2.30b. The same quantities are shown in Figure 2.31 in polar coordinates. Above several MeV, the electron angular distribution is extremely peaked in the forward direction.

\subsubsection{Scattered Photon Angular Distribution}

The photon differential scattering cross section $\mathrm{d} \sigma / \mathrm{d} \Omega_{\gamma}$ (barn/ster) is shown in Figure 2.32a, and the distribution in polar angle $\theta_{\gamma}, \mathrm{d} \sigma / \mathrm{d} \theta_{\gamma}=2 \pi \sin \theta_{\gamma} \mathrm{d} \sigma / \mathrm{d} \Omega_{\gamma}$ (barn/rad) in Figure 2.32b. Again, the same quantities are shown in Figure 2.33 in polar coordinates. In the forward direction $\left(\theta_{\gamma}=0\right)$, the photon differential cross section is $r_{\mathrm{o}}^{2}=0.0794$ barn at all energies. This is true only for scattering from a free electron. Angular distributions near $\theta_{\gamma}=0$ are much different when scattering from an atom, as discussed in the next section.

\subsubsection{Cumulative Angular Distributions}

Figure 2.34 gives the fraction of all electrons and photons that exit at a polar angle less than $\theta$. At $h \nu_{0}=3 \mathrm{MeV}$, half of all Compton electrons come out at 


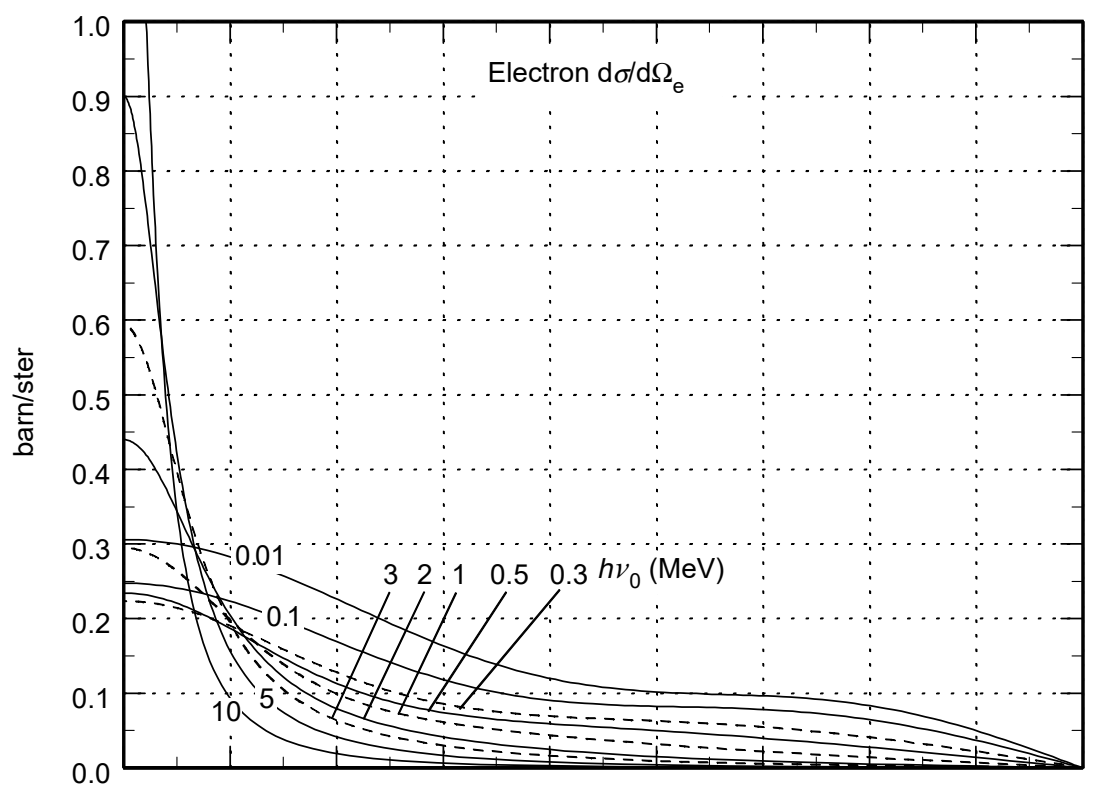

(a)

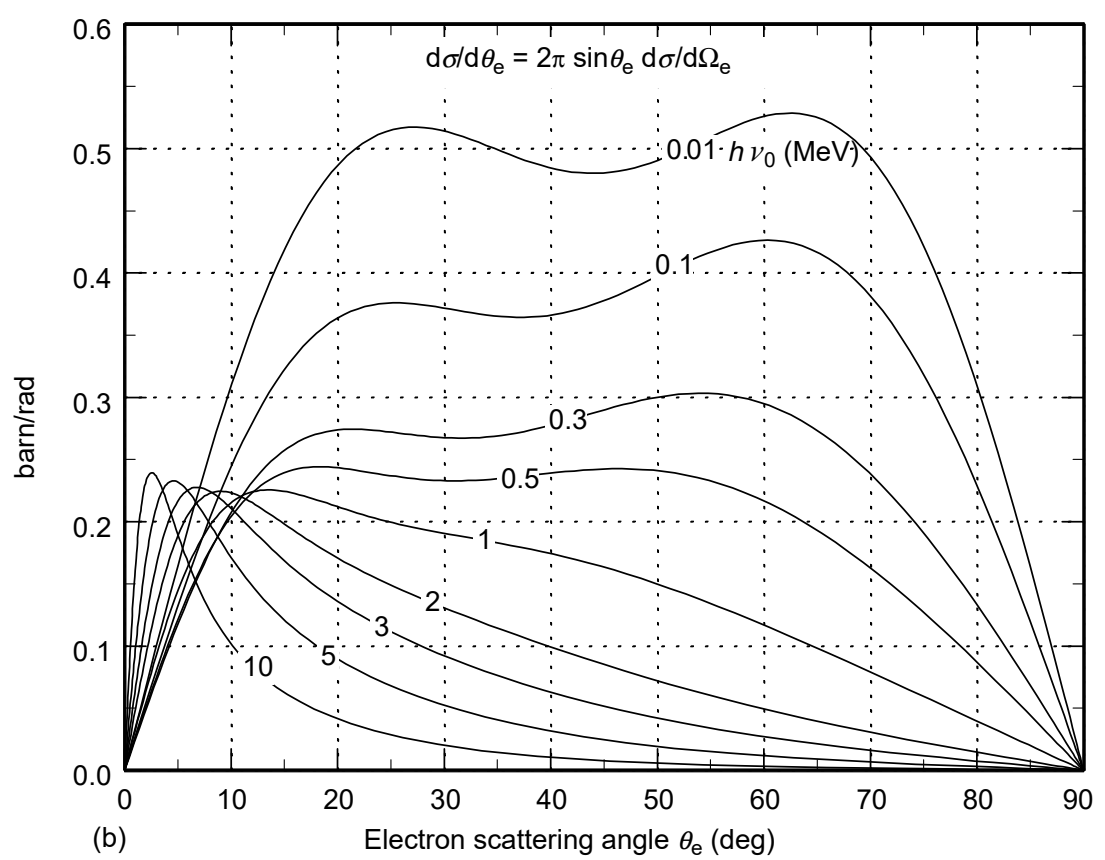

FIGURE 2.30 Compton electron differential scattering cross section on a free electron vs. outgoing electron polar angle $\theta_{\mathrm{e}}$. 


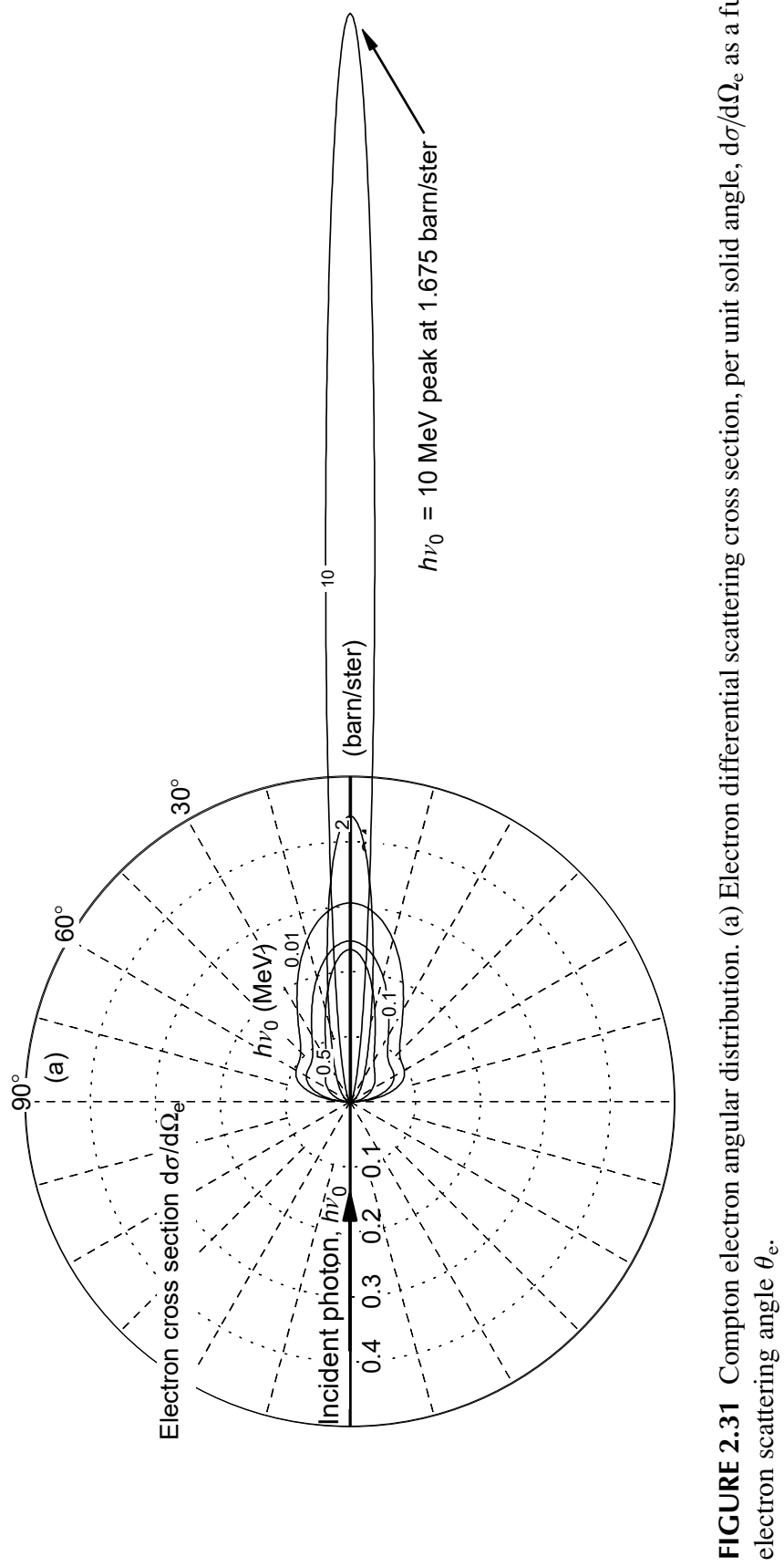




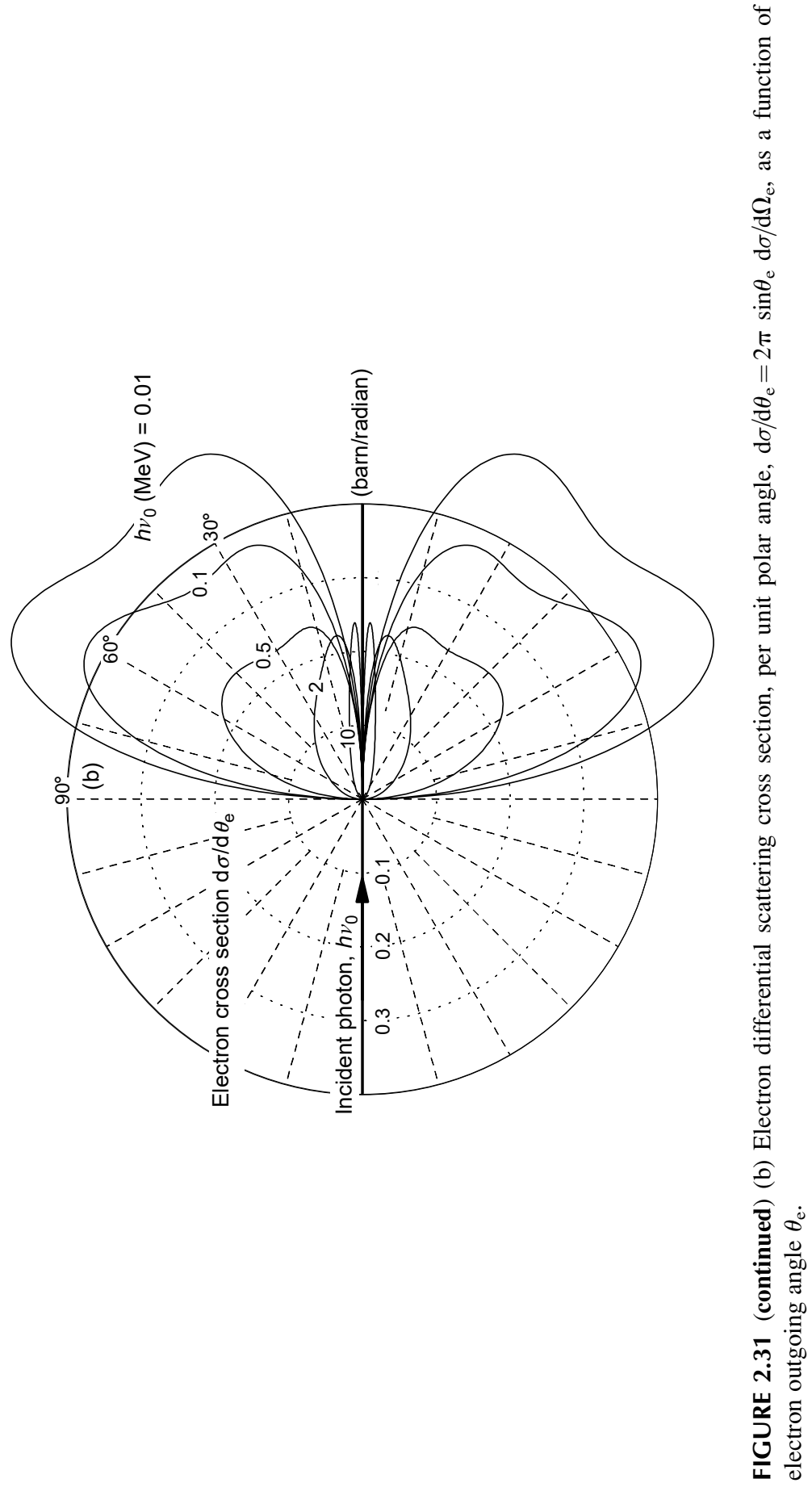




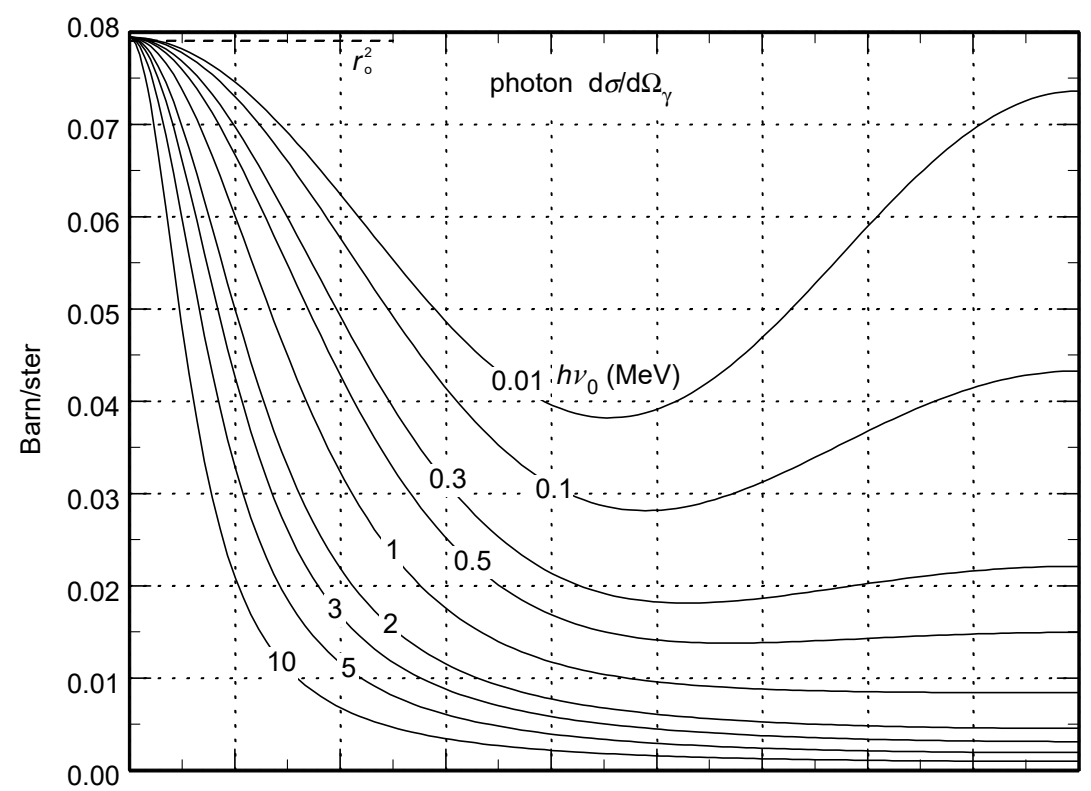

(a)

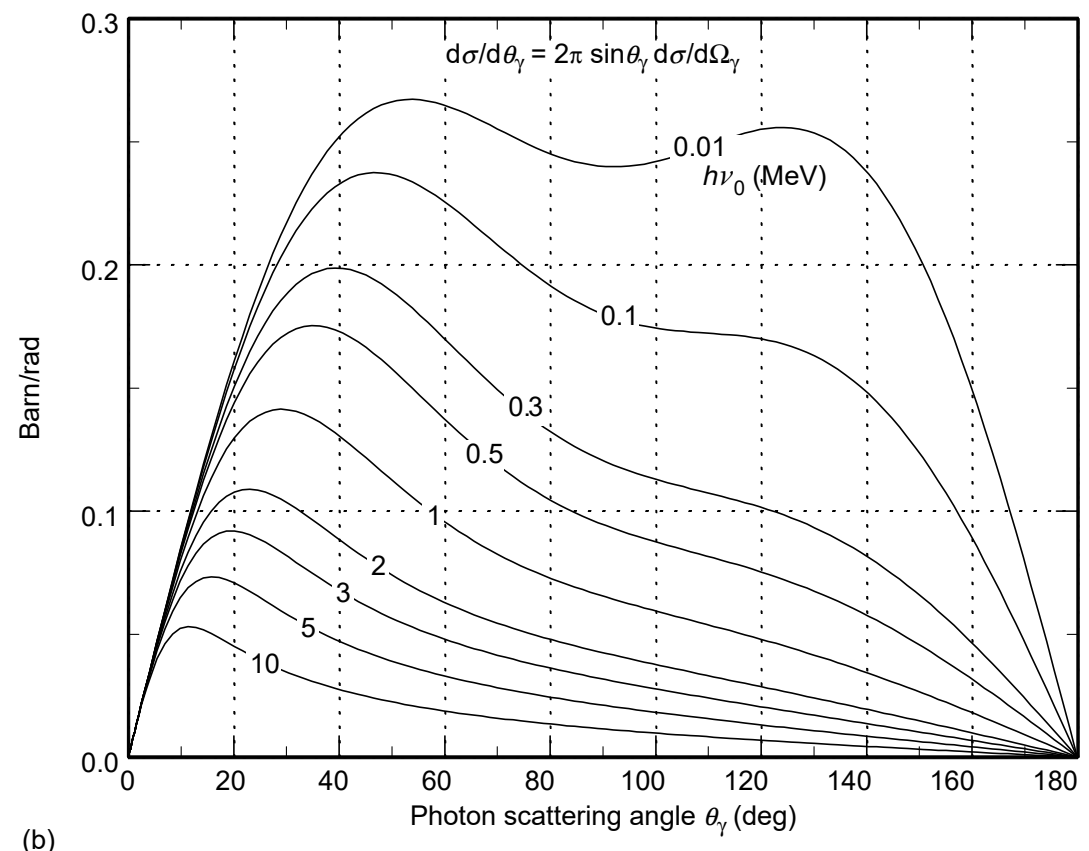

FIGURE 2.32 Compton scattered photon differential cross section on a free electron vs. scattering angle. 


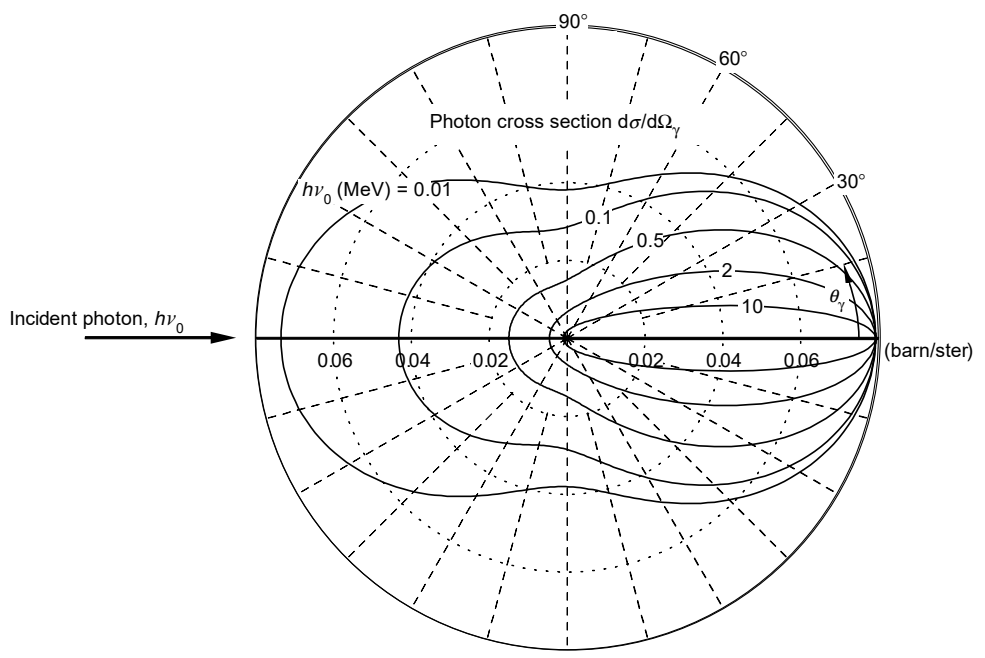

(a) Photon differential scattering cross section, per unit solid angle, $\mathrm{d} \sigma / \mathrm{d} \Omega_{\gamma}$ as a function of scattering angle $\theta_{\gamma}$.

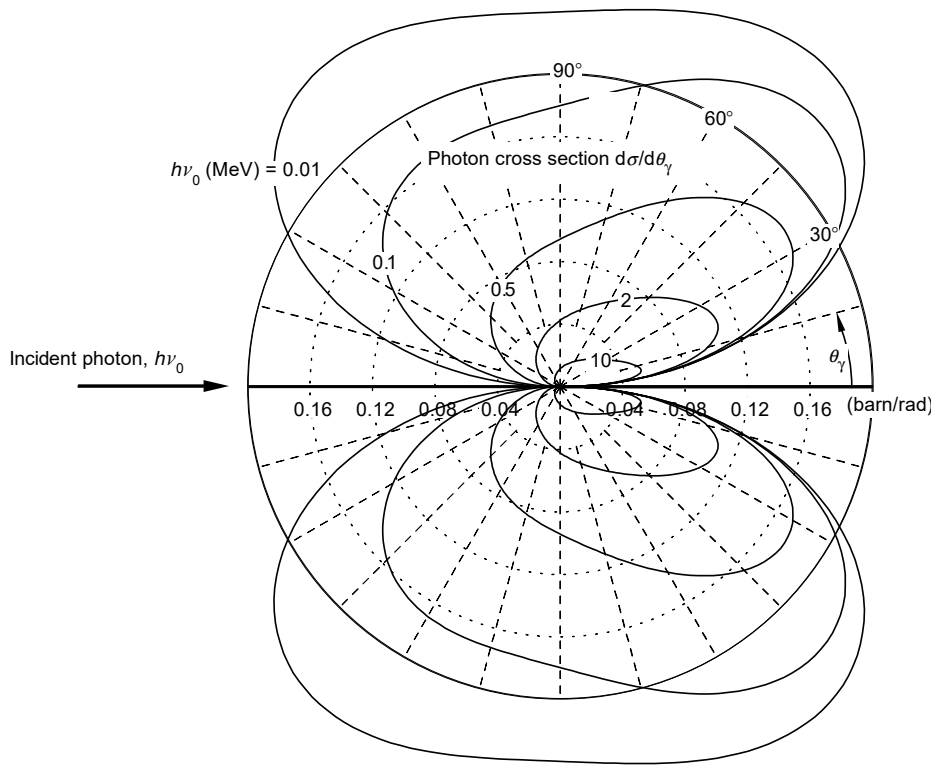

(b) Photon differential scattering cross section, per unit polar angle, $\mathrm{d} \sigma / \mathrm{d} \theta_{\gamma}$ as a function of scattering angle $\theta_{\gamma}$ $=2 \pi \sin \theta_{\gamma} \mathrm{d} \sigma / \mathrm{d} \Omega_{\gamma}$

FIGURE 2.33 Angular distribution of scattered photon in Compton scattering from a free electron. 

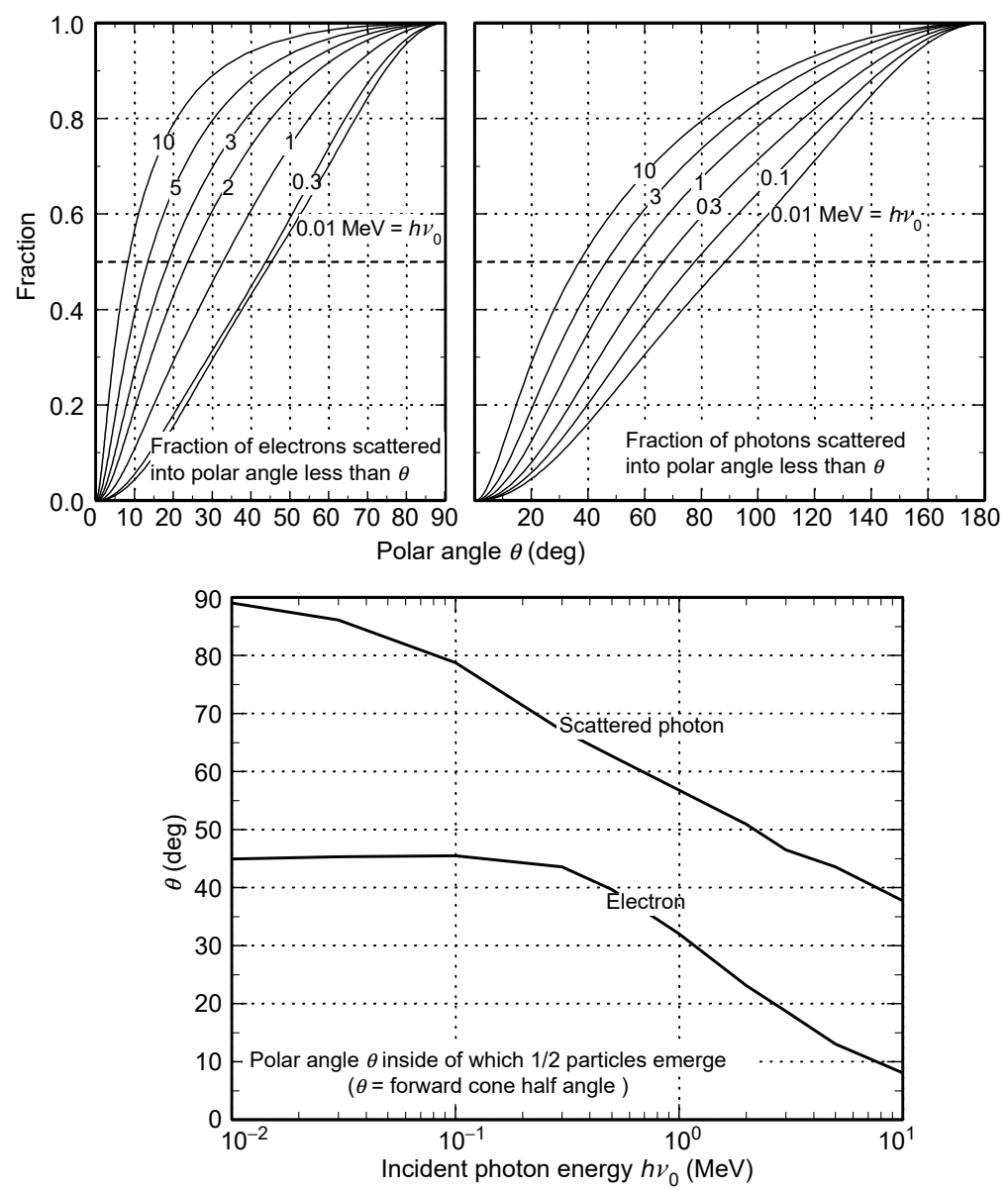

FIGURE 2.34 Compton scattering from a fee electron. Cumulative angular distributions.

less than or greater than about $19^{\circ}$, whereas half of the photons exit at less than or greater than about $47^{\circ}$.

The bottom graph in Figure 2.34 shows the angle inside of which $1 / 2$ of the electrons or photons exit, as a function of $h \nu_{0}$. For a $2 \mathrm{MeV}$ photon, $1 / 2$ of all electrons exit at less than $22^{\circ}$, and $1 / 2$ of the scattered photons exit at less than $51^{\circ}$.

\subsubsection{Comment on Angular Distribution}

Kinematics requires that the scattered photon and the ejected Compton electron cannot both exit the interaction in the forward direction. If the photon comes out near $\theta_{\gamma}=0$, the electron comes out near $\theta_{\mathrm{e}}=90^{\circ}$. If the electron comes out near $\theta_{\mathrm{e}}=0$, the photon comes out near $\theta_{\gamma}=180^{\circ}$. 
Since the photon angular distribution is peaked in the forward direction, one might therefore expect the electron distribution must peak at some large angle, or, at least, could not also peak in the forward direction. In fact, however, both the electron and photon angular distributions do peak in the forward direction.

This does not violate kinematics, since the two particles do not both exit in the forward direction in the same event.

Kinematically, because electrons cannot backscatter but photons can, scattered photons have available twice the phase space that is available to electrons; the electrons tend to be compressed in the forward direction. When scattered at a large angle, the photon has relatively little energy. Momentum conservation then throws the electron forward. Electron angular distribution is significantly more compressed at higher energies, as seen in Figure 2.30 or Figure 2.31.

\subsubsection{Application to a Gamma Spectrometer}

Detectors of high-energy photons rely on the interaction of the gamma with the material of the detector itself. Bolometers measure the temperature rise of a small mass as it absorbs the energy of some of the photons that enter it. Scintillators are excited to emit light when a photon deposits energy in it. Semiconductor detectors measure the charge of electron-hole pairs created by photon energy deposition.

At nuclear gamma ray energies, the Compton effect is usually the most probable process; a gamma entering the detector is more likely to Compton scatter than to be absorbed by the photoelectric effect or undergo pair production. The short-range Compton electron then deposits its energy in the detector.

Equation 2.37 for the Compton electron energy spectrum is therefore useful in studies of the performance of liquid- or solid-state detectors. A solid scintillator (e.g., NaI, CsI, or Bismuth Germanate (BGO)) senses the energy deposited by the Compton electron (or photoelectron) by measuring the energy in light (essentially visible) emitted when excited and ionized atoms de-excite or recombine. This light enters a photomultiplier tube that converts it to an electrical pulse, which is then amplified. Except for relatively small nonlinearities, the electrical output of the scintillator is proportional to the energy deposited in the crystal [Kn00].

Another common detector is a solid semiconductor (e.g., GaAs, CdTe, $\mathrm{CdZnTe}$, or $\mathrm{HgI}_{2}$ ) in which the energy deposited by the photon creates electron-hole pairs that are collected at electrodes attached to the crystal. The number of pairs, and hence the magnitude of the charge pulse, is proportional to the energy deposited in the crystal by the photon.

The photon deposits its energy by any one of the following ways:

1. Suffering a photoelectric absorption, ejecting a photoelectron that has a short range and deposits its energy by further ionization

2. Undergoing Compton scattering from an atomic electron in the detector, ejecting a short-range Compton electron with some fraction 
of the original photon energy, while the reduced energy scattered photon continues on

3. Undergoing pair production (if the photon energy exceeds $1.02 \mathrm{MeV}$ ), ejecting an electron and positron that then ionize further

Figure 2.35 schematically shows the typical geometry of a semiconductor detector in a metal housing, with three illustrative photons incident normally on the crystal. The upper photon is absorbed in the crystal by the photoelectric effect; essentially all its energy is deposited in the crystal. The second photon passes through the crystal and is Compton backscattered from solid material in the rear; the backscattered photon is absorbed in the crystal. The third photon Compton scatters in the crystal; the electron deposits its energy and the scattered photon continues on, leaving the crystal. These processes are discussed later.

The range of the Compton electron is almost always a small fraction of the detector linear dimensions, and so deposits all its energy in the detector. For example, in $\mathrm{CdZnTe}(\mathrm{CZT})$, the mean forward range (against stopping power and multiple scattering) of a $1 \mathrm{MeV}$ electron is $0.2 \mathrm{~g} / \mathrm{cm}^{2}=0.035 \mathrm{~cm}$. However, the scattered photon continues to a much greater distance. If the crystal is not too large, the scattered photon often exits the crystal without interacting again. As detector response is proportional to energy deposited, a Compton event leads to a fraction of the photon energy being registered by the detector, as energy deposited by the Compton electron. The energy deposited is therefore $T_{\max }$ or less. Equation 2.37 gives the pulse height distribution resulting

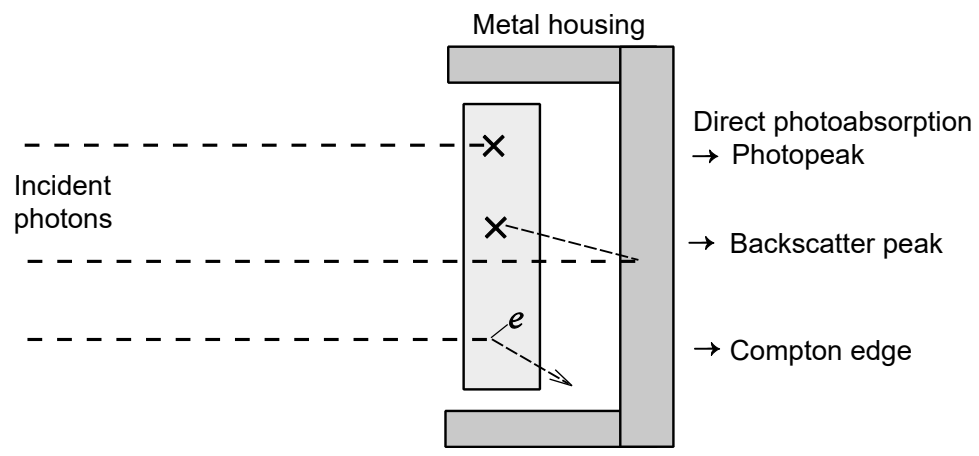

FIGURE 2.35 Illustrating three kinds of photon interactions in a crystal gamma detector. The upper photon is directly absorbed by the photoelectric effect; all its energy is deposited in the crystal (except possibly for fluorescence x-rays), contributing to the photopeak. The center photon passes through the crystal and Compton scatters through a large angle from the housing; the scattered photon is absorbed in the crystal, leading to the backscatter peak. The lower photon undergoes a Compton scattering in the crystal; the electron stops in the crystal contributing its energy to the Compton shoulder. 
from Compton electrons when gammas enter the detector material and Compton scatter once. That whole process is quite common, particularly for small crystals.

Instead of a single Compton scattering, the gamma ray may be absorbed directly by the photoelectric effect, depositing essentially all its energy $h \nu_{0}$ in the crystal. For low- $Z$ materials, the electron binding energy is recovered in the form of Auger electron kinetic energy. However, for high $Z$, electron binding energy is lost to fluorescence photons, so there are counts on the lowenergy side of the full photon energy (in $Z=50$, Figure 2.13 shows that if the gamma is photoabsorbed in the $K$ shell, $86 \%$ of absorptions produce a fluorescence $\mathrm{x}$-ray). Alternatively, once Compton scattered, the lower energy scattered photon may be photoabsorbed before exiting, with the result that again all the initial energy $h \nu_{0}$ is deposited in the crystal. In either of these cases or in a number of other processes or sequence of processes, the entire gamma ray energy is deposited in the crystal. As a result, the detector will measure the full gamma energy $h \nu_{0}$ and display a peak at that energy. The magnitude of the peak, called the photopeak, is determined by the source intensity. The sharpness of the peak is determined by the energy resolution of the detector; the count rate falls off rapidly away from the center of the peak.

Nevertheless, because of the relatively high probability of a single Compton scattering followed by escape of the scattered photon, at an energy $T_{\max }$ there will be a relatively sudden rise in the pulse height distribution as the energy of Compton electrons is detected. This rise is called the Compton edge. At energies below the edge, the pulse height distribution is determined by the cross section Equation 2.37.

Figure 2.36 shows an example of a relatively clean gamma ray spectrum taken by a CZT crystal detector. The crystal is $5 \times 10 \times 10 \mathrm{~mm}$ and was exposed for $20 \mathrm{~h}$ to a $\mathrm{Cs}^{137}$ source, which emits $h \nu_{0}=661.6 \mathrm{keV}$ gammas. The photons were incident normally on the $10 \times 10$-mm face; the longest path available to them was $5 \mathrm{~mm}$. The photopeak is very pronounced. Natural environmental background gammas were also present, of course, and have not been subtracted from the data.

As one proceeds down in energy below the photopeak, at an energy $T_{\max }=477 \mathrm{keV}$ (or $E_{\mathrm{s}}=184 \mathrm{keV}$ below the peak) the signal rises, registering Compton electrons created when the gamma Compton scattered in the crystal and the scattered photon exited without further interaction; that is the Compton edge. In the figure, along with the measured spectrum, the ideal Compton electron energy spectrum (Equation 2.37), at and below the Compton edge, is drawn (dotted line). This is the spectrum of Compton electrons and the detector response that would occur if the gamma scattered from free electrons and in the absence of detector imperfections or background noise.

The actual shape of the measured spectrum in the vicinity of the Compton edge is not a sharp discontinuity, but is smoothed and rounded. The reasons are: (1) the natural resolution of the detector (here about $3 \%$ or about $15 \mathrm{keV}$ ) broadens the response to any one energy; (2) thermal drift of the detector 


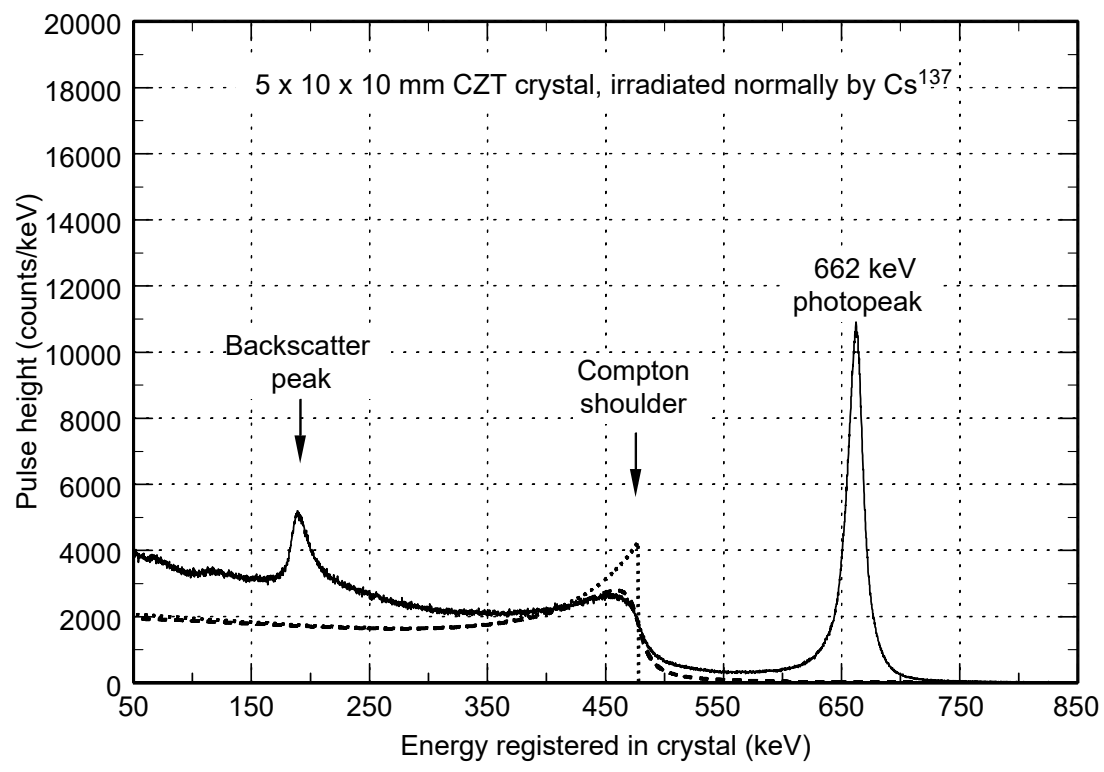

FIGURE 2.36 Gamma ray spectrum measurement of a $\mathrm{Cs}^{137}$ source in a CdZnTe crystal. The main Cs peak at $661.6 \mathrm{keV}$ is pronounced. The Compton shoulder is clear at $T_{\max }=477 \mathrm{keV}$. The backscatter peak is near a minimum energy of $h \nu^{\prime}\left(180^{\circ}\right)=$ $184 \mathrm{keV}$. The data include ambient environmental background noise. The dotted curve is the ideal Compton electron spectrum, with a sharp cutoff at $T_{\max }$. The dashed curve is after broadening by a detector resolution function with FWHM $=18 \mathrm{keV}$. (Data courtesy of John Baker, Mission Research Corporation.)

calibration during the long measurement spreads the response; (3) Compton scattering on the bound crystal electrons is not the same as that from free electrons; and (4) background gammas have not been subtracted in the figure, and they provide a nonuniform background noise. The rounded Compton edge is referred to as the Compton shoulder.

To illustrate the effects of the four broadening mechanisms, Equation 2.37 has been filtered through a typical detector resolution function with an $18 \mathrm{keV}$ full width at half maximum (about $3.8 \%$ at $477 \mathrm{keV}$ ). The result is shown as the dashed line in the figure. Above $500 \mathrm{keV}$, this broadened line falls below the data because the measurements include background noise not represented in the calculation. The shape of the broadened spectrum is quite close to the measured spectrum near the Compton edge at $477 \mathrm{keV}$. Below that energy, background noise and back- or side-scattering makes the data higher than the computed ideal spectrum.

Also seen in Figure 2.36 is a peak at $190 \mathrm{keV}$ labeled backscatter peak. Gammas will most likely penetrate the 5 -mm thick crystal without interacting 
(the mfp against any interaction of a $662 \mathrm{keV}$ photon in CZT is $2.1 \mathrm{~cm}$ ). In the structure, there is always some solid material behind and around the crystal. After passing through or nearby the crystal, the gamma can Compton scatter from this material through a large angle, and the reduced-energy scattered photon will propagate back to the crystal where it is likely to be photoabsorbed. From Equation 2.26 the energy of the backscattered photon is 184 $\mathrm{keV}$ or greater, depending on the angle through which it scattered. As this is the minimum energy of a scattered photon, and scattering occurs through a wide range of angles from surrounding material, a broad peak is produced by these scattered photons that enter the crystal from many angles and get absorbed. The peak is steep on the low-energy side, because $184 \mathrm{keV}$ is the strict minimum photon energy, but the distribution of smaller scattering angles and larger energies gives the peak a wider slope on the high-energy side. In CZT, near $190 \mathrm{keV}$, photoelectric absorption is more probable than Compton scattering, so the full energy of the backscattered photon is recorded. A backscatter peak such as this one is commonly seen in gamma detectors.

Information from both the photopeak and the Compton shoulder can be used to increase confidence in photospectroscopy over a simple measurement of the photopeak alone. In addition, since the Compton scattered photon can easily be arranged to exit the crystal (by making it small), a second crystal can be positioned to capture some scattered photons. By measuring time coincidence between the two, confident photospectroscopy can be performed [Sh64, Si65, Qu72, Kn00].

\subsubsection{Application to Penetration of Scattered Photons through a Shield}

Relative to photoelectric absorption, low- $Z$ materials scatter (Rayleigh or Compton) more than high- $Z$ materials. Complete cross-section plots (Figure 2.50 in Section 2.8) show, for example, that the Rayleigh and Compton cross sections in low $Z$ exceed photoelectric absorption at energies above a few $\mathrm{keV}$, whereas in high $Z$ they do so only above a few hundred keV. Wood and plastic are more annoying in X-ray laboratories than heavier elements. Because of the forward peaking of both Rayleigh and Compton scattering, both high and low $Z$ may scatter much at grazing incidence. However, although high- $Z$ elements generally have larger cross sections than low $Z$, they are not always preferred for shielding.

Rayleigh or Compton scattered photons can penetrate a slab of material, and may do so more readily than unscattered photons. Let $\sigma_{\mathrm{R}}, \sigma_{\mathrm{pe}}, \sigma_{\mathrm{C}}$, and $\sigma_{\mathrm{pp}}$ be the Rayleigh, photoelectric, Compton, and pair-production cross sections, respectively, of a material, and $\sigma_{\mathrm{Tot}}=\sigma_{\mathrm{R}}+\sigma_{\mathrm{pe}}+\sigma_{\mathrm{C}}+\sigma_{\mathrm{pp}}$ be the total cross section. For a slab of thickness $d$ and density $\rho$, the probability that a photon will penetrate unscattered is $p_{\text {unsc }}=\exp \left(-\sigma_{\text {Tot }} \rho d\right)$. 
Now consider a photon that Rayleigh scatters at depth $(x, x+\mathrm{d} x)$. For forward scattering, the probability that it exits the slab after one scattering is $\mathrm{d} p_{\text {exit }}=P\{$ it gets to $x$ without scattering $\} \cdot \mathrm{P}\{$ it Rayleigh scatters in $(x$, $x+\mathrm{d} x)\} \cdot P\{$ it gets to $d$ without further scattering $\}$, which is

$$
\begin{aligned}
\mathrm{d} p_{\text {exit }} & =\exp \left(-\sigma_{\mathrm{Tot}} \rho x\right) \cdot \sigma_{\mathrm{R}} \rho \mathrm{d} x \cdot \exp \left(-\sigma_{\mathrm{Tot}} \rho(d-x)\right) \\
& =\sigma_{\mathrm{R}} \rho \mathrm{d} x \cdot \exp \left(-\sigma_{\mathrm{Tot}} \rho d\right) \\
& =\sigma_{\mathrm{R}} \rho \mathrm{d} x \cdot p_{\mathrm{unsc}}
\end{aligned}
$$

The $x$ dependence drops out, and the probability that a once-Rayleighscattered photon penetrates the slab is

$$
p_{\text {exit }}=\int \mathrm{d} p_{\text {exit }}=\sigma_{\mathrm{R}} \rho d p_{\text {unsc }}=\frac{\rho d}{\lambda_{\mathrm{R}}} p_{\text {unsc }}
$$

where $\lambda_{\mathrm{R}}=1 / \sigma_{\mathrm{R}}$ is the mfp against Rayleigh scattering. Thus, if the slab is thicker than $\lambda_{\mathrm{R}}$, the probability that a Rayleigh scattered photon penetrates is greater than the probability that an unscattered photon penetrates. (Of course, as $d$ increases, both $p_{\text {unsc }}$ and $p_{\text {exit }}$ decrease.) As the scattered photon is of the same energy and predominantly scatters in or near the forward direction, the emerging flux is greater than the unscattered flux by the factor $1+\rho d / \lambda_{\mathrm{R}}=1+\sigma_{\mathrm{R}} \rho d$. Thus, depending on the slab thickness, one may have to include Rayleigh scattered photons when computing the penetrating flux. An accurate value for the penetrating flux requires a Monte Carlo photon transport calculation.

As $p_{\text {unsc }}=\mathrm{e}^{-s}$ is a function only of $s \equiv \sigma_{\text {Tot }} \rho d, p_{\text {exit }}$ may be written

$$
p_{\text {exit }}=\frac{\sigma_{\mathrm{R}}}{\sigma_{\mathrm{Tot}}} s \mathrm{e}^{-s}
$$

Then, for a given total attenuation $p_{\text {unsc }}$, photon scattering when passing through a slab will be least important when $\sigma_{\mathrm{R}} / \sigma_{\mathrm{Tot}}$ is as small as possible.

A similar effect for Compton scattering must also be taken into account, although the scattered photon is of lower energy. (Near forward scattering, more probable at higher energies, the scattered photon energy is only slightly below the incident energy.) $\sigma_{\mathrm{R}}$ given earlier can be replaced by $\sigma_{\mathrm{R}}+\sigma_{\mathrm{C}}$, and the cross-section ratio $\left(\sigma_{\mathrm{R}}+\sigma_{\mathrm{C}}\right) / \sigma_{\mathrm{Tot}}$ is a measure of the importance of scattering (Rayleigh + Compton) relative to total attenuation. It is shown in Figure 2.37 at several energies for all elements.

For example, to shield $80 \mathrm{keV}$ and minimize scattered x-rays, it is best to use elements $Z \sim 70-78$, for which the $K$ edge is just below $80 \mathrm{keV}$. At that energy, the transmission of unscattered $\mathrm{x}$-rays through $0.18 \mathrm{~mm} \mathrm{~W}$ $\left(0.35 \mathrm{~g} / \mathrm{cm}^{2}\right)$ or $1 \mathrm{~mm} \mathrm{~Pb}\left(1.13 \mathrm{~g} / \mathrm{cm}^{2}\right)$ is $p_{\text {unsc }}=6.7 \%$. However, W will 


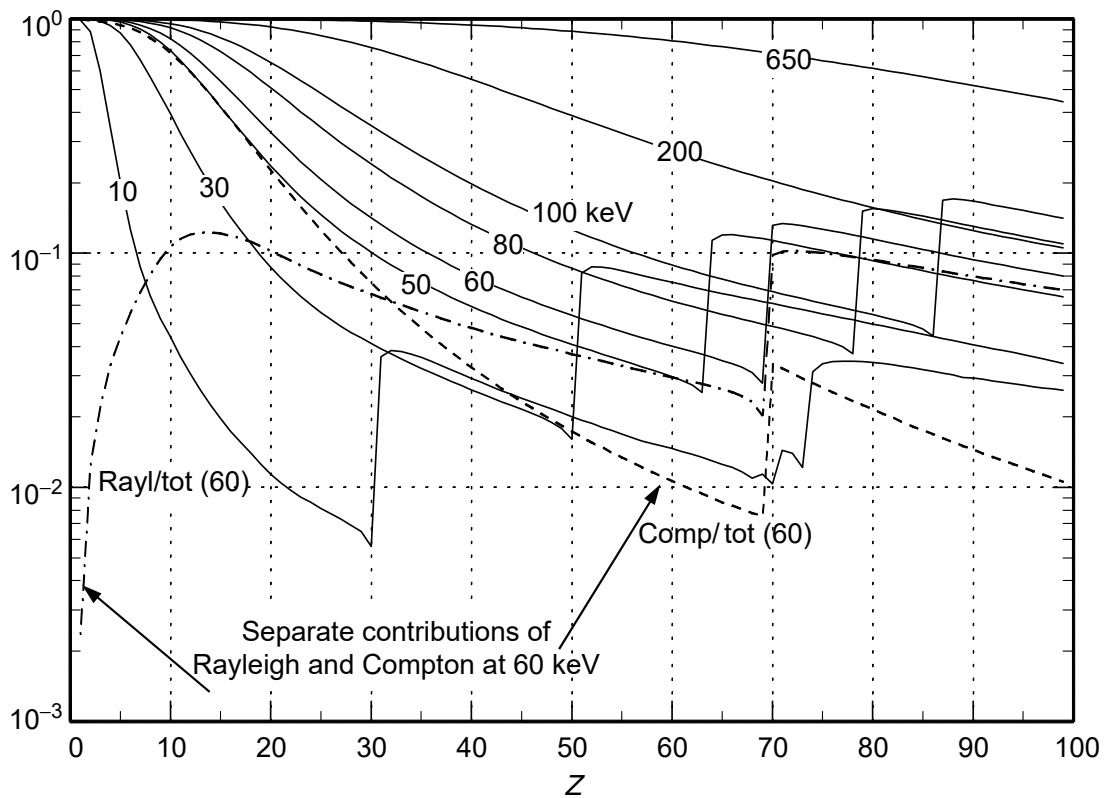

FIGURE 2.37 The ratio of scattering cross section (Rayleigh + Compton) to total cross section in all elements at selected energies. The ratio is a measure of the importance of scattering (a photon exits the interaction) to attenuation. At $60 \mathrm{keV}$, the separate contributions of Rayleigh/total and Compton/total are shown. The discontinuities in the curves are due to passing over the $\mathrm{K}$ edge of the corresponding element. The best $\mathrm{x}$-ray shield at, say, $30 \mathrm{keV}$, that minimizes scattering for given attenuation is one made of elements $Z \sim 45-50$. Lead is best near $100 \mathrm{keV}$.

create many fewer scattered photons. Although estimated here for Rayleigh alone, when Compton scattered photons are included, the transmitted flux increases by (no more than) $1+\left(\sigma_{\mathrm{R}}+\sigma_{\mathrm{C}}\right) \rho d$. The second term is 0.4 for $\mathrm{Pb}$, but only 0.06 for $\mathrm{W}$, indicating that for the same attenuation of unscattered photons, the $\mathrm{Pb}$ shield would add nearly $40 \%$ in scattered photons, whereas $\mathrm{W}$ would add only $6 \%$. In many applications, shield weight and thickness are not of great concern, and one simply uses a suitably thick shield. Nevertheless, if weight and thickness were important (as, e.g., in satellites), in this example, a thinner and lighter $\mathrm{W}$ shield would be preferred over $\mathrm{Pb}$.

\subsubsection{Compton Scattering from Atoms}

The difference between the total (angle integrated) Compton cross section on an atom, relative to that on a free electron, was mentioned earlier in connection 
with the incoherent scattering function $S(q, Z)$. Figure 2.23 shows examples of the difference for aluminum and lead; the difference is prominent at low energies. It is mostly owing to the different angular distribution near the forward direction for an atom relative to a free electron and to the effects of binding energies.

\subsubsection{Angular Distribution}

The angular distribution of scattered photons in Compton scattering from atoms is given by Equation 2.24, and is different from the angular distribution in Compton scattering from a free electron. The difference is caused by the binding of electrons, and the incoherent scattering function $S(q)$ is intended to quantify that effect.

At a high-momentum transfer to the atomic electrons (and so high-energy transfer), the binding energy of electrons makes no difference, and the cross section for scattering from an atom of atomic number $Z$ is simply $Z$ times the Klein-Nishina cross section: $\mathrm{d} \sigma_{\text {atom }} / \mathrm{d} \Omega=Z \mathrm{~d} \sigma_{\mathrm{KN}} / \mathrm{d} \Omega$. That is, $S(q)=Z$ for large $q$. The momentum transfer, however, is very small for forward or near forward photon scattering, even at high incident energy, and in exactly the forward direction $\left(\theta_{\gamma}=0\right)$ one has $q=0$ for all energies. If there is no momentum transferred to the electrons in the atom, no electron can be ejected, and Compton scattering does not occur. The differential cross section for the outgoing photon direction in Compton scattering from an atom vanishes in the exact forward direction, $\mathrm{d} \sigma_{\text {atom }}\left(\theta_{\gamma}=0\right) / \mathrm{d} \Omega=0$, and for very small angles (the differential cross section for electron ejection in the forward direction does not vanish).

This is illustrated in Figure 2.38, which shows the photon differential cross section in Fe for three incident energies. The solid lines are the atomic cross sections, computed by Equation 2.24 with $S(q)$ taken from EPDL97. The dashed lines are $Z$ times the Klein-Nishina cross section (Equation 2.33 or Equation 2.34). The Klein-Nishina cross section peaks smoothly in the forward direction, but the atomic cross sections, although generally peaking forward, have a sharp dip at small angles. The cross section vanishes from $\theta_{\gamma}=0$ out to a small finite angle. This behavior is explained as follows.

The minimum energy that must be transferred to an electron in order for Compton scattering to occur (i.e., in order for an electron to be ejected) is the atom's ionization potential, roughly of the order $10 \mathrm{eV}$. The minimum energy for Compton scattering to occur on a $K$ shell electron is the $K$ edge energy $E_{K}$. Now, from Equation 2.30 when the electron receives energy $T$, the photon scatters at angle $\theta_{\gamma}$ given by

$$
1-\cos \theta_{\gamma}=\frac{m c^{2} T}{\left(h \nu_{0}\right)^{2}} \frac{1}{1-T / h \nu_{0}}
$$




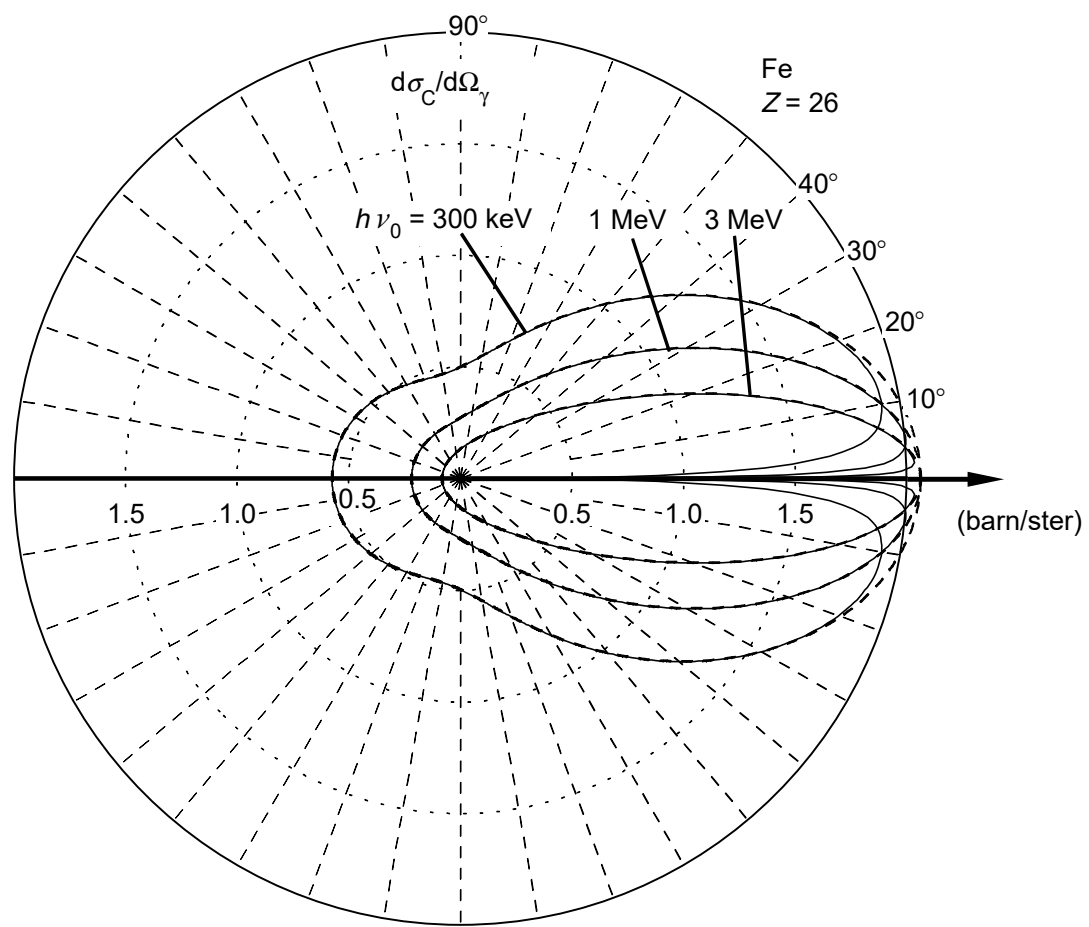

FIGURE 2.38 Compton differential cross section for the scattered photon on atomic $\mathrm{Fe}$ at three incident energies. Solid lines are computed from Equation 2.24 using data on $S(q)$ from EPDL97. Dashed lines are $Z \mathrm{~d} \sigma_{\mathrm{KN}} / \mathrm{d} \Omega$.

For small $\theta_{\gamma}$, and for $T \ll h \nu_{0}$, this becomes

$$
\theta_{\gamma}\left(h \nu_{0}, T\right)=\frac{\sqrt{2 m c^{2} T}}{h \nu_{0}}=10^{-3} \frac{\sqrt{T(\mathrm{eV})}}{h \nu_{0}(\mathrm{MeV})}
$$

Thus, at $E=h \nu_{0}=1 \mathrm{MeV}$, the photon must scatter through $3.2 \mathrm{mrad}=0.18^{\circ}$ in order to impart $10 \mathrm{eV}$ to an electron. A Compton scattered photon cannot exit at an angle smaller than this on an atom with an ionization potential of $10 \mathrm{eV}$.

The atomic cross section is not equal to $Z \mathrm{~d} \sigma_{\mathrm{KN}} / \mathrm{d} \Omega$ until every electron can be ejected, including the most tightly bound electrons; that is, until $T=E_{K}$. In Fe, the $K$ edge is $E_{K}=7.08 \mathrm{keV}$. Using this value for $T$ in Equation $2.43, \theta_{\gamma}\left(h \nu_{0}, T\right)$ at $E=1 \mathrm{MeV}$ is $0.084=4.8^{\circ}$. Figure 2.38 shows that this is the angle at which $\mathrm{d} \sigma_{\text {atom }} / \mathrm{d} \Omega$ returns to the value $Z \mathrm{~d} \sigma_{\mathrm{KN}} / \mathrm{d} \Omega$. When the energy transfer to an electron, $T$, exceeds $E_{K}$, Compton scattering from an atom reverts to Klein-Nishina scattering at angles greater than $\theta_{\gamma}\left(h \nu_{0}, T\right)$, given by Equation 2.43 . 


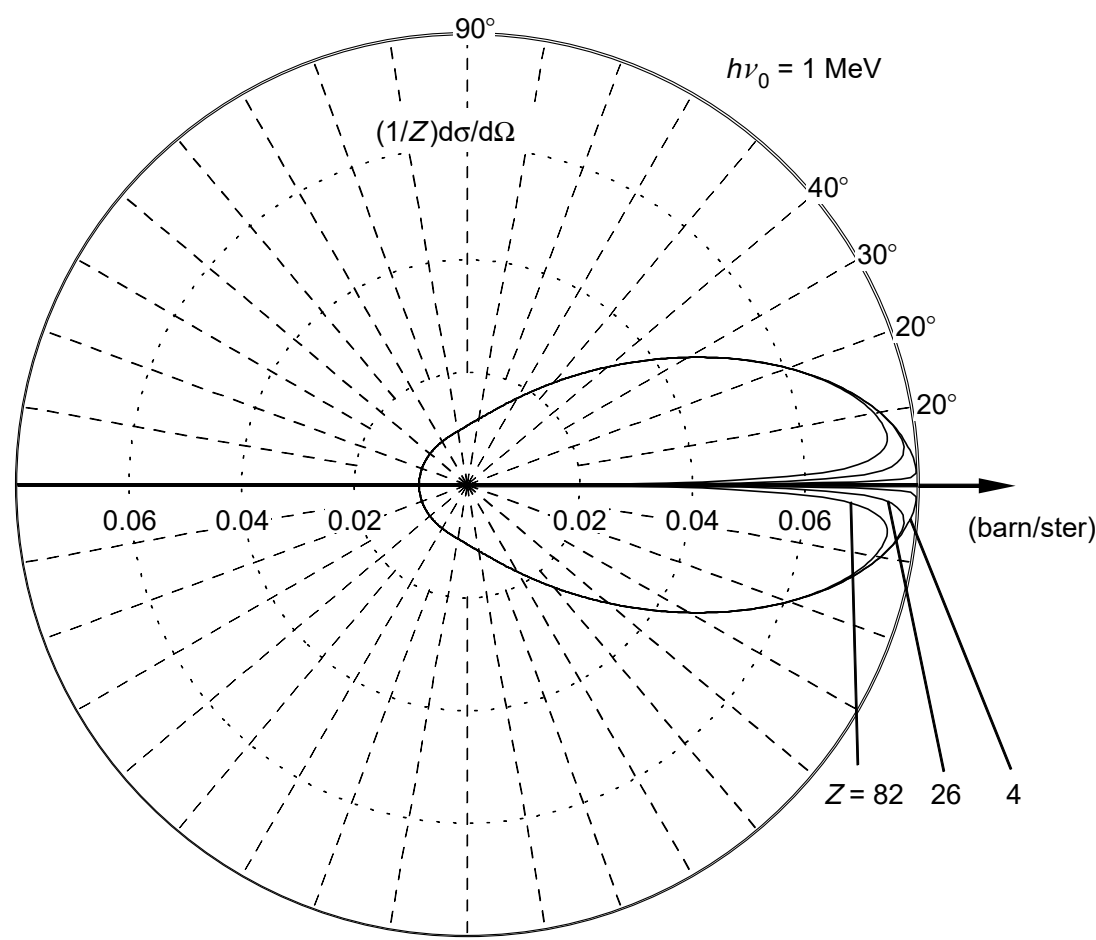

FIGURE 2.39 Compton differential cross section for the scattered photon, divided by the atomic number $\mathrm{Z}$, on $\mathrm{Be}, \mathrm{Fe}$, and $\mathrm{Pb}$ at $1 \mathrm{MeV}$ incident energy.

For fixed incident energy $E$, the variation with $Z$ of the differential cross section near the forward direction is then controlled merely by the $K$ shell energy $T=E_{K}(Z)$, as it determines $\theta_{\gamma}\left(h \nu_{0}, T\right)$. This is illustrated in Figure 2.39 , which shows, at $h \nu_{0}=1 \mathrm{MeV}$, the angular distribution of the scattered photon on $\mathrm{Be}, \mathrm{Fe}$, and $\mathrm{Pb}$. The differential cross section has been divided by $Z$ so that they become equal away from the forward direction. The cross sections differ only at angles less than $\theta_{\gamma}\left(h \nu_{0}, T\right)$.

A scattered photon can emerge at $\theta_{\gamma}=0$, but it cannot be accompanied by an ejected electron, and so, by definition, the process is not Compton scattering. Coherent scattering peaks at $\theta_{\gamma}=0$, but the scattered photon has the same energy as the incident photon. Inelastic scattering can also occur at $\theta_{\gamma}=0$, with the atom left in an excited, unionized state.

In contrast to the angular distribution of the scattered photon, the angular distribution of the outgoing electron is essentially the same for an atom as for the single-electron Klein-Nishina formula, at least when $E \gg E_{K}$. The reason is that when a photon scatters into angles near forward, the electron is ejected near $\theta_{\mathrm{e}}=\pi / 2$, with little energy and little range in the material. However, whenever 
the electron is ejected with a substantial energy, the photon must impart substantial momentum to the atom, and scatter through a large angle. In these cases, the photon and electron distributions follow the Klein-Nishina distribution.

\subsubsection{Compton Scattering in the Elements}

As with all photon interaction cross sections, the Compton cross section in the elements is tabulated in various databases, as mentioned in Section 2.12.

\subsubsection{Total Compton Cross Section}

If the angular distribution for atomic Compton scattering were the same as that of the Klein-Nishina formula, that is, if $S(q)=Z$ for all $q$, the total Compton cross section for scattering from an atom would be just $Z \sigma_{\mathrm{KN}}$. In the differential cross section, however, the actual deviation of $S$ from $Z$ at small $q$, as just discussed, makes the atomic Compton cross section less than $Z \sigma_{\mathrm{KN}}$, especially at relatively low energies.

The Compton cross section vs. $E=h \nu_{0}$ for several elements is shown in Figure 2.40. When expressed in $\mathrm{cm}^{2} / \mathrm{g}$ as in this figure, the approximate

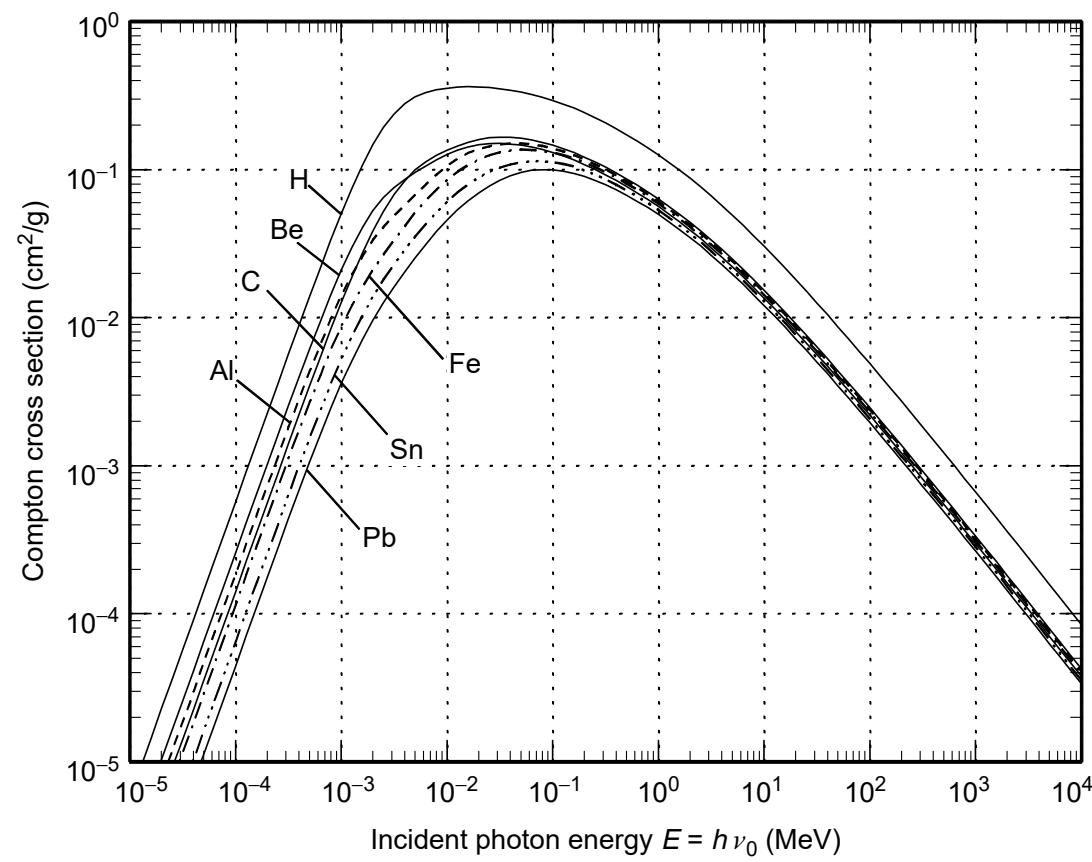

FIGURE 2.40 Total Compton cross section on several elements, $\mathrm{cm}^{2} / \mathrm{g}$. (Data from EPDL97.) 


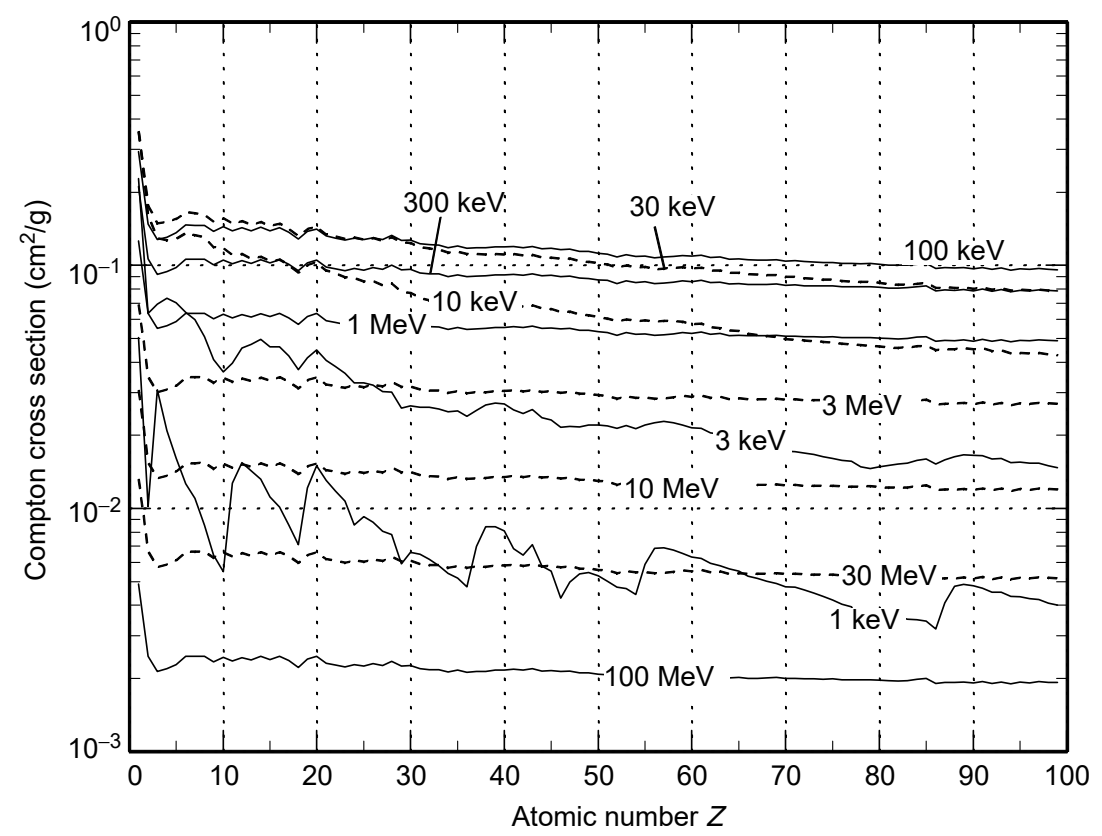

FIGURE 2.41 Total Compton cross section for all elements at several energies, $\mathrm{cm}^{2} / \mathrm{g}$. (Data from EPDL97.)

proportionality to $Z$ is largely removed, and the bulk cross section is only slowly varying with $Z$.

The slow variation with $Z$ is also seen in Figure 2.41, which shows the bulk Compton scattering cross section $\left(\mathrm{cm}^{2} / \mathrm{g}\right)$ for selected energies as a function of $Z$. The Compton cross section alone peaks near $100 \mathrm{keV}$ in all elements. Data are taken from EPDL97.

Collecting these data for the elements, the total atomic Compton interaction cross sections (barn/atom) in all elements for energies from $1 \mathrm{keV}$ to $100 \mathrm{MeV}$ are shown in the contour plot of Figure 2.42. The corresponding bulk total Compton cross sections $\left(\mathrm{cm}^{2} / \mathrm{g}\right)$ in all elements are shown in the contour plot in Figure 2.43. Here, because contour values are fairly closely spaced, the variation of atomic weight $A$ with $Z$ makes very visible irregularities in the shape of contour lines. These were first discussed in the context of the photoelectric contour plots (Figure 2.7 and Figure 2.9), in which the irregularities are not pronounced because of the relatively large spacing between contours.

The atomic Compton cross section is proportional to $Z$ and hence essentially to $A$. Mass density $\rho$ is also proportional to $A$. Therefore, at energies where the Compton effect dominates, the cross section expressed as $\mathrm{cm}^{2} / \mathrm{g}$ is 


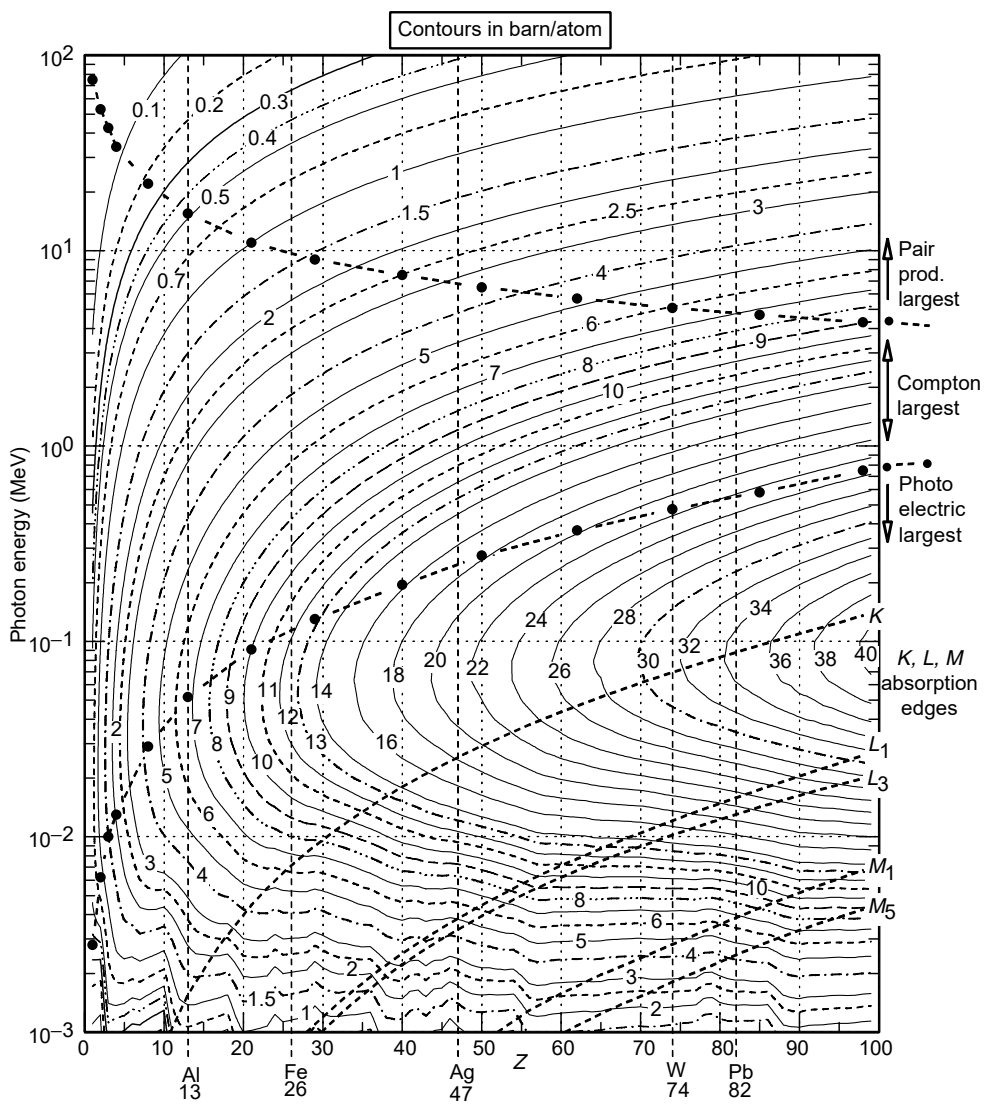

FIGURE 2.42 Contours of Compton cross section in all elements, from $1 \mathrm{keV}$ to 100 $\mathrm{MeV}$, in barn/atom. (Data from LLNL EPDL97.)

nearly independent of atomic number. This is clearly seen in Figure 2.43. Near $1 \mathrm{MeV}$, for example, the Compton cross section is about $0.06 \mathrm{~cm}^{2} / \mathrm{g}$ in all elements. In the vicinity of $1 \mathrm{MeV}$, Compton scattering is the dominant process; therefore, the photon-matter total interaction cross section in all elements is also about $0.06 \mathrm{~cm}^{2} / \mathrm{g}$. This will be seen again in the total crosssection graph of Figure 2.54 in Section 2.8.1. It is not true far from $1 \mathrm{MeV}$, where the photoelectric or pair production effects dominate.

\subsection{PAIR PRODUCTION}

At energies above about $1 \mathrm{MeV}$, another photon-atom process can occur in which the photon disappears and an electron-positron pair emerges from the 


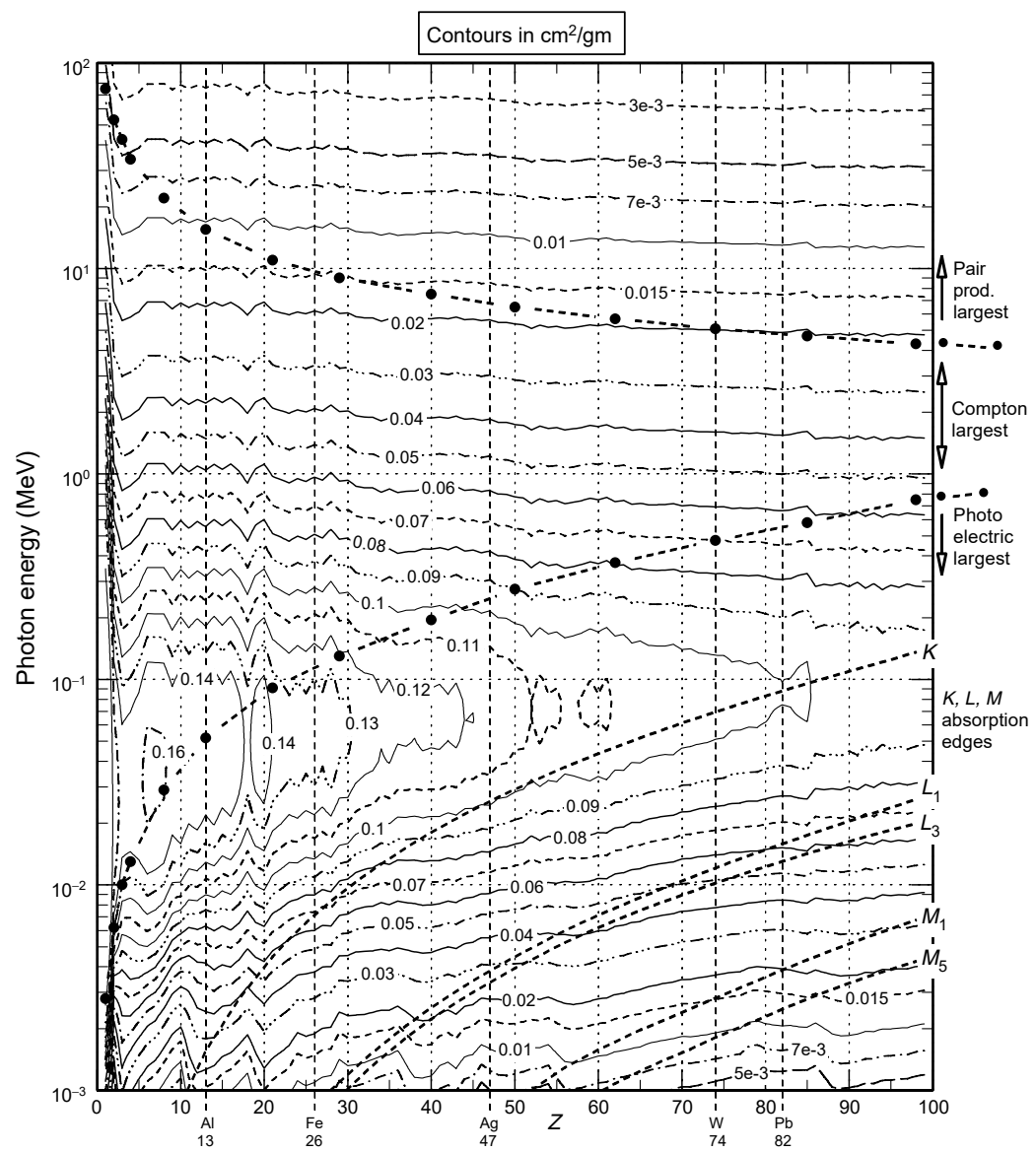

FIGURE 2.43 Contours of Compton cross section in all elements, from $1 \mathrm{keV}$ to 100 $\mathrm{MeV}$, in $\mathrm{cm}^{2} / \mathrm{g}$. (Data from LLNL EPDL97.)

atom. This pair production occurs by the photon interacting with the (static) electric field surrounding the nucleus or an electron. As is the case for Delbrück scattering, enormous fields are required for this process to occur. As mentioned previously, the static nuclear field at a distance $r=100 \mathrm{fm}=10^{-3} \AA$ from a high- $Z$ nucleus is of the order $10^{19} \mathrm{~V} / \mathrm{m}$.

For $\gamma$ rays of less than several $\mathrm{MeV}$, pair production is seldom the dominant process. At $3 \mathrm{MeV}$ in aluminum, for example, the cross section is only $0.002 \mathrm{~cm}^{2} / \mathrm{g}$, whereas the Compton cross section is $0.034 \mathrm{~cm}^{2} / \mathrm{g}$. However, by $20 \mathrm{MeV}$ in $\mathrm{Al}$, or at only $5 \mathrm{MeV}$ in $\mathrm{W}$, the cross section for pair production is as large as the cross section for Compton scattering. At still higher energies, pair production is the dominant process for a photon to interact with matter, and remains so at all higher energies. 


\subsubsection{Threshold Energies for Pair Production}

In pair production in the Coulomb field of a nucleus, the heavy nucleus can recoil, absorbing momentum but essentially no energy. Thus, for all practical purposes, energy is conserved among the incident photon and the two members of the created pair, but momentum is not conserved among those three particles alone (of course, both energy and momentum are rigorously conserved among the photon, the nucleus, and the created pair). To create the pair and conserve energy, the photon needs to have only $2 m c^{2}$ of energy, just sufficient to create both members of the pair at rest. Thus, the threshold photon energy for pair production in the field of a nucleus is $2 m c^{2}=1.022 \mathrm{MeV}$.

Pair production can also occur in the field of an electron in the atom. Here, the created positron and electron and the struck electron are all ejected from the atom; the process is referred to as triplet production. In this case, the recoiling electron absorbs both momentum and energy, and the energy it absorbs is not negligible. As the target electron carries some energy away, the threshold photon energy for pair production in the field of an electron will be greater than $2 m c^{2}$. To obtain the threshold energy, it is convenient to consider the conservation laws in the center-of-momentum (CM) frame of reference.

Figure 2.44 sketches the geometry. Results will be obtained for a target particle of any mass $M$, but we are primarily interested in the threshold when the target particle is an electron, $M=m$.

For the discussion of threshold kinematics, we work with units in which $h=c=1$. In the laboratory frame, where the target mass $M$ is at rest, the photon has energy $\nu_{0}$. In the CM system, the photon has energy $\nu_{\mathrm{CM}}$,

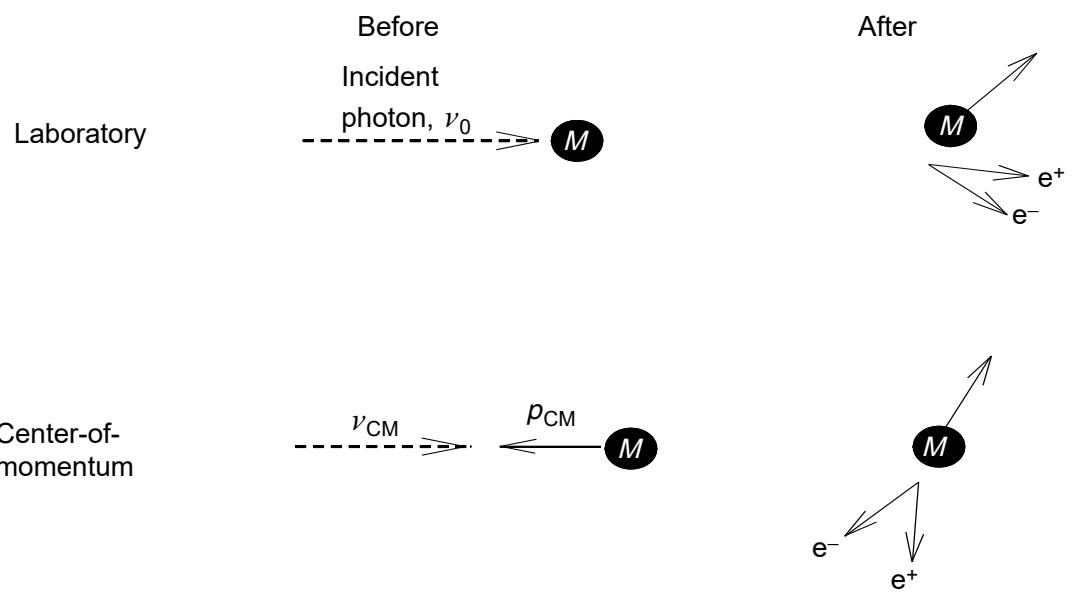

FIGURE 2.44 Pair production by a photon in the Coulomb field of a charged particle of mass $M$. Depicted before and after the interaction, and as seen in the laboratory and in the center-of-momentum frames of reference. 
and the target has momentum $p_{\mathrm{CM}}=-\nu_{\mathrm{CM}}$. The target energy is $E_{\mathrm{CM}}=\sqrt{ }\left(M^{2}+p_{\mathrm{CM}}^{2}\right)=\sqrt{ }\left(M^{2}+\nu_{\mathrm{CM}}^{2}\right)$.

At the threshold of pair production in the CM system, each member of the pair is created at rest. The photon has energy $\nu_{\mathrm{CM}}^{\text {th }}$ to be determined. As the total momentum vanishes, the target particle is also at rest, and the only energies are the three rest masses. Consequently, equating the incident system energy to the final energy, the CM threshold photon energy for pair production is given by

$$
\nu_{\mathrm{CM}}^{\mathrm{th}}+\sqrt{M^{2}+\nu_{\mathrm{CM}}^{\mathrm{th}}{ }^{2}}=M+2 m
$$

or

$$
\nu_{\mathrm{CM}}^{\mathrm{th}}=2 m \frac{M+m}{M+2 m}
$$

To obtain the threshold photon energy $\nu_{0}^{\text {th }}$ in the laboratory system, we need only to transform the energy $\nu_{\mathrm{CM}}^{\text {th }}$ back to the lab frame in which $M$ is at rest.

To do this, let the velocity of the CM system, measured in the lab frame, be $V$. Then, in the CM the photon has energy

$$
\nu_{\mathrm{CM}}=\nu_{0} \gamma(1-V)
$$

where

$$
\gamma=\frac{1}{\sqrt{1-V^{2}}}
$$

and the target has velocity $-V$ and momentum $p_{\mathrm{CM}}=-\gamma M V$. However by definition, the CM system is the one in which the photon momentum equals in magnitude the target momentum

$$
\nu_{\mathrm{CM}}=p_{\mathrm{CM}}=\gamma M V
$$

Thus, $V$ is determined by equating

$$
\nu_{\mathrm{CM}}=\nu_{0} \gamma(1-V)=\gamma M V
$$

from which one obtains

$$
V=\frac{\nu_{0}}{M+\nu_{0}}
$$

Inserting this expression for $V$ in Equation 2.46, we express $\nu_{\mathrm{CM}}$ in terms of $\nu_{0}$ 


$$
\nu_{\mathrm{CM}}=\nu_{0} \sqrt{\frac{1-V}{1+V}}=\frac{\nu_{0}}{\sqrt{1+2 \nu_{0} / M}}
$$

At threshold, this is to be equated to Equation 2.45, and we have

$$
\nu_{\mathrm{CM}}^{\mathrm{th}}=\frac{\nu_{0}^{\mathrm{th}}}{\sqrt{1+2 \nu_{0}^{\mathrm{th}} / M}}=2 m \frac{M+m}{M+2 m}
$$

which is the desired relation determining the laboratory threshold energy $\nu_{0}^{\text {th }}$ in terms of the target mass $M$ and electron mass $m$.

Clearly, if the target is very heavy, $M \gg m$, the threshold energy is $2 m$, as stated for pair production in the field of a nucleus. But if the target is an electron, $M=m$, Equation 2.52 becomes

$$
\nu_{\mathrm{CM}}^{\mathrm{th}}=\frac{4}{3} m
$$

Reverting to normal units, the CM threshold photon energy for pair production in the field of an electron is $(4 / 3) m c^{2}$. In the laboratory frame, Equation 2.52 says that the threshold photon energy for pair production is

$$
h \nu_{0}^{\text {th }}=4 m c^{2}
$$

Pair production in the field of a nucleus starts at $h \nu_{0}^{\text {th }}=2 m c^{2}$, whereas pair production in the field of an electron starts at $h \nu_{0}^{\text {th }}=4 m c^{2}=2.044 \mathrm{MeV}$.

In pair production from an electron, energy and momentum are conserved among the incident photon, the created pair, and the original electron, but neither energy nor momentum is conserved among just the photon and the created pair.

\subsubsection{Z Dependence and Approximate Value of Cross Section}

Heitler [He54] gives a thorough derivation of the pair production cross section. Bethe and Ashkin [Be53] and Evans [Ev55] review the theory and experimental basis. Hubbell et al. [Hu80] present a discussion and data in all elements.

In the Coulomb field of an exposed nucleus, the amplitude for the process is proportional to the field magnitude and hence to the nuclear charge $\mathrm{Ze}$ and to the electron-positron charge $e$. Then the amplitude is proportional to $Z e^{2}$, and the pair production cross section on an unscreened nucleus is proportional to $Z^{2} e^{4} \propto Z^{2} r_{\mathrm{o}}^{2}$. Screening by atomic electrons is important when both the outgoing positron and electron are relativistic. At these high energies, screening makes the $Z$ dependence slightly less than the second power. In general, for all elements and at all incident photon energies above $3 \mathrm{MeV}$, the total 
pair production cross section from the (screened) nucleus rises from about 1 to less than about 15 times $\sigma_{0}$ as $Z$ increases, where

$$
\sigma_{0} \equiv \alpha Z^{2} r_{\mathrm{o}}^{2}=5.795 \times 10^{-4} Z^{2}(\text { barn })
$$

The contribution to the total cross section due to pair production from atomic electrons is proportional to $Z r_{\mathrm{o}}^{2}$, hence the total (nuclear plus electronic) pair production cross section is approximately proportional to $\alpha Z(Z+1) r_{\mathrm{o}}^{2}=(1+1 / Z) \sigma_{0}$, at energies greater than $4 m c^{2}$ in which both processes are possible. Again, screening reduces this value at high energies. Figure 2.45 illustrates the approximate proportionality to $Z(Z+1)$. The reduction at high energies is about a factor of two in high- $Z$ elements.

Heitler [He54] showed that the nuclear pair production cross section $\sigma_{\mathrm{pp}}^{\text {nuc }}(Z, E)$, at very high photon energies $E$, approaches a constant value independent of $E$, given approximately by

$$
\sigma_{\mathrm{pp}}^{\mathrm{nuc}}(E \rightarrow \infty) \rightarrow \frac{28}{9}\left[\ln \left(\frac{183}{Z^{1 / 3}}\right)-\frac{1}{42}\right] \sigma_{0}
$$

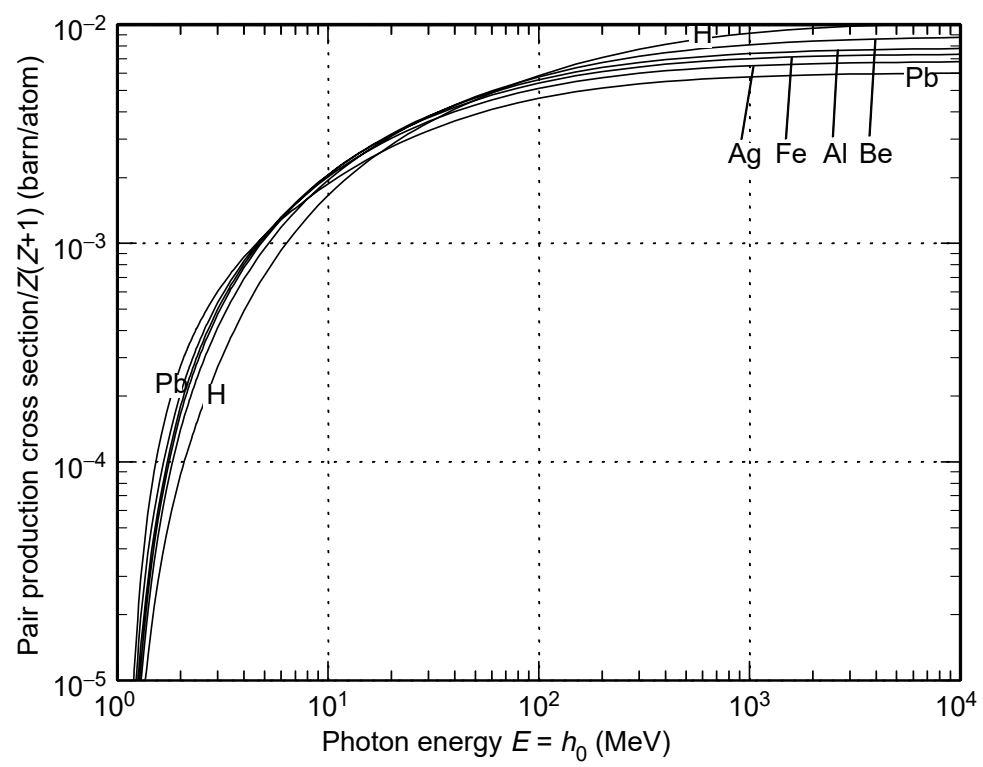

FIGURE 2.45 The total (nuclear plus electronic) pair production cross section divided by $Z(Z+1)$ in several elements. Cross section in barn/atom. Between the lightest and heaviest elements, at high energies, the cross section departs from proportionality to $Z(Z+1)$ by about a factor of 2. (Data from EPDL97.) 
More correctly, above $1 \mathrm{GeV}$, both the nuclear and electronic pair production cross sections from an atom are given by an expression of the form

$$
\sigma_{\infty}+\left[c_{2}+c_{3} \ln (E)\right] / E
$$

$\sigma_{\infty}$ is the infinite energy cross section. $\sigma_{\infty}$ and the coefficients $c_{2}$ and $c_{3}$ are tabulated for each element in, for example, the EPICSHOW data set distributed with LLNL's TART code.

In $\mathrm{Al}$, the right-hand side of $\sigma_{\mathrm{pp}}^{\text {nuc }}(E \rightarrow \infty)$ is 1.32 barn. The infinite energy number $\sigma_{\infty}$ for the nuclear cross section is given by the LLNL database EPDL97 as 1.3097, very close to the theoretical number. With the contribution from atomic electrons included, the total pair production cross section at 100 $\mathrm{GeV}$ on $\mathrm{Al}$ is given in EPDL97 as $1.428 \mathrm{~b}$ and on $\mathrm{Pb}$ at the same energy as 41.183 barn.

The foregoing is the "classic" discussion of pair production. Tsai [Ts74] has summarized more recent developments. He reports that the more accurate calculations put the infinite energy $\mathrm{Al}$ pair production cross section at 1.443 barn and that of $\mathrm{Pb}$ at $41.72 \mathrm{~b}$. In the LLNL database, the infinite energy number $\sigma_{\infty}^{\text {nuc }}+\sigma_{\infty}^{\text {elec }}$ for $\mathrm{Al}$ is 1.429 and that for $\mathrm{Pb}$ is 41.244 barn, slightly less than Tsai's value.

\subsubsection{Graphs of Pair Production Cross Sections}

Tabulations of pair production cross section data are available separately for the nuclear and electronic processes (e.g., in NIST XCOM or in EPDL97), but here we plot only their sum, the total pair production cross section.

Figure 2.46 shows cross sections as a function of $E=h \nu_{0}$ for seven elements, while Figure 2.47 plots the cross sections as a function of $Z$ for selected photon energies.

In Figure 2.48, contours of the total pair production cross section vs. $Z$ and $E$ in barn/atom are shown. The variation with both independent variables is, of course, quite smooth.

Again converting to $\mathrm{cm}^{2} / \mathrm{g}$, contours are shown in Figure 2.49. The irregularity in these contours introduced by the irregular variation of $A(Z)$ is evident. It is especially pronounced near $Z=85-86$, where $A(Z)$ has a significant discontinuity (Figure 2.8).

\subsection{TOTAL PHOTON CROSS SECTIONS}

Figure 2.50a through Figure 2.50o present all cross sections (photoelectric, Rayleigh (coherent), Compton (incoherent), pair production and total) vs. incident photon energy in a number of elements of increasing Z. Aluminum was shown in Figure 2.4. Plots such as these, for all elements, are conveniently available in LLNL's display package, EPICSHOW, distributed with the 


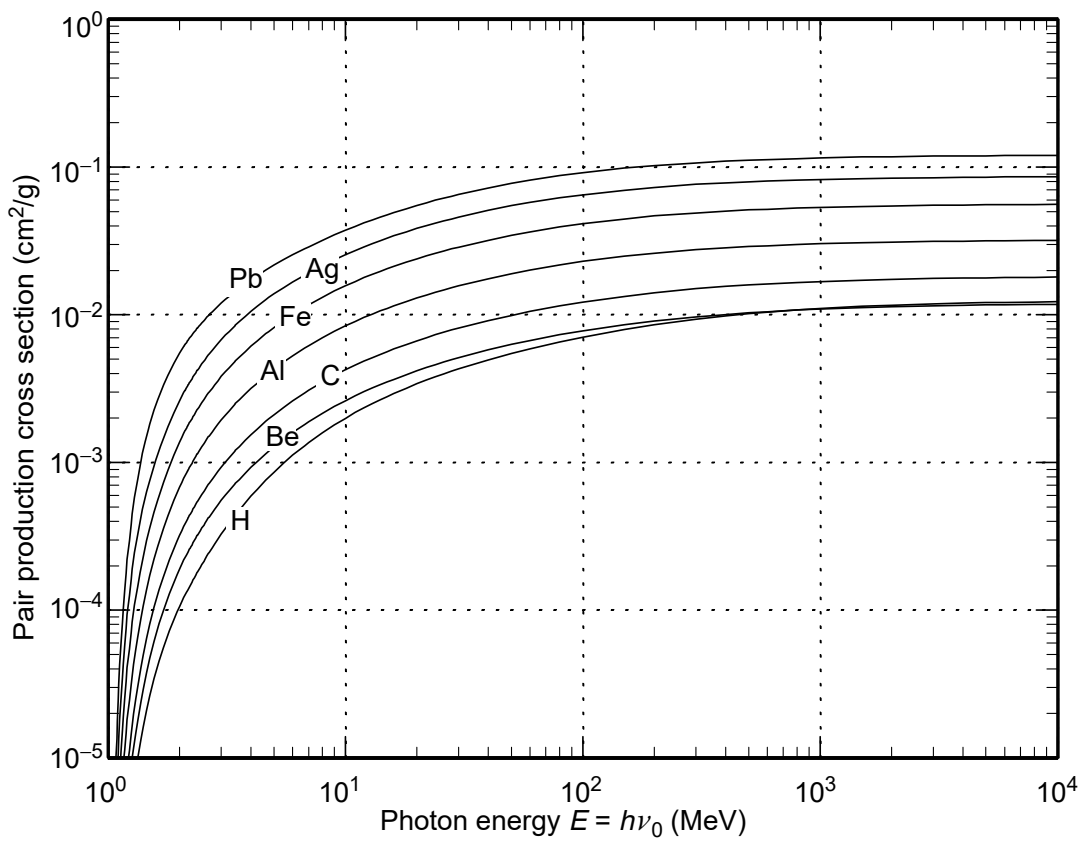

FIGURE 2.46 Total (nuclear plus electronic) pair production cross section for selected elements vs. incident photon energy, in $\mathrm{cm}^{2} / \mathrm{g}$.

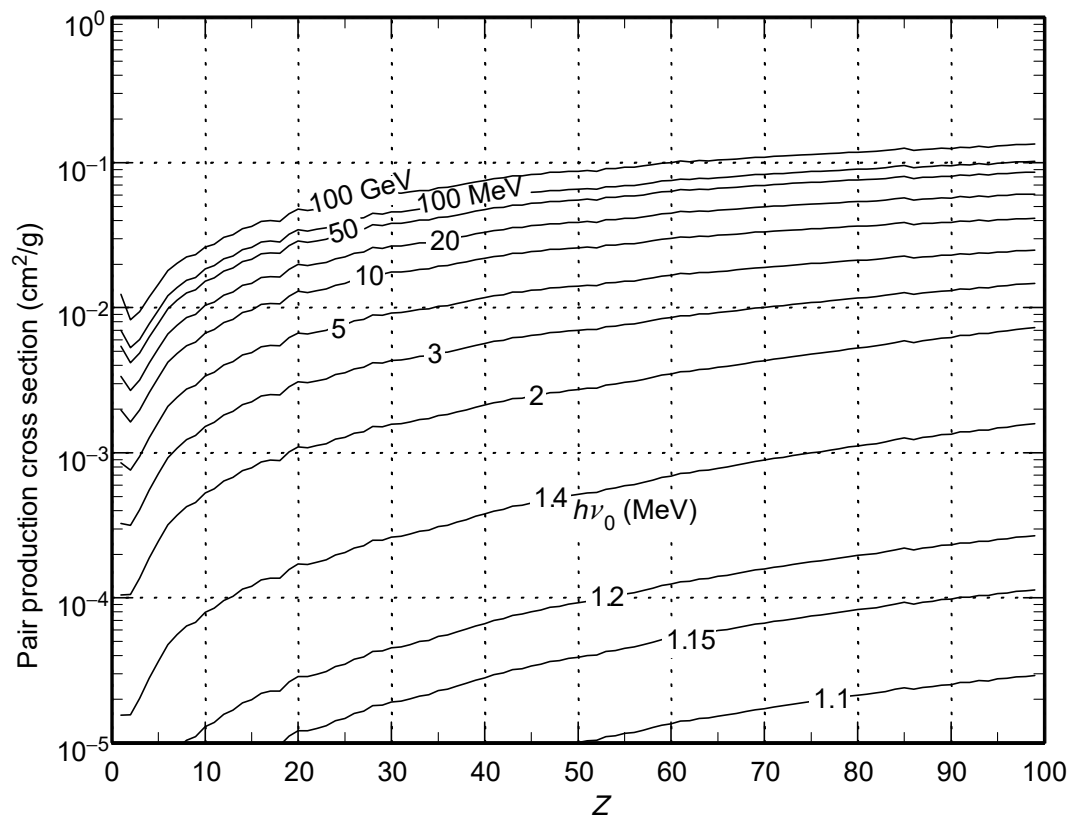

FIGURE 2.47 Total (nuclear plus electronic) pair production cross section for selected incident photon energies vs. $Z$, in $\mathrm{cm}^{2} / \mathrm{g}$. 


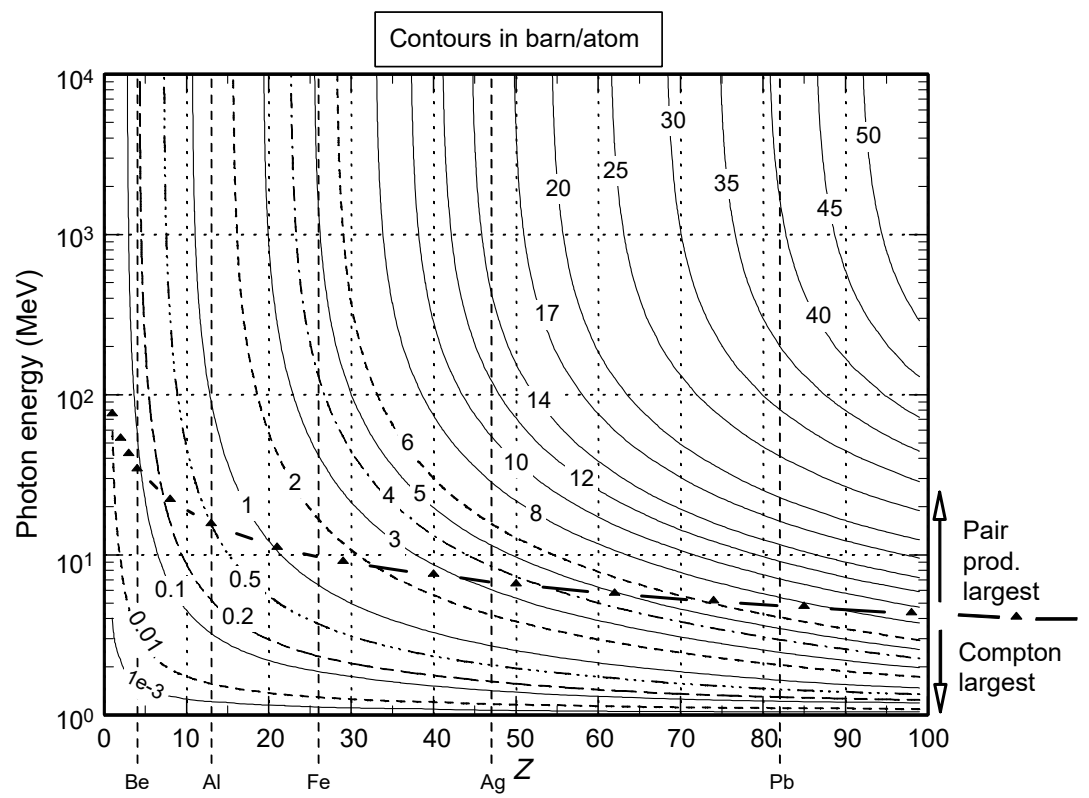

FIGURE 2.48 Contours of total (nuclear plus electronic) pair production cross section vs. incident photon energy and $Z$, in barn/atom.

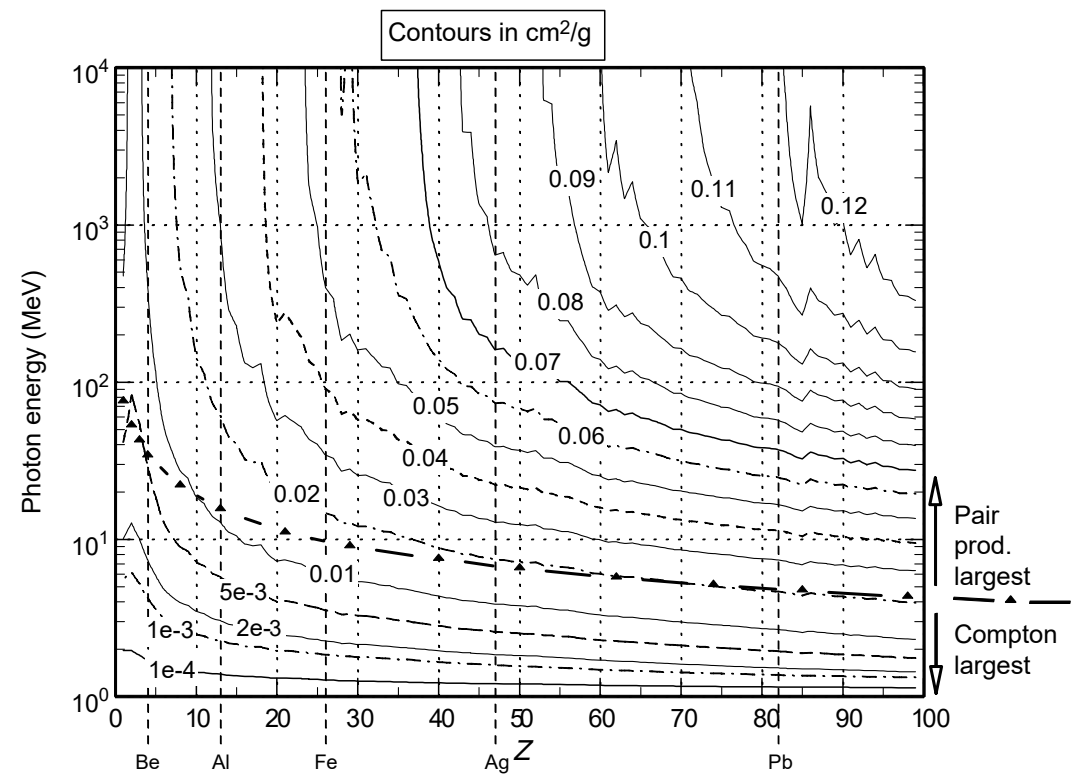

FIGURE 2.49 Contours of total (nuclear plus electronic) pair production cross section vs. incident photon energy and $Z$, in $\mathrm{cm}^{2} / \mathrm{g}$. 


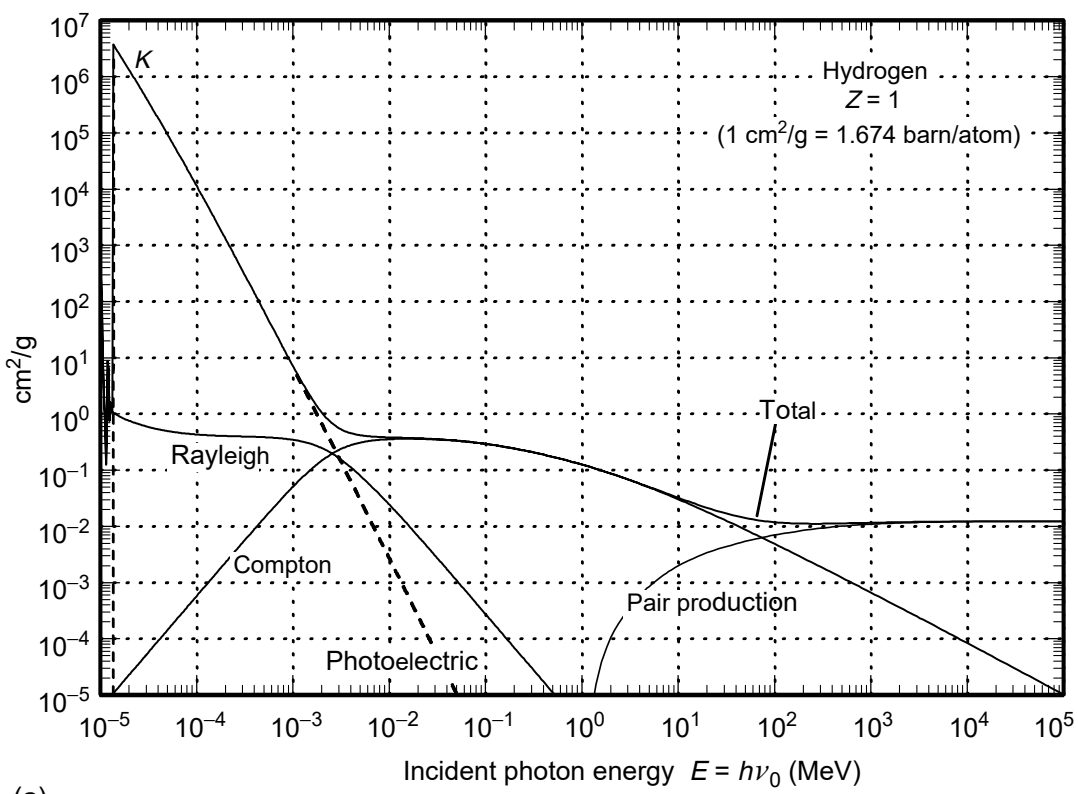

(a)

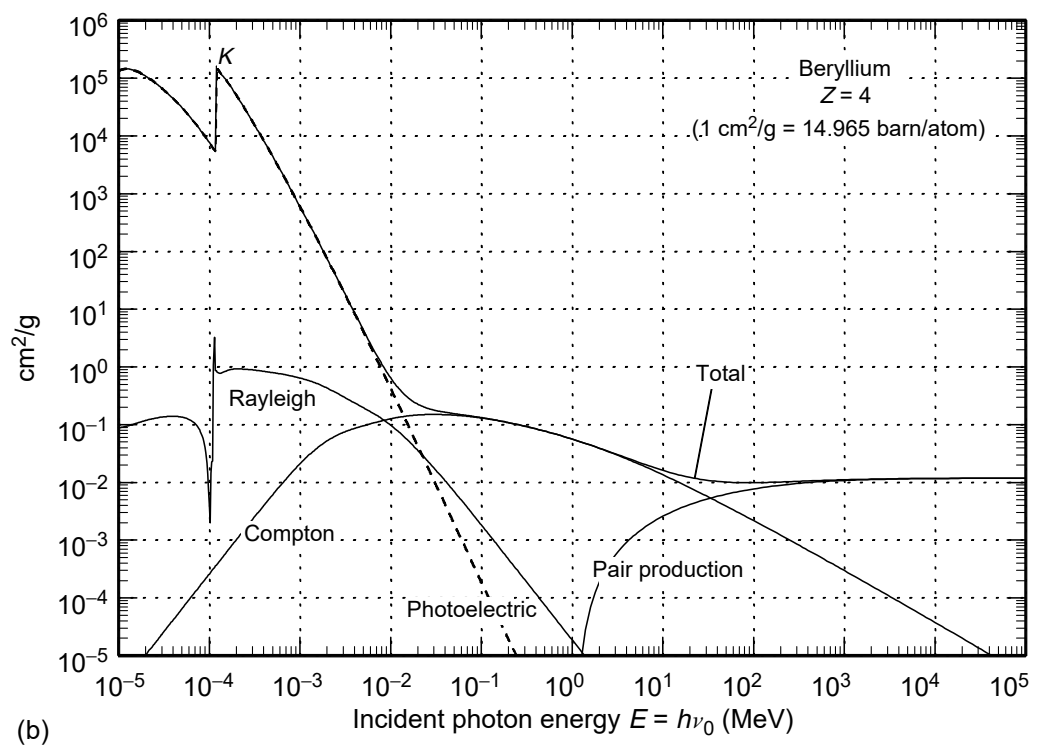

FIGURE 2.50 Photon interaction cross sections on isolated, cold atoms. (a) hydrogen (H). (b) beryllium (Be). (Data from EPDL97.) 


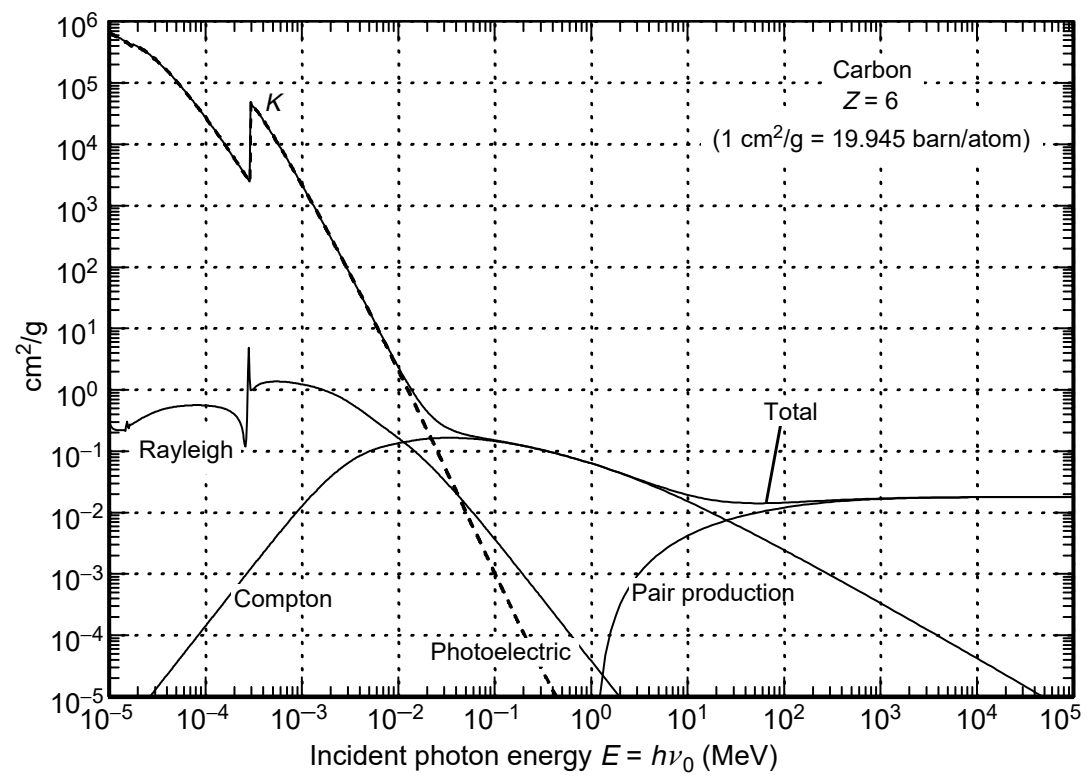

(c)

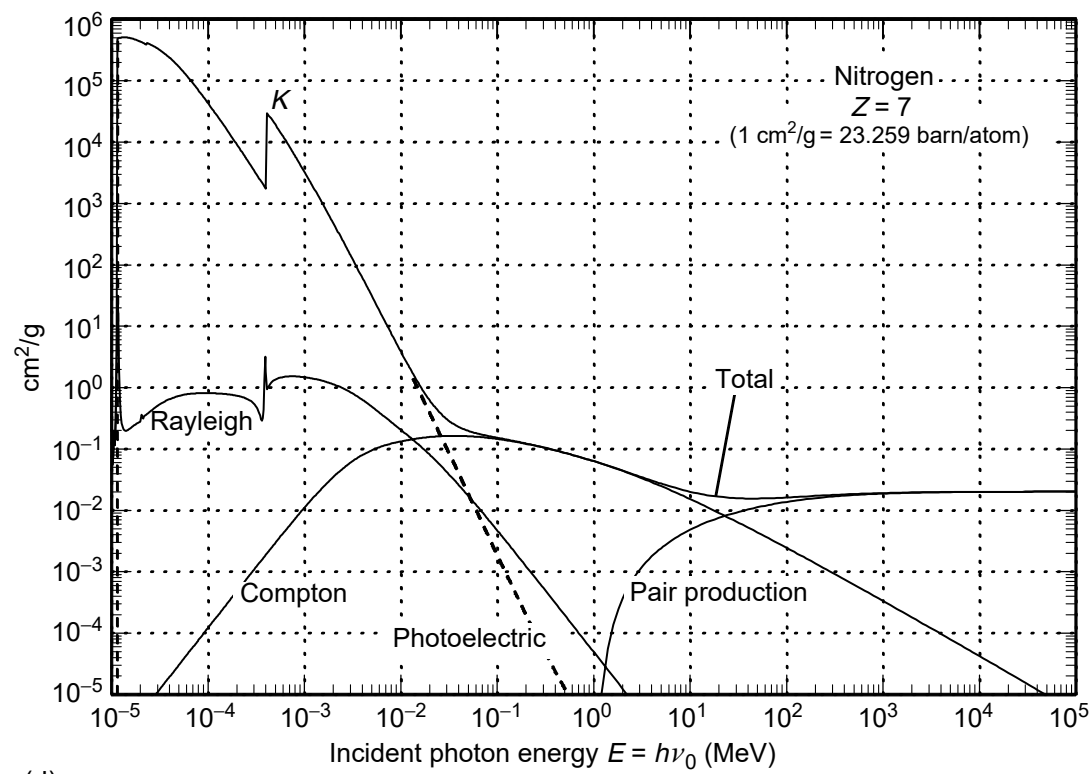

(d)

FIGURE 2.50 (continued) Photon interaction cross sections on isolated, cold atoms. (c) carbon (C). (d) nitrogen (N). (Data from EPDL97.) 


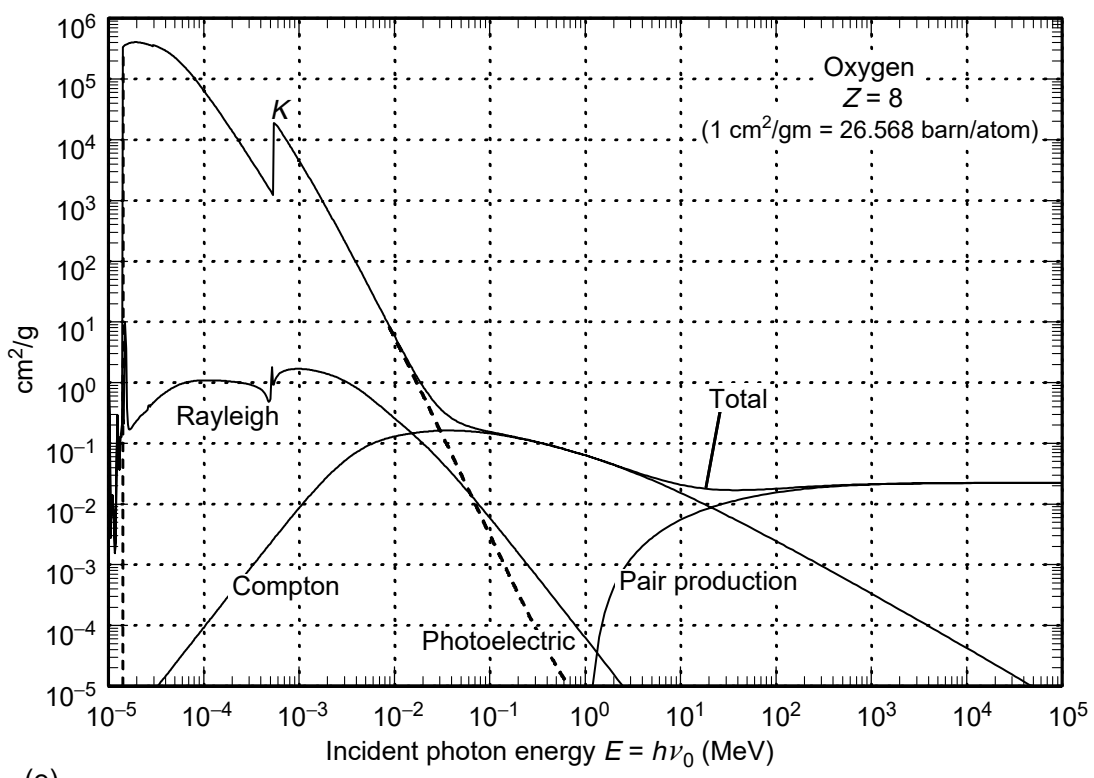

(e)

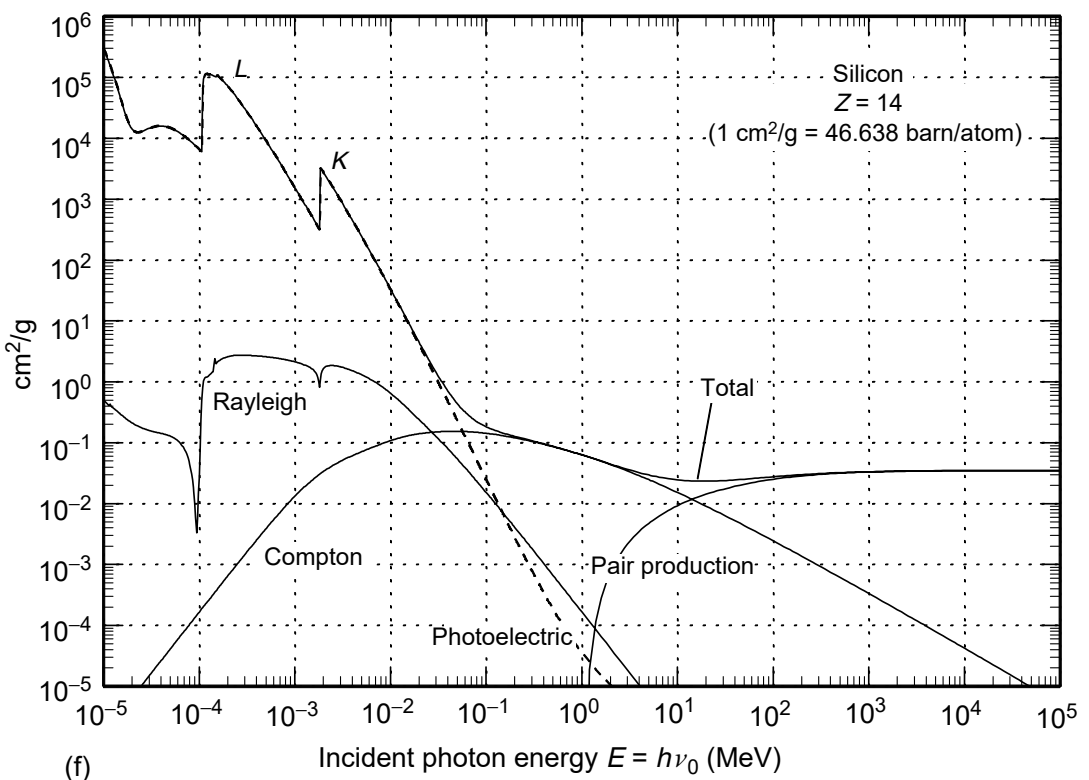

FIGURE 2.50 (continued) Photon interaction cross sections on isolated, cold atoms. (e) oxygen (O). (f) silicon (Si). (Data from EPDL97.) 


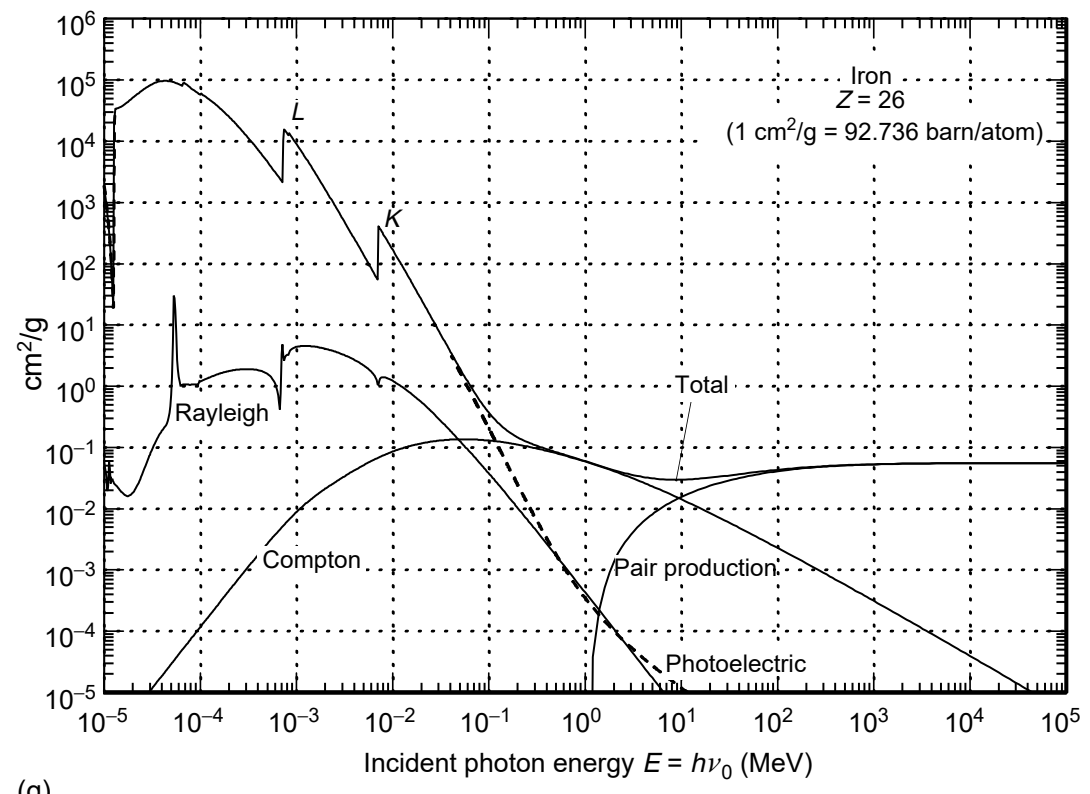

(g)

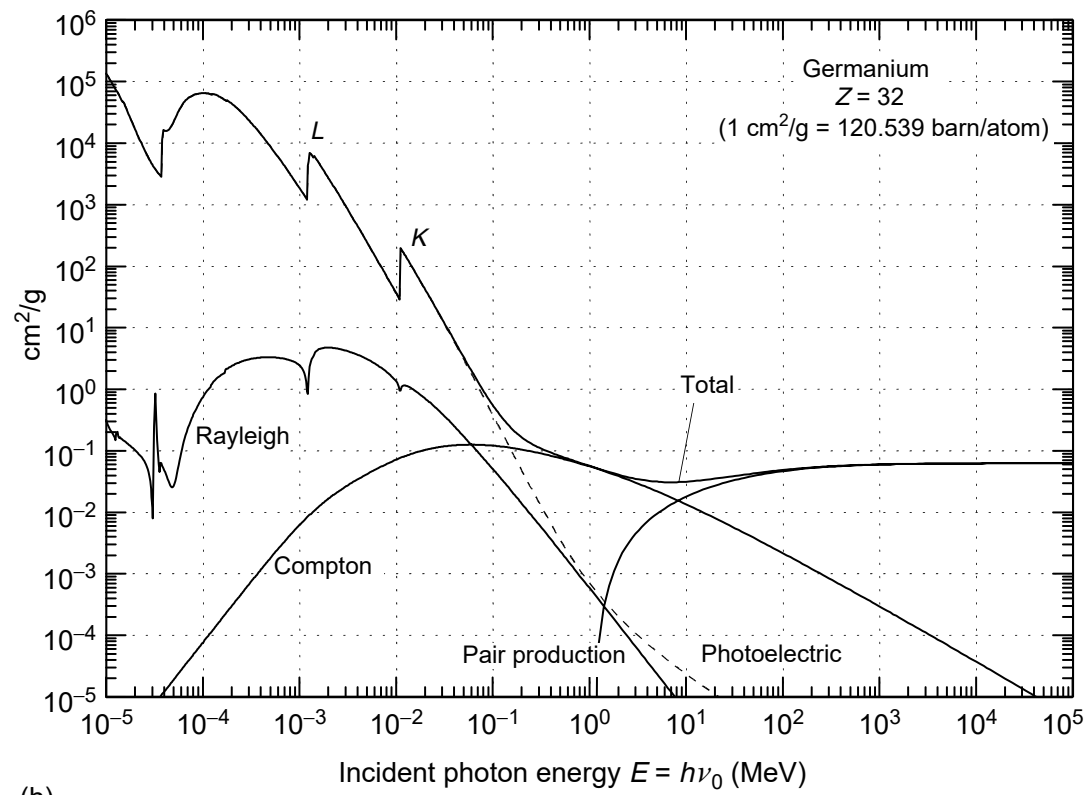

FIGURE 2.50 (continued) Photon interaction cross sections on isolated, cold atoms. (g) iron (Fe). (h) germanium (Ge). (Data from EPDL97.) 


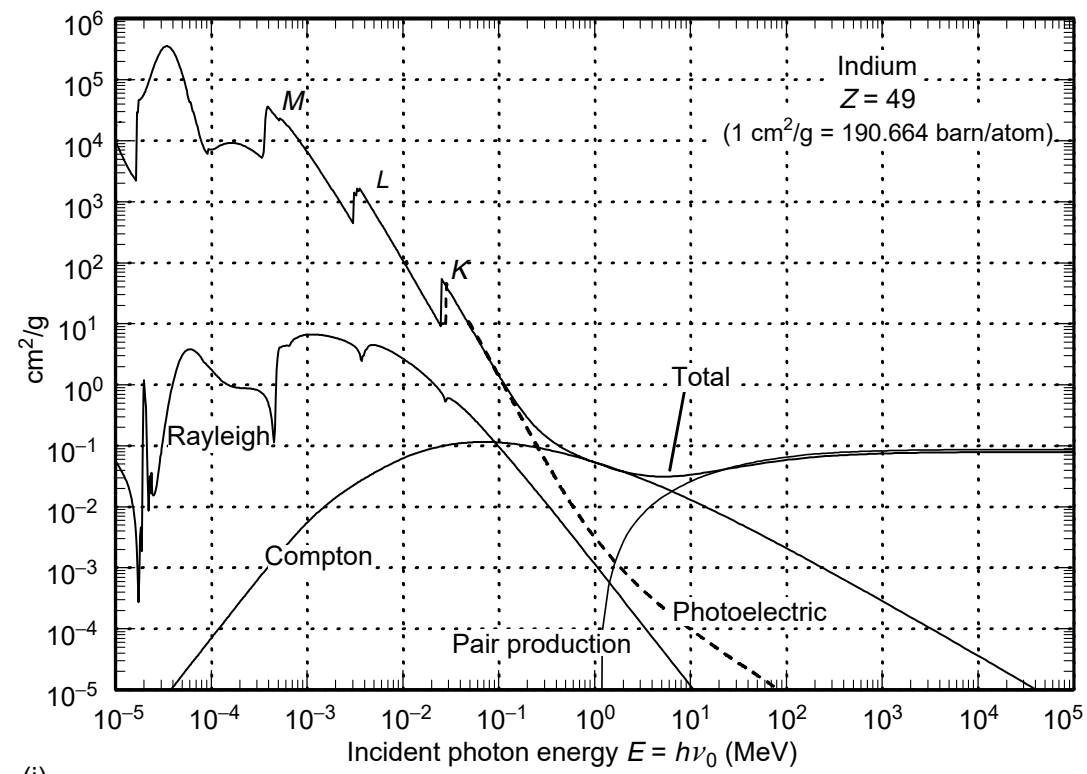

(i)

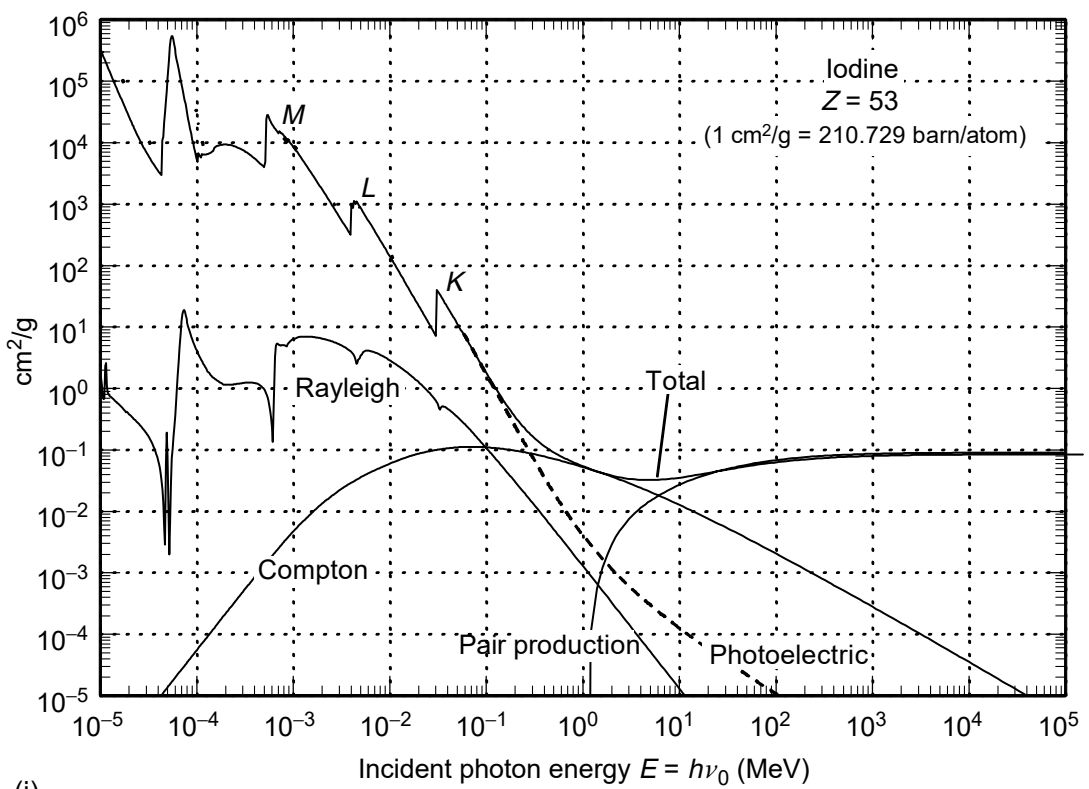

(j)

FIGURE 2.50 (continued) Photon interaction cross sections on isolated, cold atoms. (i) indium (In). ( j ) iodine (I). (Data from EPDL97.) 


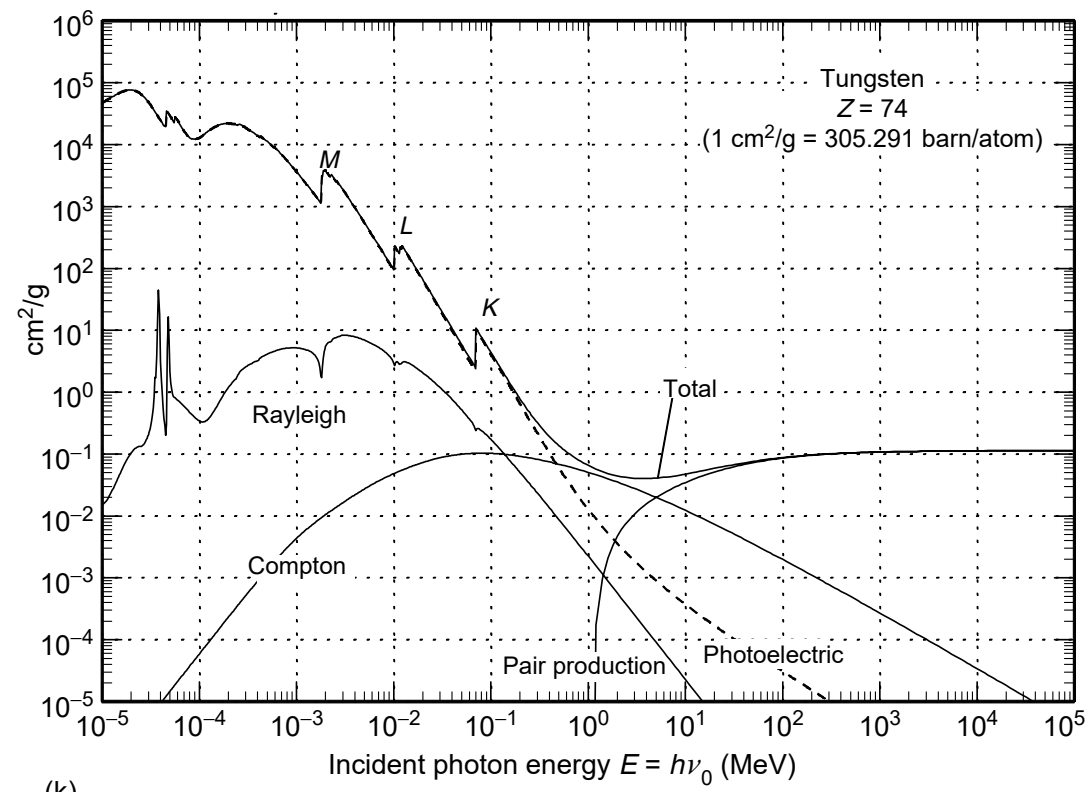

(k)

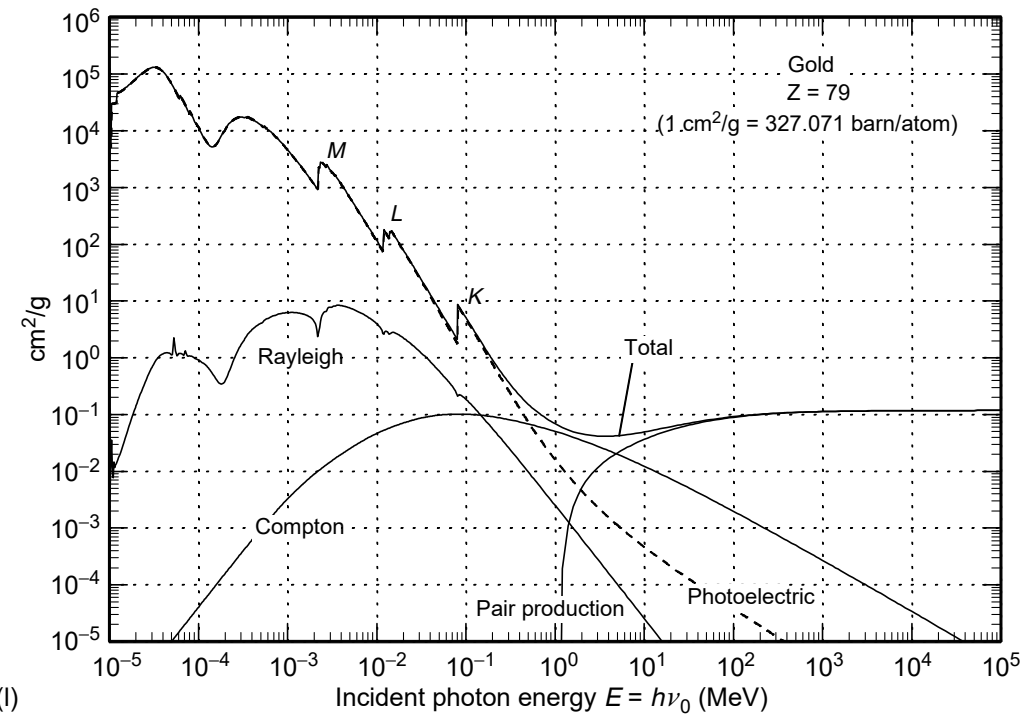

FIGURE 2.50 (continued) Photon interaction cross sections on isolated, cold atoms. (k) tungsten (W). (1) gold (Au). (Data from EPDL97.) 


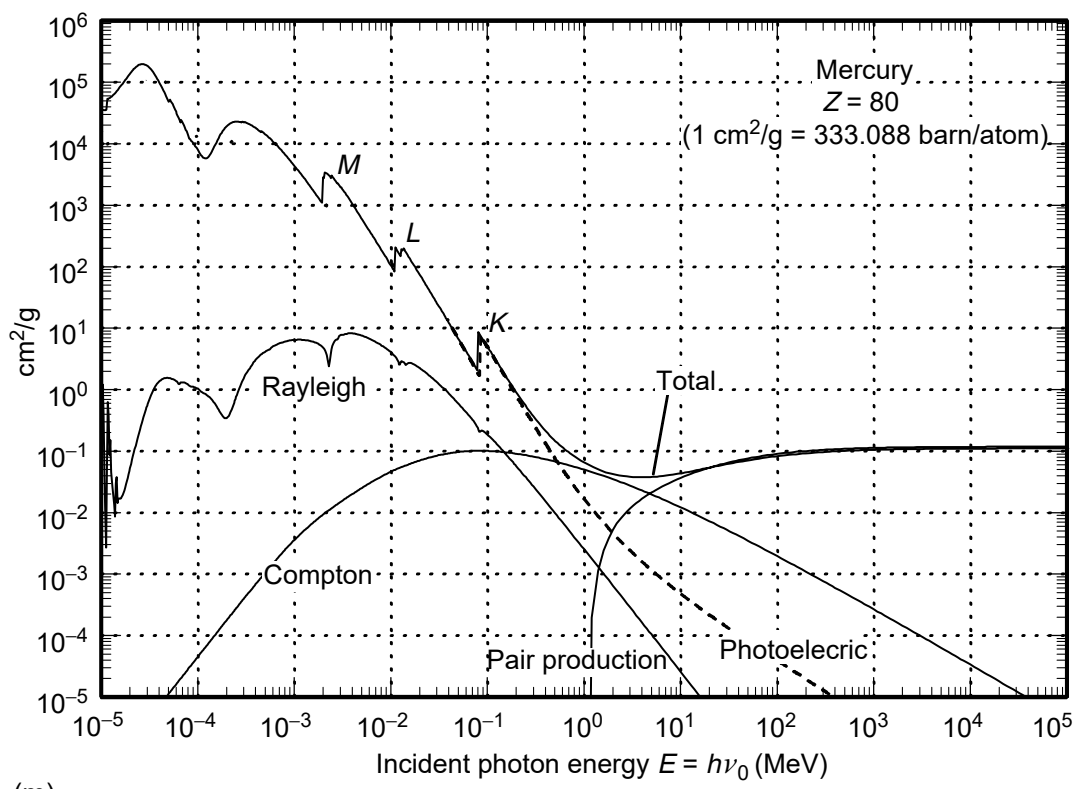

(m)

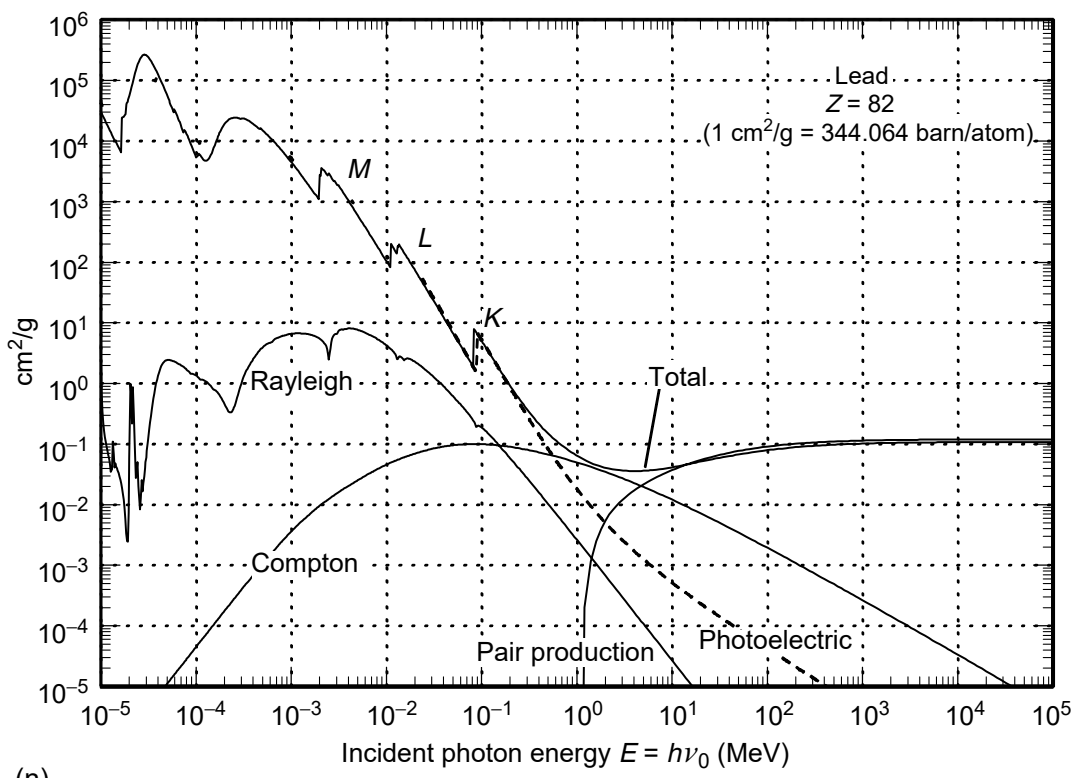

(n)

FIGURE 2.50 (continued) Photon interaction cross sections on isolated, cold atoms. (m) mercury $(\mathrm{Hg})$. (n) lead $(\mathrm{Pb})$. (Data from EPDL97.) 


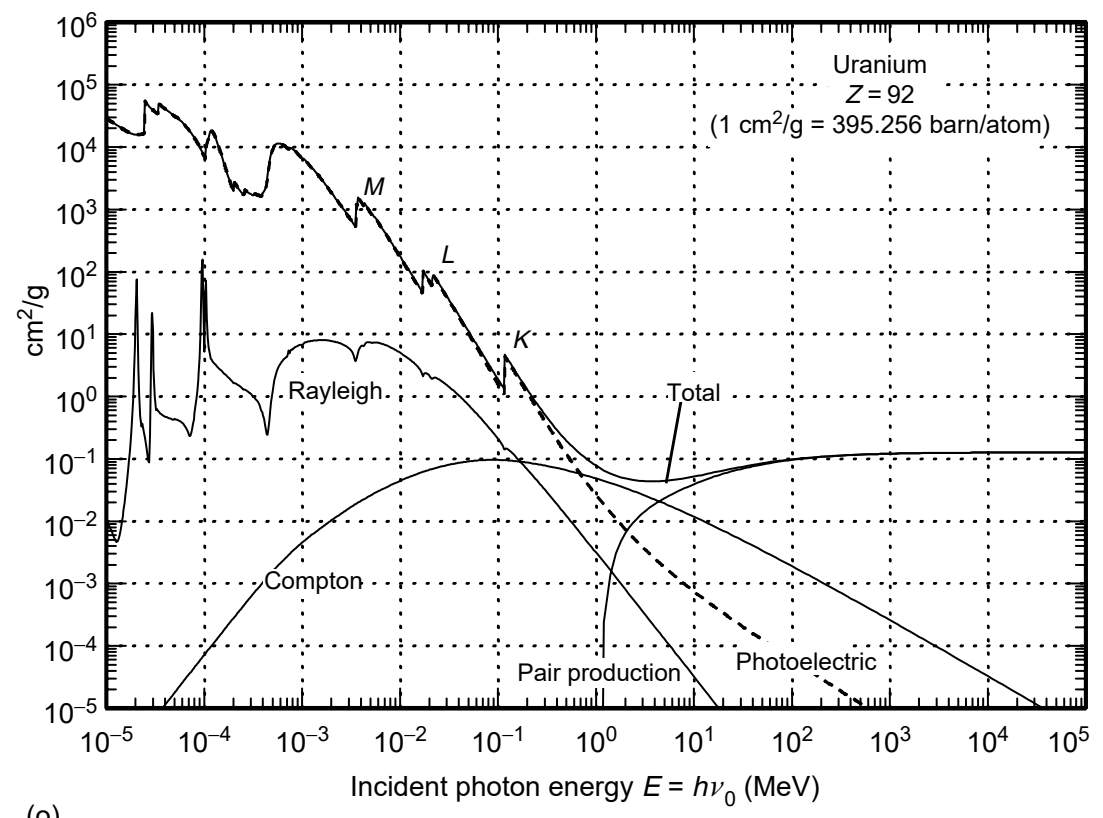

FIGURE 2.50 (continued) Photon interaction cross sections on isolated, cold atoms. (o) uranium (U). (Data from EPDL97.)

neutron-photon Monte Carlo code TART. Similar graphs can also be generated by the Janis software from the Nuclear Energy Agency, or by the ZVView program at the IAEA (see Section 2.12).

It is worth noting that in hydrogen, Compton scattering is the main process from $3 \mathrm{keV}$ to $70 \mathrm{MeV}$, essentially the full range of $\mathrm{x}$-rays and $\gamma$ rays. Photoelectric absorption does not dominate until below $3 \mathrm{keV}$, and pair production does not dominate until above $70 \mathrm{MeV}$. And between about 10 $\mathrm{keV}$ and $10 \mathrm{MeV}$, Compton scattering is an order of magnitude more probable than any other process. This is significant as $\mathrm{H}$ is a major constituent of organic materials; scattering from $\mathrm{H}$ is more probable than absorption in $\mathrm{H}$.

Likewise, in carbon, Compton scattering is the main process from about $25 \mathrm{keV}$ to $25 \mathrm{MeV}$. Low- $Z$ materials tend to scatter $\mathrm{x}$-rays (if sufficiently energetic) more than absorb them.

Total cross sections for several elements as a function of photon energy $E$ are also shown in Figure 2.51, and at selected energies as a function of $Z$ in Figure 2.52.

Finally, contours of total cross section (barn/atom) in all elements for all energies are shown in Figure 2.53, and in $\left(\mathrm{cm}^{2} / \mathrm{g}\right)$ in Figure 2.54. Its inverse, the mfp against any scattering $\left(\mathrm{g} / \mathrm{cm}^{2}\right)$, is separately useful; it is shown in Figure 2.55. Large, color contour plots of these cross sections, in both barn/atom and 


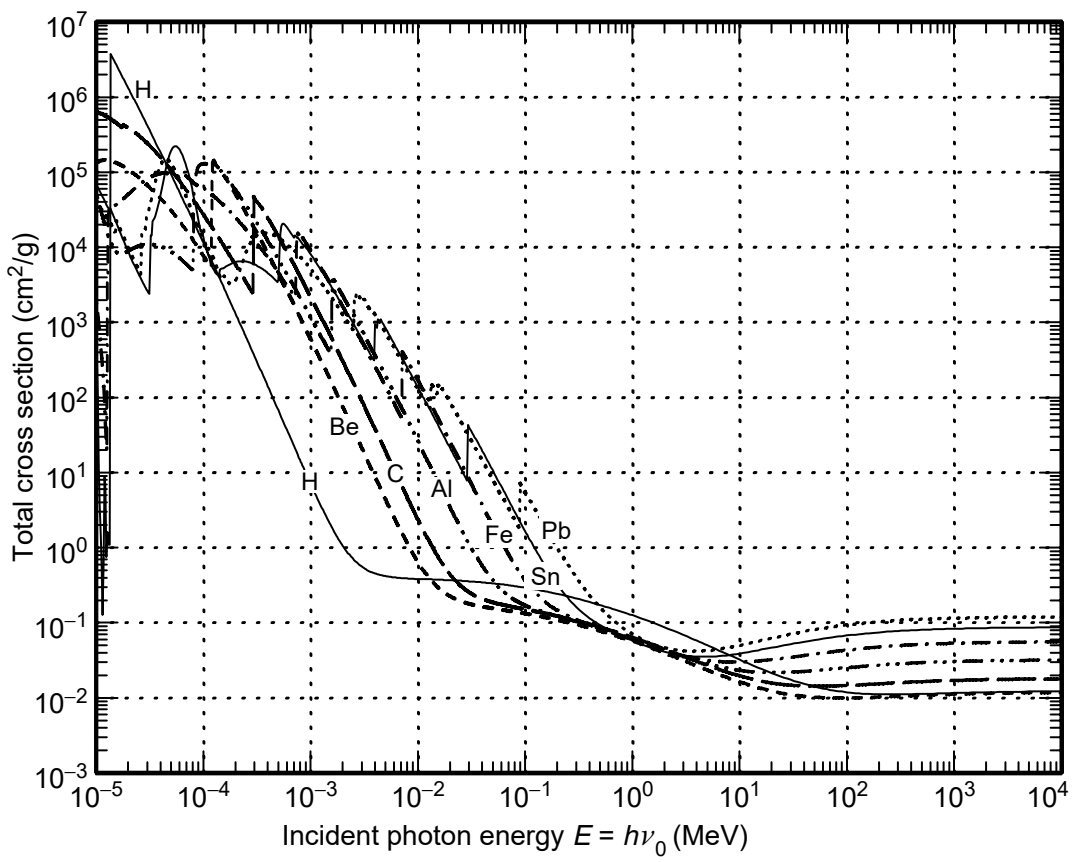

FIGURE 2.51 Total photon interaction cross section (including coherent) on several elements, $\mathrm{cm}^{2} / \mathrm{g}$. (Data from EPDL97.)

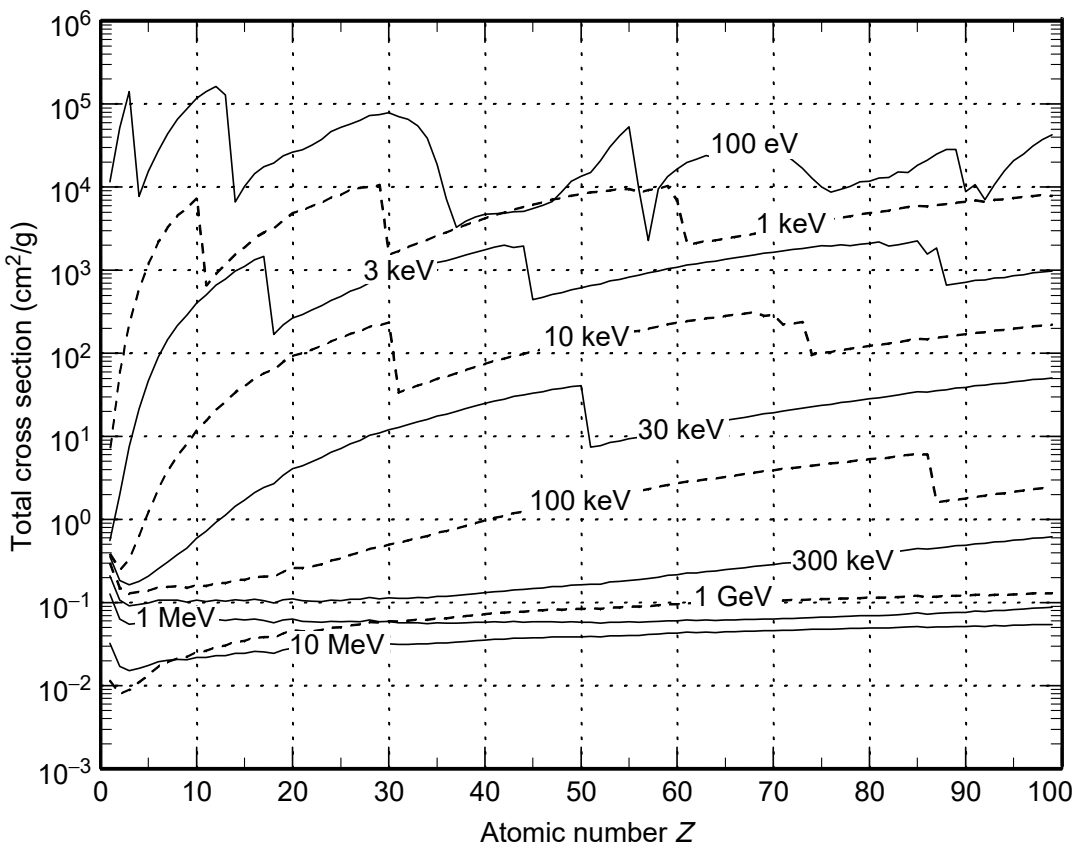

FIGURE 2.52 Total photon interaction cross section (including coherent) at several energies on all elements, in $\mathrm{cm}^{2} / \mathrm{g}$. (Data from EPDL97.) 


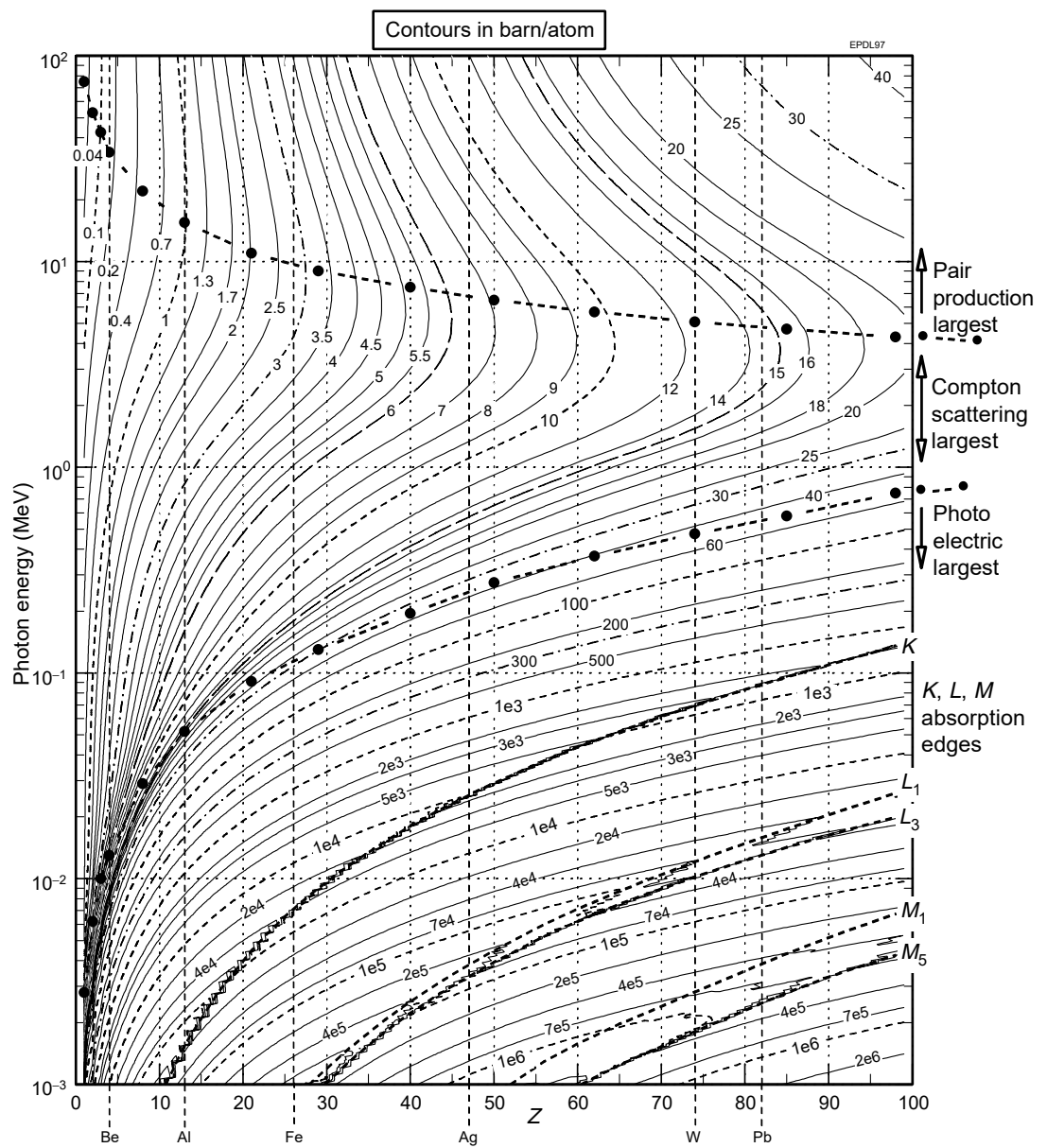

FIGURE 2.53 Contours of total photon cross section in all elements, from $1 \mathrm{keV}$ to $100 \mathrm{MeV}$, in barn/atom. (Data from EPDL97.)

in $\mathrm{cm}^{2} / \mathrm{g}$, and of the mfp are three of seventeen graphs included as Adobe portable document files (PDF) on the accompanying CD-ROM. These are intended to be printed on $11^{\prime \prime} \times 17^{\prime \prime}$ paper. In that form, they are easily read to an accuracy acceptable for many practical applications.

From Figure 2.54 or Figure 2.55 one can see, for example, that in high- $Z$ materials the largest photon $\mathrm{mfp}$, that is, the most penetrating radiation, occurs at $3-5 \mathrm{MeV}$, whereas in low- $Z$ materials, it is somewhat higher, near $10 \mathrm{MeV}$ or more.

\subsubsection{Use of Contour Plots in Shielding Applications}

Besides conveying an appreciation of overall trends in $E$ and $Z$, the contour plots are useful in applications, especially in their $11^{\prime \prime} \times 17^{\prime \prime}$ form. They 


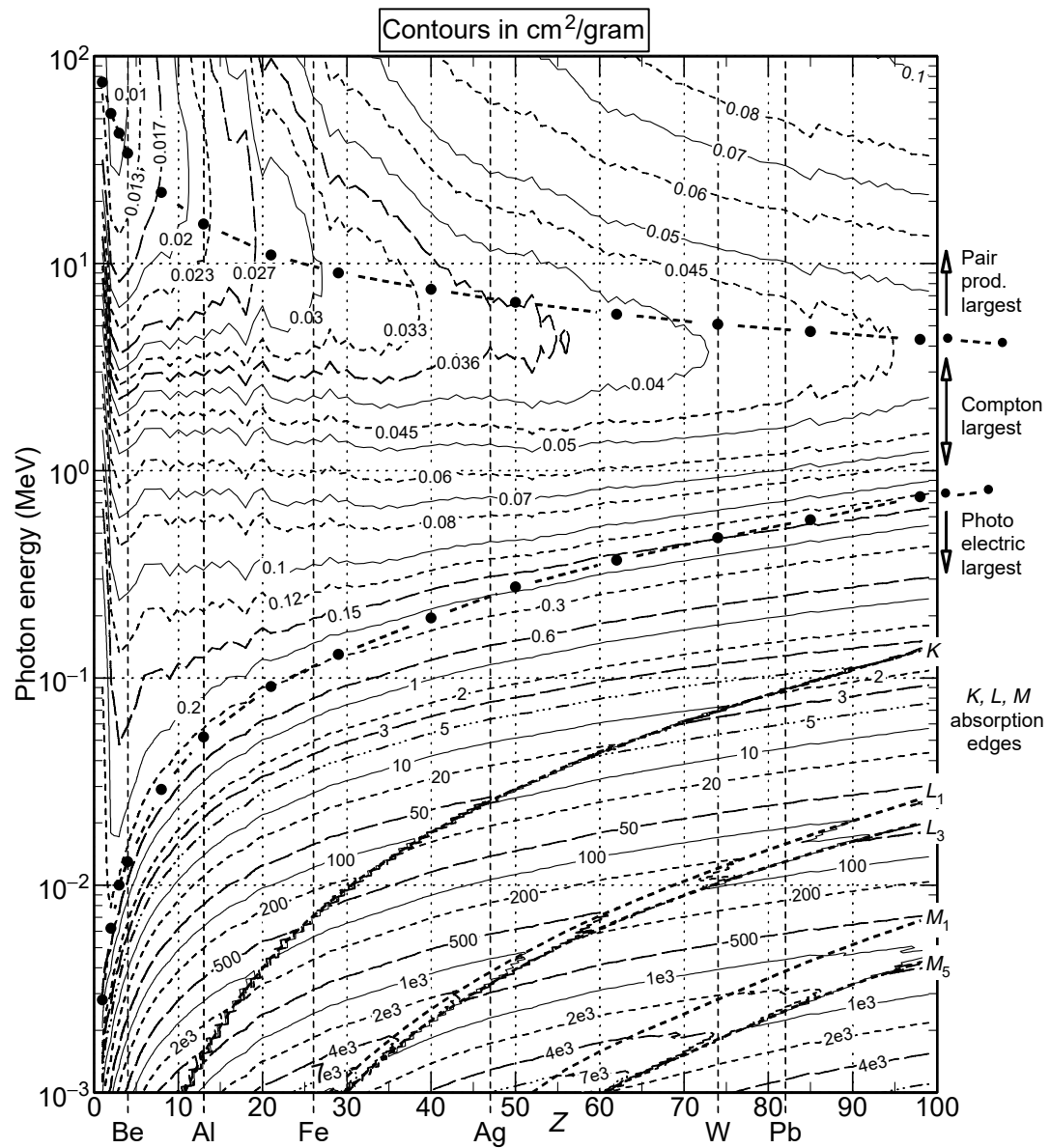

FIGURE 2.54 Contours of total photon cross section in all elements, from $1 \mathrm{keV}$ to $100 \mathrm{MeV}$, in $\mathrm{cm}^{2} / \mathrm{g}$. (Data from EPDL97.)

can, for example, help in selecting shielding material. If we wish to shield, in general, $x$-rays of energy $\lesssim 100 \mathrm{keV}$, with the least mass of material, Figure 2.55 shows that, as expected, high- $Z$ elements are to be favored over low $Z$, both because the photoelectric effect dominates in high $Z$ with a large cross section and small $\mathrm{mfp}$, and because low- $Z$ materials preferentially Compton scatter.

As discussed in regard to Equation 2.39 through Equation 2.41 and Figure 2.37 , if we wish to absorb as well as possible, with the least mass of material, all photons with energy less than $80 \mathrm{keV}$, we should not necessarily merely select the highest $Z$. Figure 2.55 shows that the mfp of an $80 \mathrm{keV}$ photon in $\mathrm{U}$ is $0.3 \mathrm{~g} / \mathrm{cm}^{2}$, whereas in $\mathrm{Nd}(Z=60)$ it is only $0.2 \mathrm{~g} / \mathrm{cm}^{2}$ because we are 


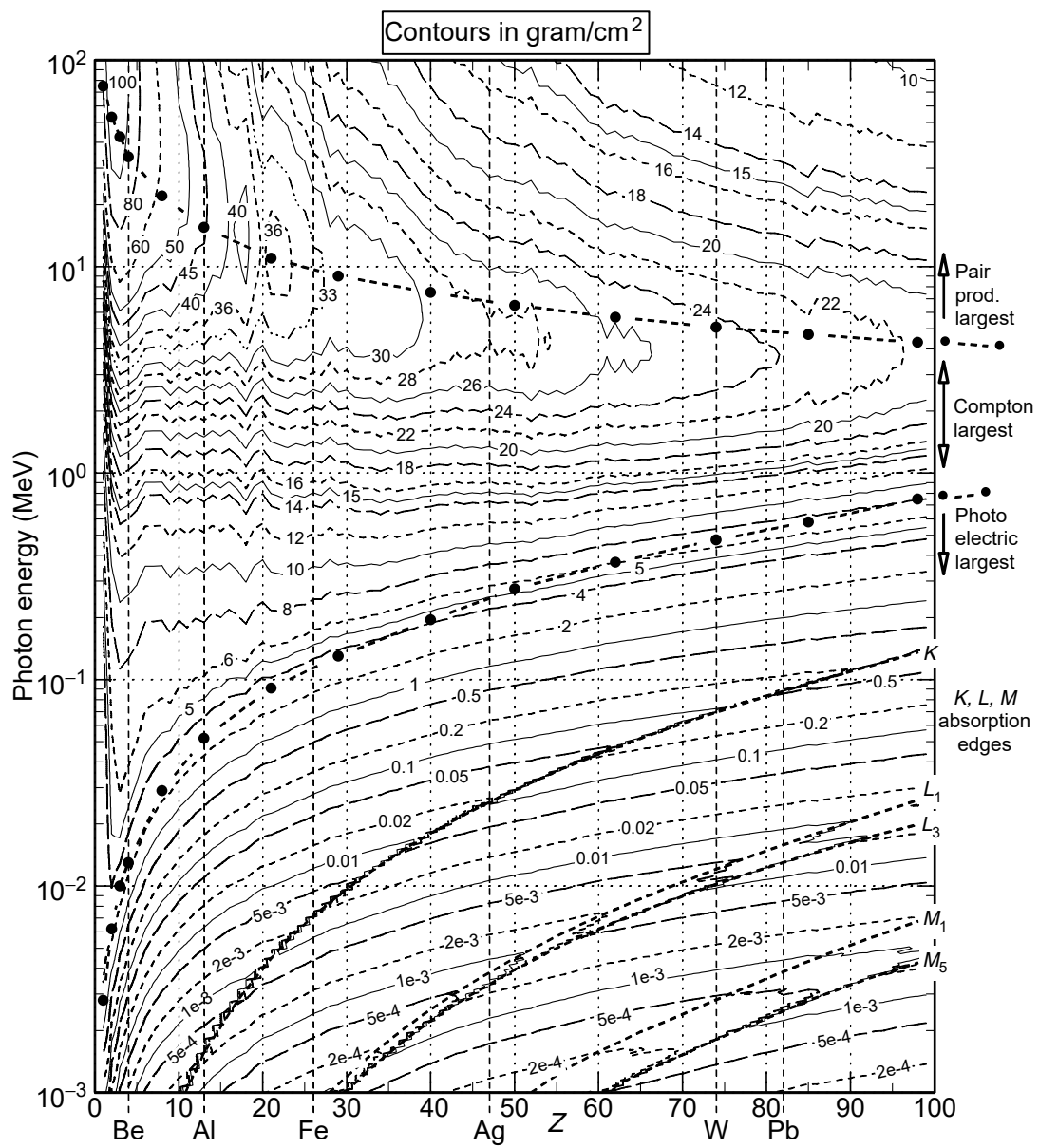

FIGURE 2.55 Contours of photon mean free path against total scattering in all elements, from $1 \mathrm{keV}$ to $100 \mathrm{MeV}$, in $\mathrm{g} / \mathrm{cm}^{2}$. (Data from EPDL97.)

above the $\mathrm{Nd} K$ edge but below the $\mathrm{U} K$ edge. $Z=58$ to 60 are the best absorbers for the purpose, because at all energies the $\mathrm{mfp}$ is less than $0.2 \mathrm{~g} / \mathrm{cm}^{2}$. Although other elements may be better absorbers of some energies less than $80 \mathrm{keV}$, all elements other than 58 to 60 have smaller cross sections for some energy less than $80 \mathrm{keV}$. Note that elements near $\mathrm{W}$ are the best absorbers of $80 \mathrm{keV}$, but they are not the best at $65 \mathrm{keV}$ or below. The choice of shielding an element when the penetration of Rayleigh or Compton scattered photons may be important was discussed in Section 2.6.

If the photon spectrum to be absorbed has a certain feature, other elements may be more appropriate. Even if all energies are below $80 \mathrm{keV}$, it may be 
that the energy in the x-rays is concentrated in a narrower band of lower energies. In that case, to minimize energy transmission, it may be best to choose elements that best absorb in that narrow band, even though they do not absorb energy below $80 \mathrm{keV}$ as well as other elements.

If a specific energy photon is to be shielded, photoabsorption edges can be used to good advantage. One tries to select a material with the $K$ edge just below the energy to be absorbed. The $K$ edge of element $Z=70(\mathrm{Yb})$ is 61.33 $\mathrm{keV}$, whereas that of $Z=69(\mathrm{Tm})$ is $59.39 \mathrm{keV}$.* Thus, Tm is a better absorber of the $59.54 \mathrm{keV} \gamma$ of $\mathrm{Am}^{241}$ than $\mathrm{Yb}$. Figure 2.54 shows that the absorption cross section in elements ..., 68, 69 is about $12 \mathrm{~cm}^{2} / \mathrm{g}$, whereas in elements $70,71, \ldots$ it is only $3 \mathrm{~cm}^{2} / \mathrm{g}$. Hence, for the same mass Tm is four times as good an absorber as $\mathrm{Yb}$. What is not so easy to recognize without a contour plot is that not only are elements ..., 68, 69 better than $70,71, \ldots$ but also that no other element is as good as $Z=69$. For absorbing $60 \mathrm{keV}$, Tm requires less mass than any other element. Figure 2.55 shows that the $\mathrm{mfp}$ in $\mathrm{Tm}$ is about $0.08 \mathrm{~g} / \mathrm{cm}^{2}$, whereas that in $\mathrm{Pb}$ is 0.2 . Thus, to absorb $99 \%$ of the photons (4.6 mfps), one would need about $0.37 \mathrm{~g} / \mathrm{cm}^{2}$ of Tm, but $0.92 \mathrm{~g} / \mathrm{cm}^{2}$ of $\mathrm{Pb}$.

For final detailed work, after consulting contour plots for estimates, cross section numbers for the selected elements should be checked in the numerical tables on the accompanying CD-ROM or other sources.

\subsection{ATTENUATION AND ENERGY DEPOSITED IN TARGET MATERIAL}

\subsubsection{Attenuation of a Beam}

An incident photon beam is attenuated whenever a photon is absorbed or scattered out of the beam. Thus, the intensity $I(E, x)$ of the remaining unscattered photons in a beam of photons of energy $E$ depends on the distance penetrated, $x$, according to

$$
I(E, x)=I_{0} \exp \left(-N \sigma_{\text {Tot }} x\right)
$$

where $N$ is the atom number density $\left(\mathrm{cm}^{-3}\right)$ or density of scattering centers, $I_{0}$ is the initial intensity, and

$$
\sigma_{\mathrm{Tot}}=\sigma_{\mathrm{Tot}}(E)=\sigma_{\mathrm{pe}}+\sigma_{\mathrm{C}}+\sigma_{\mathrm{Ray} 1}+\sigma_{\mathrm{pp}}
$$

\footnotetext{
*These cross sections are from NIST XCOM. The LLNL EPDL97 database uses 61.44 and $59.49 \mathrm{keV}$, respectively.
} 
is the total cross section for the photon to interact with the atom (or scattering center), the sum of photoelectric absorption $\sigma_{\mathrm{pe}}$, Compton scattering* $\sigma_{\mathrm{C}}$, coherent Rayleigh scattering $\sigma_{\text {Rayl }}$, and pair production $\sigma_{\mathrm{pp}}$. Other minor processes, such as photonuclear scattering, should in principle be included in the total cross section, but are conventionally excluded as negligible. As Rayleigh scattering is small and tends to peak in the forward direction, it is sometimes not included in the total cross section to define attenuation.

The quantity

$$
\mu_{0}(E) \equiv N \sigma_{\text {Tot }}(E)
$$

is the linear (total) attenuation coefficient, with dimensions of $\mathrm{cm}^{-1}$, so that $I(x)=I_{0} \exp \left(-\mu_{0} x\right)$. When one specifies distance penetrated in a material of density $\rho\left(\mathrm{g} / \mathrm{cm}^{3}\right)$ in terms of the mass penetrated, as $\rho x\left(\mathrm{~g} / \mathrm{cm}^{2}\right)$, it is convenient to define the mass (total) attenuation coefficient

$$
\mu_{0} / \rho\left(\mathrm{cm}^{2} / \mathrm{g}\right)
$$

so that $I(x)=I_{0} \exp \left(-\left(\mu_{0} / \rho\right) \rho x\right)$. The linear attenuation coefficient $\mu_{0}$ is the total cross section per unit volume of material, and the mass attenuation coefficient $\mu_{0} / \rho$ is the total cross section per unit mass, that is, the total bulk cross section; it is the quantity plotted in Figure 2.54 . The mean free path in a material against total scattering is $1 / \mu_{0}=1 / N \sigma_{\text {Tot }}$, expressed here in centimeters or $\rho / \mu_{0}$ in $\mathrm{g} / \mathrm{cm}^{2}$. The mfp against a single process, say Compton scattering, is $1 / N \sigma_{\mathrm{C}}>1 / N \sigma_{\text {Tot }}$.

The phrase "or density of scattering centers" above allows for compounds or mixtures of elements. Then, instead of a single element, the scattering center may be a molecule or a crystal cell, or any repeated unit of several elements in an arbitrary mixture. In this case, each of $\sigma_{\mathrm{pe}}, \sigma_{\mathrm{C}}, \sigma_{\mathrm{Rayl}}$, and $\sigma_{\mathrm{pp}}$ is the cross section for scattering from that scattering center; for water $\sigma_{\mathrm{C}}=2 \sigma_{\mathrm{C}}(\mathrm{H})+\sigma_{\mathrm{C}}(\mathrm{O})$.

\subsubsection{Energy Transferred to the Target Material: Kerma}

Neutral particles (photons or neutrons) passing through matter are referred to as indirectly ionizing particles. Charged particles (electrons, protons, and ions) are directly ionizing. The obvious reason is that photons or neutrons do not directly ionize the material. They do so only after interacting with an

\footnotetext{
*As mentioned previously, our Compton cross section, $\sigma_{\mathrm{C}}$, is what Evans [Ev55] calls the collision cross section. His symbol for it on a single electron is ${ }_{\mathrm{e}} \sigma$.
} 
atom and kicking out an electron (or recoil target nucleus), and that moving charged particle then ionizes by direct ionizing stopping power.

Thus, the process by which an indirectly ionizing particle deposits energy in a target material is a two-step process. In the first step, the neutral particle, in the present case a photon, interacts with an atom and ejects one or more charged particles (directly ionizing particles), and perhaps a secondary neutral particle (e.g., Compton scattered photon). The charged particles, in the present case one or more electrons, are ejected with some kinetic energy. In the second step, those charged particles deposit much of their energy directly in the target by ionization and excitation collisions with target atoms (by collisional stopping power).

The quantity kerma, or the energy-transfer coefficient that is directly related to it, is introduced to parameterize the first step. The quantity dose, or the directly related energy-absorption coefficient, is introduced to parameterize the second step [ICRU80, Ca85].

The first step, the initial ejection of charged particles, takes place at some small volume of interest in the target medium where the photon first interacts. The second step, the ionization energy loss of those charged particles, occurs over some distance beyond the first volume, distributed over the ranges of the produced electrons. Dose does not occur at the same point at which kerma occurs.

Here, we summarize the energy transfer from an incident photon beam to the target material. In Section 2.4 on the photoelectric effect, we mentioned the energy-transfer coefficient and kerma appropriate to that effect. Of course, Compton scattering and pair production also create charged particles in the target material and deposit energy.

In a beam of photons of energy $E(\mathrm{MeV})$, number fluence $\varphi$ (photons $/ \mathrm{cm}^{2}$ ), the number of photoelectric interactions per gram in an elemental target of atom number density $N$, density $\rho$, atom mass density $N_{\mathrm{g}}=N / \rho($ atoms $/ \mathrm{g})$ is

$$
\left.\sigma_{\mathrm{pe}}(E) N_{\mathrm{g}} \varphi \text { (photoelectric interactions } / \mathrm{g}\right) \text {. }
$$

Each interaction ejects a kinetic energy $f_{\mathrm{pe}} E=E-X$ in electrons, and so the kerma by the photoelectric effect is

$$
k_{\mathrm{pe}}=f_{\mathrm{pe}} E \sigma_{\mathrm{pe}}(E) N_{\mathrm{g}} \varphi
$$

or

$$
k_{\mathrm{pe}}=(E-X) \sigma_{\mathrm{pe}}(E) N_{\mathrm{g}} \varphi(\mathrm{MeV} / \mathrm{g}),
$$

where $X$ is the average fluorescence $\mathrm{x}$-ray energy per photoelectric absorption event. 
Recall that the separate quantity

$$
\mu_{\mathrm{tr}, \mathrm{pe}}=f_{\mathrm{pe}} \sigma_{\mathrm{pe}} N\left(\mathrm{~cm}^{-1}\right)
$$

or

$$
\mu_{\mathrm{tr}, \mathrm{pe}} / \rho=f_{\mathrm{pe}} \sigma_{\mathrm{pe}} N_{\mathrm{g}}=(1-X / E) \sigma_{\mathrm{pe}} N_{\mathrm{g}}\left(\mathrm{cm}^{2} / \mathrm{g}\right)
$$

was defined as the energy-transfer coefficient (by the photoelectric effect). More generally, for any process, the kerma is the energy-transfer coefficient times the photon energy fluence $E \varphi$

$$
k=\left(\mu_{\mathrm{tr}} / \rho\right) E \varphi(\mathrm{MeV} / \mathrm{g})
$$

In general, the kerma is the sum of the kinetic energies of all charged particles ejected in the neutral particle interaction. Here, with photons incident, these charged particles are electrons. For incident neutrons, the kerma is the sum of the kinetic energies of the recoil nucleus together with protons and $\alpha$ particles, ... that may exit the interaction. Kerma is the kinetic energy of charged particles (i.e., directly ionizing radiation) resulting from the interaction of neutral particles (indirectly ionizing radiation) with a material target.

In Compton scattering of a photon of energy $E$, each Compton electron would have an average energy $f_{\mathrm{C}} E$ if the photon scattered from a free electron ( $f_{\mathrm{C}}$ is plotted vs. $E$ in Figure 2.24). Its actual energy is reduced by its atomic binding energy $E_{\mathrm{B}} . E_{\mathrm{B}}$ is recovered as kinetic energy of more electrons if the atom relaxes by ejecting Augers. However, $E_{\mathrm{B}}$ appears as more photons if the atom relaxes by fluorescence. As $E_{\mathrm{B}}$ depends on the shell from which the photon scattered, it is simpler to parameterize with the average energy $X$ of fluorescence photons per Compton scattering, and the average energy $E^{\prime}$ of the Compton scattered photon. The scattered photon continues on to some (relatively) distant point. The kerma by Compton scattering is therefore

$$
k_{\mathrm{C}}=\left(E-E^{\prime}-X\right) \sigma_{\mathrm{C}}(E) N_{\mathrm{g}} \varphi(\mathrm{MeV} / \mathrm{g})
$$

Energy-transfer coefficients can be defined separately for the Compton effect and for pair production, for example,

$$
\mu_{\mathrm{tr}, \mathrm{C}} / \rho=\left(1-\left(E^{\prime}+X\right) / E\right) \sigma_{\mathrm{C}} N_{\mathrm{g}}\left(\mathrm{cm}^{2} / \mathrm{g}\right)
$$

is the mass energy-transfer coefficient appropriate for Compton scattering. The kerma by Compton scattering is $k_{\mathrm{C}}=\left(\mu_{\mathrm{tr}, \mathrm{C}} / \rho\right) E \varphi$.

In pair production from the nucleus, an energy $E-2 m c^{2}$ appears as kinetic energy of the electron and positron. Thus, the kerma caused by nuclear pair production is 


$$
k_{\mathrm{ppn}}=\left(E-2 m c^{2}\right) \sigma_{\mathrm{ppn}}(E) N_{\mathrm{g}} \varphi(\mathrm{MeV} / \mathrm{g})
$$

where $\sigma_{\mathrm{ppn}}$ is the nuclear pair production cross section.

Pair production from atomic electrons (triplet production) is similar, except that fluorescence $\mathrm{x}$-rays are again possible. The kerma as a result of electronic pair production is

$$
k_{\mathrm{ppe}}=\left(E-2 m c^{2}-X\right) \sigma_{\mathrm{ppe}}(E) N_{\mathrm{g}} \varphi(\mathrm{MeV} / \mathrm{g}),
$$

where $\sigma_{\mathrm{ppe}}$ is the electronic pair production cross section, and $X$ is the average fluorescence x-ray energy.

In Rayleigh scattering, the photon leaves no energy behind, and so produces no kerma.

Together, all photon interactions produce a kerma

$$
k_{\mathrm{tot}}=k_{\mathrm{pe}}+k_{\mathrm{C}}+k_{\mathrm{ppn}}+k_{\mathrm{ppe}}(\mathrm{MeV} / \mathrm{g})
$$

The total kerma per unit fluence (specific kerma) is

$$
\begin{aligned}
k_{\mathrm{tot}}^{\prime} \equiv \frac{k_{\mathrm{tot}}}{\varphi}= & {\left[(E-X) \sigma_{\mathrm{pe}}+\left(E-E^{\prime}-X\right) \sigma_{\mathrm{C}}+\left(E-2 m c^{2}\right) \sigma_{\mathrm{ppn}}\right.} \\
& \left.+\left(E-2 m c^{2}-X\right) \sigma_{\mathrm{ppe}}\right] N_{\mathrm{g}}\left(\mathrm{MeV}-\mathrm{cm}^{2} / \mathrm{g}\right)
\end{aligned}
$$

Here, the $X$ in each process is different, as it depends on the distribution of electron shells with which the photon interacted, and must be evaluated separately for photoelectric absorption, Compton scattering and electronic pair production. Though units indicated are $\mathrm{MeV}-\mathrm{cm}^{2} / \mathrm{g}$, kerma per unit fluence is equally often expressed in erg- $\mathrm{cm}^{2} / \mathrm{g}$ or $\operatorname{rad}-\mathrm{cm}^{2}\left(=\operatorname{rad}\right.$ per photon $\left./ \mathrm{cm}^{2}\right)$. $1 \mathrm{rad} \equiv 100 \mathrm{erg} / \mathrm{g}$. SI unit purists must use $\mathrm{J}-\mathrm{m}^{2} / \mathrm{kg}=\mathrm{Gy}-\mathrm{m}^{2} ; 1 \mathrm{~Gy} \equiv$ 1 Gray $\equiv 1 \mathrm{~J} / \mathrm{kg} \equiv 100 \mathrm{rad}$.

Kerma is a point quantity, as it is the photon energy immediately transferred to kinetic energy of charged particles in a small volume, in the limit as the volume becomes infinitesimally small. Therefore, only the first photon interaction in that volume is included in its definition. For example, the subsequent photoelectric absorption of a Compton-scattered photon is not included. All energy in scattered photons is treated as nonlocal and is not included in the definition of kerma.

Even though not all the kinetic energy of charged particles released in the material is deposited locally (i.e., not all kerma turns into dose, partly because of nonionizing Bremsstrahlung energy loss), kerma is useful because, unlike dose, it is independent of target geometry and is directly related to basic cross sections. It is also an approximate upper limit to dose; except for attenuation and finite electron range effects discussed later, the dose is never larger than the kerma. 
The kerma in a compound or mixture of elements is the simple additive sum of its constituents by weight fraction. If a compound consists of elements $i$ and $j$, in weight fractions $w_{i}$ and $w_{j}$, and $k_{i}^{\prime}$ and $k_{j}^{\prime}\left(\mathrm{MeV}-\mathrm{cm}^{2} / \mathrm{g}\right)$ are the specific kermas in each element separately, then the kerma in the compound is

$$
k^{\prime}=w_{i} k_{i}^{\prime}+w_{j} k_{j}^{\prime}
$$

As discussed later, the dose in a compound does not add so simply.

\subsubsection{Photon Fluence-to-Kerma Conversion Factors}

As kerma depends only on basic cross sections, it is a function only of photon energy $E$ for a given elemental target material. Photon kerma as a function of $E$ for several elements is shown in Figure 2.56 and for selected semiconductors in Figure 2.57. It is shown as a function of $Z$ for several energies in Figure 2.58. As was the case for cross sections, it may be displayed for all elements as contours in $Z-E$ space. Figure 2.59 shows photon kerma in all elements. The units chosen, $\operatorname{rad}$ (element) per unit photon fluence (taken as $10^{9}$ photon $/ \mathrm{cm}^{2}$ ),

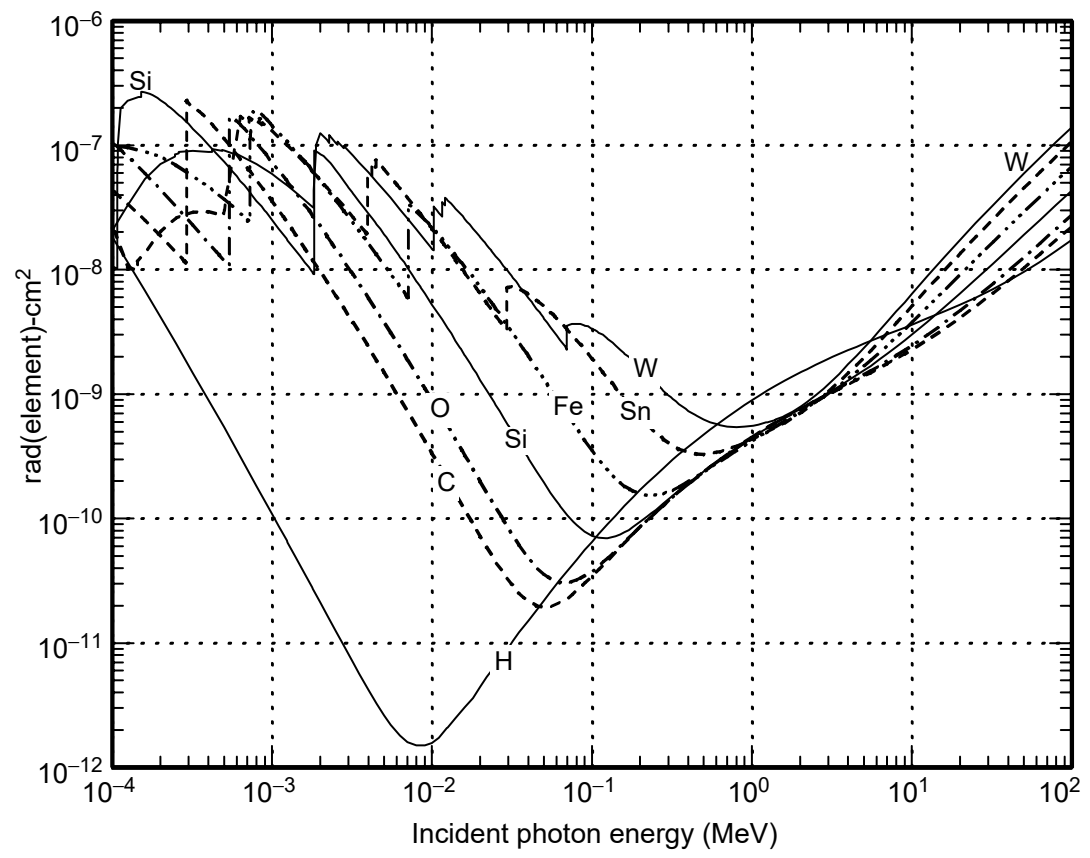

FIGURE 2.56 Photon number fluence-to-kerma conversion factor for several elements, as a function of incident photon energy. Units are $\operatorname{rad}\left(\right.$ element) per photon $/ \mathrm{cm}^{2}$. At $2 \mathrm{MeV}$ in almost all elements except $\mathrm{H}$, about $1.3 \mathrm{E} 9$ photons $/ \mathrm{cm}^{2}$ delivers $1 \mathrm{rad}$. 


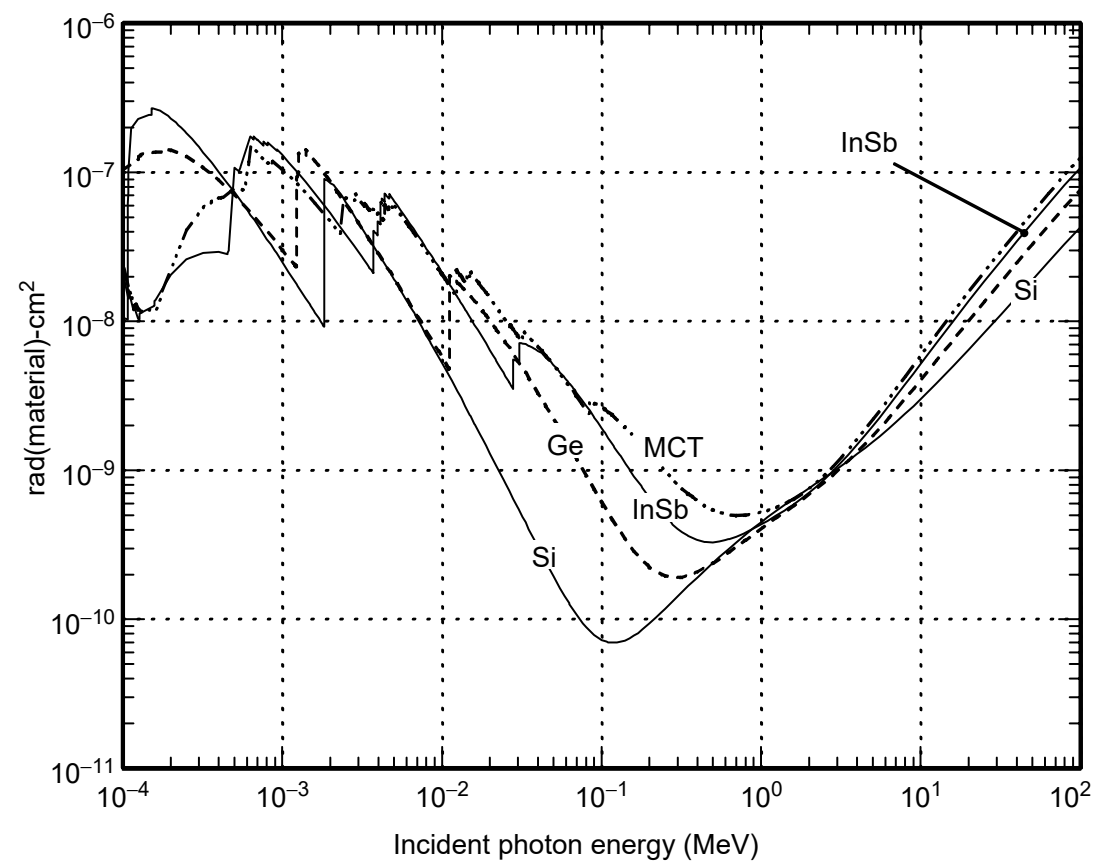

FIGURE 2.57 Photon number fluence-to-kerma conversion factor for four semiconductor materials. Units are rad (material) per photon $/ \mathrm{cm}^{2}$. GaAs differs only slightly from Ge. MCT is $\mathrm{Hg}_{0.8} \mathrm{Cd}_{0.2} \mathrm{Te}$.

are the units in which one commonly specifies dose. These curves are the photon fluence-to-kerma conversion functions. To the extent that kerma is the same as dose, they are also the fluence-to-dose conversion functions, or the material response function, giving energy deposited in any element for incident photons of any energy and any fluence. A large, color version of Figure 2.59 , intended for printing on $11^{\prime \prime} \times 17^{\prime \prime}$ paper, is on the accompanying CDROM, in PDF format.

The figures show that at $E=1 \mathrm{MeV}$, all elements from $Z=2$ to about 60 experience approximately $4.5 \times 10^{-10} \mathrm{rad}$ per photon $/ \mathrm{cm}^{2}$. Conversely, it requires some $2.2 \times 10^{9}$ photons $/ \mathrm{cm}^{2}$ of $1 \mathrm{MeV}$ to deliver $1 \mathrm{rad}$ to any element with $Z<60$ (except $H$ ). Higher $Z$ experiences a slightly higher kerma, up to about $8 \times 10^{-10} \mathrm{rad}$ per photon $/ \mathrm{cm}^{2}$. At 0.8 to $4 \mathrm{MeV}$, for all elements, the responsible process is Compton scattering. At a fixed photon energy $E$ below a few $\mathrm{MeV}$, the response function increases somewhat as $Z$ increases. The reason is twofold. First, the bulk cross section increases as $Z$ increases (Figure 2.54) because at high $Z$ the larger photoelectric cross section becomes nonnegligible compared with the Compton cross section. Second, the average photon energy converted to electron energy in the Compton effect is less than or of the order 


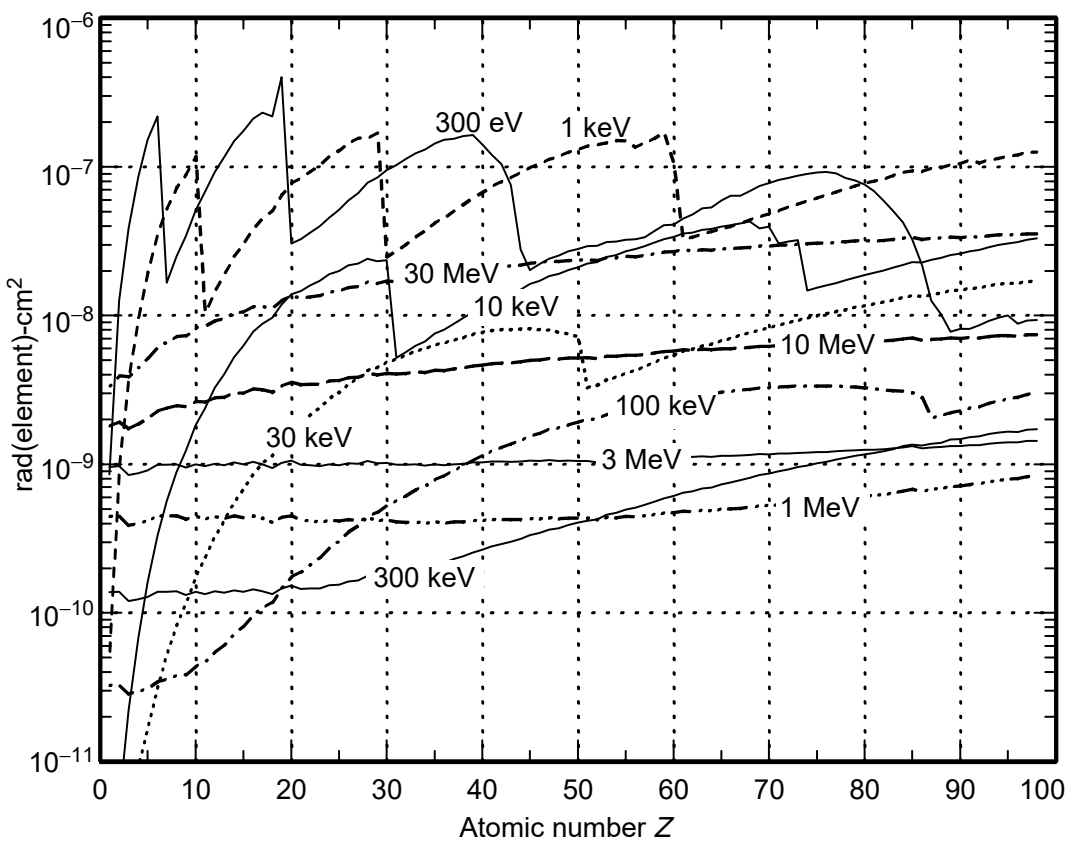

FIGURE 2.58 Photon number fluence-to-kerma conversion factor for all elements at selected energies. Units are rad(element) per photon $/ \mathrm{cm}^{2}$.

$E / 2$, whereas for the photoelectric effect it is essentially $E$. For example, at the $\mathrm{Cs}^{137}$ line, $E=0.662 \mathrm{MeV}$, the cross section rises from about $0.08 \mathrm{~cm}^{2} / \mathrm{g}$ at low $Z$ to 0.14 at high $Z$, not quite factor of 2 . However, the kerma per unit fluence increases by more, from 0.3 to $1 \mathrm{rad}$ per $10^{9}$ photons $/ \mathrm{cm}^{2}$ because of the increased fraction of photon energy converted to kerma as the photoelectric effect becomes relatively more important at higher $Z$.

\subsubsection{Energy Deposited in the Target Material: Dose}

Once the first step is completed, the photon ejects electrons (and possibly positrons) from parent atoms. The kinetic energy in those electrons and positrons is the kerma. As the electrons move, they lose their energy along their track length to collisional excitation and ionization and to Bremsstrahlung. The energy lost per gram of target material to excitation and ionization (collisional stopping power) is taken to be deposited locally, and is the dose. Since the loss to Bremsstrahlung is nonzero, the dose is apparently less than kerma. Nevertheless, because the electrons move away from their point of origin, the dose occurs at points different from where the original kerma occurs. This may allow dose, as a point function 


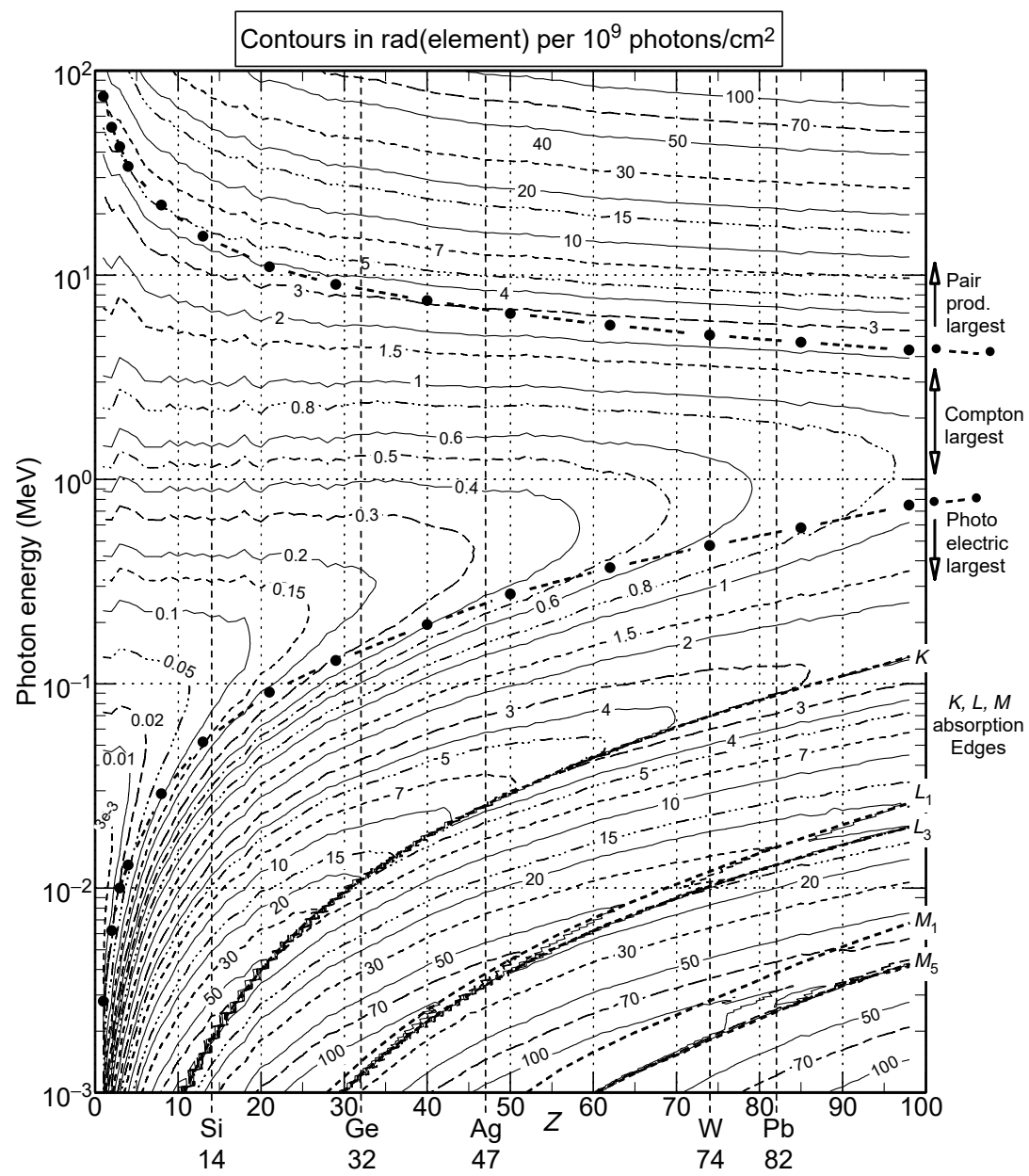

FIGURE 2.59 Contours of photon number fluence-to-kerma conversion factor in all elements, from $1 \mathrm{keV}$ to $100 \mathrm{MeV}$. In $\operatorname{rad}\left(\right.$ element) per $10^{9}$ photon $/ \mathrm{cm}^{2}$. $1 \mathrm{rad}=100 \mathrm{erg} / \mathrm{g}$.

of position, to exceed kerma occasionally, even in a uniform medium (see the following section).

Although the spatial distribution of dose and kerma may differ, their spatial integrals would be equal if no energy were lost to Bremsstrahlung. The energy lost to Bremsstrahlung prevents the dose from equaling kerma, even when integrated over all positions. In pair production, the positron loses energy by ionization and excitation just as the electron does, usually before annihilating with another electron. Hence, except for positron Bremsstrahlung, its full kinetic energy is also part of the dose. 
Let $g$ be the fraction of an electron's energy eventually lost to photon emission during the course of its slowing down to rest. In the first approximation, $g$ is just the Bremsstrahlung radiative yield $Y_{\mathrm{R}}$ (as discussed in Chapter 3) as it is the fraction of energy lost to Bremsstrahlung radiation from an energetic electron. Positron annihilation in flight is a small correction to $g$ [Hi92]. Another correction comes from how one treats energy lost to atomic relaxation fluorescence photons following electron collisional (excitation and ionization) energy losses with atoms. There seems to be no universal agreement as to whether the energy of these fluorescence photons should be included in the dose [ICRU80, Hu95], but for consistency their energy should be excluded as well. The compilation by Hubbell and Seltzer [Hu95] has the best account of photon energy.

For any target material, then, the energy-absorption coefficient $\mu_{\mathrm{e}}$ is smaller than the energy-transfer coefficient $\mu_{\text {tr }}$ by $g$

$$
\mu_{\mathrm{e}}=(1-g) \mu_{\mathrm{tr}}\left(\mathrm{cm}^{-1}\right)
$$

Likewise, the mass energy-absorption coefficient $\mu_{\mathrm{e}} / \rho$ is related to the mass energy-transfer coefficient $\mu_{\mathrm{tr}} / \rho$ by

$$
\mu_{\mathrm{e}} / \rho=(1-g) \mu_{\mathrm{tr}} / \rho\left(\mathrm{cm}^{2} / \mathrm{g}\right)
$$

The dose is the mass energy-absorption coefficient times the photon energy fluence $E \varphi$

$$
\text { dose }=\left(\mu_{\mathrm{e}} / \rho\right) E \varphi \quad(\mathrm{MeV} / \mathrm{g})
$$

or the dose per unit fluence (the specific dose)

$$
\operatorname{dose} / \varphi=\left(\mu_{\mathrm{e}} / \rho\right) E\left(\mathrm{MeV}-\mathrm{cm}^{2} / \mathrm{g}\right)
$$

This definition of dose, in terms of $\mu_{\mathrm{e}}$, parallels that of kerma in terms of $\mu_{\mathrm{tr}}$. It is valid more conceptually than in detail in a specific application because of the above caveat that, because of electron range, dose occurs at a different point from where kerma occurs.

In addition, if the target is made of thin layers of different materials, the kerma occurring in one layer may cause dose in another layer as the electrons move. That other layer may have a different energy-transfer coefficient and a different electron stopping power from the first layer. Thus, dose also depends on geometry. Therefore, because of the angular scattering of electrons and complicated materials geometry, in principle the only way to accurately compute dose in a realistic problem is by a photon-electron transport calculation, for example, a Monte Carlo simulation. 


\subsubsection{Kerma and Dose at a Material Interface}

Figure 2.60 sketches a side view of photons entering a uniform slab of material from vacuum. The photons may interact with any atom along the way, so kerma occurs immediately near the surface; kerma at a point is directly proportional to the beam fluence and material density at that point. As the beam proceeds, attenuation reduces its fluence (and its kerma) exponentially. However, the dose at shallow depth $d$ near the surface is a result of only those electrons produced in the preceding thin layer $0<x<d$ (and backscattered electrons, which we ignore here). There are few of these, so the dose for small $x$ is small. It builds up linearly with increasing depth $d$ as kerma adds more electrons. Dose saturates at a depth equal to the range $R_{\max }$ of the most energetic electron. At depths greater than that, electrons are ejected anew from atoms at about the same rate at which previous electrons come to rest, causing a "steady-state" balance between kerma and dose. The balance is not equal, however, because the local dose at depth $d$ is caused by electrons created back to $d-R_{\max }$, where intensity and kerma were greater. Thus, at a given depth it is possible for dose to exceed kerma. Usually, $R_{\max }$ is much less than the beam attenuation length (or $\mathrm{mfp}$ ), hence dose closely approximates kerma. For example, in $\mathrm{Si}$, the $\mathrm{mfp}$ of a $1 \mathrm{MeV} \gamma$ ray is about $16 \mathrm{~g} / \mathrm{cm}^{2}$, whereas $R_{\max }$ of a $1 \mathrm{MeV}$ electron is less than $0.6 \mathrm{~g} / \mathrm{cm}^{2}$. At $\gamma$-ray energies $(\gtrsim 1 \mathrm{MeV})$, Compton electrons are generally energetic enough $(\gtrsim 0.5 \mathrm{MeV})$ and produced more nearly in the forward direction so that their backscattering is a small correction. At x-ray energies, however, in which photoelectric absorption dominates, electrons are produced in the backward hemisphere almost as much as in the forward hemisphere and are of lower energy so that scattering is more important. Then, electron backscatter is not negligible.

The term charged particle equilibrium is introduced as the condition for dose to equal kerma. It means that electrons must be ejected from their parent

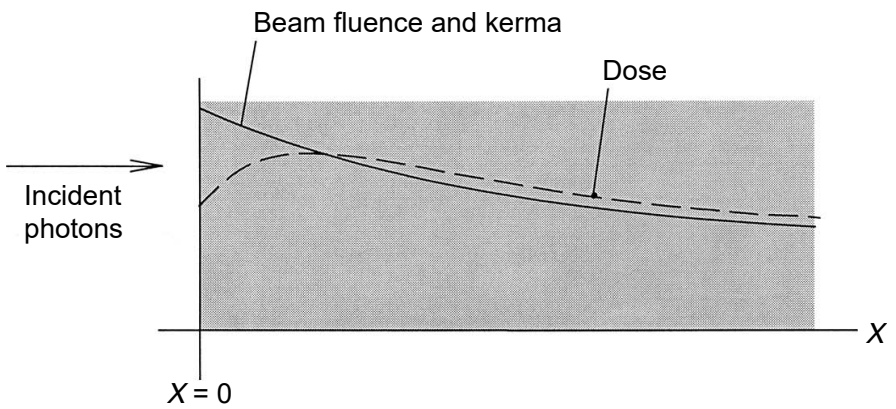

FIGURE 2.60 Photon beam entering matter from vacuum. Photon fluence and kerma attenuate exponentially. Dose may build up to slightly exceed kerma. (Adapted from [ICRU69]. With permission.) 
atoms uniformly over a distance $R_{\max }$. It does not merely mean that a new charged particle enters the (infinitesimal) volume of inquiry whenever one leaves it; rather, the stopping power of the exiting particle must equal that of the entering particle (which effectively means their energies must be equal) in order for the dose to be compensated. When charged particle equilibrium holds, dose equals kerma.

The dose profile depicted in Figure 2.60 holds only when the preceding medium is vacuum. If, say, the medium were air, then photoelectrons and Compton electrons produced in the air would enter the slab at $x=0$, and dose at $x=0+\varepsilon$ would be higher than shown.

A second, common situation occurs at the interface between two materials, as sketched in Figure 2.61, where charge particle equilibrium is clearly violated. Material 1 and material 2 are each taken to be slabs thinner than the photon $\mathrm{mfp}$, so beam attenuation can be neglected, and the photon fluence is a constant through both slabs. Likewise, the kerma is a constant in material 1 and constant at a different value in material 2. As they are of different $Z$, the mass energy-transfer coefficients $\mu_{\mathrm{tr}} / \rho$ and kermas of the two materials are different, and so the kerma is discontinuous across the interface, as indicated by the two different levels in the figure. Farther than an electron range from the interface, the dose is also constant and equal to the kerma.

The figure is drawn for the case $\mu_{\mathrm{tr}} / \rho(2)>\mu_{\mathrm{tr}} / \rho(1)$, so that kerma is greater in material 2 than in material 1 . Some of the electrons produced in 2 near the interface scatter back into 1, adding to the dose there. Other electrons are produced in 1 near the interface and move into 2 . Together, these make the dose near the interface in 1 greater than its distant, constant value and that in 2 near the interface less than its distant, constant value. This scattering tends to smooth out the discontinuity in dose, leaving the discontinuity in kerma. The electron number flux across the interface is continuous. Thus, the dose would be exactly continuous if the electron stopping power were the same in both media. As the stopping powers are not equal, a small discontinuity in the dose

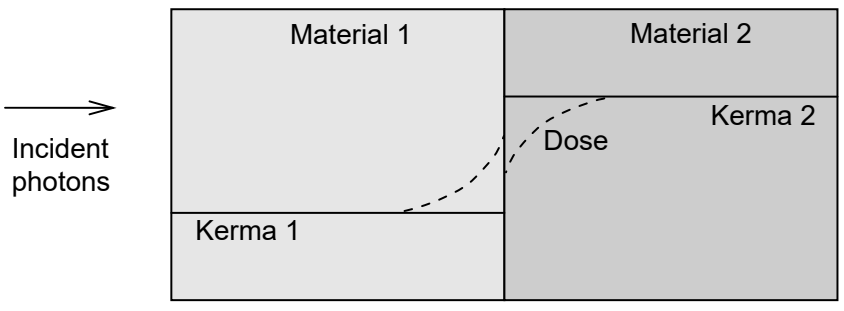

FIGURE 2.61 Illustrating photon beam entering matter of two thin layers of two different materials. Photon fluence is assumed to be constant throughout. Kerma in material 1 is constant, and kerma in 2 is constant. Dose spreads out over the interface due to finite electron range and scattering. (Adapted from [ICRU69]. With permission.) 
results. The sketch is drawn for the case that the stopping power in material 1 is greater than that in material 2, so the dose in 1 at the interface is greater than that in 2 . If the stopping power in 1 were smaller than in 2 , the dose in 1 at the interface would be less than that in 2 .

Clearly, whereas kerma depends only on material cross sections, dose depends on material geometry and electron transport as well. Thus, no plots of dose vs. $Z$ or $E$ can be prepared comparable to those of kerma vs. $Z$ or $E$.

As the fraction $g$ of energy lost to radiative processes depends on the elements in the target material, the dose in a compound material is not the simple weight-fraction additive sum that kerma is. Dose in one element is computed using a radiative yield $Y_{\mathrm{R}}$ appropriate only to that element.

\subsubsection{Attenuation, Energy Transfer, and Energy Absorption}

Photon beam attenuation is due to the total cross section $\sigma_{\text {tot }}$, with attenuation rate $\sigma_{\text {Tot }} N_{\mathrm{g}}\left(\mathrm{cm}^{2} / \mathrm{g}\right)$. The energy-transfer rate $\mu_{\mathrm{tr}} / \rho$ is smaller than attenuation because of energy scattered out in the form of other photons. The energy-absorption rate $\mu_{\mathrm{e}} / \rho$ is smaller than the energy-transfer rate because of Bremsstrahlung and other radiative losses.

A pictorial way of seeing the flow of energy from the incident photons to final deposition, caused by each process, photoelectric, Compton, Rayleigh, and pair production, has been suggested by Hubbell [Hu77, Hu99b].

The Hubbell Diagram, Figure 2.62, depicts photons entering from the left and interacting with a target material via one of the processes. The upward-branching arrows depict energy going off after the interaction in photons, leaving the local deposition region; these photons are either the scattered incident photon, Bremsstrahlung, fluorescence, or those from positron annihilation. All processes together contribute to beam attenuation. All but coherent Rayleigh scattering contribute some energy transfer and deposition. After scattering of the incident photon and rapid fluorescence, but before Bremsstrahlung, is the energy-transfer coefficient $\mu_{\mathrm{tr}}$. After losses to Bremsstrahlung and positron annihilation in-flight is the actual energy absorption, $\mu_{\mathrm{e}}$. An alternate graphical visualization of energy path to deposition has been suggested by Berger [Be61].

Older literature [Ev55] considers only the energy of first scattered photons to be removed from the photon beam, the remainder to be deposited and refers to the resulting coefficient as the mass absorption coefficient, $\mu_{\mathrm{a}}$. Modern treatments account for energy lost from the charged particles to Bremsstrahlung, fluorescence photons, and positron annihilation radiation before attributing the remainder as deposited; the result is the mass energy-absorption coefficient $\mu_{\mathrm{e}}$.

As an example, Figure 2.63 shows the attenuation and energy-transfer coefficients, $\mu_{0} / \rho$ and $\mu_{\mathrm{tr}} / \rho$, respectively, for photons in Si and in $\mathrm{Pb}$, from $100 \mathrm{eV}$ to $100 \mathrm{MeV}$. As the fluorescence yield in $\mathrm{Si}$ is small, there is little difference between the two coefficients at the $K$ edge. The difference is 


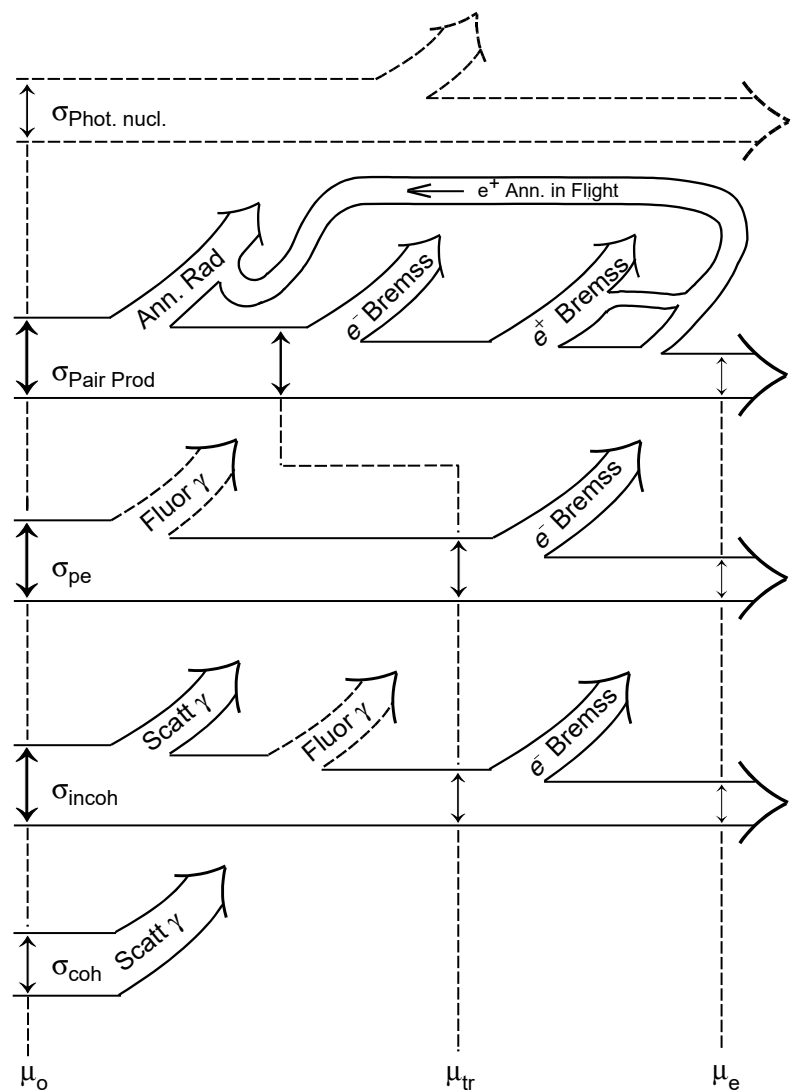

FIGURE 2.62 Hubbell diagram depicting contributions by each of the five possible interactions to the attenuation coefficient $\mu_{0}$, the energy transfer coefficient $\mu_{\mathrm{tr}}$, and the energy absorption coefficient $\mu_{\mathrm{e}}$. Energy escaping the local region by photons is indicated by upward going arrows. In pair production, energy lost to Bremsstrahlung from positrons is reduced because some positrons annihilate in flight. (Adapted from [Hu77]. With permission.)

significant in high- $Z$ elements, as seen in $\mathrm{Pb}$. The energy-absorption coefficient $\mu_{\mathrm{e}} / \rho$ is not shown in the figure. It is smaller than $\mu_{\mathrm{tr}} / \rho$ only at high energies $(\gtrsim 100 \mathrm{MeV})$ when the radiative yield of produced electrons and positrons becomes significant.

\subsection{ELECTRON FLUX FROM PHOTON FLUX-THE 1\% RULE}

Most of the effects on target materials of the passage of photons are due to dose, the energy deposited by the photons. However, some targets, notably pixilated semiconductor devices such as charge-coupled devices (CCDs), or focal plane arrays (FPAs), are sensitive to the passage of individual electrons 


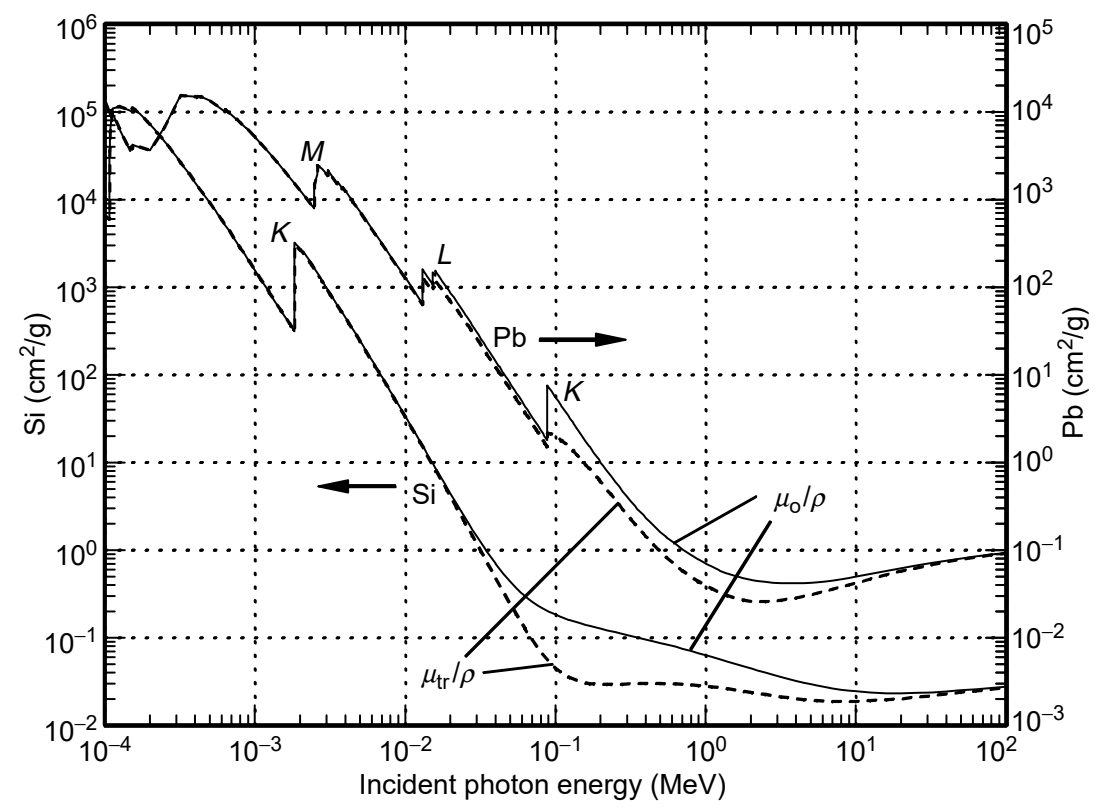

FIGURE 2.63 Total attenuation coefficient $\mu_{\mathrm{o}} / \rho$, (=total cross section per gm), and energy-transfer coefficient $\mu_{\mathrm{tr}} / \rho$ in $\mathrm{Si}$ and in $\mathrm{Pb}$, in $\mathrm{cm}^{2} / \mathrm{g}$.

through the pixels, even one energetic electron through one pixel. Actually, this sensitivity is a matter of dose, but only the dose left by electrons that pass through a pixel (whose sensitive volume may be only microns thick) and create electron-hole pairs. In addition to their energy deposition per se, one is interested in the number of electrons that pass per $\mathrm{cm}^{2}$, because that determines noise. Therefore, one is interested in the number of electrons that are created by an incident photon beam, not merely their dose.

Electrons are created from a photon beam by photoelectric absorption, by Compton scattering, and by pair production. In common situations involving nuclear $\gamma$ rays with energies in the order of an $\mathrm{MeV}$, these electrons are usually Compton electrons.

In a material of atom number density $N$, the rate at which incident photons of energy $E$ and flux $f_{\gamma}=f_{\gamma}(E)$ interact by process $i[i=$ pe (photoelectric absorption), $C$ (Compton scattering), or pp (pair production)] is

$$
\eta_{i} \equiv \text { number of interactions of process } i \text { per } \mathrm{cm}^{3} \text { per sec }=\frac{f_{\gamma}}{\lambda_{i}}
$$

where $\lambda_{i}=1 / N \sigma_{i}$ is the $\operatorname{mfp}$ for process $i$, and $\sigma_{i}(E)$ is the cross section for that process.

Electrons are produced with some energy distribution, but with average energy $E_{\mathrm{e} i}$ when created by process $i$. Electrons with energy $E_{\mathrm{e} i}$ have a range 
$R=R\left(E_{\mathrm{e} i}\right)$ against their total stopping power in the medium. In steady state, therefore, the flux of electrons is obtained by integrating their production rate over a distance equal to their range,

$$
\text { electrons } / \mathrm{cm}^{2} / \mathrm{sec} \text { by process } i \equiv f_{\mathrm{ei}}=\eta_{i} R=N \sigma_{i} f_{\gamma} R=\left(R / \lambda_{i}\right) f_{\gamma}
$$

$f_{\mathrm{e} i}$ is the omnidirectional flux of electrons caused by process $i$, the flux of interest for the ionization subsequently caused by the electrons. This expression for $f_{\mathrm{e} i}$ assumes that there is material in an electron range $R$ before the point of interest to create the electrons that arrive at the point. That is, we assume charged particle equilibrium. As the range of a $1 \mathrm{MeV}$ electron is less than $0.8 \mathrm{~g} / \mathrm{cm}^{2}$ in all materials $(<0.3 \mathrm{~cm} \mathrm{Al}$ or $6.4 \mathrm{~m} \mathrm{STP} \mathrm{air})$, charged particle equilibrium will often hold in practical situations. At a depth $x<R$ in a material at a vacuum-matter interface, with the photons entering from vacuum, the electron flux $f_{\mathrm{e} i}$ depends on $x$ approximately as $f_{\mathrm{e} i}=\eta_{i} x$.

The total cross section is $\sigma_{\mathrm{Tot}}=\sigma_{\mathrm{pe}}+\sigma_{\mathrm{C}}+\sigma_{\mathrm{pp}}$, and the omnidirectional electron flux is

$$
f_{\mathrm{e}}=\sum f_{i} i=f_{\mathrm{epe}}+f_{\mathrm{eC}}+f_{\mathrm{epp}}=N\left[\sigma_{\mathrm{pe}} R\left(E_{\mathrm{epe}}\right)+\sigma_{\mathrm{C}} R\left(E_{\mathrm{eC}}\right)+\sigma_{\mathrm{pp}} R\left(E_{\mathrm{epp}}\right)\right] f_{\gamma}
$$

The cross section for each process is weighted by the electron range at the average energy $E_{\text {epe }}, E_{\mathrm{eC}}$, or $E_{\text {epp }}$ appropriate for that process.

The dependence of $f_{\mathrm{e}}$ on photon energy $E$ can be estimated. Photon energies above $100 \mathrm{keV}$ are primarily of interest. The electron energy $E_{\mathrm{e}}$ is roughly proportional to the photon energy $E$. The electron range $R$ is roughly proportional to $E_{\mathrm{e}}^{2}$ for $E_{\mathrm{e}}$ less than a few hundred $\mathrm{keV}$, is roughly proportional to $E_{\mathrm{e}}$ up to about $10 \mathrm{MeV}$, and increases only logarithmically above $10 \mathrm{MeV}$ (due to Bremsstrahlung). The photoelectric cross section is roughly proportional to $1 / E^{3}$. Thus, $f_{\text {epe }}$ scales between $1 / E$ and $1 / E^{2}$ when $0.1<E<1$ $\mathrm{MeV}$. For Compton scattering, $\sigma_{\mathrm{C}}$ is only slowly decreasing with $E$ up to $\sim 1$ $\mathrm{MeV}$; at higher energies, it drops off roughly as $1 / E$. Thus, $f_{\mathrm{eC}}$ increases with $E$ when below $\sim 1 \mathrm{MeV}$, and then tapers off to be nearly independent of $E$ above $10 \mathrm{MeV}$. The pair production cross section approaches a constant above several tens of $\mathrm{MeV}$, while the electron range continues to increase; thus, $f_{\text {epp }}$ continues to increase roughly linearly with $E$.

The ratio of electron flux $f_{\mathrm{e} i}$ to incident photon flux $f_{\gamma}$ is

$$
\xi_{i}=f_{\mathrm{e} i} / f_{\gamma}=R / \lambda_{i}=N \sigma_{i} R
$$

When $E \sim 1 \mathrm{MeV}$ (and so the Compton effect dominates), this ratio is often of the order $10^{-2}$ for most materials;

$$
f_{\mathrm{e}} \approx 0.01 f_{\gamma} \quad(E \sim 1 \mathrm{MeV})
$$


The Compton electron flux is about 0.01 times the photon flux. This is the origin of the name "The $1 \%$ Rule." However, the ratio differs substantially from 0.01 at other energies. For example, as $E$ increases from 0.1 to $10 \mathrm{MeV}$, $E_{\mathrm{e}}$ and $R$ increase by more than two orders of magnitude, whereas $\sigma_{\mathrm{C}}$ decreases by only one order of magnitude. Thus, the ratio continues to increase. Above $10 \mathrm{MeV}$, where pair production dominates, the pair production cross section saturates, but $E_{\mathrm{e}}$ and $R$ continue to increase, similarly increasing the ratio. A better rule can be constructed.

\subsubsection{THE 1\% RULE}

From the previous estimates of the scaling behavior of $f_{\text {epe }}, f_{\mathrm{eC}}$, and $f_{\text {epp }}$ with $E$, the ratio

$$
\xi_{E}=f_{\mathrm{e}} / E f_{\gamma}
$$

is more nearly constant with increasing $E$ than the ratio $f_{\mathrm{e}} / f_{\gamma}$. The quantity

$$
\xi_{E} \equiv f_{\mathrm{e}} / E f_{\gamma}=N\left[\sigma_{\mathrm{pe}} R\left(E_{\mathrm{epe}}\right)+\sigma_{\mathrm{C}} R\left(E_{\mathrm{eC}}\right)+\sigma_{\mathrm{pp}} R\left(E_{\mathrm{epp}}\right)\right] / E\left(\mathrm{MeV}^{-1}\right)
$$

that is, the ratio of the omnidirectional electron number flux $f_{\mathrm{e}}$ to the photon energy flux $E f_{\gamma}$, turns out to be nearly independent of $E$ over a wide range of $E$.

Figure 2.64 through Figure 2.68 show the ratios $\xi=f_{\mathrm{e}} / f_{\gamma}$ and $\xi_{E}=f_{\mathrm{e}} / E f_{\gamma}$ in air, silicon, germanium, InSb, and $\mathrm{Hg}_{1-x} \mathrm{Cd}_{x}$ Te with $x=0.2$, together with the contribution to $\xi_{E}$ from Compton scattering. In $\mathrm{Si}$, the Compton effect dominates from $0.2 \mathrm{MeV}$ to $10 \mathrm{MeV}$, and above that energy the Compton contribution to $\xi_{E}$ drops off, but pair production keeps the ratio up near 0.01. Below about 0.2 $\mathrm{MeV}$, most electrons are created by photoelectric absorption. In Ge, Compton scattering dominates only from about $0.4 \mathrm{MeV}$ to $4 \mathrm{MeV}$. In both $\mathrm{Si}$ and $\mathrm{Ge}, \xi$ is close to 0.01 only near $1 \mathrm{MeV}$. Some values are given in Table 2.1.

The numbers for GaAs are essentially the same as for Ge because of the proximity of atomic numbers. Differences arise only immediately near X-ray edges.

In these results, the positron from pair production has been included because it ionizes much as an electron does. Thus, $f_{\mathrm{e}}$ is really the flux of electrons and positrons. * At $30 \mathrm{MeV}$ in $\mathrm{Ge}$, where $\xi_{E}=0.0199$, the positron flux is very nearly equal to the electron flux, and it happens that the ratio of each to photon energy flux is very nearly exactly $0.01 \mathrm{MeV}^{-1}$.

For photon energies $E>100 \mathrm{MeV}$, the corresponding electron (and positron) energies are above $\sim 50 \mathrm{MeV}$ (mostly from pair production), and their range exceeds $\sim 20 \mathrm{~g} / \mathrm{cm}^{2}$. In practical devices, charged particle equilibrium is much

\footnotetext{
*Positrons ionize at about the same rate as electrons and usually come to rest before annihilating. Positron annihilation in-flight is a small correction. See, e.g., Heitler [He54] or Berger and Seltzer [Be82].
} 


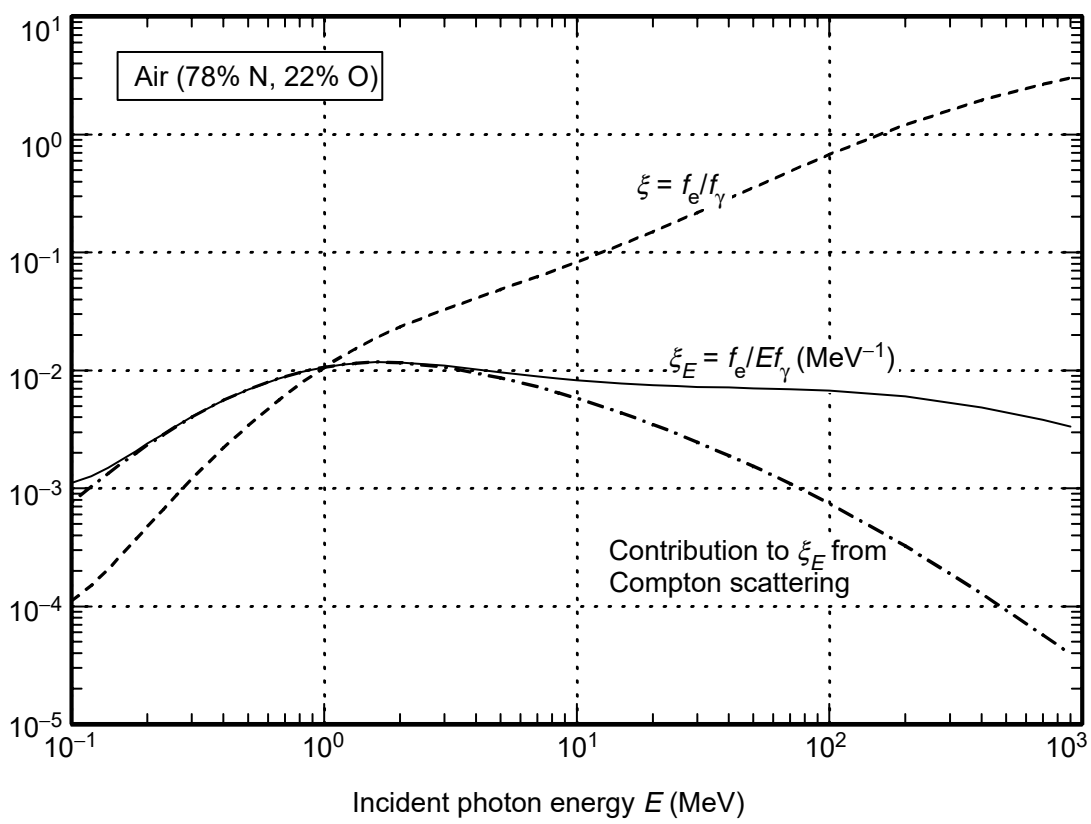

FIGURE 2.64 The $1 \%$ rule in air, the ratio of omnidirectional electron and positron flux $f_{\mathrm{e}}$ [(electrons + positrons $) / \mathrm{cm}^{2} / \mathrm{sec}$ due to photoelectric, Compton, and pair production] to photon number flux $f_{\gamma}$, $\xi$, or to photon energy flux $E f_{\gamma}, \xi_{E}=\xi / E$. Assumes charged particle equilibrium.

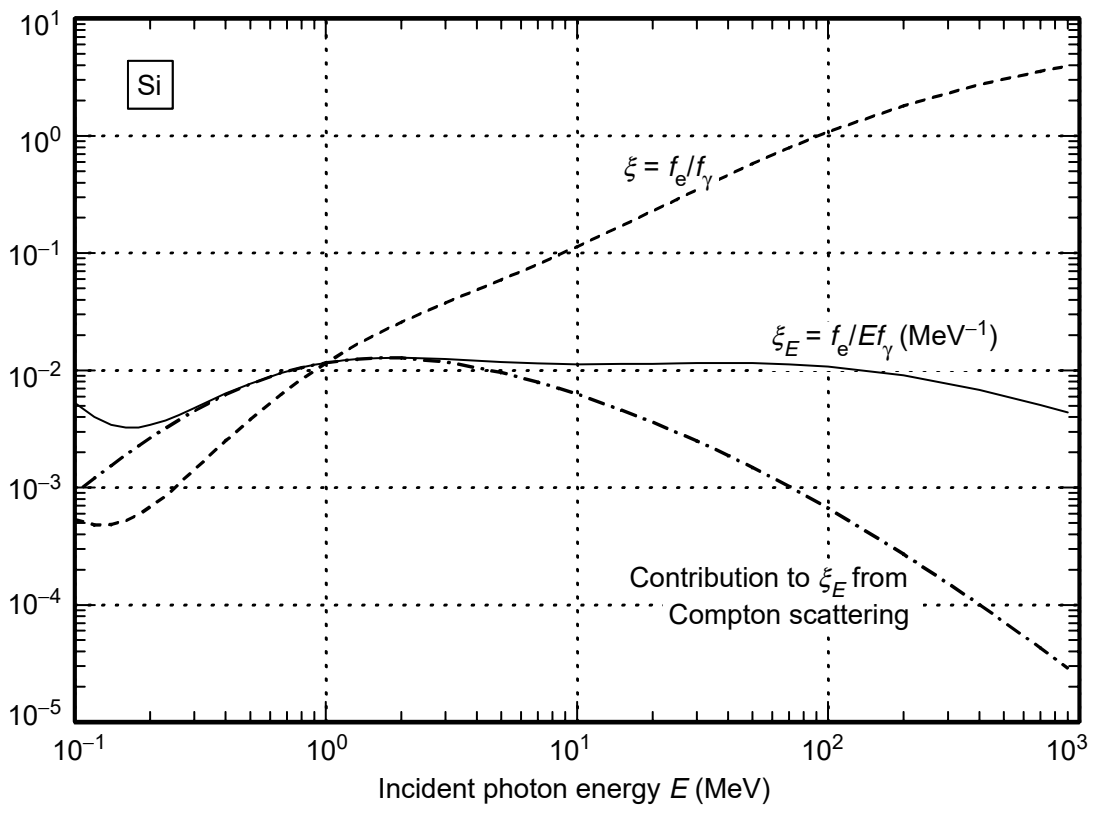

FIGURE 2.65 The $1 \%$ rule in Si. Assumes charged particle equilibrium, and includes positron flux from pair production. 


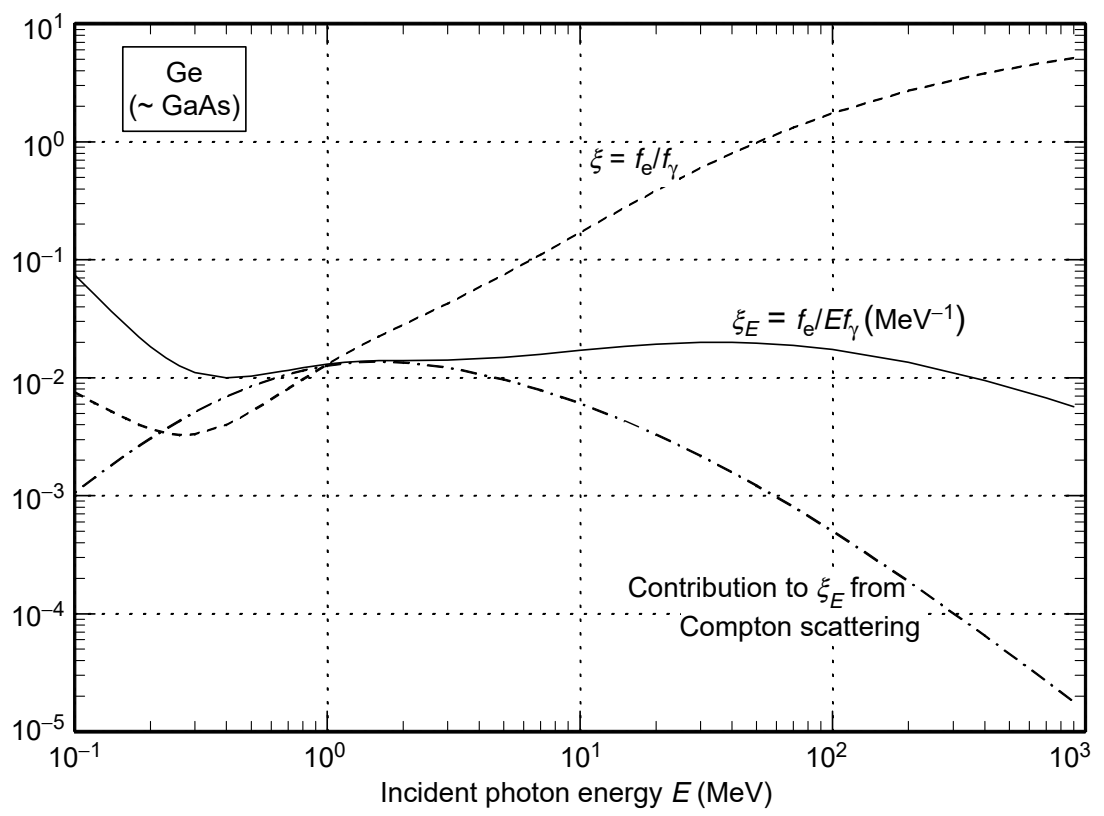

FIGURE 2.66 The $1 \%$ rule in Ge. Assumes charged particle equilibrium, and includes positron flux from pair production. GaAs is essentially the same, due to the proximity in $Z$.

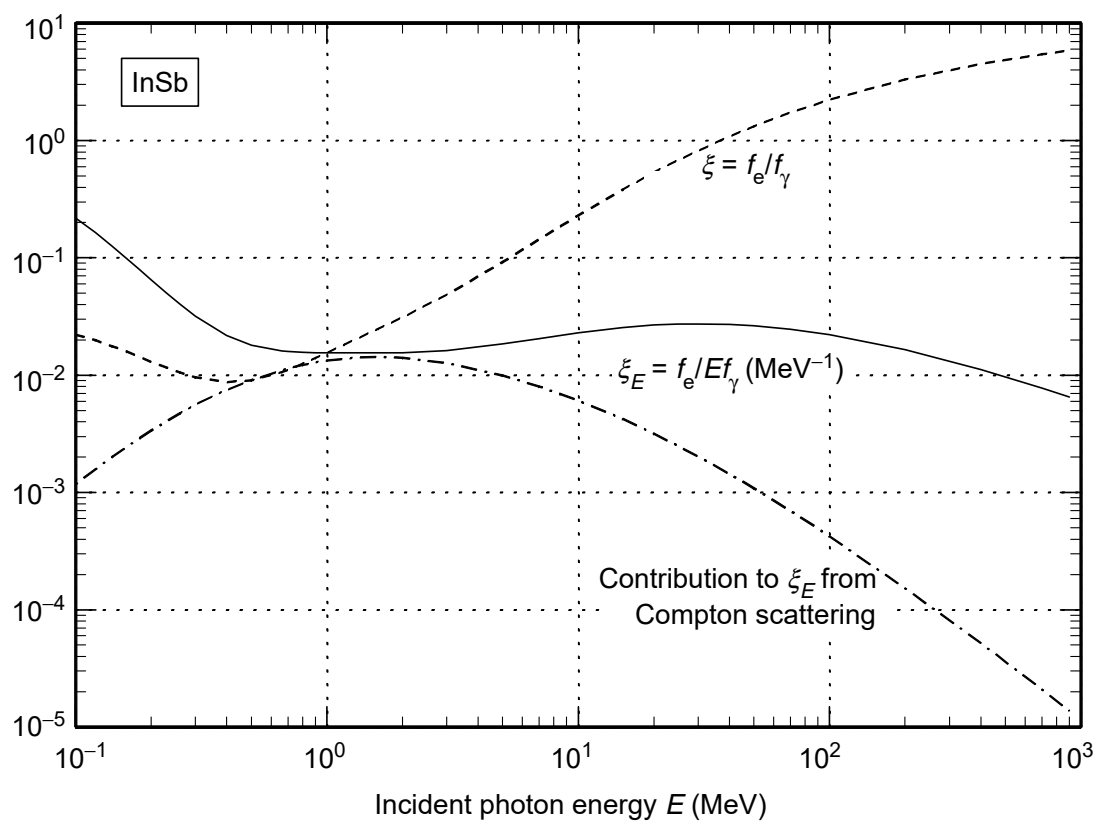

FIGURE 2.67 The 1\% rule in InSb. Assumes charged particle equilibrium, and includes positron flux from pair production. 


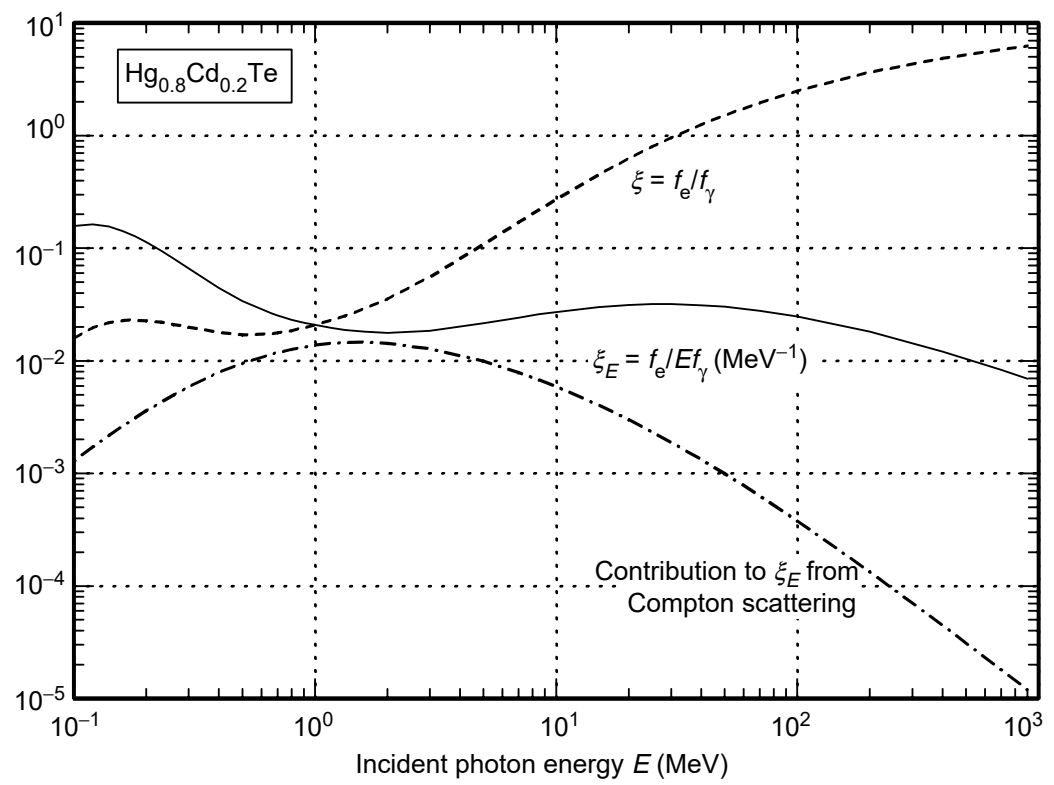

FIGURE 2.68 The $1 \%$ rule in $\mathrm{Hg}_{1-x} \mathrm{Cd}_{x} \mathrm{Te}$ for $x=0.2$. Assumes charged particle equilibrium, and includes positron flux from pair production. Results are not very sensitive to $x$ near $x=0.2$.

less likely to hold at these energies, and the actual ratio of (electron + positron) flux to photon flux will likely be less than in the table or the figures.

The Compton cross section $\left(\mathrm{cm}^{2} / \mathrm{g}\right)$ is only slightly smaller for high- $Z$ elements than for low $Z$. The electron range $\left(\mathrm{g} / \mathrm{cm}^{2}\right)$ is only slightly larger in high- $Z$ elements than in low $Z$. Thus, the Compton contribution to $\xi$ or to $\xi_{E}$ is nearly independent of $Z$, as can be seen by comparing air with $\mathrm{HgCdTe}$.

The photoelectric cross section near $100 \mathrm{keV}$ is some two orders of magnitude larger for $Z \approx 80$ than for $Z \approx 8$. Thus, the photoelectric contribution to $\xi$ or to $\xi_{E}$ is two orders of magnitude greater in $\mathrm{HgCdTe}$ than in air or Si.

The pair production cross section is several times larger for high $Z$ than for low $Z$. Thus, above several $\mathrm{MeV}$, the ratios $\xi$ and $\xi_{E}$ are several times larger in $\mathrm{HgCdTe}$ than in air or $\mathrm{Si}$. These dependencies on $Z$ can be seen in the figures from air to $\mathrm{HgCdTe}$.

Thus, The $1 \%$ Rule may be taken as the observation that the ratio $\xi_{E}$ of electron ( + positron) omnidirectional number flux to photon energy flux remains near $0.01 \mathrm{MeV}^{-1}$ over a wide range of energies, assuming charged particle equilibrium. In $\mathrm{Si}$, the ratio is between $\sim 0.9 \%$ and $1.3 \%$ for all energies in $0.6 \lesssim E(\mathrm{MeV}) \lesssim 200$. In Ge, it is between $1 \%$ and $2 \%$ over the same energies.

As mentioned, charged particle equilibrium becomes valid only at a depth beyond the range of the most energetic electrons. Because of a relatively long 


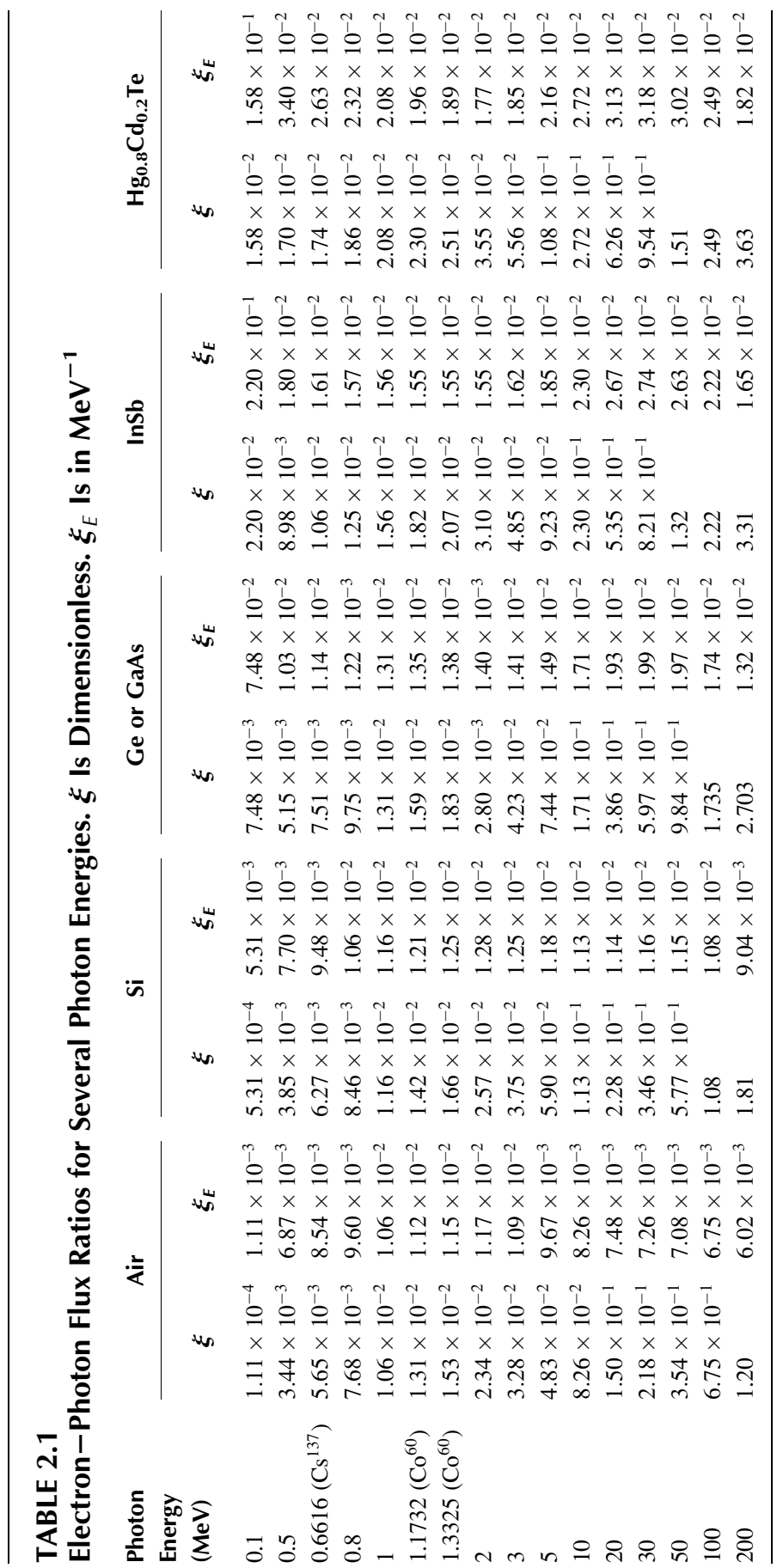


electron range, this condition is stressed in practice in a single medium for high-photon energies, where Compton or pair production dominates. For the case $E \approx 1 \mathrm{MeV}$, typical electron energies are $\sim 0.5 \mathrm{MeV}$. The range of a 0.5 $\mathrm{MeV}$ electron is approximately $0.25 \mathrm{~g} / \mathrm{cm}^{2}$ in most materials. In $\mathrm{Si}$, therefore, the table and figures for the $1 \%$ rule are not strictly applicable above $1 \mathrm{MeV}$ until one reaches depths of more than $0.1 \mathrm{~cm}$. However, equally often in practice the target is not directly exposed to photons through vacuum. Instead, the photons must penetrate some other solid before striking the target material, such as an Al or stainless steel housing, or air, and so on. The table and figures are applicable in the chosen target material if that material is preceded by a thickness of other matter equal to the range of the most energetic electrons produced (near $1 \mathrm{MeV}$ that is about $0.2 \mathrm{~g} / \mathrm{cm}^{2}$ or more of aluminum or other material). To the extent that the target material of interest is surrounded by sufficient columnar mass density of other matter, the table and figures will be approximately correct, as the ratios $\xi$ and $\xi_{E}$ are only slightly varying with atomic number.

\subsection{DOSIMETRIC QUANTITIES FOR GAMMAS AND X-RAYS}

Although we have discussed dose, there are other photon-matter interaction parameters in common use. We present here an introduction to gamma and $\mathrm{X}$-ray dosimetric quantities, skipping details to focus on concepts.

Laboratory survey dosimeters may display in $\mathrm{mR} / \mathrm{h}$ (unit of Exposure) or perhaps in mrem/h (units of dose equivalent). Another instrument may read in counts per minute. A vial of a radioactive substance is likely labeled in microcuries. Film badge results are given in millirems. An ion counter reads out in Roentgens. Here, we try to clarify commonly confusing points about Roentgen, rem, exposure, dose equivalent, and so on.*

There are at least three measures of the effects of radiation on matter that are in common use:

1. The amount of ionization it produces (Exposure). The Roentgen ${ }^{\dagger}(\mathrm{R})$ is a unit of Exposure.

2. The energy it deposits (dose).

The $\operatorname{Rad}^{\ddagger}$ and Gray ${ }^{\S}$ (Gy) are units of dose.

\footnotetext{
*Fuller treatments may be found in, e.g., J.E. Turner [Tu95]; N. Tsoulfanidis [Ts95]; F. Attix [At86]; S. Green [Gr06]; S.K. Jani [Ja93], and references listed later.

${ }^{\dagger}$ Wilhelm Konrad Roentgen (1845-1923), German physicist. Discovered X-rays in 1896. (The same year, Antoine Henri Becquerel (1852-1908) discovered radioactivity.) Roentgen received the Nobel Prize in 1901, the first year it was awarded; Becquerel shared it with Pierre and Marie Curie in 1903.

${ }^{\ddagger}$ Radiation Absorbed Dose

${ }^{\S}$ Louis Harold Gray (1905-1965), English medical physicist.
} 
3. Various measures of its damage to biological organisms (dose equivalent). The Sievert* (Sv) and rem are units of dose equivalent.

Exposure is defined only for photons. Dose and dose equivalent are defined for any type of radiation (photons, electrons, neutrons, ions, etc.).

The radiation field itself is characterized by its flux (particles $/ \mathrm{cm}^{2} / \mathrm{sec}$ ) and particle energy. Exposure, dose, dose equivalent, flux, and energy are all measures of the strength of a radiation field; one needs to understand relations among them. For inanimate targets, Exposure, fluence, and dose are the most important quantities.

\subsubsection{Brief HISTORY}

Exposure and Roentgen are old, historical ideas and terms, dating from the early 1900s. They were an attempt to quantitatively specify exposure to x-rays. Historically, Exposure was the first measure of quantity of radiation introduced. The first unit for it was the erythema dose, the amount of x-rays that would barely cause reddening of the skin. In 1928, the International Commission on Radiation Protection (ICRP) was established. The erythema dose unit was then replaced by the Roentgen, a less subjective measure. The Roentgen specifies an amount of ionization the X-rays produce.

However, Exposure and Roentgen had their limitations. It was found that Exposure did not correlate well with the damage x-rays inflicted on targets (biological or otherwise). Rather, damage correlated more closely with the energy deposited per unit mass. Therefore, the concept of dose, and a unit for it, was introduced. Dose is simply the energy deposited by any radiation per unit mass of the target. Whereas Roentgen is defined only for air, dose could be defined in any material. Dose was also preferred because it could be extended to include energy deposited by any type of radiation, not just photons. The rad (100 erg/g) and the Gray (J/kg) are its common units.

Refinements were introduced to reflect the effects of radiation on biological systems. Biological effects were found to be not merely proportional to dose, but were worse for protons and alpha particles than for gammas of the same dose. The first parameter introduced was the Relative Biological Effectiveness (RBE). It attempted to account for different radiations, different organs, and different biological endpoint effects. It turned out, however, to be too complicated to put all those effects in one number. Workers backed off and parameterized the radiation field separately from the exposed organ. That led to the introduction of the Quality factor $Q$ for different radiations and energies. $Q$ was assigned a larger value for alphas and protons than for gammas, roughly in proportion to their perceived biological effect. Later, the

\footnotetext{
*Rolf Maximilian Sievert (1896-1966), Swedish radiologist.
} 
radiation weighting factor $w_{\mathrm{R}}$ was introduced as a generalization of $Q$ for the average dose on an organ. Further refinements accounted for effects on different types of biological tissues (bone, muscle, organs, etc.) produced by the same radiation and the same dose. They also accounted for the average dose on an internal organ. Parameters such as the tissue weighting factor $w_{\mathrm{T}}$, the organ-averaged dose equivalent, and the effective dose equivalent are attempts to quantify responses of different types of tissues.

\subsubsection{Nonbiological Measures}

\subsubsection{Exposure, Dose, and Fluence}

A Roentgen is a unit of Exposure, an unfortunate name for the ionization produced in air by exposure to X-rays or gamma rays.

By definition:

$1 R \equiv 1$ Roentgen $\equiv$ that amount of photons that produces in dry air an ionization of 1 esu of charge separated (i.e., electron-ion pairs) per $\mathrm{cm}^{3}$ of STP air.

Today, the same definition is usually stated in SI units:

$1 R \equiv$ that amount of photons that produces in dry air an ionization of $2.58 \times 10^{-4} \mathrm{C} / \mathrm{kg}$ of air $=1.61 \times 10^{15}$ electron-ion pairs per $\mathrm{kg}$ STP air.

The SI numbers appear arbitrary until one realizes their origin in esu. The concepts of Exposure and of Roentgen were originally intended for mediumenergy x-rays, say photon energies $E_{\gamma}$ in the range of $10 \mathrm{keV} \lesssim E_{\gamma} \lesssim 300 \mathrm{keV}$, but can be extended to $\sim 3 \mathrm{MeV}$.

If someone says, "I was exposed to 1 Roentgen of x-rays," it means that the photons that hit him, if sent into air, would produce $1.61 \times 10^{15}$ electron-ion pairs in $1 \mathrm{~kg}$ air.

Although defined in terms of e-i pairs, Exposure can be expressed in terms of energy deposited (i.e., dose) if we know how much energy is required to produce $1 \mathrm{e}-\mathrm{i}$ pair.

It takes about $34 \mathrm{eV}$ to create one $\mathrm{e}-\mathrm{i}$ pair in air. Thus, the dose produced in air by an Exposure of $1 \mathrm{R}$ is $\left(34 \mathrm{eV} /\right.$ pair) $\times 1.61 \times 10^{15}$ (pairs per $\mathrm{kg}$ ) = $5.47 \times 10^{16} \mathrm{eV} / \mathrm{kg}=87.7 \mathrm{erg} / \mathrm{g}=0.877 \mathrm{rad}$ (air). Hence, $1 \mathrm{R}$ of photons of any energy is that amount of photons that produces a dose of $0.877 \mathrm{rad}$ in air. For photons, $1 \mathrm{R}$ and $0.877 \mathrm{rad}$ (air) are synonymous.* Thus, there is a direct relation between Exposure and photon fluence ("amount of photons"). Because energy deposition differs for different photon energies, the relation is a function of energy.

When one speaks of dose (due to x-rays or gammas), one must specify the material, as rad (Si) or rad (air). When one speaks of Exposure, one need not

*As $34 \mathrm{eV}$ is not a firm number, some sources quote a number slightly different from 0.877 . 


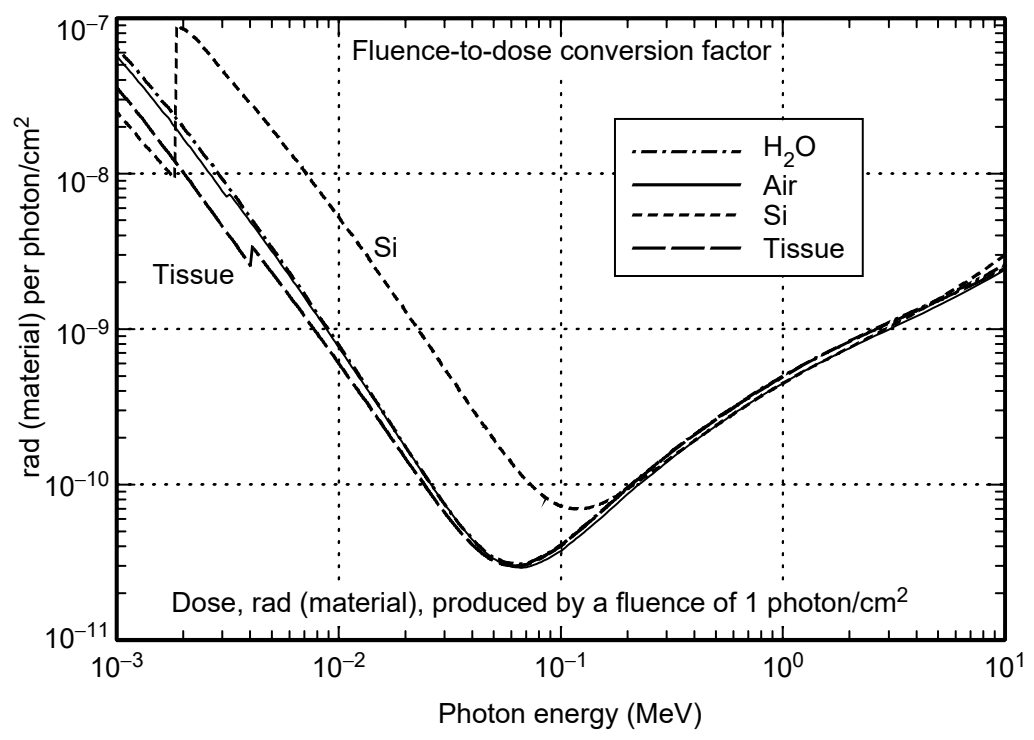

FIGURE 2.69 Fluence-to-dose conversion factor. Dose in several materials produced by 1 photon $/ \mathrm{cm}^{2}$.

specify the material, as it is always referenced to air. That is one reason the old concept is still in use.

$1 \mathrm{R}$ of $\mathrm{x}$-rays produces different dose in different materials. It is of interest to obtain the dose in selected materials when exposed to $1 \mathrm{R}$, that is, to construct Exposure-to-dose conversion tables. As fluence-to-dose conversion factors are generally available, it is easiest to first convert Exposure to photon fluence, and then fluence to dose, obtaining Exposure-to-dose conversion. We discuss fluence-to-dose conversion first.

\subsubsection{Fluence-to-Dose Conversion}

Photon energy deposition by photoelectric absorption, Compton scattering, and pair production was discussed in Section 2.9. Figure 2.69 shows the energy deposited per gram of material by unit fluence, that is, the fluenceto-dose conversion curves, $\operatorname{rad}\left(\right.$ material) per photon $/ \mathrm{cm}^{2}$. Selected values of the inverse (fluence per unit dose) are in Table 2.2. This quantity, of course, has nothing to do with Exposure or Roentgen. (Tissue here is actually A-150 tissue-equivalent plastic.*)

\footnotetext{
*ICRU A-150 plastic. Atomic composition H: 10.133, C:77.55, N:3.506, O:5.232, F:1.742, Ca:1.838 weight \%. See http://physics.nist.gov/ > Radiation Dosimetry Data > aSTAR > Material Composition Data.
} 
TABLE 2.2

Photon Fluence-to-Dose Conversions in Several Materials

Photon Energy (keV)

10

30

$60(\mathrm{Am} 241)$

100

200

300

$662(\mathrm{Cs} 137)$

1000

1173 (Co60)

1332 (Co60)

1461 (K40)

2000

3000

Fluence $\left(\gamma / \mathrm{cm}^{2}\right)$ to Produce $1 \mathrm{rad}$ in

\begin{tabular}{ccc}
\hline Air & Si & Tissue (A150 Plastic) \\
$1.31 \times 10^{9}$ & $1.91 \times 10^{8}$ & $1.67 \times 10^{9}$ \\
$1.33 \times 10^{10}$ & $1.79 \times 10^{9}$ & $1.51 \times 10^{10}$ \\
$3.36 \times 10^{10}$ & $7.24 \times 10^{9}$ & $3.34 \times 10^{10}$ \\
$2.68 \times 10^{10}$ & $1.38 \times 10^{10}$ & $2.48 \times 10^{10}$ \\
$1.15 \times 10^{10}$ & $1.06 \times 10^{10}$ & $1.05 \times 10^{10}$ \\
$7.18 \times 10^{9}$ & $7.03 \times 10^{9}$ & $6.53 \times 10^{9}$ \\
$3.20 \times 10^{9}$ & $3.20 \times 10^{9}$ & $2.91 \times 10^{9}$ \\
$2.24 \times 10^{9}$ & $2.24 \times 10^{9}$ & $2.03 \times 10^{9}$ \\
$1.97 \times 10^{9}$ & $1.97 \times 10^{9}$ & $1.79 \times 10^{9}$ \\
$1.78 \times 10^{9}$ & $1.78 \times 10^{9}$ & $1.62 \times 10^{9}$ \\
$1.66 \times 10^{9}$ & $1.66 \times 10^{9}$ & $1.51 \times 10^{9}$ \\
$1.33 \times 10^{9}$ & $1.32 \times 10^{9}$ & $1.21 \times 10^{9}$ \\
$1.01 \times 10^{9}$ & $9.75 \times 10^{8}$ & $9.22 \times 10^{8}$ \\
\hline
\end{tabular}

Above $200 \mathrm{keV}$, where Compton scattering dominates, the dose is about the same in all materials because Compton scattering per unit mass is proportional to the number of electrons $/ \mathrm{g}, N_{\mathrm{g}}$. In turn, $N_{\mathrm{g}}$ is proportional to $Z / A$. As $Z / A$ is nearly independent of $Z, N_{\mathrm{g}}$ and Compton scattering and fluence-to-dose conversion are also nearly independent of material.

Below about $100 \mathrm{keV}$ photoelectric absorption dominates, with its very large cross section and large deposition in a short distance, meaning the same fluence creates a larger dose. The bulk photoelectric cross section $\sigma_{\mathrm{pe}}\left(\mathrm{cm}^{2} / \mathrm{g}\right)$ is roughly proportional to $1 / E_{\gamma}^{3}$. The full energy is deposited, so the dose of a given fluence is proportional to $\sigma_{\mathrm{pe}} E_{\gamma} \propto 1 / E_{\gamma}^{2}$, as seen in the figure. In silicon, the photoelectric cross section and dose are an order of magnitude larger than in the other materials because of its relatively high $Z$.

The ledge in tissue at $4 \mathrm{keV}$ is the $K$ edge in $\mathrm{Ca}$ in this tissue-equivalent plastic.

\subsubsection{Exposure-to-Fluence Conversion}

For this conversion, we need the fluence of photons of energy $E_{\gamma}$ that produces an Exposure of $1 \mathrm{R}$. It is the fluence that deposits $0.877 \mathrm{rad}$ in air. This is just the inverse of the air curve in Figure 2.69 and is graphed in Figure 2.70, with selected values in Table 2.3. As Exposure is proportional to dose in air, the fluence per $\mathrm{R}$ is small at low energies as just discussed for fluence-to-dose.

Table 2.3 shows, for example, that at $1 \mathrm{~m}$ from $1 \mathrm{Ci}$ of $\mathrm{Cs}^{137}(=0.85 \times$ $\left.3.7 \times 10^{10}=3.145 \times 10^{10} \mathrm{\gamma} / \mathrm{sec}\right)$, the Exposure is $0.85 \times 378=321 \mathrm{mR} / \mathrm{h}$. 


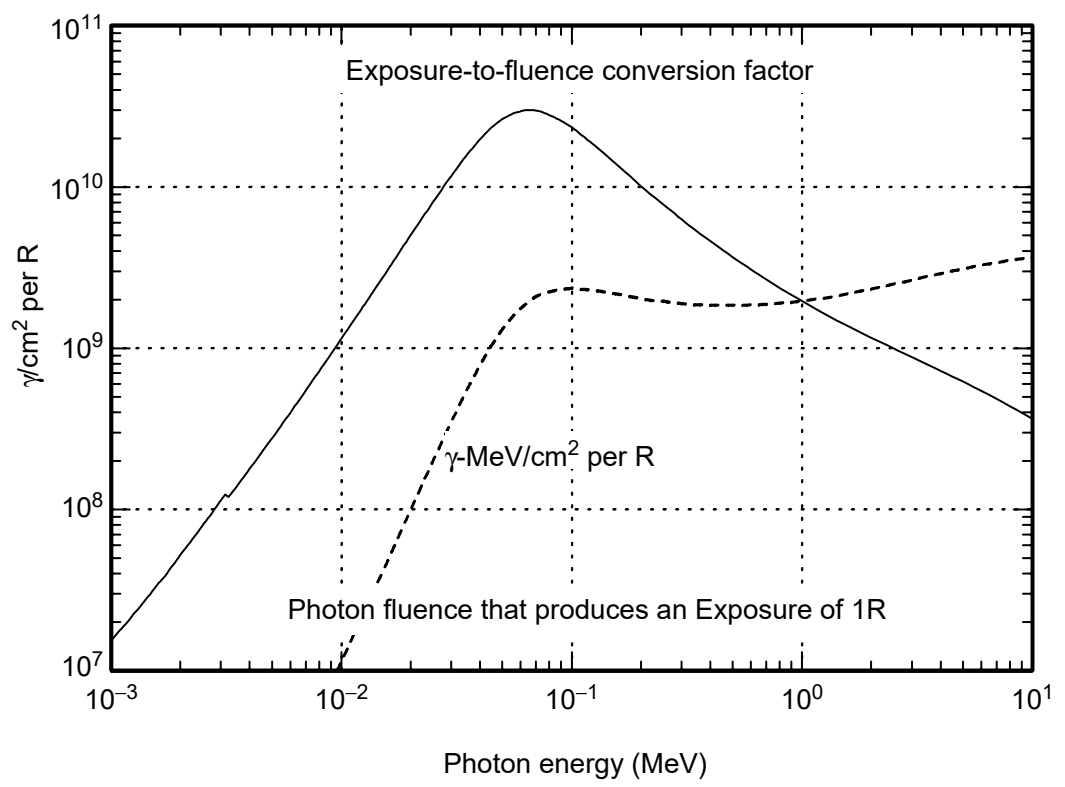

FIGURE 2.70 Solid curve: photon fluence that produces an Exposure of $1 \mathrm{R}$; that is, photon fluence that produces a dose of $0.877 \mathrm{rad}$ air. When multiplied by photon energy there results the dashed curve, the photon energy fluence per R; above $100 \mathrm{keV}$ it is slowly varying, near $2 \times 10^{9} \gamma-\mathrm{MeV} / \mathrm{cm}^{2}$ per $\mathrm{R}$.

\section{TABLE 2.3}

\section{Relation between Photon Fluence and Exposure}

$\begin{array}{lccc}\begin{array}{l}\text { Photon } \\ \text { Energy (keV) }\end{array} & \begin{array}{c}\text { Fluence to Produce } \\ \mathbf{1} \boldsymbol{\mu R}\left(\boldsymbol{\gamma} / \mathbf{c m}^{2}\right)\end{array} & \begin{array}{c}\text { Flux to Produce } \\ \mathbf{1} \boldsymbol{\mu R} / \mathbf{h}\left(\boldsymbol{\gamma} / \mathbf{c m}^{\mathbf{2}} / \mathbf{s e c}\right)\end{array} & \begin{array}{c}\text { Exposure Rate at } \\ \mathbf{1} \mathbf{~ m} \text { from } \\ \mathbf{3 . 7 E 1 0} \boldsymbol{\gamma} / \mathbf{s e c}(\mathbf{m R} / \mathbf{h})\end{array} \\ 10 & 1,147 & 0.32 & 920 \\ 30 & 11,670 & 3.24 & 90.9 \\ 60(\text { Am241) } & 29,500 & 8.19 & 36 \\ 100 & 23,490 & 6.53 & 45.1 \\ 200 & 10,110 & 2.81 & 105 \\ 300 & 6,301 & 1.75 & 168 \\ 662(\mathrm{Cs} 137) & 2,808 & 0.780 & 378 \\ 1000 & 1,960 & 0.544 & 541 \\ 1173(\mathrm{Co60}) & 1,720 & 0.478 & 616 \\ 1332(\mathrm{Co60}) & 1,557 & 0.433 & 680 \\ 1461(\mathrm{~K} 40) & 1,454 & 0.404 & 729 \\ 2000 & 1,163 & 0.323 & 912 \\ 3000 & 881 & 0.245 & 1,202\end{array}$


The small ledge at $3.18 \mathrm{keV}$ is the $K$ edge in Ar. Ar is only $1 \%$ of air by particle number, but its cross section per atom just above its $K$ edge is 15 times that of an $\mathrm{N}_{2}$ or $\mathrm{O}_{2}$ molecule.

\subsubsection{Exposure-to-Dose Conversion}

The desired Exposure-to-dose factor is the product (Exposure-tofluence) $\times$ (fluence-to-dose). This is shown in Figure 2.71 and Table 2.4 for
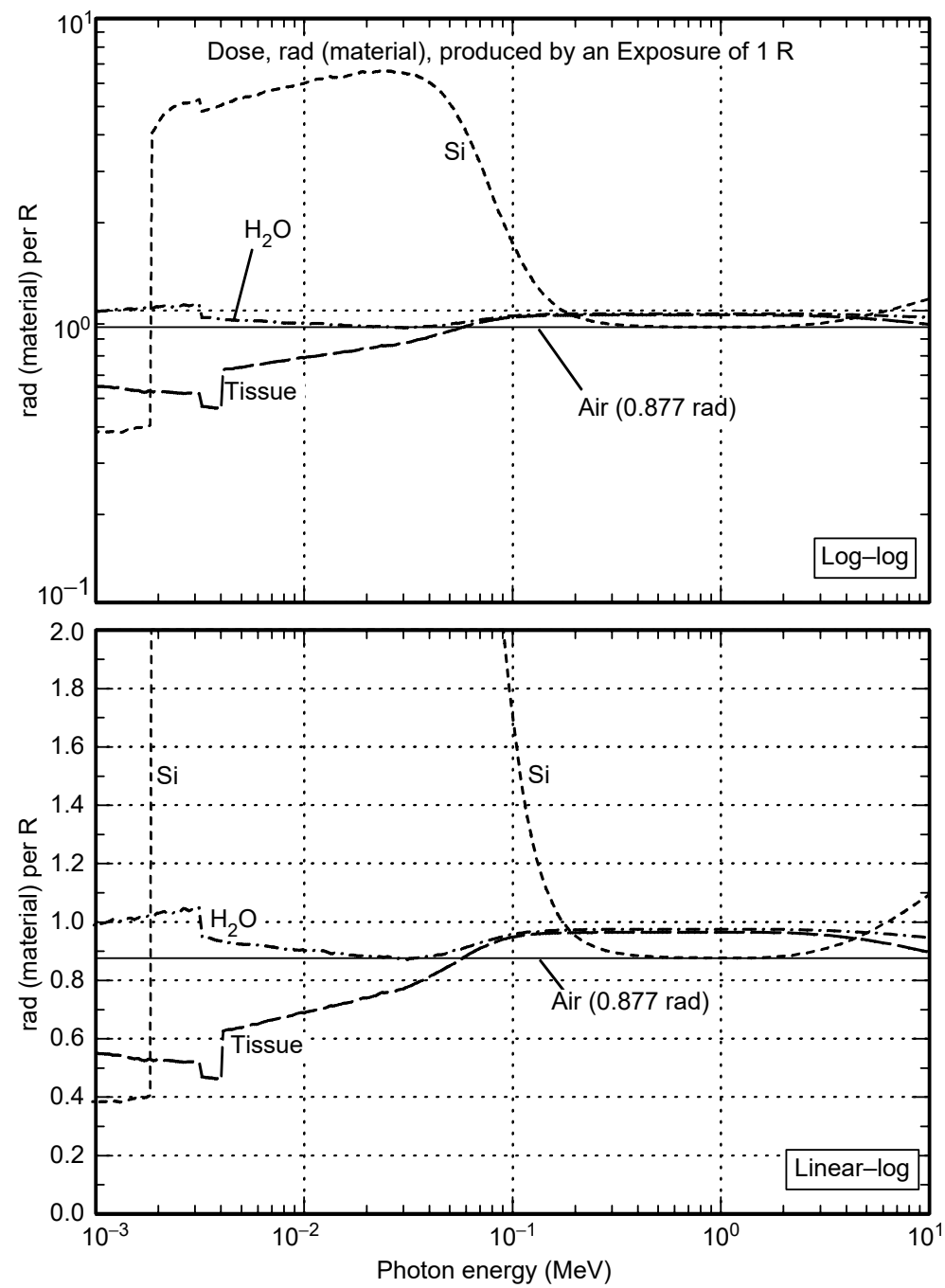

FIGURE 2.71 Exposure-to-dose conversion factor. Dose in several materials produced by an Exposure of $1 \mathrm{R}$. 


\section{TABLE 2.4}

\section{Exposure-to-Dose Conversion. Dose in Several Materials Produced by an Exposure of $1 R$, in rad (material) per $R$}

\begin{tabular}{|c|c|c|c|c|}
\hline \multirow{2}{*}{$\begin{array}{l}\text { Photon } \\
\text { Energy (keV) }\end{array}$} & \multicolumn{4}{|c|}{ Material } \\
\hline & Air & Water & Si & Tissue \\
\hline 10 & 0.877 & 0.902 & 6.01 & 0.689 \\
\hline 30 & & 0.873 & 6.51 & 0.772 \\
\hline $60(\mathrm{Am} 241)$ & & 0.914 & 4.07 & 0.885 \\
\hline 100 & & 0.957 & 1.70 & 0.948 \\
\hline 200 & & 0.973 & 0.953 & 0.964 \\
\hline 300 & & 0.974 & 0.897 & 0.965 \\
\hline $662(\mathrm{Cs} 137)$ & & 0.975 & 0.878 & 0.965 \\
\hline 1000 & & 0.975 & 0.877 & 0.965 \\
\hline 1173 (Co60) & & 0.975 & 0.876 & 0.965 \\
\hline $1332(\mathrm{Co} 60)$ & & 0.975 & 0.876 & 0.965 \\
\hline 1461 (K40) & & 0.975 & 0.877 & 0.964 \\
\hline 2000 & & 0.974 & 0.883 & 0.963 \\
\hline 3000 & & 0.971 & 0.904 & 0.956 \\
\hline
\end{tabular}

several materials, in rad(material) per R. It is, of course, constant at $0.877 \mathrm{rad}$ for air. Between about $100 \mathrm{keV}$ and $3 \mathrm{MeV}$, it is $0.96 \mathrm{rad}$ in tissue, slightly larger than air because the hydrogen raises the average $Z / A$. At low energies, the photoelectric effect dominates Compton scattering in most elements (except $\mathrm{H}$ ), and the $\mathrm{O}$ in water makes $\mathrm{H}_{2} \mathrm{O}$ absorb more than the $\mathrm{C}$ in tissue.

Again between $100 \mathrm{keV}$ and $3 \mathrm{MeV}, \mathrm{Si}$ is the same as air because they have the same $Z / A$, and Compton dominates. At low energies, where photoelectric absorption dominates, the dose in $\mathrm{Si}$ is much larger because of its large cross section. Below $100 \mathrm{keV}$, Roentgen-to-rad conversion is not a convenient parameter for $\mathrm{Si}$.

Thus, for a given material, a single, constant rad per $\mathrm{R}$ can be assigned only over a limited energy range, say from 0.1 to $3 \mathrm{MeV}$. However, as a rule of thumb, soft tissue exposed to $1 \mathrm{R}$ of intermediate energy x-rays produces a dose of about $0.96 \mathrm{rad}$, or nearly $1 \mathrm{rad}$.

\subsubsection{Dose in Hydrogenous Material}

Generally, more photon energy is deposited in a mass of hydrogenous material than in the same mass of nonhydrogenous material. Above about $50 \mathrm{keV}$, where Compton scattering dominates in low- $Z$ materials, absorption is proportional to the number of electrons per gram. A gram of $\mathrm{H}$ contains twice as many electrons as a gram of anything else (because $Z / A=1$ for $\mathrm{H}$, but $\approx 1 / 2$ for other elements) and so absorbs more energy. 
Biological material contains much hydrogen. In nominal tissue (often A-150 tissue-equivalent plastic), the dose produced by an Exposure of $1 \mathrm{R}$ is about $10 \%$ higher than in air because of the $\mathrm{H}$. The atomic composition and dose further vary somewhat with the type of tissue (muscle, fat, bone, internal organs, etc.).

Dose, and sometimes dose rate, seems to be sufficient to characterize most radiation effects on inanimate matter. But the response of biological organisms is more complicated. In an attempt to quantify biological effects, further parameters are introduced. Some were mentioned earlier in Section 2.11.1.

\subsubsection{Biological Measures}

Dose is directly related to the physics of particle interaction with atoms; it is relatively easy to compute and measure. But the important effects of a fixed dose in biological tissue are more difficult to quantify. It turns out that different radiations (photons, electrons, neutrons, heavy ions, etc.), when arranged to produce the same dose in a biological target, cause different consequences. A dose of 1 rad produced by photons is less damaging than a dose of $1 \mathrm{rad}$ produced by heavy ions or by neutrons. Neutrons, though neutral, produce their dose and biological effect largely by the dose of recoil protons and ions from their elastic scattering off hydrogen, carbon, and other atoms in tissue.

The main reason for the difference of photons and electrons relative to heavy ions and neutrons is that the former are relatively lightly ionizing particles, losing their energy to ionization over a long path length. Heavy ions, however, lose kinetic energy to ionization over a shorter path. Their stopping power or linear energy transfer (LET) is larger, and damage increases with LET. Photons and electrons are considered low LET particles. Protons, alphas, heavier ions, and neutrons are considered high LET particles. Even though higher LET particles have higher doses, the difference in biological effects between electrons and ions is more than can be accounted for by different dose. That is, the response of biological organisms to energy deposited by radiations is found to be not merely proportional to dose, but to depend on the type of radiation as well.

\subsubsection{Relative Biological Effectiveness}

The biological response also differs if the entire body is exposed or if only a particular organ or a particular selection of tissue is exposed. An early parameter to account for different types of radiation and for the responses of different organs was the relative biological effectiveness, RBE. Most radiations cause more biological damage than photons of the same dose, so photons are taken as a reference radiation. The RBE of a different radiation and a particular organ or tissue is defined so that if a radiation produces a dose $D$ in a tissue, it produces the same biological effect as a dose $\mathrm{RBE} \times D$ of a 
reference photon source.* RBE is a number almost always greater than 1 . The reference photons were those readily achievable, typically the output of a $250-\mathrm{kVp} x$-ray machine. The RBE then is a function of the type of radiation, its energy, the organ or tissue exposed, and the biological effect considered.

The RBE turned out to be too complicated for simple parameterization or as a basis for regulations for radiation protection. Instead, it was deemed more sensible to separate parameters characterizing the radiation from those characterizing the exposed organ and its vulnerability to radiation. The first step in this direction was to introduce the quality factor to characterize different radiations. The second step was to introduce parameters for the various tissues and organs and the various biological effects of interest.

\subsubsection{Quality Factor and Dose-Equivalent}

To account for the effects of different radiations (largely different LET) and to relate biological response of different types of radiation to dose, a parameter $Q$, called the quality factor, was introduced. As particle LET also depends on its energy, $Q$ is a function of the radiation type and its energy.

$Q$ is chosen to be $Q=1$ for all photons and for electrons and positrons of energy greater than about $30 \mathrm{keV}$. Lower energy electrons have a larger LET. For other radiations, $Q$ is generally larger than 1 and varies with particle energy and type of radiation. A measure of biological effects is both statistical and imprecise; human judgment is involved in defining $Q$. Although subjective, a specific definition has evolved, defined by an international committee. The committee will change the definition as new experimental evidence and pressure from the medical community or environmentalists dictate.

An incomplete sampling of $Q$ values is**

$\begin{array}{ll}\text { Radiation } & Q \\ \text { x-rays } & 1 \\ \gamma \text { rays } & 1 \\ \text { Electrons }>30 \mathrm{keV} & 1 \\ \text { Electrons }<30 \mathrm{keV} & >1 \\ \alpha \text { particles } & 20 \\ \text { Heavy ions } & 20 \\ \text { Slow neutrons } & 2.5 \\ \text { Fast neutrons } & 20\end{array}$

Thus, $Q$ is defined such that if a radiation produces a dose $D$ in a tissue, it produces the same biological effect as a dose $Q D$ of photons. As the effect on

\footnotetext{
*See Shultis and Faw [Sh00], Tsoulfanidis [Ts95], and Turner [Tu95].

**A complete definition can be found in, e.g., [Sh98].
} 
humans is often of prime interest, one defines the dose-equivalent $H$ (for human-equivalent dose)

$$
H=Q D
$$

Personnel dosimeters therefore usually display output in dose-equivalent. Some gamma and beta dosimeters directly measure the physical dose $D$, but display dose-equivalent. On the other hand, a laboratory ion chamber counts ions (accumulated charge) and therefore measures Roentgen. Others, such as the Navy's Snoopy neutron rem-meter, have internal baffles and absorbers surrounding the actual detector, designed so that the response of the internal sensitive element $\left(\mathrm{He}^{3}\right.$ tube in the case of Snoopy) is proportional to dose-equivalent.

The definition of $Q$ is similar to that of RBE except that it applies to a generic biological material. There is not a separate $Q$ for different tissues or organs. Additional parameters, discussed presently, are introduced to characterize different targets.

Dose-equivalent has the same dimensions as dose, but is given a new unit. In SI, the unit of dose is the Gray, while the unit of dose-equivalent is the Sievert (Sv). The older unit of dose, the rad, is still widely used; its corresponding unit of dose-equivalent is the rem (Roentgen equivalent in man). The connection is

$$
\begin{aligned}
1 \mathrm{~Gy} & \equiv 100 \mathrm{rad} \\
1 \mathrm{~Sv} & \equiv 100 \mathrm{rem}
\end{aligned}
$$

For photons or energetic electrons or positrons, or any radiation at an energy for which $Q=1$, one has $1 \mathrm{~Sv}=1 \mathrm{Gray}$, and $1 \mathrm{rem}=1 \mathrm{rad}$. And as in tissue, $1 \mathrm{R} \approx 0.96 \mathrm{rad} \approx 1 \mathrm{rad}$, as a rule of thumb, one has

$$
1 R \approx 1 \mathrm{rad}=1 \mathrm{rem} \text { (photons in biological tissue) }
$$

The exact number varies from 1 depending on the particular tissue.

Exposure and dose, and their units Roentgen, rad, and Gray, are defined with no reference to biological effects. The quality factor, dose-equivalent, $\mathrm{Sv}$, and rem are defined in terms of their biological effects. Dose-equivalent $H$ is a first attempt to account for varying biological effects of a given physical dose.

Dose $D$ and dose-equivalent $H$ are point functions. As photon flux attenuates with depth and particle energy and LET vary with depth, $D$ and $H$ are functions of depth when a given radiation is incident.

To specify standards for health effects and radiation protection, refinements need to be added to simple dose-equivalent. 
To date, these refinements have been based on the following:

- The depth in tissue (or organs) at which one considers the dose (shallow dose-equivalent vs. deep dose-equivalent)

- The nature and vulnerability of the target material (tissue or a specific organ)

- An average dose-equivalent over an entire organ rather than at a point.

- Whether the exposure is localized or whole-body

The following is a brief summary.*

\subsubsection{Shallow vs. Deep Dose-Equivalent}

A distinction is drawn between the dose at the surface of exposed tissue (taken as a depth less than $70 \mu \mathrm{m}$, the assumed depth of basal cells) and the dose down to a depth of $1 \mathrm{~cm}\left(1 \mathrm{~g} / \mathrm{cm}^{2}\right)$. The shallow-dose equivalent is for the protection of the skin and basal cell layer. The deep dose-equivalent is only representative of deep exposure, and is not intended to account for exposure to organs, which may lie much deeper below the body surface. Deep dose-equivalent is intended to apply to external whole-body exposure. In the literature, one commonly finds curves of deep dose-equivalent or shallow dose-equivalent as a function of incident particle energy.

\subsubsection{Dose Equivalent Averaged over an Organ}

To account for depth dependence on a particular organ or tissue $T$, two parameters are defined. The first accounts for the type of radiation $R$ (photon, electron, ion, etc.), and is called the radiation weighting factor $w_{\mathrm{R}}$. It is similar to $Q$. The second accounts for the vulnerability and sensitivity of particular organs or tissue, and is the tissue- or organ-weighting factor $w_{\mathrm{T}}$.

Then, instead of dose and dose-equivalent at a point, one defines the dose $D_{\mathrm{T}, \mathrm{R}}$, due to radiation of type $\mathrm{R}$, averaged over the volume of an organ T. In the current discussion, this quantity replaces ordinary $D$. Hence, the organaveraged dose-equivalent $H_{\mathrm{T}, \mathrm{R}}$ is defined by

$$
H_{\mathrm{T}, \mathrm{R}}=w_{\mathrm{R}} D_{\mathrm{T}, \mathrm{R}}
$$

$w_{\mathrm{R}}$ takes the place of $Q$, and multiplies the organ-averaged dose rather than the point dose. The organ-averaged dose-equivalent and $w_{\mathrm{R}}$ are replacing point dose-equivalent and $Q$ in radiological health physics. Values of $w_{\mathrm{R}}$ are given in, for example, Shleien et al. [Sh98]. Typical values are

Radiation: Photons Electrons, Muons Protons Heavy Ions Neutrons

$\begin{array}{llllll}w_{\mathrm{R}} & 1 & 1 & 5 & 20 & 5-20\end{array}$

\footnotetext{
*A full discussion is in, e.g., Shleien et al. [Sh98]. See also Knoll [Kn00] and Shultis and Faw [Sh00].
} 
The neutron value depends on energy. If several types of radiation are present, the organ-averaged dose-equivalent is

$$
H_{\mathrm{T}}=\Sigma_{\mathrm{R}} w_{\mathrm{R}} D_{\mathrm{T}, \mathrm{R}}
$$

$H_{\mathrm{T}}$ is called the equivalent dose. The organ- (or tissue-) averaged quantities $D_{\mathrm{T}, \mathrm{R}}$ and $H_{\mathrm{T}}$ were formally introduced in 1990 by the ICRP [ICRP91].

Just as $Q$ accounts only for the different radiations and does not account for the different responses of various tissues or organs exposed to them, so $w_{\mathrm{R}}$ does not account for the responses of different organs. An organ-weighting factor, or tissue-weighting factor, $w_{\mathrm{T}}$ is therefore introduced to account for the various target organs and effects. Thus, the equivalent dose must be further modified by $w_{\mathrm{T}}$, defining a quantity $E$ called the effective dose, in units of $\mathrm{Sv}$,

$$
E=\Sigma_{\mathrm{T}} w_{\mathrm{T}} H_{\mathrm{T}}
$$

$E$ is usually understood to pertain to whole-body exposure, and $w_{\mathrm{T}}$ is the relative importance of the different organs and tissues in producing various biological effects (hereditary defects, cancer, etc.) under that exposure. $w_{\mathrm{T}}$ is supposed to depend only on the organ or tissue and on the endpoint effect and not on the radiation; $w_{\mathrm{R}}$ is supposed to depend only on the radiation type, and not on the organ or effect. The $w_{\mathrm{T}} \mathrm{s}$ for all body organs, tissues, bones, and so on, are defined to sum to 1 , so that uniform equivalent dose on all organs $\left(H_{\mathrm{T}}\right.$ independent of $T$ ) produces an effective dose equal to $H_{\mathrm{T}}$. Bone marrow is assigned $w_{\mathrm{T}}=0.12$; bone surface 0.01; liver 0.05; and so forth (Shleien et al. [Sh98]).

The ICRP tissue and radiation weighting factors are coming into wider use. However, in contrast to the discussion just given based on ICRP definitions, the ANSI/ANS standard [ANS91] introduces slightly different radiobiological quantities, also in general use. Here, an effective dose-equivalent $H_{\mathrm{E}}$ is defined directly from a given incident fluence $\Phi$ (of photons or neutrons) by

$$
H_{\mathrm{E}}=h_{\mathrm{E}} \Phi
$$

again applicable to whole-body exposure. $H_{\mathrm{E}}$ is an overall effective dose equivalent $(\mathrm{Sv})$ of the body, taking into account the various vulnerabilities of different organs and their depth. $h_{\mathrm{E}}$ is then the conversion factor from fluence to dose-equivalent for full body exposure. It is different for different directions of body exposure. Figure 2.72 shows $h_{\mathrm{E}}$ for lateral exposure (LAT) and for frontal exposure (AP) and compares them with the simple fluenceto-dose conversion factor.

The dose accounts for basic energy deposition; $Q$ or $w_{\mathrm{R}}$ accounts for effects of different radiations and $w_{\mathrm{T}}$ accounts for specific biological target 


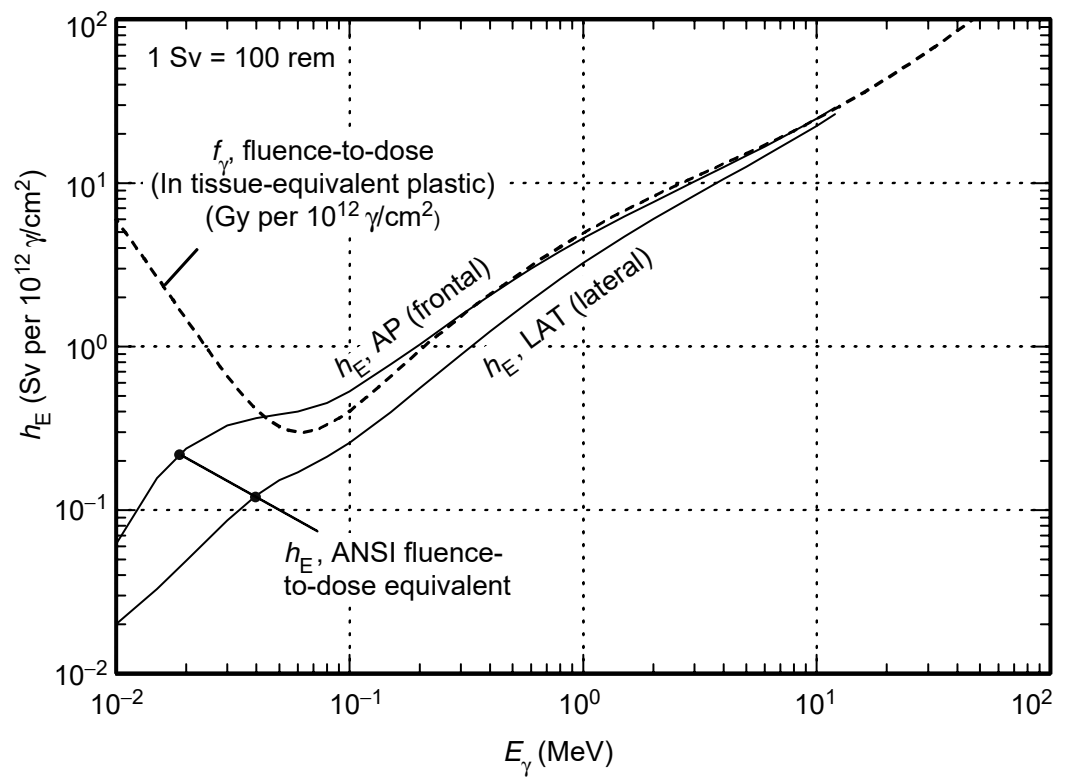

FIGURE 2.72 Fluence-to-dose in tissue-equivalent plastic, and fluence to effective dose-equivalent conversion factors $h_{\mathrm{E}}$, for gammas. $h_{\mathrm{E}}$ is defined by standard ANSI/ ANS 6.1.1-1991 for whole body exposures from various directions.

features. Because of the wide variations in biological responses, radiobiology is not an exact science.

\subsubsection{Dose-Equivalent for Photons}

Since $Q=w_{\mathrm{R}}=1$ for photons, the dose-equivalent vs. energy curve differs from the dose vs. energy curve only because of $w_{\mathrm{T}}$. Those factors can make a large difference, so that curves of human dose-equivalent or equivalent dose that appear in health physics contexts are not the same as dose. Published graphs may be of the effective dose-equivalent conversion factor $h_{\mathrm{E}}$ for various geometries of whole-body exposure.

As mentioned, Figure 2.72 shows the effective dose-equivalent conversion factor $h_{\mathrm{E}}$ under whole-body exposure from two different directions by a given photon fluence [ANS91].* Radiation is more damaging for frontal body exposure than if exposed from the side. Other directions have an $h_{\mathrm{E}}$ conversion factor mostly between the two graphed. Also shown is the fluenceto-dose conversion factor for photons in tissue-equivalent plastic, reproduced 
from Figure 2.69. Its rise at low energy is caused by the short range of lowenergy photons. The $h_{\mathrm{E}}$ curves are for the entire body and thus deep dose equivalent; internal organs may be very sensitive, but would experience less dose, making the overall damage sensitive to the direction of exposure but insensitive to low-energy photons.

\subsection{SOURCES OF CROSS-SECTION DATA}

\subsubsection{Sources of Photon Cross-Section Data}

The two principal photon-atom cross-section databases have been compiled by NIST and by LLNL. They are available at no cost on the Web. Both are for unpolarized incident photons only, and contain no polarization data. (It is possible for polarized photons to follow from unpolarized incident photons, for example, in Rayleigh scattering near $90^{\circ}$. The NIST and LLNL compilations provide no information for applications involving incident or outgoing polarized photons.)

NIST cross sections may be found in the XCOM database at the NIST physics reference data site, http://physics.nist.gov/PhysRefData/Xcom/ Text/XCOM.html. All cross sections on the elements (photoelectric, Rayleigh, Compton, nuclear, and electronic pair production) are tabulated at incident photon energies from $1 \mathrm{keV}$ to $10^{5} \mathrm{MeV}$. Any compound or mixture may be entered, and its cross sections will be returned over the same energies. The incoherent scattering function and the coherent scattering form factors are provided not directly in XCOM, but in another tabulation called FFAST, also on the NIST site [Ch95b], so angular distributions can be constructed. The databases are updated periodically. Saloman et al. [Sa88] summarizes almost all the data up to 1988 .

Over the years, NIST has developed a series of Monte Carlo transport codes primarily for the transport of photons and electrons, under the generic name ETRAN. ETRAN algorithms form the basis for a number of later codes, such as CYLTRAN, ACCEPT, Sandia's SANDYL, TIGER and its outgrowth ITS (Integrated Tiger Series), and so forth [Se88]. Electron energies are typically $1 \mathrm{keV}$ to $1 \mathrm{GeV}$. The ETRAN-based codes provide the transporting and sampling algorithms; various cross-section databases may be used.

The LLNL photon cross-section database is the Evaluated Photon Data Library (EPDL), updated every few years. The current version, EPDL97, is from 1997. This compilation contains all cross sections on the elements at photon energies from $1 \mathrm{eV}$ to $10^{5} \mathrm{MeV}$. In addition to the total cross sections (integrated over solid angle) as a function of incident photon energy, the incoherent scattering function and coherent scattering form factors are given, allowing one to construct angular distributions (as were used to construct the graphs in this chapter). In addition, partial photoelectric cross sections for ionization from individual electron shells are given. 
EPDL97 is documented in Cullen [Cu97] and the format of its files can be found in [McL01]. Both documents are included on the distribution CDROM, and are separately available as discussed later.

The EPDL97 data library is stored in ASCII text documents, readable by almost any editor on any computer, with the numbers arrayed in one of two formats, that of the ENDF library (six columns per line), or that of the earlier ENDL library (two columns per line). The ENDL format is being phased out in favor of the more popular ENDF format.* These are summarized separately later. On the other hand, the NIST site presents online columnar data that can be downloaded immediately into any text file or spreadsheet.

The EPDL compilation of photon cross sections is available in the ENDF and ENDL formats via any one of the following four sites:

- National Nuclear Data Center, Brookhaven National Laboratory, http://www.nndc.bnl.gov/

- Los Alamos National Laboratory, http://t2.lanl.gov/data/atomic.html

- The International Atomic Energy Agency, Vienna, http://wwwnds.iaea.org/epd197/

- The International Nuclear Energy Agency, Paris, http://www.nea.fr/

The site http://www.llnl.gov/cullen1/ also serves as a portal to these data.

\subsubsection{Sources of General Nuclear and Particle Data}

Although it departs from the current topic of photons, we digress to present sources of data of more general interest.

Commonly needed material data include:

- Data on particle interactions with atoms and nuclei (photoatomic and electroatomic cross sections, neutron cross sections, angle and energy distributions, etc.)

- Radioactive nuclear decay data

- Fission product yields

The international network for compiling, maintaining, and making available these and related data comprises four primary centers:

\footnotetext{
*The ENDF format is now the de facto world standard for tabulating cross sections. It was originally devised by BNL to tabulate neutron cross sections. Meanwhile, LLNL had been compiling electron and photon cross sections in their own ENDL-format library. Most of the electron and photon data in ENDL has been transcribed to ENDF format. However, the ENDF format has no provision for a few quantities; they remain only in ENDL format. Examples are photoatomic excitation cross sections and the average energy of outgoing particles.
} 
- The U.S. National Nuclear Data Center (NNDC) at Brookhaven National Laboratory (BNL), Brookhaven, NY, http://www.nndc.bnl.gov/

- The International Atomic Energy Agency (IAEA), Vienna, Austria, http://www-nds.iaea.org/

- The International Nuclear Energy Agency (NEA), headquartered in Paris, France, http://www.nea.fr/

- The Russia Nuclear Data Center (Institute of Physics and Power Engineering, IPPE), Obninsk, Russia, http://www.ippe.obninsk.ru/ and http://www.rssi.ru/IPPE/

Other locations, primarily the

- Los Alamos National Laboratory T2 site, http://t2.lanl.gov/, maintain access to most of these data; in addition,

- The U.S. Idaho National Laboratory (INL, formerly INEEL) maintains radioactive decay gamma spectroscopy data, http://www.inl.gov/, http://www.inl.gov/gammaray/catalogs/catalogs.shtml

- The U.S. National Institute of Standards and Technology (NIST) provides photon and charged particle atom interaction data, and its own measurements of radioactive nuclide half-lives, http://physics.nist.gov/PhysRefData/

Particle cross-section data for photon, electron, protons, heavier ions, and neutron projectiles, and particle decay data, often but not always from ENDF, are available at five main organizations:

LANL, NNDC, IAEA, NEA, and NIST,

at their web sites listed earlier.

Most sites that provide particle data do so in three major categories:

1. Raw experimental data

2. Evaluated data

3. Bibliography of experimental measurements

A typical user will be interested in category 2, a collection of best experimental measurements and calculations that have been reviewed and stand as recommended values. Experts will be interested in categories 1 and 3 .

Over the years, several countries have produced many nuclear data libraries. Most are accessible through the NEA or IAEA at the above web sites. A partial list is given here. All are in ENDF-6 format unless otherwise specified.

\section{General purpose libraries}

BROND Russian (and FSU) evaluated neutron data library.

CENDL Chinese evaluated neutron data library.

ENDF/B-VI U.S. evaluated nuclear data library. The library is divided into the following sublibraries; incident neutron data (general purpose library), radioactive decay data, fission 
yields data, thermal scattering law data, photoatomic interaction data, electroatomic data, incident chargedparticle data, activation data, and dosimetry data.

JEFF The joint evaluated fission and fusion file is an evaluated library produced via an international collaboration of data bank member countries. It supersedes the outdated JEF compilation.

JENDL Japanese evaluated neutron data library.

\section{Special purpose libraries:}

ADL Russian activation data library. The library contains excitation functions of reactions on targets for neutron energies up to $20 \mathrm{MeV}$. The library is in a pseudo ENDF-6 format, that is, it generally follows ENDF formatting rules, but with some modifications to allow the description of reactions leading to metastable states.

EADL The evaluated atomic data library of the LLNL, U.S., in the ENDL format. It contains evaluated atomic subshell and relaxation data for isolated neutral atoms, including fluorescence yields, subshell parameters (e.g., binding energies), both radioactive and nonradioactive transition probabilities, and energy deposition terms.

EAF The European activation file is designed for activation, transmutation, and gas-production calculations. The file, in EAF format, contains data on neutron-induced reactions on stable and unstable targets.

EEDL The evaluated electron interaction data library of the LLNL, U.S.

EFF The European fusion file contains data for 80 materials of interest for fusion neutronic design calculations, from $\mathrm{H}$ to $\mathrm{Bi}^{209}$. The library format conforms mostly to the legal ENDF-6 format, but there are deviations.

EPDL The evaluated photon interaction data library of the LLNL, U.S.

FENDL A comprehensive nuclear data library for fusion applications, developed by an international effort coordinated by the IAEA nuclear data section. Sublibraries exist for activation (FENDL/A), decay (FENDL/D), dosimetry (FENDL/DS), fusion (FENDL/C), transport applications (FENDL/E), and validation (VALIDATION).

IRDF The international reactor dosimetry file. It contains neutron cross-section data for reactions used for reactor dosimetry by foil activation. It also contains radiation damage cross sections for a few materials in the older ENDF-V format.

JENDL-ACT Japanese library of activation reactions. 
MENDL The medium energy nuclear data library, a neutron reaction data library for nuclear activation and transmutation at intermediate energies (up to $100 \mathrm{MeV}$ ). It generally follows ENDF formatting rules, but with different MT numbers.

UKFY The UK fission product yield evaluated data.

UKHEDD UK heavy element decay data file. It contains evaluations for 125 heavy element nuclides of interest in nuclear fuel cycle calculations, including some spontaneous fission data.

UKPADD The UK activation product decay data library. It contains radioactive decay data for 328 activation products (including some fission products).

IAEA Photonuclear data.

It contains photoexcitation, photoabsorption, photoneutron production, and photofission cross sections. It is available at http://wwwnds.iaea.org/photonuclear/ and provides the Russian BOFOD library (from IPPE, Obninsk, Russia), Russian EPNDL library (from CDFE, Moscow), Chinese (CNDC), Japanese (JENDL), South Korean (KAERI), and U.S. (LANL) photonuclear data libraries, with evaluated, recommended values.

These and others may be accessed through the NEA, http://www.nea.fr/.

\subsubsection{The ENDF Data Library and Its Format}

One of the major data evaluations is the U.S. evaluated nuclear data file library (ENDF), a massive compilation of atomic and nuclear cross sections and nuclear decay rate data. It was originally designed by the national nuclear data center (NNDC) at Brookhaven National Laboratory for recording neutron cross-section data. Over the decades, it has expanded to include other projectiles. Data are updated periodically; the current version is ENDF/B-VI. $\mathrm{ENDF} / \mathrm{B}-\mathrm{VII}$ is due to be released soon.

The term ENDF refers mainly to the format in which the data are recorded. However it is loosely applied to the data compilation itself. Other compilations may also use the ENDF format.

An introduction to ENDF is available online [LA98]. To quote from it:

ENDF-format libraries are computer-readable files of nuclear data that describe nuclear reaction cross sections, the distributions in energy and angle of reaction products, the various nuclei produced during nuclear reactions, the decay modes and product spectra resulting from the decay of radioactive nuclei, and the estimated errors in these quantities.

ENDF-format libraries are intended to be used for a wide variety of applications that require calculations of the transport of neutrons, photons, and charged particles through materials, the enumeration of the interactions of this radiation with the materials and their surroundings, and the time evolution of the radioactivity associated with the nuclear processes. 
Examples of uses for ENDF-based libraries include fission and fusion reactor calculations, shielding and radiation protection calculations, criticality safety, nuclear weapons, nuclear physics research, medical radiotherapy, radioisotope therapy and diagnostics, accelerator design and operations, geological and environmental work, radioactive waste disposal calculations, and space travel calculations.

The ENDF library now includes photons, electrons, neutrons, protons, and alpha particles as projectiles. It is continually updated and expanded. The library containing cross sections for incident photons is called the evaluated photon data library (EPDL), and the database for incident electrons is the evaluated electron data library (EEDL).

After excitation or ionization of atoms by photon or incident electron collisions, the atom may de-excite by x-ray emission (fluorescence) or other electron emissions (Auger or Coster-Kronig). Probabilities for these atomic relaxation modes are tabulated in a separate library, the evaluated atomic data library (EADL).

EPDL includes cross sections for photoionization from individual atomic subshells, but it does not tabulate photoexcitation cross sections. The latter are contained separately in the evaluated excitation data library (EXDL). EXDL is distributed with EPDL97, but maintained as a separate file of data. At present, EXDL is available only in the older ENDL format. This format is documented in the LLNL report [Pe02], and libraries in that format are included with the complete EPDL library.

The library containing cross sections for incident neutrons or protons or alpha particles has no separate name and is loosely known as ENDF.

\subsubsection{Structure of the ENDF Format}

This discussion addresses all incident projectiles, not just photons. ENDF data files are nuclear data evaluated (category 2 given earlier) by the cross section evaluation working group. They are kept as computer-readable ASCII files. The ENDF format refers to the way numbers are arranged on the records in the file. The ENDF layout format has become one of the choice worldwide for almost all nuclear reaction cross-section data and photoatomic data. In this format, every record is 80 -characters wide. Numerical data are presented in pointwise pairs (e.g., energy-cross section), three pairs (six numbers) to a line, consuming 66 of the 80 columns. For example, 300 data points fit on 100 rows. The numerical data are preceded by rows identifying the element, what process the following data are for (whether it is the coherent scattering cross section vs. incident photon energy; a form factor vs. momentum transfer, etc.), interpolation algorithms, and so on. The ENDF format has been documented by members of the cross section evaluation working group in [McL01]; this document has come to be known as ENDF-102. 
To parameterize all possible interactions, one must specify the incident projectile, the target, the kind of data one is working with (a total cross section vs. energy, a differential cross section, decay data, etc.), and the specific reaction of interest (e.g., $\mathrm{Al}^{27}(n, n p) \mathrm{Mg}^{27}$, etc.). In $\mathrm{ENDF}$, these four quantities are controlled by four numerical parameters, NSUB, MAT, MF, and MT, as follows.

The entire ENDF library is divided into sublibraries, one for each incident particle. More precisely, there may be several sublibraries for a given projectile; for example, for incident photons there is one sublibrary for photonuclear data, one for photoinduced fission product yields, and one for photoatomic interaction data (this latter being EPDL). A FORTRAN parameter, NSUB, is defined to identify the sublibrary. For example, EPDL for photoatomic cross sections is NSUB $=3$.

A target material is identified by a four-digit integer variable given the name MAT, ranging from 1 to 9999 . For any projectile, target materials may be either individual nuclear isotopes or elements in their natural isotopic compositions or in some cases, other mixtures of isotopes. To accommodate all possibilities, the assignment conventions of the MAT numbers may be only partially transparent (and the numbering convention allows for two excited isomeric nuclear states of nuclides). The first two of the four digits in MAT are the element atomic number. The last two identify the isotope or the natural element. The two isotope identification digits do not necessarily correspond to atomic weight or neutron number. An element $Z$ in its natural isotopic composition has MAT number 100Z; MAT for natural $\mathrm{Fe}$ is 2600 . The lightest stable isotope is assigned number MAT $=100 Z+25$; $\mathrm{MAT}=2625$ for ${ }_{26} \mathrm{Fe}^{54}$. The (stable) isotope ${ }_{26} \mathrm{Fe}^{57}$ has MAT number 2634. The list of all MAT numbers can be found in the format document ENDF-102 referred to earlier.

For a given projectile and target, one must specify the class of information desired about that projectile on that material, such as a reaction cross section as a function of incident projectile energy, or angular distributions for emitted particles, or neutron resonance parameters, and so on. Each of these quantities was originally kept in a separate file; but now each quantity is assigned an identifying integer variable MF; the " $F$ " for file and the preceding $M$ to make it a FORTRAN integer, harmonious with the $\mathrm{M}$ in MAT. The two-digit MF variable ranges from 1 to 99 . For a reaction cross section as a function of incident projectile energy, $\mathrm{MF}=3$; neutron resonance parameters are in the file $\mathrm{MF}=2$; angular distribution for emitted particles is in $\mathrm{MF}=4$, and so on. Though earliest compilations had each MF value in a separate file, today all MF values are included in a single, much larger file for a given target material. A class of data for a given MF is still loosely referred to as a file.

For a given projectile, target, and quantity of interest, one must specify a particular reaction of interest, or the type of quantity desired. This is identified by the three-digit type variable MT. It ranges from 1 to 999 . MT $=2$ is the total elastic scattering cross section; $\mathrm{MT}=18$ is the total projectile-induced 
fission cross section; $\mathrm{MT}=102$ is the neutron radiative capture cross section and is defined only for incident neutrons. In the early days, each type of quantity was kept in a certain section of a file. Today, a particular reaction is still referred to as a section of a "file," meaning a particular MT in a particular MF part of the complete file for that material.

Thus, the projectile is specified by NSUB, the chosen sublibrary (e.g., EPDL97 for a photon). The target material is specified by MAT (e.g., MAT $=1300$ for natural Al). The quantity desired is specified by MF (e.g., $M F=3$ for a cross section vs. energy). The particular type of reaction for that quantity (i.e., the specific cross section) is specified by MT (e.g., $\mathrm{MT}=522$ for photoelectric cross section).

Every ENDF file contains the number NSUB in the first few lines. Every record of every ENDF file contains the numbers MAT, MF, and MT in columns 67 to 75 , and a five-digit record sequence number in columns 76 to 80. Some special numbers are set aside for incident photons or electrons. For example, $\mathrm{MT}=522$ is the photoelectric absorption cross section, meaningful only for incident photons; MT $=540$ is the cross section for photoelectric ionization from the $\mathrm{M}_{3}\left(3 \mathrm{p}^{3 / 2}\right)$ subshell, and so forth. With some exceptions, most (but not all) cross sections and processes of interest can be made to fit into these ENDF conventions, and are tabulated in the library.

The first few MF numbers in the ENDF format are defined as follows:

\begin{aligned} & \hline MF \multicolumn{1}{c}{ Description of File Contents (MF Number) } \\ & 1 General information \\ & 2 Resonance parameter data \\ & 3 Reaction cross sections \\ & 4 Angular distributions for emitted particles \\ & 5 Energy distributions for emitted particles \\ & 6 Energy-angle distributions for emitted particles \\ & 7 Thermal neutron scattering law data \\ & 8 Radioactivity and fission-product yield data \\ & 9 Multiplicities for radioactive nuclide production \\ & 10 Cross sections for radioactive nuclide production \\ & 12 Multiplicities for photon production \\ & 13 Cross sections for photon production \\ & 14 Angular distributions for photon production \\ & 15 Energy distributions for photon production \\ & 23 Photo- or electroatomic interaction cross sections \\ & \hline\end{aligned}

The definitions of all MF values are given in document ENDF-102 [McL01].

With some exceptions, the MT numbers can be broken down approximately as follows: 
MT

\section{Description of Reaction Types (MT Numbers)}

$\begin{array}{cl}10-100 & \text { Reactions in which at least } 1 \text { neutron is emitted } \\ 10-49 & \text { One or more neutrons, and at least one other particle, are produced } \\ 50-91 & \text { Just one neutron is produced, with residual nucleus left in excited state } \\ 101-150 & \text { Reactions in which no neutron is in the final state } \\ 151-200 & \text { Resonance region information } \\ 201-450 & \text { Quantities derived from the basic data, such as particle production cross sections, } \\ \text { average energies, etc. } & \text { Miscellaneous quantities, such as fission nubar, charged particle stopping powers, } \\ 451-599 & \text { etc., and photon cross sections } \\ 600-891 & \begin{array}{l}\text { Particle production with residual nucleus in excited state } \\ 600-649\end{array} \\ 650-699 & \begin{array}{l}\text { Proton is produced, with residual nucleus left in excited state } \\ 700-749\end{array} \\ 750-799 & \text { Triton is produced, with residual nucleus left in excited state } \\ 800-849 & \alpha \text { particle is produced, with residual nucleus left in excited state }\end{array}$

Specific MT values are too numerous to list here. Full descriptions are in document ENDF-102 [McL01]. In addition, the first records of every ENDF data file contain descriptive text and include MF and MT definitions for all the data quantities in that file.

\subsubsection{ENDF Preprocessing and Postprocessing Codes}

ENDF stores basic data in a rather compressed form. Further processing may be necessary to obtain the desired quantity. In neutron reactions, for example, cross sections in the resonance region are not stored as energy-cross section pairs; rather the resonance parameters are given. To get the cross section vs. energy, the resonance parameters must be expanded.

One should use the preprocessing codes PREPRO2004 (or latest version) to prepare the raw data in the basic ENDF data files. For all projectiles (i.e., for all ENDF data files), the PREPRO codes check for and correct internal inconsistencies, make sure partial cross sections add up to the proper total, check for negative cross sections, and so on. A capability for combining elements into compounds and mixtures is provided. For neutrons, it also expands resonance parameters to produce pointwise cross sections as a function of neutron energy, and Doppler broadens the cross sections to any specified temperature. PREPRO is available at no cost through the major organizations listed earlier, for example, at the IAEA website: http://wwwnds.iaea.org/ndspub/endf/prepro/.

A more comprehensive postprocessing group of codes is NJOY, which provides extensive capabilities for manipulating ENDF files and data. Authorized users may obtain it for a fee through the Radiation Safety Information 
Computational Center: http://www-rsicc.ornl.gov/ or at the NEA Data Bank: http://www.nea.fr/. NJOY introductory documentation is freely available through Los Alamos National Laboratory: http://t2.lanl.gov/njoy/, and http://t2.lanl.gov/codes/.

\subsubsection{Extracting or Plotting a Cross Section FROM AN ENDF FILE}

It is often convenient to have cross-section data in simple parallel column format, rather than in the compressed ENDF format.

There are several ways to extract a cross section from an ENDF file, short of writing one's own code to read the file.

- Most sites (especially LANL T2 and NNDC) permit downloading ENDF data automatically extracted in parallel column format, usually six or ten columns wide.

- A utility program LISTEF, written by C.L. Dunford at BNL-NNDC, extracts all data from an ENDF file and expands them into parallel columns. A version of this code is included in the CD-ROM accompanying this book.

- To extract one-dimensional data only (i.e., a cross section vs. energy, but not a differential cross section as a function of incident particle energy and outgoing particle angle), one can use the simple code ENDF1D included on the CD-ROM.

- The NEA maintains a free, useful utility, Janis, which can extract, display, and compare data.

Plots of cross sections are available at the LANL-T2 site. The NEA Janis routine also produces plots. The LLNL Monte Carlo transport codes TART and EPIC, discussed presently, include a good display package, EPICSHOW, to plot photon, electron, light ion, and neutron cross sections. The ZVView program at the IAEA will create any plot with many options, http://wwwnds.iaea.org/ndspub/zvview/.

\subsubsection{The Evaluated Photon Data Library in ENDF Format}

After the digression to general data, we now again focus on incident photons and discuss the EPDL database. Owing to the popularity of the ENDF format, the EPDL library, originally in ENDL format, has been made available in ENDF format. In that format, it contains the following quantities:

- Photoionization cross sections from individual electron subshells $\left(K, L_{1}, L_{2}, L_{3}, M_{1}, M_{2}\right.$, etc.) 
- For each subshell, the photoionization edge energy and the fluorescence yield

- Atomic photoionization cross section (as distinct from photoexcitation), which is the sum of all subshell photoionization cross sections

- Coherent (Rayleigh) scattering cross section

- Incoherent (Compton) scattering cross section

- Nuclear-field, electron-field, and total pair production cross sections

- Total cross section (= sum of photoelectric, coherent, incoherent, nuclear pair, and electronic pair)

- Incoherent scattering function $S(q)$, from which one can construct the incoherent differential cross section

- Coherent scattering form factor $F(q)$

- Real and imaginary parts of coherent anomalous scattering factors $f_{1}(E)$ and $f_{2}(E)$, from which, together with $F(q)$, one can construct the coherent differential cross section

Functions of incident photon energy $E$ (i.e., all quantities except $S(q)$ and $F(q))$ are given for energies from $1 \mathrm{eV}$ to $100 \mathrm{GeV}$. As reaction identifiers in the ENDF format were established long ago with no idea that the ENDL library would ever be transcribed to it, some parameters in ENDL format could not be carried over. Thus, the EPDL library in the ENDL format still contains some quantities not in the ENDF format.

\subsubsection{The ENDL Data Library and Its Format}

The Evaluated Nuclear Data Library has been compiled over many years, starting in the late 1950s. It has been documented in many paper reports, carrying the general number UCRL 50400, but recently has moved to electronic form. The EPDL is only one part of ENDL. ENDL also contains electron-atom cross sections and energy loss (EEDL), atomic relaxation data (EADL), and atomic excitation data (EXDL).

The EPDL97 database in its original ENDL format contains all the quantities put in ENDF format (given earlier) and the following additional quantities on all 100 elements:

- Average energy of outgoing particles (e.g., average Compton electron energy or average positron and electron energies following pair production as a function of incident photon energy).

- Average energy left in the residual atom following photoelectric absorption. From this and the average energy of outgoing particles one can construct the energy transfer and kerma.

- Photoexcitation cross sections and photoionization cross sections. 
LLNL has also constructed a Monte Carlo electron-photon transport code, EPIC, using the EPDL database, and a Monte Carlo neutron-photon transport code TART. TART dates from the 1970s, spearheaded largely by E.F. Plechaty, but the current versions of EPIC and TART were written by Dermott E. (Red) Cullen. TART is distributed by RSICC at ORNL. In addition to neutron cross sections, the EPDL database is provided with the TART code at a reduced set of energies, suitable for plotting. A good, fast plotting routine, EPICSHOW, is included, enabling one to quickly view all major and minor cross sections as a function of energy and print them out in tabular form at the included energies. Forthcoming versions of EPICSHOW should include all energies of the original ENDL database.

\subsubsection{Comparison of NIST and LLNL Photon Data}

Experimental measurements do not exist for all interaction processes on all elements at all energies. Cross-section databases must therefore be compiled from what data do exist and from calculations. Such calculations are required for photon interaction cross sections on ions in their ground states or on neutral atoms and ions in excited states, for which there are few, if any, measurements. These latter cross sections are needed, for example, for astrophysical and laboratory plasma applications.

Therefore, databases, even for neutral atoms, can differ based on those experiments and calculations in which the compilers have faith and choose for their foundation. Each database represents one possible interpretation of the available data and computations.

Even within NIST, two different compilations below about $400 \mathrm{keV}$, XCOM and FFAST, each developed for different purposes, may differ in total cross section by several percent [NISTb]. In general, however, we have found EPDL97 and XCOM to agree in the total cross section and in each major cross section (that process which is dominant at a given energy) to better than about $1 \%$.

In addition to the comparison of Rayleigh scattering angular distribution in Figure 2.15 and Figure 2.16, we mention here a quick comparison of angleintegrated cross sections between the two major compilations, EPDL97 and NIST XCOM.

In general, the two datasets agree to significantly better than $1 \%$ on total cross section (sum of photoelectric, coherent, incoherent, and pair production). This means that at any one energy, the two compilations for the dominant cross section at that energy also agree as well. However, the lesser cross sections differ by more. As an example, Figure 2.73 shows both compilations on aluminum. The solid lines are the EPDL97 data, shown here, as in Figure 2.4, from $10 \mathrm{eV}$ to $100 \mathrm{GeV}$. The dots are the NIST data, which are given on the NIST web site from $1 \mathrm{keV}$ to $100 \mathrm{GeV}$. Below about 


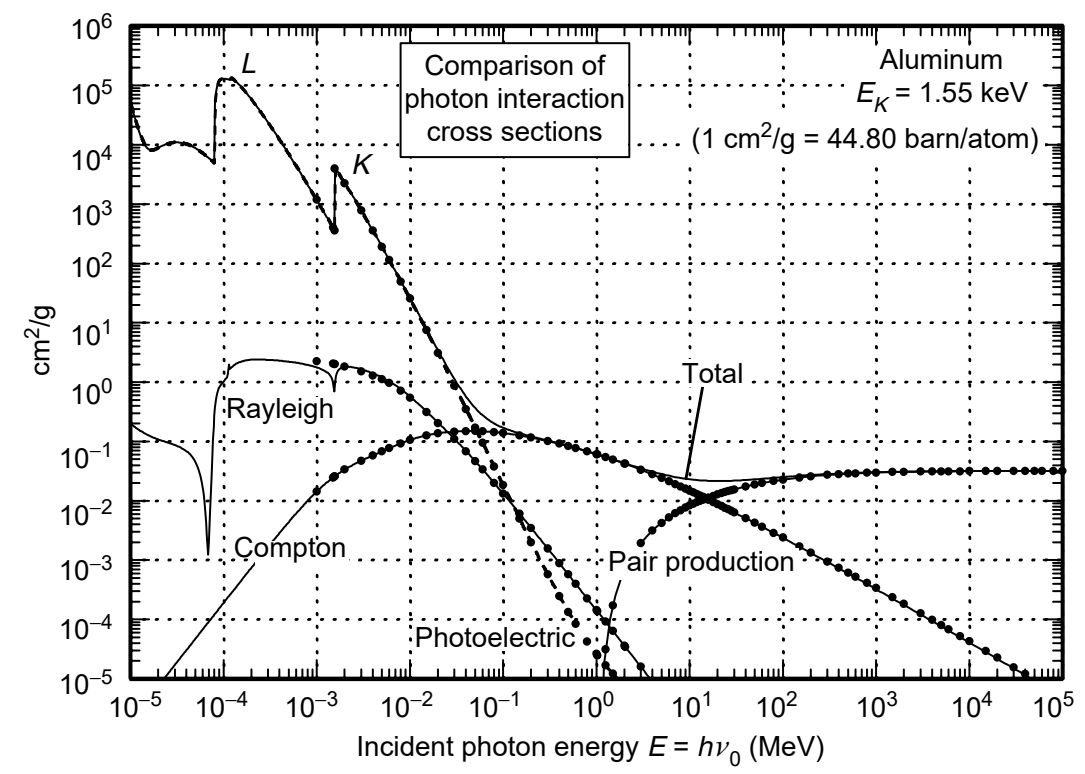

FIGURE 2.73 Comparison of $\mathrm{Al}$ cross sections in two databases. (Solid lines: EPDL97. Dots: http://physlab.nist.gov/PhysRefData/Xcom/Text/XCOM.html.)

$30 \mathrm{keV}$, the photoeffect dominates, and the two compilations agree very well on that cross section. Nevertheless, the Rayleigh cross section at low energies disagrees considerably, at least near x-ray edges. The NIST XCOM model does not yet fully account for the anomalous scattering that causes significant, sometimes wild, variations in the coherent cross section near edges.

Likewise, above a few hundred $\mathrm{MeV}$, the Compton cross sections disagree by an ever-increasing amount, growing to nearly $5 \%$ at tens of GeV. Thus, if an application requires accurate cross sections for a nondominant process, such as Compton scattering at hundreds of $\mathrm{MeV}$, or Rayleigh scattering below tens of $\mathrm{keV}$, the user must inquire more deeply as to which data compilation is more nearly correct for that process at those energies. Table 2.5 shows numerical comparisons from the two main libraries at a few energies. Both databases are continually updated. Websites should be checked for the latest release.

\subsubsection{Other Data Sources}

Links to photon-atom cross-section data can be found at the Lawrence Berkeley National Laboratory Center for X-Ray Optics sites: http://www-cxro. lbl.gov/optical_constants/ and http://www-cxro.lbl.gov/optical_constants/ web.html. Various tabulations of form factors and scattering functions can be 


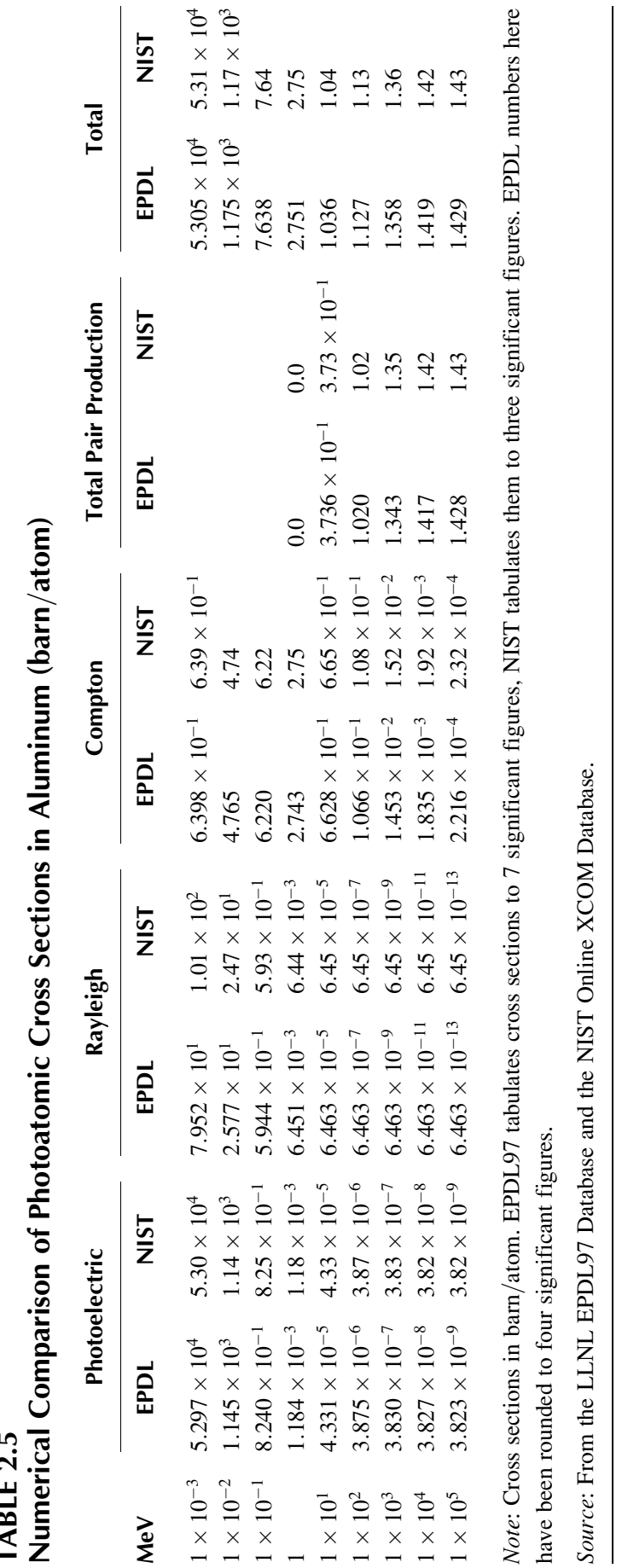


found at these sites. As mentioned, current work on elastic photon-atom scattering is compiled at http://www-phys.llnl.gov/Research/scattering/. Other available data sources are summarized in [Cu97].

\subsection{LARGE CONTOUR PLOTS}

Four large, color contour plots $\left(11^{\prime \prime} \times 17^{\prime \prime}\right)$ are available that graph useful photon parameters. They are in high-resolution PDF format on the accompanying $\mathrm{CD}$ (and would require a printer capable of handling $11^{\prime \prime} \times 17^{\prime \prime}$ paper). They are

Total photon cross section. Photoelectric + Rayleigh (三 "elastic" $\equiv$ "coherent") + Compton (三 "Incoherent") + pair production, in two units:

- Total photon cross section in barn/atom. (1barn $\left.\equiv 10^{-24} \mathrm{~cm}^{2}\right)$. A smooth function of $Z$ and $E$.

- Total photon cross section in $\mathrm{cm}^{2} / \mathrm{g}$. While $\sigma$ (barn/atom) is smooth, contours are jagged here because $A(Z)$ is a jagged function of $Z$.

The Compton cross section $\sigma_{\mathrm{C}}$ is proportional to the number of electrons in the material. This causes the $\mathrm{H}$ cross section $\left(\mathrm{cm}^{2} / \mathrm{g}\right)$ to be nearly twice that of He. This fact would cause contour lines to bunch near $Z=1$. To avoid this problem, the $\mathrm{H}$ cross section graphed in $\mathrm{cm}^{2} / \mathrm{g}$ is $1 / 2$ the actual $\mathrm{H}$ cross section. All other elements are graphed at their correct value. The same ploy is used on graphs of charged particle stopping powers.

- Mean free path against total scattering $\left(\mathrm{g} / \mathrm{cm}^{2}\right) \equiv 1 / \sigma\left(\mathrm{cm}^{2} / \mathrm{g}\right)$. This duplicates information in the previous graph. However, displayed contour values are not always the inverse of those on the $\sigma\left(\mathrm{cm}^{2} / \mathrm{g}\right) \mathrm{graph}$, and hence are not in the same position. Therefore, the two graphs allow more precise interpolation than one graph alone.

- Photon number fluence-to-kerma conversion factor. Units of $\mathrm{rad}$ (element) per $10^{9}$ photons $/ \mathrm{cm}^{2}$. Dose is geometry dependent and must be calculated separately; dose equals kerma under "charged particle equilibrium."

These graphs duplicate Figure 2.53 through Figure 2.55 and Figure 2.59 of the text, but on a larger and more accurate scale. When printed in color, they are easy to read accurately enough for use in simple calculations.

\subsection{DATA ON THE CD-ROM}

Although EPDL97 contains cross sections down to $1 \mathrm{eV}$, we have included on the CD-ROM cross sections only at energies above $100 \mathrm{eV}$. There are several reasons: 
- The libraries EPDL, EEDL, EADL, and EXDL have been compiled by LLNL to be a self-consistent set for use in electron-photon transport codes. In this application, self-consistency is more important than absolute accuracy. (Hence, some photoelectric edges differ from other standard compilations.) The libraries are not intended to be accurate cross sections for arbitrary applications. Nevertheless, the cross sections at the energies presented are believed to be reliable for free atoms.

- Notwithstanding applications to atomic gases, as mentioned in Section 2.1, free atom cross sections at low energies give inaccurate results in applications in solids, in liquids, and in diatomic or molecular gases.

- EPDL97 cross sections at low energies were compiled and intended only to support calculations of coherent anomalous scattering factors for use in electron-photon transport calculations, not as true, evaluated atom cross sections for arbitrary application.

- Documentation [Cu97] blatantly states, "Between $10 \mathrm{eV}$ and $100 \mathrm{eV}$ it is neither safe nor accurate to use these data" in arbitrary applications. And below $10 \mathrm{eV}$, the warnings are even sterner.

- Users desiring cross sections below $100 \mathrm{eV}$ can get them by actually downloading EPDL97 from one of the sources listed earlier. The pain of this chore may encourage them to read the documentation, where there is sufficient warning about the data's uncertainties. Were the lowenergy cross sections provided on the CD-ROM accompanying this book, the temptation to use them would be too great to prevent their use in inappropriate applications.

Paraphrasing the discussion in Cullen et al. [Cu97], above about $1 \mathrm{keV}$ the cross sections appear to be reliable and safe to use. From $100 \mathrm{eV}$ to $1 \mathrm{keV}$, the experimental and theoretical cross-section data contain some uncertainties. However, in this energy span, their application appears to be limited only by that uncertainty, at least in noncondensed atomic media. In noncondensed molecular media, the cross sections are again inappropriate because of level shifts from interactions with neighboring atoms. In condensed media, their application is further limited by interactions with neighboring atoms, as per the caveats in Section 2.1. Whereas the LLNL group chose to include the lowenergy data anyway in EPDL97, hoping some numbers would be better than none (and requesting user feedback), the NIST group has chosen to present numbers in XCOM only above $1 \mathrm{keV}$. Another difference between the two compilations is that numerical cross-section values are presented in EPDL97 to seven significant figures, whereas the NIST tabulation presents them to three significant figures. Recall that the purpose of the EPDL cross-section set is for use in electron-photon transport calculations and is designed to be consistent with electron cross sections in the separate EEDL electron-atom cross-section database. 
The cross sections in the NIST and EPDL97 databases are for photons incident on a single, isolated, cold atom. Differences in $K$ and $L$ edge energies between gas phase and in a solid are compiled by Deslattes et al. [De03].

In spite of the reservations about the use of free atom cross sections below a few hundred $\mathrm{eV}$ we have chosen to present numbers down to $100 \mathrm{eV}$ in the hope that the reader will apply them responsibly. That we have chosen to use the LLNL EPDL97 numbers rather than NIST XCOM numbers is in no way to be construed as a technical judgment about the correctness of one set over another. Rather, it is because only EPDL97 has a consistent set both below and above $1 \mathrm{keV}$. Above $1 \mathrm{keV}$, one should feel just as free and secure using the NIST cross sections.

As documented in [Cu97] the partial cross sections are to be interpolated $\log x-\log y$, that is, power-law interpolation between tabulated points. The energies at which the cross sections are tabulated in the CD-ROM files are chosen to adequately resolve energy dependence.

The included data are

- Fluorescence yields of $K$ shell and average fluorescence yields of $L$ shell and $M$ shell for all elements

- Fluence-to-kerma conversion functions in all elements as a function of photon energy

- Photoatomic cross sections in all elements as a function of photon energy (both in units of barn/atom and in $\mathrm{cm}^{2} / \mathrm{g}$ )

- Total photoelectric ionization cross section (from all shells)

- Coherent scattering (Rayleigh elastic scattering) cross section

- Incoherent scattering (Compton scattering) cross section

- Cross section for pair production from the nucleus

- Cross section for pair production from atomic electrons

- Total pair production cross section (nuclear + electronic)

- Total cross section (photoelectric + coherent + incoherent + pair production)

\section{REFERENCES}

[Åb85] Åberg, T. and Tulkki, J., "Inelastic x-ray scattering including resonance phenomena," Chapter 10 in Crasemann, Ed., Atomic Inner-Shell Physics, Plenum Press, 1985.

[ANS91] The American Nuclear Society, Report ANSI/ANS-6.1.1-1991, Neutron and Gamma-Ray Fluence-to-Dose Factors, 1991.

[At86] Attix, F.H., Introduction to Radiological Physics and Radiation Dosimetry, Wiley-Interscience, 1986.

[Ba72] Bambynek, W., Crasemann, B., Fink, R.W., Freund, H.-U., Mark, H., Swift, C.D., Price, R.E. and Venugopala Rao, P., X-ray fluorescence yields, Auger, and Coster-Kronig transition probabilities, Rev. Mod. Phys. 44(4): 716-813, 1972; 46:853, 1974. 
[Be53] Bethe, H.A. and Ashkin, J., "Passage of radiations through matter," in Segre, E. Ed., Experimental Nuclear Physics, Vol. 1, Wiley, 1953.

[Be61] Berger, R.T., The x- or gamma-ray energy absorption or transfer coefficient: tabulations and discussion, Radiat. Res. 15(1): 1-29, July, 1961.

[Be67] Bearden, J.A. and Burr, A.F., Reevaluation of x-ray atomic energy levels, Rev. Mod. Phys. 39(1): 125, 1967.

[Be79] Berkowitz, J., Photoabsorption, Photoionization, and Photoelectron Spectroscopy, Academic Press, 1979.

[Be82] Berger, M.J. and Seltzer, S.M., Stopping Powers and Ranges of Electrons and Positrons, 2nd edn, NBS publication NBSIR 82-2550-A, December 1982.

[Be93] Bergstrom, P.M. Jr, Surić, T., Pisk, K. and Pratt, R.H., Compton scattering of photons from bound electrons: full relativistic independent-particle-approximation calculations, Phys. Rev. A 48(2): 1134-1162, 1993.

[Be97] Bergstrom, P.M. and Pratt, R.H., An overview of the theories used in Compton scattering calculations, Radiat. Phys. Chem. 50: 3-29, 1997.

[Bi99] Biémont, E., Frémat, Y. and Quinet, P., Ionization potentials of atoms and ions from lithium to tin $(Z=50)$, At. Data Nucl. Data Tables 71(1):117-146, January 1, 1999.

[Ca85] Carlsson, G.A., "Theoretical basis for dosimetry," Chapter 1 in The Dosimetry of Ionizing Radiation, Vol. 1, Kase, K.R., Bjärngard, B.E. and Attix, F.H., Eds., Academic Press, 1985.

[Ca03] Campbell, J.L., Fluorescence yields and Coster-Kronig probabilities for the atomic L subshells, At. Data Nucl. Data Tables, 85: 291-315, 2003.

[Ch95a] Chantler, C.T., Theoretical form factor, attenuation and scattering tabulation for $Z=1-92$ from $E=1-10 \mathrm{eV}$ to $E=0.4-1.0 \mathrm{MeV}, J$. Phys. Chem. Ref. Data 24(1): 71, 1995.

[Ch95b] FFAST: NIST $\mathrm{x}$-ray form factor, attenuation, and scattering tables, http://physics.nist.gov/PhysRefData/FFast/Text/cover.html.

[Ch95c] Chantler, C.T., Erratum on theoretical form factor, attenuation and scattering tabulation for $Z=1-92$ from $E=1-10 \mathrm{eV}$ to $E=0.4-1.0 \mathrm{MeV}, J$. Phys. Chem. Ref. Data 24(1): 71, 1995. http://physics.nist.gov/PhysRefData/ FFast/Text/erratum.html.

[Ch98] Chatterjee, B.K. and Roy S.C., Tables of elastic scattering cross sections of photons in the energy range $50 \mathrm{keV}$ to $1500 \mathrm{keV}$ for all elements in the range $13<Z<104$, J. Phys. Chem. Ref. Dat. 27(6): 1011, 1998.

[Ch00] Chantler, C.T., Detailed tabulation of atomic form factors, photoelectric absorption and scattering cross section, and mass attenuation coefficients in the vicinity of absorption edges in the soft $x$-ray $(z=30-36, z=60-89$, $E=0.1 \mathrm{keV}-10 \mathrm{keV})$, addressing convergence issues of earlier work, J. Phys. Chem. Ref. Data 29(4), 2000.

[Cr85] Crasemann, B., Ed., Atomic Inner-Shell Physics, Plenum Press, 1985.

[Cu95] Cullen, D.E., A simple model of photon transport, Nucl. Instrum. Methods Phys. Res. B 101: 499-510, 1995.

[Cu97] Cullen, D.E., Hubbell, J.H. and Kissel, L., EPDL97: The Evaluated Photon Data Library, '97 Version, UCRL-50400, Vol. 6, Rev. 5, September 19, 1997. 
[Da52] Davisson, C.M. and Evans, R.D., Gamma-ray absorption coefficients, Rev. Mod. Phys. 24: 79, 1952.

[De99] Derevianko, A., Johnson, W.R. and Cheng, K.T., Non-dipole effects in photoelectron angular distributions for rare gas atoms, At. Data Nucl. Data Tables, 73(2): 153-211, November 1999.

[De00] Devereaux, T.P. and Kampf, A.P., A consistent picture of electronic Raman scattering and infrared conductivity in the cuprates, Phys. Rev. B 61, 1490, 2000.

[De03] Deslattes, R.D., Kessler, E.G. Jr, Indelicato, P., de Billy, L., Lindroth, E. and Anton, J., X-ray transition energies: new approach to a comprehensive evaluation, Rev. Mod. Phys. 75(1): 35, 2003.

[Ev55] Evans, R.D., The Atomic Nucleus, McGraw-Hill, 1955.

[Ev58] Evans, R.D., Compton effect, in Encyclopedia of Physics, Vol. XXXIV, Part II, Springer-Verlag, 1958.

[Gr06] Green, S., Fundamentals of Radiation Dosimetry, 2nd edn, Taylor \& Francis, 2006.

[He54] Heitler, W., The Quantum Theory of Radiation, 3rd edn, Oxford University Press, 1954.

[Ha58] Hayward, R.W. Nuclear electromagnetic radiations, Chapter 9-6 in Handbook of Physics, in Condon, E.U. and Odishaw, H., Eds., McGraw-Hill, New York, 1958.

[He93] Henke, B.L., Gullikson, E.M. and Davis J.C., X-ray interactions: photo-absorption, scattering, transmission, and reflection at $E=50-30000 \mathrm{eV}, Z=1-92, A t$. Data Nucl.Data Tables, 54 (2):181-342, July 1993. (These tables are available at http://www-cxro.lbl.gov/optical_constants/asf .html).

[Hi92] Higgins, P.D., Attix, F.H., Hubbell, J.H., Seltzer, S.M., Berger, M.J. and Sibata, C.H., Mass energy-transfer and mass energy-absorption coefficients, including in-flight positron annihilation for photon energies $1 \mathrm{keV}$ to $100 \mathrm{MeV}$, National Institute of Standards and Technology, Report NISTIR 4812, 1992.

[Hu04] Hubbell J.H. and Bergstrom, P.M. Jr, Delbrück scattering: a bibliography, and some numerical comparisons with other photon interaction processes, National Institute of Standards and Technology, Report NISTIR 7115, May 2004.

[Hu77] Hubbell, J.H., Photon mass attenuation and mass energy-absorption coefficients for $\mathrm{H}, \mathrm{C}, \mathrm{N}, \mathrm{O}, \mathrm{Ar}$, and seven mixtures from $0.1 \mathrm{keV}$ to $20 \mathrm{MeV}$, Radiat. Res. 70: 58-81, 1977.

[Hu80] Hubbell, J.H., Gimm, H.A. and Overbo, I., Pair, triplet, and total atomic cross sections (and mass attenuation coefficients) for $1 \mathrm{MeV}-100 \mathrm{GeV}$ photons in elements $Z=1$ to 100, J. Phys. Chem. Ref. Data 9(4): 10231147, 1980.

[Hu89] Hubbell, J.H., Bibliography, and current status of $K, L$, and higher shell fluorescence yields for computations of photon energy-absorption coefficients, National Institute of Standards, Report NISTIR 89-4144, August 1989.

[Hu94] Hubbell, J.H., Trehan, P.N., Singh, N., Chand, B., Mehta, D., Garg, M.L., Garg, R.R., Singh, S. and Puri S., A review, bibliography, and tabulation of $K, L$, and higher atomic shell x-ray fluorescence yields, J. Phys. Chem. Ref. Data, 23(2): 339-364, 1994 (Errata: 33(2): 621, 2004). 
[Hu95] Hubbell, J.H. and Seltzer, S., Table of x-ray mass attenuation coefficients and mass energy-absorption coefficients from $1 \mathrm{keV}$ to $20 \mathrm{MeV}$ for elements $Z=1$ to 92 and 48 additional substances of dosimetric interest, National Institute of Standards and Technology, Report NISTIR 5632, May 1995.

[Hu99a] Hubbell, J.H., An examination and assessment of available incoherent scattering S-matrix theory, also Compton profile information, and their impact on photon attenuation coefficient compilations, National Institute of Standards Report NISTIR 6358, July 1999.

[Hu99b] Hubbell, J.H., Review of photon interaction cross section data in the medical and biological context, Phys. Med. Biol. 44:R1-R22, 1999.

[IAEApn] IAEApnRecommended photo-nuclear cross sections: http://wwwnds.iaea.org/photonuclear/recommended/

[ICRP91] 1990 Recommendations of The International Commission On Radiological Protection, ICRP Pub 60, Ann. ICRP 21:1-3, 1991.

[ICRU69] International Commission on Radiation Units and Measurements, Report 13, Neutron Fluence, Neutron Spectra and Kerma, September 15, 1969.

[ICRU76] International Commission on Radiation Units and Measurements, Report 25, Conceptual Basis for the Determination of Dose Equivalent, 1976.

[ICRU80] International Commission on Radiation Units and Measurements, Report 33, Radiation Quantities and Units, 1980.

[ICRU93] International Commission on Radiation Units and Measurements, Report 51, Quantities and Units in Radiation Protection Dosimetry, 1993.

[Ja93] Jani, S.K., Handbook of Dosimetry Data for Radiotherapy, CRC Press, 1993.

[Ka98] Kahane, S., Relativistic Dirac-Hartree-Fock photon incoherent scattering functions, At. Data Nucl. Data Tables 68(2):323-347, March 1, 1998.

[Ki80] Kim, Y.S., Pratt, R.H., Ron, A. and Tseng, H.K., Phys. Rev. A 22: 567-576, 1980. See also Tseng, H.K., Pratt, R.H., Yu., S. and Ron, A., Ibid 17(3): 1061-1079, 1978.

[Ki85] Kissel, L. and Pratt, R.H., Rayleigh scattering: elastic photon scattering by bound electrons, Chapter 11 in Crasemann, Ed., Atomic Inner-Shell Physics, Plenum Press, 1985.

[K191] Klock, P., Ed., "Handbook of Infrared Optical Materials”, Marcel Dekker, 1991.

[Kn00] Knoll, G.F., Radiation Detection and Measurement, 3rd edn, Wiley, 2000.

[Kr79] Krause, M.O., Atomic radiative and radiationless yields for $K$ and $L$ shells, J. Phys. Chem. Ref. Data 8(2): 307-322, 1979.

[LA98] Los Alamos National Laboratory report LA-UR-98-1779, http://t2lanl. gov/endf/title.html.

[LLNL] D.E. Cullen, EPICSHOW module in the TART code, summarizing cross sections.

[Ly85] Lynch, D.W., Interband absorption-mechanisms and interpretation, Chapter 10 in Palik, E.D., Ed., Handbook of Optical Constants of Solids, Academic Press, Vol. 1, 1985. 
[McL01] McLane, V., Ed., ENDF-102, Data formats and procedures for the evaluated nuclear data file ENDF-6, National Nuclear Data Center, Brookhaven National Laboratory, Report BNL-NCS-44945-01/04-Rev. Revised April, 2001 (Available at: http://www.nndc.bnl.gov/nndcscr/documents/endf /endf102/).

[Mi85] Mitra, S.S., Optical properties of nonmetallic solids for photon energies below the fundamental band gap, Chapter 11 in Palik, E.D., Ed., Handbook of Optical Constants of Solids, Academic Press, Vol. 1, 1985.

[NCRP90] National Council on Radiation Protection and Measurements, Report 104, The Relative Biological Effectiveness of Radiations of Different Quality, 1990.

[Ne96] Newton, R.G., Scattering Theory of Waves and Particles, McGraw-Hill, 1966.

[NIST] http://physlab.nist.gov/PhysRefData/Xcom/Text/XCOM.html.

[NISTb] http://physics.nist.gov/PhysRefData/XrayNoteB.html.

[NIST20] NIST X-ray Photoelectron Spectroscopy (XPS) Database, (NIST Standard Reference Database 20) http://srdata.nist.gov/xps/.

[NNDC] National Nuclear Data Center http://www.nndc.bnl.gov/.

[Pa98] Palik, E.D., Ed., Handbook of Optical Constants of Solids, Academic Press, Vol. 1: 1985, Vol. 2: 1991, Vol. 3: 1998.

[Pe02] Perkins, S.T. and Cullen, D.E., ENDL type formats for the LLNL Evaluated Atomic Data Library, EADL, for the Evaluated Electron Data Library, EEDL, and for the Evaluated Photon Data Library, EPDL, LLNL Report UCRL-ID-117796, Rev. 1, May 17, 2002.

[P175] Plechaty, E.F., Cullen, D.E. and Howerton, R.J., Tables and graphs of photon interaction cross sections from $1 \mathrm{keV}$ to $100 \mathrm{MeV}$ derived from the LLL evaluated nuclear data library, LLNL Report UCRL-50400, Vol. 6, Rev. 1, October 31, 1975.

[Pu95] Puri, S., Chand, B., Mehta, D., Garg, M.L., Singh, N. and Trehan, P.N., K and $L$ shell x-ray fluorescence cross sections, At. Data Nucl. Data Tables 61(2): 289-311, November 1, 1995.

[Qu72] Quittner, P., Gamma-Ray Spectroscopy: with Particular Reference to Detector and Computer Evaluation Techniques, Hilger, Bristol, England, 1972.

[Qu91] Querry, M.R., Wieliczka, D.M. and Segelstein, D.J., Water $\left(\mathrm{H}_{2} \mathrm{O}\right)$, in Palik, Ed., Handbook of Optical Constants of Solids, Academic Press, Vol. 2 pp. 1059-1078, 1991.

[Sa31] Sauter, F., Ann. Phys. (Paris) 11: 454 (1931)

[Sa88] Saloman, E.B., Hubbell, J.H. and Scofield, J.H., X-ray attenuation cross sections for energies $100 \mathrm{eV}$ to $100 \mathrm{keV}$ and elements $Z=1$ to $Z=92$, Atom. Data Nucl. Data Tables 38(1):1-197, January 1988.

[Se88] Seltzer, S.M., "An overview of ETRAN Monte Carlo methods," Chapter 7 in Jenkins, T.M., Nelson, W.R. and Rindi, A. Eds., Monte Carlo Transport of Electrons and Photons, Plenum Press, New York, 1988.

[Sh64] Shafroth, S.M., Ed., Scintillation Spectroscopy of Gamma Radiation, Gordon \& Breach, 1964.

[Sh98] Shleien, B., Slaback, L.A. Jr, and Birky, B.K., Eds, Handbook of Health Physics and Radiological Health, 3rd edn, Williams \& Wilkins, 1998. 
[Sh00] Shultis, J.K. and Faw, R.E. Radiation Shielding, Section 5.2, American Nuclear Society, 2000.

[Si65] Siegbahn, K., Alpha, Beta and Gamma Ray Spectroscopy, Elsevier Science, 1965.

[Sm85] Smith, D.Y., Shiles, E. and Mitio Inokuti, "The optical properties of metallic aluminum," in Palik, Ed., Handbook of Optical Constants of Solids, Academic Press, Vol. 1, pp. 369-406,1985.

[Te97] Ter-Mikaelyan, M.L., Simple atomic systems in resonant laser fields, Physics-Uspekhi 40(12): 1195, 1997 (Full text at: http://ufn.ioc.ac.ru /abstracts/abst97/abst9712.html).

[Tr01] Trzhaskovskaya, M.B., Nefedov, V.I. and Yarzhemsky, V.G., Photoelectron angular distribution parameters for elements $Z=1$ to $Z=54$ in the photoelectron energy range 100-5000 eV, At. Data Nucl. Data Tables, 77(1): 97-159, January 1, 2001.

[Tr02] Trzhaskovskaya, M.B., Nefedov, V.I. and Yarzhemsky, V.G., Photoelectron angular distribution parameters for elements $Z=55$ to $Z=100$ in the photoelectron energy range 100-5000 eV, At. Data Nucl. Data Tables, 82(2): 257-311, November, 2002.

[Ts74] Yung-Su Tsai, Pair production and Bremsstrahlung of charged leptons, Rev. Mod. Phys. 46(4): 815-851, October 1974.

[Ts95] Tsoulfanidis, N., Measurement and Detection of Radiation, 2nd edn, Taylor \& Francis, 1995.

[Tu95] Turner, J.E., Atoms, Radiation, and Radiation Protection, 2nd edn, Wiley, 1995.

[Wa93] Wang, J.H., Sagar, R.P., Schmider, H. and Smith, V.H., X-ray elastic and inelastic scattering factors for neutral atoms $Z=2-92$, At. Data Nucl. Data Tables 53(2): 233-269, March 1, 1993.

[Wi58] Winans J.G. and Seldin, E.J., Fluorescence and phosphorescence, Part 6, Chapter 7 in Condon E.U. and Odishaw, H., Eds., Handbook of Physics, McGraw-Hill, 1958.

[Wi77] Williams, B., Ed., Compton Scattering, McGraw-Hill, 1977.

[Wo85] Wolfe, W.L. and Zissis, Eds., "The Infrared Handbook”, Revised edition, Environmental Research Institute of Michigan, 1985. 



\section{Electrons}

DOI: $10.1201 / 9781420012378-3$

\subsection{INTRODUCTION}

In striking an atom, an electron may

- Elastically scatter without loss of energy and leave the atom undisturbed. Above a few hundred keV, elastic scattering is sharply peaked in the forward direction.

- Emit a photon while scattering from the nucleus; the electron continues with reduced energy (Bremsstrahlung). The projectile may also emit a photon while scattering from an atomic electron.

- Inelastically scatter by raising an atomic electron to an excited state, while the original projectile continues with reduced energy (excitation).

- Kick out one of the atomic electrons and ionize the atom. Two electrons exit the interaction region. The more energetic of the two is considered the primary electron (ionization).

Bremsstrahlung and the excitation and ionization processes contribute to the energy loss of the projectile. Energy loss by excitation and ionization is referred to as collisional energy loss. Energy loss to Bremsstrahlung photons is referred to as radiative energy loss.

In passing through matter, as a result of the single-atom interactions given above, an electron will interact with many atoms along its path, giving rise to the following:

- Many successive elastic scatterings without loss of energy, which substantially deviates the electron from its original straight-line path (multiple scattering)

- An overall mean energy loss rate by excitation and ionization and Bremsstrahlung that will eventually bring the electron to rest (mean collisional or radiative stopping power)

These two effects control the behavior of an electron in passing through matter.

Apart from these two effects a third effect is the creation of and energy loss to Cerenkov radiation in optical materials if the electron velocity exceeds the phase velocity of light in the medium. This, however, turns out to be a small contributor 
to energy loss. Jelley [Je53] found the stopping power due to Cerenkov radiation to be only about $0.1 \%$ that due to collisional energy losses for velocities near $c$. We therefore neglect Cerenkov radiation as a contributor to stopping power. Jackson [Ja75] and Birkhoff [Bi58] discuss energy loss to Cerenkov radiation as a function of the material optical index of refraction.

The range of electrons of energy $\lesssim 1 \mathrm{keV}$ in most matter is less than $10^{-4} \mathrm{~g} / \mathrm{cm}^{2}$. In solids, this is a sufficiently small distance $\left(\lesssim 10^{-4} \mathrm{~cm}\right)$ that details of the motion of such low-energy electrons are seldom of interest. In gases, however, especially at low pressure, these low energies may indeed be of interest; in Section 3.9 we discuss slow electrons in air. In most of this chapter we concentrate on energies higher than about $1 \mathrm{keV}$.

Transport of electrons through matter is the subject of a number of codes. EPIC, ITS (Integrated Tiger Series), MCSet, Penelope, and Casino are some of them. The SRIM site, primarily intended for the transport of ions, is developing a collection of electron transport tools (http://www.srim.org/SREM.htm).

We first consider elastic scattering on a single atom; multiple scattering is discussed later.

\subsection{ELASTIC SCATTERING OF AN ELECTRON FROM AN ATOM}

We present order of magnitude estimates for the elastic scattering of an unpolarized electron from a neutral atom at any incident velocity (relativistic or nonrelativistic) and then show the compiled data.

At high energies and relatively large scattering angles, the incident electron scatters from the nucleus by Coulomb scattering. Screening by the atomic electrons affects scattering only at small angles when the electron passes at a large impact parameter. At lower energies, screening is important even at large scattering angles.

The theory of elastic and inelastic scattering of structureless charged particles from atoms has been presented in a number of studies (e.g., [Be32, Be33, Ma56a, Ma56b, Be64, Mo65, In71, Mo85]). Here we review only enough to remind the reader of the basic theory and to make understandable the magnitude of numerical data based on much more careful calculations with detailed atomic wave functions.

\subsubsection{Coulomb Scattering from a Bare Nucleus}

Consider an electron of momentum $\hbar \vec{k}$ incident on an atom in state $\psi_{0}$, usually the ground state. It interacts via the Coulomb potential

$$
V=-\frac{Z e^{2}}{r}+\sum_{j=1}^{Z} \frac{e^{2}}{\left|\vec{r}-\vec{r}_{j}\right|},
$$


with the nucleus at the origin (first term) and with the $Z$ atomic electrons at $\vec{r}_{j}$ (second term). Consider it to elastically scatter through angle $\theta$ to final momentum $\hbar \vec{k}^{\prime}, k^{\prime}=k, \vec{k}^{\prime} \cdot \vec{k}=k^{2} \cos \theta$. In first-order perturbation theory (the Born approximation), and ignoring electron spin, the amplitude is [Be64]

$$
f=-\frac{1}{4 \pi} \frac{2 m}{\hbar^{2}} \int \psi_{0}^{*}(\tau) e^{-i \vec{k}^{\prime} \cdot \vec{r}} V(\tau, \vec{r}) e^{i \vec{k} \cdot \vec{r}} \psi_{0}(\tau) \mathrm{d} \tau \mathrm{d}^{3} r,
$$

where $\tau=\vec{r}_{1}, \vec{r}_{2}, \ldots \vec{r}_{Z}$ represents the coordinates of the $Z$ atomic electrons, $\mathrm{d} \tau=\mathrm{d}^{3} r_{1} \mathrm{~d}^{3} r_{2} \ldots \mathrm{d}^{3} r_{Z}$

The nonrelativistic Equation 3.2 will be relativistically correct if the factor $2 m / \hbar^{2}$ is replaced by $2 \gamma m / \hbar^{2}$, where $\gamma=\left(1-v^{2} / c^{2}\right)^{-1 / 2}$ is the electron relativistic factor and $v$ the electron velocity, so that the momentum is $\hbar k=\hbar k^{\prime}=\gamma m v$. This factor arises from the relativistic expression for the density of states and may be incorporated in the definition of $f$ to preserve the usual relation $\mathrm{d} \sigma / \mathrm{d} \Omega=|f|^{2}$ expressing the differential cross section in terms of the scattering amplitude [Fe61, §23; Ne66, Ch.8; Mo85].

Temporarily ignoring the term in the atomic electrons in $V$, consider scattering from only a bare nucleus, $V=-Z e^{2} / r$. The factors in $\tau$ integrate to 1 , and $f$ becomes

$$
f=-\frac{1}{4 \pi} \frac{2 \gamma m}{\hbar^{2}} Z e^{2} \int e^{i \vec{q} \cdot \vec{r}} \frac{1}{r} \mathrm{~d}^{3} r
$$

where $\vec{q}=\vec{k}-\vec{k}^{\prime}$ is the momentum transferred to the atom, and we have inserted $\gamma$, as mentioned, to make it relativistically correct. The integral evaluates to $4 \pi / q^{2}$.

Therefore, in the laboratory system, the Rutherford differential cross section for the classical scattering through an angle $\theta$ of an electron (here of zero spin) of kinetic energy $E=(\gamma-1) m c^{2}$ on a heavy bare nucleus of charge $Z e$ is

$$
\left(\frac{\mathrm{d} \sigma}{\mathrm{d} \Omega}\right)_{\mathrm{R}}=|f|^{2}=\frac{Z^{2} r_{\mathrm{o}}^{2}}{\gamma^{2} \beta^{4}(1-\cos \theta)^{2}},
$$

where $\beta=v / c$, and $r_{\mathrm{o}}=e^{2} / m c^{2}=2.818 \mathrm{fm}$ is the classical electron radius. The cross-section magnitude is set by $r_{\mathrm{o}}^{2}=0.0794$ barn. Electron spin is not included in the classical Rutherford formula. When spin is taken into account, the equation is replaced by the Mott formula

$$
\left(\frac{\mathrm{d} \sigma}{\mathrm{d} \Omega}\right)_{\mathrm{M}}=\frac{Z^{2} r_{\mathrm{o}}^{2}}{\gamma^{2} \beta^{4}(1-\cos \theta)^{2}}\left[1-\beta^{2} \sin ^{2} \frac{1}{2} \theta+\pi \alpha \beta Z\left(1-\sin \frac{1}{2} \theta\right) \sin \frac{1}{2} \theta\right]
$$


[Mo65]. The factor $1-\beta^{2} \sin ^{2} \frac{1}{2} \theta$ accounts for electron spin in the first Born approximation, and arises automatically upon using Dirac spinors for the incident and outgoing electron wave functions. The term in $\pi \alpha \beta Z$ is one term beyond the Born approximation in the quantity $\alpha Z$, where $\alpha=e^{2} / \hbar c=1 / 137$ is the fine structure constant.*

At large angles the Mott cross section is smaller than the Rutherford. In Fe at $E=1 \mathrm{MeV}$, the differential cross section at $\theta=\pi / 2$ is about 0.62 times the Rutherford expression. Nuclear recoil, important at hundreds of $\mathrm{MeV}$ where the electron relativistic mass becomes comparable to the nuclear mass, and interaction with the nuclear magnetic moment are also not contained in the Rutherford or Mott formula [Mo65]. In addition, at a few hundred MeV the electron probes the nuclear charge distribution, which is characterized by a nuclear form factor that is not included. Both the Rutherford and Mott differential cross sections diverge in the forward direction, and their respective total cross sections, $\sigma=\int_{4 \pi} \mathrm{d} \Omega(\mathrm{d} \sigma / \mathrm{d} \Omega)$, also diverge.

\subsubsection{Scattering from a Neutral Atom}

Shielding by atomic electrons, however, prevents the Coulomb divergence in the forward direction; for scattering on a neutral atom the total cross section and the differential cross section at all angles are finite. An estimate for elastic scattering from a neutral atom is obtained by approximating the potential of the nucleus and the atomic electrons by a screened Coulomb potential $V(r)=Z e \exp (-r / a) / r$ in place of the nuclear Coulomb potential itself (and in place of the actual potential equation 3.1), where $a$ is a screening distance comparable to the atomic radius. The magnitude $q$ of the momentum transfer is given by $q^{2}=2 k^{2}(1-\cos \theta)=4 k^{2} \sin ^{2}(\theta / 2)$. Maximum momentum transfer is $q_{\max }=2 k$. The amplitude is then

$$
f=-\frac{1}{4 \pi} \frac{2 \gamma m}{\hbar^{2}} \int \mathrm{d}^{3} r e^{i \vec{q} \cdot \vec{r}} e V(r)=-\frac{2 \gamma m Z e^{2}}{\hbar^{2} q^{2}} \frac{q^{2} a^{2}}{1+q^{2} a^{2}}
$$

and the cross section for incident, relativistic, spin $\frac{1}{2}$ particles is [Mo65]

$$
\begin{aligned}
\frac{\mathrm{d} \sigma}{\mathrm{d} \Omega} & =|f|^{2}\left(1-\beta^{2} \sin ^{2} \frac{1}{2} \theta\right) \\
& =\left(\frac{2 \gamma m Z e^{2}}{\hbar^{2} q^{2}}\right)^{2}\left(\frac{q^{2} a^{2}}{1+q^{2} a^{2}}\right)^{2}\left(1-\beta^{2} \sin ^{2} \frac{1}{2} \theta\right) .
\end{aligned}
$$

\footnotetext{
*Equation 3.5 is McKinley and Feshbach's approximation to the complete Mott formula. [Mc48].
} 
The first factor in the second line is just the Rutherford cross section. The factor $\left(1-\beta^{2} \sin ^{2} \frac{1}{2} \theta\right)$ is a result of electron spin as before. The factor containing $q^{2} a^{2}$ is unity for an unscreened charge $(a \longrightarrow \infty)$ or for a large momentum transfer; but at low-energy or small-momentum transfer (large impact parameter) it reduces the cross section to below the Rutherford value, and it makes finite both the differential cross section in the forward direction and the total cross section.

\subsubsection{Total Cross Section}

Integrating Equation 3.7 over all solid angles, one obtains an estimate for the total elastic electron-atom scattering cross section:

$$
\begin{aligned}
\sigma & =\int_{4 \pi} \frac{\mathrm{d} \sigma}{\mathrm{d} \Omega} \mathrm{d} \Omega \\
& =4 \pi\left(\frac{\gamma Z a}{k a_{\mathrm{o}}}\right)^{2}\left\{\frac{\beta^{2}+(2 a k)^{2}}{1+(2 a k)^{2}}-\frac{\beta^{2}}{(2 a k)^{2}} \ln \left[1+(2 a k)^{2}\right]\right\},
\end{aligned}
$$

where $a_{\mathrm{o}}=\hbar^{2} / m e^{2}=0.529 \AA$ is the Bohr radius. Using $\hbar k=\gamma \beta m c$, the maximum momentum-transfer parameter $(2 a k)^{2}=\left(q_{\max } a\right)^{2}$ can be written as $(2 a k)^{2}=4\left(\gamma^{2} \beta^{2} / \alpha^{2}\right)\left(a^{2} / a_{\mathrm{o}}^{2}\right)$, and so is a large number except for $\beta \lesssim \alpha(E \lesssim$ $15 \mathrm{eV})$. Ignoring such low energies, we have approximately

$$
\sigma=4 \pi\left(\frac{\gamma Z a}{k a_{o}}\right)^{2}=4 \pi \frac{(\alpha Z a)^{2}}{\beta^{2}}
$$

At nonrelativistic energies, $\sigma$ scales as $1 / E$, and at relativistic energies saturates to $4 \pi(\alpha Z a)^{2}$. As $a \lesssim a_{\mathrm{o}} / Z^{1 / 3}$ (see later), the order of magnitude of $\sigma$ above a few hundred $\operatorname{keV}(\beta \approx 1)$ is $\sigma \lesssim 4 \pi\left(\alpha Z^{2 / 3} a_{\mathrm{o}}\right)^{2} \approx 1.9 \times 10^{4} Z^{4 / 3}$ barn.

A better estimate for the numerical coefficient is obtained by using the Thomas-Fermi model of the atom to judge the magnitude of $a$. In this model [Sc55],

$$
a=\frac{0.885 a_{\mathrm{o}}}{Z^{1 / 3}} .
$$

Therefore we have

$$
\sigma=1.47 \times 10^{4} \frac{Z^{4 / 3}}{\beta^{2}}(\text { barn }) .
$$

This discussion provides a qualitative guide to realistic cross sections. The scaling with energy and the magnitude turn out to be reasonably accurate. 


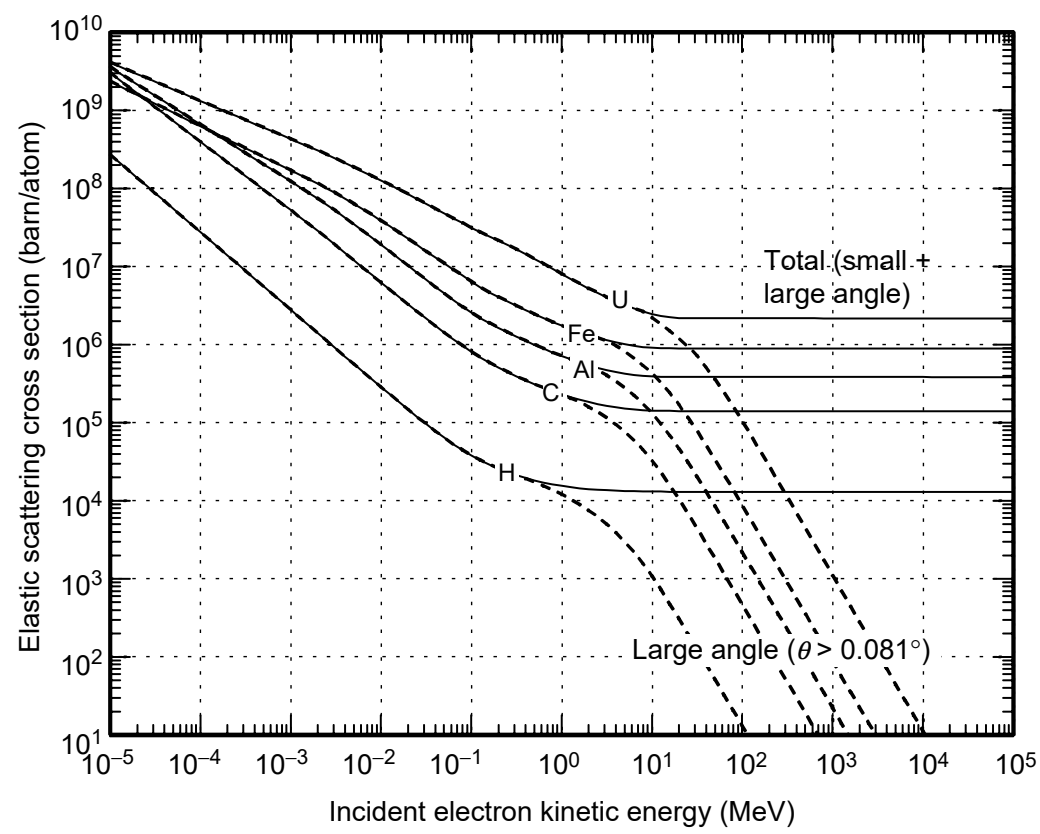

FIGURE 3.1 Electron-atom total (solid) and large-angle (dashed) elastic cross sections on five atoms as a function of incident electron energy. (Data from EEDL.)

In $\mathrm{Al}$, for example, from Equation 3.11 we expect $\sigma$ at relativistic energies to saturate to $\sim 4 \times 10^{5}$ barn, and at $1 \mathrm{keV}\left(\beta^{2}=3.9 \times 10^{-3}\right)$ Equation 3.11 predicts about $1 \times 10^{8}$ barn. Both estimates are borne out by data and more accurate calculations. Figure 3.1 presents data on the total elastic cross sections of five elements (solid curves) from the Evaluated Electron Data Library (EEDL) compiled by Lawrence Livermore National Laboratory (LLNL) [Pe91].

However, for large $Z$ or low energies the Born approximation becomes invalid. As seen in Figure 3.1 for U, the low-energy behavior is not well approximated by $1 / E$, and the high-energy total elastic cross section is about $2 \times 10^{6}$ barn, whereas Equation 3.11 predicts $6 \times 10^{6}$ barn.

\subsubsection{Differential Cross Section}

Combining the $q^{2}$ dependence in Equation 3.7, we obtain

$$
\begin{aligned}
\frac{\mathrm{d} \sigma}{\mathrm{d} \Omega} & =\left(\frac{2 \gamma Z a^{2} / a_{\mathrm{o}}}{1+q^{2} a^{2}}\right)^{2}\left(1-\beta^{2} \sin ^{2} \frac{1}{2} \theta\right) \\
& =\left(\frac{Z r_{\mathrm{o}}}{\gamma \beta^{2}}\right)^{2} \frac{1-\beta^{2} \sin ^{2} \frac{1}{2} \theta}{\left[1-\cos \theta+\frac{1}{2} \theta_{\mathrm{s}}^{2}\right]^{2}} .
\end{aligned}
$$


Except for the spin factor $\left(1-\beta^{2} \sin ^{2} \frac{1}{2} \theta\right)$, this differs from the Rutherford cross section, Equation 3.4 , only by the screening angle $\theta_{\mathrm{s}}$

$$
\begin{aligned}
\theta_{\mathrm{s}}^{2} & =\frac{1}{k^{2} a^{2}}=\frac{\alpha^{2}}{\gamma^{2} \beta^{2}}\left(\frac{a_{\mathrm{o}}}{a}\right)^{2} \\
& =\frac{\alpha^{2}}{\gamma^{2} \beta^{2}} \frac{Z^{2 / 3}}{(0.885)^{2}}
\end{aligned}
$$

The last form here has used the Thomas-Fermi expression for $a$. At relativistic energies, $\theta_{\mathrm{s}}$ is very small, so the differential cross section differs substantially from the Rutherford or Mott expression only near the forward direction. For $\theta \ll 1$, Equation 3.12 reduces to

$$
\frac{\mathrm{d} \sigma}{\mathrm{d} \Omega}=4\left(\frac{Z r_{\mathrm{o}}}{\gamma \beta^{2}}\right)^{2} \frac{1}{\left(\theta^{2}+\theta_{\mathrm{s}}^{2}\right)^{2}}
$$

When written in this form, $\theta_{\mathrm{s}}$ is sometimes called a cutoff angle, but we reserve this term for $\theta_{\mathrm{c}}$ introduced later for the separation between smallangle scattering and large-angle scattering.

\subsubsection{Differential Cross Section at $\theta=0$}

In exactly the forward direction, the differential cross section becomes

$$
\frac{\mathrm{d} \sigma}{\mathrm{d} \Omega}(\theta=0)=4\left(\frac{Z r_{\mathrm{o}}}{\gamma \beta^{2} \theta_{\mathrm{s}}^{2}}\right)^{2}=2.45 \gamma^{2} Z^{2 / 3} a_{\mathrm{o}}^{2}
$$

For relativistic energies, the forward scattering amplitude increases as $E^{2}$. At $100 \mathrm{MeV}$ on $\mathrm{Fe}$, it is $2 \times 10^{13}$ barn/ster. This enormous differential cross section persists only out to the small, energy-dependent angle $\theta_{\mathrm{s}}$. At $100 \mathrm{MeV}$ on $\mathrm{Fe}, \theta_{\mathrm{s}}=1.2 \times 10^{-4}=0.007^{\circ}$. From Equation 3.12 the cross section for scattering at angles less than $\theta_{\mathrm{s}}$ is

$$
\sigma\left(<\theta_{\mathrm{s}}\right)=2 \pi \int_{0}^{\theta_{\mathrm{s}}} \mathrm{d} \theta \sin \theta \frac{\mathrm{d} \sigma}{\mathrm{d} \Omega}=2 \pi\left(\frac{Z r_{\mathrm{o}}}{\gamma \beta^{2} \theta_{\mathrm{s}}}\right)^{2}=2 \pi \frac{(\alpha Z a)^{2}}{\beta^{2}}
$$

just one half the total cross section (Equation 3.9). Nearly the entire remaining half occurs at angles less than a few $\theta_{\mathrm{s}}$. Essentially all the scattering occurs at angles less than or of the order $\theta_{\mathrm{s}}$, into a solid angle of the order $\Delta \Omega \approx \pi \theta_{\mathrm{s}}^{2}$. At $100 \mathrm{MeV}$ in $\mathrm{Fe}, \Delta \Omega \approx 5 \times 10^{-8}$ ster. 


\subsubsection{Differential Cross Section at large and Small Angles}

At relativistic energies, the screening angle $\theta_{\mathrm{s}} \propto 1 / \gamma$. As just seen, for $\theta$ less than $\theta_{\mathrm{s}}$, the differential cross section increases in proportion to $\gamma^{2}$, whereas the total cross section is independent of $\gamma$. However, for a given $\theta$ larger than $\theta_{\mathrm{s}}$, the differential cross-section, Equation 3.12, drops off as $1 / \gamma^{2}$. Thus, as energy increases there is a larger separation between the total cross section and the cross section for scattering at large angles. The high-energy total cross section is essentially all due to scattering at angles $\lesssim \theta_{\mathrm{s}}$. For scattering at angles greater than $\theta_{\mathrm{s}}$ and at relativistic energies, $\theta_{\mathrm{s}}^{2}$ in Equation 3.12 is negligible, and the angular distribution is given approximately by the Mott expression Equation 3.5.

The elastic scattering cross section is $\gtrsim 10^{5}$ barn (Figure 3.1). As an electron moves through solid matter of atom density $N \sim 10^{23} \mathrm{~cm}^{-3}$, its mean free path (mfp) against elastic scattering is $1 / N \sigma \lesssim 10^{-4} \mathrm{~cm}$. This means it will elastically scatter from atoms about once every time it progresses $1 \mu \mathrm{m}$ in low- $Z$ targets at relativistic energies and will scatter many times in $1 \mu \mathrm{m}$ in high- $Z$ targets or at low energies. In codes that compute electron transport, one cannot compute each of these scatterings. Because small-angle scattering is so dominant and yet deflects the particle so little, it is inefficient to compute each electron-atom scattering in transport codes. Rather, for efficient computation, small-angle scattering is handled by analytic multiple scattering algorithms over a convenient path length, while large-angle scattering can be treated conventionally with each interaction [Be63, Se88].

For this reason, compilations of electron-atom elastic cross sections tabulate the large-angle cross section separately from the total cross section, at least at high energies. The LLNL compilation of electron cross sections, the EEDL, chooses as the transition point between small-angle and large-angle scattering a cutoff angle $\theta_{c}$ defined by

$$
1-\mu_{\mathrm{c}} \equiv 1-\cos \theta_{\mathrm{c}}=1 \times 10^{-6}
$$

$\left(\theta_{c}=1.414 \times 10^{-3}=0.081^{\circ}\right)$. Its chosen value is somewhat arbitrary. Scattering at angles greater than $\theta_{c}$ is considered large-angle scattering, and EEDL digitally tabulates its angular distribution $\mathrm{d} \sigma_{\mathrm{LA}} / \mathrm{d} \cos \theta$, as well as its contribution to the total scattering

$$
\sigma_{\mathrm{LA}}=\int_{-1}^{\mu_{\mathrm{c}}} \frac{\mathrm{d} \sigma_{\mathrm{LA}}}{\mathrm{d} \cos \theta} \mathrm{d} \cos \theta
$$

Scattering at angles less than $\theta_{\mathrm{c}}$ is considered small-angle scattering. In this region, the differential cross section is not tabulated from data. Rather, the functional form given in Equation 3.12 is used, but with a different overall 
normalization, determined by matching to the large-angle distribution at their common angle $\theta_{\mathrm{c}}$ to assure that $\mathrm{d} \sigma / \mathrm{d} \Omega$ is continuous at $\theta=\theta_{\mathrm{c}}$. Moreover, instead of Equation 3.13, an improved expression for the screening angle is employed as follows:

$$
\theta_{\mathrm{s}}^{2}=\frac{\alpha^{2}}{\gamma^{2} \beta^{2}} \frac{Z^{2 / 3}}{(0.885)^{2}}\left[1.13+3.76(\alpha Z / \beta)^{2}\left(\frac{E}{E+m c^{2}}\right)^{1 / 2}\right] .
$$

This is Seltzer's empirical adjustment to Molière's screening angle [Se88, Se91]. The actual differential cross section at small angles is then obtained by matching the small-angle expression to the large-angle differential crosssection data at its smallest angle $\theta_{\mathrm{c}}$. This provides the normalization for the small-angle formula and fixes the differential cross section at all angles. The total cross section is the sum of the large-angle cross section $\sigma_{\mathrm{LA}}$ (for $\theta>\theta_{\mathrm{c}}$ ) and the small-angle cross section $\sigma_{\mathrm{SA}}$ (for $\theta<\theta_{\mathrm{c}}$ ). $\sigma_{\mathrm{LA}}$ is the large-angle cross section tabulated from data (and calculations). $\sigma_{\mathrm{SA}}$ is the analytic integral on $\mu \equiv \cos \theta$ from $\mu_{\mathrm{c}}$ to 1 (a span of only $1.0 \times 10^{-6}$ on $\mu$ ) of the small-angle differential cross section Equation 3.12 using Equation 3.17 for $\theta_{\mathrm{s}}$.

Within the context of the screened Coulomb potential of the form $\exp (-r / a) / r$, Seltzer's semiempirical screening angle is tantamount to an expression for the screening distance $a$, which is an improvement on the standard Thomas-Fermi expression Equation 3.10. Because $\theta_{\mathrm{s}}$ and $a$ are related by the first equation in Equation 3.13, we have, using Equation 3.17 for $\theta_{\mathrm{s}}$, the effective Seltzer screening distance

$$
a_{S}=\frac{1}{k_{i} \theta_{\mathrm{s}}}=\frac{0.885 a_{\mathrm{o}}}{Z^{1 / 3}}\left[1.13+3.76(\alpha Z / \beta)^{2}\left(\frac{E}{E+m c^{2}}\right)^{1 / 2}\right]^{-1 / 2},
$$

instead of the Thomas-Fermi Equation 3.10.

It would therefore be more appropriate to use $a_{S}$ in place of $a$ in Equation 3.6 through Equation 3.12. However, the improvement in the value of the cross section is modest at best, and other factors, primarily the inadequacy of the Born approximation for low energies and high $Z$, have a larger effect on the differential and integrated cross sections. Seltzer's $\theta_{\mathrm{s}}$, in Equation 3.17, should be used in the simple, adequate form of Equation 3.14 for the differential cross section at small angles, but it is best to obtain the normalization from the fitting procedure discussed earlier. Figure 3.1 shows the total and large-angle elastic scattering cross sections on several elements as a function of energy, in barn/atom. Figure 3.2 shows the same quantities in $\mathrm{cm}^{2} / \mathrm{g}$. 


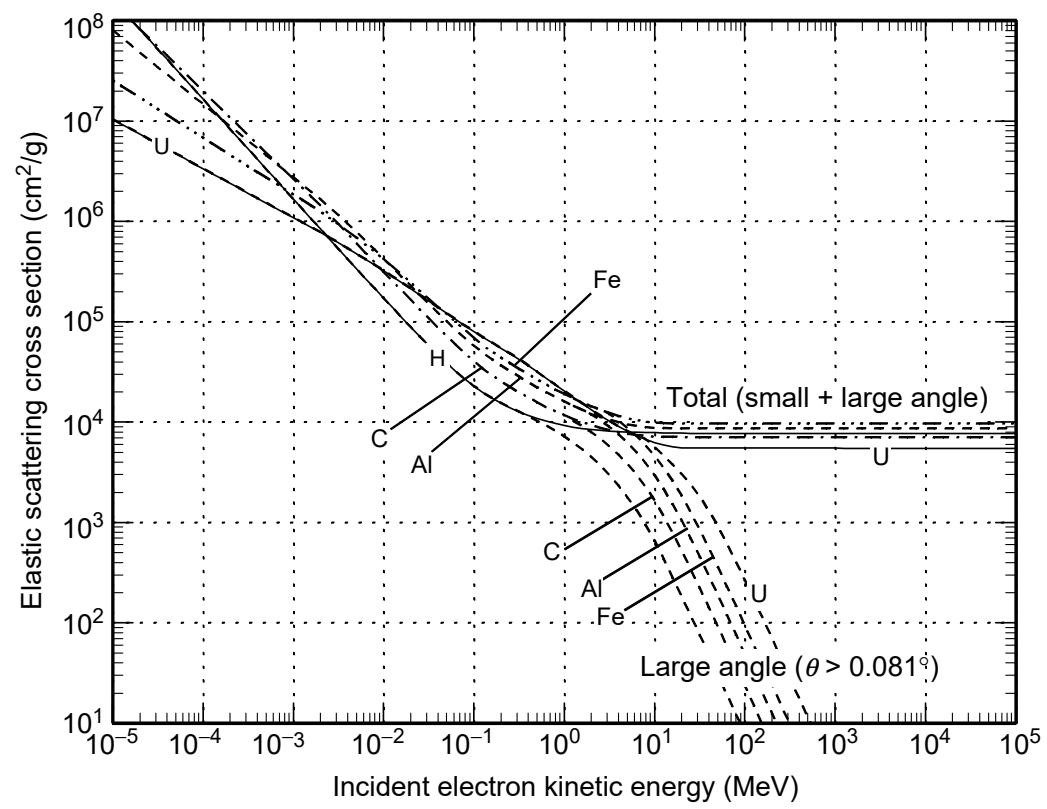

FIGURE 3.2 Electron-atom total (solid) and large-angle (dashed) elastic cross sections on five atoms as a function of incident electron energy. In $\mathrm{cm}^{2} / \mathrm{g}$. (From EEDL.)

\subsubsection{Angular Distribution}

At high energies, the differential cross section is remarkably peaked in the forward direction. For example, in $\mathrm{Fe}$ at $100 \mathrm{MeV}$, Figure 3.1 shows that the total cross section is $10^{2}$ times the large-angle cross section. Thus, $99 \%$ of the scattering is due to small-angle scattering and occurs in a forward cone comprising only $\sim 10^{-8}$ of a sphere. The differential cross section drops more than four orders of magnitude in less than $1.4 \mathrm{mrad}$, from $\theta=0$ to $\theta \approx 0.08^{\circ}$.

At large angles $\left(\theta>\theta_{\mathrm{c}}\right)$, the shape of the angular distribution in $\mathrm{Al}$ is shown in Figure 3.3. Plotted is $f(\mu)=\left[\mathrm{d} \sigma_{\mathrm{LA}} / \mathrm{d} \cos \theta\right] / \sigma_{\mathrm{LA}}=2 \pi\left[\mathrm{d} \sigma_{\mathrm{LA}} / \mathrm{d} \Omega\right] / \sigma_{\mathrm{LA}}$, $\mu \equiv \cos \theta$, normalized to $\int_{-1}^{\mu_{\mathrm{c}}} f(\mu) \mathrm{d} \mu=1$. Scattering is nearly isotropic at 10 $\mathrm{eV}$ and becomes increasingly forward peaked at higher energies.

As an illustration, Figure 3.4 shows the absolute differential cross section on $\mathrm{Al}$ at four energies. It includes all angles, showing the extremely rapid drop-off $\left(\sim 1 / \theta^{4}\right)$ at high energies, as $\theta$ increases. At $100 \mathrm{MeV}$, the differential cross section at $\theta=1^{\circ}$ is down 9 orders of magnitude, and at $\theta=10^{\circ}$ is down 13 orders of magnitude, from its peak at $\theta=0$.

As noted in Chapter 2, compilers of databases select, from all those available, measurements and computations believed worthy of contributing to the particular library. Each database represents one possible interpretation 


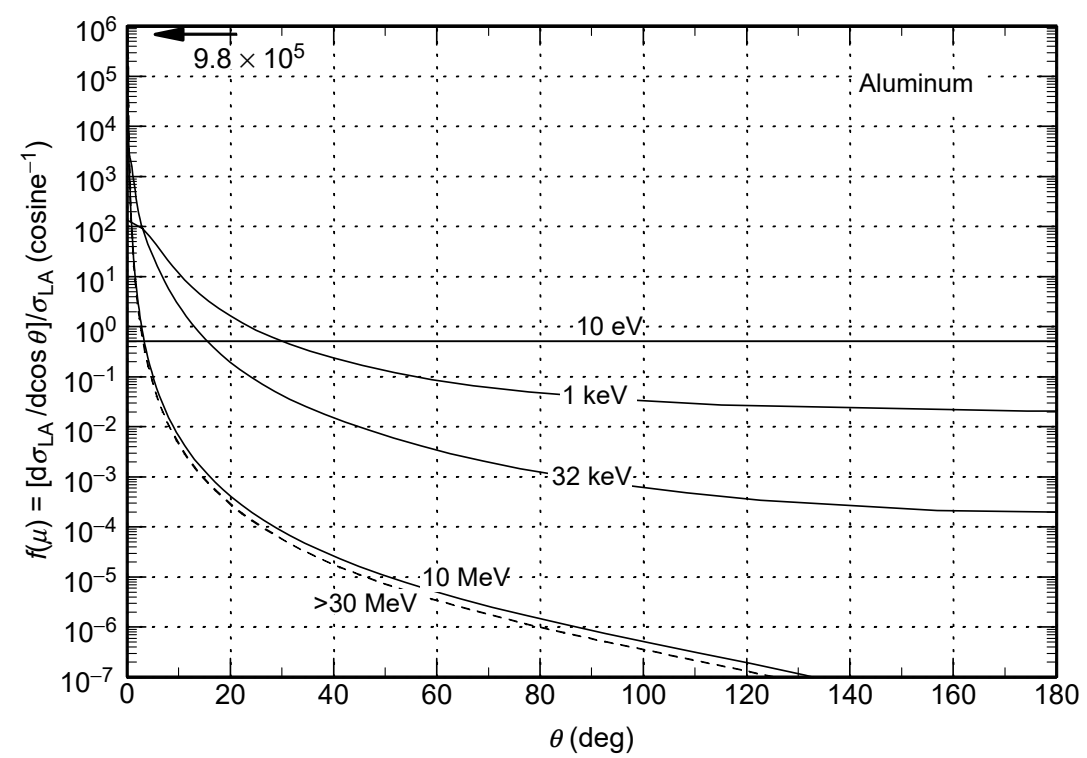

FIGURE 3.3 Normalized angular distribution of electron-atom elastic scattering on isolated Aluminum atom at several energies, for $\theta>0.08^{\circ}$ (large-angle scattering). Isotropic at $10 \mathrm{eV}$, and increasingly more forward-peaked at increasing energies. Above $30 \mathrm{MeV}, f$ is about $9.8 \times 10^{5}$ at $0.08^{\circ}$. (Data from EEDL.)

of the data and calculations. Elastic electron differential scattering cross sections have also been compiled by NIST as well as by LLNL. The latest NIST collection is Standard Reference Database 64, NIST Electron ElasticScattering Cross-Section Database, which presents differential cross sections vs. scattering angle for incident electron energies from $50 \mathrm{eV}$ to $300 \mathrm{keV}$ (http://www.nist.gov/srd/nist64.htm). These NIST data appear to be in excellent agreement with the EEDL data. Recently, Jablonski, Salvat, and Powell have presented a comparison of electron scattering calculations [Ja04].

\subsection{INELASTIC SCATTERING OF AN ELECTRON FROM AN ATOM}

The inelastic scattering cross section is determined by the off-diagonal matrix elements of the interaction Hamiltonian. The nonrelativistic scattering amplitude for exciting the atom from state 0 to state $n$ is, instead of Equation 3.2,

$$
f_{n 0}=-\frac{1}{4 \pi} \frac{2 m}{\hbar^{2}} \int \psi_{n}^{*}(\tau) e^{-i \vec{k} \cdot \vec{r}} V(\tau, \vec{r}) e^{i \vec{k} \cdot \vec{r}} \psi_{0}(\tau) \mathrm{d} \tau \mathrm{d}^{3} r
$$




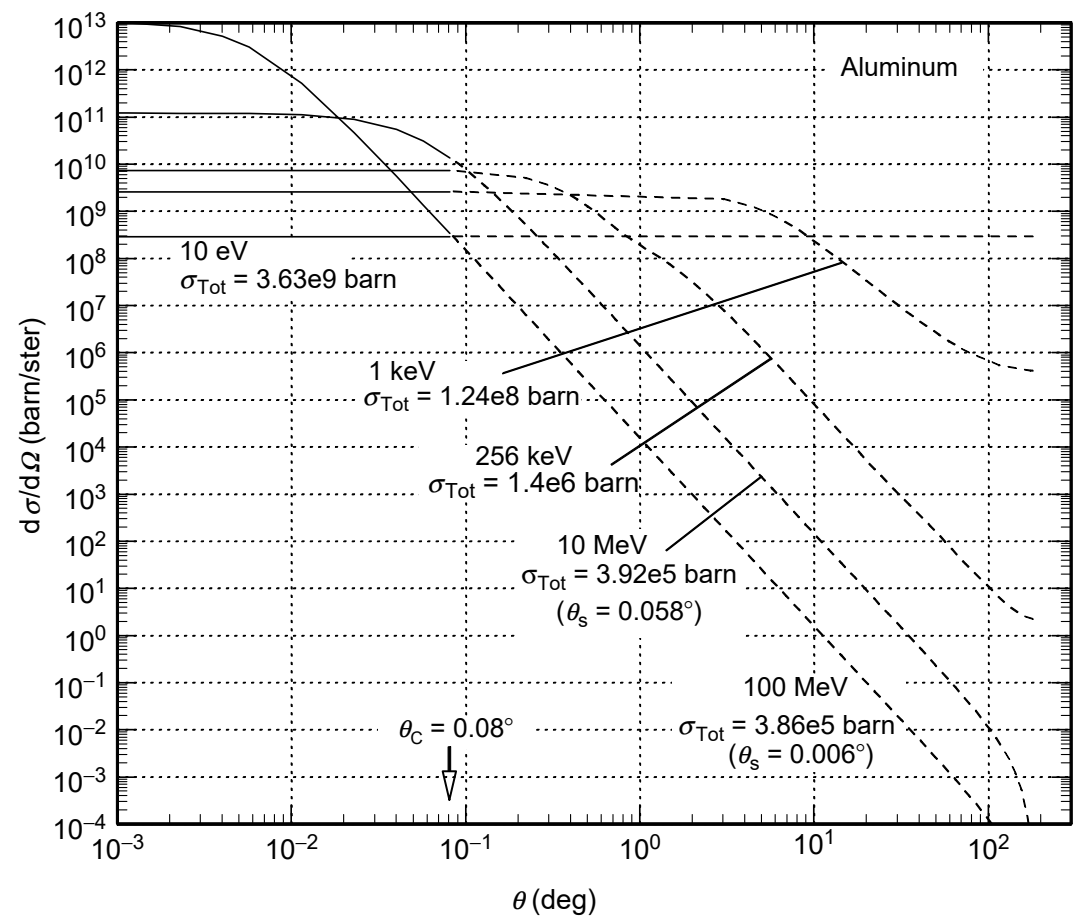

FIGURE 3.4 Absolute differential cross section for electron-atom elastic scattering on isolated aluminum atom at five energies, for all angles. Dashed curve is tabulated digital data at $\theta>0.08^{\circ}$. Solid is analytic fit of form equation 3.9. $\sigma_{\text {Tot }}$ is the $4 \pi$ integrated elastic cross section. (Data from EEDL.)

For excitation, both $\psi_{0}$ and $\psi_{n}$ are bound. For ionization, $\psi_{n}=e^{i \vec{k}^{\prime \prime}} \cdot \vec{r}$, where $k^{\prime \prime}$ is the ionized electron momentum. In all cases, the $\tau$ dependence of $\psi_{0}$ and $V$, and $\psi_{n}$ for excitation, limit the integration region, and the off-diagonal matrix elements between the atom ground state and an excited or ionized state are not very different in value from the diagonal matrix elements corresponding to elastic scattering. Thus at all energies, the excitation or ionization cross sections are not widely different from the elastic cross section, and at high energies inelastic scattering also approaches a constant value not very different from the elastic scattering cross section. Excitation and ionization cross sections tend to be comparable to one another, and are slightly lower than elastic cross sections in most elements. The theory of excitation and ionization of atoms by fast electrons was worked out by Bethe [Be32, Be33] and is summarized by Massey [Ma56b], Bethe [Be64], Mott and Massey [Mo65], Inokuti [In71], Moiseiwitsch [Mo85], and by Schattschneider [Sc86]. Recent ionization cross-section data are reviewed by Bartlett and Stelbovics [Ba04]. 
The cross sections for excitation or ionization of a particular electron shell are generally of interest only in applications in which one needs details of the subsequent relaxation of the atom. More often one needs the total excitation or ionization cross sections from the atom as a whole and energy lost by the electron in each interaction. The EEDL compiled by LLNL tabulates both the total excitation cross sections and subshell ionization cross sections.

Figure 3.5 shows excitation and ionization cross sections for $\mathrm{Al}$ and $\mathrm{Pb}$, in barn/atom, taken from EEDL. Below $1 \mathrm{MeV}$, excitation is generally about twice as large as ionization, and above $1 \mathrm{MeV}$, it is comparable to ionization. The excitation cross section is that for excitation from any level to any other. The ionization cross section is the total ionization cross section, the sum of the ionization cross sections from all shells.

Figure 3.6 shows the mean energy lost per collision in an excitation or ionization collision, in $\mathrm{Al}$ and $\mathrm{Pb}$, in $\mathrm{eV} /$ collision. In an ionization collision, the energy lost from the primary goes into the ionization potential of the ejected secondary electron and its subsequent kinetic energy. By excitation, a typical energy loss per collision for all elements is of the order $10 \mathrm{eV}$. By ionization, a typical energy loss per collision for all elements is an amount

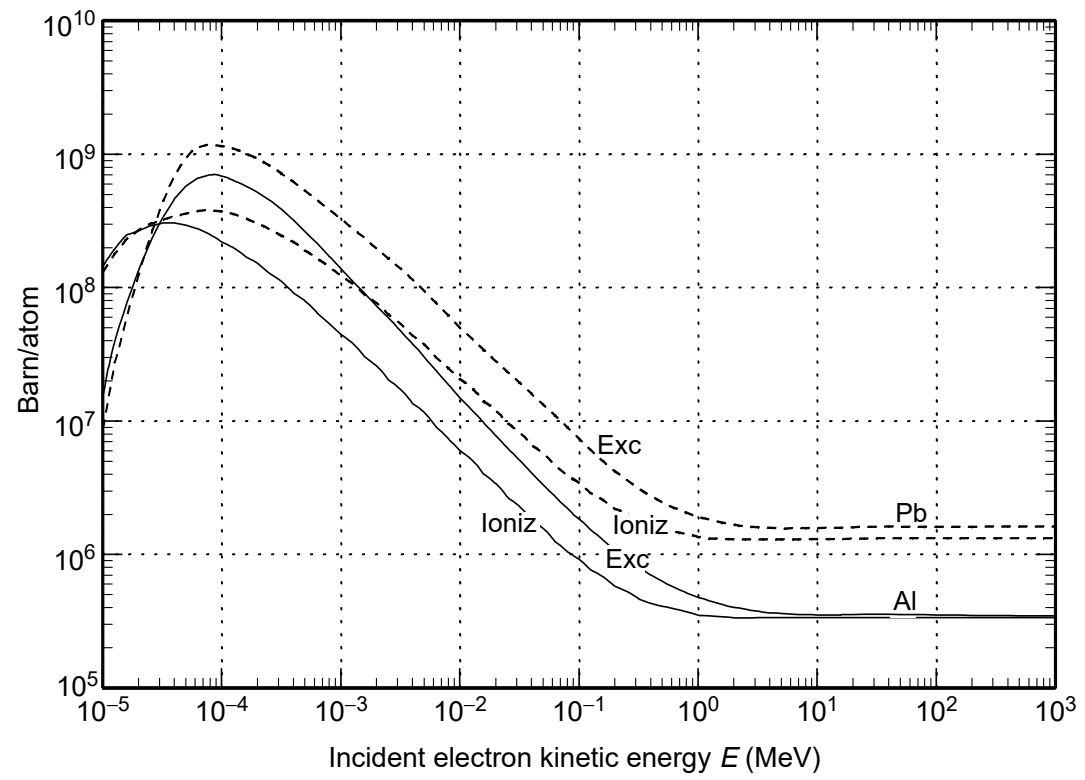

FIGURE 3.5 Cross section for ionization and excitation on an isolated atom. $\mathrm{Al}$ and $\mathrm{Pb}$. (Data from EEDL.) 


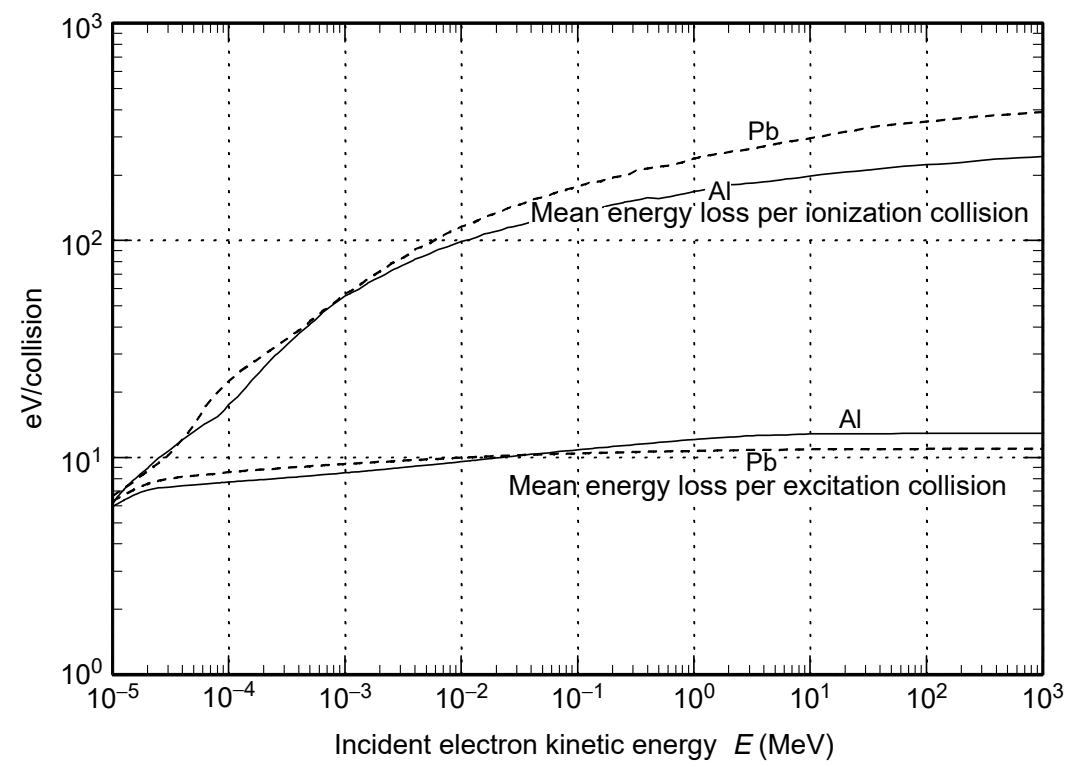

FIGURE 3.6 Mean energy loss per ionization collision or per excitation collision in $\mathrm{Al}$ and $\mathrm{Pb}$. (Data from EEDL.)

that slowly increases from less than $10 \mathrm{eV}$ when incident electron energy $E \lesssim$ $30 \mathrm{eV}$ to only a few hundred $\mathrm{eV}$ when $E \gtrsim 100 \mathrm{MeV}$.

Of greater interest is energy lost per unit columnar mass traversed, $\mathrm{MeV}$ per $\mathrm{g} / \mathrm{cm}^{2}$. The relations for ionization and excitation collisions are

$$
\begin{aligned}
& \mathrm{d} E /\left.\mathrm{d} s\right|_{\text {ion }}\left(\mathrm{MeV} \mathrm{cm}^{2} / \mathrm{g}\right)=N_{\mathrm{g}} \sigma_{\text {ion }} E_{\text {ion }}, \\
& \mathrm{d} E /\left.\mathrm{d} s\right|_{\text {exc }}\left(\mathrm{MeV} \mathrm{cm}^{2} / \mathrm{g}\right)=N_{\mathrm{g}} \sigma_{\mathrm{exc}} E_{\text {exc }},
\end{aligned}
$$

where $N_{\mathrm{g}}=N / \rho=N_{A} / A$ is the number of target atoms per gram, $N=$ atom number density $\left(\mathrm{cm}^{-3}\right), \rho$ is the material density $\left(\mathrm{g} / \mathrm{cm}^{3}\right), N_{A}$ is Avogadro's number, $A$ is the material atomic weight, $\sigma_{\text {ion }}=\sigma_{\text {ion }}(E)$ is the ionization cross section, $\sigma_{\text {exc }}=\sigma_{\text {exc }}(E)$ is the cross section for excitation, $E_{\mathrm{ion}}(\mathrm{MeV} /$ collision $)$ is the mean electron energy loss per ionization collision, and $E_{\text {exc }}$ is the mean energy loss per excitation collision.

When expressed in $\mathrm{MeVcm}^{2} / \mathrm{g}$, the curves take the shape shown in Figure 3.7 for $\mathrm{Al}$ and $\mathrm{Pb}$. Per unit mass, more is lost in low- $Z$ elements than in high- $Z$ by both excitation and ionization, whereas the cross section per atom, shown in Figure 3.5, is larger in high Z. Although in a given element $\sigma_{\text {exc }}$ and $\sigma_{\text {ion }}$ are comparable, above $\sim 1 \mathrm{keV}$ the energy loss is dominated by ionization, as there, $E_{\text {ion }}>E_{\text {exc }}$. 


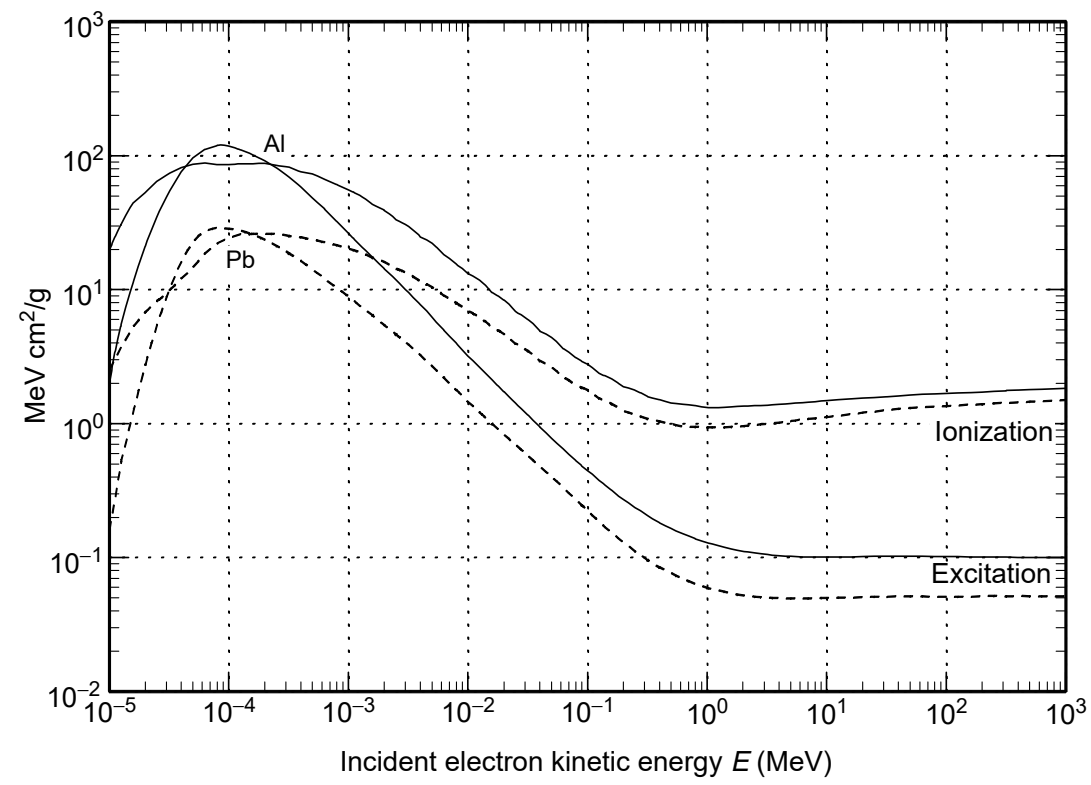

FIGURE 3.7 Mean energy loss rate by ionization or by excitation in $\mathrm{Al}$ and $\mathrm{Pb}$. (Data from EEDL.)

\subsection{ELECTRON ENERGY LOSS}

Electrons lose energy by interacting with both nuclei and atomic electrons. From elastic scattering off nuclei they lose little energy, but may scatter through large angles. From atomic electrons, they both lose energy (to excitation and ionization) and scatter in angle. In addition, at high energies, when scattering from either nuclei or electrons, they lose energy by radiative processes (Bremsstrahlung), emitting photons.

As an electron passes through matter, the mean rate of losing energy, $\mathrm{d} E / \mathrm{d} s$, is its (mean) stopping power. It has lost all energy and stops after a track length called the (mean) range

$$
R=\int(\mathrm{d} E / \mathrm{d} s)^{-1} \mathrm{~d} E
$$

In $\mathrm{Si}$, a $1 \mathrm{MeV}$ electron loses energy at a rate of about $1.5 \mathrm{MeVcm}^{2} / \mathrm{g}$ and has a range $R=0.55 \mathrm{~g} / \mathrm{cm}^{2}=0.23 \mathrm{~cm}$. In sea-level air, a $1 \mathrm{MeV}$ electron has $R=4 \mathrm{~m}$.

Energy loss of a charged particle is a probabilistic process; particles lose energy at more or less than the average rate $\mathrm{d} E / \mathrm{d} s$. Spread about the mean 
energy loss rate is called straggling. According to Equation 3.20, the range $R$ is defined as the track length of a particle that always loses energy at the mean rate; an actual particle may travel somewhat more or less than $R$. As a result, energy loss straggling leads to range straggling.* Working with only the mean rate is called the continuous slowing down approximation (CSDA). Thus, the mean range $R$ is also called the CSDA range $R_{\mathrm{CSDA}}$. Electron range straggling amounts to about $10 \%$ to $15 \%$ of the CSDA range [Be53]. The theory of straggling for electrons, mesons, protons, and alpha particles, and its effects in Silicon, has been presented by Bichsel [Bi88].

In addition to losing energy to atomic ionization and excitation, electrons that are energetic enough also lose energy to Bremsstrahlung, the radiation from their slowing down. This process is more important than collisional processes above the critical energy, the energy at which collisional and radiative energy losses are equal. The critical energy is about $95 \mathrm{MeV}$ in air and $10 \mathrm{MeV}$ in $\mathrm{Pb}$ (to be seen in Figure 3.10 or Figure 3.11 in Section 3.5.1, dashed curve labeled 0.5).

The term stopping power refers to the effect of the medium in slowing down the projectile; it is the energy lost by the electron in passing through the medium, and is usually expressed in $\mathrm{MeVcm}^{2} / \mathrm{g}$ or $\mathrm{MeV} / \mathrm{cm}$. The term collisional stopping power is the sum of the energy losses caused by excitation and ionization processes alone (not including Bremsstrahlung). The energy deposited along the track would be the same as stopping power if all secondary particles stopped in the medium. However, the term usually means energy deposited locally, that is, within a small distance of the electron track. Energy lost by the primary to ionization is taken up first by the struck electron binding energy, and then goes into its kinetic energy, which ionizes further and hence deposits its energy within a short distance, contributing to local energy deposition. The atomic shell vacancy left by the struck electron may be filled by capturing an electron outside the atom itself or by Auger transitions within the atom. If the vacancy is filled by a free electron, a fluorescence $\mathrm{X}$-ray is emitted; it either escapes the (small) target or is captured at a relatively long distance, and hence does not contribute to local energy deposition. If the vacancy is filled by Auger or Coster-Kronig transitions, the ejected electrons are of low energy and deposit their energy locally.

As most secondary electrons have a range short compared with the primary, local energy deposited is approximately the same as the collisional stopping power minus the average energy in fluorescence $\mathrm{x}$-rays. It is often the quantity of interest (as opposed to ionization or excitation losses separately,

\footnotetext{
*The literature uses the word straggling in various ways. Early experiments passed monoenergetic $\mathrm{MeV}$ electrons through thin slabs of metal. The exiting electrons had a wide range of energies. This is sometimes referred to as energy straggling, but it is primarily due to elastic scattering causing each electron to undergo a random walk, which produces a spread in track length, and therefore in residual energy, before the electron exits.
} 
and as opposed to the total stopping power, which includes Bremsstrahlung). However, in addition to the fact that fluorescence x-rays deposit their energy at some distance, energetic secondary electrons (called delta rays) from a direct ionization collision may actually travel some distance before losing all their energy, thus, local is a relative term. In a target larger than the mfp of fluorescence $\mathrm{x}$-rays and the range of delta rays, global energy deposition is the same as (global) stopping power. However, as delta rays and fluorescence photons deposit their energy at a point removed from where they were born, energy deposition occurs at a different point from where the original energy was lost.

To focus attention on local energy deposition, one may define a restricted collisional stopping power as collisional energy loss to a charged projectile caused by atomic energy transfers less than a specified value $\Delta$. The definition specifies an energy cutoff, not a range cutoff. But then, all energy loss by restricted stopping power is deposited about the projectile track within the range $R(\Delta)$ of an electron of energy $\Delta$, and hence all energy loss by restricted stopping power is deposited locally. Berger and Seltzer [Be82] discuss restricted stopping power and present some data.

The term Linear Energy Transfer (LET) is sometimes used, often in engineering and biological disciplines. Its precise definition has evolved over the years since first introduced by Zirkle [Zi40] and by Zirkle et al. [Zi52] in a biological context. The ICRU has devoted a report to its specification [ICRU70]. The usefulness of a certain definition is context-dependent. The current definition of LET is the same as that of restricted collisional stopping power, the energy deposited by transfers of less than some value $\Delta$. Unfortunately, the energy deposited by energy transfers less than $\Delta$ is only part of the energy deposited locally; secondaries more energetic than $\Delta$ may also deposit some energy at a radius less than $R(\Delta)$, because of scattering. However, since very energetic secondaries are relatively rare, only those with energy moderately greater than $\Delta$ would contribute significantly. Very often, LET is taken simply to be the (unrestricted) collisional stopping power. A new quantity, the energy loss of an energetic primary that results in energy deposited within a specified cylindrical radius from the particle track, would be a useful quantity for applications (such as the effects of energetic charged particles on microelectronic components, where distances less than a micron matter; or the ionization by charged particles in biological materials). Its computation would be considerably more complicated than that of restricted stopping power, as one would have to follow the trajectory of more energetic secondaries. It is tantamount to determining the radial profile of energy deposited by an energetic primary electron by all generations of secondary, tertiary, and other, ionizations.

As in the two previous equations relating $\mathrm{d} E /\left.\mathrm{d} s\right|_{\text {ion }}$ to $E_{\text {ion }}$ and $\mathrm{d} E /\left.\mathrm{d} s\right|_{\text {exc }}$ to $E_{\text {exc }}$, when the cross section is multiplied by the energy loss for that process, and all processes summed, one has the mean energy loss per $\mathrm{g} / \mathrm{cm}^{2}$ of the electron. 
$R$ defined in Equation 3.20 is the electron track length against mean stopping power. As mentioned in Section 3.2 and discussed further later, electrons scatter a great deal; by the time they come to rest, their average displacement in their original direction of motion can be considerably less than $R . R$ is the mean range as regards stopping power, but is essentially the extreme range as regards multiple scattering.

The introductory sections of the much used Berger, Seltzer Tables [Be82] contain a useful summary of the physics and approximations in the theory of electron and positron stopping power. Stopping of muons is discussed in, for example, Groom et al. [Gr01] and is summarized in the biennial Particle Data Group summary [PDG04].

\subsection{COLLISIONAL ENERGY LOSS}

Collisions are caused by the Coulomb field of the incident electron acting on the atomic electrons and nuclei in the target material. The energy transfer in Coulomb collisions and estimates of the resulting energy loss to the projectile are summarized clearly by Jackson [Ja75], in the vein of the classical discussion of the early Bohr, with heuristic quantum mechanical corrections.

\subsubsection{Accuracy of the Bethe Mean Stopping Power Formula}

Shortly after quantum mechanics solidified in the late 1920s, Bethe used the theory to compute the mean rate at which an electron loses energy by collisional processes in passing through matter [Be30, Be32, Be33]. His result is the Bethe mean stopping power formula for an electron of kinetic energy $E$,

$$
\begin{aligned}
L_{B}(E) & \equiv-\frac{\mathrm{d} E}{\mathrm{~d} s} \\
& =\frac{2 \pi N Z e^{4}}{m v^{2}}\left\{\ln \left[\frac{(\gamma+1) E^{2}}{2 I^{2}}\right]-\left(\frac{2}{\gamma}-\frac{1}{\gamma^{2}}\right) \ln 2+\frac{1}{\gamma^{2}}+\frac{1}{8}\left(1-\frac{1}{\gamma}\right)^{2}-\delta\right\},
\end{aligned}
$$

where $N$ is the density of material atoms, $\gamma=1+E / m c^{2}=1 /\left(1-v^{2} / c^{2}\right)^{1 / 2}$ is the usual electron relativistic factor, $I$ is the material mean excitation energy, and other symbols have their usual meaning. Since atomic binding energies are not accounted for in Equation 3.21, the expression is intended for energies greater than a few $\mathrm{keV}$. Figure 3.8 graphs Equation 3.21 (with $\delta=0$ ) for $\mathrm{Al}$ and $\mathrm{Pb}$, along with current best calculations of mean stopping power. For $\mathrm{Pb}$, $L_{B}(E)$ is accurate only above $100 \mathrm{keV}$ (deviations above $1 \mathrm{MeV}$ are due to the density effect, discussed later). For lower energies, more accurate calculations are used; see the summary in Berger and Seltzer [Be82]. If $N$ is $\mathrm{cm}^{-3}, L_{\mathrm{B}}$ is in $\mathrm{MeV} / \mathrm{cm}$; if $N$ is $\mathrm{g}^{-1}, L_{\mathrm{B}}$ is in $\mathrm{MeV} \mathrm{cm} / \mathrm{g}$. 


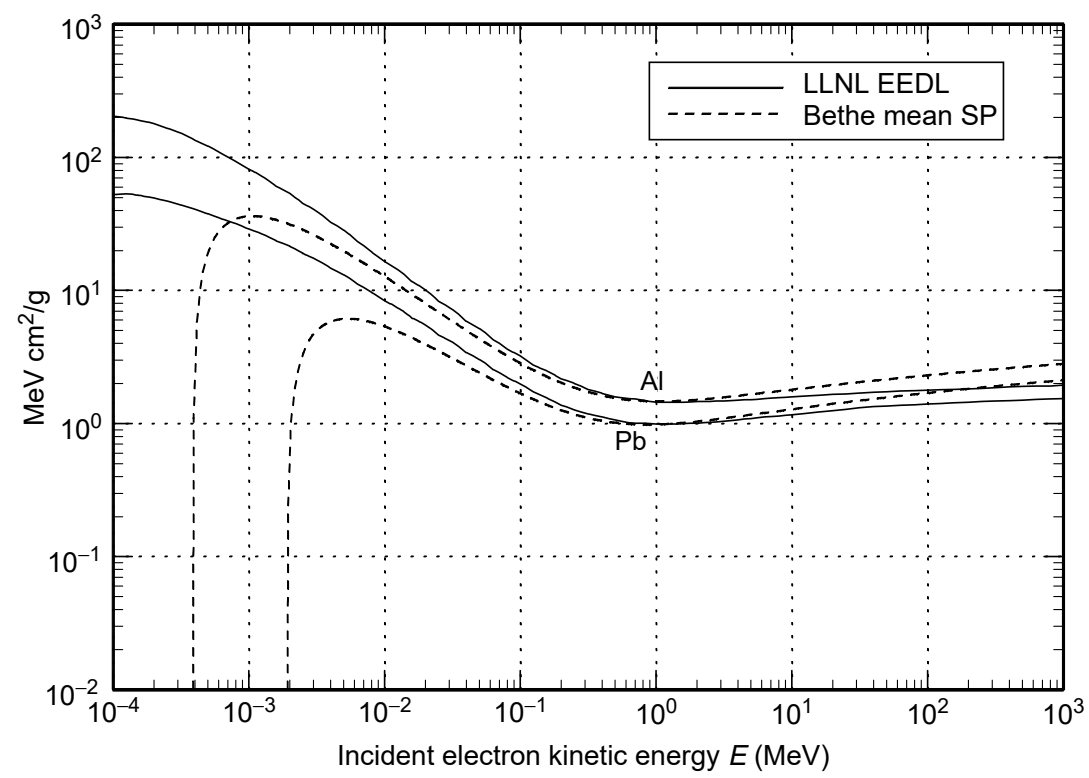

FIGURE 3.8 Electron mean collisional stopping power (excitation + ionization) in Al and $\mathrm{Pb}$. Solid: tabulated data in LLNL EEDL electron cross-section database. Dashed: Bethe mean stopping power formula.

Equation 3.21 is based on the Møller cross section (electron scattering from a free electron) and hence is intended to be valid only when energy transfer is larger than binding energies, that is, at relatively high incident energies. As $K$ shell binding in $\mathrm{Al}$ is $1.55 \mathrm{keV}, L_{B}$ becomes inaccurate below a few $\mathrm{keV}$ in low- $Z$ elements, and below some tens of $\mathrm{keV}$ in high- $Z$ elements. $\delta$ is the density effect parameter introduced later by Swann [Sw38] and Fermi [Fe40]. Bethe's original equation had $\delta=0$. A concise summary of the theory and approximations is in the introductory sections of [Be82].

The general behavior as a function of electron energy can readily be understood. For nonrelativistic velocities, $L_{B}$ behaves as $1 / v^{2}$, the logarithmic terms varying only slowly. The momentum impulse $\Delta p$ delivered by the projectile's field $e / r^{2}=e /\left(b^{2}+v^{2} t^{2}\right)$ to a target electron at a given impact parameter $b$ is $\Delta p=\int\left[e^{2} /\left(b^{2}+v^{2} t^{2}\right)\right] \mathrm{d} t \propto 1 / b v$, because the time over which the field acts is $\propto 1 / v$. As a result, the energy transfer to an atom at $b, \Delta E \propto$ $\Delta p^{2} \propto 1 / b^{2} v^{2}$, and the energy loss behaves as $1 / v^{2}$.

The logarithmic terms arise in summing the energy transfer contributions to all neighboring atoms. With $N$ atoms $/ \mathrm{cm}^{3}$, the number of atoms per linear centimeter in the annular ring between $b$ and $b+\mathrm{d} b$ to which the projectile can transfer energy is $N 2 \pi b \mathrm{~d} b$, and the energy transfer to all of them is 
proportional to $\int \Delta E N 2 \pi b \mathrm{~d} b \propto \int(1 / b)^{2} b \mathrm{~d} b \propto \ln \left(b_{\max } / b_{\min }\right) . b_{\max }$ is the maximum impact parameter at which the projectile can impart at least the atomic excitation or ionization energy and therefore transfer more than zero energy. $b_{\min }$ is the minimum impact parameter which can be considered consistent with the uncertainty principle. Jackson [Ja75] shows that $b_{\max } \sim \gamma v /\left\langle\omega_{\mathrm{o}}\right\rangle$, where $\left\langle\omega_{\mathrm{o}}\right\rangle$ is the logarithmic average of the atomic shell frequencies, and that $b_{\min } \sim$ the larger of $e^{2} / \gamma m v^{2}$ and $(\hbar / m c) \sqrt{2 /(\gamma-1)}$.

Consequently, the stopping power behaves as $1 / v^{2}$ for nonrelativistic energies, reaches a minimum as $v \longrightarrow c(E \sim 1 \mathrm{MeV})$, and thereafter increases only logarithmically with energy. The actual increase with increasing energy is somewhat slower because of the density effect discussed presently, parameterized by the quantity $\delta$ in Equation 3.21.

$I$, the target mean excitation energy, is an average over all atomic shells. Different contributions from individual electron shells introduce shell corrections to the Bethe theory. These corrections are especially important at low energies and high $Z$, where inner shell ionizations do not contribute. Crude estimates of the error introduced by omitting shell corrections at $100 \mathrm{keV}$ range from less than $1 \%$ for low $Z$ to $\sim 3 \%$ in $\mathrm{Au}$. At $10 \mathrm{keV}$, errors appear to be a few percent at low $Z$ to $\sim 20 \%$ in $\mathrm{Au}$ [Be82].

In spite of the low-energy $1 / v^{2}$ behavior, accurate calculations and data above $10 \mathrm{keV}$ show that on a given element the electron collisional stopping power varies by only a factor of 10 from $10 \mathrm{keV}$ to tens of $\mathrm{GeV}$, with a minimum of $1 \mathrm{MeV} \mathrm{cm} / \mathrm{g}-1.5 \mathrm{MeV} \mathrm{cm}^{2} / \mathrm{g}$ near $1 \mathrm{MeV}$ in most materials. In addition, with the exceptions of $\mathrm{H}$ and $\mathrm{He}$, at a given energy the collisional stopping power varies by only a factor of 3 as $Z$ varies from 3 to 99 . Figure 3.9a plots the collisional stopping power for $\mathrm{CH}_{2}$ and six elements vs. $E$, and Figure $3.9 \mathrm{~b}$ for selected energies as a function of $Z$. Data for the figures were taken from standard tables [NIST].

For electrons, minimum ionization occurs near $1 \mathrm{MeV}$ or kinetic energy $\sim 2 m c^{2}$. As the Bethe formula, Equation 3.21, holds with only slight modifications for ions as well, where it is known as the Bethe-Bloch formula, ions also exhibit their minimum ionization near the same velocity or kinetic energy $\sim 2 M c^{2}$, where $M$ is the ion mass. Electrons or ions (or muons, etc.) at energies near the minimum of the collisional stopping power, for instance, with kinetic energy near twice their rest energy, are known as minimum ionizing particles. A name for such particles evolved because in practice many electrons and heavy ions occur near that energy (nuclear betas, cosmic rays, etc.). Electrons near $1 \mathrm{MeV}$, protons near $2 \mathrm{GeV}$, and alpha particles near $8 \mathrm{GeV}$ are minimum ionizing.

$\mathrm{d} E / \mathrm{d} s$ is a function of $Z$ and $E$, and so forms a surface over the $Z, E$ plane. Collisional mean stopping power in all elements at all energies can be displayed in a contour plot similar to the photon cross section contour plots of Chapter 2. This graph is shown in Figure 3.10, for energies from 
Electron mean collisional stopping power

(a)
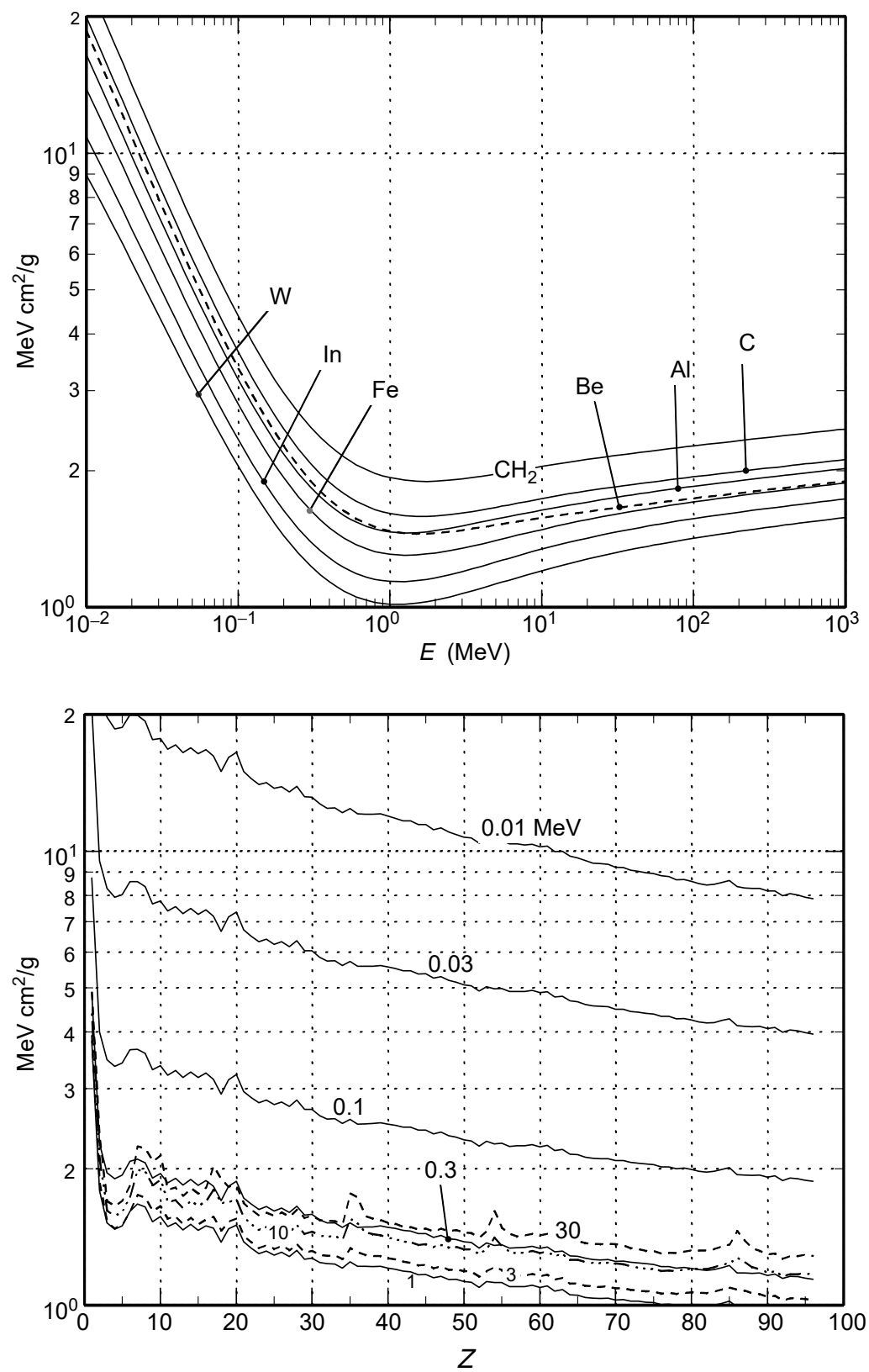

FIGURE 3.9 (a) Mean collisional stopping power vs. $E$ in several elements; (b) vs. $Z$ at several energies. Electrons near $1 \mathrm{MeV}$ are minimum ionizing particles. (Data from [NIST].) 


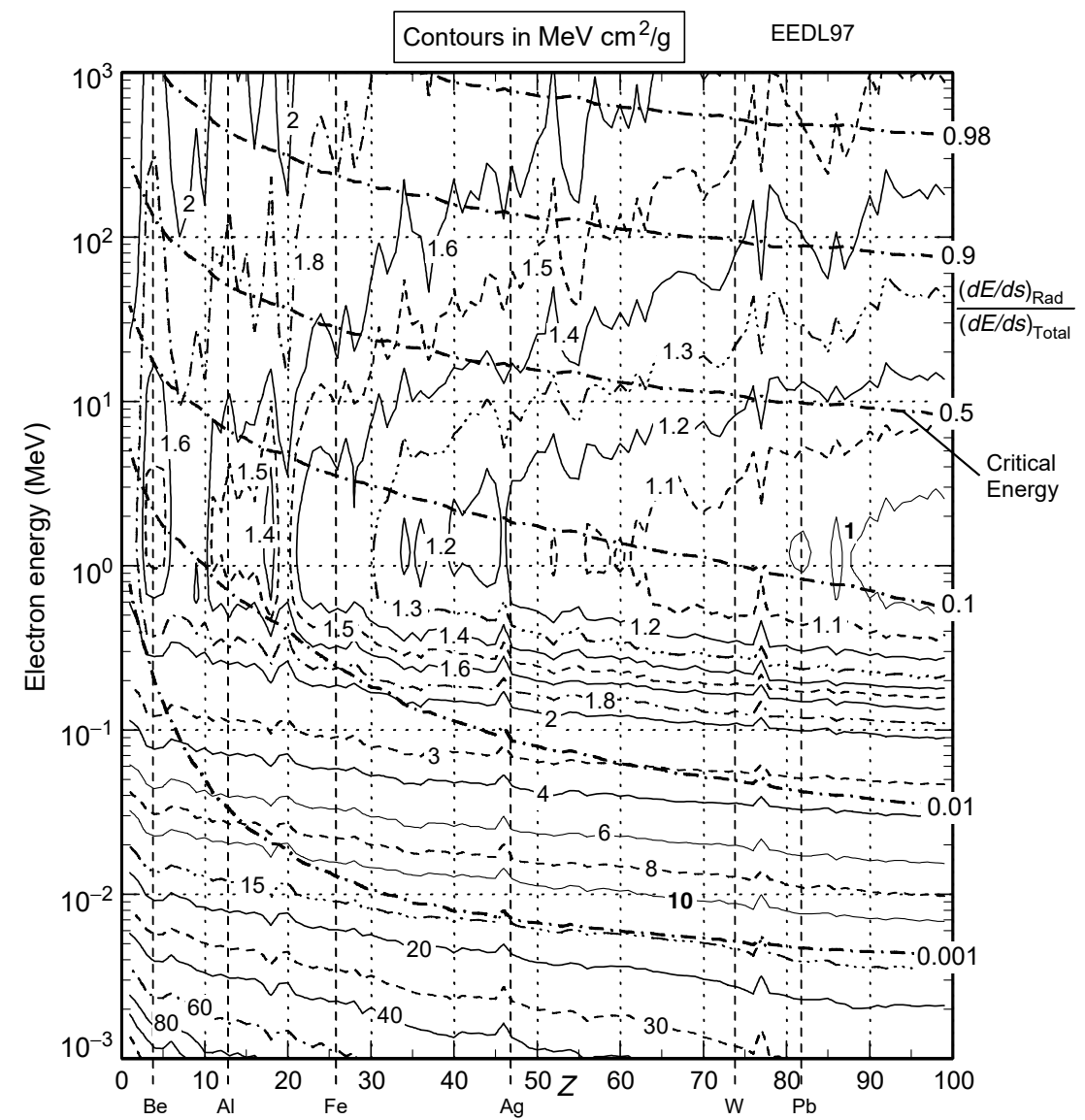

FIGURE 3.10 Mean collisional stopping power contours (ionization + excitation). Superimposed in heavy dot-dash lines are contours of the fraction (radiative stopping power)/(total stopping power). (Data from EEDL.) This graph, in color and 11" $\times 17 "$ paper, is available in high-resolution Adobe pdf format on the accompanying CD-ROM. See Section 3.11.

$1 \mathrm{keV}$ to $1 \mathrm{GeV}$. Data for this figure are taken from the EEDL compilation. The minimum energy loss plateau near $1 \mathrm{MeV}$ in all elements $\left(1 \mathrm{MeV} \mathrm{cm}^{2} / \mathrm{g}\right.$ for high $Z$ to $\sim 1.7 \mathrm{MeV} \mathrm{cm}^{2} / \mathrm{g}$ for low $Z$ ) is evident. $\mathrm{H}$ and $\mathrm{He}$ reach $3 \mathrm{MeV} \mathrm{cm} / \mathrm{g}$ or $4 \mathrm{MeV} \mathrm{cm}^{2} / \mathrm{g}$. The same quantity is shown in Figure 3.11 with data taken from the tabulation by NIST, from $10 \mathrm{keV}$ to 1 $\mathrm{GeV}$.

Irregular structure at $Z=86$ and at several other elements in both figures is caused by the jump in atomic weight at those elements. When energy loss is expressed as $\mathrm{MeV}$ per collision, there is no irregularity. At a fixed energy 


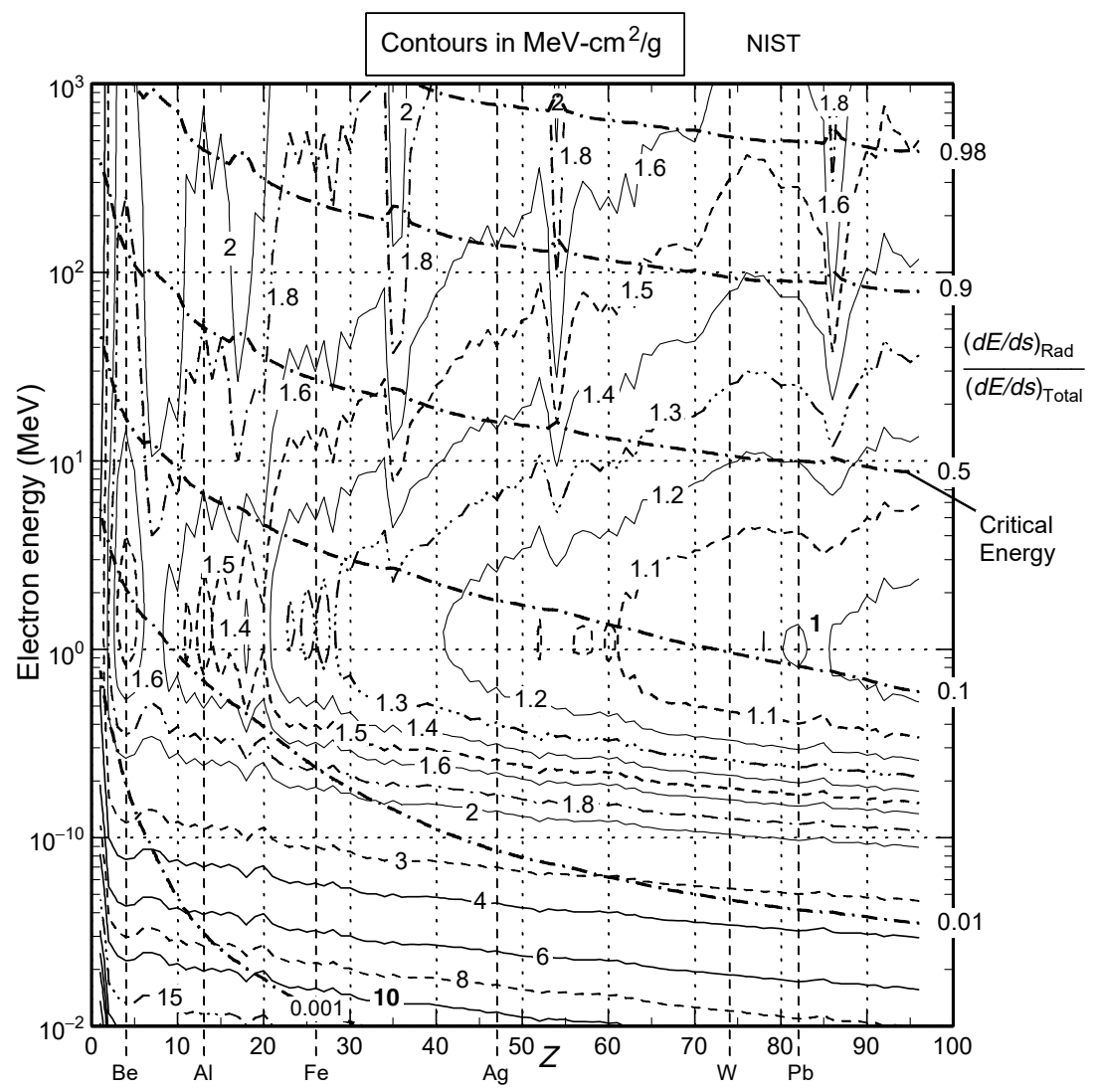

FIGURE 3.11 Mean collisional stopping power contours (ionization + excitation). Superimposed in dot-dash lines are contours of the fraction (radiative stopping power)/(total stopping power). (Data from [NIST].) This graph, in color and $11 " \times 17$ " paper, is available in high-resolution Adobe pdf format on the accompanying CD-ROM. See Section 3.11.

below $1 \mathrm{MeV}$, as a function of $Z$, Figure 3.10 shows an approximate $10 \%$ jump in energy loss in two elements, $Z=46(\mathrm{Pd})$ and 77 (Ir), relative to their immediate neighbors. This is peculiar to the LLNL data set; it does not occur in NIST data.

In Figure 3.11 one sees an increase in collisional stopping power at the gases, $Z=1,2,7,8,9,10,17,18,36,54$, and 86 , not seen in the LLNL data in Figure 3.10. This is because NIST has computed the density effect parameter $\delta$ (see later) and the stopping power of gases at their STP densities, $\sim 10^{-3} \mathrm{~g} / \mathrm{cm}^{3} . \delta$ is therefore smaller and the stopping power larger, than neighboring solids. However, LLNL, in the EEDL database, has presented 
the stopping power for gases at a density near $1 \mathrm{~g} / \mathrm{cm}^{3}$. This database is intended for use in electron transport codes. When elements that are gases at STP $(\mathrm{H}, \mathrm{N}, \mathrm{O}$, etc.) occur in solid or liquid compounds, a nominal density for them of the order $1 \mathrm{~g} / \mathrm{cm}^{3}$ is appropriate in those cases of transport through the condensed media in which they are constituents. This high density is not appropriate for actual gases. As discussed later, this choice causes at most a few tens of percent difference in energy deposited (by collisional stopping power) and causes very little difference in total stopping power because Bremsstrahlung dominates at high energies where the density effect is important.

The same effect, the increase in collisional stopping power in gases where the density effect is small, increases the total stopping power (collisional + radiative). Therefore, the ratio (radiative)/(total) has dips at the gases. This causes the local rises in the ratio curves in the NIST Figure 3.11 but not in the LLNL Figure 3.10. The same will be seen in later contour graphs.

\subsubsection{Density Effect}

Overall, the mean collisional stopping power $L_{B}$ is proportional to the atom number density $N$. All other factors in curly brackets in Equation 3.21 are independent of $N$ except the quantity $\delta$.

Experiments on high-energy cosmic rays in the 1920s and 1930s showed the actual stopping power in condensed media to be less than the original Bethe formula (Equation 3.21 without $\delta$ ) or its ion counterpart, the BetheBloch formula given in Chapter 4. Swann pointed out [Sw38] that the dielectric constant in solids will weaken the field of the projectile and reduce the projectile's ability to transfer energy to target atoms in distant collisions. This would reduce the stopping power.

The radial Coulomb field in vacuum of an electron of velocity $v$, in the plane normal to its velocity and passing through the electron, at radius $r$ is

$$
E_{0}=\frac{\gamma e}{r^{2}}
$$

A few atoms distant from a nonrelativistic electron $(\gamma=1)$ passing through a solid, say, at impact parameter $r=b_{1}=10 \AA$, this field is $E_{0}=1.4 \times 10^{9}$ $(\mathrm{V} / \mathrm{m})$. Nearby atoms are polarized by the field, so that the actual field at $b_{1}$ is $E=E_{0} / \varepsilon_{\mathrm{r}}$, where $\varepsilon_{\mathrm{r}}$ is the material relative dielectric constant. $E$ applies a force $e E=0.144 / \varepsilon_{\mathrm{r}}(\mathrm{eV} / \AA)$ to an electron in an atom at lateral distance $b_{1}$. A force of this magnitude does not distort the atom very much; the atom experiencing this force will not rob the projectile of kinetic energy. However, when the projectile is relativistic, $\gamma \gg 1$, and $e E$ can be many eV per $\AA$, enough to excite or ionize atoms near the electron path, contributing to the electron excitation and ionization stopping power. Then, $E_{0}, E$, and their difference can be substantial. The effect of this difference on collisional stopping 
power is known as the density effect, since it becomes important at relatively high material densities (and at high energies). The effect was first pointed out by Swann [Sw38] and calculated by Fermi (Fe39, Fe40), with later developments by Wick [Wi43] and by Halpern and Hall [Ha40, Ha48]. A brief account is given by Jackson [Ja75] (see also Bethe and Ashkin, [Be53] Crispin and Fowler, [Cr70]; Berger and Seltzer, [Be82]; and Sternheimer et al., [St82, St84].)

This polarizing density effect is conveniently incorporated in the Bethe stopping power formula via the parameter $\delta . \delta$ is a function of the element (through the ionization potential $I$ ), material density, and particle energy, $\delta=\delta(I, \rho, E)$. Thus, tables of electron collisional stopping power must specify the target density at which the stopping power is tabulated and should specify what value is used for $I$.

$\delta$ can be expressed in terms of the material dielectric constant over all frequencies or in terms of the atomic energy levels and oscillator strengths [St52, St82], but the general expressions are not very illuminating. The expression simplifies in the relativistic limit, $\gamma \gg 1$, to

$$
\delta=\ln \left[\frac{\gamma^{2}\left(\hbar \omega_{p}\right)^{2}}{I^{2}}\right]-1
$$

[Be82] where $\omega_{p}$ is the plasma frequency of all electrons, $\omega_{p}^{2}=4 \pi e^{2} \mathrm{NZ} / \mathrm{m}$, and $N$ is the atom number density. $\hbar \omega_{p}$ is between $10 \mathrm{eV}$ and $100 \mathrm{eV}$ for elemental solids and $\lesssim 1 \mathrm{eV}$ in STP gases.

For $\gamma \gg 1$, the logarithm term in Equation 3.21 is $\ln \left[\gamma^{3}\left(m c^{2}\right)^{2} / 2 I^{2}\right]$; at high energy, the largest term in curly brackets in Equation 3.21 increases as $3 \ln \gamma$ except $\delta$. When $\delta$ (Equation 3.23) is subtracted from it, the $I$ dependence drops out and a term $2 \ln \gamma$ is subtracted. One is left with $\ln \left[\gamma\left(m c^{2}\right)^{2} / 2\left(\hbar \omega_{p}\right)^{2}\right]$, and at high energy the curly brackets increase only as $\ln \gamma$ instead of $3 \ln \gamma$, making it appear that the density effect should reduce the stopping power by a factor of 3 . However, because $\left(m c^{2}\right)^{2} / 2\left(\hbar \omega_{p}\right)^{2} \gtrsim 10^{7}$, its log is greater than or comparable to $\ln \gamma$ up to $\sim 5000 \mathrm{GeV}$, and the reduction in stopping power due to the density effect is actually modest, rather than a factor of 3 . In $\mathrm{Al}$, for example, $\hbar \omega_{p}=32.8 \mathrm{eV}$, and $I=166 \mathrm{eV}$. At $1 \mathrm{GeV}, \delta=10.92$, whereas the logarithm term in Equation 3.21 is 38.1. All terms included, in this example the collisional stopping power including the density effect is 0.69 times the Bethe value without the density effect correction. In gases, when the density effect is taken into account, near $1 \mathrm{GeV}$, the collisional stopping power may be $\sim 0.9$ times the Bethe value with $\delta=0$.

As energy increases, the stopping power of electrons is reduced by the density effect but it continues to rise slowly. As will be seen in Chapter 4, for ions the density effect reduces stopping power, and at a sufficiently high energy, levels it off to a constant value, the Fermi plateau. The reason for the difference between electrons and ions lies in the maximum energy the 
projectile can impart to an atomic electron. An electron can impart half its full energy to a target electron, but a heavy ion can impart only a small fraction of its own energy.

The effect of $\delta$ on collisional stopping power can be tens of percent, but the effect on electron total stopping power (collisional plus radiative) is minor. Figure 3.8 shows the collisional stopping powers in $\mathrm{Al}$ and $\mathrm{Pb}$, computed by the Bethe mean stopping power with $\delta=0$ (dashed lines) and with $\delta$ at its correct value for normal solid densities (solid lines). In each element, differences above $1 \mathrm{MeV}$ are caused by the density effect. At $1 \mathrm{GeV}$, the difference is $\sim 30 \%$. However, at these high energies where $\delta$ is important, the primary energy loss mechanism is Bremsstrahlung. At $1 \mathrm{GeV}$ in $\mathrm{Al}, 93 \%$ of the stopping power is radiative; in $\mathrm{Pb}, 99 \%$ is radiative. The density effect therefore has greater consequences on energy deposited in the material than on energy lost to the projectile.

\subsection{3 $\delta$-RAYS}

In an ionization event caused by a primary electron of energy $E$, the struck electron may be ejected with energy anywhere from 0 to $E / 2$ (neglecting binding energy). The more energetic of the two outgoing particles is considered the primary. Therefore, the ejected electron (called the knock-on electron) may have sufficient energy itself to travel some distance. Energetic knock-ons are called $\delta$-rays, or (first-generation) secondary electrons, and can do further ionization at some distance from the primary electron track. The number and energy distribution of $\delta$-rays themselves are therefore of interest.

\subsubsection{Energy and Number Distribution of $\delta$-Rays}

The production cross section on a single target electron for a secondary of kinetic energy $w$, large compared with its binding energy (Møller scattering), is approximately [Be53]

$$
\frac{\mathrm{d} \sigma}{\mathrm{d} w}=\frac{\sigma_{0} m c^{2}}{\beta_{0}^{2} w^{2}},
$$

where $\beta_{0}=v / c$ of the primary, and $\sigma_{0}=2 \pi\left(e^{2} / m c^{2}\right)^{2}=0.499$ barn. Many more low-energy secondaries are produced than high-energy ones. Maximum secondary energy is $w_{\max }=E / 2$.

Per primary electron of energy $E$, we seek the number of secondaries produced per $\mathrm{g} / \mathrm{cm}^{2}$ that have energy greater than $w$, when $w$ is much greater than binding energy. The energy loss rate to secondaries with energy greater than $w$ is

$$
\left.\frac{\mathrm{d} E}{\mathrm{~d} s}\right|_{>w}=N_{g} \int_{w}^{w_{\max }} w \frac{\mathrm{d} \sigma}{\mathrm{d} w} \mathrm{~d} w=\frac{N_{g} \sigma_{0} m c^{2}}{\beta_{0}^{2}} \ln \left(w_{\max } / w\right),
$$


so that the energy lost to secondaries with energy between $w_{1}$ and $w_{2}>w_{1}$ is

$$
\left.\frac{\mathrm{d} E}{\mathrm{~d} s}\right|_{w_{1}-w_{2}}=\frac{N_{g} \sigma_{0} m c^{2}}{\beta_{0}^{2}} \ln \left(w_{2} / w_{1}\right)
$$

In the $\delta$-ray spectrum, there is the same amount of energy in each decade of $w$. Here, $N_{g}$ is the number of electrons per gram.

Energy dependence drops out of Equation 3.25 if we ask for the loss to $\delta$-rays with energy greater than a fixed fraction of $E$ or of $w_{\max }, w=$ $\xi w_{\max }=\xi E / 2$, with $\xi<1$. It is

$$
\left.\frac{\mathrm{d} E}{\mathrm{~d} s}\right|_{>\xi w_{\max }}=\frac{N_{g} \sigma_{0} m c^{2}}{\beta_{0}^{2}} \ln \left(\xi^{-1}\right)
$$

All relativistic primary electrons of energy $E$ lose energy at the same rate to knock-on secondaries with energy greater than a fixed fraction of $E$.

Arbitrarily choosing $\xi=1 / e=0.368$, and noting that $N_{g} \sigma_{0} m c^{2}=$ $(Z / A) N_{A} \sigma_{0} m c^{2}=0.154(Z / A)\left(\mathrm{MeV} \mathrm{cm}^{2} / \mathrm{g}\right)$, we have

$$
\left.\frac{\mathrm{d} E}{\mathrm{~d} s}\right|_{>0.184 E}=\frac{2 Z}{A} 0.077 \quad \mathrm{MeV} \mathrm{cm}^{2} / \mathrm{g}
$$

As $2 Z / A \approx 1$, all relativistic primary electrons lose energy to $\delta$-rays with energy greater than about $0.18 E$ at rate about $0.08 \mathrm{MeV} \mathrm{cm} / \mathrm{g}$.

The average energy of a secondary with energy greater than $w$ is

$$
\bar{w}=\int_{w}^{E / 2} w \frac{\mathrm{d} \sigma}{\mathrm{d} w} \mathrm{~d} w / \int_{w}^{E / 2} \frac{\mathrm{d} \sigma}{\mathrm{d} w} \mathrm{~d} w=\frac{E}{2} \frac{\ln (E / 2 w)}{(E / 2 w)-1}=\frac{w w_{\max }}{w_{\max }-w} \ln \left(\frac{w_{\max }}{w}\right)
$$

The number $\eta(>w)$ of secondaries produced with energy greater than $w$ is of interest in applications, for it determines the maximum extent of ionization radially from a narrow beam. $\eta(>w)$ is defined by direct integration of Equation 3.24, or by

$$
\bar{w} \eta(>w)=\mathrm{d} E /\left.\mathrm{d} s\right|_{>w}
$$

Then, from Equation 3.25 and Equation 3.29,

$$
\eta(>w)=\frac{\mathrm{d} E /\left.\mathrm{d} s\right|_{>w}}{\bar{w}}=\frac{N_{g} \sigma_{0} m c^{2}}{\beta_{0}^{2}}\left(\frac{1}{w}-\frac{2}{E}\right)
$$




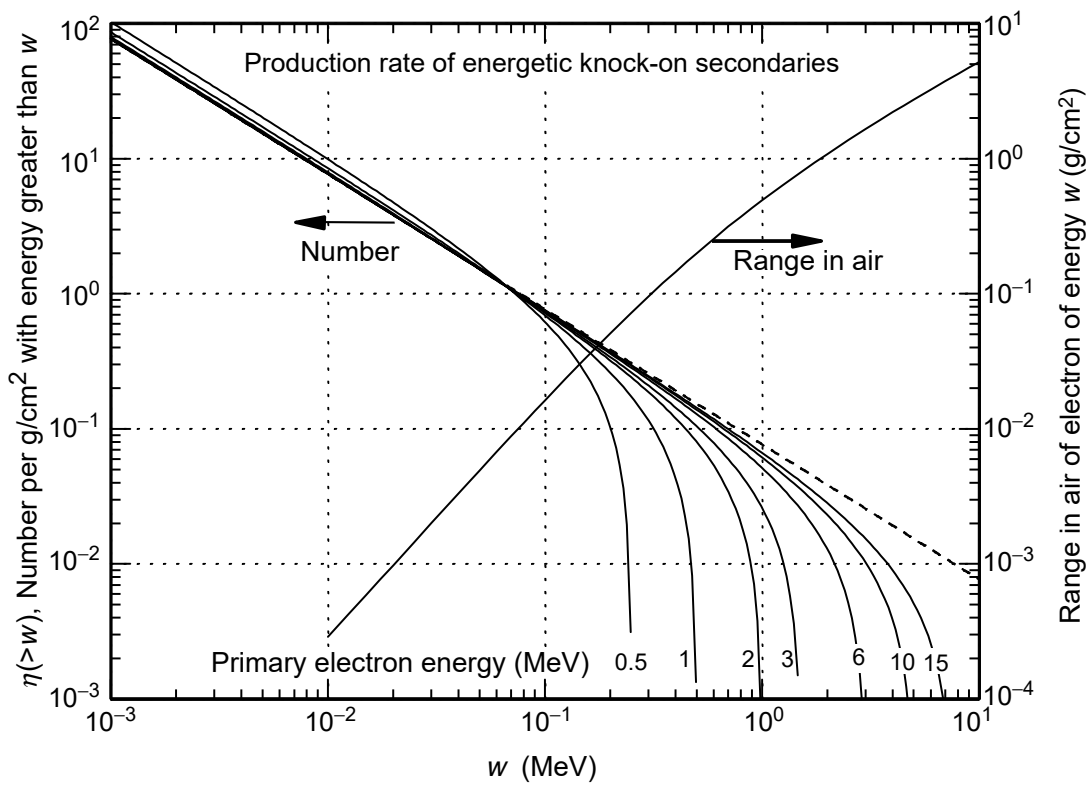

FIGURE 3.12 Number of $\delta$-rays produced per $\mathrm{g} / \mathrm{cm}^{2}$ with energy greater than $w$, per primary electron, in any material.

$\eta(>w)$ is plotted in Figure 3.12. For example, for any primary with $E \gtrsim 1$ $\mathrm{MeV}$, there is about 1 secondary produced per $\mathrm{g} / \mathrm{cm}^{2}$ with energy greater than $0.07 \mathrm{MeV}$, and about 0.2 secondary with energy above $0.3 \mathrm{MeV}$. In the figure, we have kept the correct value of $\beta_{0}$, but have set $Z / A=\frac{1}{2}$, so $N_{g}=N_{A} / 2$. Therefore, the graph applies to any material, but $\eta(>w)$ is slightly too high for heavier elements.

Equation 3.30 shows the number of $\delta$-rays saturates for high incident energies. No matter how energetic, no relativistic electron will produce, per $\mathrm{g} / \mathrm{cm}^{2}$, more than $N_{g} \sigma_{0} m c^{2} / w=0.154(Z / A) / w \approx 0.07 / w \delta$-rays with energy greater than $w(\mathrm{MeV})$ (dashed curve in Figure 3.12).

Consider, as a relevant example, betas streaming through air. The figure also plots on the right axis the CSDA range of electrons of energy $w$ in air. If one asks for $\delta$-rays with range greater than, say, $10 \mathrm{~cm}$ in STP air, the range curve shows their energy must exceed $0.09 \mathrm{MeV}$. The number curves then show that any primary of energy greater than about $0.5 \mathrm{MeV}$ will produce about $0.8 \delta$-rays per $\mathrm{g} / \mathrm{cm}^{2}$ with energy greater than $0.09 \mathrm{MeV}$ and hence with the requested range. Alternatively, per meter of path in sea-level air, any relativistic electron produces about $0.1 \delta$-ray with a range $\gtrsim 10 \mathrm{~cm}$. As discussed briefly, these relatively low-energy $\delta$-rays are ejected at a rather 
large angle. Thus, a narrow beam of relativistic betas in air will be enveloped in a cloud of secondary electrons extending out to many centimeters.

Similarly, the number of $\delta$-rays produced per $\mathrm{g} / \mathrm{cm}^{2}$ with energy between $w_{1}$ and $w_{2}>w_{1}$ is

$$
\begin{aligned}
\eta\left(w_{1}-w_{2}\right) & =\frac{N_{g} \sigma_{0} m c^{2}}{\beta_{0}^{2}}\left(\frac{1}{w_{1}}-\frac{1}{w_{2}}\right) \\
& =\frac{0.07}{\beta_{0}^{2}}\left(\frac{1}{w_{1}}-\frac{1}{w_{2}}\right),
\end{aligned}
$$

with $w$ in $\mathrm{MeV}$.

\subsubsection{Angular Distribution of $\delta$-Rays}

Kinematically, the equal mass collision between the primary and the atomic electron means that both electrons exit in the forward hemisphere, and nonrelativistically, when one exits at polar angle $\theta(0<\theta<\pi / 2)$, the other exits at right angles to it (in the lab frame), at $\theta^{\prime}=\pi / 2-\theta$. The energy of one is $w=E \cos ^{2} \theta$, and the other is $E \cos ^{2} \theta^{\prime}=E \sin ^{2} \theta=E-w$.

For relativistic electrons, however, the $w-\theta$ relation is

$$
w=\frac{E \cos ^{2} \theta}{1+\left(E / 2 m c^{2}\right) \sin ^{2} \theta},
$$

which throws knock-ons more forward than in the nonrelativistic case. $w / E$ vs. $\theta$ is shown in Figure 3.13. In this figure, the lower half, $w / E<0.5$, represents the $\delta$-ray of energy $w$, the upper half the scattered primary of energy $E-w=E(1-w / E)$. The $\delta$-ray always exits at a larger angle and smaller energy than the scattered primary. Nonrelativistically, the secondary always exits at $\theta>45^{\circ}$, but for a relativistic primary both the scattered primary and the secondary $\delta$-ray can exit at $<45^{\circ}$.

For $E=10 \mathrm{MeV}$, for example, if we inquire of a primary that scatters at, say, $12^{\circ}$, the figure shows it has $w_{\text {prim }} / E=0.7$, or is $7 \mathrm{MeV}$. Therefore, the secondary is $w_{\mathrm{sec}}=10-7=3 \mathrm{MeV}$. Taking $w_{\mathrm{sec}} / E=0.3$, the figure shows it exits at $25^{\circ}$. If both exiting electrons have $5 \mathrm{MeV}$, each exits at $17^{\circ}$ relative to the primary. At $10 \mathrm{MeV}, 17^{\circ}$ is the maximum angle at which the scattered primary can exit, and the minimum angle at which the $\delta$-ray can exit.

Similarly, for an $E=1 \mathrm{MeV}$ primary, Figure 3.13 shows that $\delta$-rays of energy $w=0.1 \mathrm{MeV}$ are produced at $65^{\circ}$, and will indeed penetrate to a maximum cylindrical radius of the order of their CSDA range of $10 \mathrm{~cm}$ (multiple scattering will make their average radial extent significantly less).

By inserting Equation 3.28 in Equation 3.26, we obtain the number of $\delta$-rays, per $\mathrm{g} / \mathrm{cm}^{2}$, produced at an angle less than $\theta$. This is shown in 


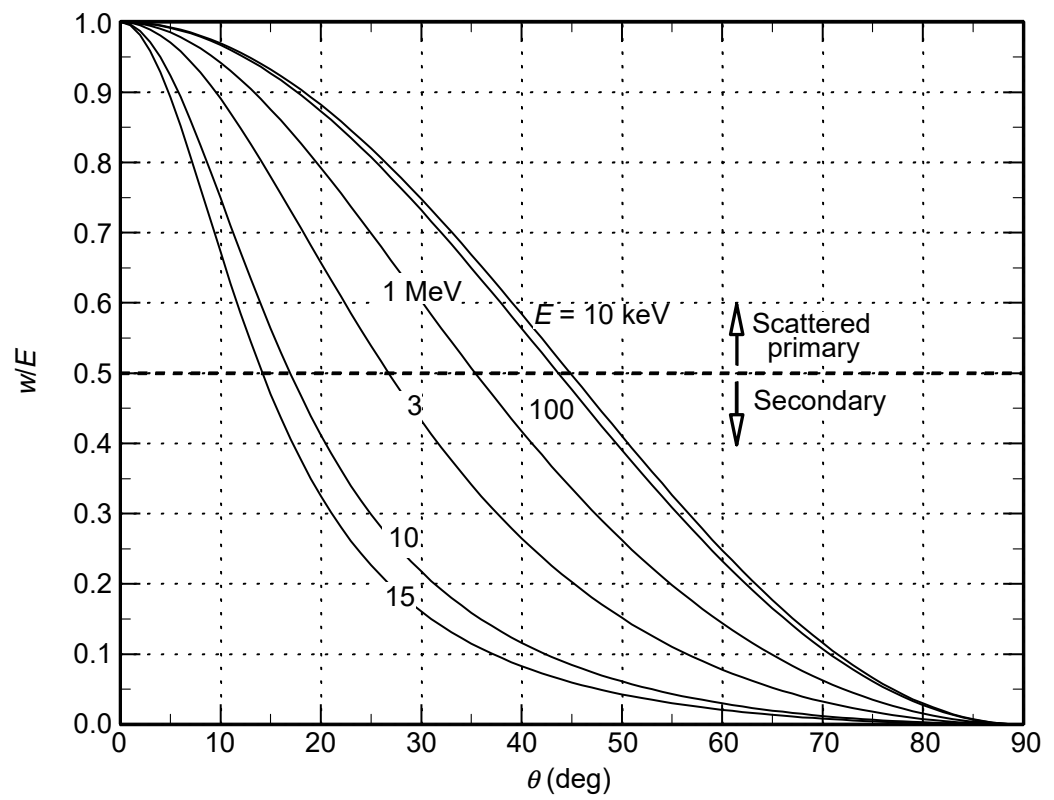

FIGURE 3.13 In electron-electron collision, the energy $w$ of the outgoing particle, primary or secondary, normalized to the incident energy $E$, as a function of the polar angle $\theta$ of the outgoing particle. For example, a $1 \mathrm{MeV}$ primary scattered to $600 \mathrm{keV}$, $w / E=0.6$, exits at $30^{\circ}$, and the $400 \mathrm{keV}$ secondary, $w / E=0.4$, exits at $41^{\circ}$.

Figure 3.14. Except for $Z / A$ differing from $\frac{1}{2}$, it holds for all materials. The figure shows that no matter what the primary energy, and no matter what the material, there is never produced more than $0.1 \delta$-ray per $\mathrm{g} / \mathrm{cm}^{2}$ at less than $45^{\circ}$. Similarly, if one wishes more than $0.01 \delta$-ray at less than $25^{\circ}$, one needs $E \gtrsim 9 \mathrm{MeV}$.

\subsection{RADIATIVE ENERGY LOSS}

When an electron is deflected by scattering from a nucleus or from an atomic electron, it is briefly accelerated and can emit radiation. Its accompanying energy loss is the radiative stopping power of the target material, and is known as Bremsstrahlung. The cross section was first presented by Bethe and Heitler [Be34] in the Born approximation and with the electron described by the Dirac equation. Thorough summaries of the underlying theory are given in [Be53, He54, Ev55, Ja75, Be82].

\subsubsection{Bremsstrahlung Cross Section}

The cross section for producing a photon of energy $k$ is commonly given for the separate cases of a nonrelativistic incident electron and for a relativistic 


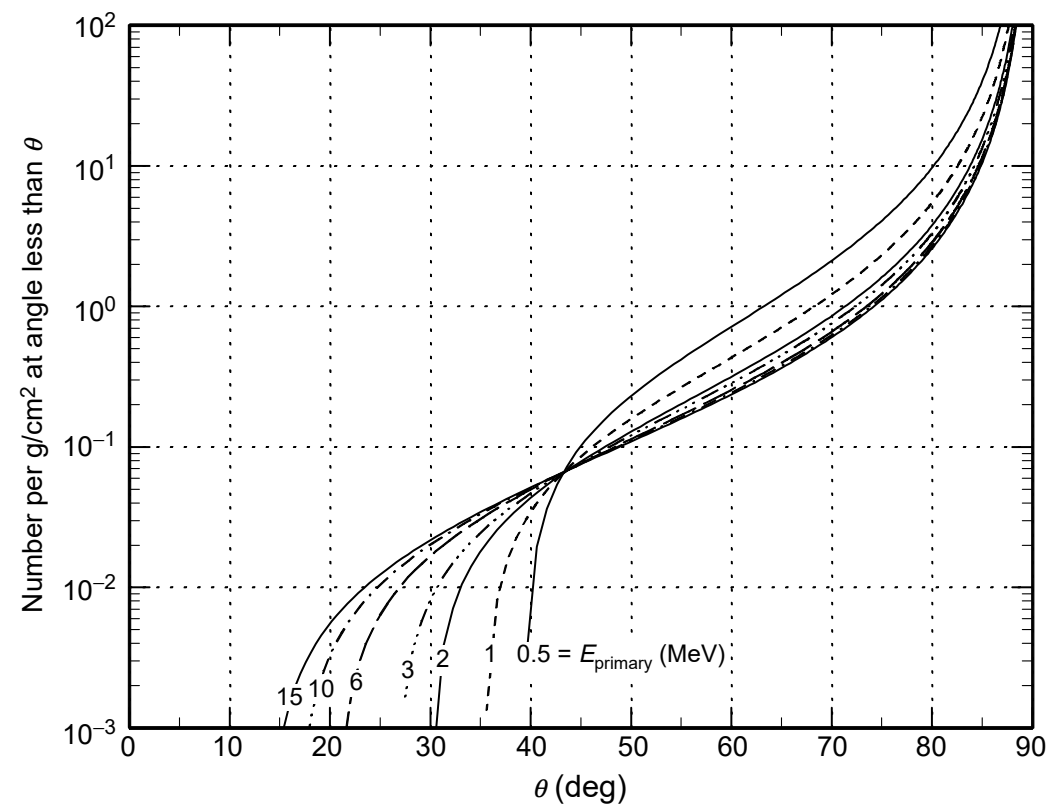

FIGURE 3.14 Number of $\delta$-rays produced, per primary electron per $\mathrm{g} / \mathrm{cm}^{2}$, at polar angle less than $\theta$, in any material.

one. Screening of the nuclear Coulomb field by atomic electrons is important. For the extreme relativistic (E.R.) case with no screening, the cross section for an electron of kinetic energy $E$ to produce a photon in energy interval $(k, k+\mathrm{d} k)$ is

$$
\frac{\mathrm{d} \sigma}{\mathrm{d} k} \mathrm{~d} k=2 \sigma_{B} \frac{\mathrm{d} k}{k}\left[1+\frac{E_{\mathrm{o}}^{\prime 2}}{E_{\mathrm{o}}^{2}}-\frac{2 E_{\mathrm{o}}^{\prime}}{3 E_{\mathrm{o}}}\right]\left[2 \ln \frac{2 E_{\mathrm{o}} E_{\mathrm{o}}^{\prime}}{m c^{2} k}-1\right] \text { (E.R.) }
$$

where $E_{\mathrm{o}}=E+m c^{2}$ is the incident electron total energy, $E^{\prime}=E-k$ is the scattered electron kinetic energy, and $E_{\mathrm{o}}^{\prime}=E^{\prime}+m c^{2}=E_{\mathrm{o}}-k$ is the scattered electron total energy. $\sigma_{B} \equiv \alpha Z^{2} r_{\mathrm{o}}^{2}=Z^{2}\left(e^{2} / m c^{2}\right)^{2} / 137$ is a convenient Bremsstrahlung cross-section unit. The cross section for producing low-energy photons diverges approximately as $1 / k$. In this case of no screening, the energy loss in $\mathrm{d} k, k(\mathrm{~d} \sigma / \mathrm{d} k) \mathrm{d} k$, diverges as $\ln k$, but this divergence no longer occurs when screening is taken into account. Cases in which there is partial or complete screening were separately computed by Bethe and Heitler, and formulas for them are summarized in Bethe and Ashkin [Be53] and Heitler [He54].

In the opposite limit, the cross section for a nonrelativistic (N.R.) electron on an unscreened nucleus is [He54] 


$$
\frac{\mathrm{d} \sigma}{\mathrm{d} k} \mathrm{~d} k=\frac{8}{3} \sigma_{B} \frac{\mathrm{d} k}{k} \frac{m c^{2}}{E} \ln \frac{[\sqrt{E}+\sqrt{E-k}]^{2}}{k} \text { (N.R.) }
$$

Here too, the cross section for producing low-energy photons diverges approximately as $1 / k$, and the energy in photons also diverges as $\ln k$. For both nonrelativistic and extreme relativistic cases, screening by atomic electrons makes the energy transfer for small $k$ finite. However, the $1 / k$ divergence of the cross section for the emission of low-energy photons (the infra-red divergence) persists in the Born approximation, and is resolved only when higher-order corrections are included [He54]. Equation 3.33 and Equation 3.34 together are known as the Bethe-Heitler formula.

Scattering with the emission of Bremsstrahlung may also occur from atomic electrons as well as from the nucleus. It is approximately the same as the previous formulas with $Z=1$. Thus, the full atomic cross section is proportional to $Z(Z+1)$, with minor corrections noted [Be53, He54].

Including screening effects on nuclear Bremsstrahlung, and scattering from atomic electrons, one can present the cross section for producing photons of energy greater than some minimum $k_{\mathrm{m}}$. This is the $k$ integral of $\mathrm{d} \sigma / \mathrm{d} k$ and behaves roughly as $\ln k_{\mathrm{m}}$. The EEDL database tabulates this integrated cross section for $k_{\mathrm{m}}=0.1 \mathrm{eV}$. Figure 3.15 shows it as a function of incident electron kinetic energy $E$ in several elements, with the factor $Z(Z+1)$ scaled out. The maximum photon energy in each case is $E$. At low energies, the $1 / E$ dependence in Equation 3.34 dominates. At energies above the minimum near $1 \mathrm{MeV}$, the cross section increases roughly as $\ln E$, as the upper limit of the integral of Equation 3.33 is $E$.

\subsubsection{Radiative Stopping Power}

For an electron of kinetic energy $E$, the number of Bremsstrahlung photons with energy between $k_{1}$ and $k_{2}$ is approximately proportional to $\int \mathrm{d} k / k \sim$ $\ln \left(k_{2} / k_{1}\right)$. That is, in Bremsstrahlung, there are roughly equal numbers of photons per energy decade (as opposed to equal energy per decade in $\delta$-rays). The distribution of photon energy, $k \mathrm{~d} \sigma / \mathrm{d} k$, is roughly a constant up to maximum energy $E$.

The mean rate at which the electron loses energy to Bremsstrahlung in a target of atom density $N_{g}\left(\mathrm{~g}^{-1}\right)$ is the radiative stopping power, $N_{g} \int_{0}^{E} k(\mathrm{~d} \sigma / \mathrm{d} k) \mathrm{d} k(\mathrm{MeV} \mathrm{cm} / \mathrm{g})$. It is shown in Figure $3.16 \mathrm{a}$ as a function of electron energy $E$ for several elements, and in Figure $3.16 \mathrm{~b}$ as a function of atomic number at selected energies. Contours for all $E$ and $Z$ are in Figure 3.17. Also in Figure 3.17 are contours (dot-dash) of the fraction of radiative to total stopping power. The critical energy corresponds to the curve labeled 0.5 . It is greater than $100 \mathrm{MeV}$ for $Z \lesssim 7$, and less than $10 \mathrm{MeV}$ for $Z \gtrsim 80$. 


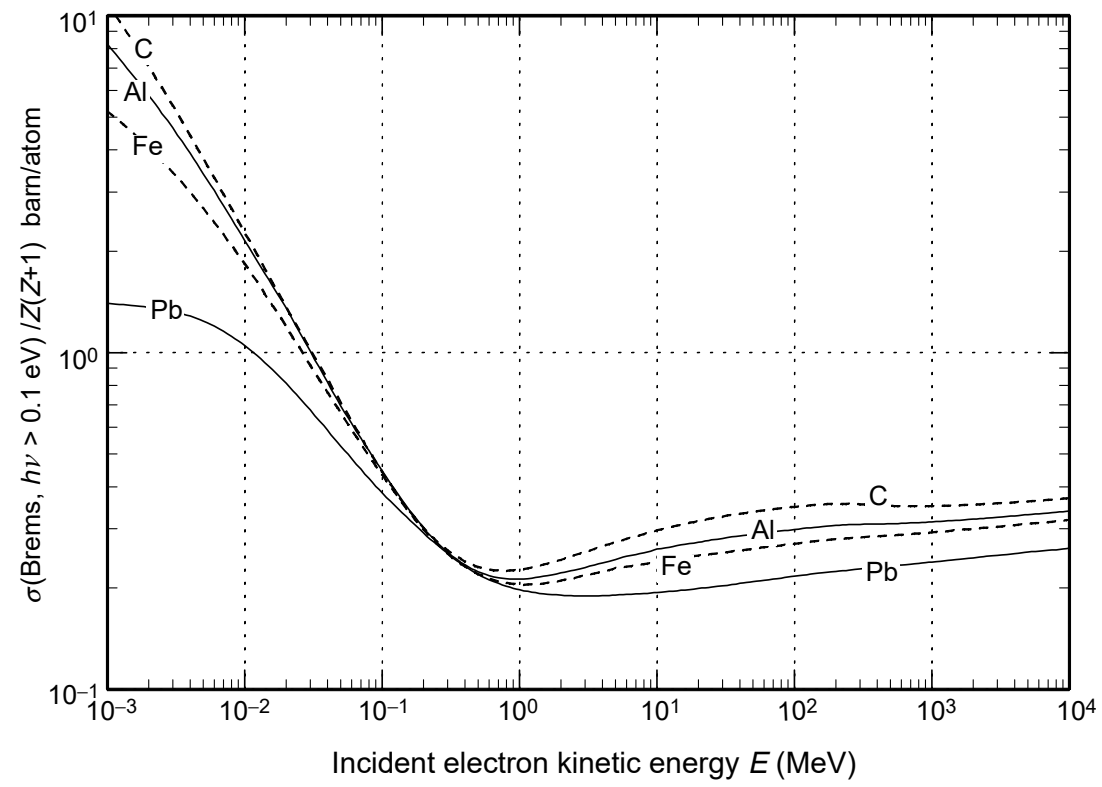

FIGURE $3.151 / Z(Z+1)$ times the cross section for producing Bremsstrahlung photons with energy greater than $0.1 \mathrm{eV}$. Maximum photon energy in each case is $E$. (Data from EEDL.)

For relativistic electrons, Equation 3.33 when integrated on $k \mathrm{~d} k$ shows that the unscreened, nuclear radiative stopping power is approximately proportional to the electron energy $E_{\mathrm{o}} \approx E$. Thus, the energy loss rate is proportional to the energy, $\mathrm{d} E / \mathrm{d} s=-E / X_{\mathrm{o}}$, and the energy drops a factor $e$ in a fixed distance. That distance is called the radiation length, usually denoted by $X_{\mathrm{o}}$. It is given approximately by [Be53]

$$
\frac{1}{X_{\mathrm{o}}}=4 N_{g} \alpha Z(Z+1) r_{\mathrm{o}}^{2} \ln \frac{183}{Z^{1 / 3}}
$$

It is an important parameter for extreme relativistic electrons, in which almost all the energy loss is to Bremsstrahlung. $X_{\mathrm{o}}$ is about $36 \mathrm{~g} / \mathrm{cm}^{2}$ in air or water, and about $5.8 \mathrm{~g} / \mathrm{cm}^{2}$ in $\mathrm{Pb}$. Thus, a $1 \mathrm{GeV}$ electron beam is reduced to about $370 \mathrm{MeV}$ in $285 \mathrm{~m}$ sea-level air, or in $36 \mathrm{~cm}$ water, or in $0.5 \mathrm{~cm} \mathrm{~Pb}$.

Per atom, the Bremsstrahlung cross section and energy loss are roughly proportional to $Z(Z+1)$, whereas collisional energy loss is $\propto Z$. As the atom density per gram, $N_{A} / A$, is roughly proportional to $1 / Z$, Bremsstrahlung energy loss in bulk matter, when expressed per $\mathrm{g} / \mathrm{cm}^{2}$, is more nearly proportional to $Z$, and collisional energy loss is roughly independent of $Z$. 
(a)
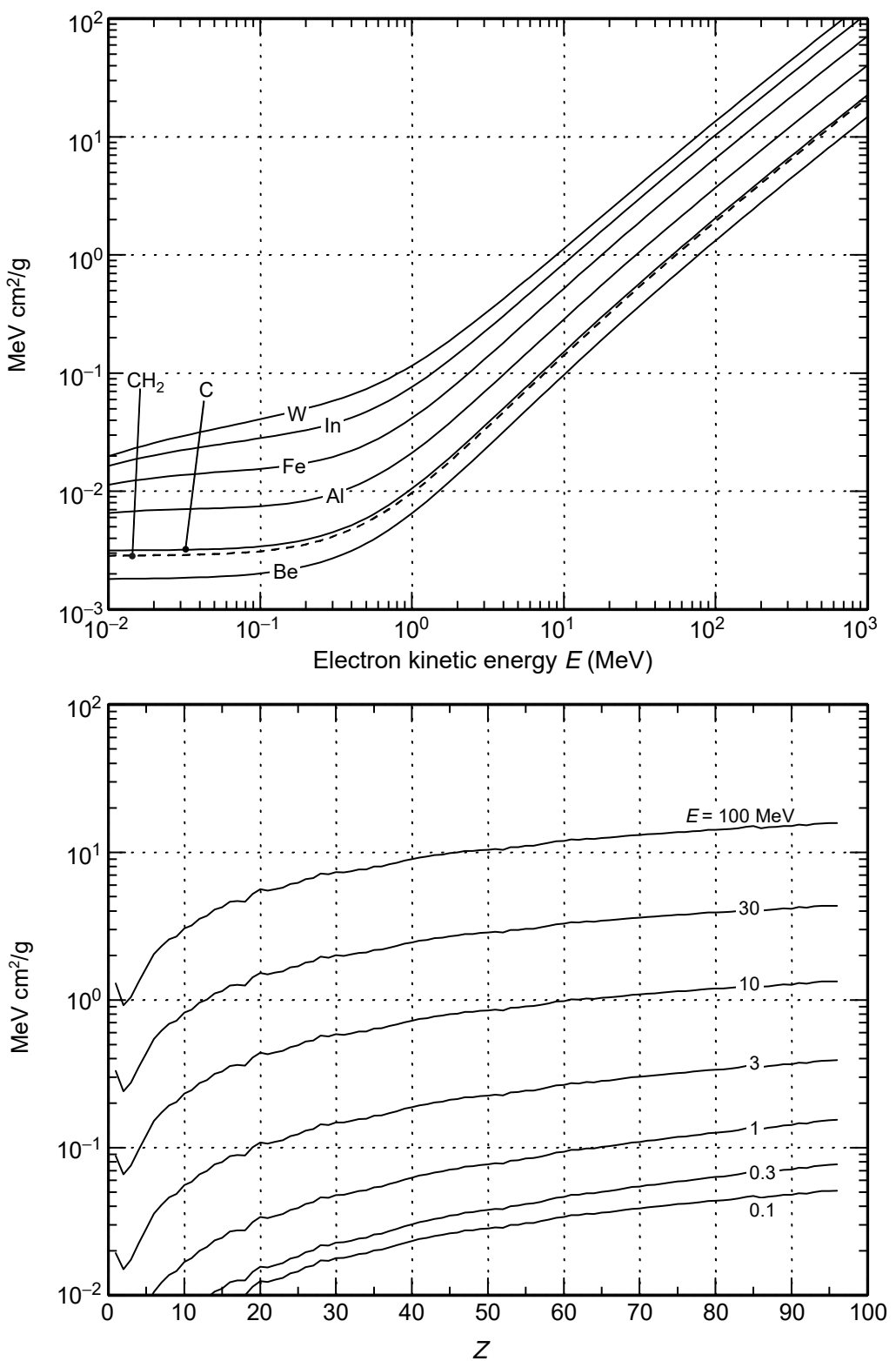

FIGURE 3.16 (a) Electron mean radiative stopping power for several elements; (b) vs. $Z$ for selected energies. (Data from [NIST].) 


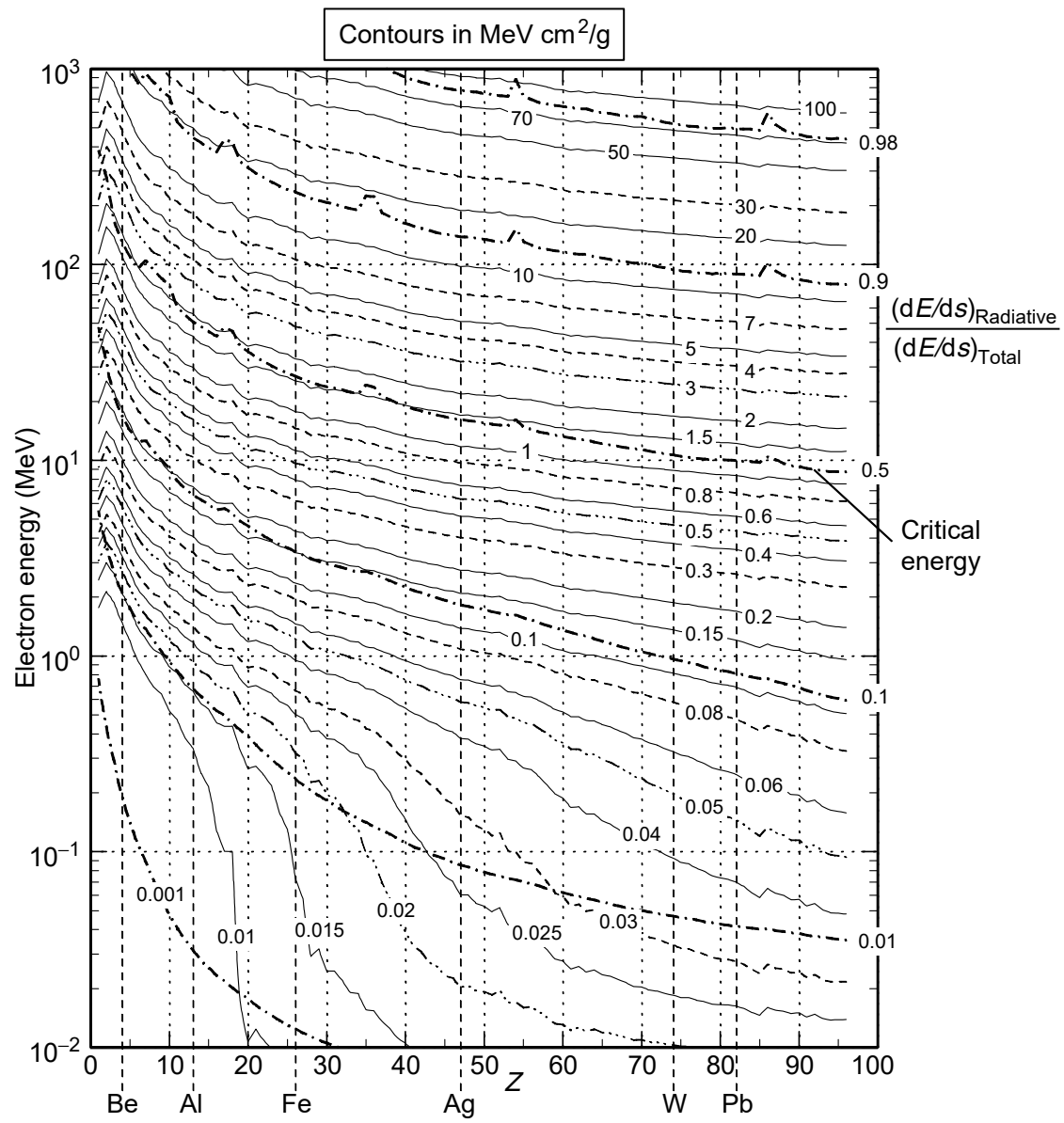

FIGURE 3.17 Electron mean radiative stopping power contours in the elements. Superimposed in heavy dot-dashed lines are contours of its fraction of total stopping power. (Data from [NIST].)

In Bremsstrahlung off a nucleus, momentum need not be conserved between the incident electron and the outgoing electron plus photon. The recoiling nucleus can take up any amount of momentum with essentially no energy penalty.

\subsubsection{Energy and Number Distribution of Bremsstrahlung Photons}

As with knock-on secondary electrons, the average number of photons produced per $\mathrm{g} / \mathrm{cm}^{2}$ and their energy distribution, or at least their average energy, is of interest in radiative energy loss. Their energy distribution is 
discussed in [Be53] and [He54]. Because of the infrared divergence, their average number is infinite, their average energy zero, the product finite, such that the energy lost from the primary electron to photons is finite.

With $k$ the Bremsstrahlung photon energy, let $\mathrm{d} \sigma / \mathrm{d} k$ be the differential (in photon energy) cross section (nuclear and electronic) on an atom, given in first approximation by Equation 3.33 or Equation 3.34. At low energies, $k \longrightarrow 0, \mathrm{~d} \sigma / \mathrm{d} k$ diverges as $1 / k$. There are approximately equal numbers of photons per decade of $k$, and therefore, most radiative energy loss goes into relatively few energetic photons. The distribution of energy in photons, $k \mathrm{~d} \sigma / \mathrm{d} k$, is only a slowly varying function of $k$.

The full energy spectrum of Bremsstrahlung is given by Heitler [He54, $\S 25]$, and is tabulated for all elements in [Se86]. Figure 3.18 shows how $k \mathrm{~d} \sigma / \mathrm{d} k$ differs from a constant for $\mathrm{Be}, \mathrm{Al}$, and $\mathrm{Pb}$, for $1 \mathrm{MeV}, 5 \mathrm{MeV}$, and $10 \mathrm{MeV}$ electrons.

The number of photons with energy greater than $k$, or between $k_{1}$ and $k_{2}$, and their average energies are of interest. The radiative energy loss of an electron of kinetic energy $E$ is

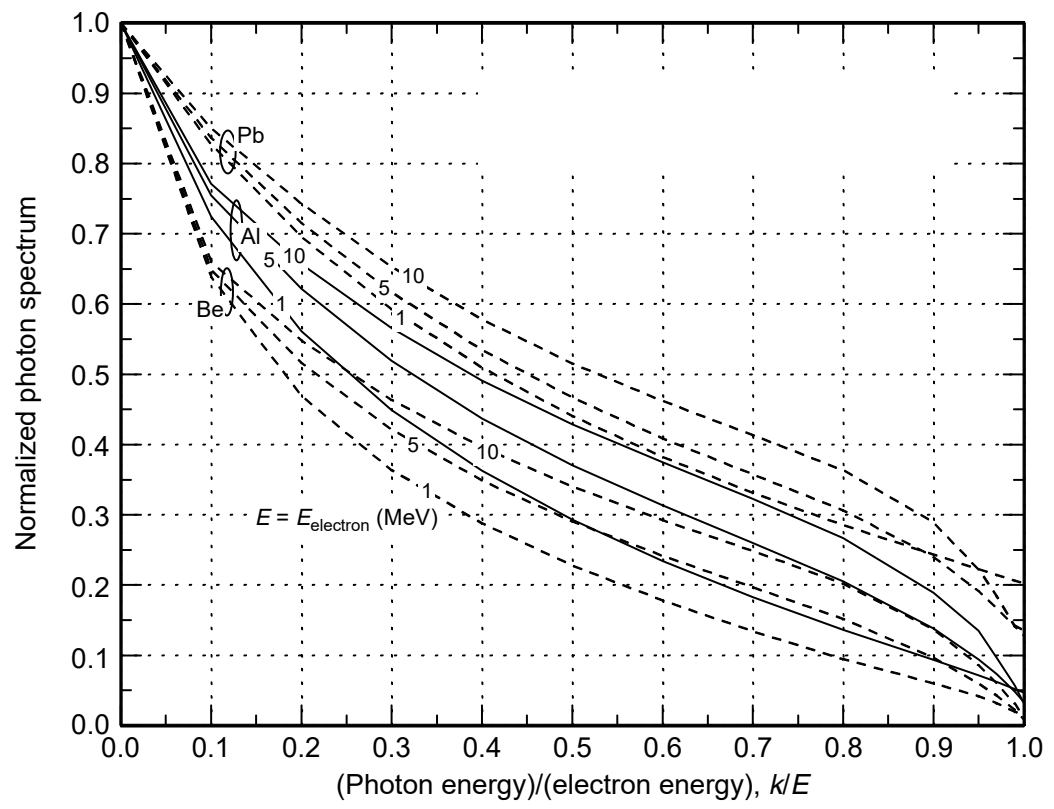

FIGURE 3.18 Bremsstrahlung energy spectra data for $\mathrm{Be}, \mathrm{Al}$, and $\mathrm{Pb}$ at incident electron energies $1 \mathrm{MeV}, 5 \mathrm{MeV}$, and $10 \mathrm{MeV}$. Plotted is $k \mathrm{~d} \sigma / \mathrm{d} k$ normalized to its value at $k=0$; only the shape is significant in the figure. (Data from [Se86].) 


$$
L_{\mathrm{rad}}(E) \equiv\left(\frac{\mathrm{d} E}{\mathrm{~d} s}\right)_{\text {radiative }}=N_{g} \int_{0}^{E} k \frac{\mathrm{d} \sigma}{\mathrm{d} k} \mathrm{~d} k,
$$

where $N_{g}$ is the number of target atoms per gram, and is the quantity plotted in Figure 3.16 and Figure 3.17. Radiation to photons of energy greater than $k$, $L_{\text {rad }}(>k)$, is given by the same expression with the lower limit replaced by $k$,

$$
L_{\mathrm{rad}}(>k)=N_{g} \int_{k}^{E} k \frac{\mathrm{d} \sigma}{\mathrm{d} k} \mathrm{~d} k
$$

The number of photons emitted per $\mathrm{g} / \mathrm{cm}^{2}$ with energy in $(k, k+\mathrm{d} k)$ is $(\mathrm{d} \eta / \mathrm{d} k)$. $\mathrm{d} k=N_{g} \mathrm{~d} \sigma / \mathrm{d} k \cdot \mathrm{d} k$, whereas the number with energy greater than $k$ is

$$
\eta(>k)=N_{g} \int_{k}^{E} \frac{\mathrm{d} \sigma}{\mathrm{d} k} \mathrm{~d} k .
$$

To generate useful plots, we now approximate the $k$ dependence of $\mathrm{d} \sigma / \mathrm{d} k$. Figure 3.18 shows $k \mathrm{~d} \sigma / \mathrm{d} k$ is more nearly proportional to $(1-k / E)$ than independent of $k$, so we set

$$
k \mathrm{~d} \sigma / \mathrm{d} k=[k \mathrm{~d} \sigma / \mathrm{d} k]_{0}(1-k / E) .
$$

Using this in Equation 3.36, we obtain its value at $k=0$

$$
\left[N_{g} k \mathrm{~d} \sigma / \mathrm{d} k\right]_{0}=\frac{2 L_{\mathrm{rad}}(E)}{E}
$$

in terms of the known radiative energy loss rate. The remaining integrals in Equation 3.37 and Equation 3.38 can now be done. The loss rate to photons of energy greater than $k$ is

$$
L_{\mathrm{rad}}(>k)=\left(1-\frac{k}{E}\right)^{2} L_{\mathrm{rad}}(E)
$$

The number of photons produced per $\mathrm{g} / \mathrm{cm}^{2}$ with energy in $(k, k+\mathrm{d} k)$ is

$$
\frac{\mathrm{d} \eta(k)}{\mathrm{d} k} \mathrm{~d} k=N_{g} \frac{\mathrm{d} \sigma}{\mathrm{d} k} \mathrm{~d} k=\frac{2 L_{\mathrm{rad}}}{E}\left(1-\frac{k}{E}\right) \frac{\mathrm{d} k}{k}
$$

while the number with energy greater than $k$ is

$$
\eta(>k)=\frac{2 L_{\mathrm{rad}}(E)}{E}[\ln (E / k)-(1-k / E)]
$$


In Figure 3.19a, we plot $\eta(>k)$ for $\mathrm{Al}$ and $\mathrm{Pb}$, using tabulated values for $L_{\mathrm{rad}}(E)$. For example, in $\mathrm{Al}$, for a $10 \mathrm{MeV}$ primary electron, there are about 0.32 Bremsstrahlung photons produced per $\mathrm{g} / \mathrm{cm}^{2}$ with energy greater than 10 $\mathrm{keV}$, but only 0.03 with energy greater than $3 \mathrm{MeV}$.

Similarly, the number of Bremsstrahlung photons produced per $\mathrm{g} / \mathrm{cm}^{2}$ with energy between $k_{1}$ and $k_{2}>k_{1}$ is

$$
\eta\left(k_{1}-k_{2}\right)=\frac{2 L_{\mathrm{rad}}(E)}{E}\left[\ln \left(k_{2} / k_{1}\right)-\frac{k_{2}-k_{1}}{E}\right] .
$$

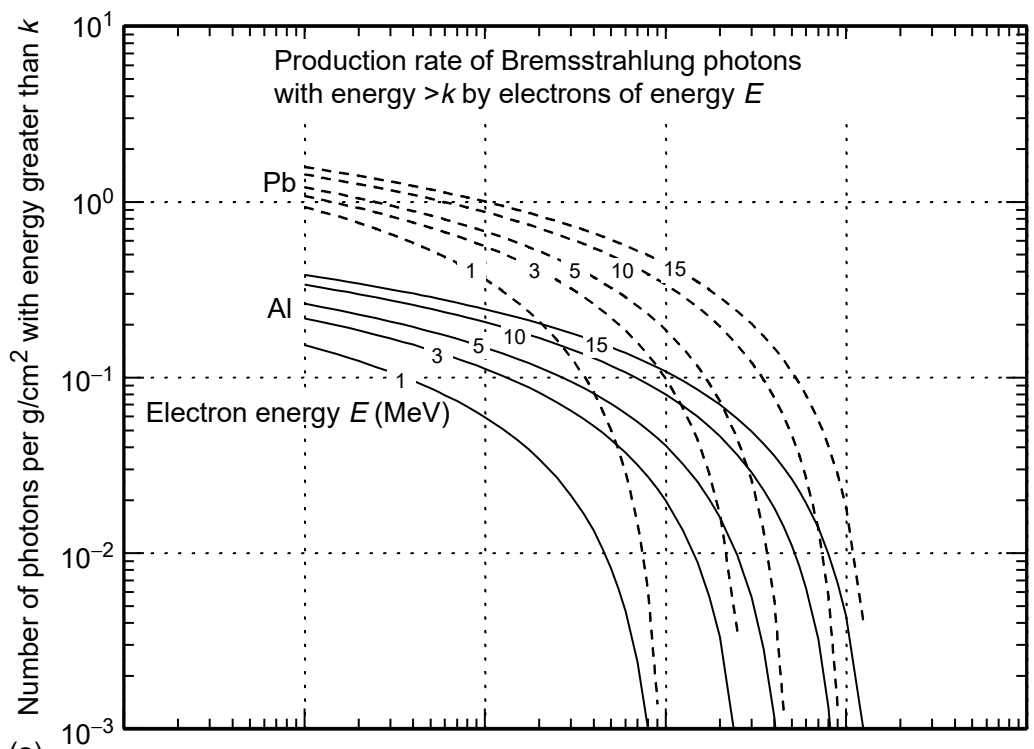

(a)

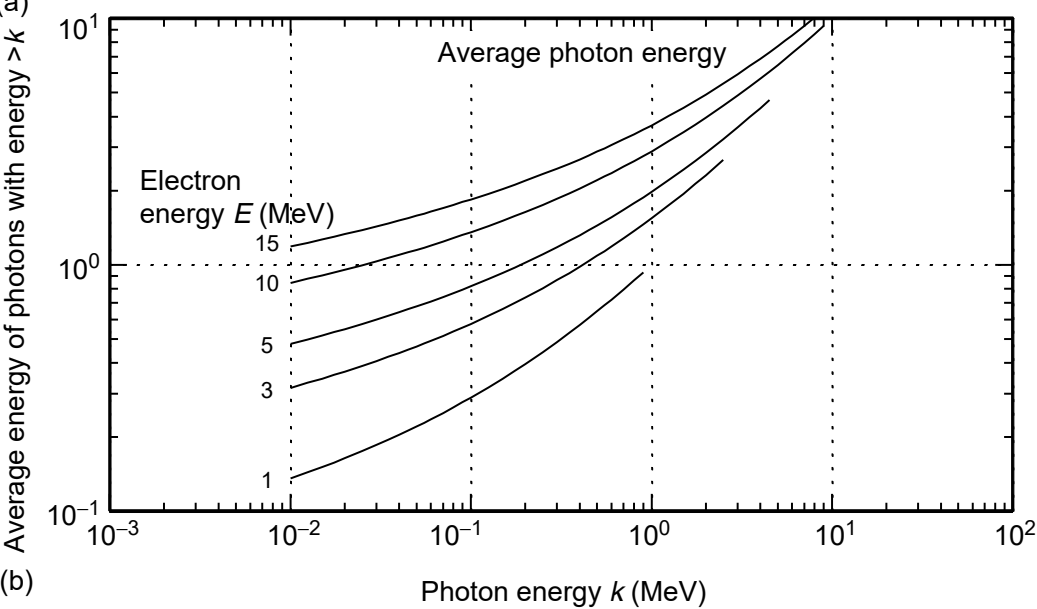

FIGURE 3.19 (a) Number of Bremsstrahlung photons with energy greater than $k$, produced per $\mathrm{g} / \mathrm{cm}^{2}$ in $\mathrm{Al}$ and $\mathrm{Pb}$. (b) Average energy of photons so produced. 
The average energy $\bar{k}$ of photons whose energy is greater than $k$ is

$$
\bar{k}=\frac{L_{\mathrm{rad}}(>k)}{\eta(>k)}=\frac{E}{2} \frac{(1-k / E)^{2}}{\ln (E / k)-(1-k / E)} .
$$

independent of material. $\bar{k}$ is shown in Figure $3.19 \mathrm{~b}$. For a $10 \mathrm{MeV}$ electron in any material, the average energy of photons with energy greater than $3 \mathrm{MeV}$ is $5 \mathrm{MeV}$.

\subsubsection{Bremsstrahlung Energy Spectrum Data}

Detailed measurements and comparisons of theories have been done by Seltzer and Berger for the energy spectrum of Bremsstrahlung photons in all elements [Se86]. Figure 3.18 plots a selected set of those data for Be, $\mathrm{Al}$, and $\mathrm{Pb}$ at three electron energies. In the figure, we have divided the tabulated spectra by their value at $k=0$, and plotted these normalized spectra.* Equation 3.40 shows how the value of $k \mathrm{~d} \sigma / \mathrm{d} k$ at $k=0$ is related to the known radiative energy loss rate under the approximation $k \mathrm{~d} \sigma / \mathrm{d} k=$ $[k \mathrm{~d} \sigma / \mathrm{d} k]_{0}(1-k / E)$. Figure 3.18 shows that the dependence is not exactly $(1-k / E)$. However, for purposes of estimating Bremsstrahlung from energetic electrons, the curves as presented in Figure 3.19 using the approximate $(1-k / E)$ dependence are quite satisfactory.

\subsubsection{Angular Distribution of Bremsstrahlung Photons}

Because of momentum take-up by the recoiling nucleus, the incident electron, the photon, and the outgoing electron need not all lie in one plane. The angular distribution of photons emitted in Bremsstrahlung is therefore complicated. It simplifies for relativistic energies, which is usually the case of interest [Be53, Section 2.B.3]. Most of the quanta emitted by an electron of high-energy $E$ are emitted at small angles. The average angle of emission is about

$$
\theta_{\text {ave }}=\frac{m c^{2}}{E+m c^{2}},
$$

independent of the energy of the emitted photon. The larger $E$, the more forward-directed are all photons.

\subsubsection{Radiative Yield}

The radiative yield $Y_{\mathrm{R}}$ is the fraction of an electron's initial energy that is lost to radiation by the time the electron has come to rest. The remainder is lost to

\footnotetext{
*Tables in [Se86] give the scaled cross section $\left(\beta^{2} / Z^{2}\right) k \mathrm{~d} \sigma / d k(\beta=v / c$ of the incident electron).
} As $k \rightarrow 0$, these have various values, not near unity, for different elements and for different $E$. 
collisional excitation and ionization. The energy lost to radiation of an electron of kinetic energy $E$ is

$$
E_{\mathrm{rad}}=\int\left(\frac{\mathrm{d} E}{\mathrm{~d} s}\right)_{\mathrm{rad}} \mathrm{d} s=\int \frac{(\mathrm{d} E / \mathrm{d} s)_{\mathrm{rad}}}{(\mathrm{d} E / \mathrm{d} s)_{\mathrm{Tot}}} \mathrm{d} E=\int_{0}^{E} \frac{L_{\mathrm{rad}}\left(E^{\prime}\right)}{L_{\mathrm{Tot}}\left(E^{\prime}\right)} \mathrm{d} E^{\prime},
$$

and the radiative yield is $Y_{\mathrm{R}}=E_{\mathrm{rad}} / E$. Here, $L_{\mathrm{rad}}=(\mathrm{d} E / \mathrm{d} s)_{\mathrm{rad}}$ is the radiative stopping power, Equation 3.36, and $L_{\mathrm{Tot}}=(\mathrm{d} E / \mathrm{d} s)_{\mathrm{Tot}}=(\mathrm{d} E / \mathrm{d} s)_{\mathrm{rad}}+$ $(\mathrm{d} E / \mathrm{d} s)_{\text {coll }}$ is the total stopping power.

$Y_{\mathrm{R}}$ is shown in Figure 3.20a for several elements vs. $E$, and in Figure $3.20 \mathrm{~b}$ at selected energies as a function of $Z$. Contours of $Y_{R}$ for all $E$ and $Z$ are shown in Figure 3.21. A $10 \mathrm{MeV}$ electron entering aluminum, for example, at first loses energy to radiation at a rate of about $0.28 \mathrm{MeV} \mathrm{cm}^{2} / \mathrm{g}$ (Figure $3.16 a$ ), about $15 \%$ of its total loss rate (collisions plus Bremsstrahlung). As it slows down, Bremsstrahlung becomes a smaller fraction of its stopping power. By the time it reaches the end of its range $\left(\sim 6 \mathrm{~g} / \mathrm{cm}^{2}\right)$, a total of only $0.75 \mathrm{MeV}$ (7.5\%) has been lost to photons (Figure 3.20a) and $9.25 \mathrm{MeV}$ to collisions.

\subsubsection{Photon Number Yield}

We have discussed the rate of production of photons of a given energy when the electron has energy $E$. The number of photons produced in energy interval $(k, k+\mathrm{d} k)$, per $\mathrm{g} / \mathrm{cm}^{2}$ traversed, is $(\mathrm{d} \eta(k) / \mathrm{d} k) \mathrm{d} k$. As the electron slows down and comes to rest, the cumulative sum of these photons is the photon number yield $Y_{\mathrm{n}}$.

In Chapter 2 we discussed the creation of an electron flux by a photon flux by photoelectric absorption, Compton scattering, and pair production (the $1 \%$ rule). Electron radiative energy loss gives rise to the opposite process: creation of a photon flux by an electron flux via Bremsstrahlung. The photon production rate is given in Equation 3.42. As the Bremsstrahlung spectrum diverges for $k \longrightarrow 0$, one can speak only of the number of photons of energy greater than a specified value.

Let $\mathrm{d} n / \mathrm{d} s=\eta(>k)$ be the number of photons with energy greater than $k$ produced per $\mathrm{g} / \mathrm{cm}^{2}$ at electron energy $E$. Then the yield of photons of energy greater than $k$ from an electron that has come to rest starting with initial kinetic energy $E$ is

$$
Y_{\mathrm{n}}(>k)=\int \mathrm{d} n=\int \frac{\mathrm{d} n}{\mathrm{~d} s} \mathrm{~d} s=\int \frac{\mathrm{d} n / \mathrm{d} s}{\mathrm{~d} E / \mathrm{d} s} \mathrm{~d} E=\int_{k}^{E} \frac{\eta(>k)}{L_{\mathrm{Tot}}\left(E^{\prime}\right)} \mathrm{d} E^{\prime},
$$

where $\eta(>k)$ is given in general by Equation 3.38 and in our approximate fit by Equation 3.43. Using the fit, $Y_{\mathrm{n}}$ is plotted in Figure 3.22 for Al. The upper panel (a) shows the total number of photons $Y_{\mathrm{n}}(>k)$ with energy greater than 
(a)
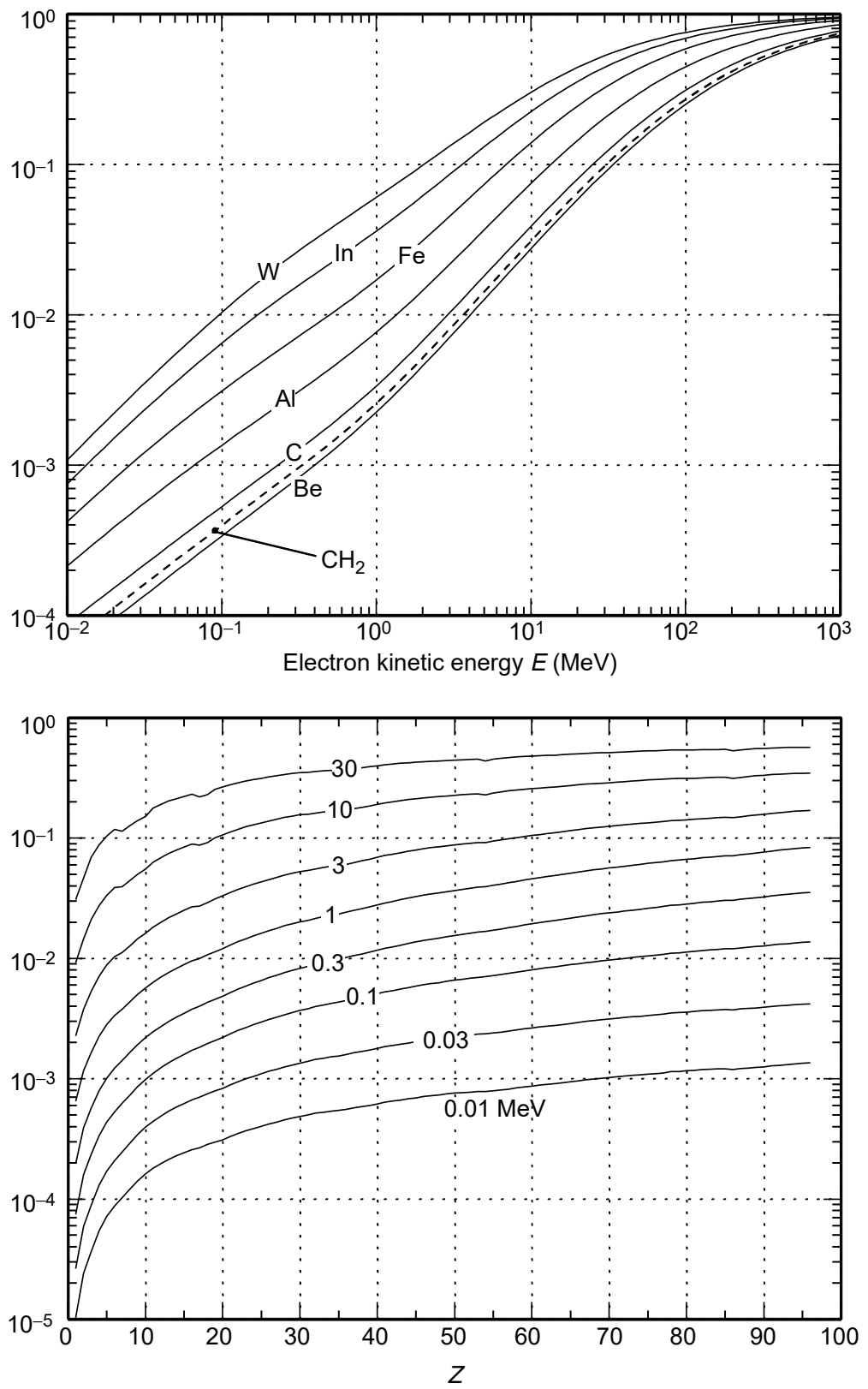

FIGURE 3.20 (a) Electron radiative yield vs. $E$ for several elements; (b) vs. atomic number $Z$ for selected energies. 


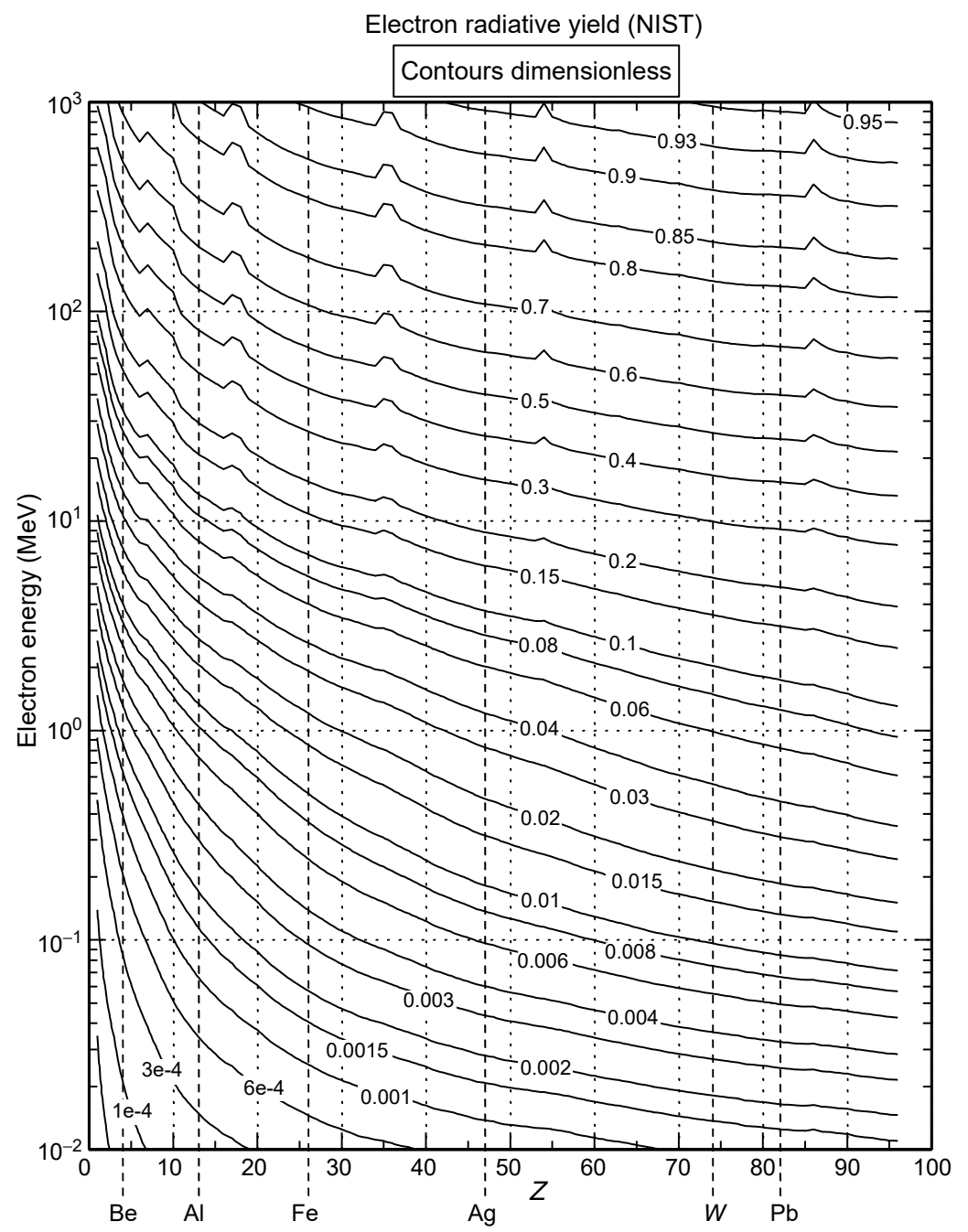

FIGURE 3.21 Contours of electron radiative yield for all elements.

$k$, for selected initial electron energies. The lower panel (b) shows the same quantity as a function of electron energy, for selected values of $k$. By the time it comes to rest, a $10 \mathrm{MeV}$ electron in $\mathrm{Al}$ has emitted about 0.22 photons of energy greater than $1 \mathrm{MeV}$.

The average energy of these photons is also of interest and is shown in Figure 3.23. The 0.22 photons of energy greater than $1 \mathrm{MeV}$ from an initial 10 $\mathrm{MeV}$ electron have an average energy of $2.3 \mathrm{MeV}$. 

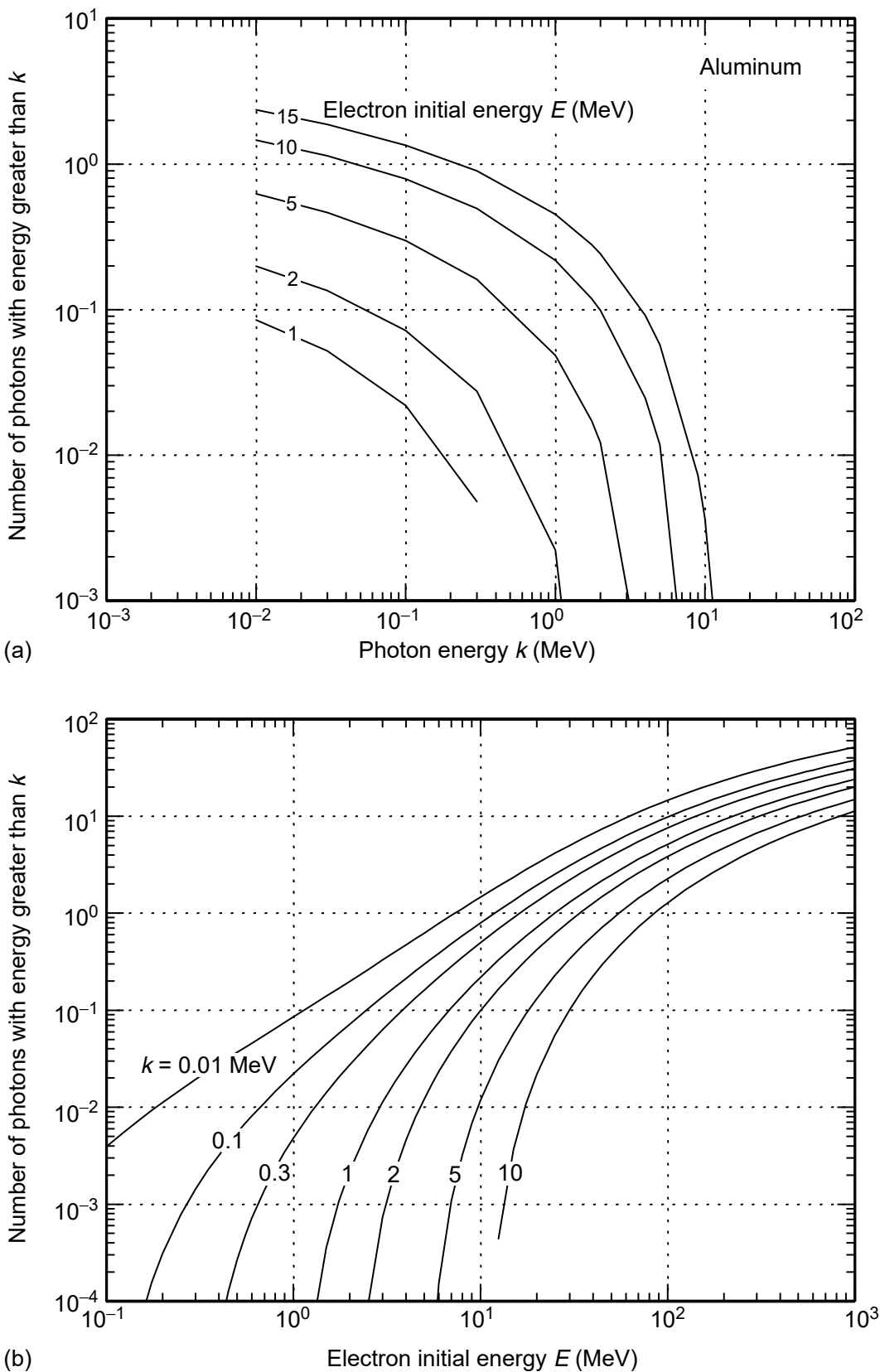

FIGURE 3.22 Bremsstrahlung photon number yield in Al. (a) Number of photons with energy greater than $k$ vs. $k$ for selected electron energies $E$; (b) vs. $E$ for selected $k$. 


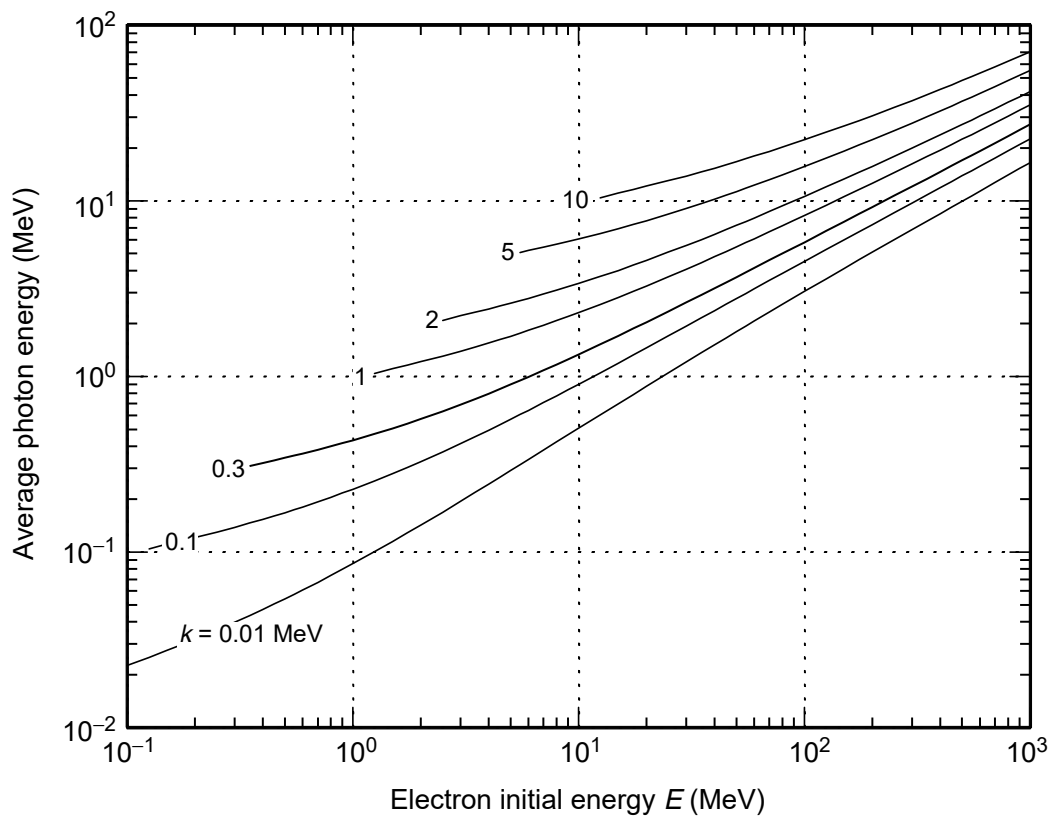

FIGURE 3.23 In the radiative yield of electrons of energy $E$, the average energy of those photons whose energy is greater than $k$.

Likewise, the number yield of photons in energy interval $(k, k+\mathrm{d} k)$ is

$$
\begin{aligned}
y_{\mathrm{n}}(k) \mathrm{d} k & =-\frac{\mathrm{d} Y_{\mathrm{n}}(>k)}{\mathrm{d} k} \mathrm{~d} k=\mathrm{d} k \int_{k}^{E} \frac{\mathrm{d} E^{\prime}}{L_{\mathrm{Tot}}\left(E^{\prime}\right)} \frac{\mathrm{d} \eta(k)}{\mathrm{d} k} \\
& =\frac{\mathrm{d} k}{k} \int_{k}^{E} \frac{\mathrm{d} E^{\prime}}{E^{\prime}} \frac{L_{\mathrm{rad}}\left(E^{\prime}\right)}{L_{\mathrm{Tot}}\left(E^{\prime}\right)} 2\left(1-k / E^{\prime}\right)
\end{aligned}
$$

For a given material, approximations or fits of $L_{\mathrm{rad}}(E) / L_{\mathrm{Tot}}(E)$ as a function of $E$ will permit a simple expression for $y_{\mathrm{n}}(k)$. For example, over a wide energy range in $\mathrm{Al}$, the ratio of radiative to total stopping powers is roughly proportional to electron energy $E$ :

$$
\frac{1}{E} \frac{L_{\mathrm{rad}}(E)}{L_{\mathrm{Tot}}(E)} \approx 0.015 \mathrm{MeV}^{-1}, \quad \mathrm{Al}, 0.1 \mathrm{MeV}<E<30 \mathrm{MeV}
$$

In $\mathrm{Be}$, this ratio is roughly constant at $0.005 \mathrm{MeV}^{-1}$ over an even wider range, $0.05 \mathrm{MeV} \lesssim E \lesssim 100 \mathrm{MeV}$, indicating that Be has a photon number yield only about one-third that of $\mathrm{Al}$ over these energies. With fits like these, one can 
obtain an approximate expression for the differential photon number yield over interesting energies.

\subsubsection{Example of the Utility of Photon Number Yield: Penetration of an Aluminum Slab}

Photon number yield $Y_{\mathrm{n}}(k)$ is discussed in relation to shower theory for extremely energetic electrons (Ro41, Be53). Here we discuss an example of its utility for less energetic electrons.

Electrons are stopped in $\mathrm{Al}$ after they have traveled about one range against total mean stopping power. If the $\mathrm{Al} \mathrm{slab}$ is thicker than the range, those incident electrons cannot penetrate to the other side.

However, energetic electrons can transfer their energy to Bremsstrahlung photons, which penetrate further than the electrons themselves, and can convert back to other electrons by Compton scattering. These second-generation electrons can then exit the far side of the Al slab. In this way, electrons can exit the far side even if the slab is thicker than the primary electron range.

Here we show a simple, approximate, analytic way to understand the process, and obtain the rough dependence on the primary electron energy.

\subsubsection{Aluminum Parameters}

Figure 3.24 shows the mean forward range of electrons in $\mathrm{Al}$ (calculation of the mean forward range is discussed later; Figure 3.30a in Section 3.8 is their CSDA range). Figure 2.55 shows the $\mathrm{mfp}$ of photons. A $5 \mathrm{MeV}$ electron, for instance, has a mean forward range of about $R_{\mathrm{mf}}=1.8 \mathrm{~g} / \mathrm{cm}^{2}$ and a maximum (CSDA) range of about $R=3 \mathrm{~g} / \mathrm{cm}^{2}$. The $\mathrm{mfp}$ of a $1 \mathrm{MeV}$ photon is $\sim 16 \mathrm{~g} / \mathrm{cm}^{2}$, much greater than $R$.

Thus in a thick slab, we have the picture sketched in Figure 3.25. Monoenergetic electrons entering from the left with kinetic energy $E_{\mathrm{o}}$ penetrate to a mean depth equal to their mean forward range $R_{\mathrm{mf}}\left(E_{\mathrm{o}}\right)$, and a maximum depth equal to their CSDA range $R$. Along the way, photons of energy less than $E_{\mathrm{o}}$ are emitted. The flux of photons builds up out to $R\left(E_{\mathrm{o}}\right)$. After that, only the photons continue, where they slowly, continuously interact by the photoelectric effect, by Compton scattering, and by pair production, ejecting new electrons. At electron energies usually of interest, Compton scattering is the dominant process.

Bremsstrahlung produces photons of energy from 0 to $E_{\mathrm{o}}$. We need not be concerned with those low-energy photons, the mfp (against the photoeffect) in $\mathrm{Al}$ of which is short compared with $R\left(E_{\mathrm{o}}\right)$, for they will be converted in a short distance to low-energy electrons, the range of which is also small compared with $R\left(E_{\mathrm{o}}\right)$ and hence will not contribute to the secondary electron flux at an interesting depth. 


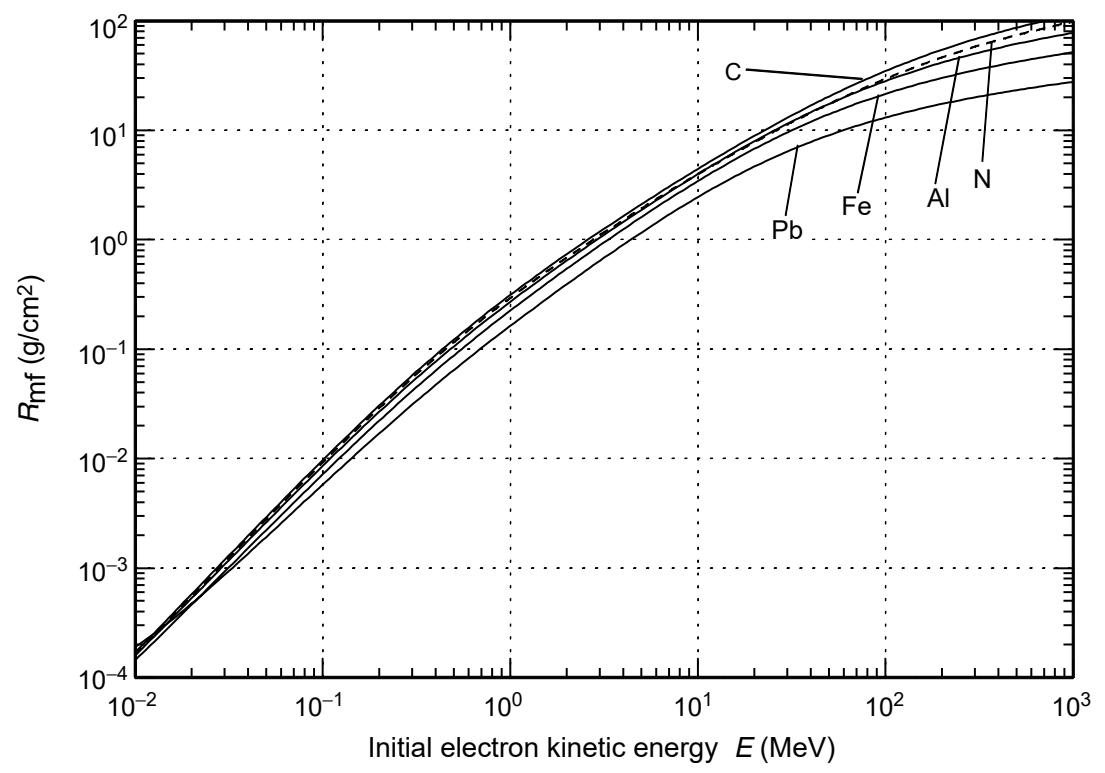

FIGURE 3.24 Approximate electron mean forward range in several elements.

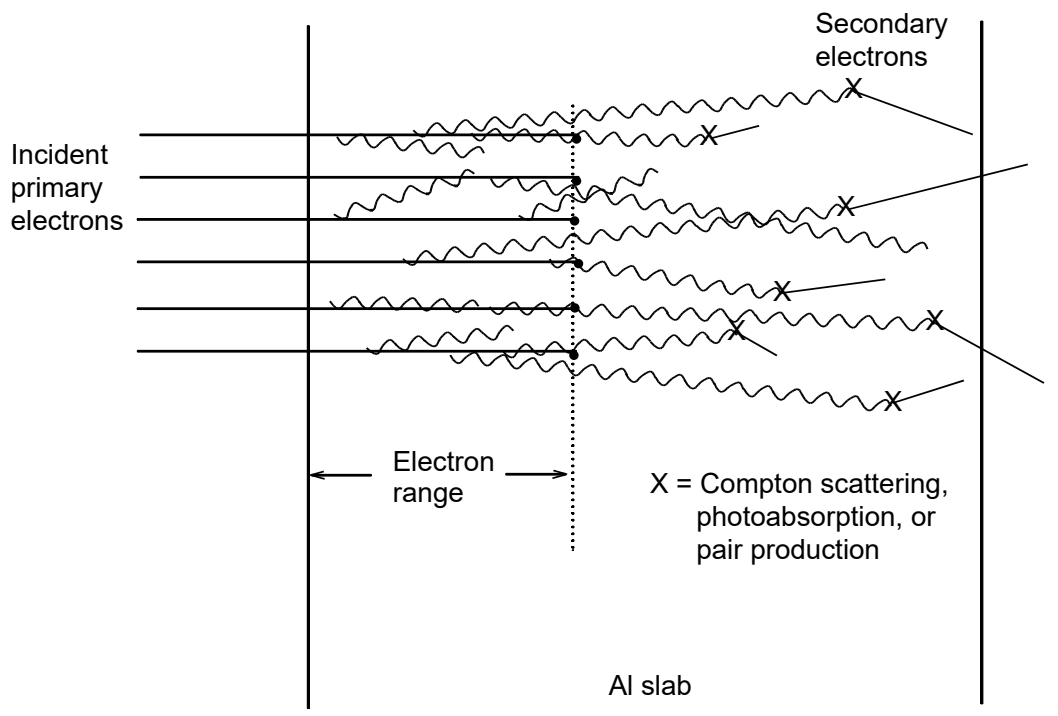

FIGURE 3.25 Depicting primary electrons producing Bremsstrahlung before the end of their range, and the photons reconverting to electrons by photoelectric, Compton, and pair production scattering. For simplicity, multiple elastic scattering of the incident electrons is not shown. 


\subsubsection{First Approximation}

Let the monoenergetic primary electron flux be $f_{\mathrm{o}}\left(E_{\mathrm{o}}\right)\left(\right.$ electrons $\left./ \mathrm{cm}^{2} / \mathrm{sec}\right)$ at kinetic energy $E_{\mathrm{o}}$, and let $Y$ be the number of photons produced per primary electron with energy great enough to penetrate to interesting depths beyond the primary electron range (at $E_{\mathrm{o}}=5 \mathrm{MeV}$ that corresponds to $k$ greater than $\left.k_{\mathrm{o}} \approx 0.03 \mathrm{MeV}\right) . Y=Y_{\mathrm{n}}\left(>k_{\mathrm{o}}\right)$ is the photon number yield of interest. Then beyond the primary range the flux of photons is

$$
f_{\gamma}=Y f_{\mathrm{o}}\left(E_{\mathrm{o}}\right)\left(\text { photons } / \mathrm{cm}^{2} / \mathrm{sec}\right)
$$

This photon flux creates Compton electrons at a rate $f_{\gamma} / \Lambda_{C}$ (electrons $/ \mathrm{g} / \mathrm{sec}$ ), where $\Lambda_{C}\left(\mathrm{~g} / \mathrm{cm}^{2}\right)$ is the mfp against Compton scattering.

In steady state, the forward-directed flux of Compton electrons (the secondaries that may exit the far surface of the Al slab) is just their mean forward range $R_{\mathrm{mf}}$ times their production rate:

$$
f_{\mathrm{s}}=R_{\mathrm{mf}} \frac{f_{\gamma}}{\Lambda_{C}}=\frac{R_{\mathrm{mf}} Y}{\Lambda_{C}} f_{\mathrm{o}}=\xi f_{\mathrm{o}},
$$

where

$$
\xi=\frac{R_{\mathrm{mf}}}{\Lambda_{C}} Y
$$

is the ratio of forward-directed secondary electron flux to primary flux. $\xi$ as a function of $E_{\mathrm{o}}$ is the quantity of interest, at least in this simplified discussion. The secondary electron flux is the primary flux times the photon number yield $Y$, times the ratio $R_{\mathrm{mf}} / \lambda_{C}$ of electron to photon ranges.

As a numerical example, consider an incident electron of $E_{\mathrm{o}}=5 \mathrm{MeV}$; its ranges $R_{\mathrm{mf}}$ and $R$ were given earlier. Present considerations apply when slab thickness exceeds $R$. Suppose slab thickness is $10 \mathrm{~g} / \mathrm{cm}^{2} \approx 3 R$.

The number of Bremsstrahlung photons with energy greater than 0.03 $\mathrm{MeV}$ is obtained from Figure $3.22 \mathrm{a}, Y=0.45$ photons per electron. Their average energy is $k_{\mathrm{av}} \approx 0.6 \mathrm{MeV}$ (Figure $3.19 \mathrm{~b}$ ). At this energy, Compton scattering dominates photoelectric absorption, and pair production is not possible; therefore, the mfp against Compton scattering is the same as the mfp against total scattering. From Figure 2.55 this $\mathrm{mfp}$ is $\Lambda_{C}\left(k_{\mathrm{av}}\right)=13 \mathrm{~g} / \mathrm{cm}^{2}$. From Figure 2.24, in Compton scattering the average electron energy at this photon energy is $T_{\mathrm{av}}=0.36 k_{\mathrm{av}}=0.22 \mathrm{MeV}$. The mean forward range of this average Compton electron is $R_{\mathrm{mf}}=0.032 \mathrm{~g} / \mathrm{cm}^{2}$ (Figure 3.24). Putting these numbers into Equation 3.53, find 


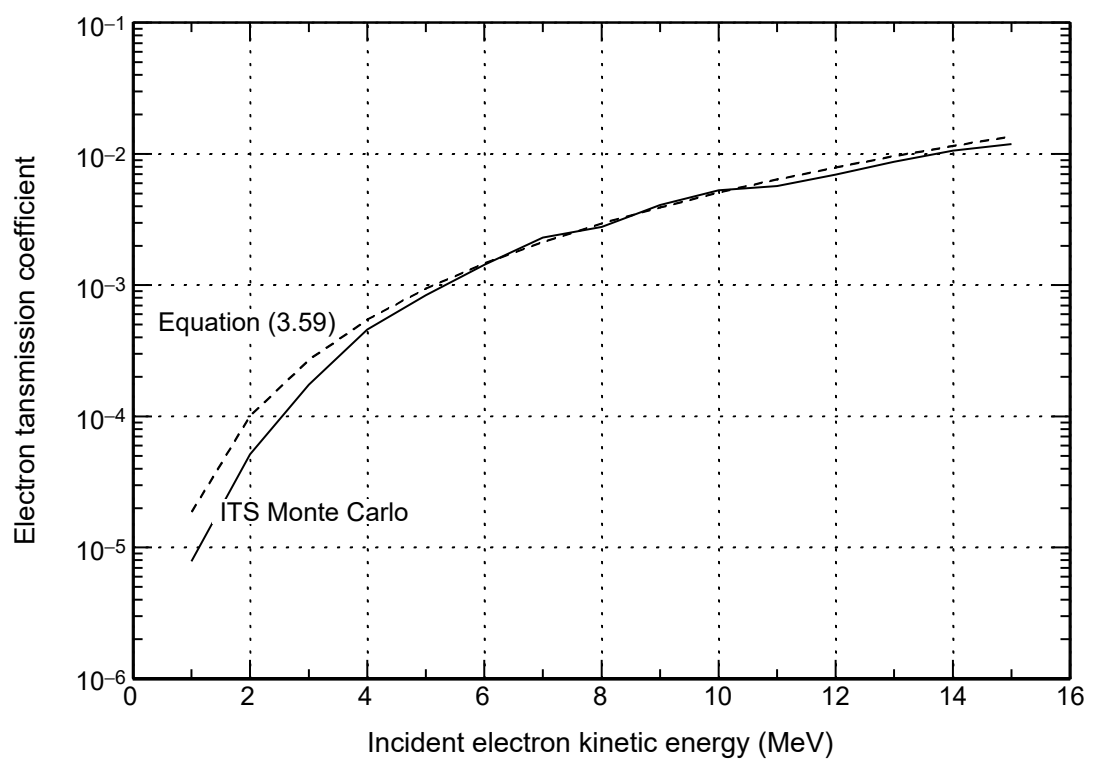

FIGURE 3.26 Number of electrons exiting a thick Al slab (1.5"), per incident electron, by conversion of incident electrons to Bremsstrahlung, and back to electrons. Solid curve is computed by the ITS Monte Carlo code (Doughty and Hawxhurst, [Do01]). Dashed curve is the analytic approximation of Equation 3.59.

$$
\xi=\frac{0.032}{13} \times 0.45=1.1 \times 10^{-3},
$$

somewhat larger than the value $8 \times 10^{-4}$ from a calculation with the Integrated TIGER Series (ITS) codes (Ha92, Do01) (see solid curve in Figure 3.26). Given the crudeness of the procedure and the use of an average Bremsstrahlung photon energy, one is pleased with better than a factor of 2 agreement.

\subsubsection{Improved Approximation}

Although the previous discussion provides a crude first estimate, it is not difficult to improve on it with a more thorough semianalytic calculation. The dependence on $E_{\mathrm{o}}$ can be obtained, and the contribution from all photon energies $k$ can be included without having to introduce a value for $k_{\mathrm{av}}$.

Equation 3.49 is the number of photons $y_{\mathrm{n}}(k) \mathrm{d} k$ in energy $(k, k+\mathrm{d} k)$. These attenuate in an $\operatorname{mfp} \Lambda_{\text {Tot }}$ against any scattering, and so at a distance $s$ beyond their birth, the photon number is reduced from $y_{\mathrm{n}}(k)$ to $y_{\mathrm{n}}(k) \exp \left(-s / \Lambda_{\text {Tot }}\right)$. As the mfp of all but the least energetic photons generally 
exceeds the electron range, $s$ may be measured from the end of the range of primary electrons.

The mfp of Bremsstrahlung photons is $\Lambda_{C}(k)$. The Compton electrons have average energy $T_{\mathrm{av}}(k)$, and a mean forward range $R_{\mathrm{mf}}\left(T_{\mathrm{av}}\right)$. Summing over all $k$, we have for the ratio $\xi$, as an improvement to Equation 3.53,

$$
\xi=\int_{k_{\min }}^{E_{\mathrm{o}}} \mathrm{d} k \frac{R_{\mathrm{mf}}\left(T_{\mathrm{av}}(k)\right)}{\Lambda_{C}(k)} e^{-s / \Lambda_{\mathrm{Tot}}(k)} \frac{1}{k} \int_{k}^{E_{\mathrm{o}}} \frac{\mathrm{d} E}{E} \frac{L_{\mathrm{rad}}(E)}{L_{\mathrm{Tot}}(E)} 2(1-k / E) .
$$

Interchanging orders of integration,

$$
\xi=\int_{k_{\min }}^{E_{\mathrm{o}}} \frac{\mathrm{d} E}{E} \frac{L_{\mathrm{rad}}(E)}{L_{\mathrm{Tot}}(E)} \int_{k_{\min }}^{E} \frac{\mathrm{d} k}{k} \frac{R_{\mathrm{mf}}\left(T_{\mathrm{av}}(k)\right)}{\Lambda_{C}(k)} 2(1-k / E) e^{-s / \Lambda_{\mathrm{Tot}}(k)} .
$$

This integral is an expression for the number of secondary electrons, per incident electron, that are created by Bremsstrahlung of the primaries. It is not yet an expression for the number of electrons that penetrate a slab; slab thickness has not entered. However, if the slab is thick compared with maximum electron range $R\left(E_{\mathrm{o}}\right)$, yet thin or comparable with the Compton mfp of an average Bremsstrahlung photon, Equation 3.56 is the number of electrons that penetrate the slab. As the Compton mfp is much greater than the electron range, a wide span of thicknesses falls in this category.

Over an interesting energy range of the incident electron, say $1<E_{\mathrm{o}}<$ $15 \mathrm{MeV}$, we may obtain a rough approximation to the integral Equation 3.56. For present purposes, to illustrate concepts, we sacrifice accuracy for simplicity, and approximate the dependencies of the various factors in the integrand by simple power laws, allowing the integral to be evaluated. However, this prepares us to expect imperfect dependence on $E_{\mathrm{o}}$.

To approximate the Compton $\operatorname{mfp} \Lambda_{C}(k)$, we appeal to the Compton cross section in Figure 2.40 , which is $1 / \Lambda_{C}$. Recalling there are many more lowenergy Bremsstrahlung photons than high-energy photons, so that our approximation to $\Lambda_{C}$ should emphasize lower energies, a rough fit to $1 / \Lambda_{C}(k)$ over the interesting energy span is proportional to $1 / \sqrt{k}$, or

$$
\Lambda_{C}(k) \approx \Lambda_{1} \sqrt{k}
$$

where $\Lambda_{1}=16 \mathrm{~g} / \mathrm{cm}^{2}$, and with $k$ in $\mathrm{MeV}$. A fit to $T_{\mathrm{av}}(k)$ is obtained from Figure 2.24 ; we use the approximation $T_{\mathrm{av}}(k) / k \approx 0.42 k^{1 / 3}$, or $T_{\mathrm{av}}(k) \approx$ $0.42 k^{4 / 3}$. We then fit the mean forward range $R_{\mathrm{mf}}(T)$ in Figure 3.24. The Compton electrons have lower energy than the Bremsstrahlung photons, so we use a fit appropriate for energies relatively low compared with $E_{\mathrm{o}}$ : $R_{\mathrm{mf}}(T) \approx 0.3 T^{1.45}$, with energies in $\mathrm{MeV}$. These crude approximations are motivated by a desire to reduce Equation 3.56 to a simple expression; better fits would result in a more accurate but more complicated expression for $\xi\left(E_{\mathrm{o}}\right)$. 
Concentrating on photons with a large enough mfp to produce Compton electrons far enough downstream, we may ignore the attenuation factor $\exp \left(-s / \Lambda_{\text {Tot }}\right)$. Then with these fits, the $k$ integral in Equation 3.56 is evaluated to be $3.0 \times 10^{-3} E^{1.433}$, so that

$$
\xi=3.0 \times 10^{-3} \int_{0}^{E_{\mathrm{o}}} \mathrm{d} E E^{0.433} \frac{L_{\mathrm{rad}}}{L_{\mathrm{Tot}}},
$$

where we have now safely replaced $k_{\min }$ by 0 . We may invoke Equation 3.50 to approximate the ratio $L_{\mathrm{rad}} / L_{\mathrm{Tot}}$, and obtain

$$
\xi=1.9 \times 10^{-5} E_{\mathrm{o}}^{2.43}
$$

with $E_{\mathrm{o}}$ in $\mathrm{MeV}$. In this approximation, $\xi$ is proportional to $E_{\mathrm{o}}^{2.43}$. Equation 3.59 is plotted in Figure 3.26 (dashed line).

This simple calculation shows that the total current transmitted through a slab thicker than the incident electron range, but thinner than the Bremsstrahlung photon $\mathrm{mfp}$, is nearly proportional to the incident electron energy to the power 2.43. Equation 3.59 agrees reasonably with the results of ITS simulations. Figure 3.26 compares expression (Equation 3.59) with the ITS calculation for slab thickness $10 \mathrm{~g} / \mathrm{cm}^{2}$ [Do01]. The simple estimate is surprisingly good above an incident electron energy of $5 \mathrm{MeV}$. Near $E_{\mathrm{o}} \sim 1 \mathrm{MeV}$ the lower-energy Bremsstrahlung photons can be absorbed too soon; the factor $\exp \left(-s / \Lambda_{\mathrm{Tot}}\right)$ should be restored and will reduce the $1 \mathrm{MeV}$ transmission to a value closer to the ITS results. The Monte Carlo simulation shows that the transmission of $1 \mathrm{MeV}$ electrons is only about half our estimate.

This exercise, of course, is not intended to exhibit much accuracy, but is to illustrate the physics of electron-photon-electron conversion and the utility of photon number yield, and to show how relatively simple analytic calculations can provide estimates and physical understanding of Monte Carlo results.

The penetration of a target thicker than the primary electron range is an (somewhat extreme) example of the different positional dependence of stopping power and kerma vs. energy deposition.

\subsection{TOTAL ENERGY LOSS AND MEAN RANGE AGAINST TOTAL STOPPING POWER}

The sum of collisional and radiative energy losses is the total stopping power. It is shown in Figure 3.27a as a function of electron energy for several elements and in Figure $3.27 \mathrm{~b}$ as a function of $Z$ for several energies. Figure 3.28 shows 

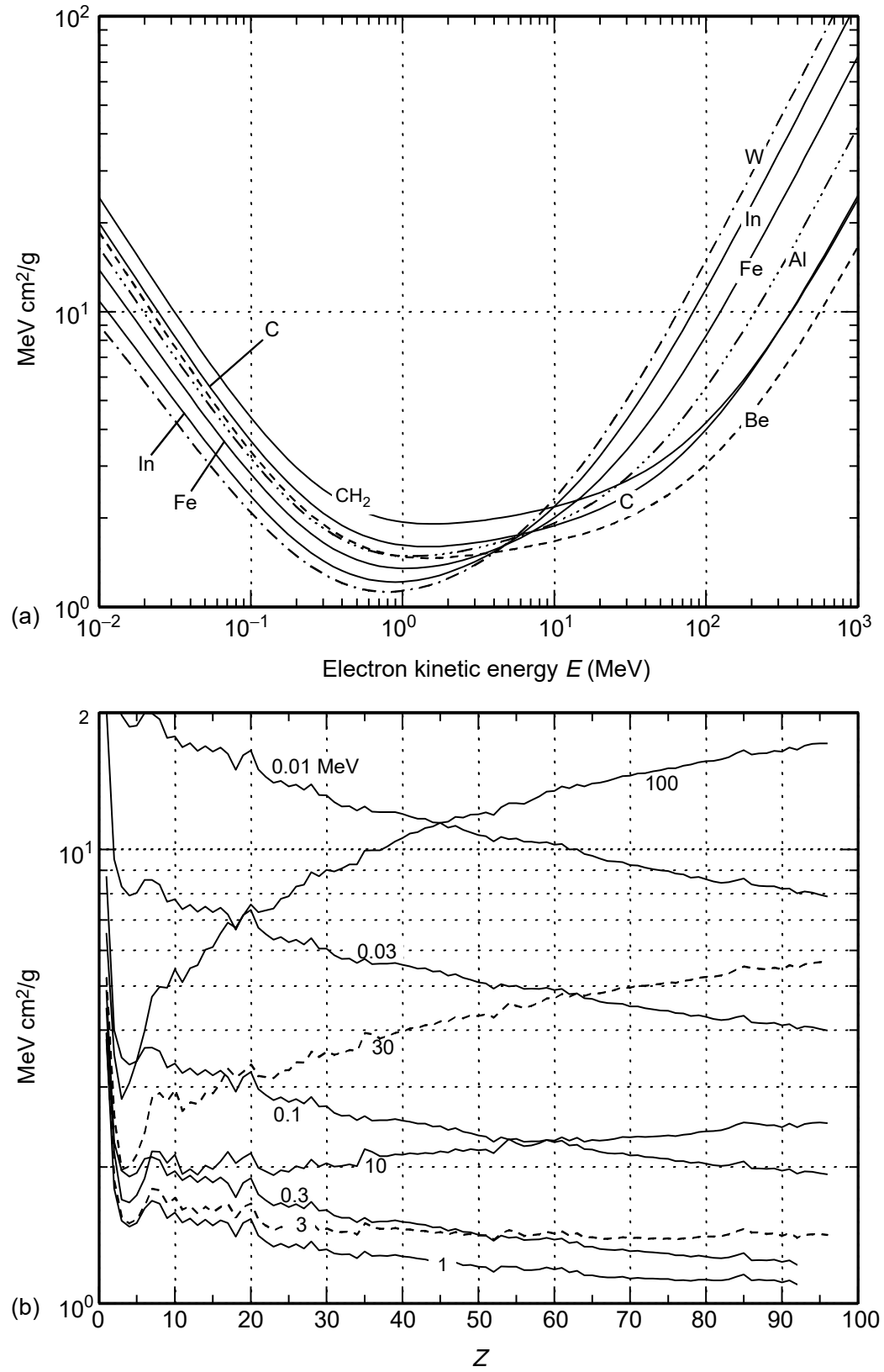

FIGURE 3.27 Electron mean total stopping power (collisional + radiative) (a) vs. $E$ for several elements; (b) vs. $Z$ for selected energies. 


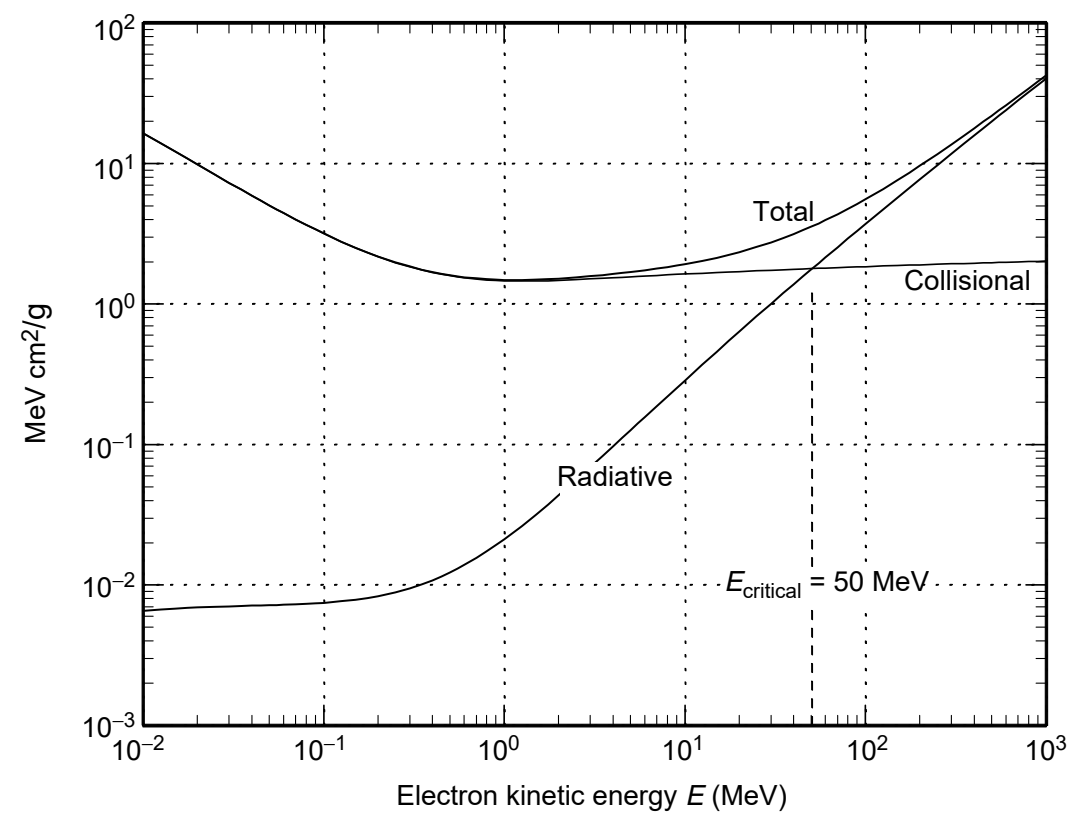

FIGURE 3.28 Electron collisional, radiative, and total stopping powers in aluminum.

the total for $\mathrm{Al}$, with its collisional and radiative components; they are equal at $50 \mathrm{MeV}$ (critical energy). Contours for all $E$ and $Z$ are shown in Figure 3.29.

The CSDA mean range $R$, Equation 3.20, against total stopping power is shown in Figure 3.30a for several elements as a function of electron energy $E$, and in Figure 3.30b for several energies as a function of $Z$.

Contours of the CSDA range $R(Z, E)$ for all elements over an interesting range of energies are given in Figure 3.31. In essentially all materials, a $1 \mathrm{MeV}$ electron has a track length of between $0.6 \mathrm{~g} / \mathrm{cm}^{2}$ and $0.8 \mathrm{~g} / \mathrm{cm}^{2}$. The ratio $R / E$ varies by only about a factor of 20 over the entire range of $Z$ and $E$.

Except for energy loss straggling, the CSDA range $R$ represents the maximum path length taken by an electron. In most instances, the spreading of the electron position owing to multiple scattering is much more important than the spreading caused by energy loss straggling, especially at lower energies and near the end of its path. Regarding the ways of using electronstopping power in Monte Carlo transport calculations, see [Be88b].

\subsection{MULTIPLE ANGULAR SCATTERING}

In addition to losing energy, electrons scatter in direction, and undergo a directed random walk. Their mean forward progress will then be less than their total track length. 


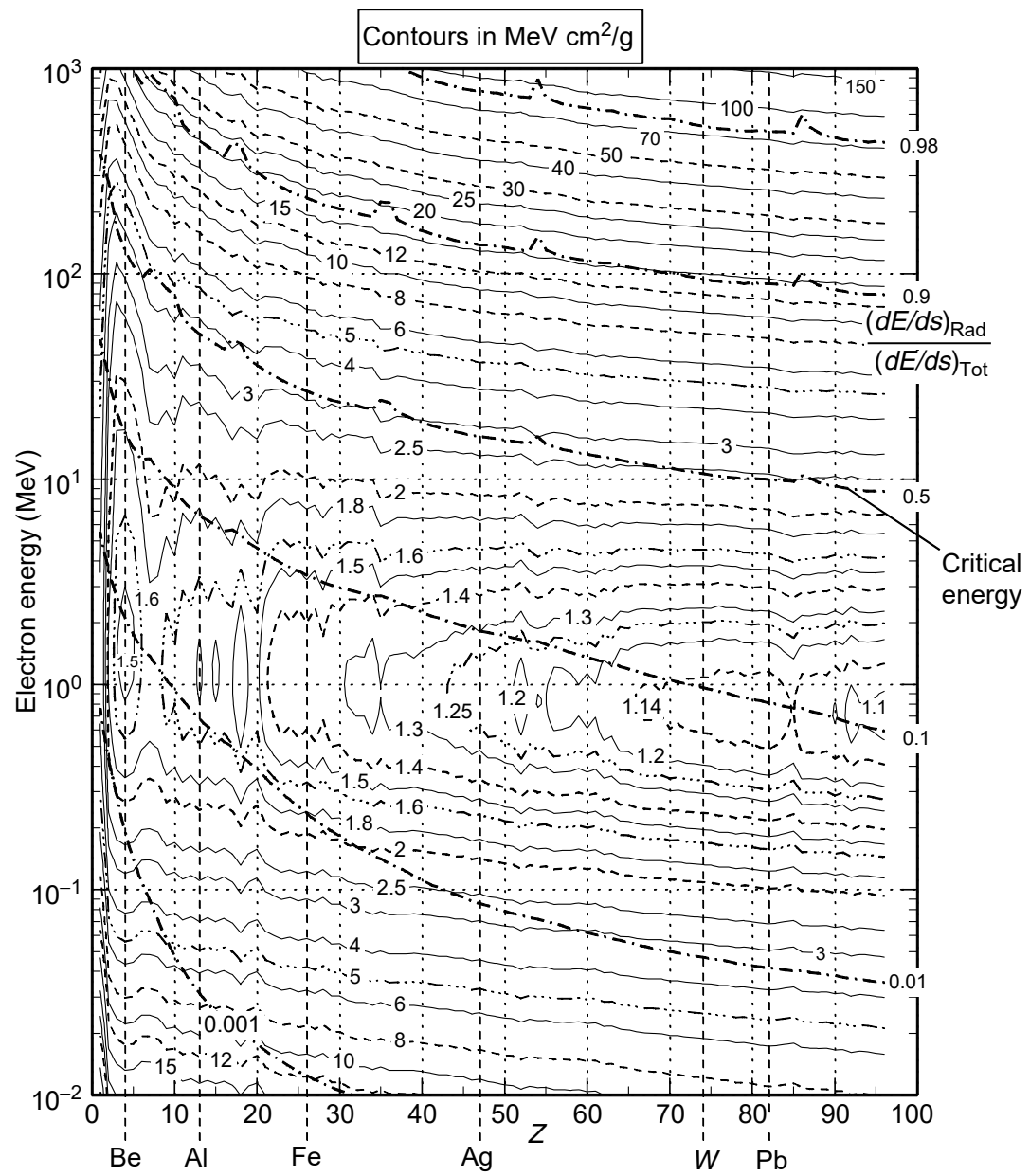

FIGURE 3.29 Electron mean total stopping power in all elements. Elements are at STP densities. Superimposed in dashed lines are contours of the fraction (radiative stopping power)/(total stopping power). Humps in these lines are at STP gases, and are caused by larger collisional stopping power in denominator. (Data from [NIST].) This graph, in color and for 11" $\times 17$ " paper, is available in high-resolution Adobe pdf format on the accompanying CD-ROM. See Section 3.11.

Multiple scattering often dominates electron motion in passing through matter. For an energetic electron, scattering effects build up as the electron slows down. At first, when still at high energies, the scattering causes a collimated beam to diverge only slowly in angle. By the time it has lost a significant fraction of its energy, the mean deviation from its original direction is no longer small and the forward progress occurs by a random walk. 

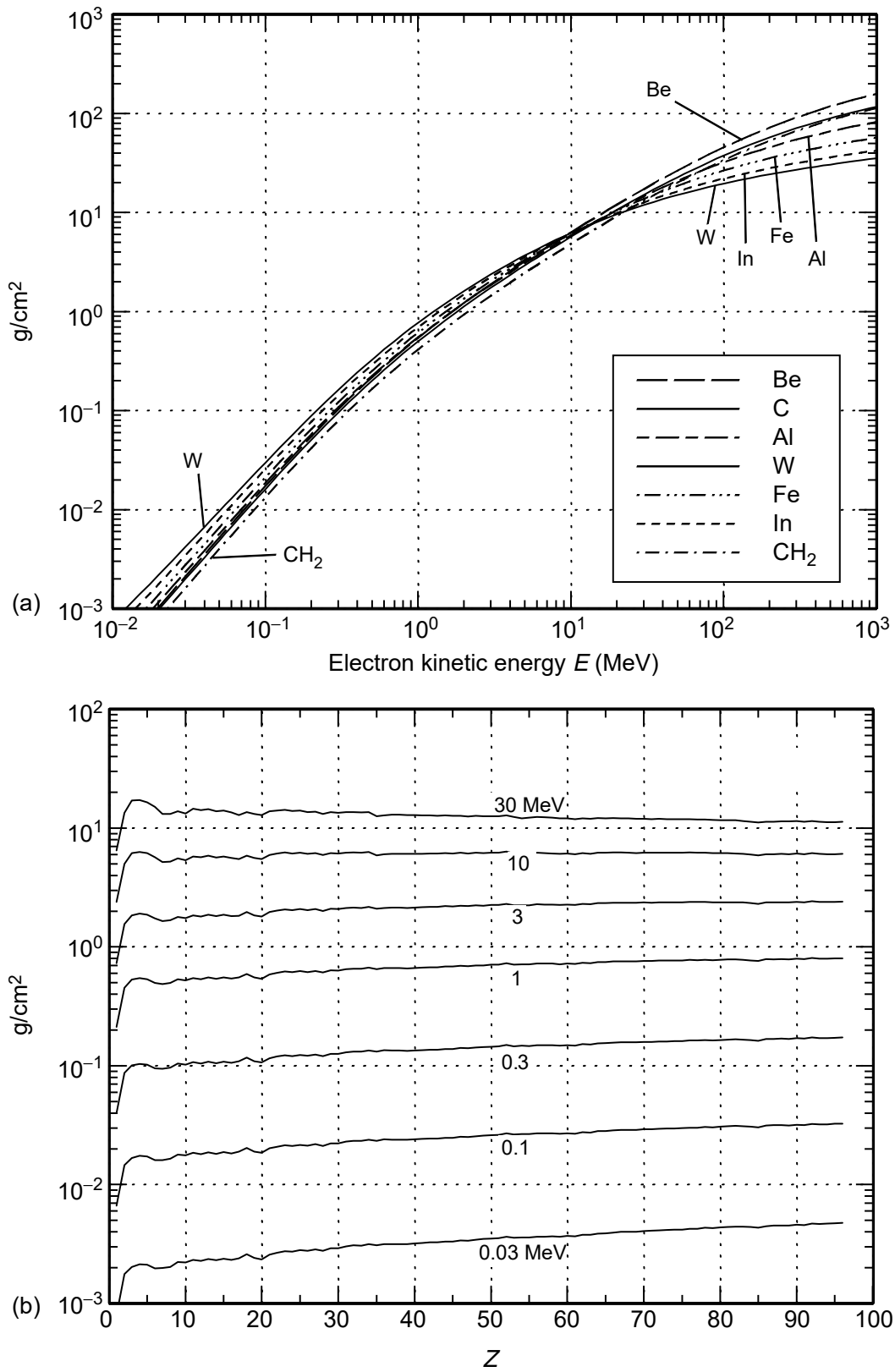

FIGURE 3.30 Electron CSDA range against mean total stopping power (a) vs. $E$ for several elements and (b) vs. $Z$ for selected energies. (Data from [NIST].) 


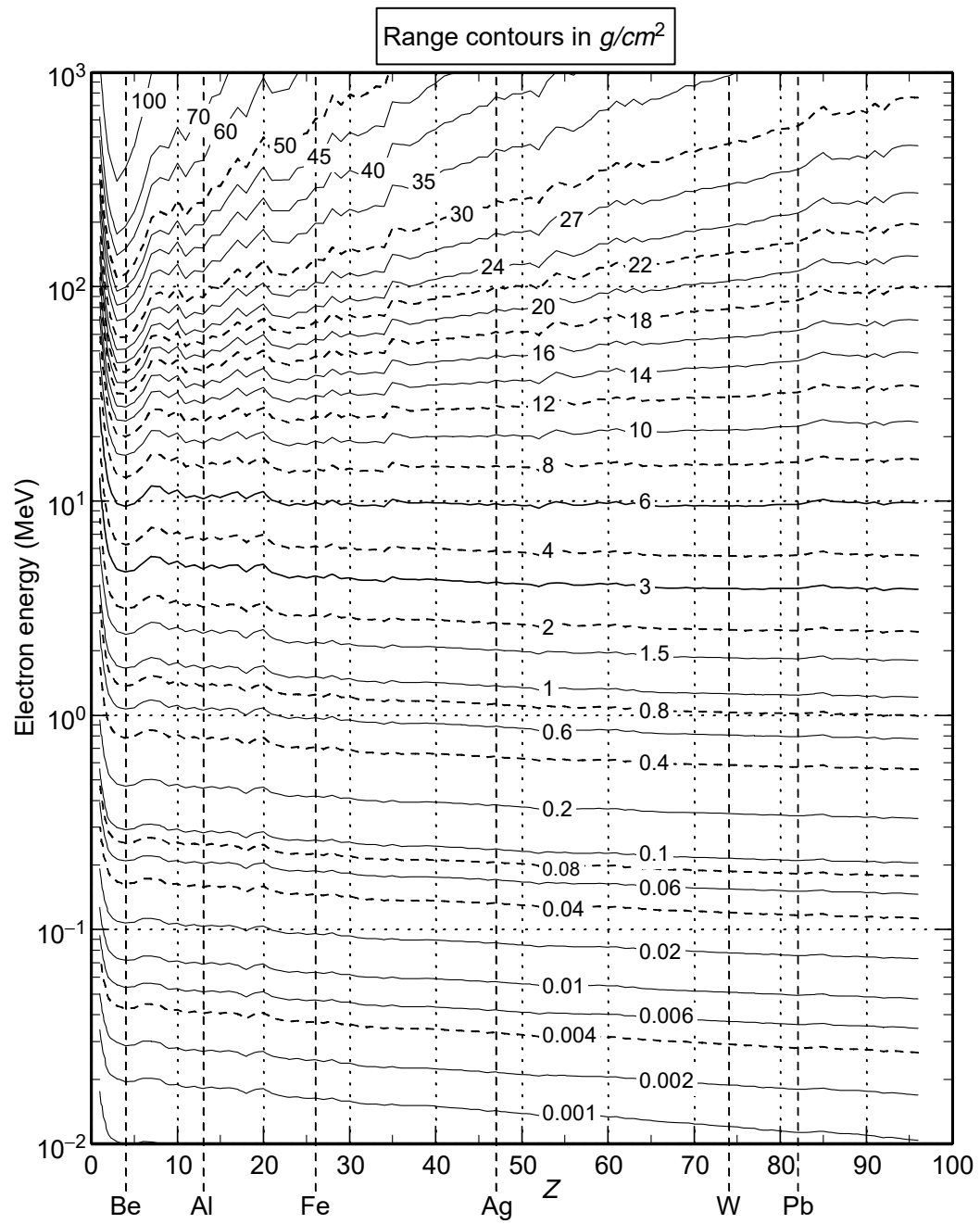

FIGURE 3.31 Contours of electron CSDA range against mean total stopping power (collisional + radiative) in all elements. (Data from [NIST].) This graph, in color and for $11 " \times 17$ " paper, is available in high-resolution Adobe pdf format on the accompanying CD-ROM. See Section 3.11.

An $800 \mathrm{keV}$ electron has barely enough energy to penetrate a $1 / 16^{\prime \prime} \mathrm{Al}$ slab. This slab thickness is $0.43 \mathrm{~g} / \mathrm{cm}^{2}$, the mean range $R=R_{\mathrm{CSDA}}$ against the total stopping power of an electron of that energy. Because of scattering, however, the vast majority of incident electrons will not exit the slab. By the time the electron is only $0.12 \mathrm{~g} / \mathrm{cm}^{2}$ into the $\mathrm{Al}$, an originally directed pencil 
beam has diverged to a mean angle of about $1 \mathrm{rad}$, and thereafter proceeds through the slab as much by diffusion caused by repeated scattering as by dint of its original momentum. Along its tortuous path, stopping power brings it to rest before exiting. Its average penetration depth is only about $0.2 \mathrm{~g} / \mathrm{cm}^{2}$, less than half the slab thickness. In order for a pencil beam to pass through a $1 / 16^{\prime \prime}$ Al slab and exit still well enough collimated to resemble a beam, with a divergence angle of no more than, say, $20^{\circ}$ (divergence cone half angle), the electron energy must be $\gtrsim 6 \mathrm{MeV}$. These statements will be substantiated in the following.

Because of scattering (and energy loss straggling) an electron will end up at the end of its track distributed in a cloud about a mean end point. The particle's mean forward progress in its original direction is called its projected range or its mean forward range (sometimes the average penetration depth [Fe96, Ta98b]. The ratio (projected range)/(CSDA range) $\leq 1$ is called the detour factor. It is a useful figure of merit indicating the relative importance of multiple scattering in relation to stopping power. Semiempirical formulas for the electron detour factor or projected range are given by Tabata and coworkers [Ta96a, Ta98b] and earlier references cited therein. For heavy ions, but not for electrons, the detour factor is tabulated in [NIST].

The track length $R$ can be larger than the projected range by a factor of 5 or more for high- $Z$ targets, even at several $\mathrm{MeV}$, but for low $Z$ at several $\mathrm{MeV}$, the track length is closer to 1.5 times the projected range. As mentioned, the mean range $R$ (Equation 3.20), is the mean range against stopping power, but is the maximum range as regards scattering. Scattering can so reduce the projected range relative to $R$, that some authors call $R$ the extreme range. Tables of the CSDA range $R$ may therefore be of limited use and must be applied carefully in a given application.

Along with mean stopping power, data on lateral and longitudinal straggling (due to both scattering and spread in energy loss rate) of ions are commonly tabulated (e.g., in the SRIM code [SRIM] or in [NIST]. Scattering affects electron motion much more severely than ion motion, but its effects are less frequently tabulated. The reasons are due to the interest in ion straggling arising from the need for accurate ion implantation in semiconductors; energy loss placement in medical ion beam therapy; and because, for electrons, it is not clear which of the many possible quantitative measures of the distributed electron cloud is the most useful. For the most part, one uses Monte Carlo computer simulations of electron transport. More recently, however, Tabata and coworkers in Japan and Austria have provided empirical analytic formulas for electron distributions and ranges in most materials [Ta96a, Ta96b, Ta98a, Ta98b, ID03]. The Monte Carlo code ITS (Halbleib et al., Ha92) is commonly employed for energetic electron transport, and the code $m c$-set (Monte Carlo Simulation of Electron 
Transport, http://www.napchan.com/), developed for application in electron microscopy, is useful for less energetic electrons $(\lesssim 30 \mathrm{keV})$. Akkerman and Gibrekhterman have presented a comparison of various Monte Carlo schemes [Ak85]. Berger and Wang discuss multiple scattering and energy loss straggling effects in Monte Carlo calculations [Be88a].

Stopping power and range-energy tables are commonly given. Standard tables of electron-stopping power and range have been prepared by LLNL, and by Berger and Seltzer [Be82], updated at [NIST], and are summarized here.

As a $10 \mathrm{keV}$ electron has a range of the order of only a few $\mu \mathrm{m}$ in solids, there is often little interest in smaller energies. But in low-pressure gases, the motion of lower-energy electrons can be of interest. Energies below $10 \mathrm{keV}$ in low-pressure air are discussed later in this chapter.

\subsubsection{Williams, Molière, and Goudsmit-Saunderson SCATTERING}

Since the elastic cross section exceeds $10^{4} \mathrm{~cm}^{2} / \mathrm{g}$ (Figure 3.2), the mean distance between scatterings above $1 \mathrm{MeV}$ is less than $10^{-4} \mathrm{~g} / \mathrm{cm}^{2}$, and less than $10^{-5} \mathrm{~g} / \mathrm{cm}^{2}$ near and below $100 \mathrm{keV}$. Thus, an electron need transit a distance only of order $10^{-3} \mathrm{~g} / \mathrm{cm}^{2}$ (a few microns of $\mathrm{Al}$ ) to suffer many scatterings, and the multiple small-angle scattering theory is applicable.

The three treatments of electron multiple small-angle scattering in wide use are that of Williams [Wi39, Wi40], who employed the Born approximation (see also Williams [Wi45], and Bethe [Be46]); that of Molière [Mo47, Mo48], who found that one could use the WKB approximation rather than the Born approximation to circumvent low-energy and large-angle limitations imposed by the latter; and that of Goudsmit and Saunderson [Go40], who use an exact expansion in spherical harmonics. Williams' is useful for estimates and analytically simple. Molière's improves on large-angle scattering. Goudsmit and Saunderson's is exact at all angles, but less tractable analytically. The general theory of multiple scattering is reviewed in a number of places [Ro52, Be53, Be63, Bi58, Mo65, Ja75] with further original references contained therein. Here we summarize Williams' treatment.

\subsubsection{Williams' TheOry}

The conventional treatment of Williams' method uses the Rutherford cross section for individual electron-atom scattering, and assumes the scattering angle $\theta \ll 1$. With it, the probability that, in passing through a thin layer $\mathrm{d} x$ of 
matter with atom number density $N$, the electron will scatter once through a small angle in the interval $(\theta, \theta+\mathrm{d} \theta)$ is

$$
g(\theta) \mathrm{d} \theta=N \mathrm{~d} x \frac{\mathrm{d} \sigma_{\mathrm{R}}}{\mathrm{d} \theta} \mathrm{d} \theta=N \mathrm{~d} x \frac{2 \pi Z^{2} r_{\mathrm{o}}^{2}}{\gamma^{2} \beta^{4}(1-\cos \theta)^{2}} \sin \theta \mathrm{d} \theta=N \mathrm{~d} x \frac{8 \pi Z^{2} r_{\mathrm{o}}^{2}}{\gamma^{2} \beta^{4}} \frac{\mathrm{d} \theta}{\theta^{3}},
$$

and, for small-angle scattering, the mean square scattering angle occurring in $\mathrm{d} x$ due to a single scattering is

$$
\begin{aligned}
\left\langle\theta^{2}\right\rangle_{\mathrm{d} x} & =\int g(\theta) \theta^{2} \mathrm{~d} \theta \\
& =N \mathrm{~d} x \int_{\theta_{\min }}^{\theta_{\max }} \frac{8 \pi Z^{2} r_{\mathrm{o}}^{2}}{\gamma^{2} \beta^{4}} \theta^{2} \frac{\mathrm{d} \theta}{\theta^{3}} \\
& =N \mathrm{~d} x \frac{8 \pi Z^{2} r_{\mathrm{o}}^{2}}{\gamma^{2} \beta^{4}} \ln \left(\frac{\theta_{\max }}{\theta_{\min }}\right) .
\end{aligned}
$$

$\theta_{\min }$ is a small-angle cutoff arising from atomic screening introduced to handle the small-angle divergence in the Rutherford cross section. It is usually taken of magnitude $\theta_{\min } \sim \lambda / a=\alpha Z^{1 / 3} / \gamma \beta$, in which $\lambda=\hbar / p$ is the electron reduced wavelength, and $a \sim a_{\mathrm{o}} / Z^{1 / 3}$ is the distance from the nucleus at which electron screening is important. $a_{\mathrm{o}}=\hbar^{2} / m e^{2}=0.529 \AA$ is the Bohr radius. But had we started with the small-angle cross section in the form of Equation 3.14 already incorporating screening effects, rather than the unscreened Rutherford cross section, the minimum angle $\theta_{\mathrm{s}}$ (Equation 3.17) arises naturally. $\theta_{\min }$ can thus be taken to be $\theta_{\mathrm{s}}$; one need not introduce it separately.

$\theta_{\max }$ is the largest angle to be considered a small angle in a single scattering. Various models differ in the evaluation of this angle. Historically, two experimental regimes were distinguished. One was the theory of multiple scattering in the passage of an electron beam through "thin foils," meaning the foil was thin enough that energy loss to the electron was negligible but that many small-angle scatterings occurred [Be53]. Then $E$ could be held constant in the calculation. The other was the opposite limit of scattering in a thick medium. Here, as mentioned, scattering is severe and builds up to a divergence angle exceeding 1.

In the thin foil, it is assumed that angles remain small. $\theta_{\max }$ is treated as that angle for which there is only one collision with $\theta>\theta_{\max }$ in traversing the entire foil of thickness $t$. Then, $\theta_{\max }^{2}$ is found to be [Be53]

$$
\theta_{\max }^{2}=t \frac{4 \pi N Z^{2} r_{\mathrm{o}}^{2}}{\gamma^{2} \beta^{4}} \equiv \theta_{1 \max }^{2},
$$

proportional to $t$. 
A second approach to setting $\theta_{\max }$, preferred here, applies to electrons continually progressing through material of any thickness. That is to notice that at high energies a maximum scattering angle occurs because of the finite radius $r_{n}$ of the nucleus. With $r_{n} \approx 0.5 r_{\mathrm{o}} A^{1 / 3}$ the approximate nuclear radius, an estimate is [Ro52, Ja75]

$$
\theta_{\max }=\lambda / r_{n} \approx 280 / \gamma \beta A^{1 / 3} \equiv \theta_{2 \max }
$$

At lower energies where $\theta_{2 \max }$ exceeds $1 \mathrm{rad}$, the maximum angle is taken instead as $1 \mathrm{rad}$. The regime in which $\theta_{2 \max }$ should be 1 is common, for in air $(A \approx 14.5$ ) or $\mathrm{Al}$ the electron must be more energetic than $50 \mathrm{MeV}$ or $60 \mathrm{MeV}$ for $\lambda / r_{n}$ to be less than unity. Thus, $\theta_{\max }$ for use in the equation is taken to be

$$
\theta_{\max }=\min \left[1, \frac{280}{\gamma \beta A^{1 / 3}}\right] \text {. }
$$

Rossi [Ro52] discusses other less common models for choosing $\theta_{\max }$.

After choosing minimum and maximum angles for single scattering, the main goal of the theory is to determine how the mean angle of scattering increases with distance traversed. As the electron progresses through the medium, successive scatterings are independent, and by general principles their variances add, and the distribution becomes normal. Thus, $\left\langle\theta^{2}\right\rangle$ at $x+\mathrm{d} x$ is equal to $\left\langle\theta^{2}\right\rangle$ at $x$ plus $\left\langle\theta^{2}\right\rangle \mathrm{d} x$, and the distribution in angles after many scatterings is Gaussian,

$$
\mathrm{d} n / \mathrm{d} \theta=\left(2 /\left\langle\theta^{2}\right\rangle\right) \theta \exp \left(-\theta^{2} /\left\langle\theta^{2}\right\rangle\right)
$$

with mean square angle $\left\langle\theta^{2}\right\rangle \cdot\left\langle\theta^{2}\right\rangle$ itself is obtained by summing the contributions $\left\langle\theta^{2}\right\rangle \mathrm{d} x$ from Equation 3.6 over all layers $\mathrm{d} x$. The result is the rate at which $\left\langle\theta^{2}\right\rangle$ grows as the electron moves through the medium,

$$
\frac{\mathrm{d}\left\langle\theta^{2}\right\rangle}{\mathrm{d} s}=\frac{8 \pi N_{A} Z(Z+1) r_{\mathrm{o}}^{2}}{A \gamma^{2} \beta^{4}} \ln \left(\frac{\theta_{\max }}{\theta_{\mathrm{s}}}\right) .
$$

Here, $N$ is replaced by $N_{A} / A$, the target density per gram, and $s$ is in $\mathrm{g} / \mathrm{cm}^{2}$. The growth in $\left\langle\theta^{2}\right\rangle$ due to elastic scattering from the nucleus is proportional to $Z^{2}$, whereas the contribution from atomic electrons is proportional to $Z$. Hence $Z^{2}$ has been replaced by $Z(Z+1)$ to include scattering from atomic electrons.

In Equation 3.63, $\theta_{\mathrm{s}}$ is given by Equation 3.17 and $\theta_{\max }$ by Equation 3.62. The expression is approximate because of the arbitrariness in defining $\theta_{\max }$, and, especially at low energies and high $Z$, because the Born approximation is of questionable validity. However, Williams' theory is valid to lower energies 
than the conventional limit of the Born approximation because that approximation is valid to lower energies at small scattering angles than it is at larger angles. The theory is useful for estimates. For an initially parallel pencil beam of electrons of energy $E$, Figure 3.32 shows the rate of growth of the mean square angle of divergence, $\mathrm{d}\left\langle\theta^{2}\right\rangle / \mathrm{d} s$, for several elements. The rate of growth in most materials is of order $1 \mathrm{rad}^{2} \mathrm{~cm}^{2} / \mathrm{g}$ for electrons of 2 to $8 \mathrm{MeV}$.

Being a function of $Z$ and $E, \mathrm{~d}\left\langle\theta^{2}\right\rangle / \mathrm{d} s$ can be represented by contours in $Z, E$ space as was the case for cross sections and stopping powers. This contour plot is shown in Figure 3.33, in units of $\mathrm{rad}^{2}$ per $\mathrm{g} / \mathrm{cm}^{2}$. In these units, $\mathrm{d}\left\langle\theta^{2}\right\rangle / \mathrm{d} s$ varies from roughly $10^{-4}$ to $10^{4}$ over most energies of interest and over all elements.

At relatively low energy, where the stopping power is large, the scattering rate builds up rapidly as the electron slows down. Therefore, in applications, the stopping power, Figure 3.29 should be consulted along with $\mathrm{d}\left\langle\theta^{2}\right\rangle / \mathrm{d} s$. If the angle growth is considered at an energy at which stopping power is large, one must take into consideration the simultaneous declining energy.

As an example, we may reconstruct the conclusion at the beginning of this paragraph, that a narrow $800 \mathrm{keV}$ electron beam will diverge by about $1 \mathrm{rad}$ in $0.12 \mathrm{~g} / \mathrm{cm}^{2}$ of Al. From the figure one sees $\mathrm{d}\left\langle\theta^{2}\right\rangle / \mathrm{d} s$ is about $8 \mathrm{rad}^{2} \mathrm{per} \mathrm{g} / \mathrm{cm}^{2}$, so that in $0.12 \mathrm{~g} / \mathrm{cm}^{2}$, the beam will grow to $\left\langle\theta^{2}\right\rangle \approx 8 \times 0.12=1 \mathrm{rad}^{2}$. At the same time, the electron is seen to be losing energy at the rate $1.5 \mathrm{MeVcm}^{2} / \mathrm{g}$,

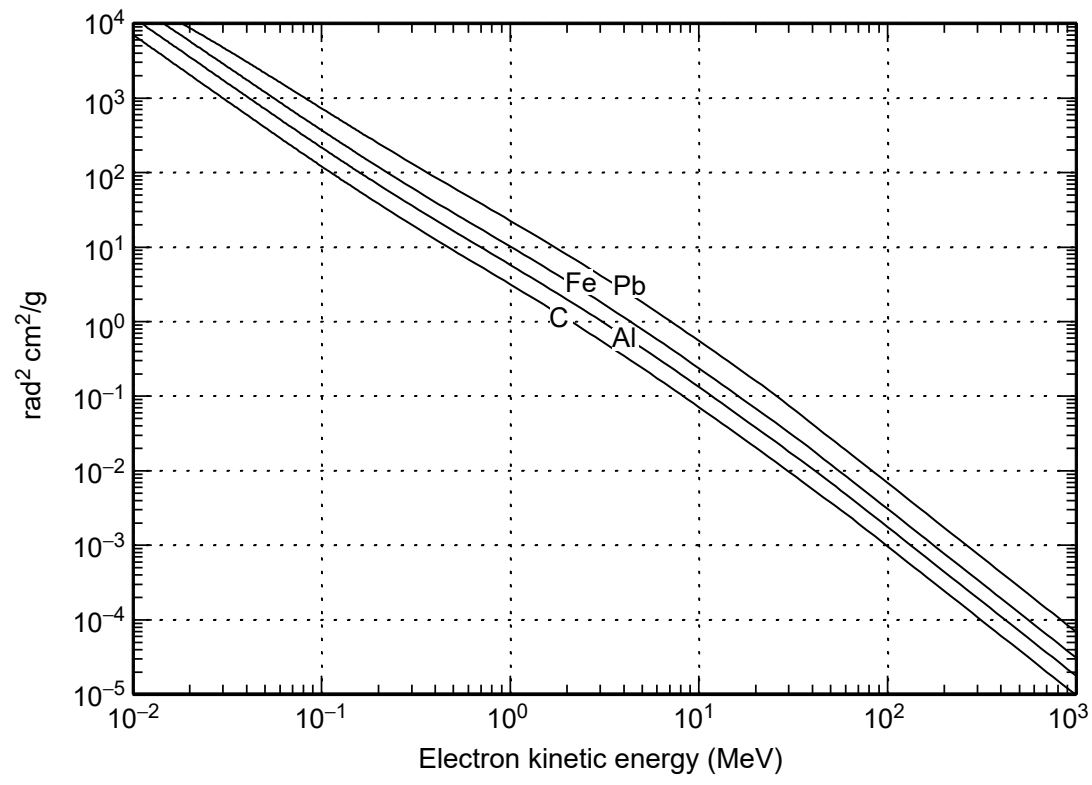

FIGURE 3.32 Rate of growth of mean square angle of deflection $\mathrm{d}\left\langle\theta^{2}\right\rangle / \mathrm{d} s$, in Williams' electron multiple scattering theory. 
Williams' election multiple scattering, growth of mean square angle

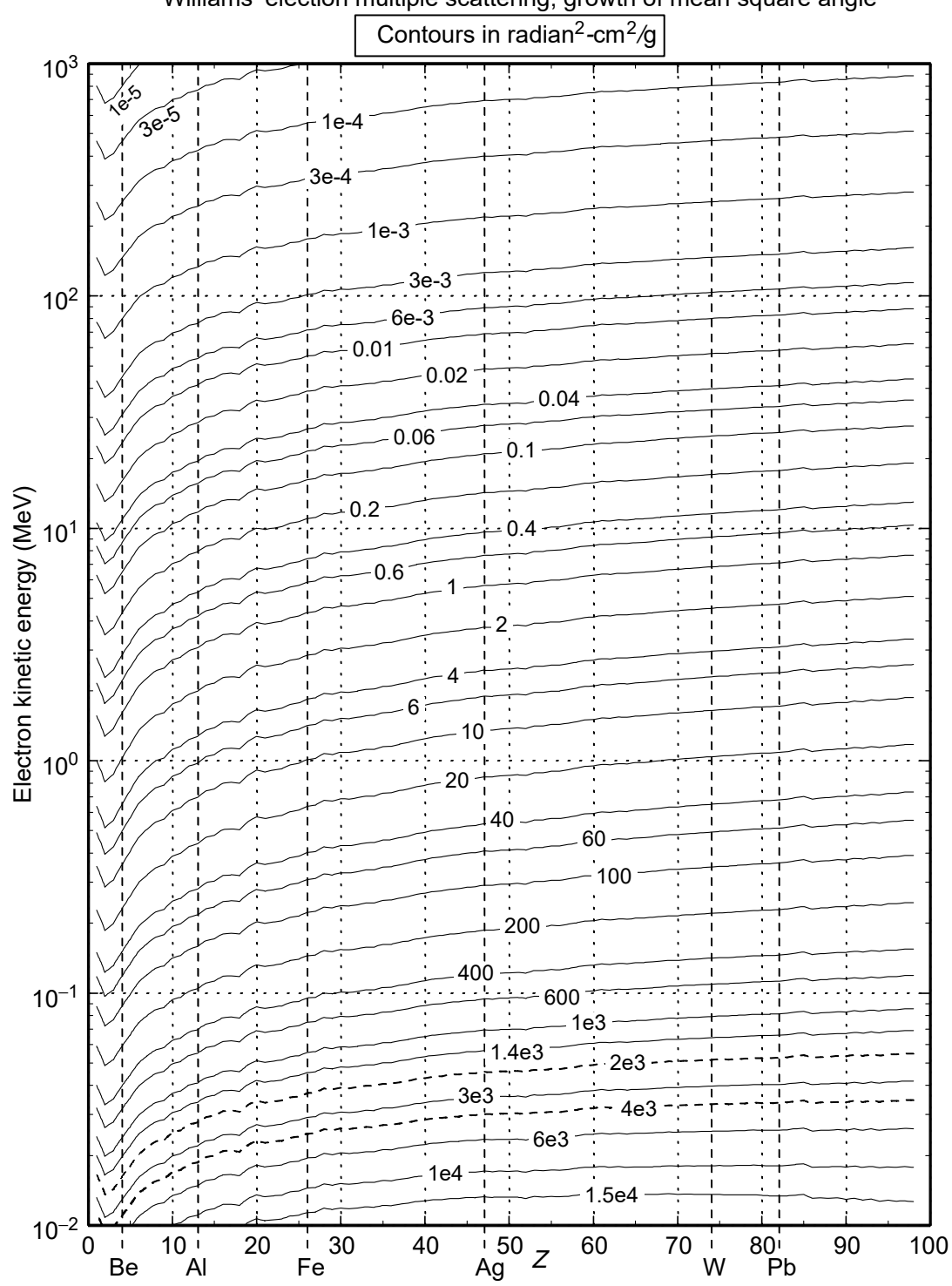

FIGURE 3.33 Rate of growth of mean square angle of divergence, $\mathrm{d}\left\langle\theta^{2}\right\rangle / \mathrm{ds}\left(\operatorname{rad}^{2} /\right.$ $\mathrm{g} / \mathrm{cm}^{2}$ ) due to electron multiple scattering according to Williams theory.

and so will lose only about $1.5 \times 0.12 \approx 0.18 \mathrm{MeV}$. If it lost much more during the distance over which the 1 rad estimate was made, we would have to take into account both that the scattering rate was increasing as the energy was 
decreasing, and that the electron path over which one needs the energy loss is greater than the linear depth of penetration.

One can also read from the graph that if we wished the beam to diverge in $0.12 \mathrm{~g} / \mathrm{cm}^{2}$ of Al by no more than $\theta_{\mathrm{rms}}=\left\langle\theta^{2}\right\rangle^{\frac{1}{2}}$ equal to say, $20^{\circ} \approx 0.3 \mathrm{rad}$, then $\left\langle\theta^{2}\right\rangle$ must grow to no more than $\sim 0.1 \mathrm{rad}^{2}$, and $\mathrm{d}\left\langle\theta^{2}\right\rangle / \mathrm{d} s$ must therefore not exceed $0.8 \mathrm{rad}^{2} \mathrm{~cm}^{2} / \mathrm{g}$. The figure then shows the beam energy must be $3.5 \mathrm{MeV}$ or greater. Alternatively, for a $1 \mathrm{MeV}$ beam to diverge by no more than $\theta_{\mathrm{rms}}=20^{\circ}$ in passing through an $\mathrm{Al}$ foil, the foil must be no thicker than $0.02 \mathrm{~g} / \mathrm{cm}^{2}=7.4 \times 10^{-3} \mathrm{~cm}$.

Although approximate, the Williams theory agrees with the more correct Goudsmit-Saunderson theory to a few percent [Mo65]. It is much simpler and useful for estimates.

\subsubsection{Angular Spread vs. Energy Loss}

As an electron progresses, its energy decreases and its angular spread increases. The rate of increase of $\left\langle\theta^{2}\right\rangle$ as $E$ decreases is given by

$$
-\frac{\mathrm{d}\left\langle\theta^{2}\right\rangle}{\mathrm{d} E}=\frac{\mathrm{d}\left\langle\theta^{2}\right\rangle / \mathrm{d} s}{|\mathrm{~d} E / \mathrm{d} s|} .
$$

Taking angular increase from Equation 3.63 and collisional stopping power $\mathrm{d} E / \mathrm{d} s$ from the Bethe formula Equation 3.21, one has

$$
\begin{gathered}
-\frac{\mathrm{d}\left\langle\theta^{2}\right\rangle}{\mathrm{d} E}=\frac{4(Z+1)}{m c^{2} \gamma^{2} \beta^{2}} \Gamma, \\
\Gamma \equiv \frac{\ln \left(\theta_{\max } / \theta_{\min }\right)}{\{\cdots\}},
\end{gathered}
$$

where $\{\ldots\}$ is the curly brackets in Equation 3.21. The Bethe formula and Equation 3.65 are valid approximately above several tens of keV. Restricting attention to energies $\lesssim 70 \mathrm{MeV}$ in low- $Z$ materials and $\lesssim 20 \mathrm{MeV}$ in high- $Z$ materials, $\theta_{\max }$ may be replaced by 1 .

Using $E=(\gamma-1) m c^{2}$, Equation 3.65 becomes

$$
-\frac{\mathrm{d}\left\langle\theta^{2}\right\rangle}{\mathrm{d} \gamma}=4 \Gamma \frac{(Z+1)}{\gamma^{2}-1}
$$

Longmire noticed that the ratio $\Gamma$ is very nearly independent of $E$ and $Z$ over a wide span of energies [Lo73]. $\Gamma=0.27 \pm 0.02$ from $1 \mathrm{keV}$ to about $50 \mathrm{MeV}$ in light elements, and from several tens of $\mathrm{keV}$ to about $20 \mathrm{MeV}$ in heavy elements. Then $\mathrm{d}\left\langle\theta^{2}\right\rangle / \mathrm{d} \gamma$ behaves as $1 / E$ for nonrelativistic energies, and as $1 / E^{2}$ for relativistic energies. 
For constant $\Gamma$ the full solution for any initial energy $\gamma_{\mathrm{o}}$ to any final energy $\gamma$ is

$$
\left\langle\theta^{2}\right\rangle=2 \Gamma(Z+1) \ln \left(\frac{\gamma_{\mathrm{o}}-1}{\gamma_{\mathrm{o}}+1} \frac{\gamma+1}{\gamma-1}\right) .
$$

In the nonrelativistic case, as a directed beam of electrons slows from $E_{\mathrm{o}}=\left(\gamma_{\mathrm{o}}-1\right) m c^{2}$ to $E$, its mean square angle grows from zero to

$$
\left\langle\theta^{2}\right\rangle=2 \Gamma(Z+1) \ln \left(\frac{E_{\mathrm{o}}}{E}\right) . \quad\left(E_{\mathrm{o}}<m c^{2}\right)
$$

The spread in angle is controlled by the fractional energy loss. Every nonrelativistic electron loses significant memory of its initial direction $\left(\left\langle\theta^{2}\right\rangle\right.$ equals, say, 1) by the time its energy has dropped to $E=\xi E_{\mathrm{o}}$, where $\xi=\exp [-1.85 /(Z+1)]$. Here we have stretched the validity Equation 3.63 by applying it for $\left\langle\theta^{2}\right\rangle \sim 1$.

In the relativistic case, as a beam slows from $\gamma_{\mathrm{o}}$ to $\gamma$, with $\gamma$ also relativistic, its mean square angle grows from zero to

$$
\left\langle\theta^{2}\right\rangle=4 \Gamma(Z+1)\left(\frac{1}{\gamma}-\frac{1}{\gamma_{\mathrm{o}}}\right)=4 \Gamma(Z+1)\left(\frac{\gamma_{\mathrm{o}}-\gamma}{\gamma \gamma_{\mathrm{o}}}\right) \cdot\left(E, E_{\mathrm{o}}>m c^{2}\right)
$$

For small energy losses, $\gamma_{\mathrm{o}}-\gamma \ll \gamma_{\mathrm{o}}$, the mean square angle is proportional to the energy loss divided by the square of the incident energy.

A simple application is to the passage of relativistic electrons through a thin foil of material $Z$. For the transmitted beam to diverge by no more than a small angle $\theta_{\mathrm{rms}}=\sqrt{ }\left\langle\theta^{2}\right\rangle$, Equation 3.69 shows its energy loss must be less than

$$
\Delta E=\frac{\left\langle\theta^{2}\right\rangle E_{\mathrm{o}}^{2}}{4 \Gamma(Z+1) m c^{2}}
$$

favoring high incident energies and low- $Z$ foils. To keep a $10 \mathrm{MeV}$ beam from diverging to no more than $\theta_{\mathrm{rms}}=0.1 \mathrm{rad}$ in passing through a typical plastic foil $(\langle Z\rangle \sim 6$ ), Equation 3.70 says its energy must drop by no more than 0.26 $\mathrm{MeV}$. Since its stopping power is about $L=2 \mathrm{MeV} \mathrm{cm}^{2} / \mathrm{g}$, the foil must be no thicker than $t=\Delta E / L=0.13 \mathrm{~g} / \mathrm{cm}^{2}$.

If the beam is nonrelativistic, and we still require $\theta_{\mathrm{rms}}=0.1$, Equation 3.68 says its energy must drop to no less than $0.9974 E_{\mathrm{o}}$, or by $0.26 \mathrm{keV}$, if $E_{\mathrm{o}}=100 \mathrm{keV}$. As its stopping power is about $L=3.6 \mathrm{MeV} \mathrm{cm} / \mathrm{g}$, the foil must be no thicker than $t=\Delta E / L=7 \times 10^{-5} \mathrm{~g} / \mathrm{cm}^{2}$. 


\subsubsection{Parameters Characterizing the Scattered Electron Distribution in a Thick Material}

In a thick target material, the mean square scattering angle $\left\langle\theta^{2}\right\rangle$ grows to exceed unity, and a theory of multiple small-angle scattering is no longer applicable. The electron motion changes from a slowly diverging directed motion to a random walk at all angles through the material, including significant backscattering. As mentioned, the electrons then stop in a diffuse cloud, caused both by the scattering and by straggling of the stopping power. The electron advance in the original direction of motion is much less than the CSDA range $R$, and the mean depth of penetration of the cloud is called the mean forward range, or the mean projected range, or sometimes the average penetration depth. We use the term mean forward range and denote it $R_{\mathrm{mf}}$.

Alternatively, if the target is of finite thickness $t$, electrons with $R$ greater than $t$ may penetrate and be transmitted. For a given energy, one inquires of the transmission coefficient $T(t)$, which is the fraction of incident electrons that are transmitted, as a function of slab thickness. For a strongly scattering target (high- $Z$ material), $R$ must greatly exceed the thickness for any appreciable fraction of electrons to fully penetrate. The difference here from the transmission through a thin slab discussed earlier is that the electron energy is not at all nearly constant.

Thus, there are a number of pertinent quantities characterizing electrons entering a thick target, and each of them is of interest in various applications. These quantities are:

1. The distribution of the stopping end points of primary electrons.

2. The distribution of deposited charge. This differs from the distribution of end points of primary electrons because energetic knock-on secondaries ( $\delta$-rays) have been displaced forward from their parent atoms and because Bremsstrahlung from primary electrons is absorbed deeper in the material, separating electrons from parent atoms at much greater depths and causing the charge distribution to be more forward-shifted than the distribution of primary end points. At high energies $(\gtrsim$ several $\mathrm{MeV}$ ), the electron and positron from pair production caused by Bremsstrahlung photons stop at different ranges, also causing charge separation. The deposited charge distribution is especially important for determining the fields created in a dielectric target material.

3. The distribution of dose of the primaries and displaced secondaries. Dose distribution is important in medical applications, in electron beam irradiation studies, in effects on electronic components, in heating of shielding material, and in various industrial applications of electron beams.

4. The already mentioned number of primaries, $T(t)$, that fully penetrate a slab of thickness $t$. 
Related to each of these quantities, one can define several weighted mean average ranges that provide a rough measure of their characteristics. Before discussing them, recall two standard ranges:

- The CSDA range $R_{\mathrm{CSDA}}$ or simply $R$ is the track length against mean stopping power, defined in Equation 3.20. Except for energy loss straggling, it is the maximum depth of penetration of a primary electron.

- The scattering range $R_{\mathrm{sc}}$ is the depth at which multiple scattering has increased a beam's angular divergence "significantly," usually taken to mean $\left\langle\theta^{2}\right\rangle=1 \operatorname{rad}^{2}$. It can thus be defined by

$$
\int_{0}^{R_{\mathrm{sc}}} \frac{\mathrm{d}\left\langle\theta^{2}\right\rangle}{\mathrm{d} s} \mathrm{~d} s=1 .
$$

With these at hand, consider first the distribution of end points of the primary electrons.

The distribution with depth $z$ at which primary electrons come to rest is the mean forward range distribution $r_{\mathrm{mf}}(z)$. Then, the mean forward range defined earlier is

$$
R_{\mathrm{mf}}=\int z r_{\mathrm{mf}}(z) \mathrm{d} z / \int r_{\mathrm{mf}}(z) \mathrm{d} z .
$$

$r_{\mathrm{mf}}(z)$ is also called the projected range distribution and commonly denoted by $r_{\mathrm{pr}}$ [Ta96a]. In the case that the electrons enter a thick material from vacuum, some electrons will scatter back into vacuum. As collisional stopping power is nearly independent of $Z$, but $\mathrm{d}\left\langle\theta^{2}\right\rangle / \mathrm{d} s$ is proportional to $Z^{2} / A \sim Z$, high- $Z$ materials can scatter electrons by large angles while they have penetrated much less than an electron range, allowing them to backscatter more readily than electrons in low- $Z$ materials. For $3 \mathrm{MeV}$ electrons, less than $1 \%$ will backscatter from a Be target, whereas some $34 \%$ backscatter from a $\mathrm{Pb}$ target.

Figure 3.34 shows the distribution of end points, $r_{\mathrm{mf}}(z)$, of $3 \mathrm{MeV}$ electrons in several thick elemental solids. The ordinate, $r_{\mathrm{mf}}$, is in units of stopped primary electrons per $\mathrm{g} / \mathrm{cm}^{2}$ per incident electron. The incident electrons that backscatter do not contribute, and so the normalization of the distributions is less than unity. The graph was generated using the very useful empirical analytic fits devised by Tabata and coworkers in Japan and Austria. They are contained in the electron-material interaction database (EMID) via an algorithm readily accessible on the Web at the Institute for Data Evaluation and Analysis [ID03].

A number of effective ranges have been defined to characterize the distribution $r_{\mathrm{mf}}(z)$ for an electron of given energy. The first five listed here 

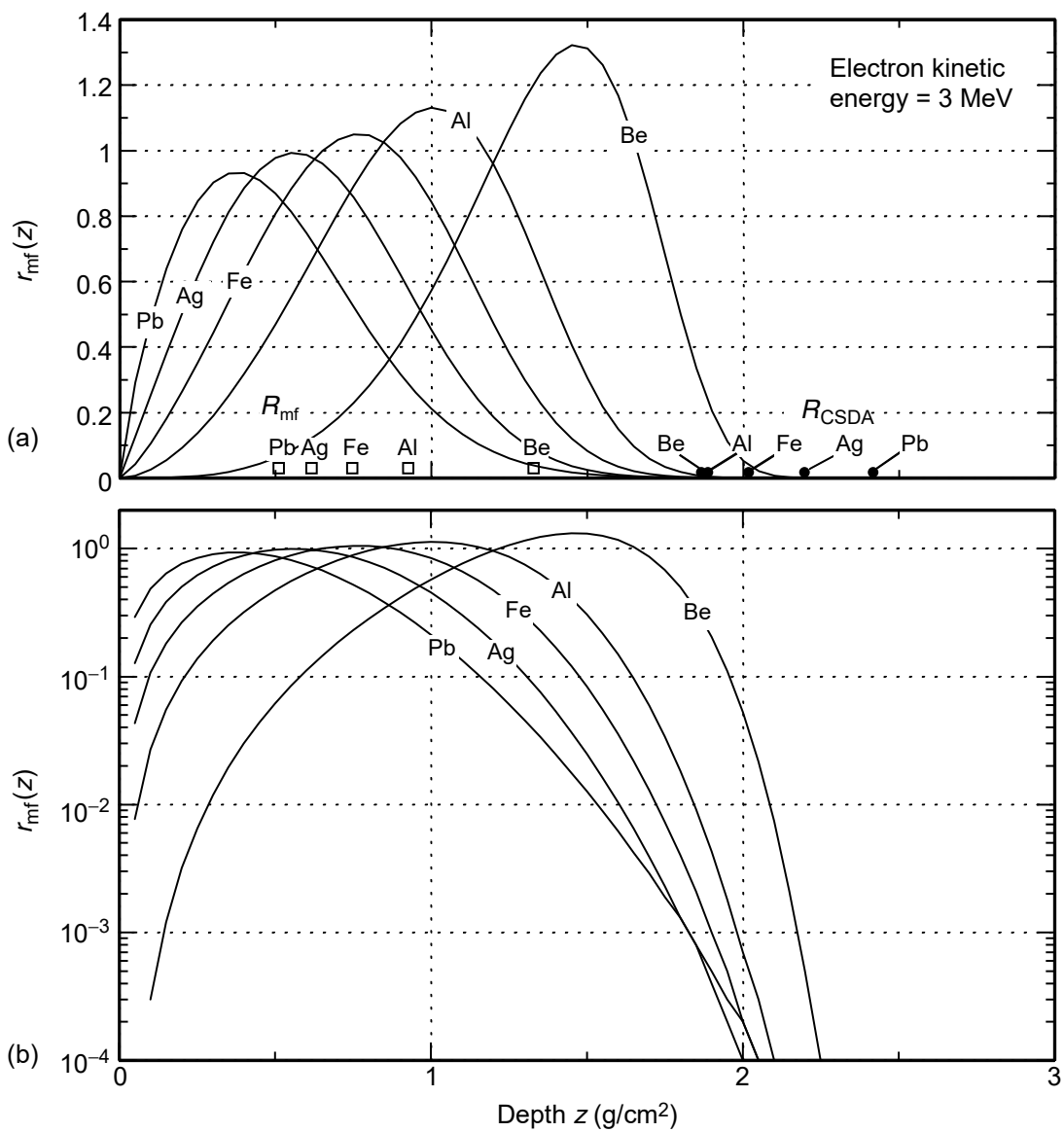

FIGURE 3.34 The mean forward range distribution $r_{\mathrm{mf}}(z)$ of electrons normally entering several elemental solids, in units of stopped primary electrons per $\mathrm{g} / \mathrm{cm}^{2}$ per incident primary electron. Backscattered electrons do not contribute. The integral under each curve is $[1-$ (backscattering coefficient)]. It is 0.66 for $\mathrm{Pb}$ at $3 \mathrm{MeV}$. The CSDA ranges are indicated as solid dots, the mean forward ranges as open squares. The detour factor for $\mathrm{Pb}$ is $R_{\mathrm{mf}} / R_{\mathrm{CSDA}}=0.21$. (Data from Tabata's EMID algorithm [ID03].) (a) Linear-linear. (b): Log-linear.

are defined directly in terms of $r_{\mathrm{mf}}(z)$ or its integral, whereas the sixth and seventh are defined in terms of dose, and the eighth from multiple scattering:

- The mean forward range $R_{\mathrm{mf}}$ was defined earlier; it is the average depth of penetration. The detour factor is the ratio $R_{\mathrm{mf}} / R_{\mathrm{CSDA}}<1$.

- The most probable depth (or most probable projected range) $R_{\mathrm{mp}}$ is the depth at the peak of the $r_{\mathrm{mf}}(z)$ curve. 
- The median depth $R_{\text {med }}$ is the depth beyond which one half of primary electrons stop:

$$
\int_{0}^{R_{\mathrm{med}}} r_{\mathrm{mf}}(z) \mathrm{d} z=\frac{1}{2} \int_{0}^{\infty} r_{\mathrm{mf}}(z) \mathrm{d} z .
$$

- The projected range straggling parameter $\Delta R$ is the full width at half maximum of $r_{\mathrm{mf}}(z)$.

The number of electrons that end up beyond $z$ is

$$
N(>z)=\int_{z}^{\infty} r_{\mathrm{mf}}\left(z^{\prime}\right) \mathrm{d} z^{\prime}
$$

$N(>z)$ is plotted along with $r_{\mathrm{mf}}(z)$ for Fe in Figure 3.35.

- The extrapolated range (or extrapolated depth) $R_{\mathrm{ex}}$ is obtained by extrapolating to the axis the tangent to $N(>z)$ at the steepest part where it is falling. As this steepest point is at $z=R_{\mathrm{mp}}, R_{\mathrm{ex}}$ may be expressed in terms of $R_{\mathrm{mp}}$,

$$
R_{\mathrm{ex}}=R_{\mathrm{mp}}+\frac{N\left(>R_{\mathrm{mp}}\right)}{r_{\mathrm{mf}}\left(z=R_{\mathrm{mp}}\right)} .
$$

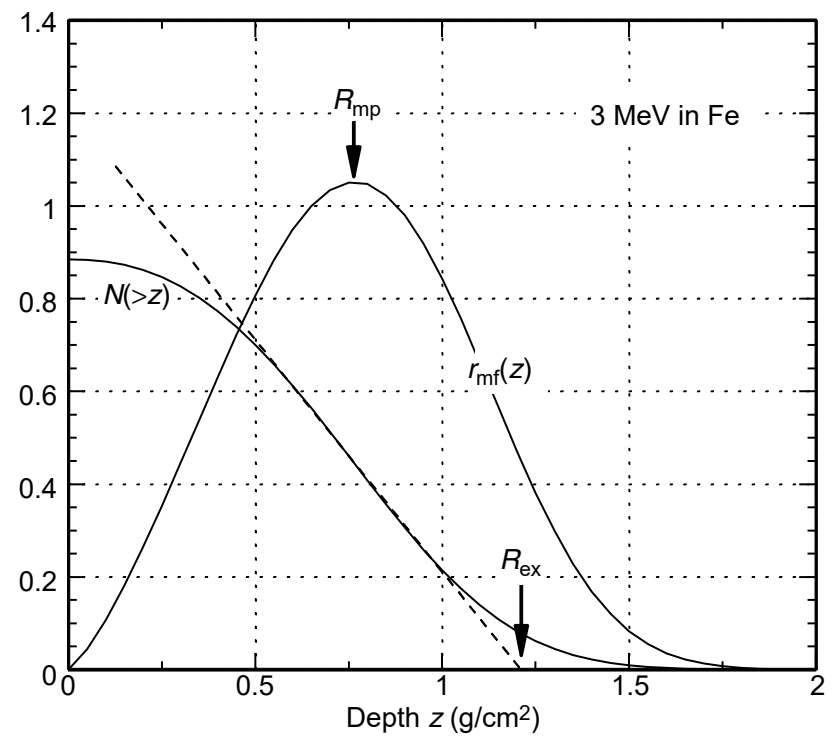

FIGURE 3.35 Mean forward range distribution $r_{\mathrm{mf}}(z)$ of $3 \mathrm{MeV}$ electrons in $\mathrm{Fe}$, and its integral $N(>z)$ from $z$ to $\infty$. The most probable range $R_{\mathrm{mp}}$ and the extrapolated range $R_{\mathrm{ex}}$ are indicated. 
It is a measure of the practical maximum depth of penetration; very few electrons penetrate beyond $R_{\text {ex }}$. Figure 3.35 shows that it is $1.23 \mathrm{~g} / \mathrm{cm}^{2}$ in $\mathrm{Fe}$ at $3 \mathrm{MeV}$. (Alternatively, the extrapolated range can be defined in terms of the number transmission curve $T(t)$ instead of $N(>z)$ [Ta96b].)

- The depth of maximum dose $R_{\mathrm{md}} . D(z)$ is the depth-dose distribution. The depth of maximum dose is the depth $z$ at which $D$ peaks.

- The practical range $R_{\mathrm{pr}}$. Whereas $N(>z)$ is the number of electrons that stop beyond $z, D(>z)$ is the dose deposited beyond $z$ (discussed presently). The practical range is obtained by extrapolating to the axis the tangent to $D(>z)$ at the steepest part where it is falling. It is a measure of the practical maximum depth at which dose is significant; little dose occurs beyond $R_{\mathrm{pr}}$. This range is of value in medical applications in which the placement of energy deposition is critical. It can be defined in terms of $R_{\mathrm{md}}, D\left(z=R_{\mathrm{md}}\right)$, and $D\left(>R_{\mathrm{md}}\right)$ just as $R_{\mathrm{ex}}$ is defined in terms of $R_{\mathrm{mp}}, r_{\mathrm{mf}}\left(z=R_{\mathrm{mp}}\right)$, and $N\left(>R_{\mathrm{mp}}\right)$. ( $R_{\mathrm{pr}}$ is not to be confused with the average of the projected range distribution (or the mean forward range distribution), which, as mentioned, is occasionally denoted as $\left.r_{\mathrm{pr}}(z)\right)$.

- An average depth of electron penetration $R_{\mathrm{av}}$ (or the average reach of electrons), which is the average of the maximum depth of penetration over all electrons. This parameter has recently been introduced [La98]. In the course of slowing down and scattering, an electron trajectory may pass a deep point and then scatter back to come to rest at a shallower point. Thus, the distribution of maximum depths of penetration is deeper than the distribution $r_{\mathrm{mf}}(z)$ of end points. This distribution, and its average, have been found useful in studies of electron beam irradiation [La98, La99].

These ranges parameterize the general behavior of the interactions of fast electrons with thick targets. In addition to the above ten range parameters, one may specify the fraction of incident electrons that scatter back through the incident surface, the backscattering coefficient $h_{\mathrm{N}}$ (or the number-reflection ratio). Also of interest is the average energy $\left\langle E_{\mathrm{bs}}\right\rangle$ of backscattered electrons when incident electrons have energy $E_{\mathrm{o}}$. The electron energy backscattering coefficient $h_{\mathrm{E}}=h_{\mathrm{N}}\left\langle E_{\mathrm{bs}}\right\rangle / E_{\mathrm{o}}$ (or the energy-reflection ratio) is sometimes specified instead. Clearly, $h_{\mathrm{E}} \leq h_{\mathrm{N}}$, as no backscattered electron has energy greater than $E_{\mathrm{o}}$. Experimentally measured backscattering coefficients are useful in their own right, and are of value because they provide a test of Monte Carlo scattering models. See the discussion in the compilation by the ICRU [ICRU96].

The backscatter coefficients apply to backscattering of the incident primary electron. The secondary emission coefficient, or the secondary yield, is the number of secondary electrons ejected per primary. Data on secondary yields and backscatter coefficients have recently been collected by Joy [Jo95]. 
TABLE 3.1

Range Parameters $\left(\mathrm{g} / \mathrm{cm}^{2}\right)$ and Dimensionless Reflection Ratios for $3 \mathrm{MeV}$ Electrons in Four Elements

\begin{tabular}{lllll} 
Range Parameters & Be & Al & Fe & Pb \\
Scattering range $R_{\mathrm{sc}}$ & 1.277 & 0.715 & 0.452 & 0.177 \\
Mean forward range $R_{\mathrm{mf}}$ & 1.32 & 0.919 & 0.739 & 0.504 \\
Average reach $R_{\mathrm{av}}$ & 1.35 & 0.996 & 0.80 & 0.52 \\
Median range $R_{\mathrm{med}}$ & 1.38 & 0.98 & 0.77 & 0.475 \\
Most probable range $R_{\mathrm{mp}}$ & 1.45 & 1.00 & 0.76 & 0.38 \\
Extrapolated range $R_{\mathrm{ex}}$ & 1.78 & 1.43 & 1.23 & 0.836 \\
Depth of maximum dose $R_{\mathrm{md}}$ & 1.00 & 0.56 & 0.39 & 0.21 \\
Practical range $R_{\mathrm{pr}}$ & 1.52 & 1.12 & 0.93 & 0.66 \\
CSDA range $R$ & 1.866 & 1.887 & 2.02 & 2.42 \\
Projected range straggling parameter $\Delta R$ & 0.71 & 0.82 & 0.83 & 0.69 \\
Number-reflection ratio $h_{\mathrm{N}}$ & 0.0045 & 0.0419 & 0.115 & 0.344 \\
Energy-reflection ratio $h_{\mathrm{E}}$ & 0.0014 & 0.0166 & 0.0525 & 0.1993 \\
\hline
\end{tabular}

Numerical values for most of these parameters at $3 \mathrm{MeV}$ in $\mathrm{Be}, \mathrm{Al}, \mathrm{Fe}$, and $\mathrm{Pb}$ are given in Table 3.1. Numerical values in Table 3.1 were obtained mostly using the online interactive EMID site [ID03].

The end point distribution $r_{\mathrm{mf}}(z)$ and the dose-depth distribution $D(z)$ are perhaps the most commonly used quantities. Figure 3.36 illustrates the dose distribution $D(z)$; the depth of maximum dose $R_{\mathrm{md}}$; the dose deposited at depths greater than $z, D(>z)$; and the practical range $R_{\mathrm{pr}}$ for a $3 \mathrm{MeV}$ electron entering Fe. Figure 3.37 compares the dose distribution $D(z)$ of $3 \mathrm{MeV}$ electrons in several thick elemental solids. These were obtained from empirical fits by Tabata et al. [Ta98a], who constructed useful analytic approximate fits to extensive Monte Carlo calculations with the ITS code for electron energies from $0.1 \mathrm{MeV}$ to $100 \mathrm{MeV}$. The calculations themselves are recorded in Tabata et al. [Ta94a], and analytic fits up to $20 \mathrm{MeV}$ are accessible in a usable form at the EMID site [ID03].

On comparing Figure 3.34 and Figure 3.37, several differences between the end point distribution $r_{\mathrm{mf}}(z)$ and the dose distribution $D(z)$ are immediately apparent. First, the dose begins immediately at $z=0$, whereas very few electrons end up at $z=0$, so that $r_{\mathrm{mf}}(0) \approx 0$. Second, partly as a result of the first, $D(z)$ peaks at a shallower depth than does $r_{\mathrm{mf}}(z)$. Third, also partly as a result of the first, dose distribution is more spread out than the end point distribution, especially for low $Z$ in which electrons penetrate more deeply. Fourth, dose occurs beyond the CSDA range $R$ as a result of Bremsstrahlung. This is more pronounced at high energies and high $Z$. 


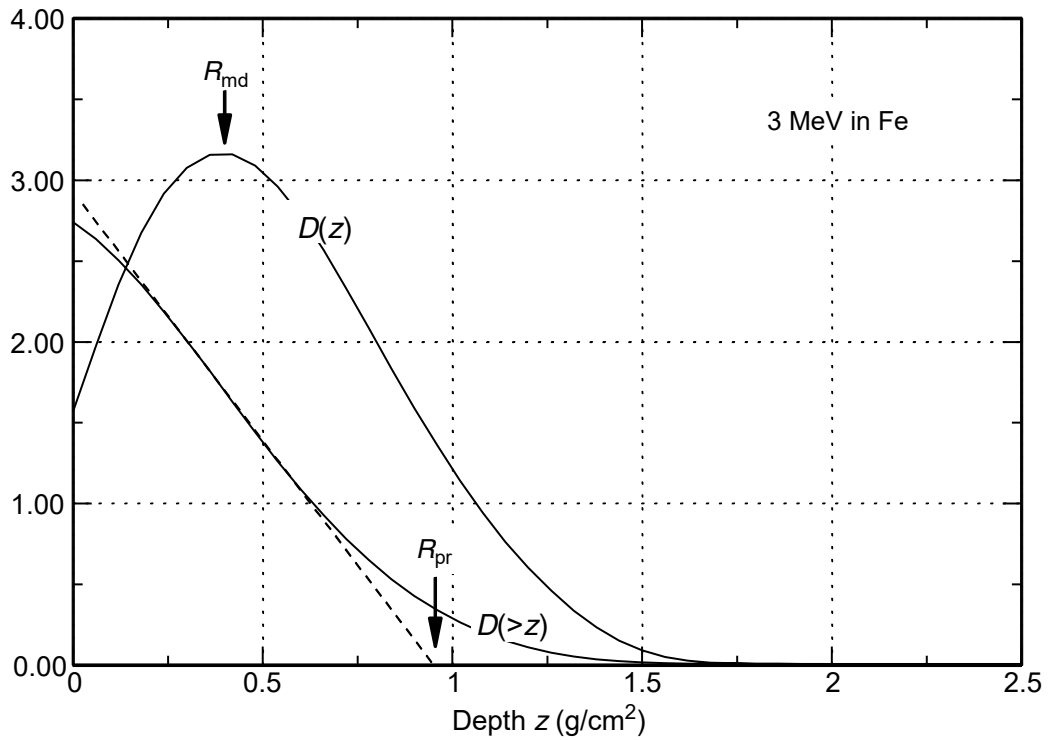

FIGURE 3.36 Dose distribution $D(z)$ of $3 \mathrm{MeV}$ electrons in $\mathrm{Fe}$, and its integral $D(>z)$ from $z$ to $\infty$. The range of maximum dose $R_{\mathrm{md}}$, and the practical range $R_{\mathrm{pr}}$ of electrons are indicated.

Tabata and coworkers have devised empirical fits to the distributions and mean ranges, obtained by careful comparison with extended Monte Carlo computations.

$D(z): \quad$ Tabata et al. [Ta98a]

$r_{\mathrm{mf}}(z): \quad$ Tabata et al. [Ta96a, Ta71]

$R_{\mathrm{mf}}: \quad$ Tabata et al. [Ta96a] and Tabata and Andreo [Ta98b]

$R_{\mathrm{CSDA}}$ : Tabata et al. [Ta96b]

Detour factor $R_{\mathrm{mf}} / R_{\mathrm{CSDA}}$ : Tabata and Andreo [Ta98b]

In addition to empirical fits, dose profiles for electrons from $0.1 \mathrm{MeV}$ to 100 $\mathrm{MeV}$ have been computed [Ta94a] with the ITS code in a number of elemental targets and mixtures of interest, such as water, tissue-equivalent plastics, air, and other plastics; and charge deposition profiles for the same energies and targets in [Ta94b].

We present here the empirical fits to the CSDA range and the detour factor.

\subsubsection{Empirical Fit to the CSDA Range}

Tabata et al. [Ta96b] present the following very useful approximate functional form $R_{\text {CSDA }}^{\mathrm{fit}}$ for the CSDA range as a function of electron kinetic energy $E \equiv m c^{2} \tau$ and $Z$ : 


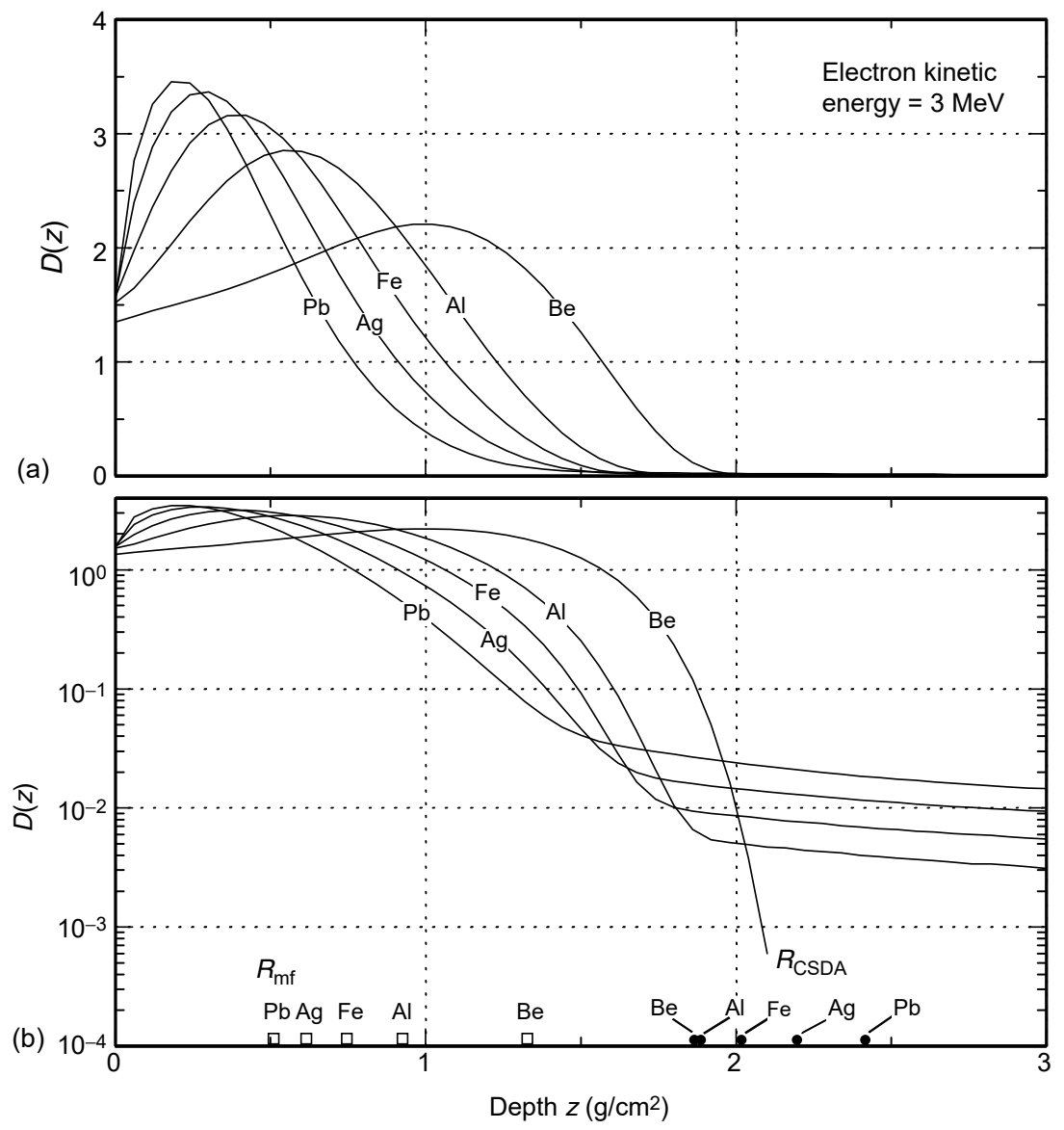

FIGURE 3.37 Dose distribution $D(z)$ of electrons normally entering several elemental solids, in units of $\mathrm{MeV} \mathrm{cm} / \mathrm{g}$ per incident primary electron. Backscattered electrons do not contribute. Within the accuracy of the formulas the integral under each curve is incident electron energy times [1-(backscattering coefficient)]. It is $2.17 \mathrm{MeV}$ for $\mathrm{Pb}$ at $3 \mathrm{MeV}$. The CSDA ranges are indicated as solid dots, the mean forward ranges as open squares. For higher $Z$, dose beyond $R_{\mathrm{CSDA}}$ is caused by Bremsstrahlung, and is effectively zero for low- $Z$ and lower incident energies. (Data from Tabata's EMID algorithm [ID03].) (a) Linear-linear. (b) Log-linear.

$$
R_{\mathrm{CSDA}}^{\mathrm{fit}}=\frac{c_{1}}{B}\left[\frac{\ln \left(1+c_{2} \tau^{c_{3}}\right)}{c_{2}}-\frac{c_{4} \tau^{c_{5}}}{1+c_{6} \tau}\right],
$$

where $B$ is similar to the stopping number:

$$
B=2 \ln \left(\frac{\tau}{I / m c^{2}+c_{7} \tau}\right)+\ln \left(1+\frac{\tau}{2}\right) .
$$


$I$ is the material mean excitation energy as it appears in the Bethe stopping power formula. $c_{1}, \ldots, c_{7}$ are functions of material atomic weight $A$ and atomic number $Z$ :

$$
\begin{array}{ll}
c_{1}=d_{1} A / Z^{d_{2}}, & c_{2}=d_{3} Z^{d_{4}}, \\
c_{3}=d_{5}-d_{6} Z, & c_{4}=d_{7}-d_{8} Z, \\
c_{5}=d_{9}-d_{10} Z, & c_{6}=d_{11} / Z^{d_{12}}, \\
c_{7}=d_{13} Z^{d_{14}} . &
\end{array}
$$

The constants $d_{n}$ are given in Table 3.2.

Figure 3.38 compares $R_{\text {CSDA }}^{\text {fit }}$ from Equation 3.71 to the CSDA range tabulated by NIST [NIST] in several elements. In the important range from $10 \mathrm{keV}$ to $100 \mathrm{MeV}$, the fit is mostly within $1 \%$ of the NIST range, which is adequate for almost all applications.

\subsubsection{Empirical Fit to the Mean Forward Range}

Tabata and Andreo [Ta98b] have also constructed a semiempirical formula, $R_{\mathrm{mf}}^{\mathrm{fit}}$, for the mean forward range, or, rather, the detour factor $R_{\mathrm{mf}} / R_{\mathrm{CSDA}}$. It is

$$
\frac{R_{\mathrm{mf}}^{\mathrm{fit}}}{R_{\mathrm{CSDA}}}=\left[1+\frac{c_{1}(Z-1)^{c_{2}}\left(1+c_{3} \tau\right)}{1+c_{4} \tau^{c_{5}}}\right]^{-1} .
$$

Here,

$$
c_{1}=0.3087, \quad c_{2}=0.6384 \text {, }
$$

\section{TABLE 3.2}

\section{Values of Constants in Fit to CSDA Range}

\begin{tabular}{llrl}
$\boldsymbol{n}$ & \multicolumn{1}{c}{$\boldsymbol{d}_{\boldsymbol{n}}$} & $\boldsymbol{n}$ & \multicolumn{1}{c}{$\boldsymbol{d}_{\boldsymbol{n}}$} \\
1 & 3.60 & 8 & $1.303 \times 10^{-4}$ \\
2 & 0.9882 & 9 & 1.02441 \\
3 & $1.191 \times 10^{-3}$ & 10 & $1.2986 \times 10^{-4}$ \\
4 & 0.8662 & 11 & 1.030 \\
5 & 1.02501 & 12 & $1.110 \times 10^{-2}$ \\
6 & $1.0803 \times 10^{-4}$ & 13 & $1.10 \times 10^{-6}$ \\
7 & 0.99628 & 14 & 0.959 \\
& &
\end{tabular}




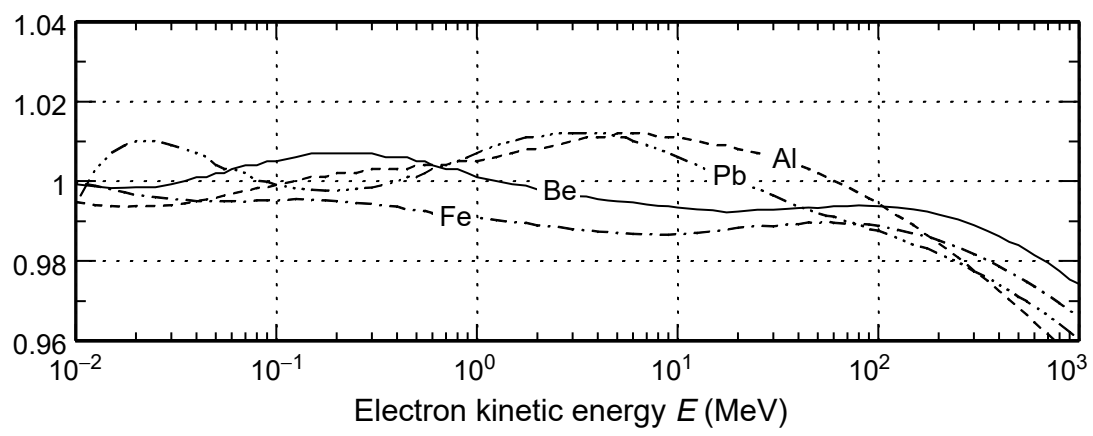

FIGURE 3.38 The ratio of $R_{\mathrm{CSDA}}^{\mathrm{fit}}$ (Equation 3.71) to the NIST CSDA range for four elements.

and $c_{3}$ to $c_{5}$ are functions of $Z$ :

$$
c_{3}=k_{1} Z^{k_{2}-k_{3} \ln Z}, \quad c_{4}=k_{4} / Z^{k_{5}}, \quad c_{5}=k_{6} Z^{k_{7}}
$$

The constants $k_{1} \cdots k_{7}$ are:

$$
\begin{aligned}
& k_{1}=0.00585, k_{2}=0.547, k_{3}=0.0571, k_{4}=0.1012, \\
& k_{5}=0.1026, k_{6}=1.0576, k_{7}=0.0234
\end{aligned}
$$

For $R_{\mathrm{CSDA}}$ in Equation 3.74, one could use $R_{\mathrm{CSDA}}^{\mathrm{fit}}$ fit or one could use the numerical data from NIST. Using the latter, Figure 3.39 shows the fit (Equation 3.74) to the detour factor for several elements. It agrees remarkably well with extensive Monte Carlo computations for $1 \mathrm{MeV}<E<50 \mathrm{MeV}$. It is intended for condensed media, not gases, since the full density effect is included in the data to which the functional form was fit.

\subsubsection{Longmire Treatment of Multiple Scattering and Mean FORWARD RANGE}

Williams' multiple scattering theory is seldom used in Monte Carlo codes because the more accurate Molière or Goudsmit-Saunderson theories are no more difficult to implement numerically. Nevertheless, the analytically simpler Williams theory is convenient for approximate analyses.

In electromagnetic applications involving Compton electrons, it is the electron mean forward range against multiple scattering that is important, for that determines both the mean charge separation and the steady-state current of Compton electrons. The analysis is made difficult because near the end of an electron's range, the angle of deviation from its original direction is not small. In seeking a simplified treatment, Longmire suggested that the Williams theory could be extended in an approximate way to obtain 


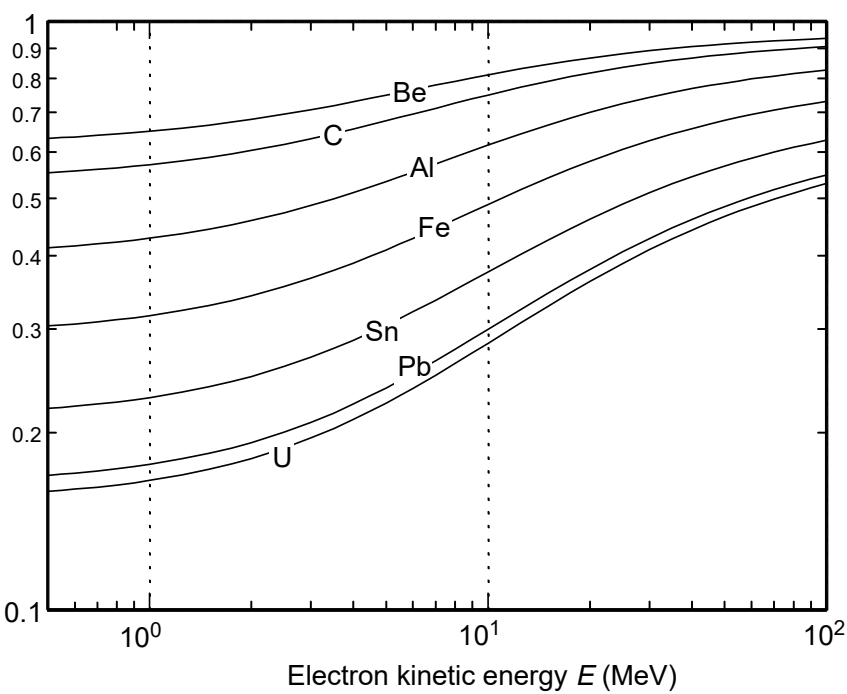

FIGURE 3.39 Fit, Equation 3.74, to detour factor, the ratio $R_{\mathrm{mf}} / R_{\mathrm{CSDA}}$ of the mean forward range to the CSDA range of electrons in several elements. Computed with the online algorithm in http://www3.ocn.ne.jp/ tttabata/[ID03]. It is intended for condensed media, and confirmed accurate for $1 \mathrm{MeV}<E<50 \mathrm{MeV}$.

an estimate for the mean forward range. Rather than using fits to Monte Carlo data, the approach is physically motivated, albeit somewhat heuristic.

Equation 3.63 was extended by Longmire and Longley [Lo73, Lo78a, Lo78b] to $\left\langle\theta^{2}\right\rangle$ not small compared with unity by introducing the obliquity factor $\eta$

$$
\begin{aligned}
\eta & \equiv \frac{1}{\langle\cos \theta\rangle} \\
& \approx 1+\frac{1}{2}\left\langle\theta^{2}\right\rangle \quad \text { for small }\left\langle\theta^{2}\right\rangle .
\end{aligned}
$$

$\eta$ has the advantage of being allowed to get large, unlike $\left\langle\theta^{2}\right\rangle . \eta$ relates the mean forward progress $\mathrm{d} z$ of a directed beam of electrons to an increment $\mathrm{d} s$ in track length by $\mathrm{d} z=(1 / \eta) \mathrm{d} s$. Following Longmire, the equation for $\eta$ is taken over from that for small $\left\langle\theta^{2}\right\rangle$,

$$
\frac{\mathrm{d} \eta}{\mathrm{d} s}=\frac{1}{2} \frac{\mathrm{d}\left\langle\theta^{2}\right\rangle}{\mathrm{d} s} .
$$

Energy loss and scattering rates can now be expressed in terms of forward progress $\mathrm{d} z$, rather than track length $\mathrm{d} s$, 


$$
\frac{\mathrm{d} E}{\mathrm{~d} z}=-\eta L, \quad \frac{\mathrm{d} \eta}{\mathrm{d} z}=\eta \frac{\mathrm{d}\left\langle\theta^{2}\right\rangle}{2 \mathrm{~d} s}
$$

Integrating the first of these gives the mean forward range

$$
R_{\mathrm{mf}}^{L}=\int \mathrm{d} z=\int_{0}^{E_{\mathrm{o}}} \frac{\mathrm{d} E}{\eta L} .
$$

Replacing $\eta$ by 1 reproduces the full CSDA track length $R=R_{\mathrm{CSDA}}$ of Equation 3.20. The required expression for $\eta(E)$ is obtained by noting

$$
\frac{\mathrm{d} \eta}{\mathrm{d} E}=\frac{1}{m c^{2}} \frac{\mathrm{d} \eta}{\mathrm{d} \gamma}=\frac{1}{2 m c^{2}} \frac{\mathrm{d}\left\langle\theta^{2}\right\rangle}{\mathrm{d} \gamma}=-\frac{2 \Gamma}{m c^{2}} \frac{(Z+1)}{\gamma^{2}-1},
$$

where Equation 3.66 has been used. Integrating with constant $\Gamma$, we obtain

$$
\eta=1+(Z+1) \Gamma \ln \left(\frac{\gamma_{\mathrm{o}}-1}{\gamma_{\mathrm{o}}+1} \frac{\gamma+1}{\gamma-1}\right)
$$

for the growth in mean square angle as an electron slows from $\gamma_{\mathrm{o}}$ to $\gamma$, similar to Equation 3.67 except that $\left\langle\theta^{2}\right\rangle$ need no longer be small compared to 1 .

The integral for $R_{\mathrm{mf}}^{L}$, Equation 3.79, with $\eta$ given by Equation 3.81 has been done numerically using the stopping power $L$ tabulated in [NIST]. The results for $R_{\mathrm{mf}}^{L}$, and the fit $\mathrm{R}_{\mathrm{mf}}^{\mathrm{fit}}$ from the EMID algorithm, Equation 3.74, using $R_{\mathrm{CSDA}}$ also tabulated by NIST, are shown in Figure 3.40. They agree remarkably well over the full span of plotted energies, including well below the original $1 \mathrm{MeV}-$ $50 \mathrm{MeV}$ for which Equation 3.74 was intended. References [Lo73, Lo78b] compare $R_{\mathrm{mf}}^{L}$ with earlier data and with Monte Carlo calculations, and show good agreement.

In view of the broad spread in $r_{\mathrm{mf}}(z)$, one seldom needs $R_{\mathrm{mf}}$ to great accuracy. Because the EMID expression $\mathrm{R}_{\mathrm{mf}}^{\mathrm{fit}}$ is known to agree very well with extensive Monte Carlo computations, and because $R_{\mathrm{mf}}^{L}$ is so close to it, one may feel comfortable using either one for the mean forward range. Because $R_{\mathrm{mf}}^{L}$ requires a numerical integration whereas $\mathrm{R}_{\mathrm{mf}}^{\mathrm{fit}}$ is a simple fit, one may prefer the latter for fast work. In simulations in which particles are advanced in time as their energy decreases, the integral equation 3.79 may be advanced as well, and may be just as simple to implement as Equation 3.74.

\subsubsection{Range to a Specified Angular Divergence}

$\Gamma$ is not truly constant, and a more general form of the obliquity factor equations allows one to use stopping power data without introducing the ratio $\Gamma$. One obtains an approximate representation for the mean square 


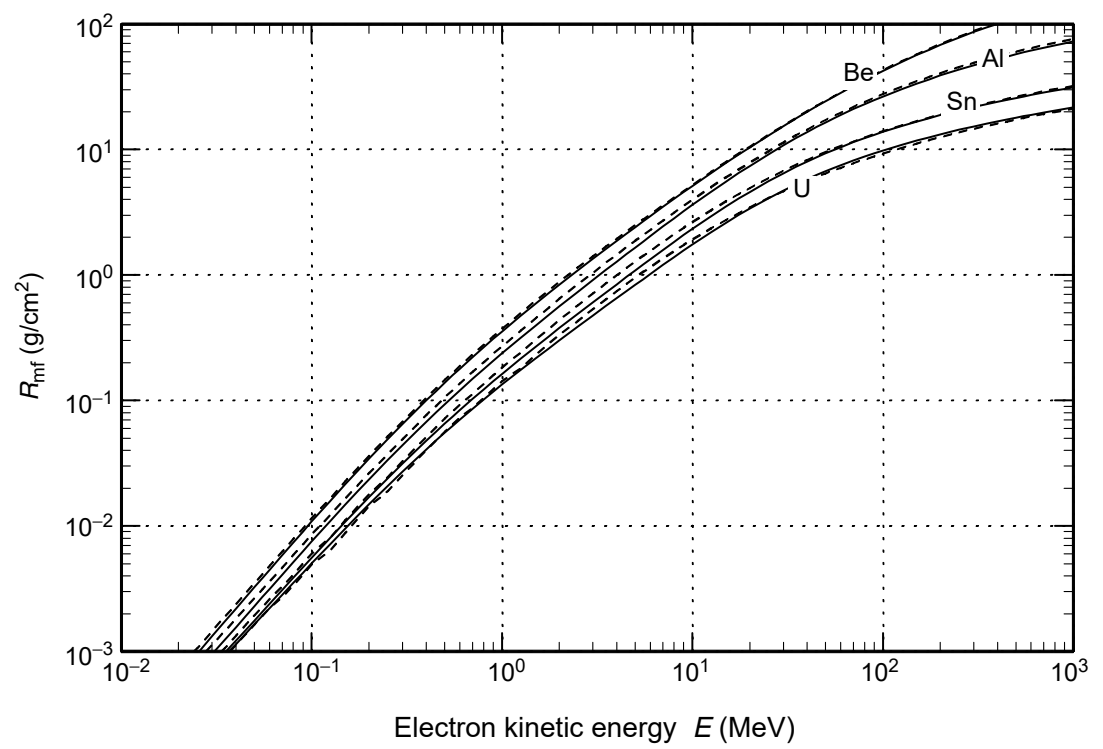

FIGURE 3.40 Comparison of Longmire model for mean forward range, Equation 3.79, (dashed) with the empirical fit of Tabata and coworkers, Equation 3.74 (solid). The latter is intended for $1 \mathrm{MeV}<E<50 \mathrm{MeV}$, but the two models agree quite well over a wider energy span. Below a few tens of $\mathrm{MeV}$, the Longmire range tends to be a few percent greater than the empirical fit.

angle $\left\langle\theta^{2}\right\rangle$ of beam divergence as a function of depth of penetration or as a function of electron energy.

Let $L=L(E) \equiv \mathrm{d} E /\left.\mathrm{d} s\right|_{\text {Total }}$ be the total electron-stopping power (collisional plus radiative), and let $W(E) \equiv \mathrm{d}\left\langle\theta^{2}\right\rangle / \mathrm{d} s$ be the growth rate of the mean square angle of divergence.

While electrons move a distance $\mathrm{d} s$ along their trajectories, they lose an energy $\mathrm{d} E=L \mathrm{~d} s$, and advance on average a forward distance $\mathrm{d} z=$ $\langle\cos \theta\rangle \mathrm{d} s=(1 / \eta) \mathrm{d} s=\mathrm{d} E / \eta L$. At the same time, $\eta$ grows at rate $\mathrm{d} \eta / \mathrm{d} s=\frac{1}{2} W$, and $\eta$ changes by $\mathrm{d} \eta=\frac{1}{2}(W / L) \mathrm{d} E$. The two equations

$$
\begin{aligned}
\mathrm{d} z & =\frac{1}{\eta L} \mathrm{~d} E \\
\mathrm{~d} \eta & =\frac{W}{2 L} \mathrm{~d} E
\end{aligned}
$$

relate the penetration depth $z$ and mean square angle of divergence $\left\langle\theta^{2}\right\rangle$ to the energy loss. Starting with an electron of energy $E_{\mathrm{o}}$, and integrating the two equations together, one obtains $z(E)=$ depth when the energy has been 


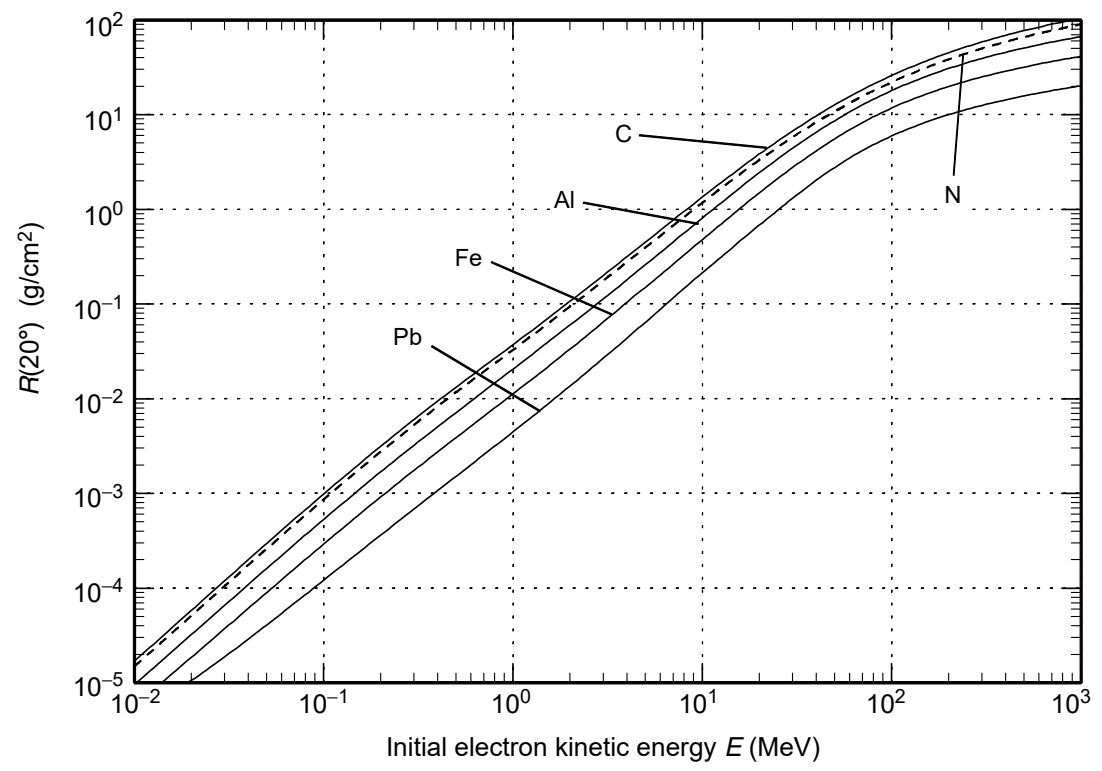

FIGURE 3.41 Approximate electron penetration depth at which a pencil beam has diverged to an rms angle of $20^{\circ}$ (cone half angle).

reduced to $E$, and $\eta(E)=$ obliquity factor when the energy has been reduced to $E$. The latter then gives the root-mean-square divergence angle $\theta_{\text {rms }}=\sqrt{ }\left\langle\theta^{2}\right\rangle=\cos ^{-1}(1 / \eta)$. $\eta$ or $\left\langle\theta^{2}\right\rangle$ may likewise be considered as a function of $z$. If the integration is continued to $E=0$, the resulting depth $z(E=0)$ is the mean forward range $R_{\mathrm{mf}}$. One need assume nothing about the behavior of the ratio $\Gamma$ or the ratio $W / L$, but the price paid is that numerical integration is required.

If the integration is continued to, say, $\theta_{\mathrm{rms}}=20^{\circ}$, one obtains an estimate for the depth of penetration at which a parallel beam has fanned out by a substantial mean angle. This range, $R\left(20^{\circ}\right)$, is shown in Figure 3.41 for several materials. In air, a pencil beam of $1 \mathrm{MeV}$ electrons will diverge to an rms angle of $20^{\circ}$ in about $0.032 \mathrm{~g} / \mathrm{cm}^{2}$ (about $26 \mathrm{~cm}$ at sea level). Similar graphs can be prepared for any angle. The full mean forward range $R_{\mathrm{mf}}$ was shown in Figure 3.24. In air, $R_{\mathrm{mf}}$ is about $0.3 \mathrm{~g} / \mathrm{cm}^{2}$ at $1 \mathrm{MeV}$. Note that at the same energy, the full CSDA range in air against stopping power alone is $0.5 \mathrm{~g} / \mathrm{cm}^{2}$.

\subsection{CROSS SECTIONS IN SELECTED ELEMENTS}

Most frequently, it is the stopping power or range of electrons that are the useful quantities. These have been presented in the preceding sections. An 
important exception occurs in Monte Carlo transport codes, which use actual cross sections.

Although most often used in those codes, it is instructive to see cross sections for representative elements. Electron-atom interaction cross sections on five elements, $\mathrm{H}, \mathrm{C}, \mathrm{Al}, \mathrm{Fe}$, and $\mathrm{U}$, are presented in this section in Figure $3.42 \mathrm{a}$ through Figure 3.42e.

We previously discussed the small-angle and large-angle elastic scattering cross sections. The distinction between the two is most important at relatively high energies, where small-angle scattering dominates. The graphs show both the large-angle cross section and the total (small angle + large angle) elastic cross section. Recall that the separation is defined at $1-\cos \theta=10^{-6}$, or $\theta=0.081^{\circ}$.

All cross sections are obtained from the LLNL library, EEDL. The ENDF format contains only the large-angle elastic cross section. One could compute the small-angle one following the rules in Section 3.2. However, the total elastic cross section is contained in the older ENDL format. As stated, the Bremsstrahlung cross section is that for producing photons of energy greater than $0.1 \mathrm{eV}$. The excitation cross section is the total, the sum over excitations from any shell to all other shells. The ionization cross section is also the total, the sum of ionizations over all shells.

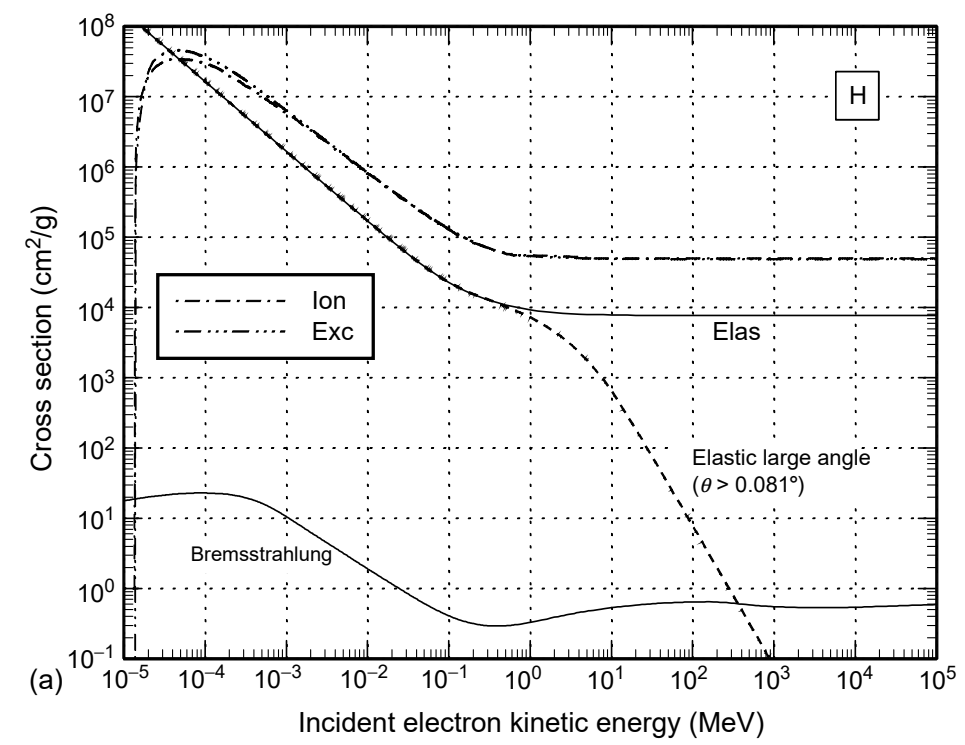

FIGURE 3.42 (a) All electroatomic cross sections on $\mathrm{H}$. Elastic large angle cross section is shown separately, along with the total elastic. Bremsstrahlung is the cross section for producing photons of energy greater than $0.1 \mathrm{eV}$. These individual cross sections are to be sharphy distinguished from energy loss rates due to each process. 

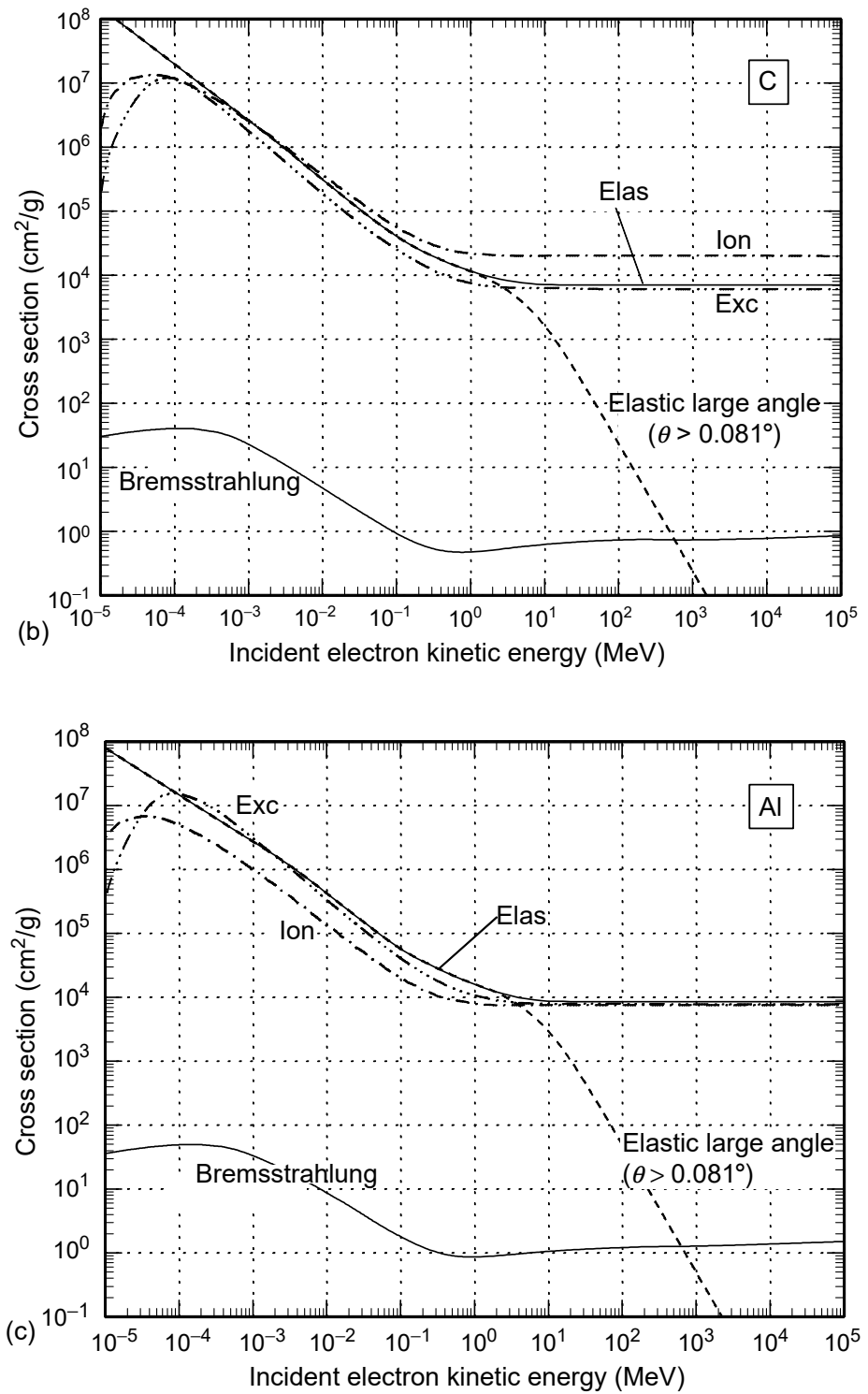

FIGURE 3.42 (continued) All electroatomic cross sections on $\mathrm{C}$ (b) and $\mathrm{Al}$ (c). Elastic large angle cross section is shown separately, along with the total elastic. Bremsstrahlung is the cross section for producing photons of energy greater than 0.1 $\mathrm{eV}$. These individual cross sections are to be sharply distinguished from energy loss rates due to each process. 

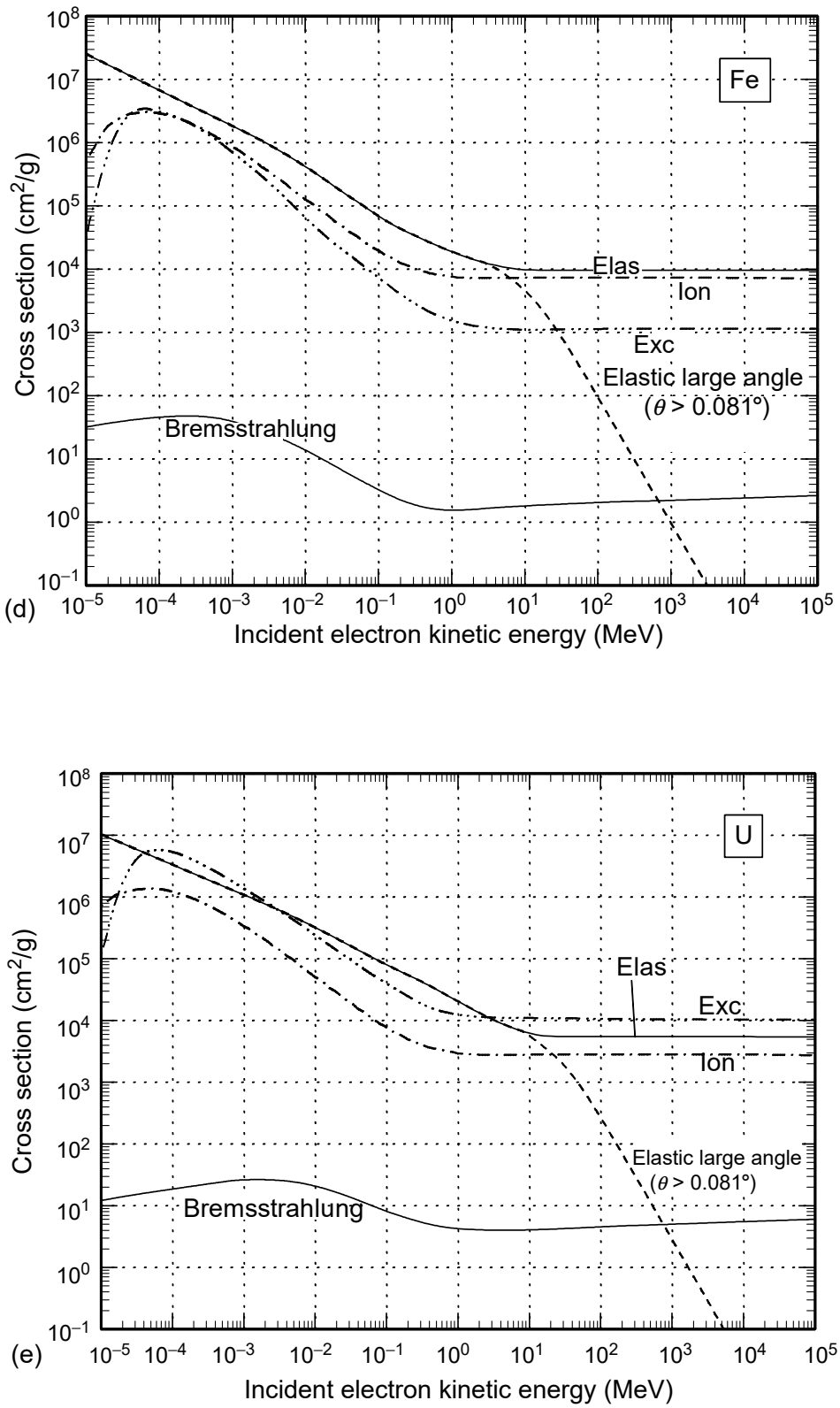

FIGURE 3.42 (continued) All electroatomic cross sections on Fe (d) and U (e). Elastic large angle cross section is shown separately, along with the total elastic. Bremsstrahlung is the cross section for producing photons of energy greater than $0.1 \mathrm{eV}$. These individual cross sections are to be sharply distinguished from energy loss rates due to each process. 


\subsection{ELECTRONS IN LOW-PRESSURE AIR}

The CSDA range of a $10 \mathrm{keV}$ electron in air is about $2.9 \times 10^{-4} \mathrm{~g} / \mathrm{cm}^{2}$, or about $0.24 \mathrm{~cm}$ at STP. Its path length before significant multiple scattering, say $\theta_{\text {rms }}=\sqrt{ }\left\langle\theta^{2}\right\rangle=1$, is of the order $0.1 \mathrm{~cm}$. Although there may be circumstances in which this short distance is important, it is more likely that the physical motion of an electron of energy of the order $10 \mathrm{keV}$ in air is of concern at low pressure in the laboratory or at high altitude in the atmosphere, where the range may be many meters.

Although it departs somewhat from our focus on more energetic electrons, the behavior in low-pressure air is not infrequently of interest. Therefore, for reference, we collect graphs for the energy loss, scattering, and ranges of electrons with kinetic energy $E \lesssim 30 \mathrm{keV}$ in air. At these energies, radiation energy loss (Bremsstrahlung) is negligible compared with collisional energy loss. Longmire has presented similar quantities for electrons from $10 \mathrm{keV}$ to $5 \mathrm{MeV}$ in air and other materials [Lo72].

\subsubsection{Energy Loss Rate}

In a diatomic gas, electrons suffer energy loss to elastic, rotational, vibrational, electronic, and ionization collisions. Including all these processes, Figure 3.43 shows the mean energy loss rate

$$
L(E) \equiv \mathrm{d} E / \mathrm{d} s\left(\mathrm{MeV} \mathrm{cm}^{2} / \mathrm{g}\right)
$$

as a function of electron energy $E$ for electrons in air (here $0.79 \mathrm{~N}_{2}+0.21 \mathrm{O}_{2}$ by number). The pronounced peak near $2.5 \mathrm{eV}$ is due to the $\mathrm{N}_{2}$ vibrational resonance. Also shown in the figure is the Bethe mean stopping power formula, Equation 3.21, in air. It is inapplicable below a few $\mathrm{keV}$, and is seen to be accurate only above $100 \mathrm{keV}$. In atmospheric applications, the stopping power is sometimes called the electron loss function.

The processes included in the correct $L$ of Figure 3.43 include all those mentioned earlier, with separate treatment of Rydberg states.

Specifically, for $\mathrm{N}_{2}$, the elastic momentum-transfer cross section above $0.01 \mathrm{eV}$ is from [Wa82], and below $0.01 \mathrm{eV}$ is from [Ph85a]. Above the available data range $(200 \mathrm{eV})$, we extrapolated with a power law $\sigma \sim 1 / E^{1.36}$. Rotational cross sections are the standard Gerjuoy-Stein cross sections [Ge55] with a quadrupole moment of 1.04 (in units of $e a_{\mathrm{o}}^{2}$, where $a_{\mathrm{o}}$ is the Bohr radius). Vibrational cross sections are from the measurements by Schulz [Sc64, Sc73] with the normalization suggestion of Haas [Ha57]. A lowenergy tail $(0.29 \mathrm{eV}-1.7 \mathrm{eV})$ for the $v=0$ to $v=1$ transition was added as suggested by Englehardt et al. [En64], with numerical values by Kieffer [Ki73] ( $v$ is the vibrational quantum number). Electronic excitation and ionization cross sections were obtained from Archer [Ar89], and agree with 


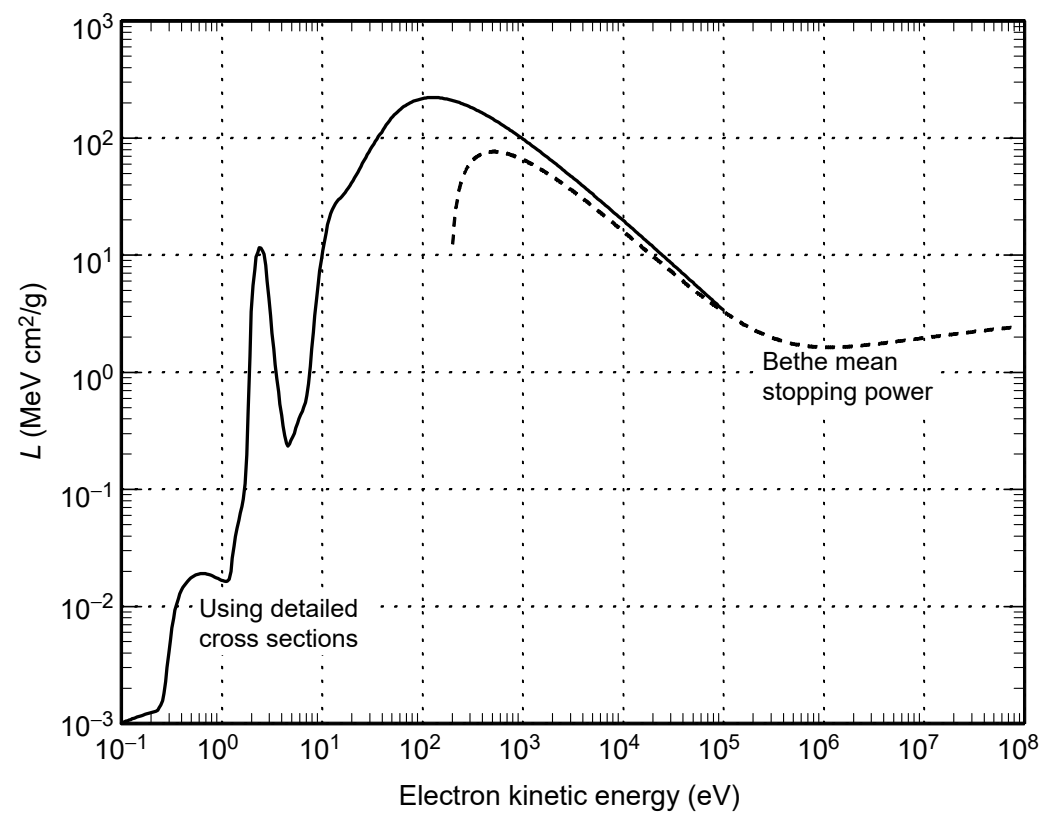

FIGURE 3.43 Electron mean energy loss rate (loss function) in air, including elastic recoil, and rotational, vibrational, and electronic excitations, and ionization (solid curve), compared with the Bethe mean stopping power formula (dashed).

those in the Defense Nuclear Agency Reaction Rate Handbook [DNA83], and with the compilation in [Wa82].

For $\mathrm{O}_{2}$, we use the momentum-transfer cross section of [Wa82] corrected by Kieffer's low-energy tail [Ki73] and renormalized as suggested by Phelps [Ph85b]. There is no definite resolution of the $\mathrm{O}_{2}$ rotational cross sections [Sc73, La80]; we again use those of Gerjuoy-Stein with quadrupole moment 1.8. Vibrational cross sections are from [Li71] and [Ph85b]. Electronic and ionization are again from Archer [Ar89]. These cross sections, and the mean stopping power, have been in common use, for example, in the auroral physics community. A more recent survey of $e-\mathrm{N}_{2}$ and $e-\mathrm{O}_{2}$ cross sections has been prepared by Majeed and Strickland [Ma97].

As electron energy increases from zero, first elastic scattering, then rotational, vibrational, electronic, and ionization excitations are available to it, and its energy loss per centimeter increases dramatically.

The electron mean track length $R$ is, as in Equation 3.20,

$$
R(E)=\int_{0}^{E} \frac{\mathrm{d} E^{\prime}}{L\left(E^{\prime}\right)}
$$


Below about $100 \mathrm{eV}$, the rapid increase of $L$ with energy means that an electron of $E \lesssim 100 \mathrm{eV}$ loses most of its energy early in its path, and $R$ is only a slowly varying function of $E$. In addition, below $100 \mathrm{eV}$, elastic scattering is the most probable process, and CSDA range is not a useful measure of actual electron motion.

\subsubsection{Range against Energy Loss}

Numerical integration of $\mathrm{d} E / L$ in Equation 3.83 from $E_{1}$ to $E$ gives an electron's path length along its trajectory, $R$, in slowing down from $E$ to $E_{1}$. $R$ is shown in Figure 3.44 for $E_{1}=0.3 \mathrm{eV}, 1 \mathrm{eV}, 3 \mathrm{eV}, 10 \mathrm{eV}, 30 \mathrm{eV}$, and 100 $\mathrm{eV}$. Below $100 \mathrm{eV}$, the stopping power behaves very roughly as $L \sim E^{2}$. Consequently, the range $\sim 1 / E_{1}-1 / E$, and is nearly independent of $E$ so long as $E \gg E_{1}$. This explains the flatness of the curves below a few hundred $\mathrm{eV}$ down to $0.3 \mathrm{eV}, 1 \mathrm{eV}$, or $3 \mathrm{eV}$. The range to $3 \mathrm{eV}$ is much greater than to 10 $\mathrm{eV}$ because there is a dramatic local drop in stopping power between $3 \mathrm{eV}$ and $10 \mathrm{eV}$, just above the $\mathrm{N}_{2}$ vibrational spike and before electronic excitation sets in. A $300 \mathrm{eV}$ electron has a track length of $10^{-6} \mathrm{~g} / \mathrm{cm}^{2}(0.6 \mathrm{~cm}$ at 1 torr $)$ before its energy is reduced to $100 \mathrm{eV}$ and then an additional $10^{-6}$ by the time its energy is down to $10 \mathrm{eV}$. By an initial energy of $10 \mathrm{keV}$, the range is independent of $E_{1} \cdot R(10 \mathrm{keV}) \approx 3 \times 10^{-4} \mathrm{~g} / \mathrm{cm}^{2}(1.8 \mathrm{~m}$ at 1 torr $)$.

However, for these low energies, the electron mean forward range $R_{\mathrm{mf}}$ is significantly less than its CSDA range $R$, because of elastic scattering. $R_{\mathrm{mf}}$ and the mfp against momentum-transfer scattering are discussed presently.

\subsubsection{Angular Scattering}

The momentum-transfer cross section due to elastic scattering (also called the diffusion cross section),

$$
\sigma_{\mathrm{m}}=\int \frac{\mathrm{d} \sigma_{\text {elas }}}{\mathrm{d} \Omega}(1-\cos \theta) \mathrm{d} \Omega
$$

is the relevant measure of change of forward momentum in an elastic collision. It provides the useful measure of a low-energy electron's motion in air. For example, in a fluid treatment of electrons, elastic collisions change the fluid velocity $u$ by $\mathrm{d} u / \mathrm{d} t=-\nu_{\mathrm{m}} u$, where $\nu_{\mathrm{m}}=N\left\langle\sigma_{\mathrm{m}} u\right\rangle$ is the momentumtransfer collision frequency, $N=$ air molecular density, and $\langle\cdots\rangle$ denotes an average over the energy spectrum.

Figure 3.45 and Figure 3.46 show all $e-\mathrm{N}_{2}$ and $e-\mathrm{O}_{2}$ cross sections below about $200 \mathrm{eV}$ [Wa82]. Elastic momentum-transfer scattering dominates below about $100 \mathrm{eV}$. Figure 3.47 shows $\sigma_{\mathrm{m}}$ more fully for $\mathrm{N}_{2}$ and for $\mathrm{O}_{2}$. The 


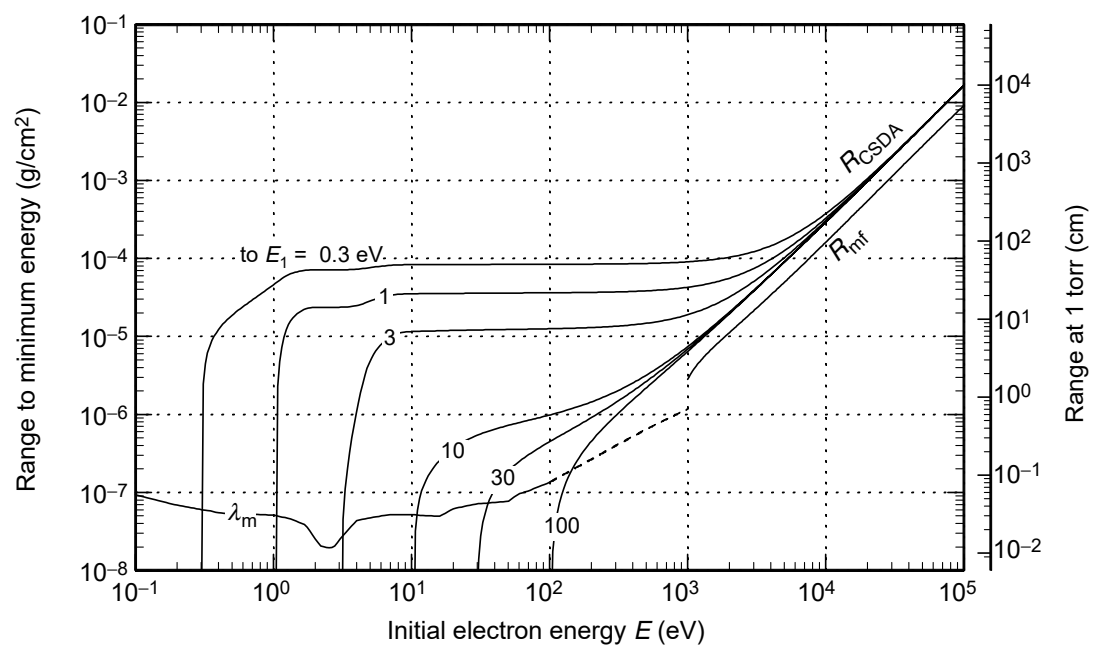

FIGURE 3.44 Trajectory path length, $R_{\mathrm{CSDA}}$, of electrons in air from high energy to various final energies $E_{1} . R_{\mathrm{mf}}$ is the mean forward range against elastic scattering of electrons of energy greater than $1 \mathrm{keV} . \lambda_{\mathrm{m}}$ is the mean free path against momentumtransfer scattering; dashed portion above $100 \mathrm{eV}$ is unreliable. At 1 torr, below about $100 \mathrm{eV}, \lambda_{\mathrm{m}}$ is less than $0.1 \mathrm{~cm}$.

$\mathrm{O}_{2}$ momentum-transfer cross sections of [Wa82] and of Phelps [Ph85b] differ below $0.7 \mathrm{eV}$ or above $8 \mathrm{eV}$, as mentioned earlier in the discussion of energy loss rate. The discrepancy is not significant in air.

For kinetic energies $E$ greater than about $1 \mathrm{keV}$, multiple small-angle scattering dominates the motion. Considering only $E<60 \mathrm{MeV}$ in air, so that we can replace $\theta_{\max }$ with unity, we have, according to Equation 3.63 , for the rate of growth of the mean square angle $\left\langle\theta^{2}\right\rangle$ relative to the original direction, per unit track length $\mathrm{d} s$

$$
\begin{aligned}
\frac{\mathrm{d}\left\langle\theta^{2}\right\rangle}{\mathrm{d} s} & =\frac{8 \pi N_{A} Z(Z+1) r_{\mathrm{o}}^{2}}{A \gamma^{2} \beta^{4}} \ln \left(\frac{1}{\theta_{\mathrm{s}}}\right) \quad \mathrm{rad}^{2} \mathrm{~cm}^{2} / \mathrm{g} \\
& =\left(\frac{384 \mathrm{keV}}{E}\right)^{2} \ln \left(\frac{E}{73 \mathrm{eV}}\right) \quad \text { nonrelativistic } \\
& =\frac{4.89}{\left(1+E / m c^{2}\right)^{2}} \ln \left[58.8\left(1+E / m c^{2}\right)\right] . \quad \beta \rightarrow 1
\end{aligned}
$$

The first form is for any electron energy and for any material, the second is for nonrelativistic electrons in air, where $A=14.5$ is the mean atomic weight of an air atom, $Z=7.2$ is the average atomic number, and the last 


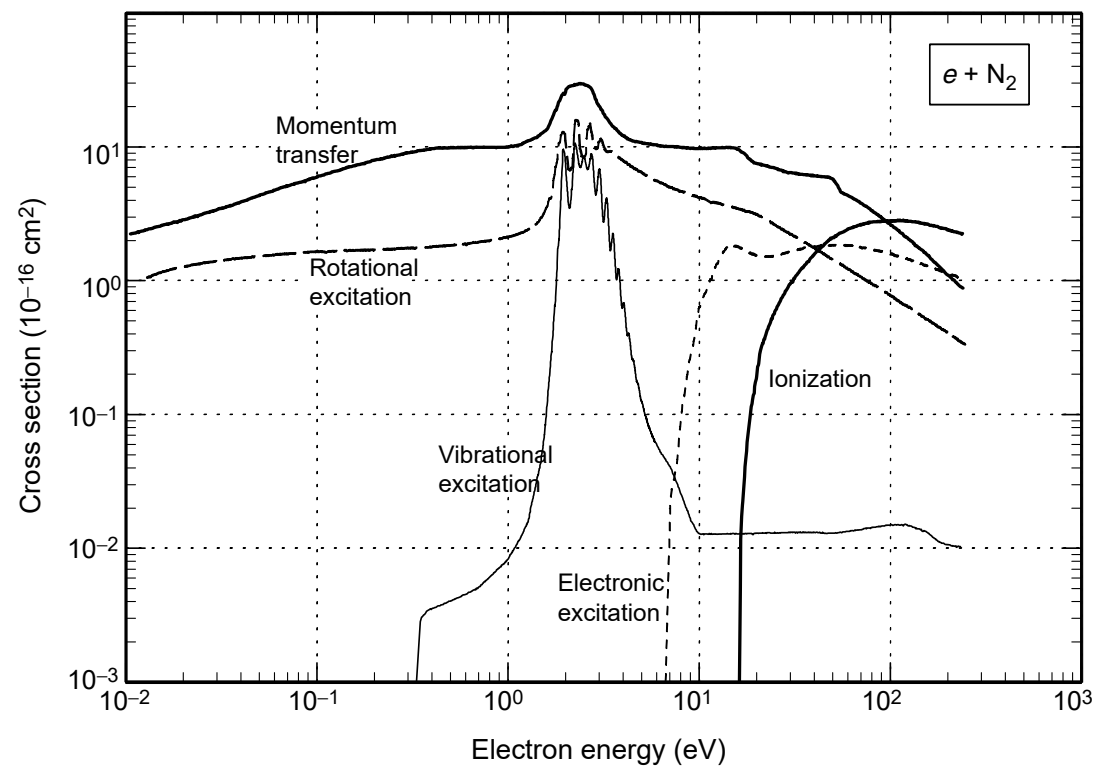

FIGURE 3.45 Cross sections for electron collisions on molecular nitrogen. (From the compilation by Wadzinski and Jasperse [Wa82].)

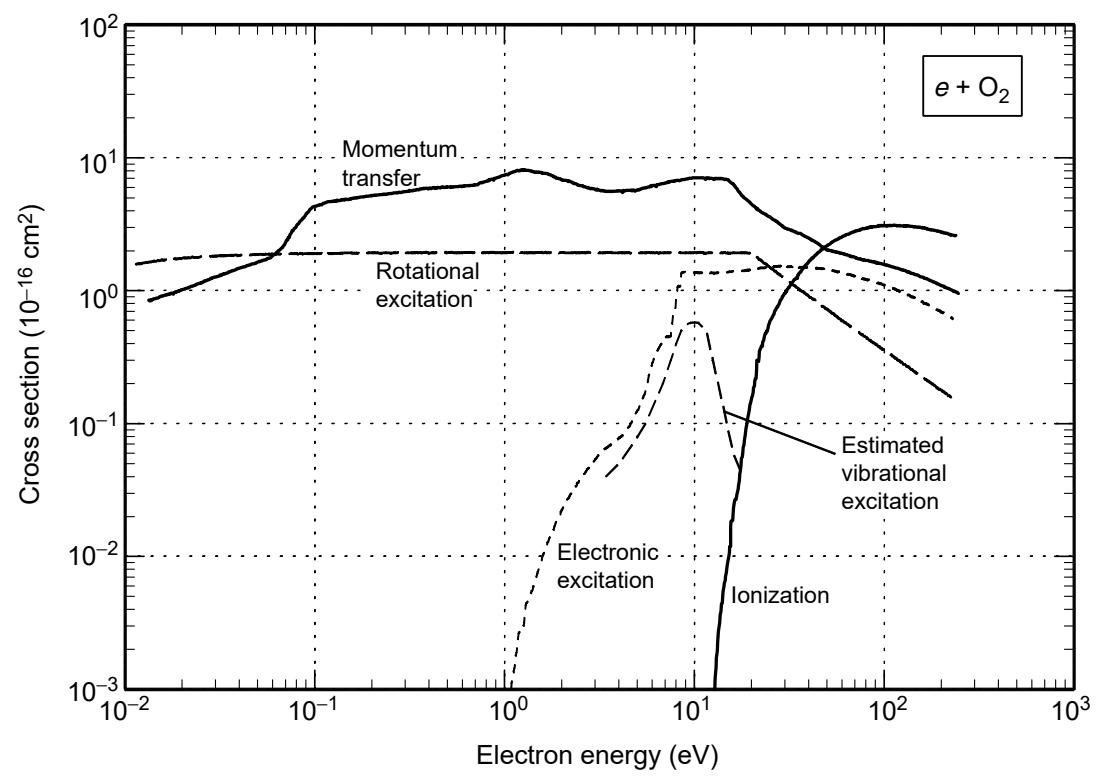

FIGURE 3.46 Cross sections for electron collisions on molecular oxygen. (From the compilation by Wadzinski and Jasperse [Wa82].) 


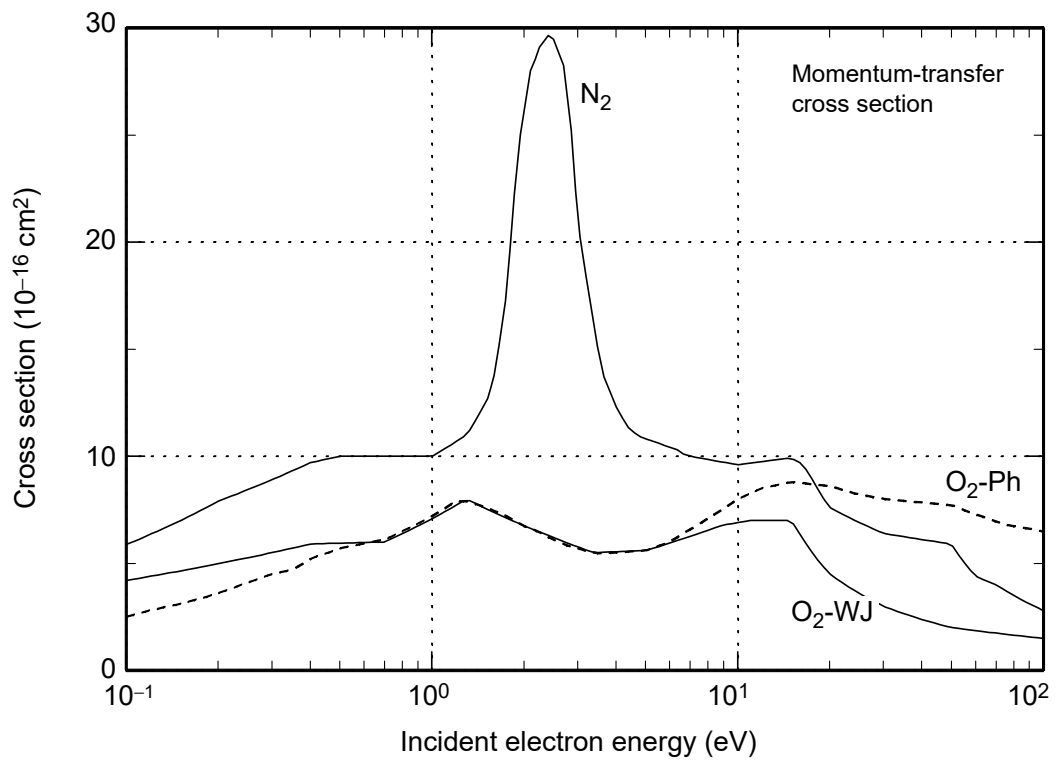

FIGURE 3.47 Momentum-transfer cross sections in $\mathrm{O}_{2}$ and $\mathrm{N}_{2} \cdot\left(\mathrm{N}_{2}\right.$ and $\mathrm{O}_{2}$-WJ from Wadzinski and Jasperse [Wa82]. $\mathrm{O}_{2}$-Ph from Phelps [Ph85b].)

form is for relativistic electrons in air (we assume only $\beta \longrightarrow 1, E$ need not be extreme relativistic). This equation is intended for $\left\langle\theta^{2}\right\rangle \ll 1$. Upon multiplying by air density, Equation 3.85 shows that $115 \mathrm{keV}$ electrons in sea-level air are growing in mean square angle at a rate of about $1 \mathrm{rad}^{2}$ in $10 \mathrm{~cm}$, whereas $3 \mathrm{MeV}$ electrons are expanding at a rate of $0.076 \mathrm{rad}^{2}$ in $1 \mathrm{~m}$.

The increasing angular divergence as energy decreases may be obtained by applying Equation 3.66 for the case $Z=7.2$. For air below $100 \mathrm{keV}$, it is safer to use the actual stopping power rather than the Bethe formula. Then, the effective $\Gamma$ is more nearly equal to 0.245 . Using that constant value, we find

$$
\frac{\mathrm{d}\left\langle\theta^{2}\right\rangle}{\mathrm{d} E}=-\frac{4.0}{E}
$$

intended for nonrelativistic electrons. Thus, as an electron of initial energy $E_{\mathrm{o}}$ slows down, its angular deviation from its original direction increases roughly as

$$
\left\langle\theta^{2}\right\rangle \approx 4 \ln \left(\frac{E_{\mathrm{o}}}{E}\right) \quad \text { nonrelativistic }
$$


and every electron loses significant memory of its initial direction $\left(\left\langle\theta^{2}\right\rangle\right.$ equals, say, 1) by the time its energy has dropped to about $0.78 E_{\mathrm{o}}$. The growth rate $\mathrm{d}\left\langle\theta^{2}\right\rangle / \mathrm{d} E$ is shown in Figure 3.48, computed both from the approximate equation and from Williams' $\mathrm{d}\left\langle\theta^{2}\right\rangle / \mathrm{d} s$ equation, with the actual air $\mathrm{d} E / \mathrm{d} s=L$ from Figure 3.43. We have again stretched the validity of Equation 3.85 by applying it for $\left\langle\theta^{2}\right\rangle \sim 1$. Equation 3.87 is approximately valid for $400 \mathrm{eV} \lesssim$ $E_{\mathrm{o}} \lesssim 600 \mathrm{keV}$ and can be used as a guide for the angular spread of nonrelativistic electrons slowing down in air.

We add that for relativistic electrons Equation 3.69 holds, and when $E \gg m c^{2}$,

$$
\begin{aligned}
\left\langle\theta^{2}\right\rangle & =4 \Gamma(Z+1) m c^{2}\left(\frac{1}{E}-\frac{1}{E_{\mathrm{o}}}\right) \quad\left(E, E_{\mathrm{o}} \gg m c^{2}\right) \\
& =4.5\left(\frac{1}{E}-\frac{1}{E_{\mathrm{o}}}\right)
\end{aligned}
$$

The last form is for air and energies are in $\mathrm{MeV}$. At sea level, a $3 \mathrm{MeV}$ electron beam grows to $\theta_{\mathrm{rms}}=\left\langle\theta^{2}\right\rangle^{\frac{1}{2}}=1 \mathrm{rad}$ by the time it has slowed down to $E=1.8$ $\mathrm{MeV}$, or after traversing about $0.7 \mathrm{~g} / \mathrm{cm}^{2}=5.8 \mathrm{~m}$.

\subsubsection{Scattering Range}

As discussed in Section 3.8, the scattering range $R_{\mathrm{sc}}$ defines how far a directed beam of electrons travels before it has undergone significant scattering. Defining significant again by $\left\langle\theta^{2}\right\rangle=1$, then according to Equation 3.87 it occurs when $E=0.78 E_{\mathrm{o}}$. Figure 3.44 shows that in air, for $E_{\mathrm{o}} \gtrsim 1 \mathrm{keV}$, the CSDA range behaves approximately as $R \propto E_{\mathrm{o}}^{5 / 3}$, at least up to $100 \mathrm{keV}$. Thus, in air

$$
R_{\mathrm{sc}} \approx\left[1-(0.78)^{5 / 3}\right] R=0.34 R
$$

for initial energy $E_{\mathrm{o}}>1 \mathrm{keV}$. At $E_{\mathrm{o}}=10 \mathrm{keV}, R_{\mathrm{sc}}=10^{-4}\left(\mathrm{~g} / \mathrm{cm}^{2}\right)$. For $E_{\mathrm{o}}<1 \mathrm{keV}, R_{\mathrm{sc}}$ is less than $0.34 R$.

\subsubsection{Momentum-Transfer Mean Free Path}

The concept of mean forward range was developed in the context of multiple small-angle scattering. But at low energies, scattering is not sharply peaked in the forward direction. Multiple small-angle scattering does not apply. Indeed, the small-angle $\theta_{\mathrm{s}}$, in Equation 3.17, exceeds 1 in air when $E<100 \mathrm{eV}$. The mean forward range is still a physically meaningful number, but it cannot be expressed in terms of small-angle scattering as it can for higher energies.

Instead, one introduces the $\mathrm{mfp} \lambda_{\mathrm{m}}=1 / N \sigma_{\mathrm{m}}$ against momentum-transfer scattering. $\lambda_{\mathrm{m}}$ is the distance an electron goes before significant change in the component of its momentum in its original direction of motion, where $N$ is the 


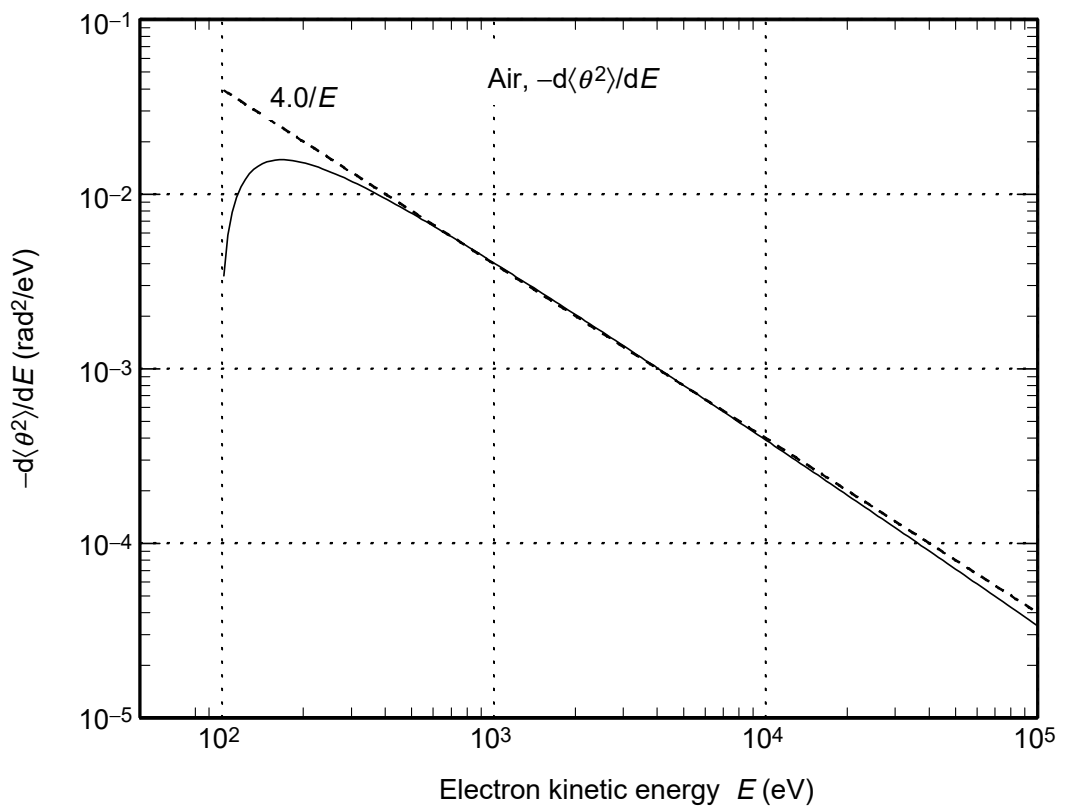

FIGURE 3.48 Incremental growth of electron mean square angle in air as energy decreases. Solid line: Actual mean stopping power and Williams' $\mathrm{d}\left\langle\theta^{2}\right\rangle / \mathrm{d} s$. Dashed line: The approximate form $4 / E$.

density of scattering centers. $\sigma_{\mathrm{m}}$ for air is $0.79 \sigma_{\mathrm{m}}\left(\mathrm{N}_{2}\right)+0.21 \sigma_{\mathrm{m}}\left(\mathrm{O}_{2}\right) . \lambda_{\mathrm{m}}$ in air is shown in Figure 3.44. Below $100 \mathrm{eV}, \lambda_{\mathrm{m}}$ is less than $0.1 \mathrm{~cm}$ at 1 torr. Above $100 \mathrm{eV}$, the required $\sigma_{\mathrm{m}}$ are obtained here by simple power-law extrapolation, and are of questionable validity.

\subsubsection{IONIZATION}

For reference, Figure 3.49 presents the total ionization cross sections on $\mathrm{N}_{2}$ and $\mathrm{O}_{2}$ [Ar89]. At energies below $10 \mathrm{keV}$, these agree well with data in the DNA Reaction Rate Handbook [DNA83] and they agree with the compilation in [Wa82], which stops at $200 \mathrm{eV}$. At energies above $1 \mathrm{keV}$, ionization is the dominant contributor (along with electronic excitation) to $L$, and $L$ and $R$ based on these cross sections agree well with the compilation by Berger and Seltzer [Be82], especially above a few keV.

\subsection{LARGE CONTOUR PLOTS}

Seven large, color contour plots $\left(11^{\prime \prime} \times 17^{\prime \prime}\right)$ are available that graph useful electron parameters. They are in high-resolution PDF format on the accompanying $\mathrm{CD}$ (and would require a printer capable of handling $11^{\prime \prime} \times 17^{\prime \prime}$ paper): 


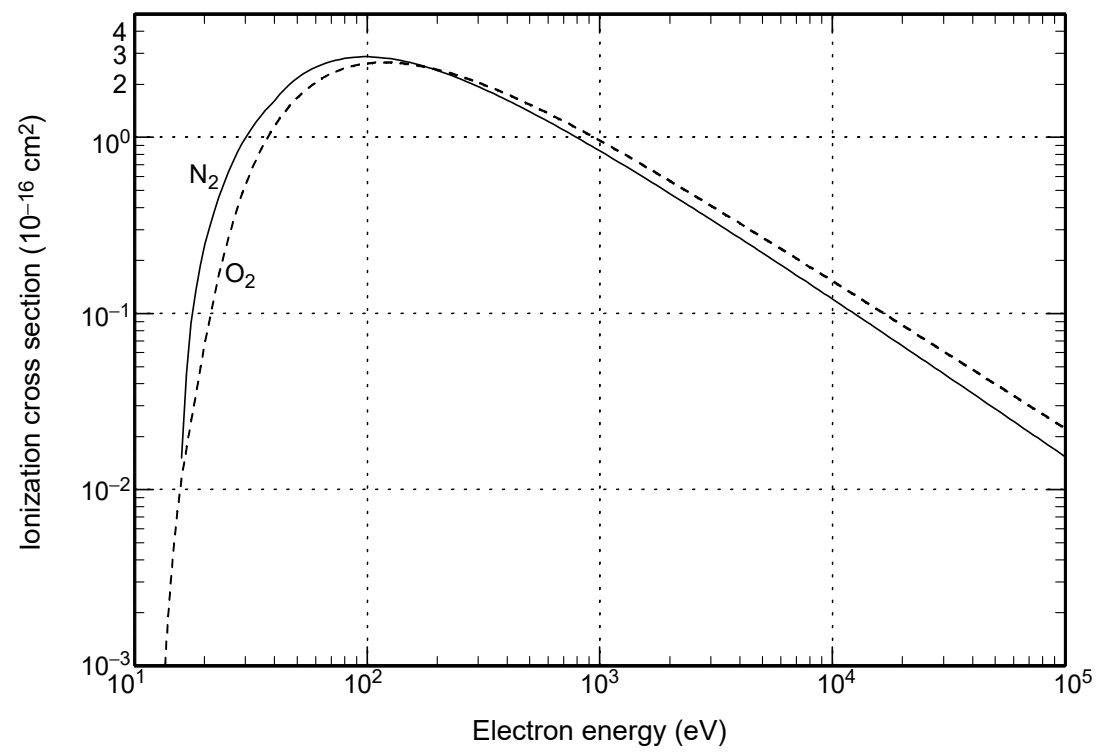

FIGURE 3.49 Total ionization cross sections on ground states of $\mathrm{N}_{2}$ and $\mathrm{O}_{2}$. (From Archer [Ar89].)

Collisional mean stopping power. Mean energy loss to excitations and ionizations $\left(\mathrm{MeV} \mathrm{cm}^{2} / \mathrm{g}\right)$ in all elements. (approximately equal to the linear energy transfer). The two main national databases are presented separately:

- Data from NIST eSTAR database (online). $10 \mathrm{keV}<E<10 \mathrm{GeV}$.

- Data from $L L N L E E D L$ database $1 \mathrm{keV}<E<1 \mathrm{GeV}$.

There is a one-decade shift in energy scale between the two graphs. For the density effect correction, NIST uses gas densities at $1 \mathrm{~atm}$ and $20^{\circ} \mathrm{C}$; in the LLNL data, densities of elements that are gases at 1 atm and $20^{\circ} \mathrm{C}$ are taken to be of the order $1 \mathrm{~g} / \mathrm{cm}^{3}$, more appropriate to solid or liquid compounds (e.g., $\mathrm{O}$ in $\mathrm{SiO}_{2}$ ). This difference in density causes visible differences in collisional stopping power between the two compilations in gases at high energy because of different density effect corrections. In the LLNL graph, the local increases at elements $Z=46$ and 77 at all energies is apparently an error in the data. Hydrogen is plotted at half its true value, as was the case for photon cross sections.

- Total (collisional + Bremsstrahlung) mean stopping power, $d E /\left.d s\right|_{\text {Total }}$. Mean energy loss of electrons caused by all processes (collisional + radiative). Here and in the two collisional stopping power 
graphs, the fraction $f_{R}$ of total stopping power that is radiative, $f_{R}=$ (Bremsstrahlung)/(collisional + Bremsstrahlung), is superimposed in a few dashed blue contour lines. In any element, the critical energy is the electron energy at which stopping power due to collisions equals stopping power due to Bremsstrahlung, that is, where the plotted fraction $f_{R}=1 / 2$. The density effect applies only to collisional stopping power, and is significant only at high energies. Since Bremsstrahlung far exceeds collisional stopping power at high energies, differences between density effect corrections as a result of differences in assumed density (as of gases) have little effect on total stopping power. Different density effect corrections do, however, affect energy deposition (actually kerma) in the material by a passing relativistic electron.

- Full electron range, $R_{C S D A}$, against total mean stopping power. The CSDA range $=R_{\mathrm{CSDA}}=\int \mathrm{d} E /\left(\mathrm{d} E /\left.\mathrm{d} s\right|_{\text {Total }}\right)=$ electron full trajectory path length.

- Approximate electron mean forward range, $R_{m f}$. The mean depth of penetration of electrons in their original direction of motion against multiple small-angle elastic scattering and total stopping power. Here and for the next two ranges, $R_{\mathrm{sc}}\left(20^{\circ}\right)$ and $R_{\mathrm{sc}}\left(57^{\circ}\right)$, the treatment is approximate, using Williams' multiple small-angle scattering formula, Longmire's obliquity factor approach to handle relatively large divergence angles, and stopping power data from NIST (collisional and radiative). However, limited comparisons with more accurate calculations show agreement to within a few percent. The scattering rate increases as the electron slows down.

- Electron scattering range to $20^{\circ}, R_{s c}\left(20^{\circ}\right)$. Range at which initially directed beam diverges to an rms scattering angle $\theta_{\mathrm{rms}} \equiv$ $\sqrt{ }\left\langle\theta^{2}\right\rangle=0.35=20^{\circ}$. Note that $\left\langle\theta^{2}\right\rangle \approx 0.1$ here. $\theta$ is cone half angle (measured from beam axis). The plot allows one to estimate beam divergence through a foil. $1 \mathrm{MeV}$ electrons are seen to diverge $\sim 20^{\circ}$ in penetrating $0.02 \mathrm{~g} / \mathrm{cm}^{2} \mathrm{Al}$, while they lose only $30 \mathrm{keV}$ energy.

- Electron scattering range to $1 \mathrm{rad}, R_{s c}\left(57^{\circ}\right)$. Range at which initially directed beam diverges to $\theta_{\mathrm{rms}}=1 \mathrm{rad}$. Here $\left\langle\theta^{2}\right\rangle$ is about ten times the previous one $\left(20^{\circ}\right)$; hence ranges are, roughly, ten times as far.

Similar graphs can be constructed for the range to any angle. Depending on one's definition, the $1 \mathrm{rad}$ range, or the $20^{\circ}$ range, or the range to any other chosen angle, may be taken as a measure of how far parallel electrons propagate before multiple small-angle scattering causes them to diverge "significantly."

At $1 \mathrm{MeV}$ in air: $R_{\mathrm{sc}}\left(20^{\circ}\right)=0.032, \quad R_{\mathrm{sc}}\left(57^{\circ}\right)=0.22, R_{\mathrm{mf}}=0.3, \quad R_{\mathrm{CSDA}}=0.5 \mathrm{~g} / \mathrm{cm}^{2}$. in Pb: $R_{\mathrm{sc}}\left(20^{\circ}\right)=0.0045, R_{\mathrm{sc}}\left(57^{\circ}\right)=0.05, R_{\mathrm{mf}}=0.16, R_{\mathrm{CSDA}}=0.79 \mathrm{~g} / \mathrm{cm}^{2}$. 


\subsection{DATA ON THE CD-ROM}

We provide in the $\mathrm{CD}$ accompanying this book numerical cross sections and stopping powers for electrons in all the elements from $1 \mathrm{keV}$ to $10 \mathrm{GeV}$. Documentation, limitations, sources of additional data, and updates on sites are in each file. The following are included:

- Cross sections from the LLNL database EEDL (barn/atom)

- Small-angle elastic cross section $\sigma_{\mathrm{SA}}(E)\left(\theta<0.081^{\circ}\right)$

- Large-angle elastic cross section $\sigma_{\mathrm{LA}}(E)\left(\theta>0.081^{\circ}\right)$

- Total elastic cross section, $\sigma_{\mathrm{SA}}+\sigma_{\mathrm{LA}}$

- Bremsstrahlung cross section for producing photons of energy $>0.1 \mathrm{eV}$

- Total excitation cross section (sum over all atomic shells)

- Total ionization cross section (sum over all atomic shells)

- Stopping powers from EEDL (to $1 \mathrm{GeV}$ only) $\left(\mathrm{MeV} \mathrm{cm}^{2} / \mathrm{g}\right.$ )

- Collisional stopping powers

(Common gases are computed at a density of the order $1 \mathrm{~g} / \mathrm{cm}^{3}$, appropriate to their presence in solids.)

- Radiative stopping powers

- Total stopping power (collisional + radiative)

- Radiative yield

- CSDA range $\left(\mathrm{g} / \mathrm{cm}^{2}\right)$

- Stopping powers from NIST $\left(\mathrm{MeV} \mathrm{cm}^{2} / \mathrm{g}\right)$

- Collisional stopping powers

(Common gases are computed at a density of 1 atm at $20^{\circ} \mathrm{C}$.)

- Density effect parameter $\delta$ used in calculation of the collisional stopping power

- Radiative stopping powers

- Total stopping power (collisional + radiative)

- Radiative yield

- CSDA range $\left(\mathrm{g} / \mathrm{cm}^{2}\right)$

At high $Z(Z \gtrsim 30)$, the NIST collisional stopping power is unreliable below a few $\mathrm{keV}$.

\section{REFERENCES}

[Ak85] Akkerman, A.F. and Gibrekhterman, A.L., Comparison of various Monte Carlo schemes for simulation of low-energy electron transport in matter, Nucl. Instr. Meth. Phys. Res. B B6, 496-503, 1985.

[Ar89] Archer, D.H., Mission Research Corporation, Private communication, 1989.

[Ba04] Bartlett, P.L. and Stelbovics, A.T., Electron-impact ionization cross sections for elements $\mathrm{Z}=1$ to $\mathrm{Z}=54$, Atomic Data Nucl. Data Tables 86, 235-265, 2004. 
[Be30] Bethe, H.A., Zur Theorie des Durchgangs schneller Korpuskularstrahlen durch Materie, Ann. d. Physik. 5: 325-400, 1930.

[Be32] Bethe, H.A., Z. Physik 76: 293, 1932.

[Be33] Bethe, H.A., Handbuch der Physik 24: 273, J. Springer, Berlin, 1933.

[Be34] Bethe, H.A. and Heitler, W., Proc. Roy. Soc. (London) A146: 83, 1934.

[Be46] Bethe, H.A., Phys. Rev. 70: 821, 1946.

[Be53] Bethe, H.A. and Ashkin, J., "Passage of Radiations through Matter," in Segre, E., Ed., Experimental Nuclear Physics, 1, J. Wiley, 1953.

[Be63] Berger, M.J., "Monte Carlo Calculation of the Penetration and Diffusion of Fast Charged Particles," in Methods in Computational Physics, Vol. 1, Alder, B., Fernbach, S., and Rotenberg, M., Eds., Academic Press, 1963, pp. 135-215.

[Be64] Bethe, H.A., Intermediate Quantum Mechanics, W.A. Benjamin, 1964.

[Be82] Berger, M.J. and Seltzer, S.M., Stopping Powers and Ranges of Electrons and Positrons (2nd edn.), NBSIR 82-2550-A (December 1982) [Issued February, 1983 as PB83-175687].

[Be88a] Berger, M.J. and Wang, R., "Multiple Scattering Angular Deflections and Energy-Loss Straggling," in Monte Carlo Transport of Electrons and Photons, Chapter 2, Jenkins, T.M., Nelson, W.R., and Rindi, A., Eds., Plenum Press, New York, 1988.

[Be88b] Berger, M.J., "Electron Stopping Powers for Transport Calculations," in Monte Carlo Transport of Electrons and Photons, Chapter 3, Jenkins, T.M., Nelson, W.R., and Rindi, A., Eds., Plenum Press, New York, 1988.

[Bi58] Birkhoff, R.D., "The Passage of Fast Electrons Through Matter," Encyclopedia of Physics, Vol. XXXIV, Springer-Verlag, 1958.

[Bi88] Bichsel, H., Straggling in thin silicon detectors, Rev. Mod. Phys. 60(3): 663-699 1988.

[Cr70] Crispin, A. and Fowler, G.N., Density effect in the ionization energy loss of fast charged particles in matter, Rev. Mod. Phys. 42: 290, 1970.

[DNA83] Bortner, M.H. and Baurer, T., Eds., Defense Nuclear Agency Reaction Rate Handbook, 2nd edn., DNA-1948H-Rev 9 and periodic chapterby-chapter revisions, 1983.

[Do01] Doughty, K. and Hawxhurst, J., Mission Research Corporation, private communication, 2001.

[En64] Engelhardt, A.G., Phelps, A.V., and Risk, C.G., Phys. Rev. 135: A1566, 1964.

[Fe39] Fermi, E., Phys. Rev. 56: 1242, 1939.

[Fe40] Fermi, E., The ionization loss of energy in gases and in condensed materials, Phys. Rev. 57: 485, 1940.

[Fe61] Fermi, E., Notes on Quantum Mechanics, University of Chicago Press, 1961.

[Fe96] Fernández-Varea, J.M., Andreo, P., and Tabata, T., Phys. Med. Biol. 41(7): 1119, 1996.

[Ge55] Gerjuoy, E. and Stein, S., Phys. Rev. 97: 1671, 1955.

[Go40] Goudsmit, S. and Saunderson, S., Phys. Rev. 57: 24 and 58: 36, 1940.

[Gr01] Groom, D.E., Mokhov, N.V., and Striganov, S.I., Muon stopping-power and range tables, $10 \mathrm{MeV}-100 \mathrm{TeV}$, Atomic Data Nucl. Data Tables 78: 183-356 2001. See also http://pdg.lbl.gov/AtomicNuclearProperties/

[Ha40] Halpern, O. and Hall, H., Phys. Rev. 57: 459, 1940.

[Ha48] Halpern, O. and Hall, H., Phys. Rev. 73: 477, 1948. 
[Ha57] Haas, R., Z. Phys. 148: 177, 1957.

[Ha92] Halbleib, J.A., Kensek, R.P., Valdez, G., Seltzer, S.M., and Berger, M.J., IEEE Trans. Nucl. Sci. 39(4): 1025, 1992.

[He54] Heitler, W., The Quantum Theory of Radiation, Section 33, 3rd edn., Oxford University Press, 1954.

[ICRU70] International Commission on Radiation Units and Measurements (ICRU), Linear Energy Transfer, Report 16, 1970.

[ICRU96] International Commission on Radiation Units and Measurements (ICRU), Secondary Electron Spectra from Charged Particle Interactions, Report 55, 1996.

[ID03] http://www3.ocn.ne.jp/ tttabata/.

[In71] Inokuti, M., Rev. Mod. Phys. 43(3): 297, 1971.

[Ja04] Jablonski, A., Salvat, F., and Powell, C.J., Comparison of electron elasticscattering cross sections calculated from two commonly used atomic potentials. J. Phys. Chem. Ref. Dat. 33(2): 409, June 2004.

[Ja75] Jackson, J.D., Classical Electrodynamics, 2nd edn., John Wiley, 1975.

[Je53] Jelley, J.V., Prog. Nucl. Phys. 3, Chapter 4. Pergamon Press, 1953.

[Jo95] Joy, D.C., A database on electron-solid interactions, Scanning 17, 270-275, 1995. Revision \#01-01: University of Tennessee, and Oak Ridge National Laboratory (2001); available at http://web.utk. edu $/ \sim$ srcutk $/$ htm $/$ interact.htm.

[Ki73] Kieffer, L.J., A compilation of electron collision cross section data for modeling gas discharge lasers, JILA Information Center Report 13, September 30, 1973.

[La80] Lane, N.F., Rev. Mod. Phys. 52: 86, 1980.

[La98] Lazurik, V., Moskvin, V., and Tabata, T., IEEE Trans. Nucl. Sci. 45(3): 626, 1998.

[La99] Lazurik, V., Moskvin, V., Rogov, Y., and Tabata, T., IEEE Trans. Nucl. Sci. 46(4): 910, 1999.

[Li71] Linder, F. and Schmidt, H., Z. Naturforsch. 26: 1617, 1971.

[Lo72] Longmire, C.L., Energy Loss of Electrons, Mission Research Corporation, unpublished (July 1972). See also AFRL Sensor and Simulation Note 194, Considerations in SGEMP Simulation, May 1974.

[Lo73] Longmire, C.L. and Longley, H.J., Improvements in the Treatment of Compton Current and Air Conductivity in EMP Problems, DNA 3192T, 25 September, 1973 (original report MRC-N-2, October 1971).

[Lo78a] Longmire, C.L., IEEE Trans. Ant. Prop. AP-26(1): 3-13, 1978.

[Lo78b] Longmire, C.L., Effect of Multiple Scattering on the Compton Recoil Current, Report MRC-R-378, February 1978.

[Ma56a] Massey, H.S.W., "Theory of Atomic Collisions," in Encyclopedia of Physics, Vol. XXXVI, 232, Springer-Verlag, 1956.

[Ma56b] Massey, H.S.W., "Excitation and Ionization of Atoms by Electron Impact," in Encyclopedia of Physics, Vol. XXXVI, 307, Springer-Verlag, 1956.

[Ma97] Majeed, T. and Strickland, D.J., New survey of electron impact cross sections for photoelectron and auroral electron energy loss calculations, J. Phys. Chem. Ref. Dat. 26(2): 335, 1997.

[Mc48] McKinley, W.A. and Feshbach, H., Phys. Rev. 74: 1759, 1948.

[Mo47] Molière, G., Z. Naturforschung 2a: 133, 1947. 
[Mo48] Molière, G., Z. Naturforschung 3a: 78, 1948.

[Mo65] Mott, N.F. and Massey, H.S.W., The Theory of Atomic Collisions, 3rd edn., Oxford, 1965.

[Mo85] Moiseiwitsch, B.L., Scattering of atomic particles at relativistic energies, Phys. Rep. 118(3): 133-177, 1985.

[Ne66] Newton, R., Scattering Theory of Waves and Particles, McGraw Hill, 1966.

[NIST] http://physlab.nist.gov/PhysRefData/Star/Text/contents.html/.

[PDG04] Particle Data Group, Review of Particle Physics, Phys. Lett. B 592, pp. 1-1109, 2004.

[Pe91] Perkins, S.T., Cullen, D.E., and Seltzer, S.M., Tables and graphs of electron-interaction cross sections from $10 \mathrm{eV}$ to $100 \mathrm{GeV}$ derived from the LLNL evaluated electron data library (EEDL), $\mathrm{Z}=1-100$, LLNL report UCRL-50400, Vol. 31, Lawrence Livermore National Laboratory November 12, 1991.

[Ph85a] Phelps, A.V. and Pitchford, L.C., Anisotropic scattering of electrons by N2 and its effects on electron transport: tabulations of cross section and results, JILA Information Center Report No. 26, May 1, 1985.

[Ph85b] Phelps, A.V., Tabulations of collision cross sections and calculated transport and reaction coefficients for electron collisions with O2, JILA Information Center Report No. 28, September 1, 1985.

[Ro41] Rossi, B. and Greisen, K., Cosmic ray theory, Rev. Mod. Phys. 13: 240309, 1941.

[Ro52] Rossi, B., High-Energy Particles, Prentice-Hall, 1952.

[Sc55] Schiff, L.I., Quantum Mechanics, 2nd edn., McGraw-Hill, 1955.

[Sc64] Schulz, G.J., Phys. Rev. 135: A988, 1964.

[Sc73] Schulz, G.J., Rev. Mod. Phys. 45: 423, 1973.

[Sc86] Schattschneider, P., Fundamentals of Inelastic Electron Scattering, Springer, New York, 1986.

[Se86] Seltzer, S.M. and Berger, M.J., Bremsstrahlung energy spectra from electrons with kinetic energy $1 \mathrm{keV}-10 \mathrm{GeV}$ incident on screened nuclei and orbital electrons of neutral atoms with $\mathrm{Z}=1-100$, Atomic Data Nucl. Data Tables 35: 345-418, 1986.

[Se88] Seltzer, S.M., "An Overview of ETRAN Monte Carlo Methods," in Monte Carlo Transport of Electrons and Photons, Chapter 7 Jenkins, T.M., Nelson, W.R., and Rindi, A., Eds., Plenum Press, New York, 1988.

[Se91] Seltzer, S.M., Electron-photon Monte Carlo calculations: the ETRAN code, Int. J. Radiat. Appl. and Instrum. Part A. Appl. Radiat. Isot. 42(10): 917-941, 1991.

[St52] Sternheimer, R.M., The density effect for the ionization loss in various materials, Phys. Rev. 88: 851, 1952.

[St82] Sternheimer, R.M., Seltzer, S.M., and Berger, M.J., Density effect for the ionization loss of charged particles, Phys. Rev. B26: 6067, 1982.

[St84] Sternheimer, R.M., Seltzer, S.M., and Berger, M.J., The density effect for the ionization loss of charged particles in various substances, Atomic. Data Nucl. Data Table 30: 261, 1984.

[Sw38] Swann, W.F.G., Theory of energy loss of high energy particles, J. Franklin Inst. 226: 598, 1938. 
[Ta71] Tabata, T., Ito, R., Okabe, S., and Fujita, Y., Japanese J. Appl. Phys. 10: 1503, 1971.

[Ta94a] Tabata, T., Andreo, P., and Ito, R., Atomic. Data Nucl. Data Table 56: 105131, 1994.

[Ta94b] Tabata, T., Andreo, P., and Ito, R., Nucl. Instr. Meth. Phys. Res. B94: 103, 1994.

[Ta96a] Tabata, T., Andreo, P., Shinoda, K., and Ito, R., Nucl. Instr. Meth. B108(1): $11,1996$.

[Ta96b] Tabata, T., Andreo, P., and Shinoda, K., Nucl. Instr. Meth. B119: 463, 1996.

[Ta98a] Tabata, T., Andreo, P., and Shinoda, K., Rad. Phys. Chem. 53: 205, 1998.

[Ta98b] Tabata, T. and Andreo, P., Rad. Phys. Chem. 53: 353, 1998.

[Wa82] Wadzinski, H.T. and Jasperse, J.R., Low energy electron and photon cross sections for $\mathrm{O}, \mathrm{N}_{2}, \mathrm{O}_{2}$ and related data, AF geophysical laboratory, Hanscom AFB, AFGL-TR-82-0008 (AD\#A118921) (4 January 1982).

[Wi39] Williams, E.J., Proc. Roy. Soc. (London) A169: 531, 1939.

[Wi40] Williams, E.J., Phys. Rev. 58: 292, 1940.

[Wi43] Wick, G.C., Nuovo Cimento 1: 302, 1943.

[Wi45] Williams, E.J., Rev. Mod. Phys. 17: 217, 1945.

[Zi40] Zirkle, R.E., J. Cell. Comp. Physiol. 16: 221, 1940.

[Zi52] Zirkle, R.E., Marchbank, D.F., and Kuck, K.D., J. Cell. Comp. Physiol. 39(Suppl. 1): 75, 1952. 



\section{Protons and}

DOI: $10.1201 / 9781420012378-4$

\subsection{BACKGROUND}

After the discovery of $\alpha$ radiation and Rutherford's use of it to probe the atomic structure of matter early in the twentieth century, the passage of $\alpha$ particles through matter became a topic of some concern. Bohr's early investigations were entirely classical; but after quantum mechanics became fully established and Bethe and Bloch obtained good quantum mechanical expressions, Bohr presented a lengthy review in 1948 [Bo48], concentrating on classical concepts but including a discussion of the Bethe formula. Bloch's corrections to Bethe's original formulation provided a bridge between Bohr's classical development and Bethe's quantum mechanical one.

After the discovery of fission, interest arose in the passage of truly heavy ions (fission fragments) through matter. The theory is difficult when the number of electrons on the ion projectile is not much smaller than the atomic number of target atoms. The question of electron stripping from the projectile, present even for protons or alpha particles, became more important. What is the charge state of the ion as it passes through the target?

Because of the huge mass difference between electrons and ions, a major difference between the passage through matter of electrons vs. ions is that multiple elastic scattering has a dominant effect on electron motion, whereas it is only a small correction to the motion of an ion. As a result of elastic scattering, the mean forward range of a $1 \mathrm{MeV}$ electron is of the order onehalf its full path length (its CSDA range) in most elements, but the mean forward range of a $1 \mathrm{MeV}$ proton or heavier ion is only a few percent less than the CSDA range. The reason is that in colliding with atomic electrons the heavier ion is hardly deflected, whereas an incident electron can be significantly altered in direction. In addition, elastic collisions between electrons and target nuclei cause multiple small-angle scattering but little energy loss, whereas nuclear collisions play a lesser role for ions. In this sense, the behavior of ions in passing through matter is simpler than that of electrons.

Over the years, there have been quite a number of reviews of the theory and quite a few tabulations of numerical stopping powers. Bohr [Bo48] presented one of the earliest reviews. Later discussions and summaries are by Rossi [Ro52], Bethe and Ashkin [Be53], Fano [Fa56, Fa63, Fa64], Northcliffe 
[No63], Uehling [Ue54], the National Academy of Sciences [Na64], Inokuti [In71], Bichsel [Bi72], Goland [Go73], Sigmund [Si75], Kumakhov and Komarov [Ku81], and Geissel et al. [Ge82]. Ahlen presents a thorough review [Ah80], whereas Evans [Ev55] presents a summary. Jackson [Ja75] includes a clear treatment of energy loss. A quick summary by Berger and Seltzer [Be82] of both electron and heavy ion energy loss is very informative. The general review by Ziegler et al. [Zi85] is widely referenced, and contains an excellent historical summary, as do Ziegler [Zi99a] and the online SRIM site [SR03]. Special attention has been given to the lightest ions, with discussion or data presented by Andersen and Ziegler [An77], Ziegler [Zi77], Kaneko [Ka93], the International Commission on Radiation Units and Measurements [ICRU93], Ziegler [Zi99a], and by NIST online [NIST].

Widely cited, older compilations are by Barkas and Berger [Ba64], Bichsel and Tschalaer [Bi67], Northcliffe and Schilling [No70], and Janni [Ja82a,b]. More recent ones are by Hubert et al. [Hu90] and by Paul and Schinner [Pa03a]. Scott [Sc63] has reviewed the effects of multiple smallangle scattering on the penetration of heavy ions. All ion projectiles are included in the widely used code SRIM [SR03], which has been used for calculations and graphs in this book. SRIM is readily available and continually updated.

Experimental measurements of stopping power, current computer codes, and tabulated data compilations do not always agree. The theory of ion stopping power is still evolving, but it is generally thought that calculated stopping powers can be believed to be about $1 \%$ for energies above $1 \mathrm{MeV}$ [Fa63], and, for light ions, a few percent at the lower energies [Zi99a]. One may follow a discussion of differences concerning two main databases, NIST and SRIM, at, for example, [Zi99b] and [Se01]. Paul and Schinner have presented a recent comparison of data with tables and codes [Pa03b], although it is not clear that the latest version of SRIM was used in their comparison. At low energies, some alpha particle stopping power calculations differed by several percent from SRIM 2000 to SRIM 2003.

High-energy muon stopping power and range tables have recently been presented by Groom et al. [Gr01]. A general review of muon, pion, and proton stopping power and ranges is regularly presented in the biennial Review of Particle Properties, for example in Bichsel et al. [Bi04].

Despite myriad experimental measurements of stopping power and ion ranges over the decades, a theory is necessary to interpolate between the energies and atomic numbers for which data are available and extrapolate to energies beyond experimental reach. In spite of the enormous amount of work devoted to the subject, it is still not possible to accurately calculate ion stopping power truly from first principles. Phenomenological parameters are required to pin the calculations to data. For example, the mean ionization potential $I$ is often adjusted empirically. There can be differences in stopping power between liquid and gaseous phases of a material, or between crystal 
and amorphous phases of a substance. For believable numbers, at some point normalization is tied to experiment. Because of the excellent reviews, we state only principal results of the theory.

\subsection{PRINCIPAL RESULTS OF ION STOPPING POWER THEORY}

Like electrons, heavy ions may interact with target atomic electrons or with atomic nuclei. The ion-nucleus collision is primarily elastic Coulomb scattering. This is true except for energetic ions that can overcome the repulsive Coulomb barrier and approach the nuclear radius (which is not uncommon and introduces nuclear elastic scattering; discussed later). Bohr noticed that by elastic Coulomb scattering of light ions from nuclei, the nucleus will recoil but relatively little energy is transferred as a result of the target's large mass. On the other hand, when the ion interacts with atomic electrons, true energy transfer can occur. The electrons may be excited or ionized and so absorb energy from the projectile. The bulk of energy loss is due to ion collisions with atomic electrons. Only for the heaviest projectiles in the lightest targets does energy loss by nuclear collisions exceed that by electronic collisions.

Bohr also had the seminal idea that the energy transfer from a passing heavy ion to an atom would depend on the velocity of the ion relative to the velocities of the outer electrons in the atom. A characteristic velocity of outer electrons is the Bohr velocity $v_{\mathrm{o}}=\alpha c$, which is the velocity of a $13.6 \mathrm{eV}$ electron or a $25 \mathrm{keV}$ proton. If the ion velocity $v$ is much less than $v_{\mathrm{o}}$, the field of the passing ion presents an adiabatic perturbation to the atomic electron orbit and little energy is transferred. If the ion velocity is comparable to $v_{\mathrm{o}}$, there is optimal opportunity to transfer energy to the atomic electrons. If $v$ is much greater than $v_{\mathrm{o}}$, the energy transfer is reduced because the momentum transfer is proportional to the time integral of the ion Coulomb field, which is proportional to $1 / v$ (Section 3.5).

Thus, in the theory of ion energy loss, ion velocity (as opposed to ion energy) plays a major role. The stopping power of an ion is nearly the same as that of an electron of the same velocity. This may be seen in the ion and electron curves in Figure 4.1 which plots the mean collisional stopping power of electrons and three ions $(p, \alpha, \mathrm{Fe})$ at the same velocity in aluminum, scaled by an effective charge $Z(v)$, to be defined. $Z(v)$ accounts for the different ion charge states at different velocities (for a proton with kinetic energy $E>1$ $\mathrm{MeV}, Z(v) \approx 1)$. The electron-stopping power is only slightly less than that of a proton of the same velocity. The speed of a $10 \mathrm{keV}$ electron is $v \approx 0.2 c$, whereas a proton of that velocity has energy about $18 \mathrm{MeV}$.

The basic expression for the energy loss rate of an ion passing through matter is known as the Bethe-Bloch equation. Bethe [Be30, Be32] made the first fully quantum mechanical calculation of the energy loss, in the first Born approximation. As the transfer of energy from a passing ion to an atom occurs 


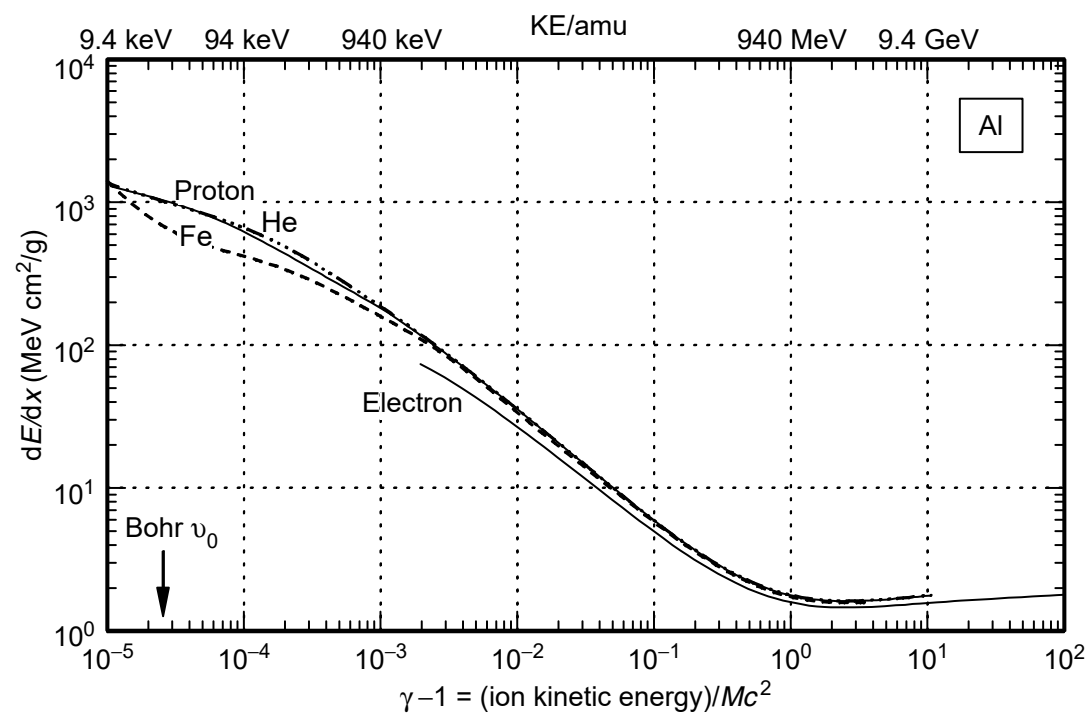

FIGURE 4.1 The stopping power of several ions in Aluminum, divided by the square of their effective charge $Z(v)$. Ions are plotted at the same velocity; on the $x$-axis, $M$ is the mass of the particular particle. Except for minor differences, ions of the same velocity, scaled by their effective charge, have the same stopping power. ( $e$ data from NIST; [SR03]. $p, \alpha$, Fe data from SRIM.)

by the Coulomb interaction between the ion charge and the atomic electrons, the classical expression for energy transfer as a function of ion parameters is the same as that for a passing electron. Let $Z$ be the ion atomic number and $M$ its mass, and let $Z_{\mathrm{t}}$ be the atomic number of the target atoms. $\beta=v / c$ is the ion velocity relative to the speed of light. Bethe's original equation for the ion collisional stopping power is now usually written [Fa63], [ICRU93, Zi99a]

$$
\begin{aligned}
\frac{\mathrm{d} E}{\mathrm{~d} x} & =\frac{4 \pi Z^{2} e^{4} Z_{\mathrm{t}}}{m v^{2}} N L_{\mathrm{o}}, \\
L_{\mathrm{o}} & =\ln \frac{2 m v^{2}}{I}-\ln \left(1-\beta^{2}\right)-\beta^{2}-\frac{C}{Z_{\mathrm{t}}}-\frac{\delta}{2} \\
& =\ln \frac{2 m c^{2} \beta^{2} \gamma^{2}}{I}-\beta^{2}-\frac{C}{Z_{\mathrm{t}}}-\frac{\delta}{2}
\end{aligned}
$$

Equation 4.1 is really an extension of the original Bethe formulation, and includes two correction terms $\left(C / Z_{\mathrm{t}}\right.$ and $\left.\delta / 2\right)$; Bethe's original equation had $C=0$ and $\delta=0$. A straightforward, brief derivation can be found in [Be82] and a more thorough treatment in [ICRU93]. $N$ is the density of target atoms 
and $\gamma=\left[1-v^{2} / c^{2}\right]^{-1 / 2}=1+E / M c^{2}$ is the usual relativistic factor. $I$ is the average excitation energy of all the electrons in a target atom. $I$ is difficult to calculate accurately; its proper definition and methods for evaluating it can be found in Fano [Fa63], Berger and Seltzer [Be82], the NAS [Na64], Ahlen [Ah80], Ziegler et al. [Zi85], and [ICRU93]. The rough value $I=11.4 Z_{\mathrm{t}}$ is sometimes employed for crude work. $I$ occurs in electron-stopping power as well. The electron mass $m$ appears in the denominator of the outer coefficient and in the logarithm rather than in the ion mass because the field of the passing particle imparts a certain momentum $\Delta p$ to the atomic electrons; hence the energy transfer is $\Delta p^{2} / 2 m$ (see discussion after the Bethe electron formula, Equation 3.21). Comparing Equation 4.1 with Equation 3.21, the main dependence on incident particle velocity is the same. (In Equation 3.21, $Z$ is the target atomic number.) The factor $L_{\mathrm{o}}$ for ions is similar to that in the curly brackets in Equation 3.21 for electrons. (It is conventional for the outer factor to contain $4 \pi$ in the ion equation and $2 \pi$ in the electron equation.) $\mathrm{d} E / \mathrm{d} x$ is per $\mathrm{cm}$ or per $\mathrm{g} / \mathrm{cm}^{2}$ according as $N$ is $\mathrm{cm}^{-3}$ or $\mathrm{g}^{-1}$. When $\mathrm{g}^{-1}, \mathrm{~d} E / \mathrm{d} x$ is referred to as the mass stopping power.

In fact, in both Equations 3.21 for electron-stopping power, and Equation 4.1 for ion stopping power, the mass of the projectile does not occur, only the projectile velocity occurs. That is, to a first approximation, all ions of the same velocity, no matter what their mass, have the same stopping power, weighted by their charge squared, $Z^{2}$. Owing to electrons stripping off the ion, an effective charge at velocity $v, Z(v)$, must really be employed instead of its inherent charge $Z$. This accounts for the near equality of all curves in Figure 4.1. A principal cause for the electron-stopping power being different from the proton is due to the different maximum energies that the different projectiles can impart to target atom electrons.

Bethe's Equation 4.1 is intended for ion velocities much greater than $v_{\mathrm{o}}$ $(25 \mathrm{keV} / \mathrm{amu})$ and was derived for interactions with a free atom, not for atoms bound to close neighbors in a solid. The energy parameter $I$ is an average excitation energy of all electrons in the atom; no account is taken of electronic shell structure. The term $C / Z_{\mathrm{t}}$ is a correction to account for the different contributions from individual atomic shells. The term $\delta / 2$ accounts for the density effect and is significant only at the highest energies (>hundreds of $\mathrm{MeV} / \mathrm{amu}$ ), and hence, usually only for the lightest ions in laboratory situations or in cosmic rays. The quantity $L_{\mathrm{o}}$, or rather its generalization $L$ discussed momentarily, is called the stopping number. The Bethe equation, with the $C$ and $\delta$ corrections and two other corrections (to $L_{\mathrm{o}}$ are added two other terms, $L_{\mathrm{o}} \longrightarrow L_{\mathrm{o}}+Z L_{1}+Z^{2} L_{2}$ ), discussed presently is the Bethe-Bloch equation and is still the basis for stopping power models for light ions at velocities above hundreds of $\mathrm{keV} / \mathrm{amu}$. The equation assumes that the ion has fixed net charge $Z$, that is, that the ion is fully stripped of all its electrons. Unless corrections are included for partial stripping, the equation is limited to velocities greater than or about $1 \mathrm{MeV} / \mathrm{amu}$, well above $v_{\mathrm{o}}$. Thus, 
with subsequent corrections, it is applicable to ions of energy sufficient to penetrate of the order $0.01 \mathrm{~g} / \mathrm{cm}^{2}$ in all elements.

The quantity $(1 / N) \mathrm{d} E / \mathrm{d} x$ has dimensions of energy $\times$ area. It is called the stopping cross section and is the stopping power per atom. It is sometimes tabulated instead of $\mathrm{d} E / \mathrm{d} x$ itself. The stopping cross section of a $1 \mathrm{MeV}$ proton in $\mathrm{Al}$ is $78.5 \mathrm{eV}-\AA^{2}$. An appreciation for the magnitude of $\mathrm{d} E / \mathrm{d} x$ can be obtained by simplifying the coefficient $4 \pi e^{4} Z_{\mathrm{t}} N / m v^{2}$ of $Z^{2} L_{\mathrm{o}}$. Its factors are readily expressed in terms of fixed constants, and the stopping power Equation 4.1 can be written as

$$
\frac{\mathrm{d} E}{\mathrm{~d} x}=0.1535 \frac{Z^{2}}{\beta^{2}} \frac{2 Z_{\mathrm{t}}}{A_{\mathrm{t}}} L_{\mathrm{o}} \quad\left(\mathrm{MeV}-\mathrm{cm}^{2} / \mathrm{g}\right)
$$

Here, the factor $Z^{2} / \beta^{2}$ depends only on the projectile. The factor $\left(2 Z_{\mathrm{t}} / A_{\mathrm{t}}\right)$, where $A_{\mathrm{t}}$ is the target atomic weight, depends only on the target and is approximately unity (except for $\mathrm{H}$ ). The factor $L_{\mathrm{o}}$ depends mildly on the target material (via $I$ and $C$ and $\delta$ ) and on the projectile velocity. It is slowly varying, generally being of the order 1 to 20 up to $\gamma \sim$ several hundred. For a proton of kinetic energy $E=3 \mathrm{MeV}, L_{\mathrm{o}}$ is about 3.7, and the proton stopping power in $\mathrm{Al}(I \sim 165 \mathrm{eV})$ is approximately $86 \mathrm{MeV}-\mathrm{cm}^{2} / \mathrm{g}$.

\subsubsection{Maximum Energy Transfer in Elastic Scattering}

When a projectile of mass $m_{\mathrm{p}}$, velocity $v=\beta c$, kinetic energy $E$, and relativistic factor $\gamma=\left(1-\beta^{2}\right)^{-1 / 2}=1+E / m_{\mathrm{p}} c^{2}$ elastically scatters from a target nucleus of mass $m_{\mathrm{t}}$ at rest in the laboratory, the maximum kinetic energy $T_{\max }=\left(\gamma_{\mathrm{t} \max }-1\right) m_{\mathrm{t}} c^{2}$ that can be transferred to the target is given by

$$
\frac{T_{\max }}{m_{\mathrm{t}} c^{2}}=\gamma_{\mathrm{t} \max }-1=\frac{2\left(\gamma^{2}-1\right)}{1+2 r \gamma+r^{2}}
$$

where $r=m_{\mathrm{t}} / m_{\mathrm{p}}$ is the ratio of target mass to projectile mass. Using $\gamma^{2}-1=\gamma^{2} \beta^{2}$, this is more conventionally written as

$$
T_{\max }=\frac{2 m_{\mathrm{t}} c^{2} \gamma^{2} \beta^{2}}{1+2 r \gamma+r^{2}}
$$

The nonrelativistic form $(\gamma \longrightarrow 1)$ is

$$
T_{\max }=4 \frac{m_{\mathrm{t}}}{m_{\mathrm{p}}} \frac{E}{(1+r)^{2}}
$$

When $r=1$, the projectile can transfer all its energy, and Equation 4.5 reduces to $T_{\max }=E$. These expressions for $T_{\max }$ hold for distinguishable particles. For 
identical particles, the less energetic of the two outgoing particles is considered to be the target and the maximum energy that can be transferred to it is $E / 2$.

For heavy ions colliding with atomic electrons $(r \ll 1)$, and when the projectile is not extremely relativistic $(2 r \gamma \ll 1)$, the denominator is approximately 1 and Equation 4.5 becomes

$$
T_{\max }=4 \frac{m}{m_{\mathrm{p}}} E\left(1+\frac{E}{2 m_{\mathrm{p}} c^{2}}\right)
$$

where the target mass $m_{\mathrm{t}}$ has been replaced by the electron mass $m$. Nonrelativistically, the target acquires twice the projectile velocity and a small fraction of the projectile energy.

The opposite case, in which the projectile is light compared with the target, applies, for example, in proton or alpha particle scattering from heavier nuclei. By pulling out $r^{2}$ in the denominator, Equation 4.5 can be rewritten as

$$
T_{\max }=2 \frac{m_{\mathrm{p}}}{m_{\mathrm{t}}} \frac{m_{\mathrm{p}} c^{2} \gamma^{2} \beta^{2}}{1+2 \gamma / r+1 / r^{2}}
$$

Considering a very heavy nucleus $(r \gg 1$ and $2 \gamma / r \ll 1)$, the denominator is again approximately 1 and

$$
T_{\max }=4 \frac{m_{\mathrm{p}}}{m_{\mathrm{t}}} E\left(1+\frac{E}{2 m_{\mathrm{p}} c^{2}}\right)
$$

In this case, for a nonrelativistic projectile, the projectile backscatters and the recoiling nucleus acquires twice the projectile momentum.

\subsubsection{Shell Corrections}

The original Bethe-Bloch formula had $C=0$, and the only material parameter was $I$. There was no accounting for atomic shell structure, and so there were inaccuracies stemming from the fact that the ion may be fast relative to atomic electrons in outer shells, but slow relative to inner shells.

That a correction due to different shell binding energies (velocities) is necessary can be seen by noting that a $5 \mathrm{MeV}$ proton has about the same velocity as a $K$-shell electron in aluminum, but is much faster than the outer electrons. Thus, the range of validity of the original Bethe-Bloch equation $\left(v \gg v_{\mathrm{o}}\right)$ is exceeded for inner electrons by ions of a few or a few tens of $\mathrm{MeV} / \mathrm{amu}$, depending on the target atomic number. This deficiency cannot be fully offset solely by proper choice of the mean excitation potential $I$.

The required corrections are included in the term $C / Z_{\mathrm{t}}$, called the shell correction term. It is written this way for historical reasons; it is not simply 
proportional to $1 / Z_{\mathrm{t}}$. Typical magnitudes and methods of computing $C / Z_{\mathrm{t}}$ are summarized by the [ICRU93] and by Ziegler [Zi99a], to which we refer for details. $C / Z_{\mathrm{t}}$ corrections are not much more than about $6 \%$ for light ions [Zi99a].

\subsubsection{The Density Effect}

As was the case for electrons, the field of a passing ion is reduced by the material's dielectric constant, reducing the field experienced by atomic electrons, and reducing energy loss at high energies. This effect is significant only at projectile velocities a significant fraction of the speed of light, i.e., at energies of hundreds of MeV per amu, when the Coulomb field is compressed and distant collisions contribute most to energy loss.

The corrective effect of $\delta / 2$ in Equation 4.1 for protons is discussed in [ICRU93]. Generally, it is most significant for low- $Z$ targets, but not always. For a $10 \mathrm{GeV}$ proton, the largest correction is in graphite and there is less than $10 \%$; in silicon it is less than $6 \%$. The theory of the density effect for ions is the same as that for electrons, and the discussions of Sternheimer et al. [St82, St84] are to be consulted.

\subsubsection{Two Further Corrections to the Bethe Formula}

In addition to the shell correction term $C / Z_{\mathrm{t}}$ and the density effect parameterized by $\delta$, two additional improvements to the original Bethe equation have been advanced. The form of the improvements is that the stopping number $L_{\mathrm{o}}$ is replaced by

$$
L=L_{0}+Z L_{1}+Z^{2} L_{2}
$$

the beginning of a power series in the projectile atomic number $Z$. The $L_{\mathrm{o}}$ term is derived in the first-order Born approximation. Both the $L_{1}$ and $L_{2}$ terms represent corrections to that approximation.

The term in $L_{1}$ is the Barkas correction, accounting for a small difference in stopping powers between positive and negative ions. It is reviewed extensively by Ziegler [Zi99a]. Barkas and coworkers [Ba56] measured that positive ions (pions in his experiment) had a slightly larger stopping power than their negative counterpart. Later, Barkas and coworkers [Ba63] proposed that the resolution was due to a departure from the first Born approximation. The effect has been calculated and reviewed often. The work is summarized in [Zi99a] and [ICRU93]. As expected from the conditions for the validity of the Born approximation, the Barkas effect is most important at relatively low energies. It is also greater in high- $Z_{t}$ targets. Calculations show [ICRU93], for example, that in $\mathrm{Ag}$ the stopping power of a $1 \mathrm{MeV}$ antiproton is $80 \%$ that of a 
proton. In addition, also in $\mathrm{Ag}$, the stopping power of a $100 \mathrm{keV}$ negative pion is $70 \%$ that of a positive pion.

The $L_{2}$ term is the Bloch correction. As mentioned, the original treatment by Bethe was in the Born approximation, valid at projectile velocities $v$ larger than target orbital velocities, $Z_{\mathrm{t}} \alpha c / v \ll 1$, where $\alpha=1 / 137$ is the fine structure constant. Bloch's treatment [B133] was quantum mechanical, but eschewed the Born approximation and allowed approximately for the distortion of target atom wave functions by the passing projectile. It provides a correction to the Bethe formula at low energies. The correction is quite small, being less than $1 \%$ in low- $Z_{\mathrm{t}}$ materials at energies above $1 \mathrm{MeV} / \mathrm{amu}$ and less than $3 \%$ in high $-Z_{t}$ materials at the same energies [ $\left.\mathrm{Zi} 99 \mathrm{a}\right]$.

When both the Barkas and Bloch corrections are added,

$$
\frac{\mathrm{d} E}{\mathrm{~d} x}=\frac{4 \pi Z^{2} e^{4} Z_{\mathrm{t}}}{m v^{2}} N L
$$

with $L$ given by Equation 4.9, is the Bethe-Bloch equation.

The Bethe-Bloch equation is for mean energy loss. The range against mean energy loss, as for electrons, is the continuous slowing down approximation range, in which the ion is considered to lose energy always at the mean rate. It is given simply as

$$
R_{\mathrm{CSDA}}=\int \frac{\mathrm{d} E}{\mathrm{~d} E / \mathrm{d} x}
$$

Fluctuations in energy loss lead to fluctuations in range, both called straggling. Straggling is much smaller for ions than for electrons and for many applications may be ignored. Lindhard and coworkers [Li63] present a generalized theory of energy and range straggling, and Bichsel has reviewed the theory in Silicon [Bi88]. Both longitudinal and lateral range straggling are computed in the code SRIM [SR03].

\subsubsection{Charge State of the Ion}

In collisions with target atoms, the projectile may lose one or more or all electrons. Only when fully stripped does it have its full nuclear charge $Z$. In further collisions, it may capture one or more electrons, becoming less positive, so there is an exchange of electrons between the ion and material atoms as the projectile advances. On balance, it will soon achieve an average charge. Capturing and stripping of electrons may occur after passing only hundreds or thousands of atoms, that is, over a range of microns in solids or less than 0.001 of the ion's range [Ev55]. That is why, for example, in ion transport calculation tools such as the SRIM code, when entering the projectile it is not necessary to specify its charge state. No matter what its original 
charge state, the ion will achieve its steady state charge at its current velocity in a short time, long before its energy changes significantly by stopping power.

The charge state can be expressed in terms of the capture and loss cross sections. However, the cross sections for losing and gaining one or more electrons in a collision are very difficult to compute. It is certain that the capture cross section is much smaller than the loss cross section. Thus, in the Bethe-Bloch equation one takes $Z$ to be the full nuclear charge of the projectile. Owing to the difficulties, only phenomenological expressions exist for the charge state as a function of velocity and target material. The most widely used one is by Northcliffe [No60], based on the ideas of Bohr:

$$
Z(v)=\left[1-\exp \left(-\frac{v}{v_{\mathrm{o}} Z^{2 / 3}}\right)\right] Z
$$

Here, $Z(v)$ is the effective (statistical average) charge of the ion at velocity $v$, $Z$ on the right-hand side is the full nuclear charge, and $v_{\mathrm{o}}=\alpha c$ is the Bohr velocity. In the Thomas-Fermi model of the atom, the electron velocity is proportional to $Z^{2 / 3}$. Thus, this expression says that ions are stripped of electrons whose velocities are less than the projectile velocity. Since the Bethe formulas are intended for high velocities, it is usual to assume fully stripped ions and set $Z(v)=Z$. However, the theory is not satisfactory, and it has been suggested that $Z(v)$ simply be that value for $Z$ in the Bethe-Bloch equation that makes it give the correct stopping power ( $\mathrm{Zi} 99 \mathrm{a})$. The problem was further discussed by Northcliffe [No63].

Therefore, $Z$ in the Bethe-Bloch Equation 4.10 is actually $Z(v)$, the current value of the ion charge. Figure 4.1 is obtained by dividing Equation 4.10 by $Z(v)$, leaving

$$
\left(\frac{\mathrm{d} E}{\mathrm{~d} x}\right)^{\prime}=\frac{\mathrm{d} E / \mathrm{d} x}{Z(v)^{2}}=\frac{4 \pi e^{4} Z_{\mathrm{t}}}{m v^{2}} N L
$$

which is a function of only velocity, with minor corrections in $L_{1}$ and $L_{2}$.

The figure may be extended to other ions. This time in $\mathrm{Si}$, Figure 4.2 shows the progression: stopping powers of several ions in $\mathrm{Si}$ as a function of ion kinetic energy, the usually desired quantity, is shown in Figure 4.2a; as a function of ion velocity, Figure 4.2b; and as a function of velocity, and dividing each stopping power by the ion $Z(v)^{2}$ in Figure $4.2 \mathrm{c}$. They collapse to nearly a single function, with variations at low velocity.

\subsubsection{The Bragg Peak}

A feature of ion stopping power noticed early in the twentieth century is that their stopping power is the greatest, and reaches a local peak, near the end of 

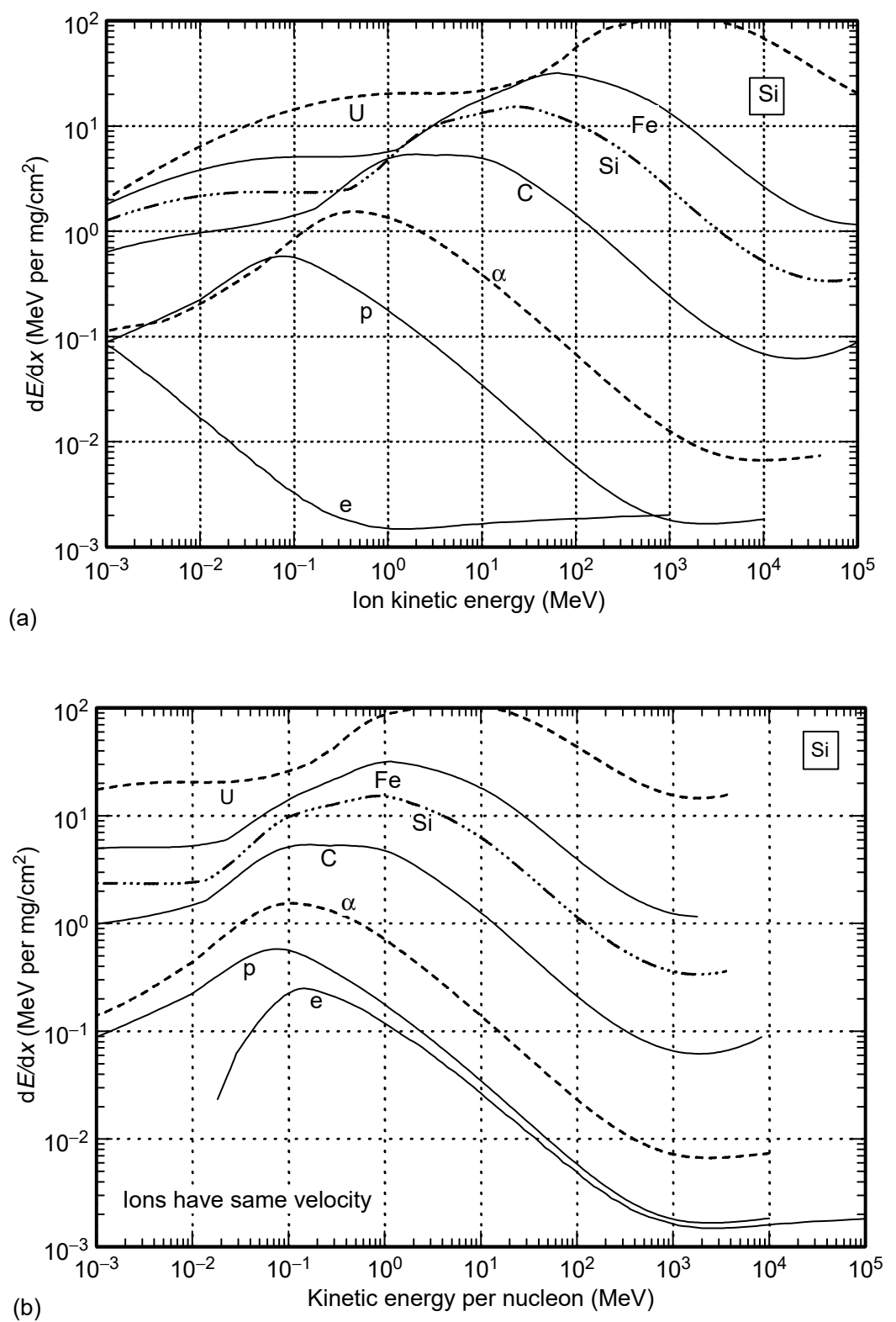

FIGURE 4.2 (a) Stopping powers of an electron, proton, alpha particle, and carbon, silicon, iron, and uranium ions in silicon, as a function of their kinetic energies. (b) As a function of their velocities. ( $e$ data from NIST. Ion data from SRIM.) 


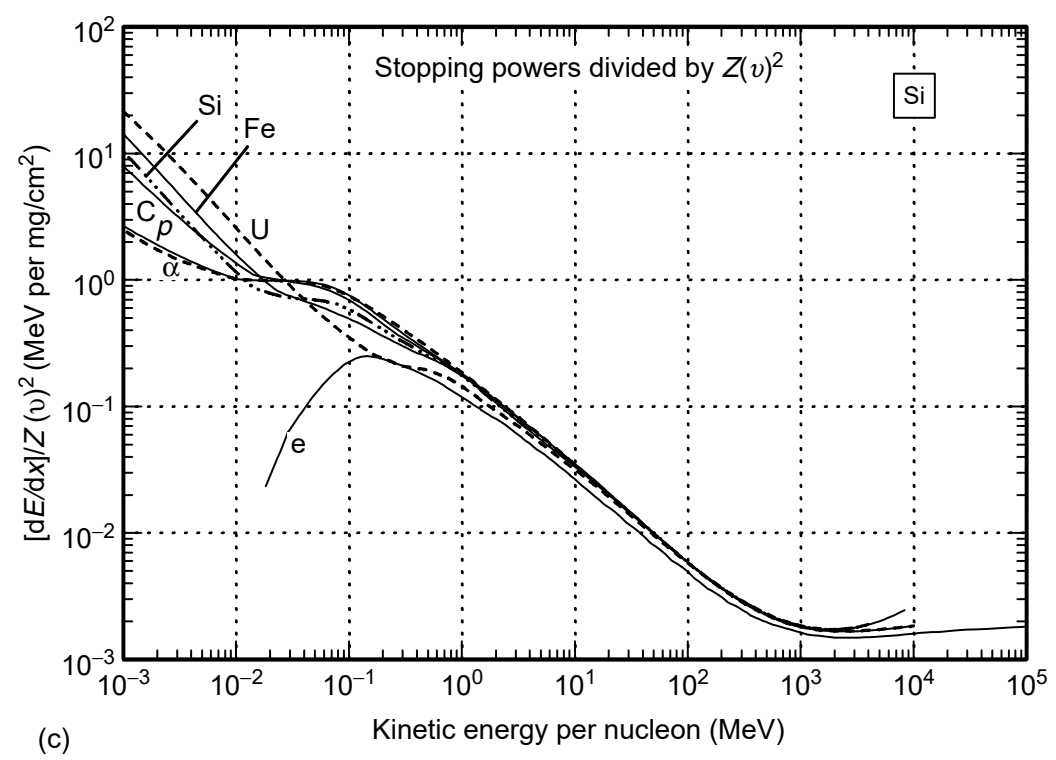

FIGURE 4.2 (continued) (c) Stopping powers divided by their effective charge, and plotted at the same velocity. ( $e$ data from [NIST]. Ion data from SRIM [SR03].)

their range. The peak is called the Bragg peak, and is illustrated for $\alpha$ particles in water in Figure 4.3. A graph of the stopping power vs. residual range is called the Bragg curve. It is valuable because it is independent of the ion starting energy and displays the maximum in stopping power. Figure 4.4 shows the Bragg curve for an $8 \mathrm{MeV}$ alpha particle in A-150 tissue-equivalent plastic, a mock up of human tissue. Figure 4.5 shows the curve for a $2 \mathrm{MeV}$ proton in the same medium.

The graphs are drawn for an individual ion. For historical and experimental reasons, purists prefer to reserve the term Bragg peak for the peak in the stopping power averaged over many ions. This differs from Figure 4.3 through Figure 4.5 because energy straggling and elastic scattering spread out the maximum and cause the peak to occur at a larger residual range. The distinction may be pedantic, but it is indeed the average that will occur in practice. Thus in proton radiotherapy, one must know the actual depth of maximum stopping power (maximum dose), and this is not accurately given by the single particle curve. A Monte Carlo calculation, such as one readily done with the SRIM code, will give a more realistic depiction of the ionization and energy deposition profile. It retains the general shape of the figures. Use may be made of the Bragg peak in selectively depositing maximum energy, and producing maximum ionization, at a desired depth, by selecting the ion energy with the range at that depth. 


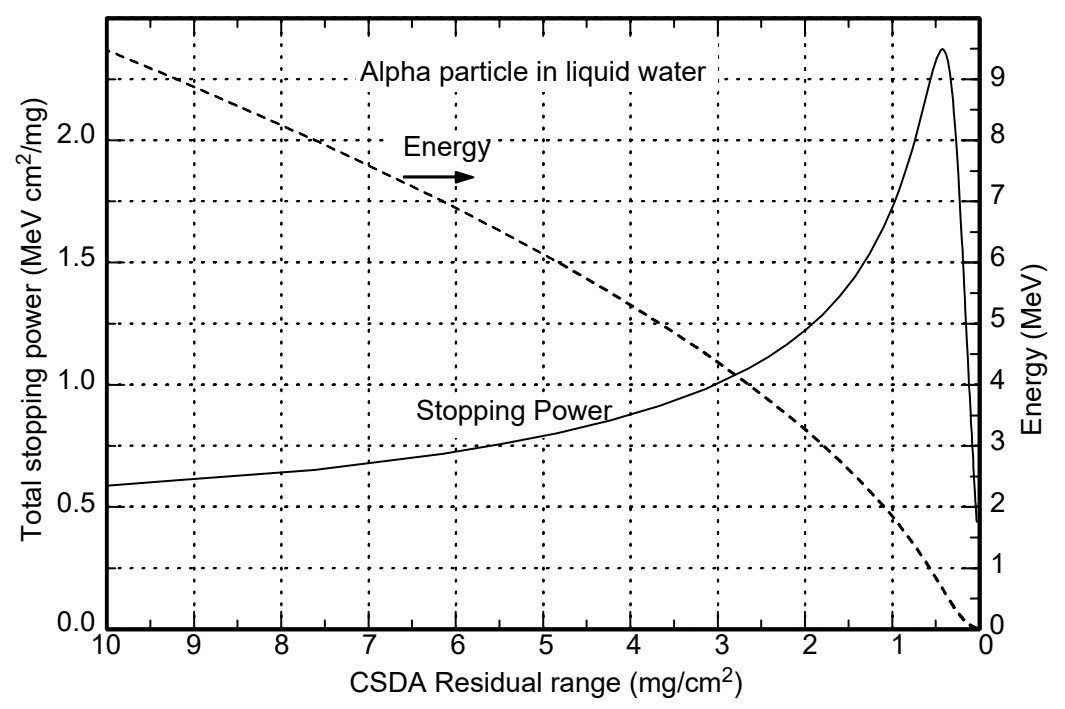

FIGURE 4.3 Bragg curve of alpha particle in liquid water. Bragg peak is the maximum of the stopping power near the end of the range. As graphed, the particle starts with kinetic energy $9.5 \mathrm{MeV}$ and CSDA range of $10 \mathrm{mg} / \mathrm{cm}^{2}=0.01 \mathrm{~cm}$. Near the end of its range, its energy loss rate peaks at four times its starting rate. It deposits about $1.8 \mathrm{MeV}$ in the last $10 \mu \mathrm{m}$ of its range. (Data from SRIM [SR03].)

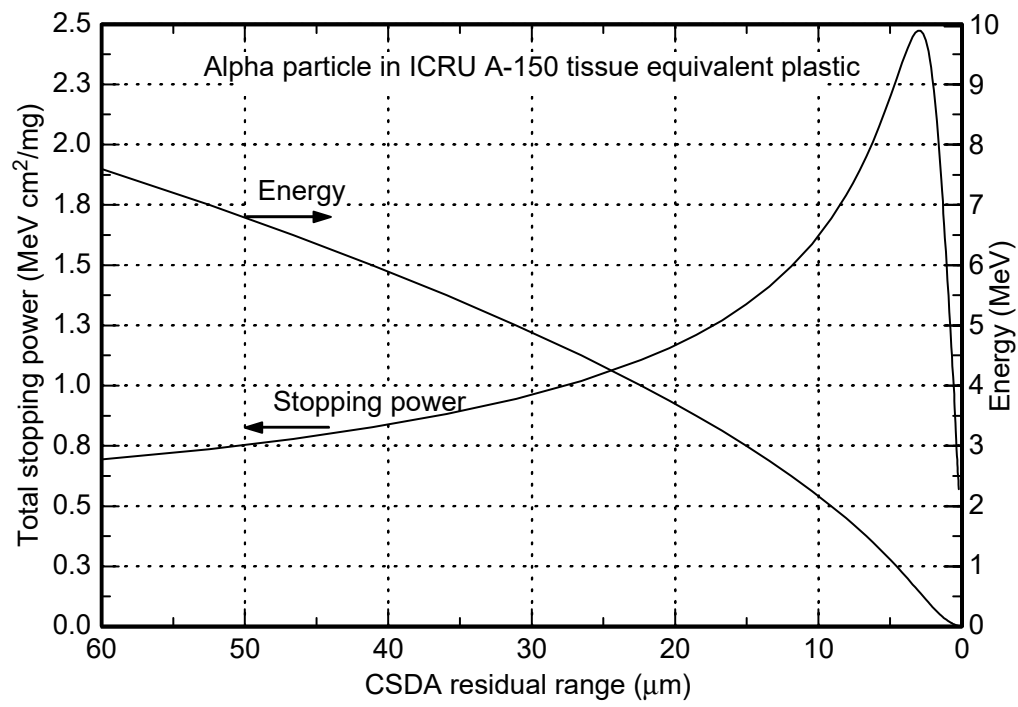

FIGURE 4.4 Bragg curve of alpha particle in ICRU A-150 tissue equivalent plastic. About $2.1 \mathrm{MeV}$ is deposited in the last $10 \mu \mathrm{m}$ of the particle range. 


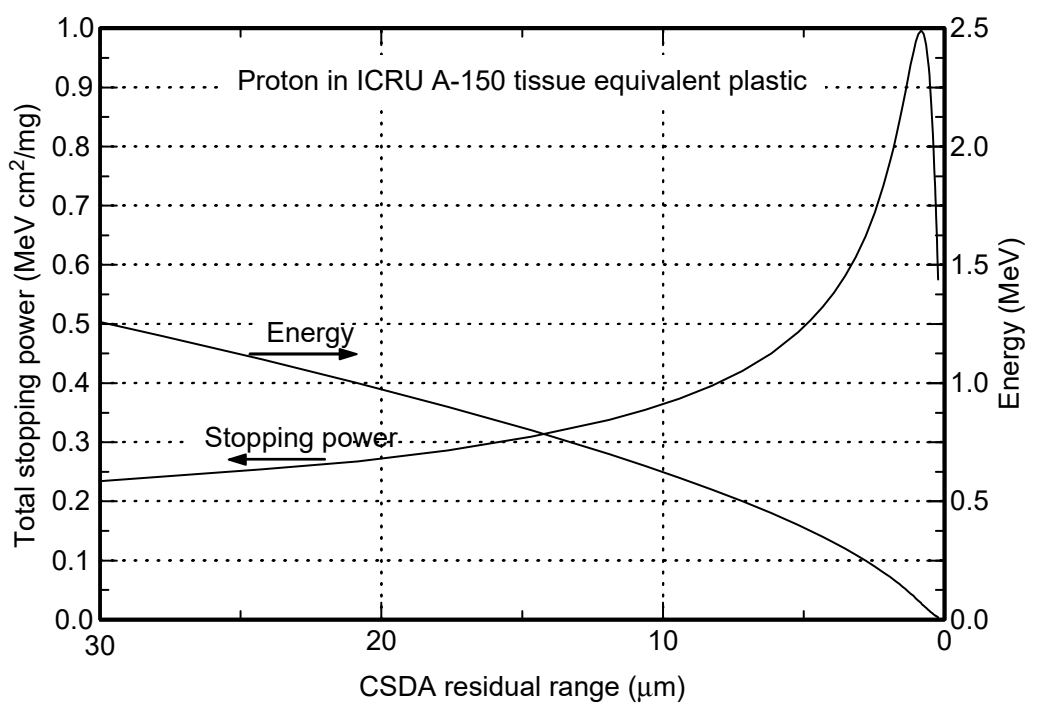

FIGURE 4.5 Bragg curve of proton in ICRU A-150 tissue equivalent plastic. About $0.4 \mathrm{MeV}$ is deposited in the last $6 \mu \mathrm{m}$ of the particle range.

\subsection{ION STOPPING POWER IN COMPOUNDS}

To a first approximation, the stopping power of an ion in a compound or mixture is simply the weighted sum of the stopping powers in its constituent elements. This is the case for electron-stopping power, and for photon cross sections. Combining ion stopping powers this way is called Bragg's rule.

However, as stated previously, energy transfer is greatest when ion velocity equals the atomic electron orbital velocity. The orbital velocity, however, is different in the gas phase from the solid or liquid phase; molecular bonding changes both the orbital structure and electron velocities and so changes binding energies and stopping powers. As a result, ion stopping power is also different in gases from solids or liquids of the same material. In addition, different intramolecular bonds have the same effect. For example, in a molecule two carbon atoms bound by the $\mathrm{C}-\mathrm{C}$ single bond will contribute a smaller stopping power to a proton than will two Carbon atoms bound by a double $\mathrm{C}=\mathrm{C}$ bond or a triple $\mathrm{C} \equiv \mathrm{C}$ bond. Calculations of the effects of this molecular bonding are difficult, and the theoretical underpinning is complicated.

There are a number of theoretical approaches to modeling the stopping power in compounds. The SRIM code [SR03] bases its model on a simplified approach, called the bond and core approach, in which separate consideration is given to stopping by energy transfers to the outer bonding electrons and to the inner core electrons. The inner core electrons are unaffected by neighboring atoms and may be treated by Bragg's rule. The bonding electrons require a 


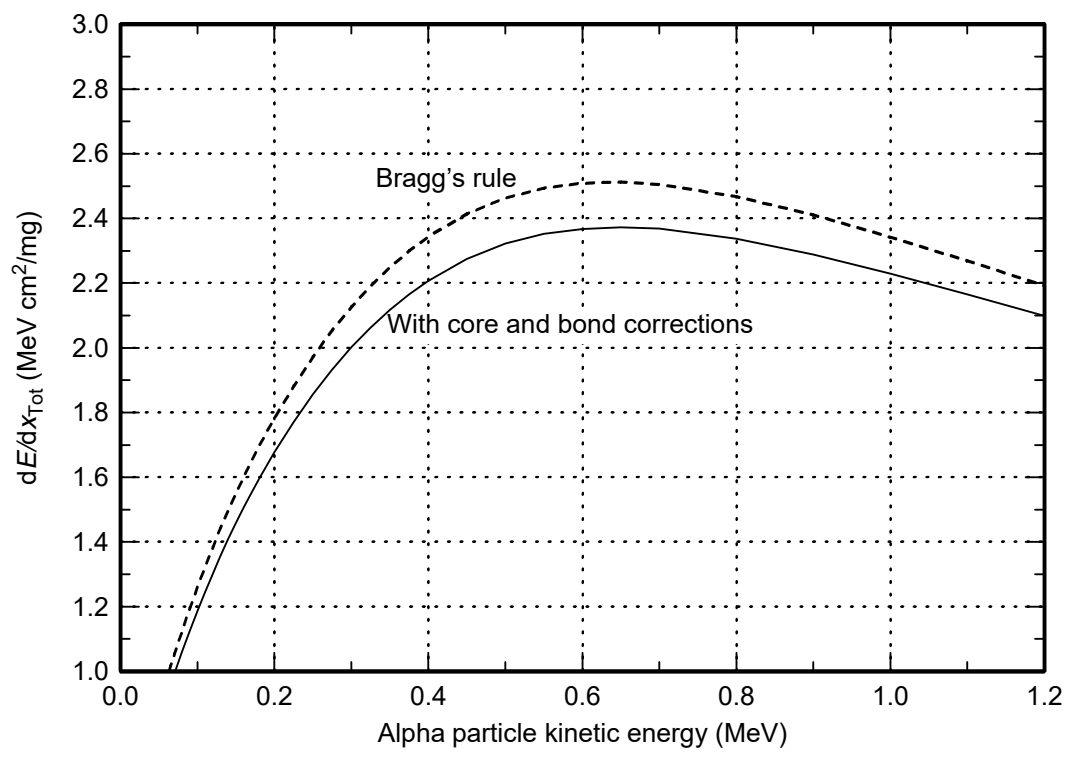

FIGURE 4.6 Alpha particle stopping power in liquid water. Dashed curve: computed by Bragg's rule from individual stopping powers of H and O. Solid curve: computed with the core and bond corrections. (All data computed with SRIM [SR03].)

model for disturbance by nearby atoms. The bonding model distinguishes between electrons that form a single bond, a double bond, or a triple bond with neighbors. It turns out that bonding in light atoms produces a greater departure from Bragg's rule than bonding in heavier atoms. See the online discussion [SR03] for details.

Figure 4.6 shows an example of alpha particles in liquid water. The stopping power computed by Bragg's rule from individual stopping powers of $\mathrm{H}$ and $\mathrm{O}$ is some $6 \%$ greater than that computed with proper corrections in the bond and core model. A larger correction occurs in ethylene, $\mathrm{C}_{2} \mathrm{H}_{4}$, as reported in [SR03]. There the difference is $12 \%$.

Bonding corrections tend to lower the stopping power. As there is no bonding in a gas, the stopping power in the gas phase is usually larger than that for the same material in a liquid or solid phase. Examples are given at SRIM online.

\subsection{PROTON AND ALPHA PARTICLE STOPPING POWERS IN SELECTED MATERIALS}

Using SRIM, it is easy to construct stopping powers and ranges for any ion in essentially any material. We present the elements and a few compounds and mixtures. 


\subsubsection{In the Elements}

\subsubsection{Protons}

For incident protons (or, effectively, hydrogen atoms), Figure 4.7 shows the stopping power in ten elements. The hydrogen target stands out with a larger mass stopping power than that of other elements. The stopping power is proportional to the number of electrons per unit mass in the target. Since Hydrogen has twice as many electrons per gram as other elements this doubles the mass stopping power in $\mathrm{H}$ over other elements even before other factors have been taken into account.

Figure 4.8 shows the stopping power in all elements at selected energies. Here and in other similar graphs, if hydrogen were plotted at its correct value, the curves would swing up dramatically at $Z=1$ relative to $Z=2$, and would be more difficult to read. For this reason, all elements are graphed at their correct stopping powers except for $\mathrm{H}$; the proton stopping power in $\mathrm{H}$ is graphed at half its true value for the sake of readability.

The proton stopping power at all energies in all elements is shown in the contour graph of Figure 4.9. The plateau (maximum stopping power) near 100 $\mathrm{keV}$ in all elements is evident. This and the following contour plots are available in color in larger scale in the Adobe portable document files (PDF) on the accompanying CD-ROM. When printed on $11^{\prime \prime} \times 17^{\prime \prime}$ paper, the graphs are easy to read to useful accuracy.

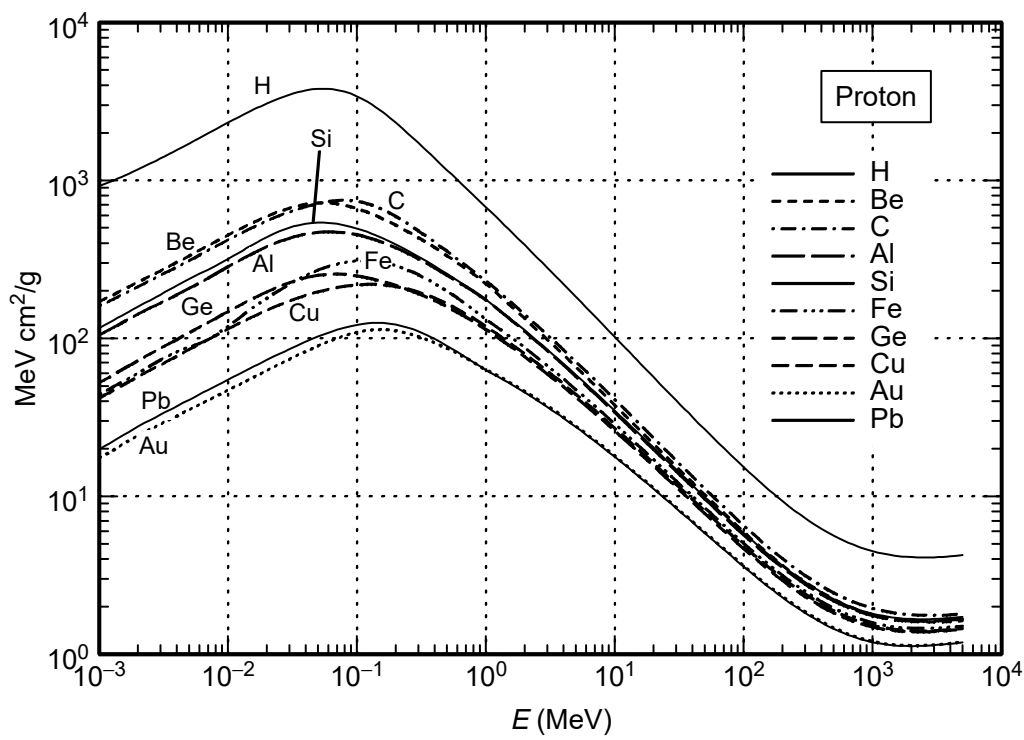

FIGURE 4.7 Proton stopping power in several elements vs. energy. Units of $\mathrm{MeV}-\mathrm{cm}^{2} / \mathrm{g}$. (Data from SRIM [SR03].) 


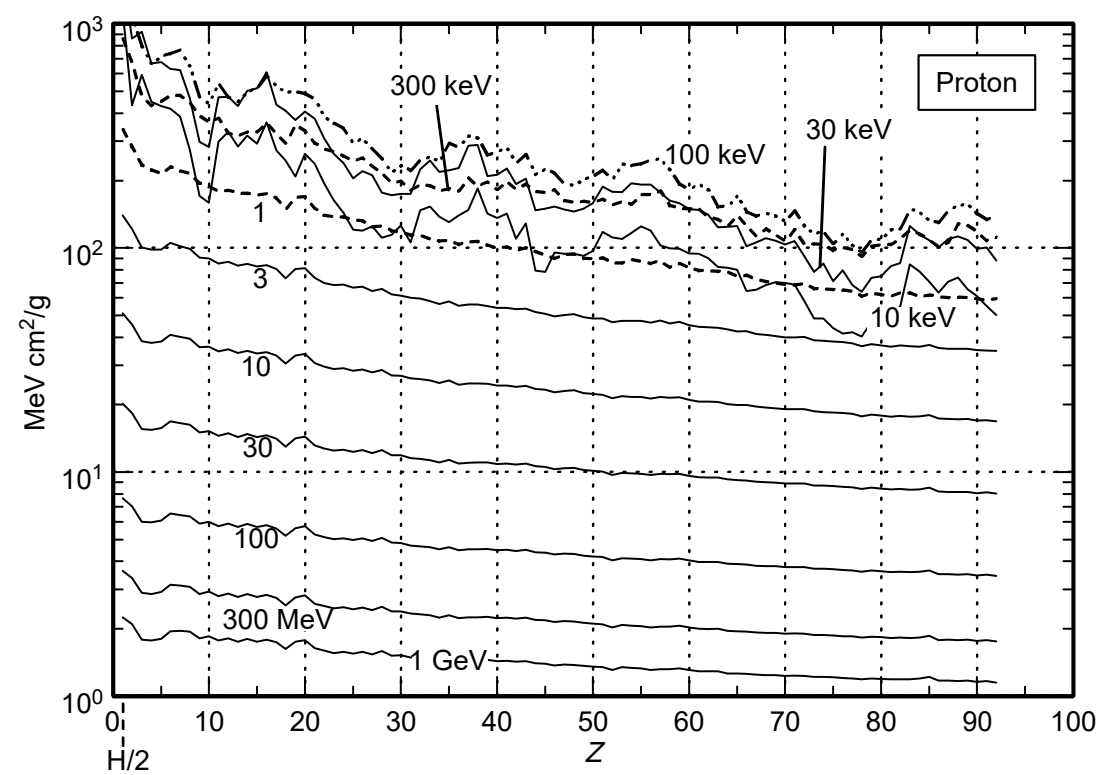

FIGURE 4.8 Proton stopping power in the elements at several energies vs. atomic number. To avoid line bunching near $Z=1$ (due to $Z / A=1$ for $\mathrm{H}$ but $1 / 2$ for other elements), the stopping power for $\mathrm{H}$ graphed here is $\frac{1}{2}$ its actual value (e.g., at $3 \mathrm{MeV}$, the graphed value is 140; its true value is 280). All other elements are graphed at their correct values. Units of $\mathrm{MeV} \mathrm{cm} / \mathrm{g}$. (Data from SRIM [SR03].)

Figure 4.10 graphs the continuous-slowing-down approximation range, $R_{\mathrm{CSDA}}$, Equation 4.11 , for protons in the same elements. It is shorter in $\mathrm{H}$ because of the increased stopping power just discussed. The range in all elements at selected energies is graphed in Figure 4.11. Hydrogen is graphed at twice its actual value for the reason given earlier. Note that a $1 \mathrm{MeV}$ proton will not penetrate $15 \mu \mathrm{m}$ of A1.

As mentioned, the effects of straggling and multiple angular scattering are less important for heavy ion projectiles than for electrons. When they are included, the mean forward progress of the ion is called the mean forward range $R_{\mathrm{mf}}$, or the projected range. It is less than $R_{\mathrm{CSDA}}$, and the ratio $R_{\mathrm{mf}} / R_{\mathrm{CSDA}}$ is called the detour factor. Scattering and straggling are most important at relatively low energies: for protons roughly below $1 \mathrm{MeV}$ in high- $Z$ targets and $100 \mathrm{keV}$ in low- $Z$ targets; for alpha particles roughly below a few $\mathrm{MeV}$ in high- $Z$ targets and a few hundred $\mathrm{keV}$ in low- $Z$ targets. This may be seen for protons in Figure 4.12, which shows the low energy ranges $R_{\mathrm{mf}}$ and $R_{\mathrm{CSDA}}$ in four elements of widely spread $Z$.

The contour plot in Figure 4.13 gives the proton $R_{\mathrm{CSDA}}$ in all elements at all energies. It is a smooth function of $Z$ at energies above about $1 \mathrm{MeV}$, 


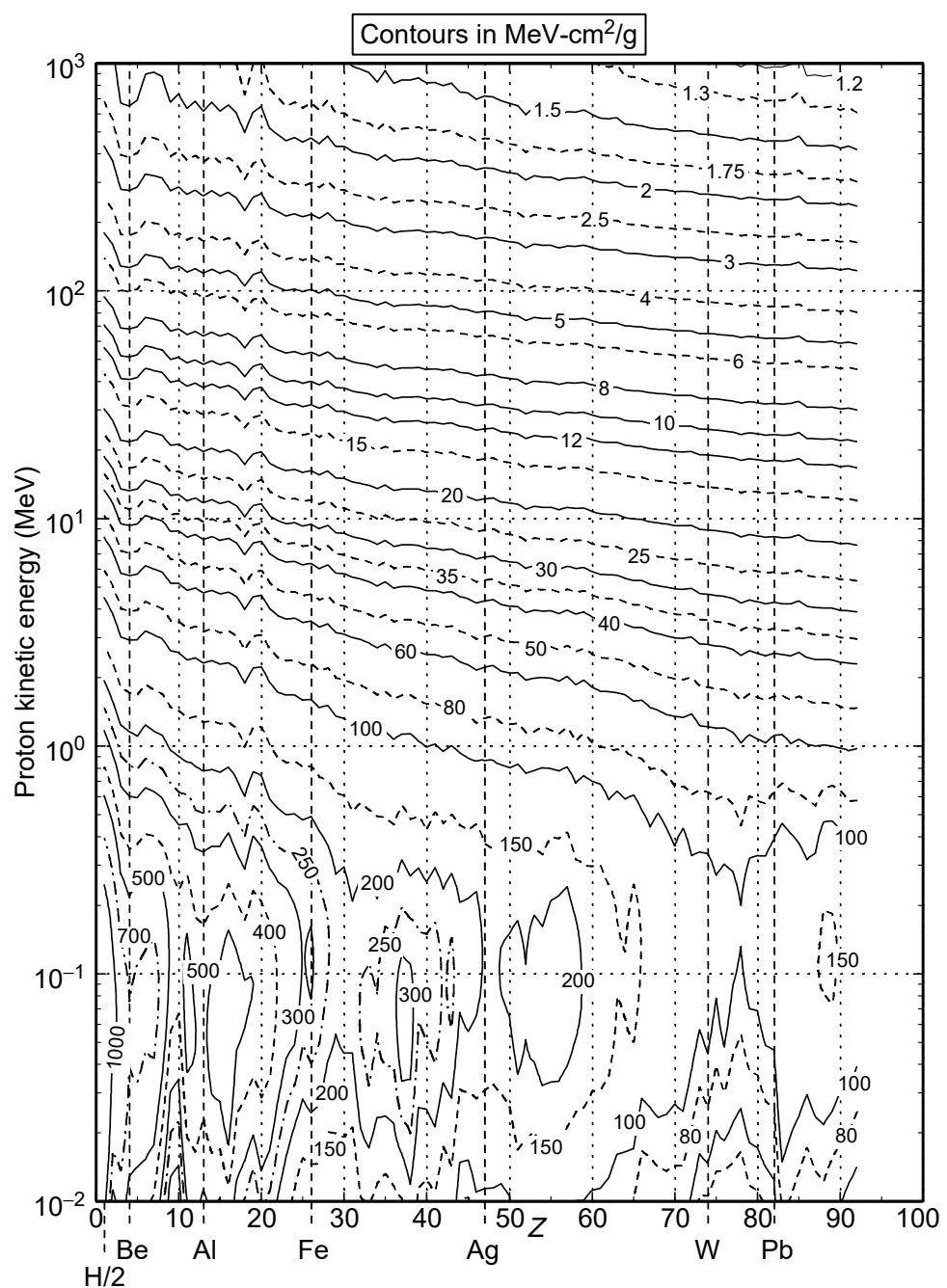

FIGURE 4.9 Total proton stopping power (electronic + nuclear) in all elements at interesting energies. To avoid line bunching near $Z=1$ (due to $Z / A=1$ for $\mathrm{H}$, but $1 / 2$ for other elements) the stopping power for $\mathrm{H}$ graphed here is $\frac{1}{2}$ its actual value (e.g., at $0.24 \mathrm{MeV}$ the graphed value is 1000; the actual value is 2000). All other elements are graphed at their correct values. Units of $\mathrm{MeV}$ per $\mathrm{g} / \mathrm{cm}^{2}$. (Data from SRIM [SR03].)

where the proton velocity exceeds the orbital velocities of most atomic electrons. Below that energy shell corrections are important. Figure 4.13 too is on the $\mathrm{CD}$ accompanying this book, in color intended for $11^{\prime \prime} \times 17^{\prime \prime}$ paper. 


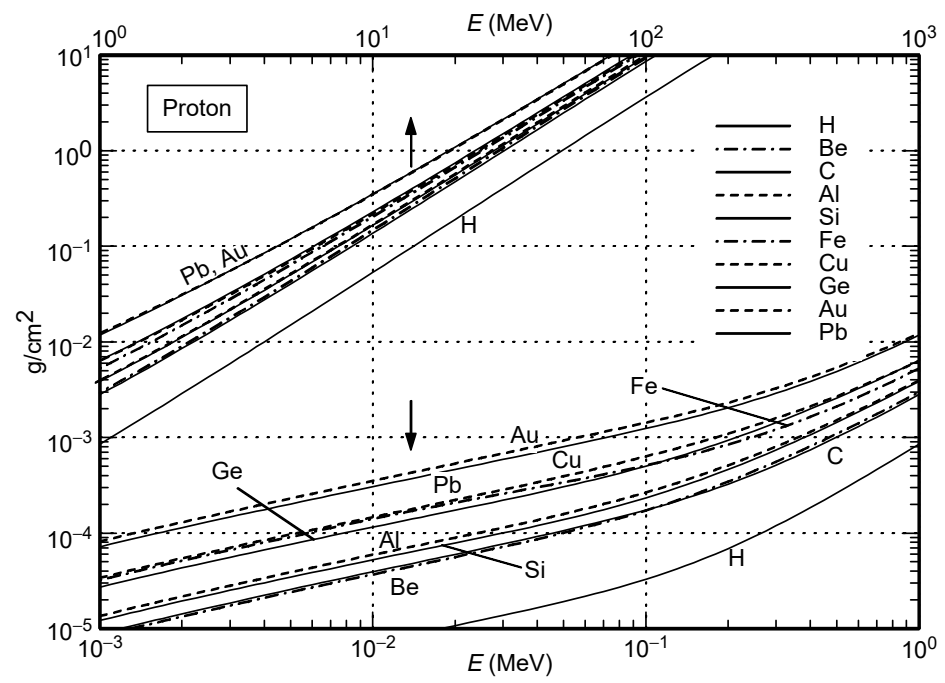

FIGURE 4.10 Proton continuous-slowing-down approximation range in ten elements, vs. energy. The range in hydrogen is plotted at its correct value. Lower curves: lower axis. Upper curves: upper axis. Range in $\mathrm{g} / \mathrm{cm}^{2}$. (Data from SRIM [SR03].)

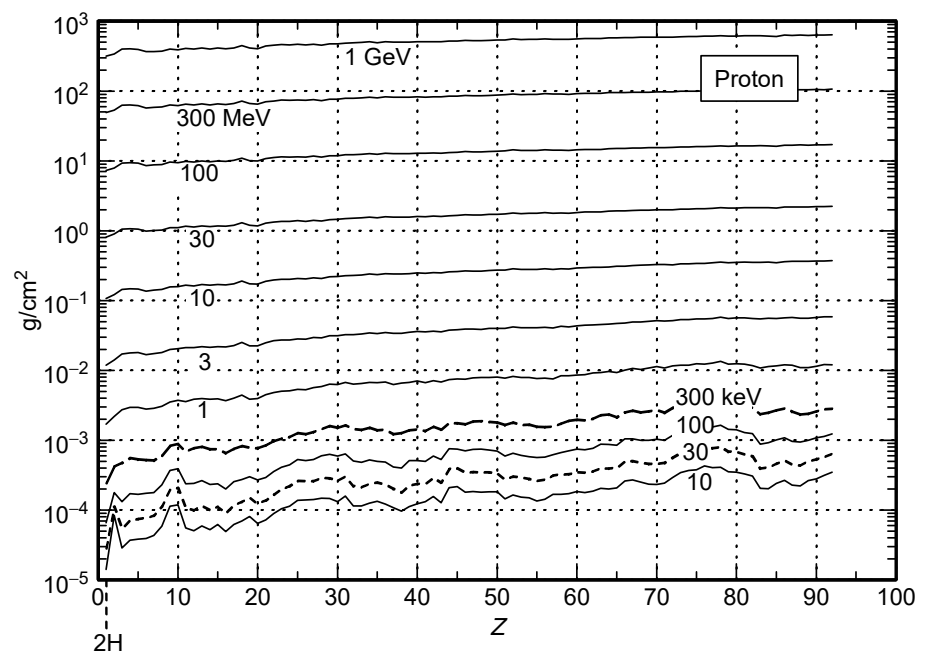

FIGURE 4.11 Proton continuous-slowing-down approximation range in the elements at several energies vs. atomic number. To avoid line bunching near $Z=1$ (due to $Z / A=1$ for $\mathrm{H}$, but $1 / 2$ for other elements), the stopping power graphed here in hydrogen is twice its true value (e.g., at $10 \mathrm{MeV}$ the graphed range is $0.106 \mathrm{~g} / \mathrm{cm}^{2}$; the actual range is $0.053 \mathrm{~g} / \mathrm{cm}^{2}$.) All other elements are graphed at their correct values. (Data from SRIM [SR03].) 


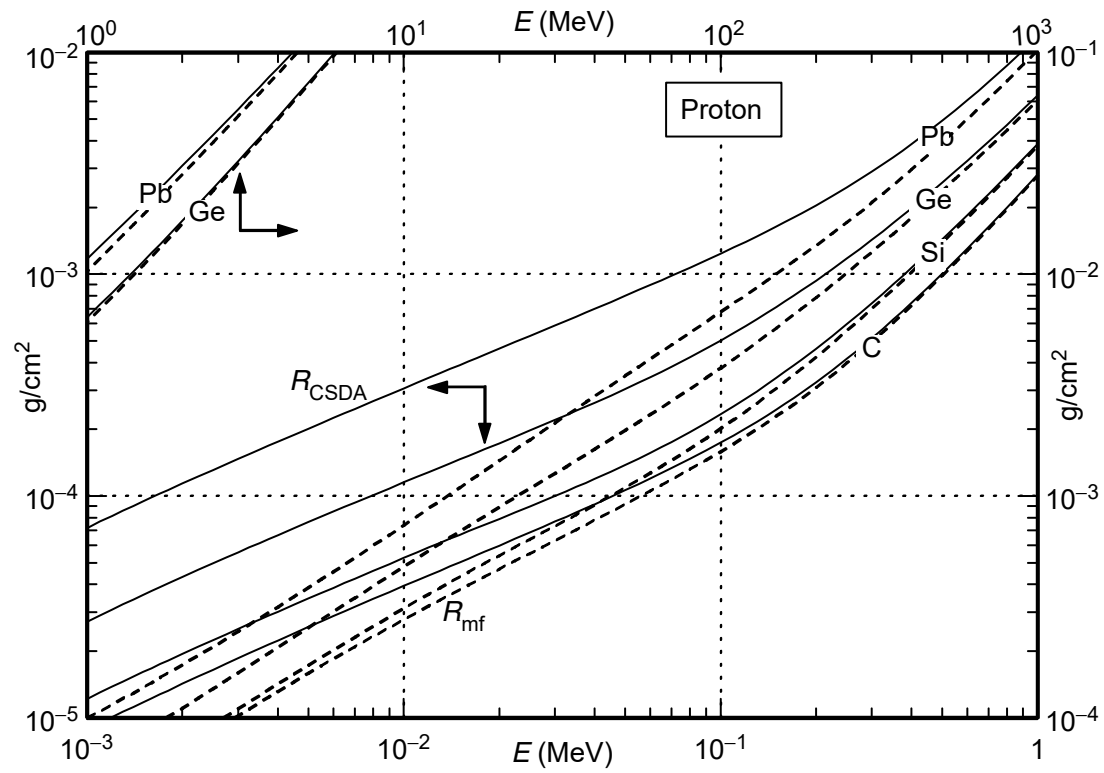

FIGURE 4.12 Comparison of proton mean forward range (against stopping power and scattering), dashed curves, and CSDA range, solid curves, in $\mathrm{C}, \mathrm{Si}, \mathrm{Ge}$, and $\mathrm{Pb}$. Lower curves: lower and left axes. Upper curves: upper and right axes. The mean forward range differs significantly from the CSDA range only below about $1 \mathrm{MeV}$ in high- $Z$ elements, and below $100 \mathrm{keV}$ in low- $Z$ elements, corresponding to ranges less than 10 $\mu \mathrm{m}$ in high $Z$ or $1 \mu \mathrm{m}$ in low $Z$. Units of $\mathrm{g} / \mathrm{cm}^{2}$. (Data from SRIM [SR03].)

\subsubsection{Alpha Particles}

Figure 4.14 shows the stopping power of an $\alpha$ in ten elements, over a wide range of energies. Again $\mathrm{H}$ stands out with having a larger mass stopping power. As was the case for protons, stopping powers among the elements differ most at lower energies. Figure 4.15 shows the stopping power in all elements as a function of $Z$, for selected energies. All elements at all energies are shown as a contour plot in Figure 4.16. Again, the plateau of maximum stopping power is evident. The $\frac{1}{2}$ hydrogen convention has been adopted in Figure 4.15 and Figure 4.16.

Turning from stopping power to ranges, Figure 4.17 shows the alpha particle $R_{\mathrm{CSDA}}$ in 10 elements as a function of projectile kinetic energy, and Figure 4.18 shows it as a function of $Z$ at selected energies. $R_{\mathrm{mf}}$ and $R_{\mathrm{CSDA}}$ are compared at low energies in Figure $4.19 . R_{\mathrm{CSDA}}$ in all elements at all energies is shown in the contour plot in Figure 4.20. Protons and alpha particles together are shown in $\mathrm{Be}$ and $\mathrm{Si}$ in Figure 4.21 and Figure 4.22. 


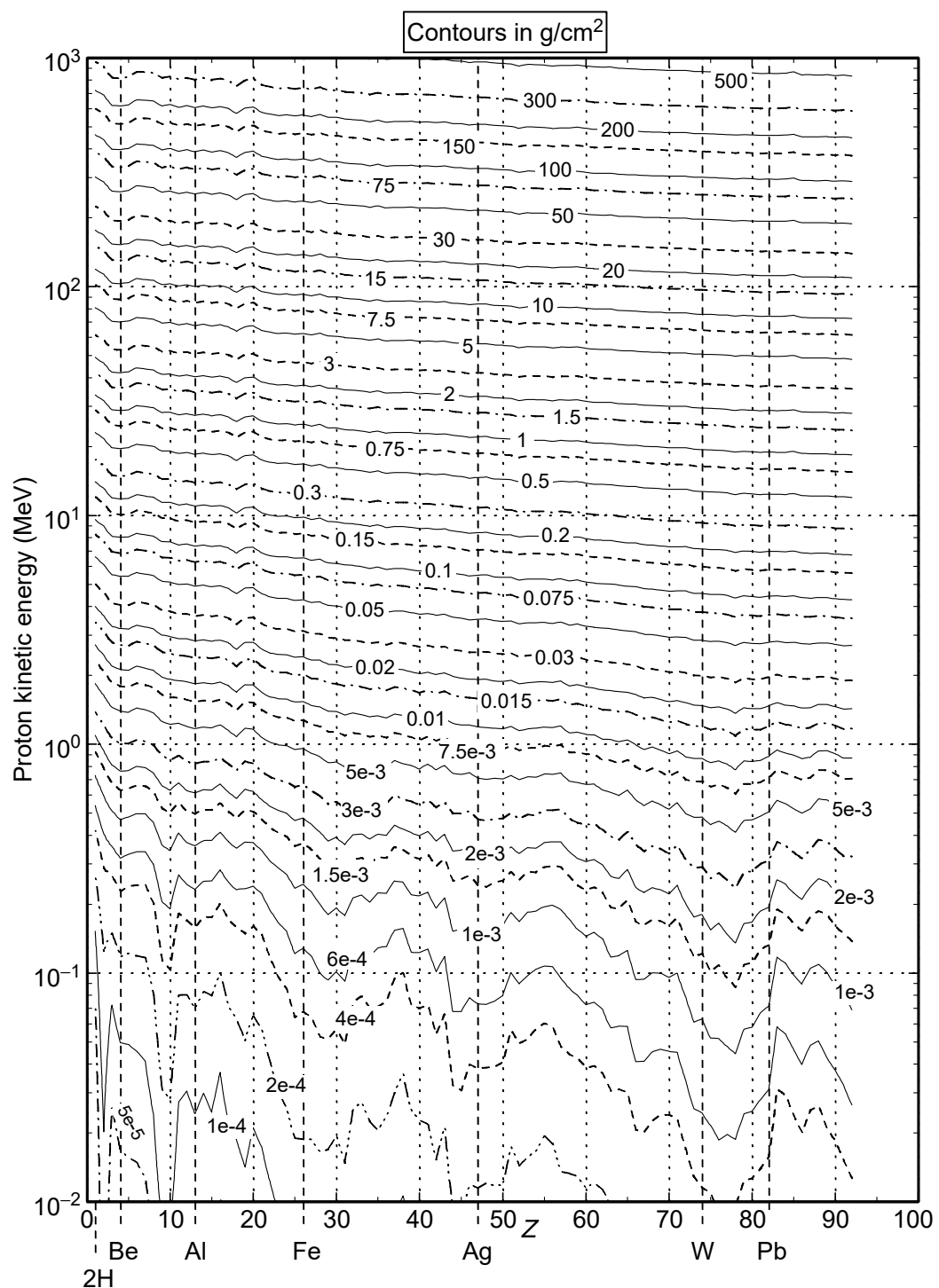

FIGURE 4.13 Proton continuous-slowing-down approximation range, $R_{\mathrm{CSDA}}$, in all elements at interesting energies. To avoid line bunching near $Z=1$ (due to $Z / A=1$ for $\mathrm{H}$ but $1 / 2$ for other elements), the graphed range in $\mathrm{H}$ is twice its actual value (e.g., at $10 \mathrm{MeV}$, the graph shows 0.106 ; its actual value is 0.053 ). All other elements are graphed at their correct values. Units of $\mathrm{g} / \mathrm{cm}^{2}$. (Data from SRIM [SR03].) 


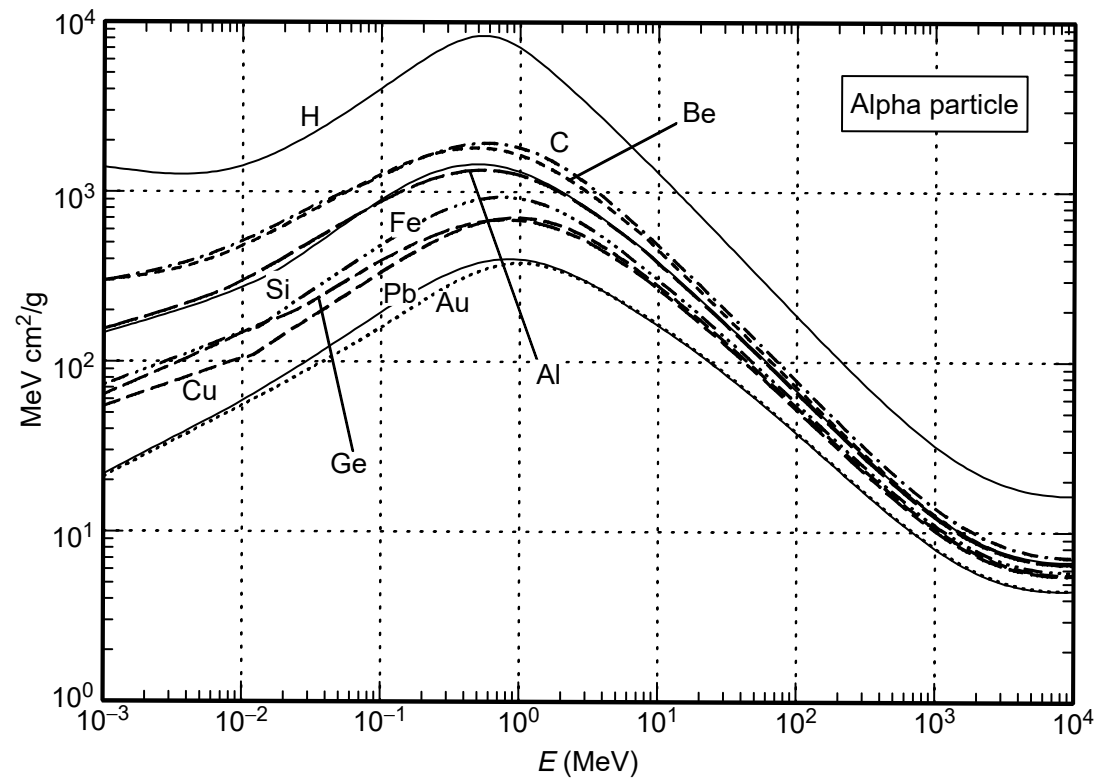

FIGURE 4.14 Alpha particle stopping power in several elements vs. energy. Units of $\mathrm{MeV}-\mathrm{cm}^{2} / \mathrm{g}$. (Data from SRIM [SR03].)

\subsection{In Compounds and Mixtures}

Proton and alpha particle stopping powers and ranges in selected materials (air, water, A150 tissue-equivalent plastic, GaAs, InSb, and $\mathrm{CdZnTe}$ ) are shown in Figure 4.23 through Figure 4.28. The plastic composition was given in Chapter 2, footnote in Section 2.11.2.2. Expressed as mass stopping powers and ranges, low- $Z$ materials are not very different from one another.

\subsection{PROTON-NUCLEUS ELASTIC SCATTERING}

Thus far we have discussed ion stopping power and range, that is, the effect of the material target on the ion. Also of concern is the ion effect on the target. It is a topic of much interest, particularly in semiconductors. Here we discuss one aspect of the problem of interest in silicon.

For light ions, the stopping power by energy transfer to atomic electrons is generally much greater than that due to energy transfer to the nucleus. Electronic transfer creates ionization in the target. However, material damage is caused by atomic displacement, which in turn is caused by nuclear recoil following ion-nuclear collisions. The recoiling nucleus is called the primary 


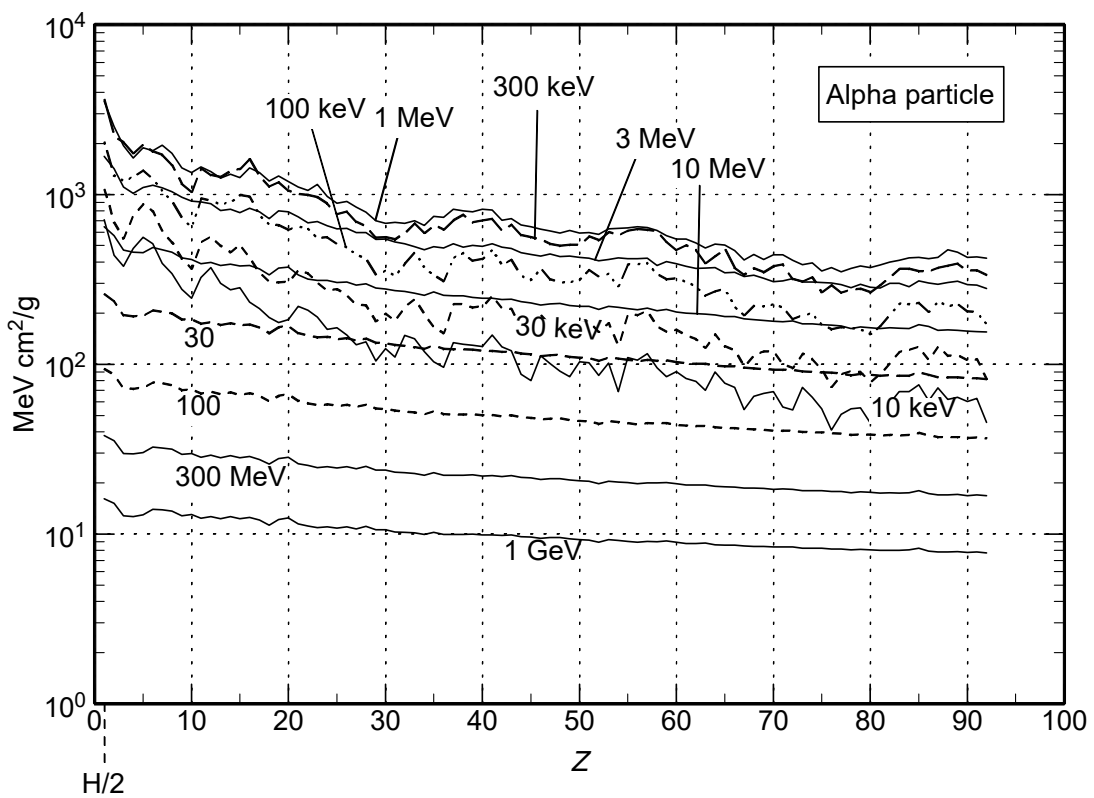

FIGURE 4.15 Alpha particle stopping power in the elements at several energies vs. atomic number. To avoid line bunching near $Z=1$ (due to $Z / A=1$ for $\mathrm{H}$, but $1 / 2$ for other elements), the stopping power graphed here in $\mathrm{H}$ is $\frac{1}{2}$ its true value (e.g., at $100 \mathrm{MeV}$, the graphed value is 94 ; its correct value is 188). All other elements are graphed at their correct values. Units of $\mathrm{MeV}-\mathrm{cm}^{2} / \mathrm{g}$. (Data from SRIM [SR03].)

knock-on atom. For damage, one needs to understand nuclear collisions, especially elastic scattering.

Nuclear recoil from elastic scattering is a major contributor to primary knock-on atoms, and is the only available process below the nuclear reaction threshold. Coulomb elastic scattering is usually the most important contributor to total elastic scattering. Until recently, many treatments included only Coulomb elastic scattering. However, nuclear elastic scattering also contributes, and its amplitude must be added coherently with Rutherford Coulomb elastic scattering, since the system is left in the same final state in either case. For an accurate assessment of nuclear recoil, especially its angular distribution, and for the cross section for a given energy transfer, one must include nuclear elastic scattering. Updated cross sections are in the ENDF library.

The complete elastic scattering amplitude then is

$$
f_{\text {elas }}=f_{\text {Coul }}+f_{\text {nuclear }}
$$




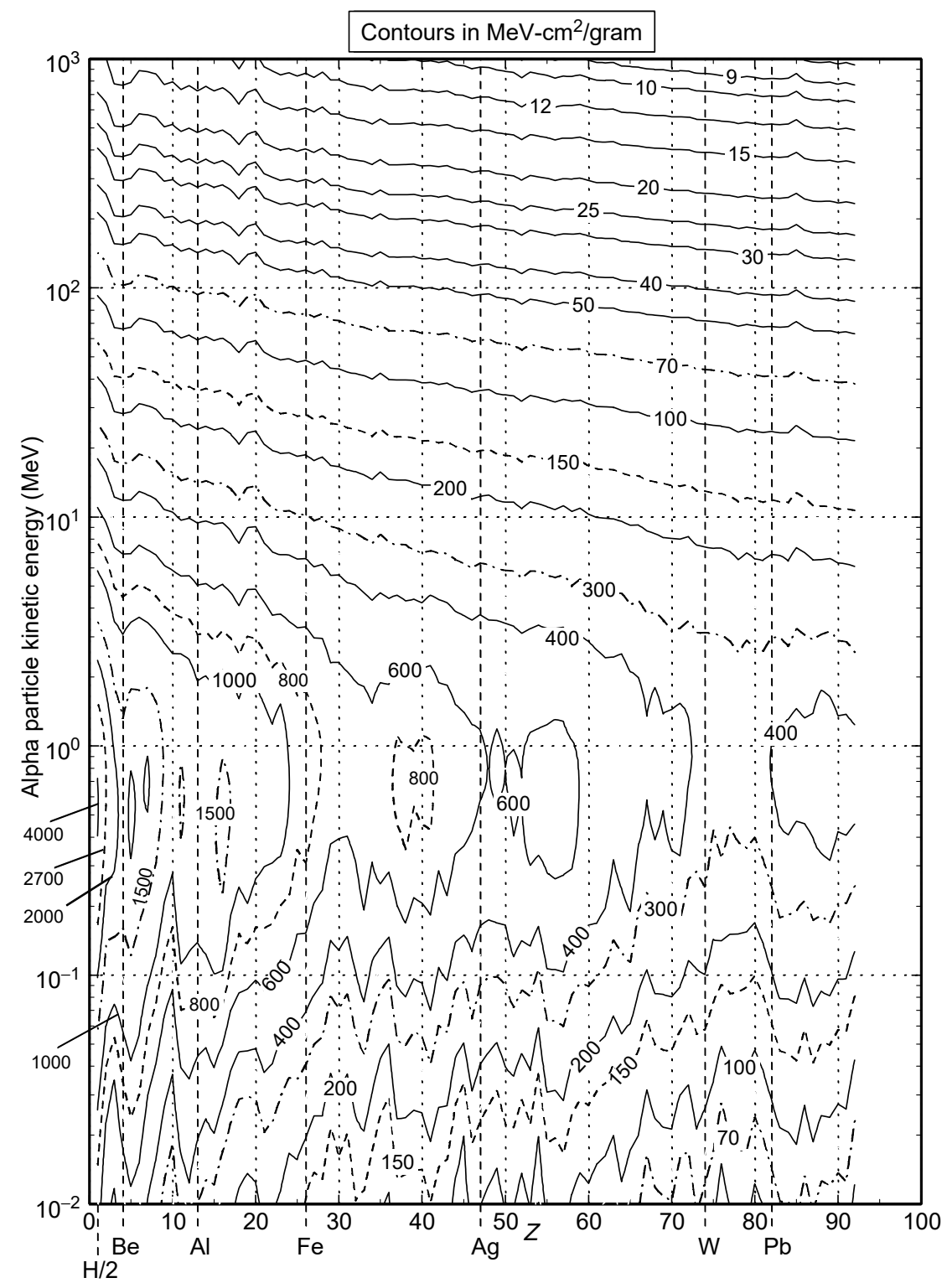

FIGURE 4.16 Total alpha particle stopping power in all elements at interesting energies. To avoid line bunching near $Z=1$ (due to $Z / A=1$ for $\mathrm{H}$, but $1 / 2$ for other elements), the stopping power for $\mathrm{H}$ graphed here is $\frac{1}{2}$ its true value (e.g., at $10 \mathrm{MeV}$ the graphed value is 649; its actual value is 1298). All other elements are graphed at their correct values. Units of $\mathrm{MeV}$ per $\mathrm{g} / \mathrm{cm}^{2}$. (Data from SRIM [SR03].) 


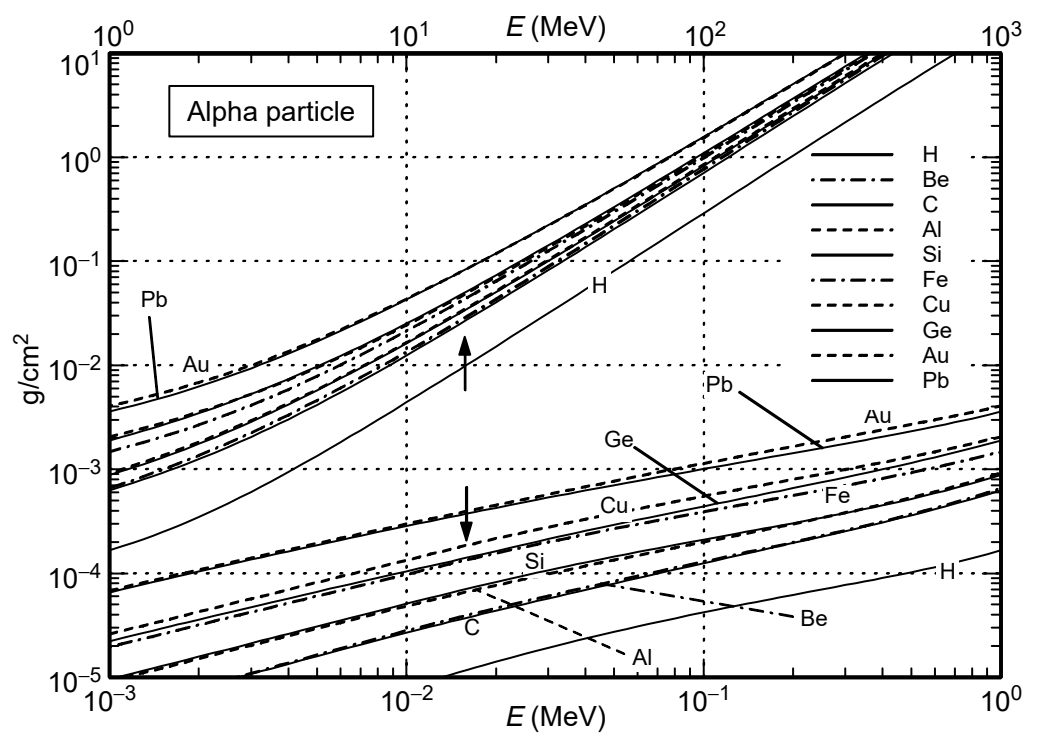

FIGURE 4.17 Alpha particle continuous-slowing-down approximation range in several elements vs. energy. Lower curves: lower axis. Upper curves: upper axis. Units of $\mathrm{g} / \mathrm{cm}^{2}$. (Data from SRIM [SR03].)

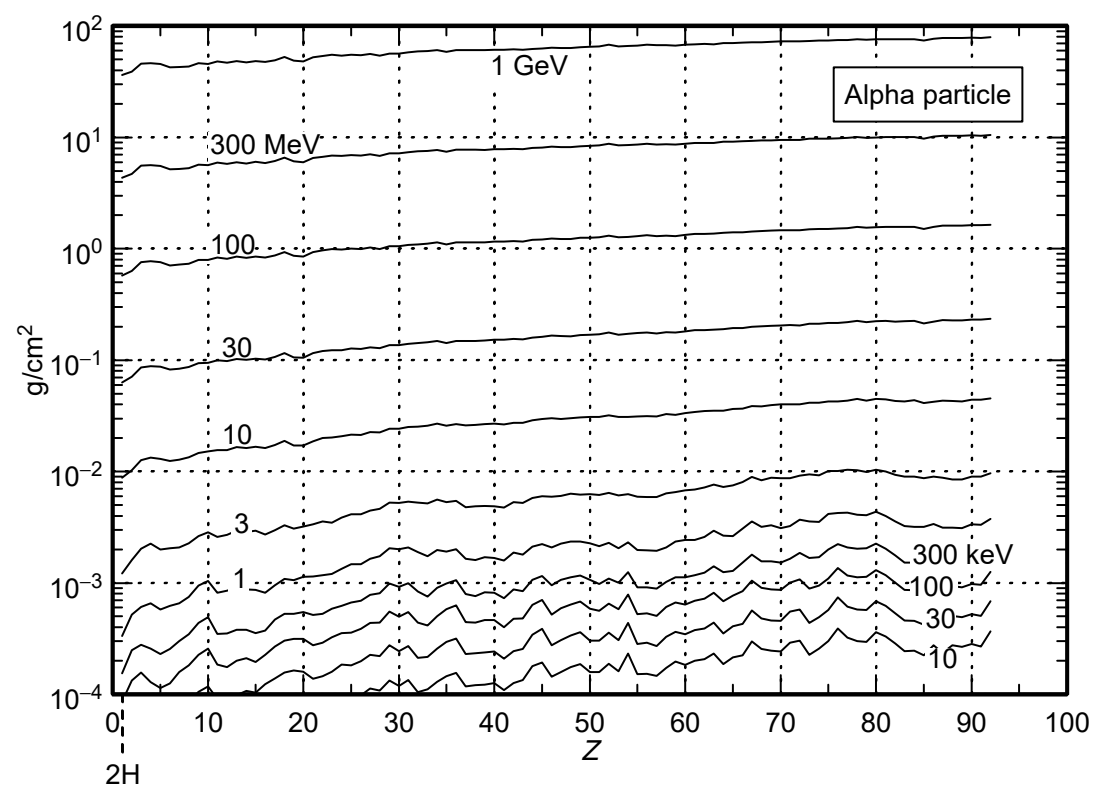

FIGURE 4.18 Alpha particle continuous-slowing-down approximation range, $R_{\mathrm{CSDA}}$, in the elements at several energies vs. atomic number. To present a cleaner graph near $Z=1$, the range in $\mathrm{H}$ graphed here is twice its actual value (e.g., at $10 \mathrm{MeV}$, the graphed range in $\mathrm{H}$ is 0.0088 ; its actual value is 0.0044 ). Units of $\mathrm{g} / \mathrm{cm}^{2}$. (Data from SRIM [SR03].) 


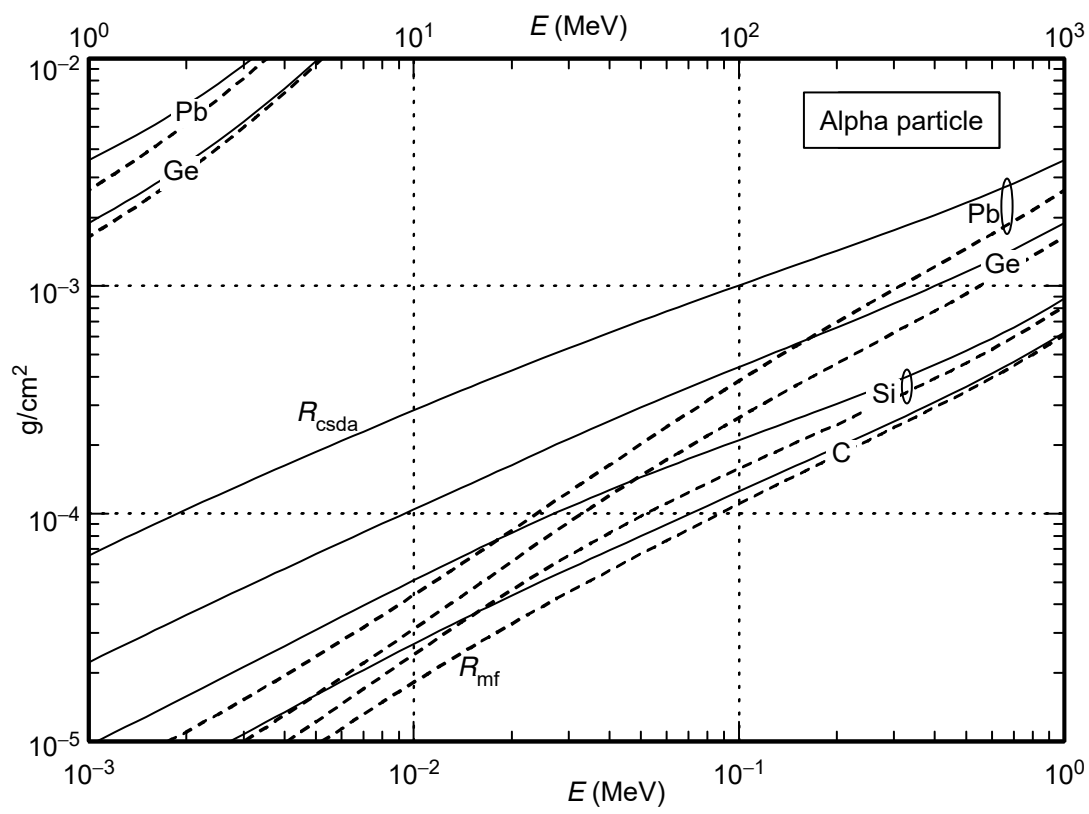

FIGURE 4.19 Comparison of alpha particle mean forward range (against stopping power and scattering), dashed curves, and CSDA range, solid curves, in solid $\mathrm{C}, \mathrm{Si}$, $\mathrm{Ge}$, and $\mathrm{Pb}$. Lower curves: lower axis. Upper curves: upper axis. The mean forward range differs from the CSDA range only below about $1 \mathrm{MeV}$, corresponding to ranges of only microns. At $1 \mathrm{MeV}$, both alpha ranges in these four solids correspond to between 2.6 and $3.6 \mu \mathrm{m}$. Units of $\mathrm{g} / \mathrm{cm}^{2}$. (Data from SRIM [SR03].)

and the elastic cross section is proportional to

$$
\left|f_{\text {elas }}\right|^{2}=\left|f_{\text {Coul }}+f_{\text {nuclear }}\right|^{2}=\left|f_{\text {Coul }}\right|^{2}+2 \operatorname{Re} f_{\text {Coul }} f_{\text {nuclear }}^{*}+\left|f_{\text {nuclear }}\right|^{2}
$$

The Rutherford Coulomb cross section $\left|f_{\text {Coul }}\right|^{2}$ is known analytically. The more difficult nuclear elastic scattering contribution enters in the cross term and in $\left|f_{\text {nuclear }}\right|^{2}$. These two terms enter only as their sum. The sum, $2 \operatorname{Re} f_{\text {Coul }}$ $f_{\text {nuclear }}^{*}+\left|f_{\text {nuclear }}\right|^{2}$, is tabulated by itself in the ENDF proton data library. This sum is to be added to the analytic Rutherford cross section to obtain the total elastic scattering cross section.

It is instructive to see effects in silicon. Using the nuclear terms from ENDF, Figure 4.29 shows the elastic scattering angular distribution with and without the nuclear contribution for a proton incident on $\mathrm{Si}^{28}$. Incident energies 24 and $28 \mathrm{MeV}$ are plotted. When nuclear elastic scattering is 


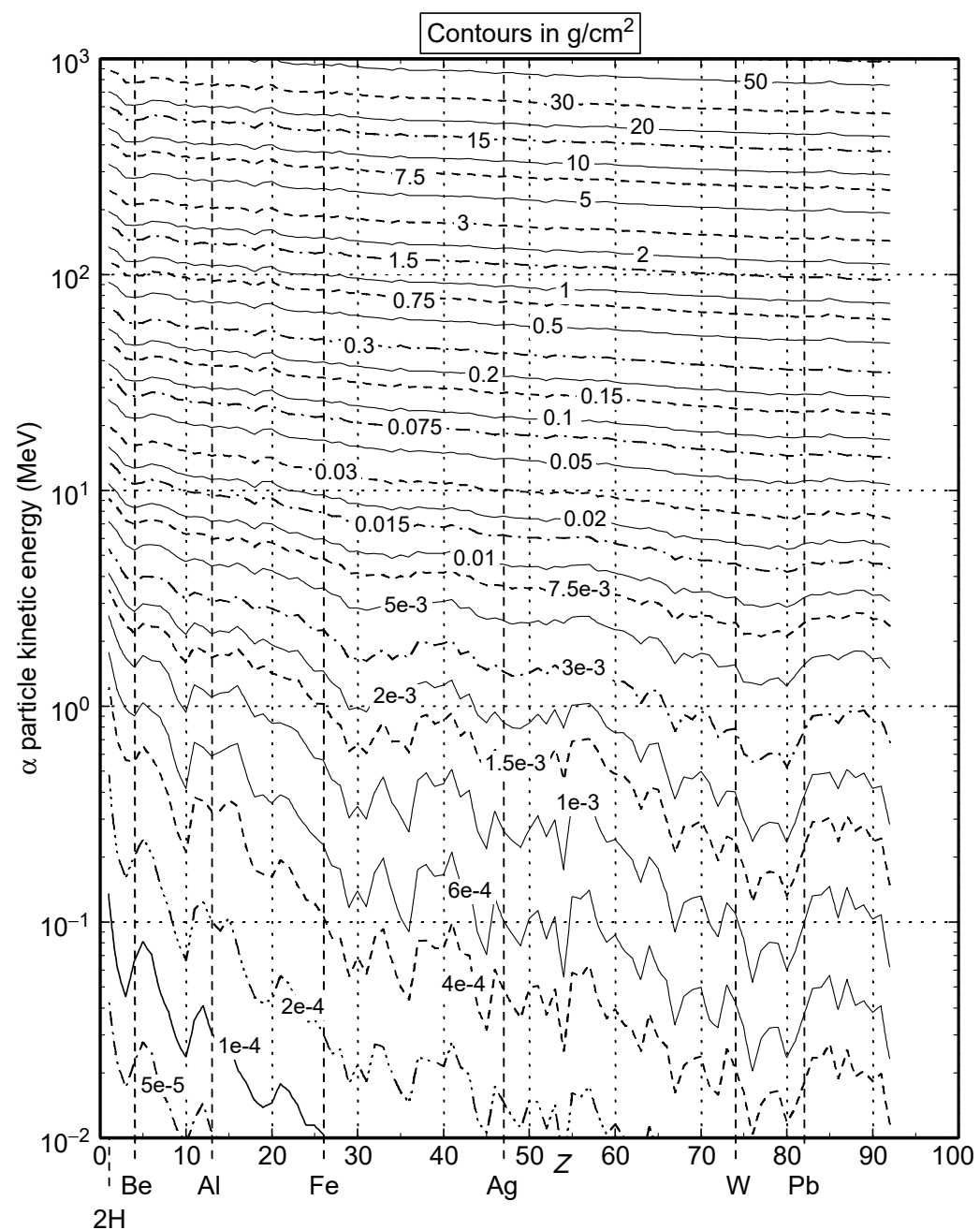

FIGURE 4.20 Alpha particle continuous-slowing-down approximation range, $R_{\mathrm{CSDA}}$, in all elements at interesting energies. To prevent line bunching near $Z=1$ (due to $Z / A=1$ for $\mathrm{H}$, but $1 / 2$ for other elements), the graphed range for $\mathrm{H}$, at all energies, is twice its true value (e.g., at $10 \mathrm{MeV}$ the graphed range is 0.0088 ; its actual value is 0.0044). Units of $\mathrm{g} / \mathrm{cm}^{2}$. (Data from SRIM [SR03].)

included, the differential cross section at angles greater than $40^{\circ}$ is several times the Coulomb cross section. As large scattering angles correspond to large energy transfers, the correct cross section produces larger energy transfers than the Coulomb cross section alone. 


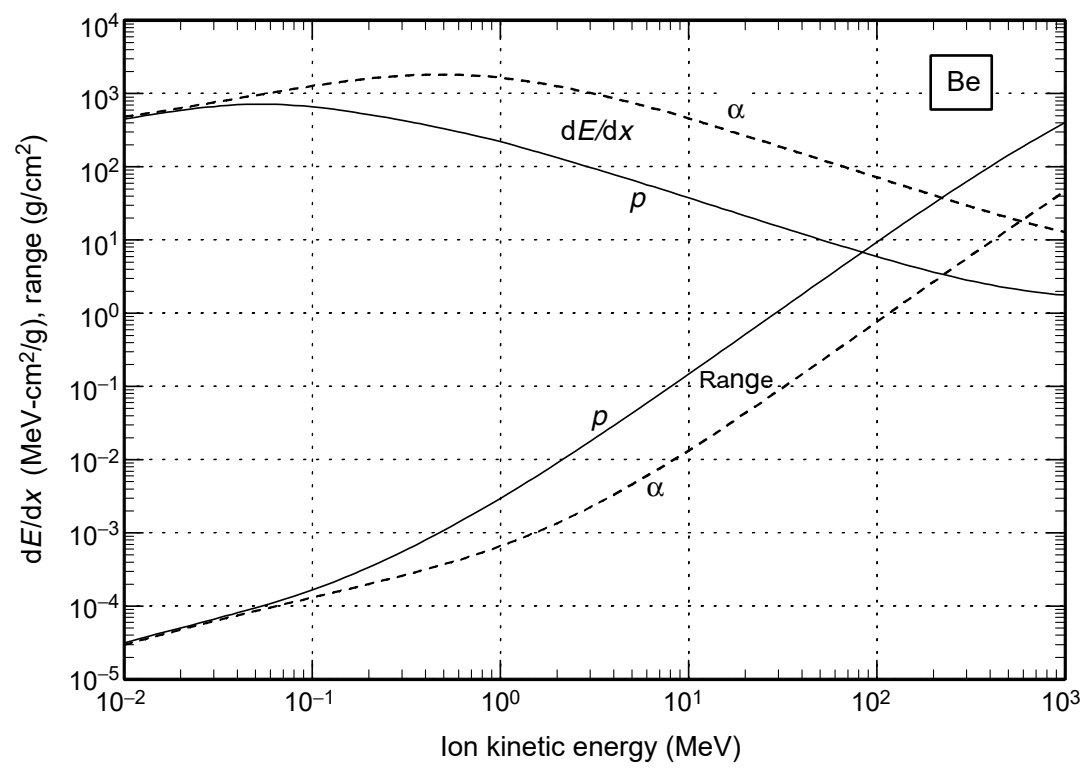

FIGURE 4.21 Stopping powers and ranges of protons and alpha particles in solid beryllium. (Data from SRIM [SR03].)

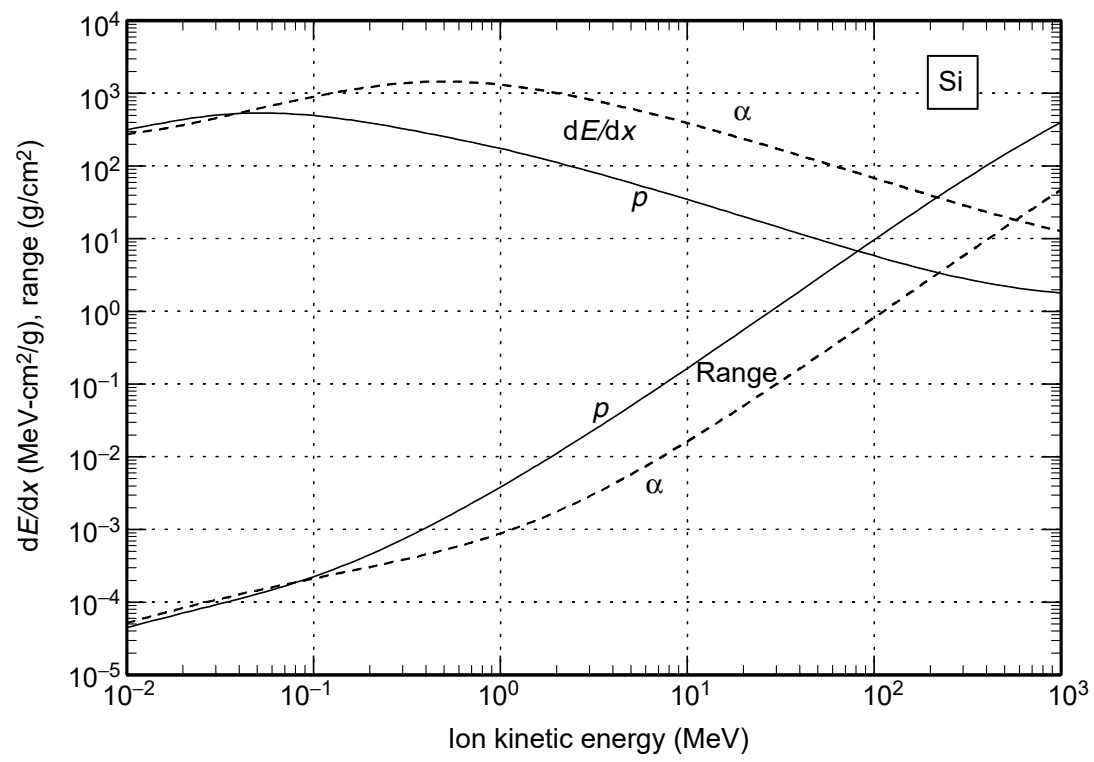

FIGURE 4.22 Stopping powers and ranges of protons and alpha particles in silicon. (Data from SRIM [SR03].) 


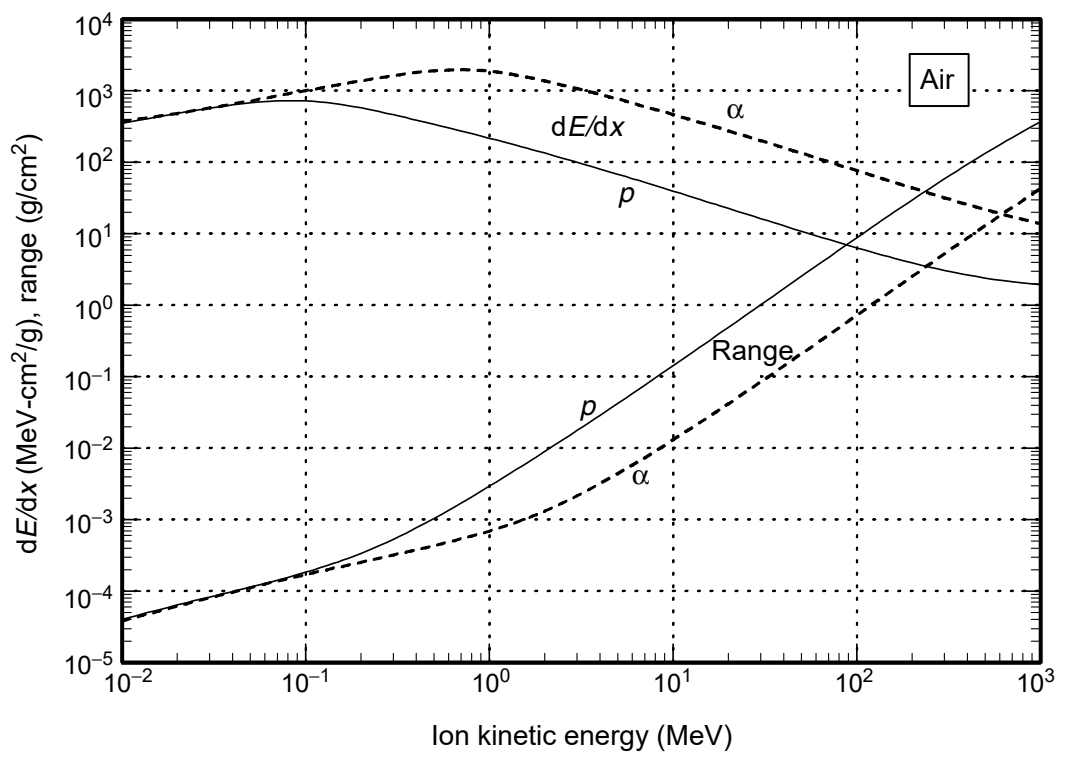

FIGURE 4.23 Stopping powers and ranges of protons and alpha particles in air. (Data from SRIM [SR03])

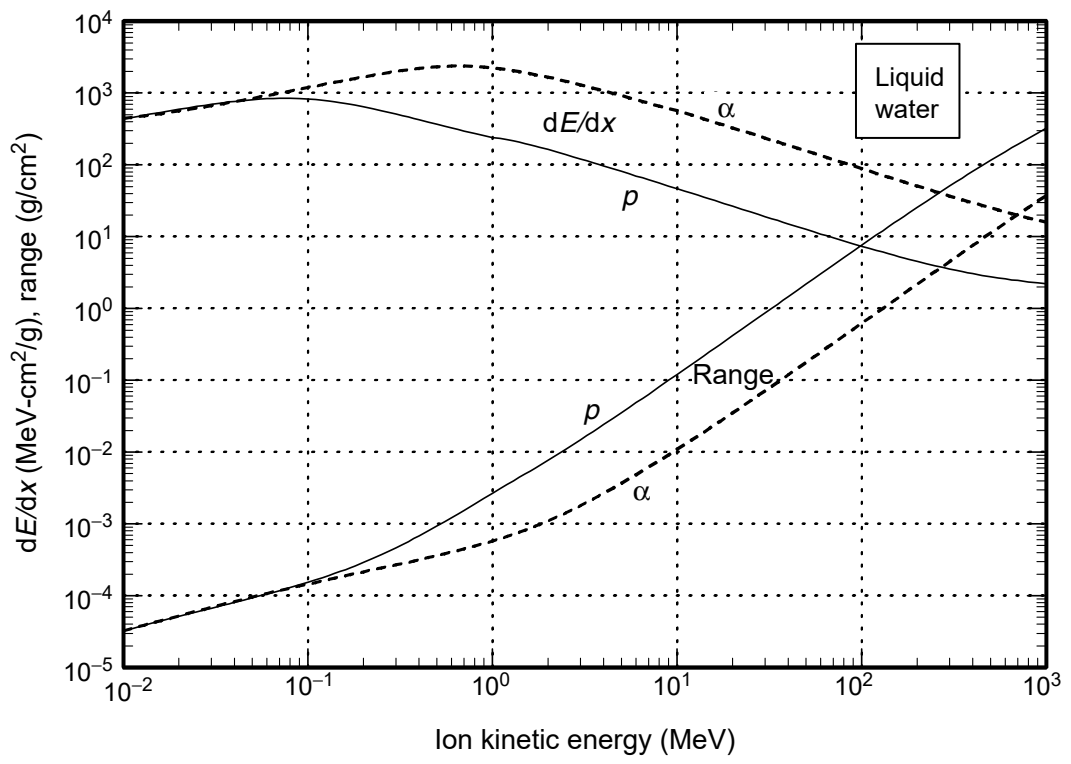

FIGURE 4.24 Stopping powers and ranges of protons and alpha particles in liquid water. (Data from SRIM [SR03].) 


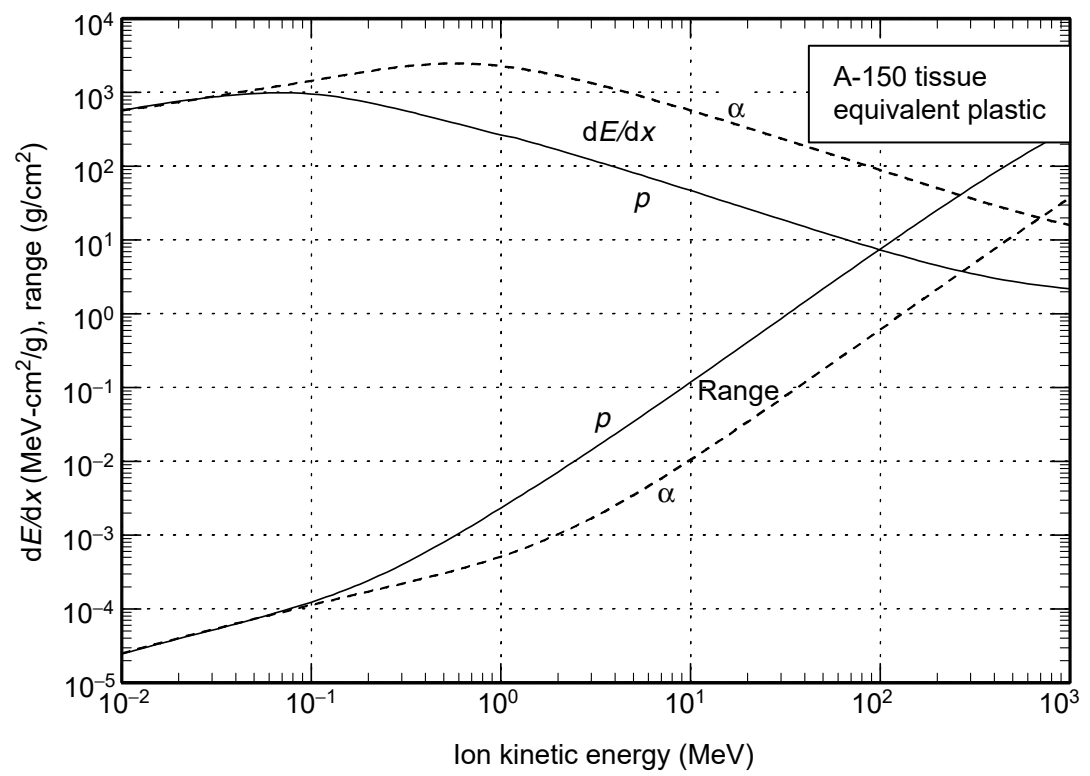

FIGURE 4.25 Stopping powers and ranges of protons and alpha particles in ICRU A-150 tissue equivalent plastic. (Data from SRIM [SR03].)

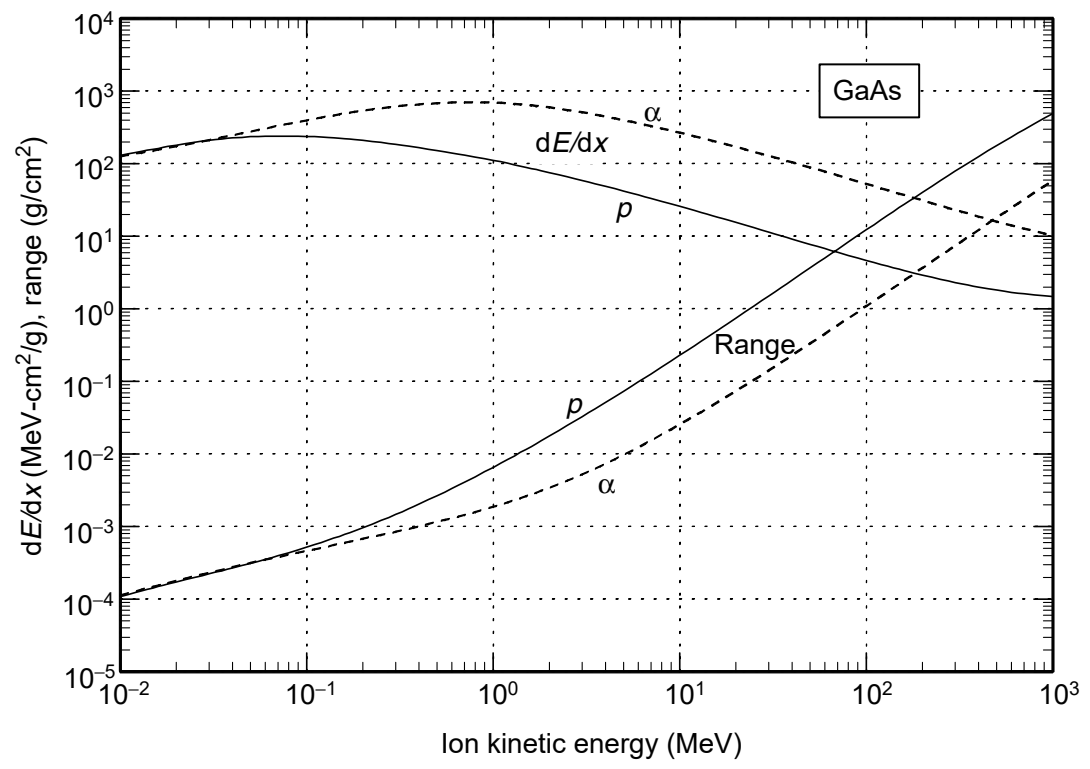

FIGURE 4.26 Stopping powers and ranges $R_{\mathrm{CSDA}}$ of protons and alpha particles in gallium arsenide. (Data from SRIM [SR03].) 


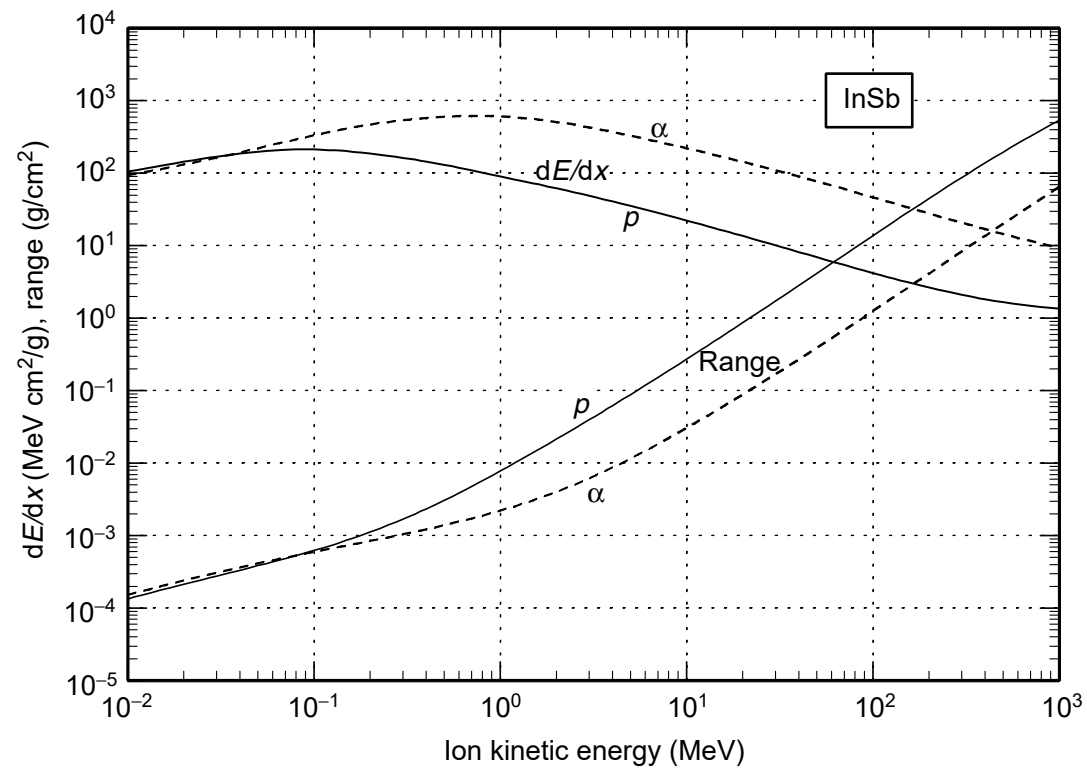

FIGURE 4.27 Stopping powers and ranges $R_{\mathrm{CSDA}}$ of protons and alpha particles in indium antimonide. (Data from SRIM [SR03].)

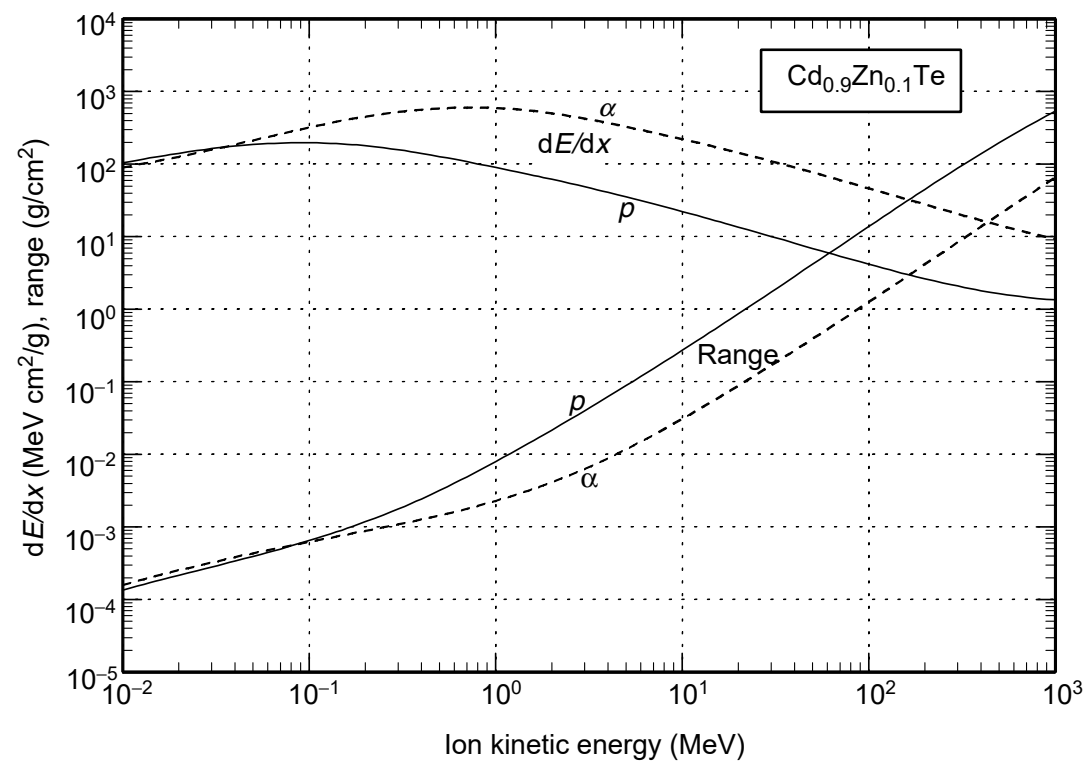

FIGURE 4.28 Stopping powers and ranges $R_{\mathrm{CSDA}}$ of protons and alpha particles in CZT. (Data from SRIM [SR03].) 


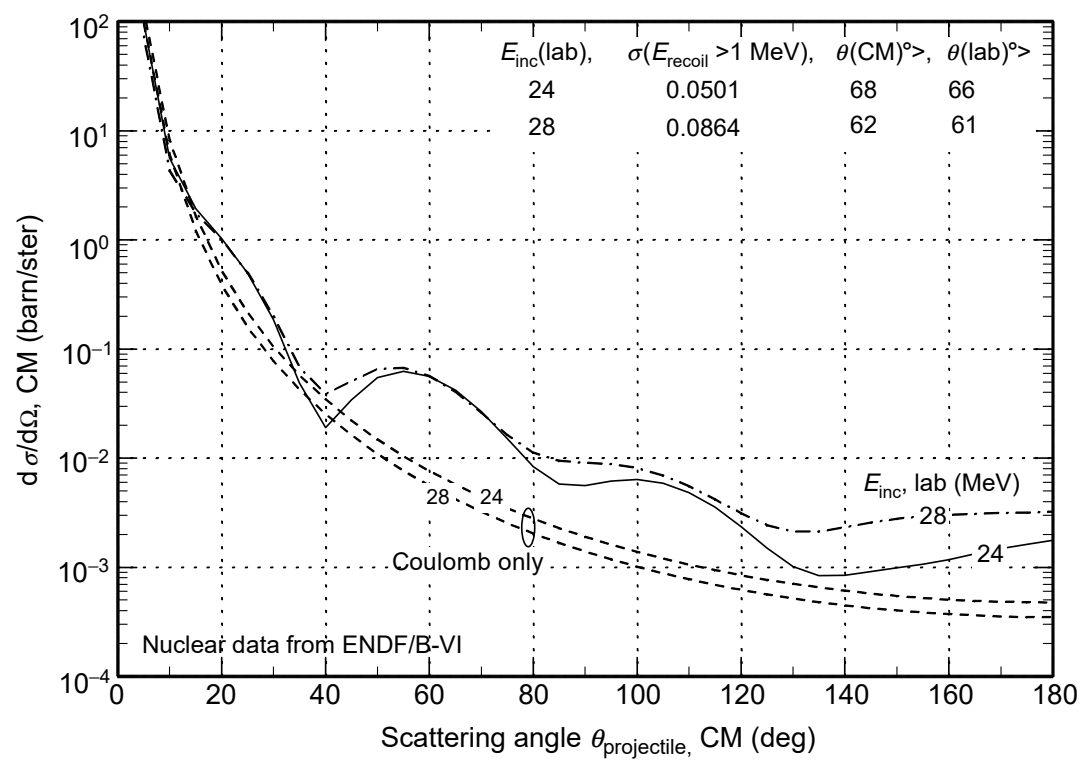

FIGURE 4.29 Proton on $\mathrm{Si}^{28}$ elastic differential cross section, at 24 and $28 \mathrm{MeV}$ incident proton energy. Rutherford Coulomb scattering only (dashed), and correct Coulomb + nuclear cross section. Nuclear data from ENDF/B-6.

The significance of nuclear scattering may also be seen by comparing the total (nuclear + Coulomb) differential cross section relative to the Coulomb cross section. Figure 4.30 shows this for $32 \mathrm{MeV}$ protons incident on silicon. At $60^{\circ}$, the complete cross section is an order of magnitude larger than the Rutherford cross section, and it is several times larger at almost all angles. In calculations involving light ion-heavy ion scattering, such as in studies of radiation damage effects in semiconductors, it is important to include nuclear elastic scattering as well as Coulomb scattering. Early studies of proton displacement damage in silicon, e.g., Burke [Bu86], used earlier experimental data for nuclear scattering, with results qualitatively the same, but quantitatively different from that in Figure 4.30, especially near maximum energy transfer (backscatter).

Similarly, the cross section for a given energy transfer is larger when nuclear scattering is included than when it is not. Figure 4.31 shows the cross section for producing a recoil Si nucleus with energy greater than $300 \mathrm{keV}$ or greater than $1 \mathrm{MeV}$ when nuclear contributions are and are not included. At a given proton energy, the cross section is several times the Coulomb cross section.

Figure 4.31 shows that nuclear elastic scattering makes a significant difference even at a proton energy $E$ of only a few $\mathrm{MeV}$. This fact can be 


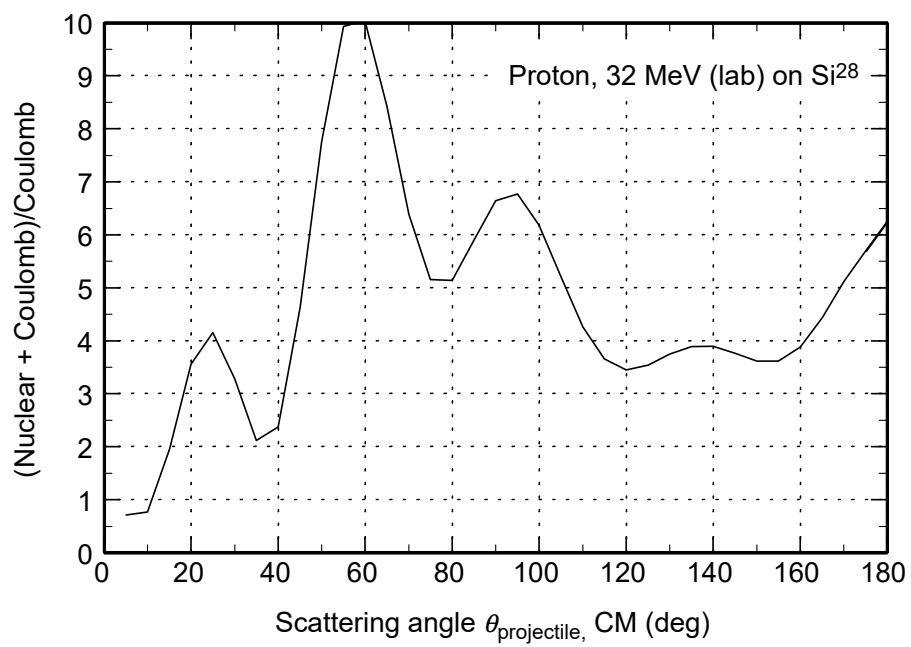

FIGURE 4.30 Ratio of Coulomb + nuclear elastic scattering differential cross section to Coulomb. $32 \mathrm{MeV}$ protons on $\mathrm{Si}^{28}$. Nuclear data from ENDF/B-6.

appreciated by noting first that the Si nuclear radius is about $4.4 \mathrm{fm}$ [Ev55]. The proton distance of closest approach is $r_{\min }=Z e^{2} / E$ (neglecting the small difference between the proton mass and the reduced mass), and the proton wavelength is $\lambda=h / \sqrt{ }\left(2 M_{\mathrm{p}} E\right)$. At, say, $E=5 \mathrm{MeV}$, find $r_{\min } \sim 4 \mathrm{fm}$, and $\lambda=12.8 \mathrm{fm}$. Thus, the proton can readily interact with the nuclear potential,

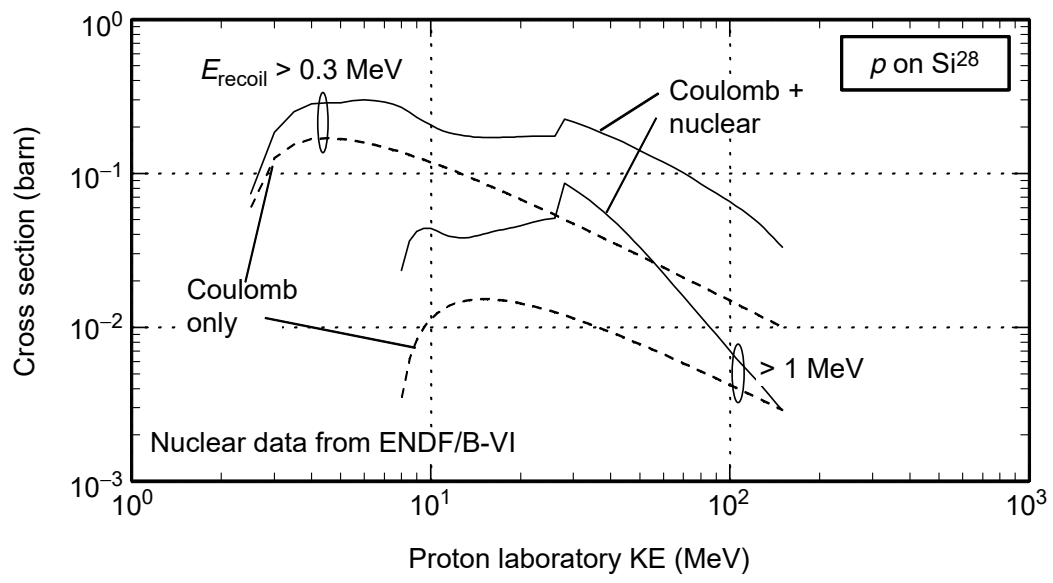

FIGURE 4.31 Proton on silicon cross section for Si recoil energy greater than 0.3 or $1 \mathrm{MeV}$. Coulomb cross section only (dashed), and nuclear + Coulomb cross section (solid). Nuclear data from ENDF/B-6. 
and nuclear elastic scattering can interfere significantly with Coulomb elastic scattering. Studies of ion damage in semiconductors must include nuclear elastic scattering with reliable angular differential cross sections.

\subsubsection{Nonionizing Energy Loss}

In addition to losing energy by knocking out electrons from target atoms, an incident ion loses some energy to target atom recoil in an elastic collision. The recoiling atom then hits others, initiating a cascade that deposits energy in the form of atom displacements, vacancies, and interstitials, rather than ionization. For incident charged particles, this form of energy deposition is traditionally referred to as NonIonizing Energy Loss, NIEL. (For neutrons, it is more often referred to as nonionizing dose or nonionizing kerma.) As displacements accumulate over time, clusters and electron trapping centers form, degrading the performance of semiconductor electronic devices. To an extent, the damage done tends to be proportional to the energy deposited, i.e., to the NIEL; however, there are many exceptions. Damage appears to be proportional to NIEL for $n$ type semiconductors at all ion energies, but in other materials it remains proportional to NIEL at high energies but becomes proportional to $(\mathrm{NIEL})^{2}$ at low energies. Summers and colleagues [Su95] summarize to what extent damage in semiconductors is proportional to NIEL. Radiation damage to electronic components is an enormous subject in its own right, presented in texts such as that of Holmes-Siedle and Adams [Ho02] and Messenger and Ash [Me92].

The basic mechanism of NIEL is the cascade following from each recoiling atom knocking out further atoms from their lattice sites. The process is pronounced in neutron irradiation, in which the projectile interacts directly with the nucleus. We postpone the discussion to Chapter 5 on neutrons, where the theory of Lindhard and coworkers is discussed.

\subsection{LARGE CONTOUR PLOTS}

Six large, color contour plots $\left(11^{\prime \prime} \times 17^{\prime \prime}\right)$ that graph useful parameters, three for protons and three for alpha particles, are available. They are in highresolution PDF format on the accompanying $\mathrm{CD}$ (and would require a printer capable of handling $11^{\prime \prime} \times 17^{\prime \prime}$ paper). They are as follows.

\subsubsection{Protons}

- Total mean stopping power, $\mathrm{d} E /\left.\mathrm{d} s\right|_{\text {Total }}$ (electronic + nuclear). Most energy transfer from ion to target is via energy loss by collisions with atomic electrons. Energy loss by collisions with nuclei is much less important (a portion of it is responsible for the small nonionizing energy loss). Bremsstrahlung is negligible for ions below a few $\mathrm{GeV} / \mathrm{amu}$. 
- Full CSDA range, $R_{\mathrm{CSDA}}$, against total mean stopping power. Full trajectory path length $\left(\mathrm{g} / \mathrm{cm}^{2}\right)$.

- Mean forward range, $R_{\mathrm{mf}}$. The mean depth of penetration against total mean stopping power and scattering. For protons, this differs significantly from the CSDA range only for $E \lesssim 0.1 \mathrm{MeV}$. The ratio $R_{\mathrm{mf}} / R_{\mathrm{CSDA}} \equiv$ detour factor $<1$.

\subsubsection{Alpha Particles}

- Total mean stopping power, $\mathrm{d} E /\left.\mathrm{d} s\right|_{\text {Total }}$ (electronic + nuclear). Nuclear exceeds electronic only in the low- $Z$, low- $E$ corner. Here and for protons, the target atomic shell structure is important at low energies, causing significant irregularities in a contour plot. Binding energies in outer electron shells (L, M, N, etc.) are typically 50 to $100 \mathrm{eV}$.

- Full CSDA range, $R_{\mathrm{CSDA}}$, against total mean stopping power. Full trajectory path length $\left(\mathrm{g} / \mathrm{cm}^{2}\right)$.

- Mean forward range, $R_{\mathrm{mf}}$. The mean depth of penetration against total mean stopping power and scattering. For alpha particles, this differs significantly from the CSDA range only for $E \lesssim$ few hundred $\mathrm{keV}$. Proton and alpha particle data computed with SRIM 2003.

\section{REFERENCES}

[Ah80] Ahlen, S.P., Theoretical and experimental aspects of the energy loss of relativistic heavily ionizing particles, Rev. Mod. Phys. 52(1): 121-173, 1980, Errata 52(3): 653.

[An77] Andersen, H.H. and Ziegler, J.F., Hydrogen stopping powers and ranges in all elements, Vol. 3, The Stopping and Ranges of Ions in Matter, Pergamon Press, 1977 (superceded by Ziegler, 1985).

[Ba56] Barkas, W.H., Birnbaum, W., and Smith, F.M., Mass-ratio method applied to the measurement of L-meson masses and the energy balance in pion dicay, Phys. Rev. 101: 778, 1956.

[Ba63] Barkas, W.H., Dyer, N.J., and Heckmann, H.H., Resolution of the $\Sigma^{-}$mass anomaly, Phys. Rev. Lett. 11: 26, 1963.

[Be30] Bethe, H.A., Ann. Physik. 5: 325, 1930.

[Be32] Bethe, H.A., Z. Phys. 76: 293, 1932.

[Be53] Bethe, H., and Ashkin, J., "1. The passage of heavy particles through matter," in Segre, E., Ed., Experimental Nuclear Physics, John Wiley, 1953.

[Be82] Berger, M.J. and Seltzer, S.M., Stopping powers and ranges of electrons and positrons, National Bureau of Standards (NIST) Report NBSIR 82-2550-A, December 1982.

[Bi67] Bichsel, H. and Tschalaer, C., A range-energy table for heavy particles in silicon, Nucl. Data Tables A 3:343, 1967.

[Bi72] Bichsel, H., Passage of charged particles through matter, Section 8d, American Institute of Physics Handbook, McGraw Hill, 1972. 
[Bi88] Bichsel, H., Straggling in thin silicon detectors, Rev. Mod. Phys. 60(3): 663-699, 1988.

[Bi04] Bichsel, H., Groom, D.E., and Klein, S.R., Passage of particles through matter, Section 27, Review of particle physics, Phys. Lett. B 592 (1-4): 1-1109, July 15, 2004.

[B133] Bloch, F., Ann. Physik 16: 285, 1933; Z. Phys. 81: 363, 1933.

[Bo48] Bohr, N., The penetration of atomic particles through matter, Det $\mathrm{Kgl}$. Danske Vid. Sels. Mat-fys. Med. XVIII, 8, 1948.

[Bu86] Burke, E.A., Energy dependence of proton-induced displacement damage in silicon, IEEE Trans. Nucl. Sci. NS-33(6): 1276-1281, December 1986.

[Ev55] Evans, R.D., The Atomic Nucleus, Chapter 22, McGraw Hill, 1955.

[Fa56] Fano, U., Differential inelastic scattering of relativistic charged particles, Phys. Rev. 102: 385; Atomic theory of electromagnetic interaction in dense materials, 103: 1202, 1956.

[Fa63] Fano, U., Penetration of protons, alpha particles, and mesons, Ann. Rev. Nucl. Sci. 13: 1-66, 1963 (reproduced as Appendix A in [Na64]).

[Ge82] Geissel, H., Laichter, Y., Schmeider, W.F.W., and Armbruster, P., Energy loss and energy loss straggling of fast heavy ions in matter, Nucl. Instr. Meth. 194: 21, 1982.

[Go73] Goland, A.N., Ed., Interaction of energetic charged particles with solids: a summer school in memory of Cavid Erginsoy, held at Robert College, Istanbul, Turkey, August 16-28, 1971. Brookhaven National Laboratory, Upton, N.Y., 1973.

[Gr01] Groom, D.E., Mokhov, N.V., and Striganov, S.I., Muon stopping power and range tables, $10 \mathrm{MeV}-100 \mathrm{TeV}$, At. Data Nucl. Data Tables 78(2): 183-356, July 1, 2001.

[Ho02] Holmes-Siedle, A. and Adams, L., Handbook of Radiation Effects, 2nd edn., Oxford University Press, 2002.

[Hu90] Hubert, F., Bimbot, R., and Gauvin, H., Range and stopping power tables for 2.5-500 MeV/nucleon heavy ions in solids, At. Data Nucl. Data Tables 46: 1, 1990.

[ICRU93] ICRU, Stopping powers and ranges for protons and alpha particles, International Commission on Radiation Units and Measurements, Report 49, May 1993.

[Ja75] Jackson, J.D., Classical Electrodynamics, 2nd edn., John Wiley, 1975.

[Ja82a] Janni, J.F., Proton range-energy tables, $1 \mathrm{keV}-10 \mathrm{GeV}$, energy loss, range, path length, time-of-flight, straggling, multiple scattering, and nuclear interaction probability. Part I. For 63 compounds, At. Data Nucl. Data Tables 27: 147-340, 1982.

[Ja82b] Janni, J.F., Proton range-energy tables, $1 \mathrm{keV}-10 \mathrm{GeV}$, energy loss, range, path length, time-of-flight, straggling, multiple scattering, and nuclear interaction probability. Part II. For 92 elements, At. Data Nucl. Data Tables 27: 341-529, 1982.

[Ka93] Kaneko, T., Partial and total electronic stopping cross sections of atoms and solids for protons, At. Data Nucl. Data Tables 53(2): 271-340, March 1, 1993. 
[Ku81] Kumakhov, M.A. and Komarov, F.F., Energy Loss and Ion Ranges in Solids, English edn., translated from Russian by Ben Teague, Gordon and Breach, N.Y, 1981.

[La78] Land, D. and Brennan, J., Electronic stopping power of low-velocity ions, ions: C-Xe, targets: H-Xe, At. Data Nucl. Data Tables 22: 235, 1978.

[Li63] Lindhard, J., Scharff, M., and Schiøtt, H.E., Range concepts and heavy ion ranges, Mat. Fys. Medd. Dan. Vid. Selsk. 33: 14, 1963.

[Me92] Messenger, G.C. and Ash, M.S., The Effects of Radiation on Electronic Systems, 2nd edn., Van-Nostrand Reinhold, New York, 1992.

[Na64] National Academy of Sciences-National Research Council, Studies in Penetration of Charged Particles in Matter, Publication 1133. Subcommittee on penetration of charged particles, Report 39. Fano, U., Subcommittee Chairman, 1964.

[NIST] NIST, http://physics.nist.gov/PhysRefData/.

[No60] Northcliffe, L.C., Energy loss and effective charge of heavy ions in aluminium, Phys. Rev. 59: 120 and 1744, 1960.

[No63] Northcliffe, L.C., Passage of heavy ions through matter, Ann. Rev. Nucl. Sci. 13: 67-102, 1963 (reproduced as Appendix B in [Na64]).

[No70] Northcliffe, L.C. and Schilling, R.F., Range and stopping power tables for heavy ions, Nucl. Data Tables A7: 233-463, 1970.

[Od84] Oddershede, J. and Sabin, J.R., Orbital and whole-atom proton stopping power and shell corrections for atoms with $Z \leq 36$, At. Data Nucl. Data Tables 31: 275, 1984.

[Pa03a] Paul, H. and Schinner, A., Empirical stopping power tables for ions from 3Li to $18 \mathrm{Ar}$ and from 0.001 to $1000 \mathrm{MeV} /$ nucleon in solids and gases, At. Data Nucl. Data Tables 85: 377-452, 2003.

[Pa03b] Paul, H. and Schinner, A., Judging the reliability of stopping power tables and programs for heavy ions, Nucl. Instrum. Methods Phys. Res. B 209: 252-258, 2003.

[Ro52] Rossi, B., High-Energy Particles, Prentice-Hall, 1952.

[Sc63] Scott, W.T., Rev. Mod. Phys. 35: 231, 1963.

[Se01] Seltzer, S.M. et al., Response to the commentary by J.F. Ziegler, Radiat. Res. 155, 378-381, 2001.

[Si75] Sigmund, P., "Energy loss of charged particles in solids," in Radiation Damage Processes in Materials, Dupuy, C.H., Ed., Noordhoff International Publishers, Leyden, 1975, pp. 3-117.

[SR03] SRIM, The Stopping and Range of Ions in Matter, http://www.SRIM.org/. Developed by J.F. Ziegler and coworkers.

[St82] Sternheimer, R.M., Seltzer, S.M., and Berger, M.J., Density effect for the ionization loss of charged particles, Phys. Rev. B 26: 6067, 1982.

[St84] Sternheimer, R., Berger, M., and Seltzer, S., Density effect for the ionization loss of charged particles in various substances, At. Data Nucl. Data Tables 30: 261, 1984.

[Su95] Summers, G.P., Burke, E.A., and Xapsos, M.A., Displacement damage analogs to ionizing radiation effects, Radiat. Meas. 24(1): 1-8, 1995.

[Ue54] Uehling, E.A., Penetration of heavy charged particles in matter, Ann. Rev. Nucl. Sci. 4: 315, 1954. 
[Zi85] Ziegler, J.F., Biersack, J.P., and Littmark, U., The stopping and range of ions in solids, Vol. 1, The Stopping and Ranges of Ions in Matter, Pergamon Press, 1985.

[Zi99a] Ziegler, J.F., The stopping of energetic light ions in elemental matter, J. Appl. Phys./Rev. Appl. Phys. 85: 1249-1272, 1999.

[Zi99b] Ziegler, J.F., Radiat. Res. 152, 219-222, 1999. 


\section{$5 \begin{aligned} & \text { Selected Topics on } \\ & \text { Neutron Interactions }\end{aligned}$}

DOI: $10.1201 / 9781420012378-5$

\subsection{INTRODUCTION}

First discovered by Chadwick in 1932 [Ch32], the neutron and its interaction with matter have ever since been the subject of intense study. Chadwick first produced neutrons by bombarding $\mathrm{Be}^{9}$ nuclei with alpha particles from Po decay, in the reaction $\alpha+\mathrm{Be}^{9} \longrightarrow \mathrm{C}^{12}+n$. The neutron, of energy several $\mathrm{MeV}$, was sent through metallic foils and elastically scattered off the metal nuclei. The recoiling metal ions entered an ionization chamber where they were identified. The $\operatorname{Be}^{9}(\alpha, n) \mathrm{C}^{12}$ reaction is still used as a neutron source today.

Much of physics in the 1930s was devoted to working out the behavior of neutrons in interaction with nuclei and in their transport through matter. Nuclear physics came into its heyday, and neutron transport and diffusion theory were worked out [Fe50, Am59, Am84].

Once the ability of a neutron to fission a heavy nucleus releasing more neutrons was discovered (1938 to 1939), it was immediately realized that a chain reaction was possible, releasing huge amounts of energy. Only a few years later, under the pressure of World War II, the process was made to have astounding consequences, culminating in August 1945, ending the war.

In the 1950s, the fission process dominated nuclear technology, and the design of nuclear power plants began [We58, Be70]; nuclear-powered submarines became a reality; and an industry flourished to protect personnel, structures, and military systems against nuclear weapons.

All these applications rely on neutron interactions with nuclei and the transport of neutrons through material.

The behavior of neutrons passing through matter is sharply distinguished from that of all other projectiles (photons, electrons, and ions) in that the latter interact primarily with atomic electrons in the target material, with their relatively small nuclear interaction a small correction. On the other hand, neutrons interact solely with atomic nuclei. Because they are neutral, they do not interact electromagnetically with electrons (or with nuclei).* They readily penetrate the atomic electron cloud and reach the nucleus.

\footnotetext{
*The neutron magnetic moment allows it to interact electromagnetically with the magnetic moment of electrons or magnetized atoms. Byrne [By94] discusses this weak interaction.
} 
Left to its own devices in vacuum, the neutron mean life $\tau$ and half-life $t_{1 / 2}$ are

$$
\begin{aligned}
\tau & =885.7 \mathrm{sec} \\
t_{1 / 2} & =613.9 \mathrm{sec}
\end{aligned}
$$

against the decay $n \rightarrow p+e+\bar{v}_{e}$ [PDG04]. However, a neutron is absorbed in most gases (e.g., air) in a fraction of a second and in many solids in milliseconds or less.

There is an enormous variety of possible interactions between a neutron and most nuclei, depending on neutron energy. The interactions differ from one isotope to another of the same element. Therefore, in this chapter we present only selected topics on neutron behavior in matter. A full treatment would require volumes.

Foremost among the interactions is simple elastic scattering, in which the neutron elastically bounces off the nucleus, and the nucleus recoils but remains in its same internal state (usually the ground state). The recoiling target carries off some energy, and the process reduces the neutron energy. Except at energies $\gtrsim 1 \mathrm{MeV}$, the neutron wavelength

$$
\lambda=\frac{h}{p}=\sqrt{\frac{1 \mathrm{MeV}}{E}} \cdot 28.6 \mathrm{fm}
$$

is large compared with nuclear radii, and so elastic scattering is mostly $\mathrm{S}$ wave and isotropic in the CM system $\left(1 \mathrm{fm}=10^{-13} \mathrm{~cm} \equiv 1\right.$ Fermi). Then, after elastic scattering from a target of atomic weight $A$, the minimum energy $E_{\min }$ (backscattering) and average laboratory energy $\bar{E}$ of a neutron with incident energy $E$ are

$$
\begin{aligned}
E_{\min } & =\left(\frac{A-1}{A+1}\right)^{2} E \\
\bar{E} & =\frac{A^{2}+1}{(A+1)^{2}} E
\end{aligned}
$$

The neutron has lost an average energy

$$
\bar{E}_{\text {lost }}=\frac{2 A}{(A+1)^{2}} E
$$

which is carried off in the kinetic energy of the recoiling nucleus.

On light nuclei, elastic scattering is usually the most probable process for incident energies below several hundred $\mathrm{keV}$. By repeated elastic 
scatterings while passing through matter, the neutron is reduced in energy (moderated) until it reaches the thermal energy of its surroundings. A moderated neutron spectrum results, extending from the original energy down to $0.01 \mathrm{eV}$ or less.

Many nuclei have internal excited states starting at a few eV up to $\mathrm{MeV}$. Therefore, at incident neutron energies sufficient to induce excitation, usually above a few $\mathrm{keV}$, it becomes increasingly likely that the neutron will scatter inelastically, in which the recoiling nucleus has been put in an excited state.

A third, common process is for a neutron to be captured by the nucleus, into an excited state of the compound nucleus. That excited state then decays, usually by emission of one or more gammas and usually in less than nanoseconds. In that case, one speaks of radiative capture. The cross section for this process is often written as $\sigma_{\text {cap }}$ or $(n, \gamma)$ or $\sigma_{\gamma}$. The first notation is not always clear because nonradiative capture may also occur [e.g., $\mathrm{N}^{14}(n, p) \mathrm{C}^{14}$ ]. The second notation runs the risk of getting confused with the special case of radiative capture in which only one gamma exits $(n, 1 \gamma) . \sigma_{\gamma}$ is perhaps the safest notation

$$
\sigma_{\gamma}=\sigma(n, 1 \gamma)+\sigma(n, 2 \gamma)+\sigma(n, 3 \gamma)+\cdots
$$

because it is the cross section for capture with only gammas in the final state. At higher energies, usually above tens of $\mathrm{keV}$, other massive particles may exit in reactions such as $(n, 2 n),(n, \alpha),(n, n p)$, and so on.

In addition to neutron-induced reactions, neutrons are spontaneously emitted by some isotopes, usually by spontaneous fission. Half-lives for spontaneous fission in most heavy elements are billions of years; an exception is the very useful isotope $\mathrm{Cf}^{252}$, with a half-life against spontaneous fission of only 85 years (half-life against $\alpha$ decay is 2.72 years, for a total half-life of 2.64 years). An exposition of gamma ray and neutron emissions and detection from radioactive elements may be found in the Passive Nondestructive Assay of Nuclear Materials manual available from Los Alamos National Laboratory [LA91].

\subsection{NEUTRON ENERGY AND REACTION NOMENCLATURE}

Over the years, a nomenclature has evolved to specify ranges of neutron energies and types of reactions.

\subsubsection{Neutron Energy Ranges}

The energy range designations are not precise. Their names are more suggestive of the consequences of reactions of neutrons in that energy range, rather than that of the range itself. A fairly standard naming system is 


$\begin{array}{ll}\text { Energy Range } & \text { Name } \\ 0-0.025 \mathrm{eV} & \text { Cold } \\ 0.025 \mathrm{eV} & \text { Thermal } \\ 0.025-0.4 \mathrm{eV} & \text { Epithermal } \\ 0.4-0.6 \mathrm{eV} & \text { Cadmium } \\ 0.6-1.0 \mathrm{eV} & \text { Epicadmium } \\ 1-10 \mathrm{eV} & \text { Slow } \\ 10-300 \mathrm{eV} & \text { Resonance } \\ 300 \mathrm{eV}-1 \mathrm{MeV} & \text { Intermediate } \\ 1-20 \mathrm{MeV} & \text { Fast } \\ >20 \mathrm{MeV} & \text { Relativistic }\end{array}$

Neutrons in thermal equilibrium with their surroundings at temperature $T$ acquire a Maxwell-Boltzmann energy distribution with mean energy $k T$ (0.025 eV at room temperature). Thus, neutrons of this energy are called thermal neutrons, even though such thermal neutrons have a distribution of energies. Neutrons with energies less than thermal are referred to as cold neutrons. Cold neutrons can be produced in large numbers from reactor neutrons (fission spectrum) by passing them down a pipe cooled by liquid nitrogen or hydrogen or helium until they come nearly to thermal equilibrium at the reduced temperature.

The next energy range, epithermal, is simply more energetic than thermal, but the upper energy to which the name applies $(0.4 \mathrm{eV})$ is defined by the neutron capture reaction in $\mathrm{Cd}^{113}$. The neutron absorption cross section of that isotope is very large below about $0.5 \mathrm{eV}$ and drops rapidly as energy increases; $0.5 \mathrm{eV}$ is commonly referred to as the cadmium cutoff. The $\mathrm{Cd}^{113}$ absorption cross section peaks at $0.17 \mathrm{eV}$ at about 55,000 barn. Its value at $0.5 \mathrm{eV}$ is $1150 \mathrm{barn}$, about midway between that of $\mathrm{B}^{10}$ and $\mathrm{He}^{3}$. Cadmium, rather than some other element or isotope, is chosen because it is widely used as a selective absorber due to its large low-energy cross section and relative absence of resonances (until $18 \mathrm{eV}$ ), and rapid drop off, preventing the absorption of more energetic neutrons. Cadmium is therefore very useful as a separator of low-energy neutrons $(<0.5 \mathrm{eV})$ and higherenergy neutrons. The energy range from 0.4 to $0.6 \mathrm{eV}$ is called the cadmium range. Figure 5.2a in Section 5.3 shows the $\mathrm{Cd}^{113}$ neutron absorption cross section as a function of energy along with the cross sections of other good absorbers.

Energies up to $1.0 \mathrm{eV}$ are referred to as epicadmium. At $1 \mathrm{eV}$ the $\mathrm{Cd}^{113}$ absorption cross section is 120 barn, having dropped one order of magnitude, whereas the neutron energy increased only from 0.5 to $1 \mathrm{eV}$.

Neutrons of energy 1 to $10 \mathrm{eV}$ are loosely called slow neutrons. Usually, but not always, nuclear resonance reactions begin above $10 \mathrm{eV}$ incident 
energy, so that little of interest occurs in the cross section as a function of energy for slow neutrons. There are many exceptions in which there are resonances below $10 \mathrm{eV}$.

From about 10 to $300 \mathrm{eV}$, most nuclei exhibit copious resonances, often spaced by only $1 \mathrm{eV}$ or less. For this reason, neutrons in that energy range are called resonance neutrons. Actually, nuclear resonances may occur up to many $\mathrm{keV}$, so the upper energy of resonance neutrons is not well defined. Not uncommonly, nuclear resonances from a few $\mathrm{eV}$ to a few $\mathrm{keV}$ are separated by an amount greater than their widths, so they can be resolved. Above a few $\mathrm{keV}$ their separation is often less, so they cannot be resolved; or, even if they are actually separated, experiments may not be able to resolve them. The energy span from the onset of resonances (generally a few eV) to the limit of resolution is called the resolved resonance region. The span above that, where resonances at least experimentally are too closely spaced, up to where resonances actually do overlap so that only an averaged smooth cross section can be determined, is called the unresolved resonance region. Examples may be seen in Figure 5.4 in Section 5.4.3 in which the neutron capture cross sections are graphed in isotopes of gadolinium. Resonances are resolved for energies of a few eV up to about $10 \mathrm{keV}$, and the cross sections are plotted as detailed, wildly oscillating functions of energy. Above $10 \mathrm{keV}$ resonances cannot be resolved, and only the smooth, average cross section is tabulated in the ENDF compilation and in the figure.

Nuclear reactions in which other particles emerge, such as $(n, p),(n, \alpha)$, $(n, p d)$, and so on, usually have a threshold at energies on the order of $1 \mathrm{MeV}$. At incident neutron energies above the resonance region, but below particle production, about $300 \mathrm{eV}$ to $1 \mathrm{MeV}$, neutrons are referred to as of intermediate energies.

The region above $1 \mathrm{MeV}$ is distinguished both because it is the interesting energy for particle production reactions to begin, and also because the neutrons produced in nuclear reactions tend to have $\mathrm{MeV}$ energies. The $\operatorname{Be}^{9}(\alpha, n) \mathrm{C}^{12}$ reaction is an example. The mass energy released in this reaction when the alpha particle has no kinetic energy (the $Q$ value) is $5.7 \mathrm{MeV}$. If the alpha particle is supplied by Po decay, as was the case in Chadwick's experiment, it has an energy about $5.3 \mathrm{MeV}$, which, when added to the Beryllium reaction $Q$ value, provides a total of about $11 \mathrm{MeV}$. Then depending on the direction of the exiting neutron relative to that of the incident alpha particle, the neutron has an energy between 0 and $11 \mathrm{MeV}$.

Neutrons from about 1 to $20 \mathrm{MeV}$ are called fast neutrons, the energy range usually found in neutrons from nuclear reactions. Above $20 \mathrm{MeV}$ they are referred to as relativistic. A $20 \mathrm{MeV}$ neutron has a velocity $20 \%$ that of light. 


\subsubsection{Neutron Reaction Nomenclature}

The myriad reactions that can occur between a neutron and target nucleus allow ample room for different categorization schemes in naming them. The following is common [Ma60].

Total Cross Section

\begin{tabular}{lllll}
\hline Elastic & \multicolumn{3}{c}{ Non-elastic } \\
\cline { 2 - 5 }$(n, n) \quad$ Inelastic & Fission & Neutron-Producing & Neutron-Disappearance \\
& $\left(n, n^{\prime}\right)$ & $(n$, fis $)$ & $(n, 2 n)\left[\right.$ e.g., $\left.\mathrm{O}^{16} \rightarrow \mathrm{O}^{15}\right]$ & Radiative Capture \\
& & & $\left(n, n^{\prime} p\right)\left[\mathrm{O}^{16} \rightarrow \mathrm{N}^{15}\right]$ & $\sigma_{\gamma},(n, 1 \gamma),(n, 2 \gamma), \ldots$ \\
& & $\left(n, n^{\prime} \alpha\right)\left[\mathrm{C}^{12} \rightarrow \mathrm{Be}^{8}\right]$ & Charged-Particle Producing \\
& & & $\left(n, n^{\prime} p \alpha\right)$ & $(n, p),(n, \alpha), \ldots \ldots \ldots$ \\
& & & \\
& & &
\end{tabular}

All processes are first divided into Elastic, and everything else, called NonElastic. The latter is divided into four main categories, one of which is the inelastic scattering mentioned above.

Alternate naming schemes exist. In the former Soviet Union, the NonElastic processes listed above have been referred to as Inelastic Interactions, whereas the Inelastic subset of them listed above has been referred to as Inelastic Scattering [Go76, Gr97].

There is the question of the time scale for the exiting particles to be created. For example, in radiative capture in nitrogen, $\mathrm{N}^{14}(n, \gamma) \mathrm{N}^{15}$, it is observed that about 2 gammas are produced per neutron capture, with a wide distribution of gamma-ray energies. It is therefore not clear whether this should be considered an $(n, 1 \gamma)$ or an $(n, 2 \gamma)$ reaction. In fact, the neutron is captured in an excited state of $\mathrm{N}^{15}$, which deexcites by decaying through a chain of lower levels to the ground state. Each transition emits a gamma. Thus, some neutron captures result in 1 gamma ray $(10.829 \mathrm{MeV})$, whereas some result in 2,3, or 4 or more gammas of lower energy. The lifetime of the intermediate excited states is of the order of picoseconds, and so all gammas exit immediately. In such a case, nuclear reaction databases usually tabulate the radiative capture cross section, and then specify probabilities for the emission of the various gammas. In other cases, it may happen that the recoiling, excited nucleus is in a longer-lived state, with a lifetime of perhaps nanoseconds or microseconds or longer. Then the particular application dictates whether one considers the reaction $(n, 1 \gamma)$, or $(n, 2 \gamma), \ldots$ An excited state with a lifetime against gamma decay longer than about $1 \mathrm{msec}$ is called an isomeric state or an isomer. Isomers exist with lifetimes from milliseconds to months.

Reduced cross sections are of interest. One defines the radiative capture gamma production cross section

$$
\sigma_{\text {prod }}^{\mathrm{rad}}(\gamma)=\sigma(n, 1 \gamma)+2 \sigma(n, 2 \gamma)+3 \sigma(n, 3 \gamma)+\cdots
$$

to be the cross section for producing a gamma when only photons exit. A neutron or particle production cross section is defined similarly. The photon 
yield, or the gamma yield, or the gamma multiplicity $\eta^{\mathrm{rad}}$ is the mean number of gammas produced on neutron capture (when no particles other than gammas are produced),

$$
\eta^{\mathrm{rad}}=\frac{\sigma_{\mathrm{prod}}^{\mathrm{rad}}(\gamma)}{\sigma_{\gamma}}
$$

where $\sigma_{\gamma}$ is the radiative capture cross section from Equation 5.4.

When the yield or multiplicity is specified for purely radiative processes, a gamma production cross section is defined for processes in which a gamma is produced along with massive particles (a probable process at higher energies):

$$
\sigma_{\text {prod }}^{\mathrm{m}}(\gamma)=\sigma\left(n, n^{\prime} \gamma\right)+2 \sigma\left(n, n^{\prime} 2 \gamma\right)+\sigma(n, p \gamma)+\sigma(n, \alpha \gamma)+\cdots
$$

The total gamma production cross section is the sum

$$
\sigma_{\text {prod }}(\gamma)=\sigma_{\text {prod }}^{\mathrm{rad}}(\gamma)+\sigma_{\text {prod }}^{\mathrm{m}}(\gamma)
$$

Any of the three gamma production cross sections may exceed the total cross section; not uncommonly, $\sigma_{\text {prod }}^{\mathrm{m}}$ does in narrow energy bands.

Energetic neutrons on light nuclei may cause the target nucleus to fragment (i.e., fission), with no neutron in the final state. In this case, fission is a neutron-absorbing process. Fission on heavy nuclei (e.g., U) usually results in more neutrons being produced. In this case, fission is both a neutron-absorbing and a neutron-producing reaction. A neutron disappearance cross section is defined as the sum of all processes in which no neutron exits:

$$
\sigma(\text { disappear })=\sigma_{\gamma}+\sigma(n, \text { charged particle })+\cdots
$$

A neutron absorption cross section is sometimes defined as either equal to $\sigma$ (disappear) or, when fission is considered a neutron-absorbing process, as

$$
\sigma(\mathrm{abs})=\sigma(\text { disappear })+\sigma(n, \text { fis })
$$

Some of these cross sections are illustrated by neutron interactions on $\mathrm{N}^{14}$, shown in Figure 5.1. The two absorption reactions $\sigma_{\gamma}$ and $(n, p)$ are exothermic and obey the $1 / v$ law up to about $100 \mathrm{keV}$. The cross section is proportional to the time the neutron spends near the nucleus and so is proportional to the inverse neutron velocity. At all energies, elastic scattering is the most probable process.

The ENDF library tabulates the inelastic cross sections for exciting the first 40 individual excited states of the recoiling nucleus and their sum $\left(n, n^{\prime}\right)$, the total inelastic cross section. The first two, $\left(n, n_{1}^{\prime}\right)$ and $\left(n, n_{2}^{\prime}\right)$, are shown in the figure for $\mathrm{N}^{14}$, along with the sum total $\left(n, n^{\prime}\right)$ for all levels. 

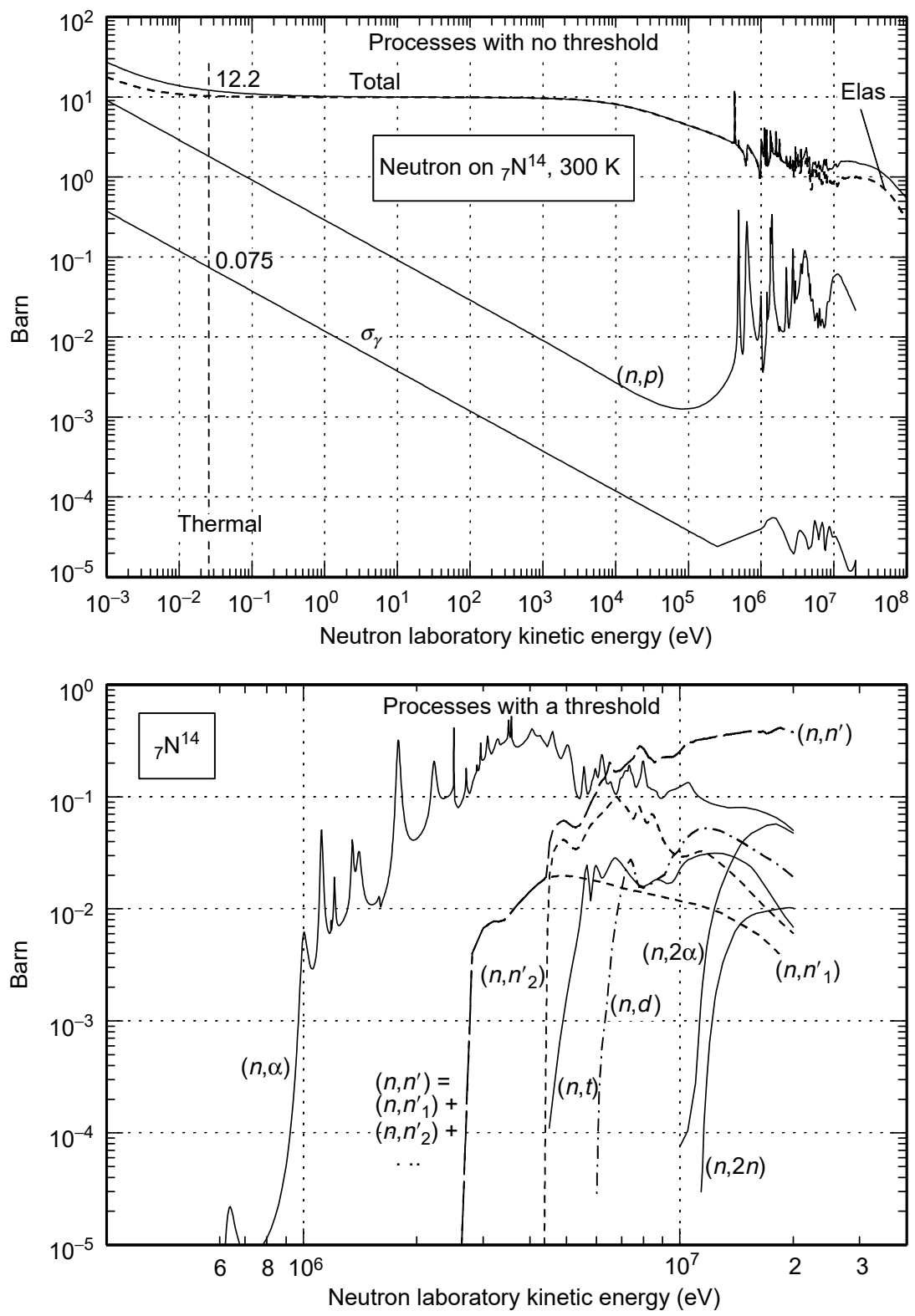

FIGURE 5.1 Principal neutron- $\mathrm{N}^{14}$ cross sections at $300 \mathrm{~K} . \sigma_{\gamma}$ is radiative capture; the gamma production cross section is larger. Other than $\sigma_{\gamma}$ or $(n, p)$ the lowest nonelastic process is $(n, \alpha)$, with a threshold of about $0.6 \mathrm{MeV}$; it is the major nonelastic process between 2 and $6 \mathrm{MeV}$. $(n, p)$ dominates below $2 \mathrm{MeV}$, whereas inelastic scattering dominates above $6 \mathrm{MeV}$. (Data from Point2004 (ENDF/B-6).) 
For a projectile of mass $m$ and laboratory kinetic energy $E_{\text {lab }}$ incident on a target of mass $M$, the center of mass energy available is (nonrelativistically)

$$
E_{\mathrm{CM}}=\frac{M}{m+M} E_{\mathrm{lab}}
$$

The first excited state of $\mathrm{N}^{14}$ is at $E_{1}=2.313 \mathrm{MeV}$ [Aj86]. To excite this state, the incident neutron must have, inverting Equation 5.10, a kinetic energy $E_{\text {lab }}=((m+M) / M) E_{1}=2.479 \mathrm{MeV}$; this is the threshold for inelastic scattering that excites the first state of the target, i.e., the reaction $\left(n, n^{\prime}{ }_{1}\right)$. The next two excited states of $\mathrm{N}^{14}$ are $E_{2}=3.958 \mathrm{MeV}$ and $E_{3}=4.915 \mathrm{MeV}$. These correspond to the next two inelastic processes, $\left(n, n^{\prime}{ }_{2}\right)$ and $\left(n, n^{\prime}{ }_{3}\right)$, with thresholds of 4.243 and $5.269 \mathrm{MeV}$, respectively.

For $\mathrm{N}^{14}$ the particle production cross section with the lowest threshold is $(n, \alpha)$. The actual threshold of the reaction is about $0.3 \mathrm{MeV}$, but the cross section is less than $10^{-5}$ barn below $0.62 \mathrm{MeV}$.

\subsection{GOOD NEUTRON ABSORBERS}

Unwanted neutrons, for example, those escaping from a nuclear reactor, can be dangerous to humans or destructive to electronic equipment if in sufficient numbers. By the time they pose such a threat they have usually been scattered many times from the surrounding material, and their spectrum is that of a moderated neutron gas, with a spectral peak at thermal energies and a characteristic $1 / E$ drop off up to $\mathrm{MeV}$ energies. Figure 1.5 is an example spectrum, but graphed only up to $1 \mathrm{keV}$. These neutrons can be removed by passing them through a material that has a high neutron absorption cross section at low energies.

Those neutrons that have not been sufficiently moderated before posing a hazard can be moderated to make them easier to absorb. Low- $Z$ material, notably polyethylene $\left(\mathrm{CH}_{2}\right)$, or water, are excellent moderators, as is low- $Z$ biological matter such as a human being, or moist soil and moist concrete.

Most nuclei absorb low-energy neutrons. $\mathrm{Cd}^{113}, \mathrm{He}^{3}, \mathrm{~B}^{10}$, and $\mathrm{Gd}^{157}$ are favored isotopes because of their especially large thermal absorption cross sections, but there are others. $\mathrm{He}^{3}, \mathrm{~B}^{10}$, and $\mathrm{Li}^{6}$ are employed in neutron detectors because the output of the capture reaction is one or more charged particles, which are then relatively easy to detect by conventional means. The capture in most other nuclei produces gammas, which are more difficult to detect.

The neutron absorption cross sections for a number of highly absorbing stable isotopes are shown in Figure 5.2a and Figure 5.2b. The isotopic abundance in the naturally occurring element, reaction products, energy released ( $Q$ value), and thermal cross section at $300 \mathrm{~K}$ are shown in Table 5.1. For comparison, the thermal neutron absorption cross section of $\mathrm{Xe}^{135}\left(t_{1 / 2}=9.1 \mathrm{~h}\right)$ is $2.65 \times 10^{6}$ barn, the largest known value of any isotope. 
(a) $\quad \square \mathrm{Xe}^{135}\left(t_{1 / 2}=9.1 \mathrm{~h}\right)$

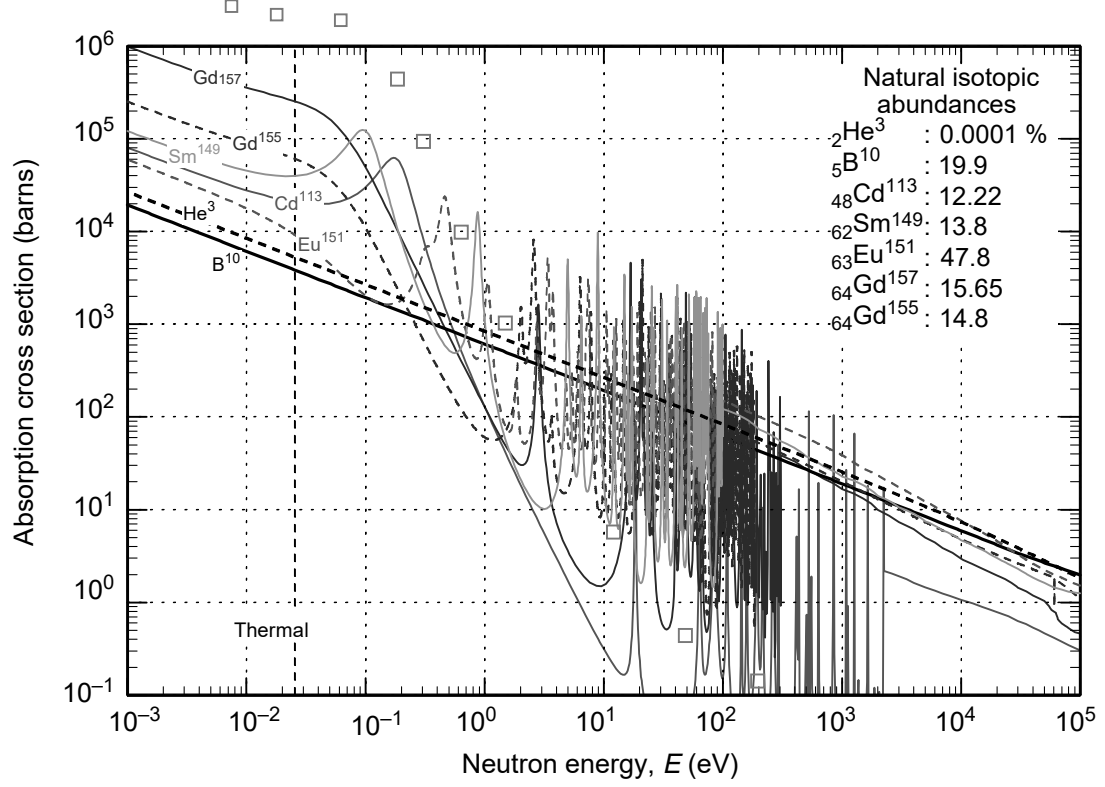

(b)

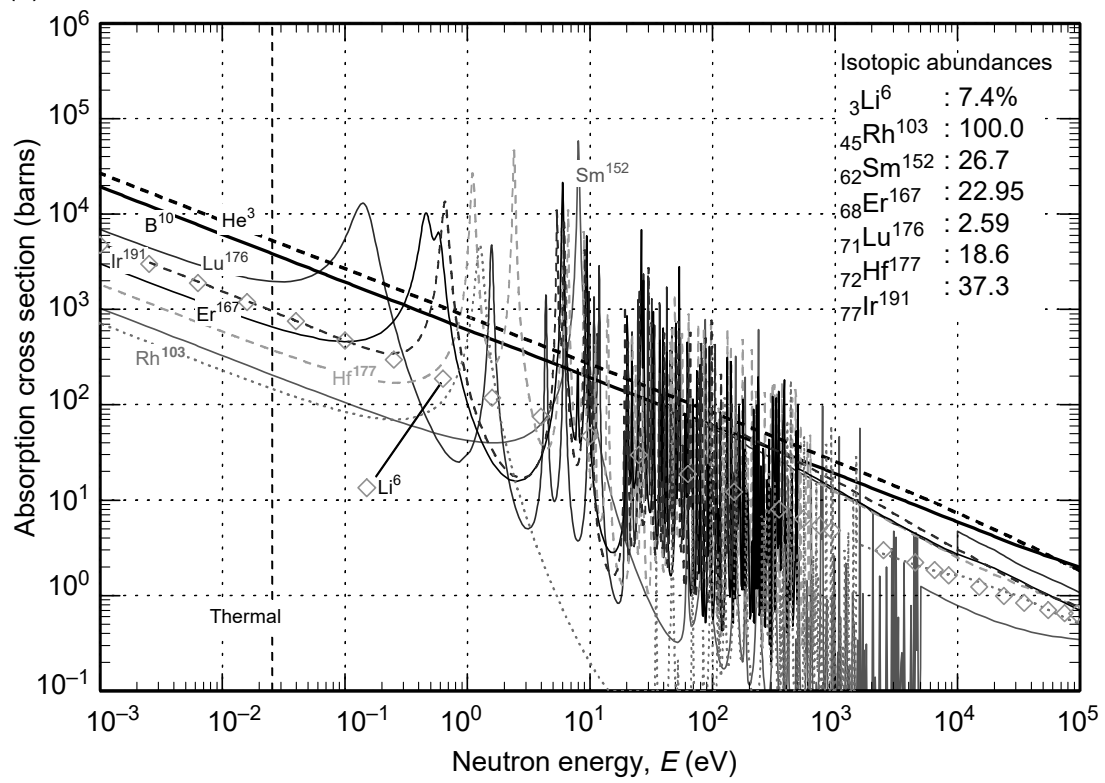

FIGURE 5.2 (See color insert following page 332.) Cross sections at $300 \mathrm{~K}$ of good absorbers of low-energy neutrons. (a) Stable isotopes with thermal cross section greater than that of $\mathrm{B}^{10}$. All reactions are $(n, \gamma)$ or $(n, c e)$ except $\mathrm{He}^{3}(n, p)$ and $\mathrm{B}^{10}(n, \alpha) . \mathrm{Xe}^{135}$ (half-life $9.1 \mathrm{~h}$ ), with thermal cross section 2.5 Mbarn, is shown in squares for comparison. (b) Stable isotopes with thermal cross section less than that of $\mathrm{B}^{10}$. All reactions are $(n, \gamma)$ or $(n, c e)$ except $\mathrm{He}^{3}(n, p), \mathrm{B}^{10}(n, \alpha)$, and $\mathrm{Li}^{6}(n, t)$. 
TABLE 5.1

Neutron Capture Reactions in Stable Isotopes with Relatively Large Thermal Cross Section

\begin{tabular}{lcllr}
$\begin{array}{l}\text { Target } \\
\text { Isotope }\end{array}$ & $\begin{array}{c}\text { Target Isotope } \\
\text { Abundance }(\%)\end{array}$ & Reaction Product & $\boldsymbol{Q}(\mathbf{M e V})$ & $\begin{array}{r}\text { Thermal Cross } \\
\text { Section (Barn) }\end{array}$ \\
$\mathrm{Gd}^{157}$ & 15.7 & $\mathrm{Gd}^{158}+(\gamma$ or ce $)$ & 7.937 & 254,000 \\
$\mathrm{Gd}^{155}$ & 14.8 & $\mathrm{Gd}^{156}+(\gamma$ or ce $)$ & 8.536 & 60,900 \\
$\mathrm{Sm}^{149}$ & 13.9 & $\mathrm{Sm}^{150}+(\gamma$ or ce $)$ & 7.985 & 40,140 \\
$\mathrm{Cd}^{113}$ & 12.22 & $\mathrm{Cd}^{114}+(\gamma$ or ce $)$ & 9.041 & 20,600 \\
$\mathrm{Eu}^{151}$ & 47.86 & $\mathrm{Eu}^{152}+(\gamma$ or ce $)$ & 6.305 & 9,200 \\
$\mathrm{He}^{3}$ & $1.38 \times 10^{-4}$ & $p+t$ & 0.7637 & 5,333 \\
$\mathrm{~B}^{10}$ & 19.9 & $\mathrm{Li}^{7}+\alpha$ & 2.79 & 3,838 \\
$\mathrm{Lu}^{176}$ & 2.59 & $\mathrm{Lu}^{177}+(\gamma$ or ce $)$ & 7.073 & 2,090 \\
$\mathrm{Ir}^{191}$ & 37.3 & $\mathrm{Ir}^{192}+(\gamma$ or ce $)$ & 6.199 & 954 \\
$\mathrm{Li}^{6}$ & 7.5 & $\alpha+t$ & 4.784 & 941 \\
$\mathrm{Er}^{167}$ & 22.9 & $\mathrm{Er}^{168}+(\gamma$ or ce $)$ & 7.771 & 659 \\
$\mathrm{Hf}^{177}$ & 18.56 & $\mathrm{Hr}^{178}+(\gamma$ or ce $)$ & 7.626 & 373 \\
$\mathrm{Sm}^{152}$ & 26.6 & $\mathrm{Sm}^{153}+(\gamma$ or ce $)$ & 5.868 & 206 \\
$\mathrm{Rh}^{103}$ & 100.0 & $\mathrm{Rh}^{104}+(\gamma$ or ce $)$ & 7.000 & 145 \\
\hline
\end{tabular}

Data in Table 5.1 are taken from the two-volume compilation by the National Nuclear Data Center at Brookhaven National Laboratory [Mu81, Mu84, McL88]. The same cross sections are also tabulated in ENDF, with slightly different values. Cross sections at selected nonzero temperatures are in a separate library Point2004. All these ENDF-based cross sections are available at http://www3.nndc.bnl.gov/point2004/, or http://www-nds.iaea.org/ point2004/ or through http://www.llnl.gov/cullen1/.

Though unstable and so not listed, $\operatorname{Sm}^{151}\left(t_{1 / 2}=90 \mathrm{y}\right)$ has a thermal absorption cross section of 15,200 barn.

\subsubsection{Internal Conversion}

Upon neutron capture, an alternate decay mode that competes with gamma emission is internal conversion. This occurs when an atomic electron, usually from the $K$ shell, interacts with the nucleus and the nucleus de-excites by ejecting the electron from the atom. The electron kinetic energy equals the nuclear transition energy minus its binding energy. The notation (ce) is used to denote a conversion electron. Internal conversion is always possible when gamma emission is possible; it is more probable for high- $Z$ nuclei and low transition energies.

\subsection{THE CASE OF Gd $^{157}$}

Upon neutron capture in $\mathrm{Gd}^{157}$ either a gamma (or several gammas) or a conversion electron may be emitted. This isotope is a favored example because of its especially high absorption cross section. We discuss its conversion electrons here in some detail. 


\subsubsection{General Observations}

In thermal neutron capture, a neutron is captured into an excited state of the next higher isotope

$$
{ }_{z} X^{A}+n \rightarrow{ }_{z} X^{A+1^{*}}
$$

$(A+1)^{*}$ then decays by radiative transitions, emitting $\gamma \mathrm{s}$ of many energies. Sometimes, however, the nucleus decays by internal conversion (IC), emitting an electron and not a photon.

The internal conversion coefficient (ICC) of a transition is defined as the number of conversion electrons (ce) emitted per emitted gamma and so may be less than or greater than 1 :

$$
\mathrm{ICC}=(\# \text { electrons }) /(\# \text { gammas })
$$

An ICC is defined for the electron that is ejected from each atomic shell, $\mathrm{ICC}_{K}, \mathrm{ICC}_{L}$, and so on. It is a function of the nucleus $Z$, the gamma transition energy, and the gamma multipolarity.

The conversion electron fraction $f_{\text {ce }}$ is defined as the fraction of transitions that produce a conversion electron:

$$
f_{\mathrm{ce}}=(\# \text { electrons }) /(\# \text { electrons }+\# \text { gammas })=\mathrm{ICC} /(1+\mathrm{ICC})
$$

so that $0 \leq f_{\text {ce }} \leq 1$.

Thermal neutron capture gamma intensities are tabulated, for example at the National Nuclear Data Center, http://www.nndc.bnl.gov/. Commonly, tables give the relative intensity, $I_{\gamma} / I_{\gamma}(\max )$, the intensity $I_{\gamma}$ of the gamma at the chosen energy relative to the most intense gamma, $I_{\gamma}(\max )$.

ICCs can be computed directly for any transition, using the tools available at the same NNDC site. All nuclear states and transition energies are in the nuclear data sheet for $\mathrm{Gd}^{158}$ [He96].

\subsubsection{GD ${ }^{157}$}

The reaction proceeds through the compound nucleus $\mathrm{Gd}^{158^{*}}$, an excited state of $\mathrm{Gd}^{158}$, which then rapidly decays to its ground state $\mathrm{Gd}^{158 \mathrm{~g}}$ and emits a $\gamma$ (or several $\gamma \mathrm{s}$ ) or a conversion electron:

$$
n+\mathrm{Gd}^{157} \rightarrow \mathrm{Gd}^{158 *} \rightarrow \mathrm{Gd}^{158 \mathrm{~g}}+\gamma \text { or }(\mathrm{ce})
$$

In $\mathrm{Gd}^{157}$ the thermal neutron is absorbed into the $7937.1 \mathrm{keV}$ excited state of $\mathrm{Gd}^{158}$ [He96]. By decay from there, many hundreds of gamma energies are possible, from a low of $79.51 \mathrm{keV}$ to a high of $7857 \mathrm{keV}$. In the cascade, 
more than one gamma may be emitted per $n$ capture. Many of the cascading transitions populate the first excited state of $\mathrm{Gd}^{158}$ at $79.51 \mathrm{keV}$. Direct decay from the capturing state to the ground state is forbidden; no $7937.1 \mathrm{keV}$ gamma is emitted.

Atomic shell binding energies in $\mathrm{Gd}$ are:

$$
\begin{aligned}
& K=50.24 \mathrm{keV} \\
& L_{1}=8.375 \mathrm{keV} ; L_{2}=7.93 \mathrm{keV} ; L_{3}=7.24 \mathrm{keV} \\
& M=1.2 \text { to } 1.9 \mathrm{keV}
\end{aligned}
$$

The kinetic energy of a conversion electron resulting from a nuclear transition of energy $E_{\text {transition }}$ is $E_{\text {transition }}$ less one of these shell energies.

Table 5.2, constructed from the nuclear data sheets and computed ICC values, shows several of the most intense $\gamma \mathrm{s}$ and conversion electrons emitted following thermal neutron capture in $\mathrm{Gd}^{157}$. For example, in the cascade decay from the capturing state, there are two lower states separated by $181.93 \mathrm{keV}$. The lower of the two is accessible from the upper by an E2 transition. For 100 neutron captures, the transition between these two states occurs 24 times. A transition can produce only a gamma or a conversion electron. Of the 24 transitions, 18.34 transitions result in a $181.93 \mathrm{keV}$ gamma being emitted, and 5.65 transitions result in a conversion electron being

\begin{tabular}{|c|c|c|c|c|c|}
\hline $\begin{array}{l}E_{\text {transition }} \\
=E_{\gamma}(\mathrm{keV})\end{array}$ & $\begin{array}{l}\text { Transitions } \\
\text { per } 100 n \\
\text { Captures }\end{array}$ & $\begin{array}{l}\text { Gammas } \\
\text { per } 100 n \\
\text { Captures }\end{array}$ & $\begin{array}{c}\text { Gamma } \\
\text { Multipolarity }\end{array}$ & $\begin{array}{c}\text { Total Number } \\
\text { of Conversion } \\
\text { Electrons from } \\
\text { All Shells } \\
\text { per } 100 n \\
\text { Captures }\end{array}$ & $\begin{array}{c}\text { Originating Shell, } \\
\text { Number, and } \\
\text { (energies, keV) } \\
\text { of Conversion } \\
\text { Electrons per } \\
100 n \text { Captures }\end{array}$ \\
\hline 79.51 & 68 & 9.75 & E2 & 58.7 & $\begin{array}{l}K 20.0(29.3) \\
L 29.8(71) \\
M 8.9(78)\end{array}$ \\
\hline 181.93 & 24.0 & 18.34 & E2 & 5.65 & $\begin{array}{l}K 3.8(132) \\
L 1.4(174) \\
M 0.4(180)\end{array}$ \\
\hline 277.54 & 1.22 & 1.13 & E2 & 0.087 & $\begin{array}{l}K 0.066(227) \\
L 0.017(269) \\
M 0.0048(276)\end{array}$ \\
\hline 780.1 & 2.51 & 2.5 & E1 & 0.0044 & $\begin{array}{l}K 0.0039(730) \\
L 0.00052(772)\end{array}$ \\
\hline 944.1 & 8.6 & 8.55 & E1 & 0.0105 & $\begin{array}{l}K 0.0093(894) \\
L 0.0012(936)\end{array}$ \\
\hline
\end{tabular}

\section{TABLE 5.2}

\section{Most Intense Electron-Producing Transitions from $n+\mathbf{G d}^{157}$}




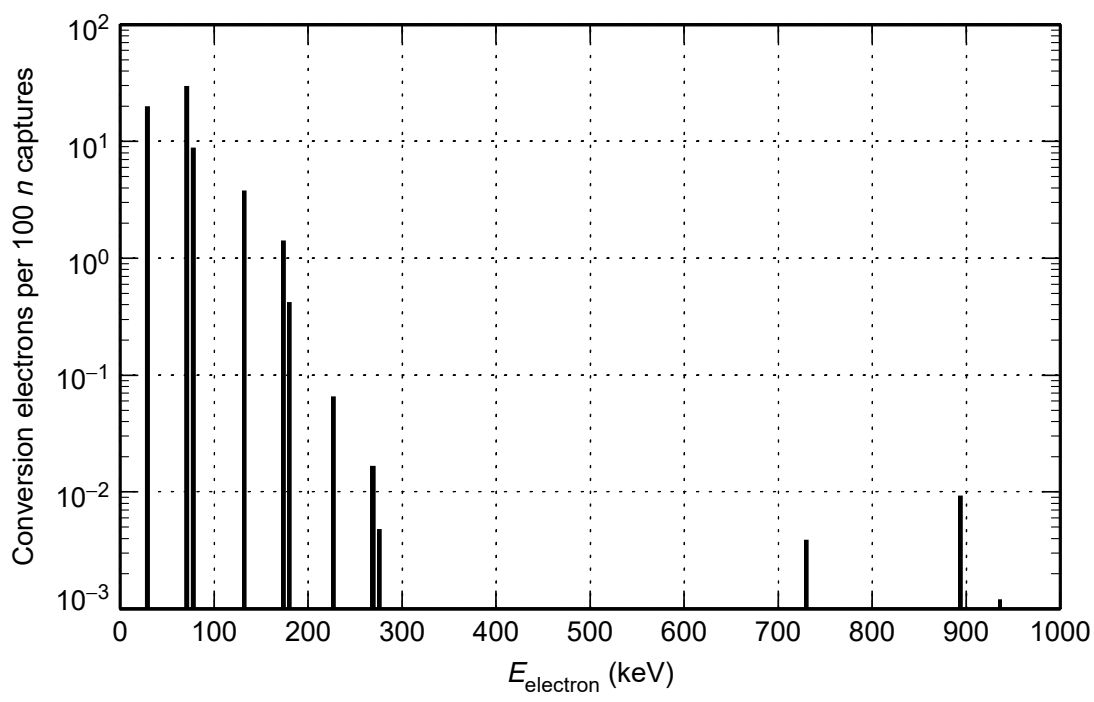

FIGURE 5.3 Principal internal conversion electrons per 100 neutron captures in $\mathrm{Gd}^{157}$.

emitted. Of these 5.65 electrons, 3.8 have $132 \mathrm{keV}, 1.4$ have $174 \mathrm{keV}$, and 0.4 are of $180 \mathrm{keV}$. As their energies indicate, the more numerous, lowest energy electrons are from $K$ capture; the next from $L$ capture, and the remainder from $M$-shell capture. The other transitions follow the same interpretation.

The three most probable electrons come from the lowest energy transition of $79.51 \mathrm{keV} .29 .8 \%$ of all neutron captures create a conversion electron of $71 \mathrm{keV}$; and $20 \%$ result in a $29.3 \mathrm{keV}$ electron. The conversion electron energy distribution for the most probable electrons is summarized in Figure 5.3.

\subsubsection{GD ${ }^{157}$ vs. $B^{10}$}

Among solids, gadolinium isotopes and $\mathrm{B}^{10}$ are the neutron absorbers with the largest cross sections that produce a charged particle in the final state. Both are in active use (as is $\mathrm{Li}^{6}$ ). There are seven stable isotopes of $\mathrm{Gd}$. The neutron capture cross section on each of them is depicted in Figure 5.4. Isotopes 155 and 157 have thermal neutron cross sections that exceed that of $\mathrm{B}^{10}$. On a per nucleus basis, either $\mathrm{Gd}^{155}$ or $\mathrm{Gd}^{157}$ is far superior to $\mathrm{B}^{10}$ in absorbing power of thermal neutrons; however, other requirements of a particular application may sway the choice away from $\mathrm{Gd}$.

First, boron is plentiful and relatively inexpensive. Due to its $10 \%$ mass difference, $\mathrm{B}^{10}$ is relatively easy to separate from natural boron. The United States prepares tons of $\mathrm{B}^{10}$ annually, specifically for use in the nuclear industry. $\mathrm{B}^{10}$ loaded polyethylene is a favored neutron moderator and absorber. Elemental Gd is rarer and more expensive. 


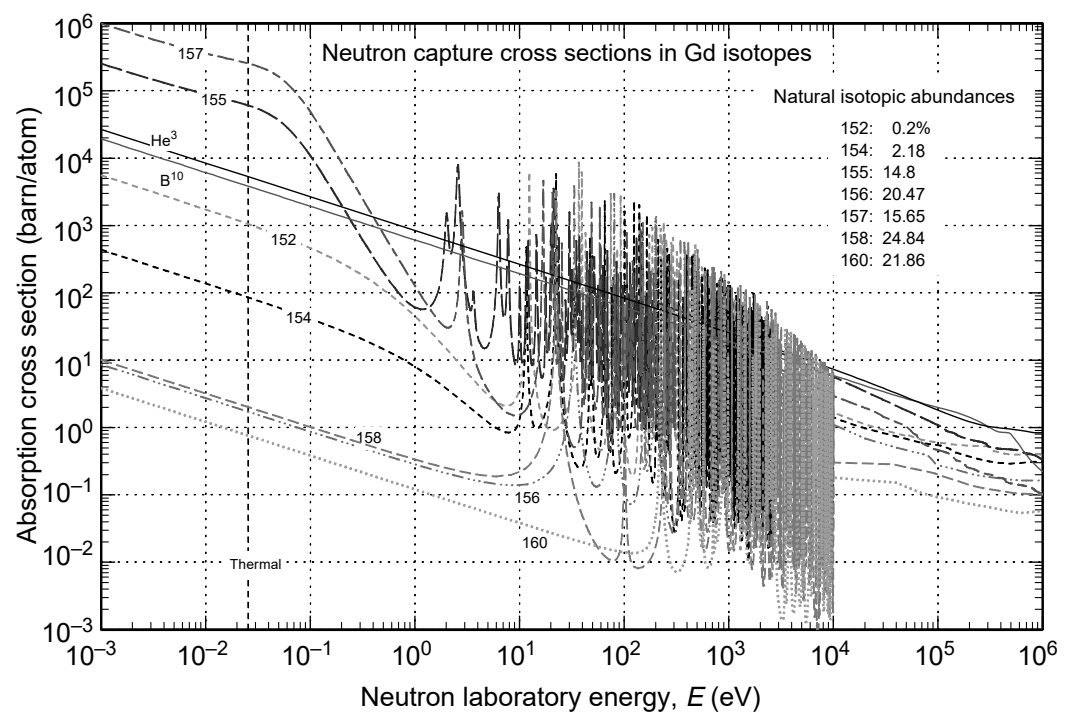

FIGURE 5.4 (See color insert following page 332.) Neutron capture cross sections on the seven stable isotopes of $\mathrm{Gd}$.

Gd isotopes differ by less than $1 \%$ in mass, and it is more difficult to separate them. Given the expense of obtaining individual isotopes from the element, it is of interest to compare the neutron-absorbing properties of natural $\mathrm{Gd}$ with those of $\mathrm{B}^{10}$. In natural $\mathrm{Gd}$, the abundance of isotope 157 is $15.65 \%$, and that of 155 is $14.8 \%$. Weighting these and the other isotopes, the thermal neutron absorption cross section of an atom of natural $\mathrm{Gd}$ is

$$
\sigma\left(\mathrm{Gd}_{\text {nat }}\right)=48,800 \text { barn }
$$

about 12.7 times that of the cross section, $\sigma\left(\mathrm{B}^{10}\right)$, on an atom of $\mathrm{B}^{10}$. On a per atom basis, natural $\mathrm{Gd}$ is an order of magnitude superior to $\mathrm{B}^{10}$ as an absorber. However, applications that are sensitive to weight, such as in spacecraft, prefer to compare on an equal mass basis: $\sigma\left(\mathrm{cm}^{2} / \mathrm{g}\right)=\left(N_{A} / A\right) \sigma\left(\mathrm{cm}^{2}\right)$, and the element atomic mass $A$ reduces the cross section relative to a single atom. As $A\left(\mathrm{Gd}_{\text {nat }}\right)=15.73 A\left(\mathrm{~B}^{10}\right)$, a gram of $\mathrm{Gd}_{\text {nat }}$ has a thermal cross section equal to $12.7 / 15.73=0.8$ that of a gram of $\mathrm{B}^{10}$ :

$$
\begin{aligned}
\sigma\left(\mathrm{Gd}_{\text {nat }}\right) & =187\left(\mathrm{~cm}^{2} / \mathrm{g}\right) \\
\sigma\left(\mathrm{B}^{10}\right) & =231\left(\mathrm{~cm}^{2} / \mathrm{g}\right)
\end{aligned}
$$

Per gram, $\mathrm{B}^{10}$ is a (slightly) better thermal absorber than natural Gd. Other features are the relatively low cost of $\mathrm{B}^{10}$; the lower $Z$ of $\mathrm{B}$; and the short 
ranges of the charged particles $\left(\alpha\right.$ and $\left.\mathrm{Li}^{7}\right)$ emitted by $\mathrm{B}^{10}$ relative to the longer ranges of a conversion electron or gamma emitted by $\mathrm{Gd}^{157}$. Given that some applications are sensitive to $\mathrm{x}$-ray and gamma absorption, and so require a low- $Z$ material, that weight may be an issue, and that it may be desirable not to have long-range particles created upon $n$ capture, there may be circumstances in which $\mathrm{B}^{10}$ is preferred to natural Gd.

\subsection{CROSS-SECTION DEPENDENCE ON TARGET TEMPERATURE}

Given the presence of the ground, buildings, concrete, walls, human bodies, air, and other low- $Z$ material, neutron moderation is inevitable, giving the neutrons a much lower energy spectrum than when they were produced, say in a reactor or in cosmic ray interactions with the atmosphere. The thermal part of the spectrum contains neutrons at the same energy (temperature) as the host medium. One therefore needs to know the spectrum averaged cross section on a medium at its own temperature and must account for Doppler broadening of cross sections.

One would think that a cross section that is independent of velocity would not be affected by target thermal motion as relative neutron-nucleus velocity does not change it. Likewise, one would expect that a velocity-dependent cross section, say the $1 / v$ dependence of a capture cross section, would deviate from simple $1 / v$ dependence when the incident neutron velocity is comparable to or smaller than target thermal velocities, since relative velocity is now important. It is at first surprising, then, that just the opposite is the case.

Figure 5.5 shows the low-energy elastic scattering and $(n, p)$ capture cross sections in $\mathrm{N}^{14}$ at 0,300 , and $1200 \mathrm{~K}$. At the higher temperatures, the constant elastic scattering cross section is increased when the neutron energy is much below thermal; the energy dependence changes from a constant to a $1 / v$ law.

The reason is as follows. The desired cross section $\sigma(v, T)$ at temperature $T$ defines the reaction rates:

$$
R=N v \sigma(v, T) \quad(\text { reactions } / \text { sec per incident neutron) }
$$

where $v$ is the incident neutron velocity, and $N$ is the target density. Target atoms of thermally oscillating velocity $V$ have a Maxwellian velocity distribution $f_{M}(V) \propto \exp \left[-M V^{2} / 2 k T\right]$. For relative velocity $v-V$, the microscopic reaction rate is $N|v-V| \sigma_{\mathrm{o}}(|v-V|)$, where $\sigma_{\mathrm{o}}=\sigma(|v-V|, 0)$ is the zero temperature cross section. Thus, the reaction rate $R$ can also be expressed as

$$
R=N \int|v-V| \sigma_{\mathrm{o}}(|v-V|) f_{M}(V, T) \mathrm{d}^{3} V
$$




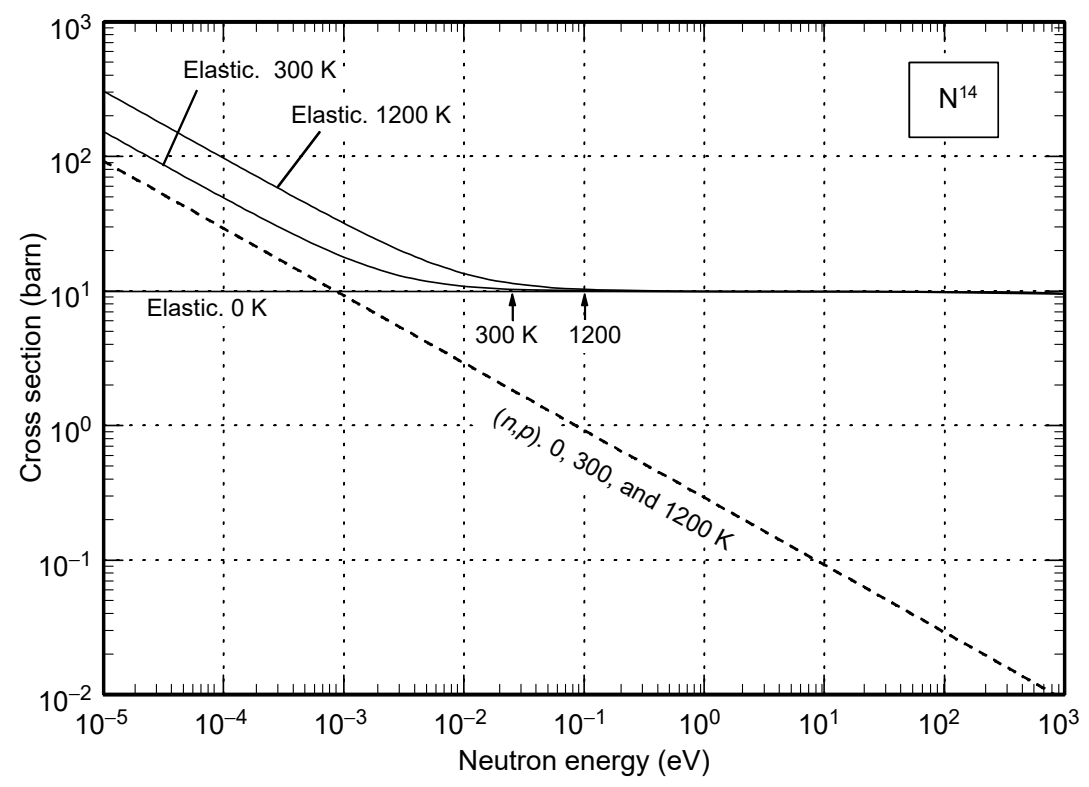

FIGURE 5.5 Low-energy elastic and $(n, p)$ cross sections on $\mathrm{N}^{14}$ at three temperatures. Illustrating the dependence of the cross section on target temperature.

or

$$
v \sigma(v, T)=\int|v-V| \sigma_{\mathrm{o}}(|v-V|) f_{M}(V, T) \mathrm{d}^{3} V
$$

This expresses the Doppler broadened cross section $\sigma(v, T)$ in terms of the stationary cross section $\sigma_{\mathrm{o}}$. When $v \ll V$, the integral is constant and $\sigma(v, T) \propto$ $1 / v$. Thus, it is clear, at least formally, that Doppler broadening turns a constant cross section into a $1 / v$ cross section, for small $v$.

The capture cross section, which behaves as $1 / v$ when the target nucleus is stationary, turns out to be unaffected by target temperature. The integrand in Equation 5.13 becomes independent of $v$, and $\sigma(v, T)$ remains proportional to $1 / v$. In spite of Equation 5.13, these behaviors are unintuitive. They are a result of the fact that target thermal agitation allows the nucleus to pass near the projectile many times, multiplying the effective cross section as the projectile passes by. It is worth understanding this explanation.

\subsubsection{Temperature Dependence of a Constant Cross Section}

Let $\sigma_{\mathrm{o}}=\pi a^{2}$ be a constant, velocity independent cross section of a neutron on a stationary nucleus $(T=0)$, such as is the case for elastic scattering on $\mathrm{N}^{14}$ for neutron energies $E$ less than about $1 \mathrm{keV}$. $a$ is the neutron-nucleus 
separation required for the interaction to occur. Let $x_{\mathrm{o}}$ be the thermal agitation amplitude and $V$ the mean thermal speed of the nucleus in a material of temperature $T$. The period of oscillation is $\tau=4 x_{\mathrm{o}} / V$. Usually, $a \ll x_{\mathrm{o}}$, except at the lowest temperatures and highest cross sections. The velocity of a subthermal neutron may be slower than that of the heavier, oscillating nucleus.

We need to consider only one degree of freedom, the oscillation parallel to the neutron velocity, and so may take $\frac{1}{2} M V^{2}=\frac{1}{2} k T$. The neutron requires a time $t_{1}=2 x_{\mathrm{o}} / v$ to travel completely past the target. When $v \ll V$, the nucleus passes close to the neutron twice on each oscillation, or $n=2 t_{1} / \tau$ times in the transit time $t_{1}$. With the above definitions, $n=2\left(2 x_{\mathrm{o}} / v\right) /\left(4 x_{\mathrm{o}} / V\right)=V / v$. Thus, the neutron has $n$ chances to interact, and $n$ is proportional to $1 / v$ and to $T^{1 / 2}$. Including the case $V=0$, the number is $n=1+V / v$, and the effective cross section is $\sigma=n \sigma_{\mathrm{o}}=(1+V / v) \sigma_{\mathrm{o}}$.

Therefore, target thermal oscillations double the cross section when $V / v=1$ or when $E \equiv \frac{1}{2} m v^{2}=(m / M) \frac{1}{2} k T$. For $\mathrm{N}^{14}$ this is at $E=k T / 28$, as is seen to be the case in the figure for both 300 and $1200 \mathrm{~K}$. At still lower energies, $V / v \gg 1$, the final cross section is

$$
\sigma \rightarrow \frac{V}{v} \sigma_{\mathrm{o}} \propto \frac{\sqrt{T}}{v}
$$

Doppler broadening turns a constant cross section into a $1 / v$ cross section. The scaling also shows the temperature dependence at a fixed energy; the cross section doubles when the temperature quadruples, say from 300 to $1200 \mathrm{~K}$.

\subsubsection{Temperature Dependence of a $1 / \boldsymbol{v}$ Cross Section}

Now consider an interaction for which the cross section on a stationary nucleus behaves as $1 / v, \sigma(v)=\sigma_{1} v_{1} / v$, such as radiative capture. Equation 5.13 shows that when $\sigma(v) \propto 1 / v$ the Doppler broadened cross section remains exactly proportional to $1 / v$.

Again let $v$ be the neutron velocity and $V$ be the mean thermal nuclear velocity parallel to $v$. On one-half an oscillation, the relative velocity is $|V-v|$, and so the cross section is $\sigma_{1} v_{1} /|V-v|$. On the other half it is $V+v$, and the cross section is $\sigma_{1} v_{1} /(V+v)$. Assuming that the transit time $t_{1}$ is longer than a period $\tau$, then over a full period the cross section is

$$
\sigma_{\tau}=\sigma_{1} v_{1}\left[\frac{1}{|V-v|}+\frac{1}{V+v}\right]
$$

To obtain the final effective cross section $\sigma$, this is to be multiplied by the number of periods $t_{1} / \tau=V / 2 v$ during the transit time, $\sigma=\sigma_{\tau} V / 2 v$. Considering the very slow neutron case, $V \gg v$, it becomes 


$$
\sigma=\sigma_{\tau} \frac{V}{2 v}=\sigma_{1} \frac{v_{1}}{v} \frac{V}{2}\left[\frac{1}{V-v}+\frac{1}{V+v}\right]=\sigma_{1} \frac{v_{1}}{v}\left[1+O\left(v^{2} / V^{2}\right)\right]
$$

Except for corrections of the order $v^{2} / V^{2}$, this is the same cross section as for a nucleus at rest. For a $1 / v$ cross section, the target temperature makes no difference, and the $1 / v$ cross sections in Figure 5.5 are unchanged at all temperatures.

The definition in Equation 5.11 used $v$ for the relative velocity. The target motion is hidden in the Maxwell distribution. However, in the explanation in Equation 5.14 through Equation 5.16, the target motion was handled directly, so that $|v-V|$ is the relative velocity. This latter argument makes the hidden motion explicit.

\subsection{NEUTRONS IN SILICON}

The fate of the energy deposited in matter by neutrons is important. Heating in reactors is the desired product, but can change mechanical, metallurgical, and structural properties of heated materials. Neutrons arising from cosmic rays pass through electronic components (usually silicon), and may cause both temporary and permanent changes to a component's electrical functioning. Here we discuss how neutrons deposit their energy in silicon, and the separation into ionizing and nonionizing dose.

Upon entering silicon, neutrons can physically knock atoms out of their lattice positions, causing longer-term displacement damage. The damage can be electronic in that the creation of vacancy-interstitial pairs and clusters can degrade component performance. Under intense fluxes, the damage can be metallurgical as well. Further, the recoil atom ionizes upon coming to rest, which creates electron-hole pairs that may temporarily upset a binary state (single event upset (SEU), or bit-flip, or neutron induced upsets (NIU)).* Over time, the displacement of atoms causes cumulative permanent damage to the device. SEUs cause a temporary problem because bits can be reset without permanent damage. One needs to understand what fraction of the total energy deposited by neutron passage contributes to displacement damage and what fraction to ionization.

This is in contrast to the passage of electrons or photons through matter, which only rarely cause atomic displacements. Rather they deposit their energy almost exclusively in the form of ionization, creating $e-h$ pairs. Protons, heavy ions, and neutrons, on the other hand, can cause both displacements and ionization. However, whereas only a small fraction of the energy deposited by a proton or ion goes into atomic displacements, the

\footnotetext{
*If the semiconductor is boron-doped, neutron capture in $\mathrm{B}^{10}$ ejects either a $1.47 \mathrm{MeV}$ or alpha $1.78 \mathrm{MeV}$ alpha particle, which can likewise cause single event upsets.
} 
energy deposited by a neutron is split between displacement and ionization in very comparable amounts. The reason is that, as discussed in Chapter 4, ions lose their energy primarily by knocking out atomic electrons, whereas neutrons lose energy only by colliding with nuclei.

The neutron-nucleus interaction results in a scattered neutron of lesser energy, a recoiling nucleus, possibly gammas, and at high energies the production of light-charged particles ( $p, \alpha$, etc). In $\mathrm{Si}$ and in many materials, a low-energy neutron may also be absorbed by the $(n, \gamma)$ reaction. In this case, the nucleus will recoil from the emitted photon.

The mean free path $(\mathrm{mfp})$ between scatterings of a neutron in $\mathrm{Si}$ is between 2 and $10 \mathrm{~cm}$, depending on its energy. As this distance is large compared with the linear dimensions of most Si targets of interest, one has to consider only the first neutron interaction.

\subsubsection{Basic Cross Sections in Silicon}

Natural silicon has the following isotopic composition:

$\begin{array}{lr}\mathrm{Si}^{28} & 92.23 \% \\ \mathrm{Si}^{29} & 4.67 \% \\ \mathrm{Si}^{30} & 3.10 \%\end{array}$

Its density is $\rho=2.32 \mathrm{~g} / \mathrm{cm}^{3}$, and its atomic weight $A=28.08$. Atom number density is

$$
\begin{aligned}
& N_{\mathrm{g}}=N_{\mathrm{A}} / A=2.145 \times 10^{22} \text { atoms } / \mathrm{g} \text { or } \\
& N=N_{\mathrm{g}} \rho=4.975 \times 10^{22} \text { atoms } / \mathrm{cm}^{3}
\end{aligned}
$$

where $N_{\mathrm{A}}$ is Avogadro's number.

The two reactions

1. Elastic scattering

2. Radiative capture $\sigma_{\gamma} \quad n+\mathrm{Si}^{28} \rightarrow \mathrm{Si}^{29^{*}} \rightarrow \mathrm{Si}^{29 \mathrm{~g}}+\delta E(8.472 \mathrm{MeV})$;

$$
n+\mathrm{Si}^{29} \rightarrow \mathrm{Si}^{30^{*}} \rightarrow \mathrm{Si}^{30 \mathrm{~g}}+\delta E(10.61 \mathrm{MeV})
$$$$
n+\mathrm{Si}^{30} \rightarrow \mathrm{Si}^{31^{*}} \rightarrow \mathrm{Si}^{31 \mathrm{~g}}+\delta E(6.6 \mathrm{MeV})
$$

Thermal $\sigma$ (barn):

$$
\begin{aligned}
& 2.08 \\
& 0.16 \\
& 0.12 \\
& 0.107
\end{aligned}
$$

occur with no threshold. The ground state of $\mathrm{Si}^{29}$ is $8.472 \mathrm{MeV}$ below that of $\mathrm{Si}^{28}$ plus the mass of a neutron. The capturing states of $\mathrm{Si}^{29}, \mathrm{Si}^{30}$, or $\mathrm{Si}^{31}$ have many levels below them, and $\gamma s$ of various energies occur with some branching ratio as the nucleus decays to its ground state. These photon emissions occur on an fs-ps time scale. The ground state of $\mathrm{Si}^{31}$ is unstable, against $\beta$ and $\gamma$ emissions (maximum energy $1.48 \mathrm{MeV}$ ), with a half-life of $2.62 \mathrm{~h}$. 
Of reactions with a threshold, the one with the lowest $\left(1.84 \mathrm{MeV}\right.$ on $\mathrm{Si}^{28}$, $1.33 \mathrm{MeV}$ on $\mathrm{Si}^{29}, 2.31 \mathrm{MeV}$ on $\mathrm{Si}^{30}$ ) is inelastic scattering $n+\mathrm{Si} \rightarrow n^{\prime}+\mathrm{Si}^{*}$. The particle production threshold is $\sim 2.8 \mathrm{MeV}$, for $n+\mathrm{Si} \rightarrow \mathrm{Mg}+\alpha$.

Figure 5.6 shows the most important cross sections in natural silicon. The top panel shows the two nonthreshold processes, together with the total cross section including threshold processes, and the gamma production cross section. (The gamma production cross section momentarily exceeds the total cross section near $12 \mathrm{MeV}$.) The bottom panel shows the principal processes with a threshold.

Below several MeV elastic scattering is the most probable process, followed by neutron capture. The curve labeled $\sigma_{\gamma}$ is the cross section for radiative capture, as discussed earlier, and $\sigma_{\text {prod }}(\gamma)$ is the gamma production cross section of Equation 5.7.

The ratio $\sigma_{\text {prod }}(\gamma) / \sigma_{\gamma}$ is then the average number of $\gamma$ s produced upon $n$ capture. Below $1 \mathrm{MeV}$ this number is about 2.3. Alpha particle and proton production cross sections, $\sigma_{\text {prod }}(\alpha)$ and $\sigma_{\text {prod }}(p)$, are also shown in the bottom panel of the figure.

Upon thermal neutron capture, the spectrum of $\gamma$ s emitted from silicon in its natural isotopic composition is shown in Figure 5.7. The highest energy is 10.61 MeV from $n+\mathrm{Si}^{29}$; the highest from $\mathrm{Si}^{28}$ is $8.472 \mathrm{MeV}$. The lowest energy is $340 \mathrm{keV}$ again from $\mathrm{Si}^{29}$; the lowest from $\mathrm{Si}^{28}$ is $397.8 \mathrm{keV}$. The two most intense lines, both from $\mathrm{Si}^{28}$, are 3.54 and $4.93 \mathrm{MeV}$.

\subsubsection{Energy Transfer during Collision}

Above about $3 \mathrm{MeV}$, inelastic scattering and nuclear reactions resulting in charged particle production are more probable than elastic scattering. Computing the mean total energy transfer, the sum of the outgoing energies of the recoil nucleus and the produced particle(s) is relatively easy. But ionization and displacement energies are the ones desired. These require the angular distributions or energy distributions of the reaction products. These are more difficult to obtain, but ENDF tabulates many of these distributions, both in angle and in energy. However, it is easier to understand by simple arguments the kerma (and so, effectively, the dose) at lower energies where elastic scattering or neutron capture dominate.

\subsubsection{Kerma Due to Elastic Scattering}

When a neutron of mass $m$ and kinetic energy $E_{n}$ strikes a Si nucleus of mass $M$, the maximum energy transfer from the neutron to the Si target atom during elastic recoil is

$$
T_{\max }=\frac{4 m M}{(m+M)^{2}} E_{n}=0.133 E_{n}
$$



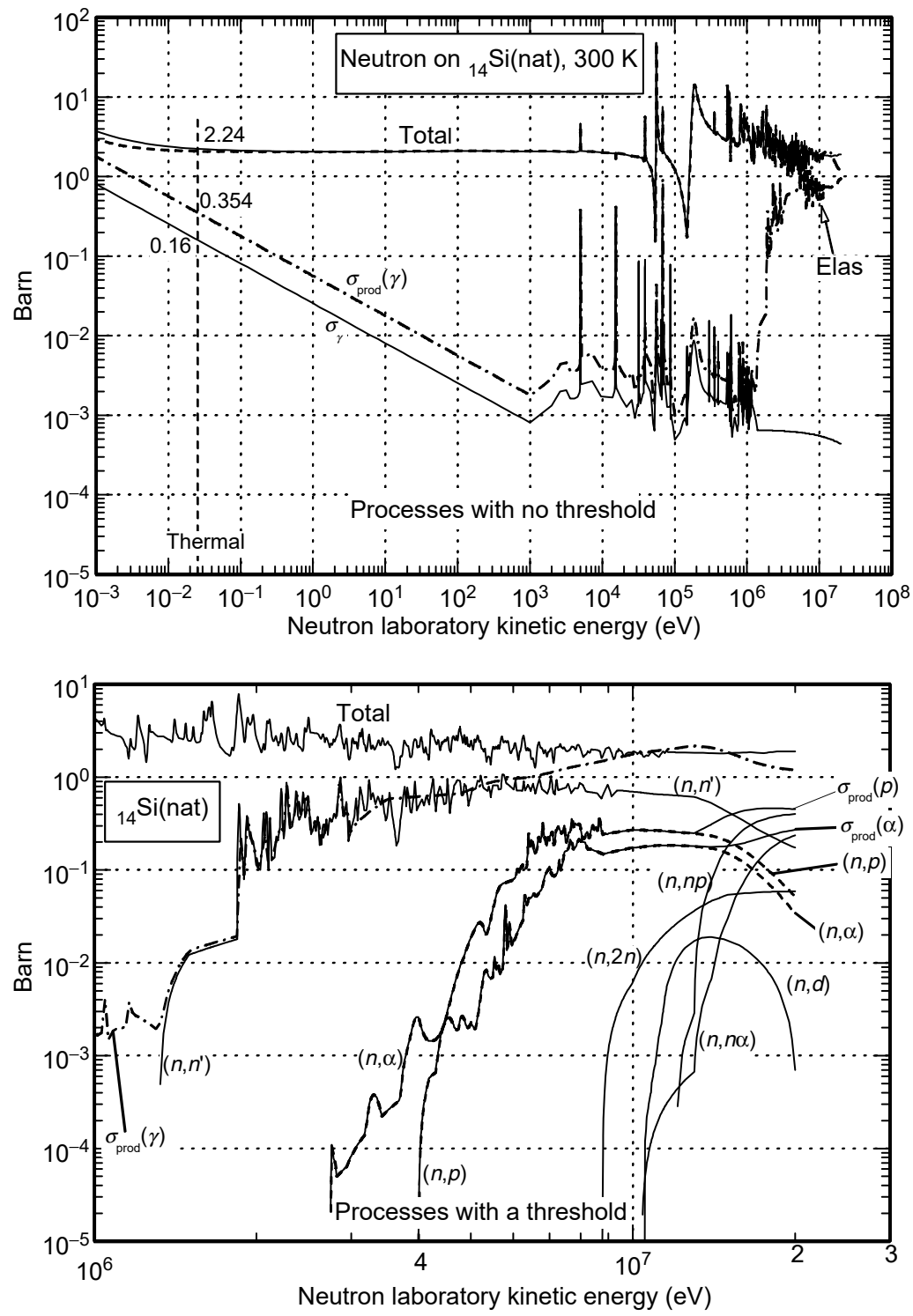

FIGURE 5.6 Principal neutron-Si(natural) cross sections at $300 \mathrm{~K}$. The first threshold process is inelastic scattering $\left(n, n^{\prime}\right)$ at about $1.3 \mathrm{MeV} . \sigma_{\text {prod }}(p)=(n, p)+2(n, 2 p)+\cdots$ is the proton production cross section. $\sigma_{\gamma}$ is radiative capture; $\sigma_{\text {prod }}(\gamma)$ is the gamma production cross section. Similarly $\sigma_{\text {prod }}(\alpha)$ is the alpha particle production cross section. (Data from ENDF/B-6 and derived files.) 


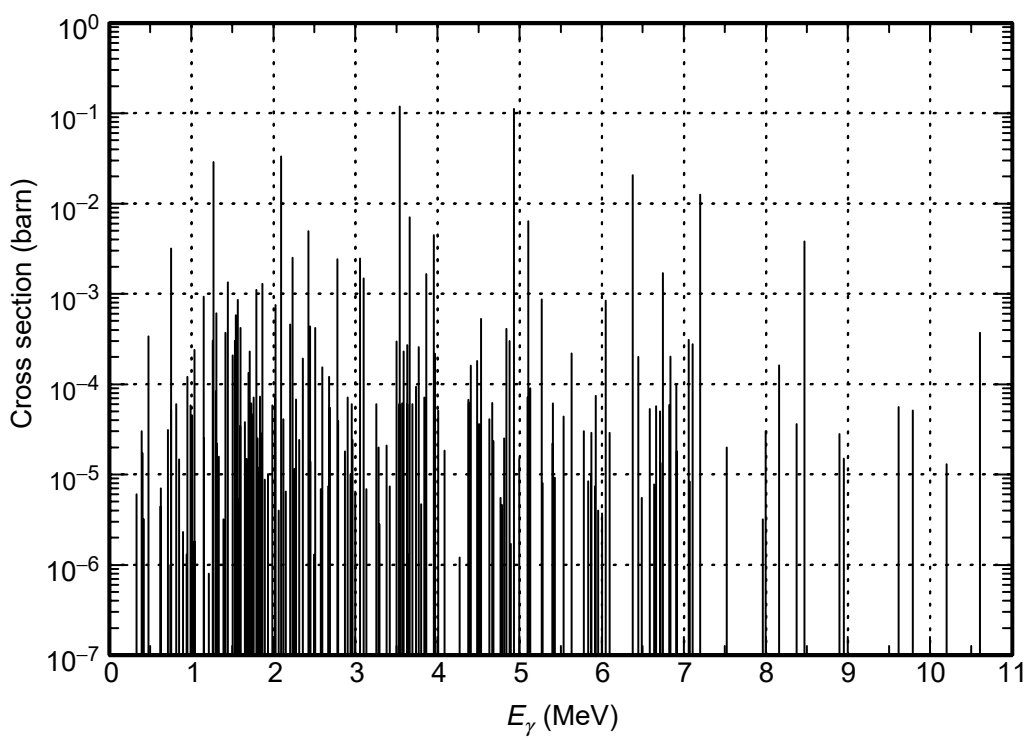

FIGURE 5.7 Cross sections for thermal neutron capture gammas in silicon (natural). The most probable gammas are 3.54 and $4.93 \mathrm{MeV}$, both from $\mathrm{Si}^{28}$, with a cross section for each of about 0.11 barn/atom. There are 183 lines. The lowest energy is $333.4 \mathrm{keV}$, the most energetic $10.61 \mathrm{MeV}$. Numerical data from the online table of radioactive isotopes (http://ie.lbl.gov/toi.htm).

The scattering is $\mathrm{S}$ wave up to about an $\mathrm{MeV}$, so the average energy transfer is about half the maximum,

$$
T_{\mathrm{av}}=\frac{1}{2} T_{\max }=\frac{2 m M}{(m+M)^{2}} E_{n}=0.067 E_{n}
$$

Thus, a $1 \mathrm{MeV}$ neutron imparts some $67 \mathrm{keV}$, on average, to the recoiling Si nucleus, and always less than $133 \mathrm{keV}$. The displacement threshold energy of $\mathrm{Si}$ in its lattice site is $E_{\mathrm{d}}=15$ to $25 \mathrm{eV},{ }^{*}$ and in $\mathrm{Si}$ the range of a $67 \mathrm{keV} \mathrm{Si}$ atom is about $1000 \AA$.

During this motion, the recoiling $\mathrm{Si}$ ion both dislodges other $\mathrm{Si}$ atoms (i.e., causes further displacements) and ionizes other atoms on the way. The partitioning of its energy loss between ionizing and further displacements is a nontrivial problem. A cascade forms in which the first recoil atom knocks

\footnotetext{
*Most recent studies favor $15 \mathrm{eV}$.
} 
others, which then dislodge others. This cascade process lends itself to analysis by integral equations; it was satisfactorily resolved only in the 1960s by Lindhard and coworkers [Li63a, Li63b, Sa66]. It is the basis for the concepts of nonionizing kerma and ionizing kerma (or nonionizing dose and ionizing dose) of neutrons and ions in matter.* The distinction is needed when inquiring into the number of electron-hole pairs or of the displacement damage produced by neutrons in a semiconductor. At least $E_{\mathrm{d}}$ is consumed for every displacement, and this amount of energy is therefore not available for ionization. Moreover, energy loss that goes into ionization is then not available to displace another atom.

Due to elastic scattering, the energy deposited per gram by a neutron fluence $\varphi\left(n / \mathrm{cm}^{2}\right)$ is $\varphi N_{g} \sigma_{\text {elas }} T_{\text {av }}=\xi_{\text {elas }} \varphi$, where $\xi_{\text {elas }}=N_{g} \sigma_{\text {elas }} T_{\text {av }}$ is the fluence-to-total kerma conversion factor $\left(\operatorname{rad} \operatorname{per} n / \mathrm{cm}^{2}\right)$ due to elastic collisions, and is proportional to the incident neutron energy for a constant $\sigma_{\text {elas. }}$. This deposited energy is in the form of the kinetic energy of the recoil Si atom. The factor

$$
k_{\text {elas }}=\sigma_{\text {elas }} T_{\text {av }}, \quad\left(\mathrm{eV}-\mathrm{cm}^{2}, \text { or eV-barn }\right)
$$

is the total kerma coefficient, or the total kerma cross section due to elastic scattering. Hence, the total kerma per unit fluence is $\xi_{\text {elas }}=N_{g} k_{\text {elas }}$. Other than capture, elastic scattering is the only available process below the inelastic threshold $(1.3 \mathrm{MeV})$, and is dominant up to $\sim 3 \mathrm{MeV}$. Si recoil from elastic scattering is the main contributor to neutron dose from $100 \mathrm{eV}$ to $3 \mathrm{MeV}$. $\xi$ for all processes at all energies, including elastic, inelastic, and scattering, is shown in Figure 5.8, the total fluence-tokerma conversion factor, from the ENDF database, converted to units of $\operatorname{rad}(\mathrm{Si})$ per $10^{11} \mathrm{n} / \mathrm{cm}^{2}$.

As charged particle ranges are so short, this total kerma is effectively the same as total dose. Thus Figure 5.8 is the fluence-to-total dose conversion factor. For neutron energies up to $\sim 100 \mathrm{keV}$, a typical elastic scattering cross section is $\sigma_{\text {elas }} \sim 2$ barn, and so at, say, $E_{n}=1 \mathrm{keV}, \xi=\xi_{\text {elas }}=2.87 \mathrm{eV} / \mathrm{g}$ per $n / \mathrm{cm}^{2}=4.6 \times 10^{-3}$ rad per $10^{11} \mathrm{n} / \mathrm{cm}^{2}$, as seen in Figure 5.8. Elastic scattering resonance cause the sharp variations about this dependence in the region $0.03 \mathrm{MeV} \lesssim E_{n} \lesssim 3 \mathrm{MeV}$.

The scattered $1 \mathrm{MeV}$ neutron, of average $933 \mathrm{keV}$, may scatter again. However, its $\mathrm{mfp}$ is $\sim 5 \mathrm{~cm}$ in $\mathrm{Si}$ and so interacts again only far from the first interaction point or outside the Si sample; its local dose or kerma is provided by its first scattering.

\footnotetext{
*The terms nonionizing energy loss (NIEL) and ionizing energy loss are more commonly used when referring to charged particles.
} 


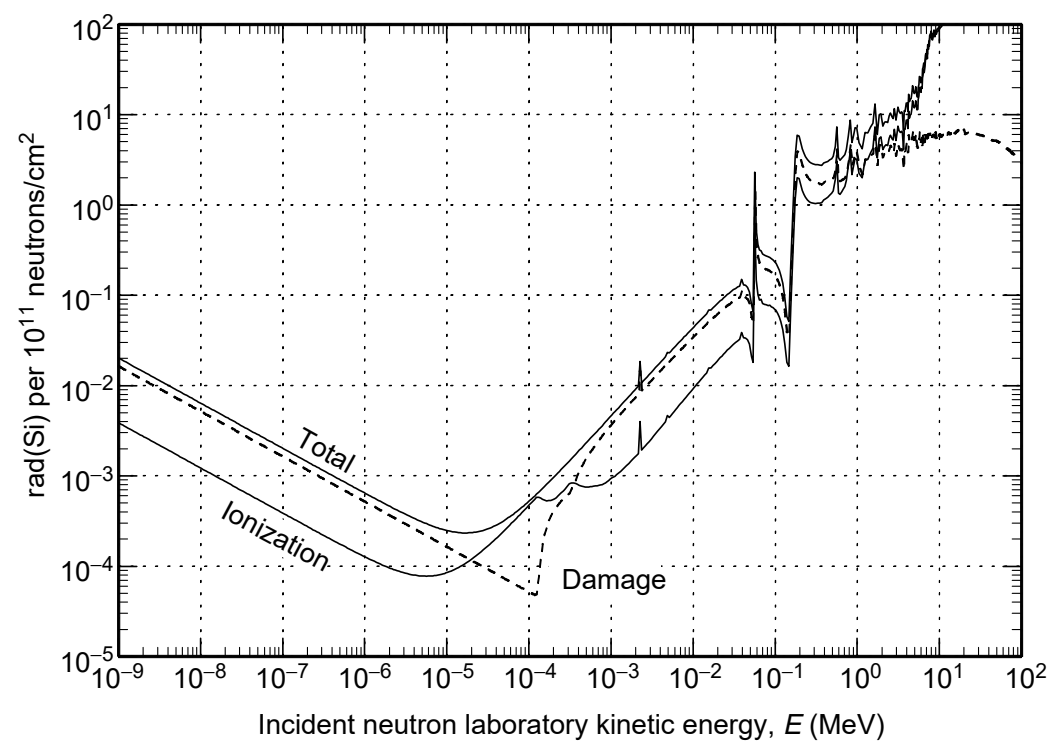

FIGURE 5.8 Neutron fluence-to-kerma conversion functions for ionizing, nonionizing, and total kermas. Kerma is essentially dose. Numerical data are on the accompanying CD-ROM. (From [Gr05], with permission, from recently released ENDF/B-7 cross sections.)

\subsubsection{Kerma Due to $n$ Capture and Gamma Emission}

Below $E_{n} \sim 50 \mathrm{eV}$, neutron capture causes more dose than elastic scattering, even though its cross section is well below that of elastic scattering. This is due to the nucleus recoil upon gamma emission.

A single emitted photon of energy $h \nu$ imparts a momentum $h \nu / c$ and an energy $E_{\mathrm{r}}=(h \nu / c)^{2} / 2 M$ to the recoiling nucleus. The recoil energy is proportional to the square of the photon energy and is independent of the incident neutron energy.

Following $n$ capture, suppose that a single photon of maximum energy is emitted, bringing the excited nucleus to its ground state. In the case of $\mathrm{Si}^{28}$, the photon is $8.472 \mathrm{MeV}$, and the nucleus recoils with kinetic energy

$$
E_{\mathrm{r}}=h v \frac{h v}{2 M c^{2}}=1.36 \mathrm{keV}
$$

At $1 \mathrm{eV}$, the capture cross section $\sigma_{\gamma}$ is 0.025 barn, and the fluence-to-total kerma by this mechanism would be

$$
\begin{aligned}
\xi_{\gamma} & =N_{\mathrm{g}} \sigma_{\gamma} E_{\mathrm{r}}=0.729\left(\mathrm{eV} / \mathrm{g} \text { per } n / \mathrm{cm}^{2}\right) \\
& =1.18 \times 10^{-14}\left(\mathrm{rad} \text { per } n / \mathrm{cm}^{2}\right)(1 \gamma \text { of maximum energy })
\end{aligned}
$$


for emission of one maximum energy photon. As a function of neutron energy $E_{n}, \xi_{\gamma}$ is proportional to $1 / \sqrt{E}_{n}$ through $\sigma_{\gamma}$.

The value in Equation 5.20 is larger than the correct value of $\xi_{\gamma}$ at $1 \mathrm{eV}$, because the excited nucleus does not always de-excite by emitting a single, maximum energy photon. If it de-excites in two steps, the first recoil imparts a smaller kinetic energy. The subsequent effect of the second photon depends on its direction of emission relative to the first. If emitted opposite the first, its recoil effectively stops the moving ion. If emitted parallel to the first, its recoil energy adds.

In addition to the relative angle of emission of the two photons, their time delay is important. A $1 \mathrm{keV} \mathrm{Si} \mathrm{atom} \mathrm{moving} \mathrm{in} \mathrm{Si} \mathrm{will} \mathrm{come} \mathrm{to} \mathrm{rest} \mathrm{in} \mathrm{about}$ $0.1 \mathrm{psec}$. If no other photon is emitted in that time, then the ion will come to rest and experience the full recoil of the next photon emitted. However, many radiative nuclear de-excitations have a lifetime less than $0.1 \mathrm{psec}$ [Fi96]. Their effect on the kinetic energy transferred to the ion after $n$ capture therefore depends on their relative angular distribution, their time delay relative to the previous decay, and the energy of the previous photon (which sets the ion velocity at the time of the next photon).

As new nuclear levels are continually discovered and lifetimes are continually refined (compare evolving editions of the Table of Isotopes), the best one can do is approximate the effects of subsequent decays, and construct an average energy transfer to the ion. This LANL has done, with little attempt at modeling the correct (and unknown) $\gamma$ cascade and angular distributions [Ma99]. The result is also seen in Figure 5.8, at $\lesssim 50 \mathrm{eV}$ where the kerma is proportional to $1 / \sqrt{E}_{n}$. At $1 \mathrm{eV}$ it is about $6 \times 10^{-4} \mathrm{rad}$ per $10^{11} \mathrm{n} / \mathrm{cm}^{2}$, smaller than Equation 5.20 as expected. Below $\sim 50 \mathrm{eV}$, Figure 5.8 must be considered approximate.

\subsubsection{Energy Partitioning and Nonionizing Dose}

It is necessary to know the fate of the energy acquired by the recoil atom. It loses its energy by ionizing other atoms and by displacing them. How is its energy partitioned between these two process?

In atom-atom collisions, Lindhard, Scharff, and Schiøtt [Li63b] obtained both the energy loss to ionization and the energy loss to kinetic energy of the recoiling target atom. A (neutral) $\mathrm{Si}$ atom collides with others by electrostatic forces. The potential is a screened Coulomb potential $\left(Z Z_{\mathrm{t}} e^{2} / r\right) f(r)$, where $f$ is a screening function. The often used $f=\exp (-r / \mathrm{a})$ is not adequate since it is too small at large $r$. Instead, [Li63b] used the Thomas-Fermi model for the screening function, and obtained a simple form for $d \sigma / d T$, the cross section for the recoil atom to have kinetic energy $T$. This then leads to the mean energy loss of a recoiling atom to kinetic energy of other atoms without ionization, i.e., the non-ionizing energy loss. It is often denoted $P\left(E_{R}\right)$, where $E_{R}$ is the primary atom recoil energy. 
Similarly, a simple form was found for the energy loss to ionization, $Q\left(E_{R}\right)$, produced by the neutral recoil atom (it is proportional to the atom velocity). Then $P\left(E_{R}\right)+Q\left(E_{R}\right)=E_{R}$. The ratio $P\left(E_{R}\right) / E_{R}$ is the fraction of the first recoil atom energy that is lost to non-ionizing displacements.

As the average recoil energy is $2 m / M$ times the incident neutron energy, where $m$ is neutron mass and $M$ is $\mathrm{Si}$ atom mass, one obtains the energy loss rates of a neutron to displacement and to ionization.

In addition to Lindhard's early theoretical curves, accurate cross sections now permit these quantities to be computed directly. Figure (5.8) has already presented these kerma, the ionizing, non-ionizing (displacement) kerma, and their sum, the total kerma, for neutrons in Silicon. The numerical data from which the figure was constructed are on the accompanying CD-ROM.

The ratio (neutron ionizing dose)/(neutron total dose) is the same as the ratio of the Lindhard ionization energy loss to total energy loss. The ratio is shown in Figure 5.9, both from the cross section data of Figure 5.8, and from Lindhard's calculation. They are quite close except above $5 \mathrm{MeV}$, where nuclear reactions begin. Below $1 \mathrm{MeV}$, most energy deposition by neutrons goes to displacement damage. Above $1 \mathrm{MeV}$, most goes to ionizing. But the difference is not dramatic unless below tens of $\mathrm{keV}$ or above $5 \mathrm{MeV}$.

Displacement damage in semiconductors by ions and by neutrons is periodically reviewed, e.g. by Srour et al. [Sr03] and by Messenger [Me92a].

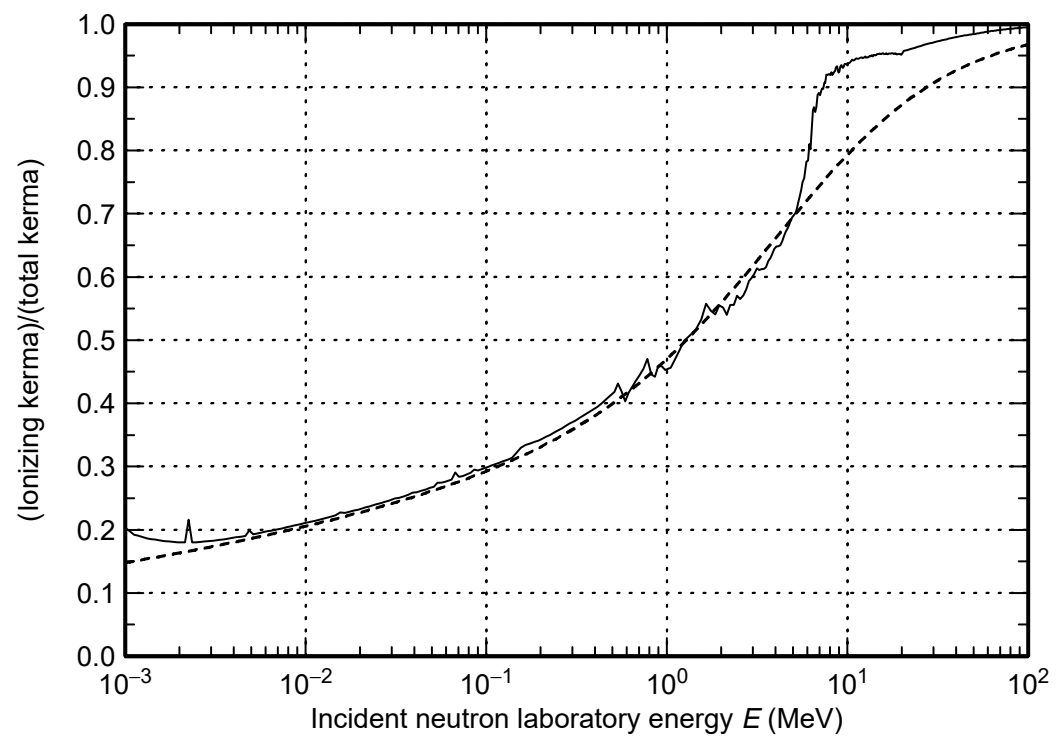

FIGURE 5.9 Fraction of neutron kerma in Si that is ionizing. Solid curve, ratio of ionizing to total kerma from recent data by [Gr05]. Dashed curve, Lindhard theoretical factor (From [Li63a].) 
It is discussed extensively in texts, for example van Lint, et al. [VL80], Messenger and Ash [Me92b], or Holmes-Siedle and Adams [Ho02.]

\subsubsection{MeV Displacement Equivalent Fluence}

It is common to express the displacement damage caused by any neutron spectrum in terms of the fluence of $1 \mathrm{MeV}$ neutrons that causes the same damage. This fluence is called the $1 \mathrm{MeV}$ displacement equivalent (silicon) fluence, or sometimes the $1 \mathrm{MeV}$ DES fluence. It has been called the $1 \mathrm{MeV}$ damage equivalent fluence. However, damage varies according to the property that is damaged and, for electronic devices, damage is not always proportional to displacement kerma. Thus, the term damage is replaced by displacement.

The displacement kerma represents structural damage to the crystal lattice and depends only on neutron interactions and Si crystalline structure. Electronic damage kerma is often nearly proportional to displacement kerma, but may further depend on device design, function, and damage parameter under consideration.

Although one speaks of a " $1-\mathrm{MeV}$ displacement equivalent fluence" in silicon, a true value for that quantity is difficult to define because displacement kerma rapidly varies near $1 \mathrm{MeV}$. To handle this problem, and to avoid a standard becoming outdated as calculations near that energy improve and change, the ASTM has defined instead a reference displacement kerma rather than a reference neutron energy. The reference kerma is $95 \mathrm{MeV}$-mbarn. This is nearly the actual $1 \mathrm{MeV}$ kerma as presently known. It is called the "1-MeV( $\mathrm{Si})$ displacement kerma." However, it is a fixed number that will not change even if refined calculations show that the actual $1 \mathrm{MeV}$ kerma is different. The problem is thus sidestepped by shifting attention from a particular neutron energy to a particular reference kerma. As calculated values are refined, the actual kerma of a $1 \mathrm{MeV}$ neutron may differ from the $1-\mathrm{MeV}$ (Si) displacement kerma (i.e., may differ from $95 \mathrm{MeV}$-mbarn), and the " $1-\mathrm{MeV}$ displacement equivalent fluence" of a $1 \mathrm{MeV}$ neutron may differ from 1 .* $^{*}$

As nearly as it can be defined, the $1-\mathrm{MeV}$ displacement equivalent fluence in silicon is given numerically on the accompanying CD-ROM. The questions of displacement vs. damage, and attempts to define a useful monoenergetic fluence, are discussed in ASTM Standard E722-94, Section 3 and Section 4 (November, 1994).

\subsection{NEUTRON LIFETIME IN AIR}

Neutrons react with nitrogen in air. Natural $\mathrm{N}$ is $99.63 \% \mathrm{~N}^{14}$ and $0.37 \% \mathrm{~N}^{15}$. Cross sections on $\mathrm{N}^{14}$ are shown in Figure 5.1, while cross sections on $\mathrm{O}^{16}$ are in Figure 5.10. Below $3 \mathrm{MeV}$, oxygen undergoes only elastic scattering

*This clarification is due to Dr. P.J. Griffin of Sandia Laboratories. 

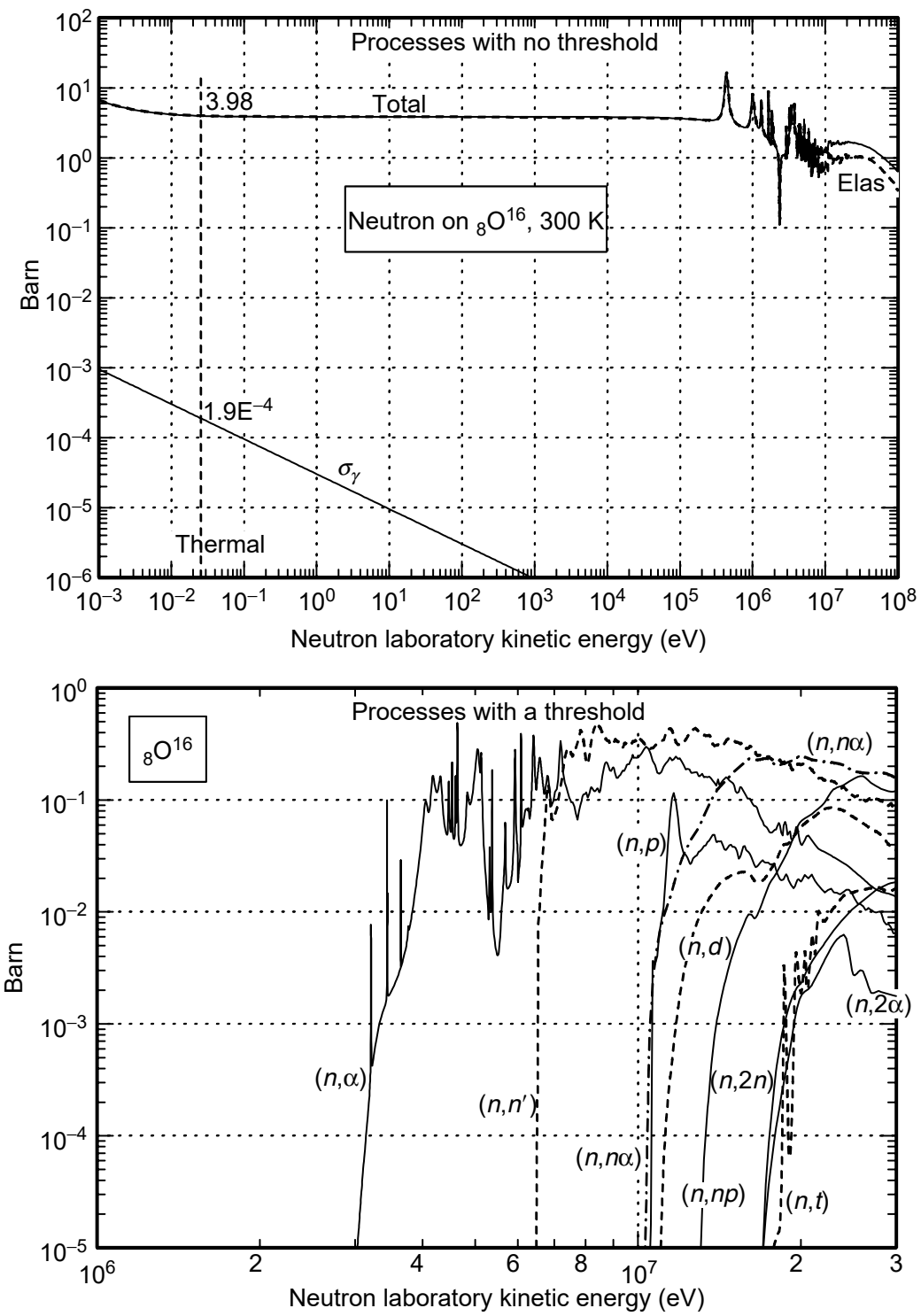

FIGURE 5.10 Principal neutron- $\mathrm{O}^{16}$ cross sections at $300 \mathrm{~K} . \sigma_{\gamma}$ is radiative capture; the gamma production cross section is larger. The first threshold process is $(n, \alpha)$ at about 2.7 MeV. (Data from Point2004 (ENDF/B-6).) 
and has a small capture cross section. $(n, p)$ capture on $\mathrm{N}$ is $10^{4}$ times larger. Below a few $\mathrm{MeV}$, nitrogen is clearly dominant in determining neutron behavior in air.

\subsubsection{Radiative Capture in Nitrogen}

In radiative capture, the state of $\mathrm{N}^{15}$ into which the neutron falls is 10.833 $\mathrm{MeV}$ above the $\mathrm{N}^{15}$ ground state (sometimes into the $10.804 \mathrm{MeV}$ state) [Aj86]. It may decay by emitting several $\gamma \mathrm{s}$ of a variety of energies. Hence, the $\gamma$ production cross section is about three times the capture cross section $\sigma_{\gamma}$. A high presence of these $\gamma \mathrm{s}$ in air is an indicator of the presence of neutrons. The highest energy $\gamma$ is $10.8291 \mathrm{MeV}$.

\subsubsection{The $(N, P)$ Reaction}

The largest capture cross section is $\mathrm{N}^{14}(n, p) \mathrm{C}^{14}$. Below $10 \mathrm{keV}$ it is

$$
\sigma(n, p)=\frac{0.291}{\sqrt{E(\mathrm{eV})}}=\frac{4.027 \times 10^{5}}{v(\mathrm{~cm} / \mathrm{sec})} \quad(\text { barn })
$$

For a thermal neutron, $\sigma(n, p)=1.8 \mathrm{~b}$. This $(n, p)$ process, when initiated by cosmic ray neutrons, is the source of atmospheric $\mathrm{C}^{14}$. A high presence of these protons is another indicator of the presence of neutrons in air. However, the $p$ energy is only $0.583 \mathrm{MeV}$, and its range in sea level air is only $1 \mathrm{~cm}$; it would therefore be difficult to observe.

\subsubsection{Neutron Mean Free Path in Air}

At sea level, the nitrogen number density is $N=3.98 \mathrm{E} 19$ nuclei $/ \mathrm{cm}^{3}$. The elastic scattering cross section at $1 \mathrm{MeV}$ is $\sim 2 \mathrm{~b}$, so the $\operatorname{mfp} \lambda=1 / N \sigma$ against elastic scattering is $\lambda \sim 125 \mathrm{~m}$. When the energy moderates to $<10 \mathrm{keV}$, $\sigma$ increases to $\sim 10 \mathrm{~b}$, and $\lambda$ decreases to $\sim 25 \mathrm{~m}$.

\subsubsection{Neutron Mean Lifetime in Air}

Capture by $(n, p)$ limits the neutron lifetime in air. As $\sigma \propto 1 / v$, the capture collision frequency

$$
\nu=N \sigma(n, p) v=16 / \mathrm{sec},
$$

where $v$ is the neutron velocity, is independent of neutron energy.

Once an energetic neutron has downscattered to about $10^{5} \mathrm{eV}$, where the $1 / v$ behavior of $\sigma(n, p)$ sets in, its collision frequency against the $(n, p)$ 
reaction will thereafter be the constant value $16 / \mathrm{sec}$. The downscatter time for a neutron of several $\mathrm{MeV}$ to be moderated by elastic scattering down to $10^{5} \mathrm{eV}$ is easily shown to be $<0.1 \mathrm{~ms}$. Therefore, the mean life in sea level air of a neutron of any initial energy is

$$
\tau=1 / \nu=0.062 \mathrm{sec}
$$

To be captured, fast neutrons need not wait to slow down; $\sigma(n, p)$ is greater than its $1 / v$ scaling when $E>10^{5} \mathrm{eV}$, so fast neutrons can be directly captured by $(n, p)$ even before $\tau$. However, as the elastic cross section exceeds $\sigma(n, p)$, a neutron is more likely to downscatter and follow the earlier discussion. This means that if at $t=0$ there are $n_{\mathrm{o}}$ neutrons of any energy in air, the number left at time $t$ is $n(t)=n_{\mathrm{o}} \exp (-t / \tau)$.

\subsection{NEUTRON CROSS SECTIONS IN SELECTED ISOTOPES}

As there are hundreds of stable isotopes, it is impractical to present cross sections on all of them. We limit ourselves to presenting graphs of cross sections for commonly important isotopes and elements in their naturally occurring isotopic composition. Nitrogen, oxygen, and silicon are in Figure 5.1, Figure 5.10, and Figure 5.6, respectively.

Figure 5.11 through Figure 5.22 show principal cross sections of the following:

$$
\begin{aligned}
& { }_{1} \mathrm{H}^{1} \text { (neutron on proton) } \\
& { }_{2} \mathrm{He}^{3} \\
& { }_{3} \mathrm{Li}^{6},{ }_{3} \mathrm{Li}^{7} \\
& { }_{4} \mathrm{Be}^{9}, \text { natural isotopic composition } \\
& { }_{5} \mathrm{~B}^{10},{ }_{5} \mathrm{~B}^{11} \\
& { }_{6} \mathrm{C} \text {, natural isotopic composition } \\
& { }_{13} \mathrm{Al}^{27} \text {, natural isotopic composition } \\
& { }_{26} \mathrm{Fe} \text {, natural isotopic composition } \\
& { }_{74} \mathrm{~W} \text {, natural isotopic composition } \\
& { }_{82} \mathrm{~Pb} \text {, natural isotopic composition }
\end{aligned}
$$

All cross sections are presented with the target at $300 \mathrm{~K}$, and all have been taken from the numerical compilation POINT2004 available at BNL and IAEA. Locations are as given after Table 5.1. In addition to the curves in McLane et al. (McL88), another valuable collection of cross-section graphs is the LANL report by Fisher (Fi89). 


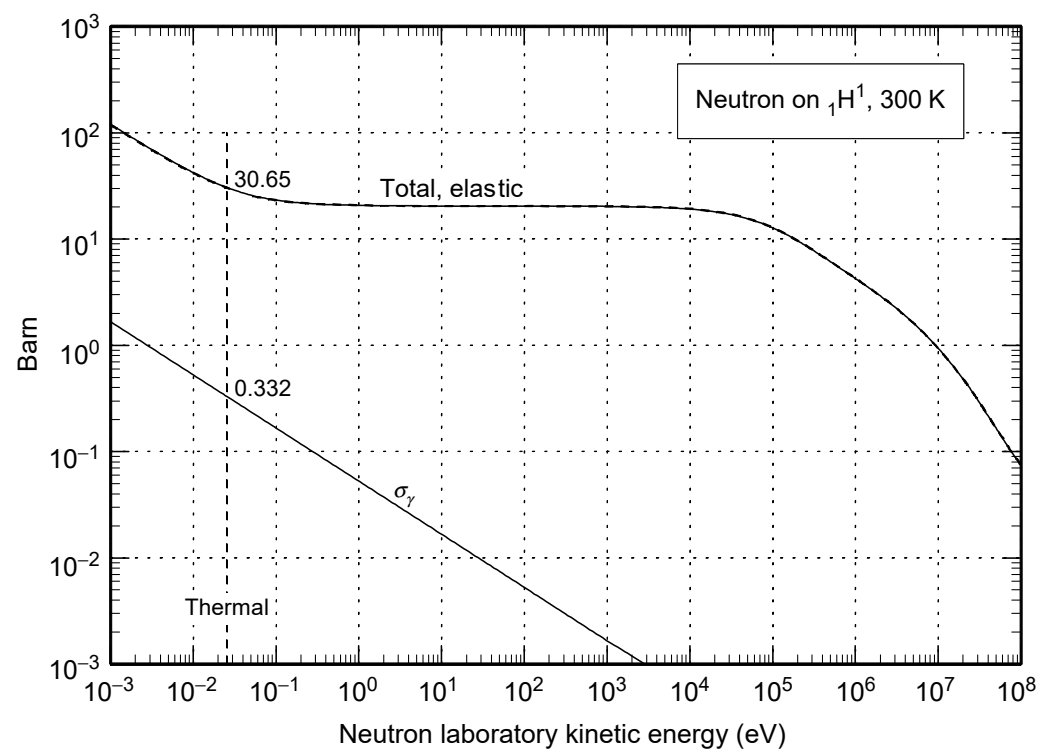

FIGURE 5.11 Neutron-proton cross sections at $300 \mathrm{~K}$. Elastic cross section (dashed) is almost equal to total cross section. Radiative capture, $\sigma_{\gamma}$, creates deuteron and one gamma of $2.223 \mathrm{MeV}$. Thermal cross section values are indicated. (Data from Point2004 (ENDF/B-6).)

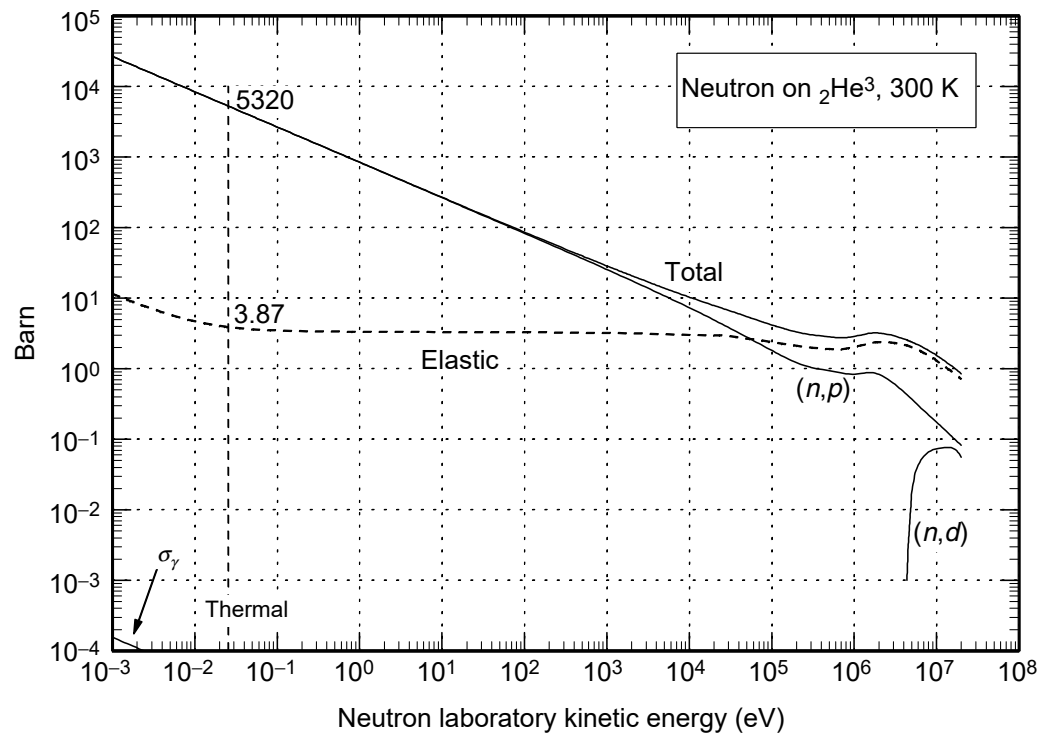

FIGURE 5.12 Neutron- $\mathrm{He}^{3}$ cross sections at $300 \mathrm{~K}$. Radiative capture $\sigma_{\gamma}$ is extremely small. The $(n, d)$ reaction is $n+\mathrm{He}^{3} \rightarrow 2 d$; its threshold is about $4.37 \mathrm{MeV}$. The deuterium production cross section is twice $\sigma(n, d)$. Thermal cross section values are indicated. (Data from Point2004 (ENDF/B-6).) 


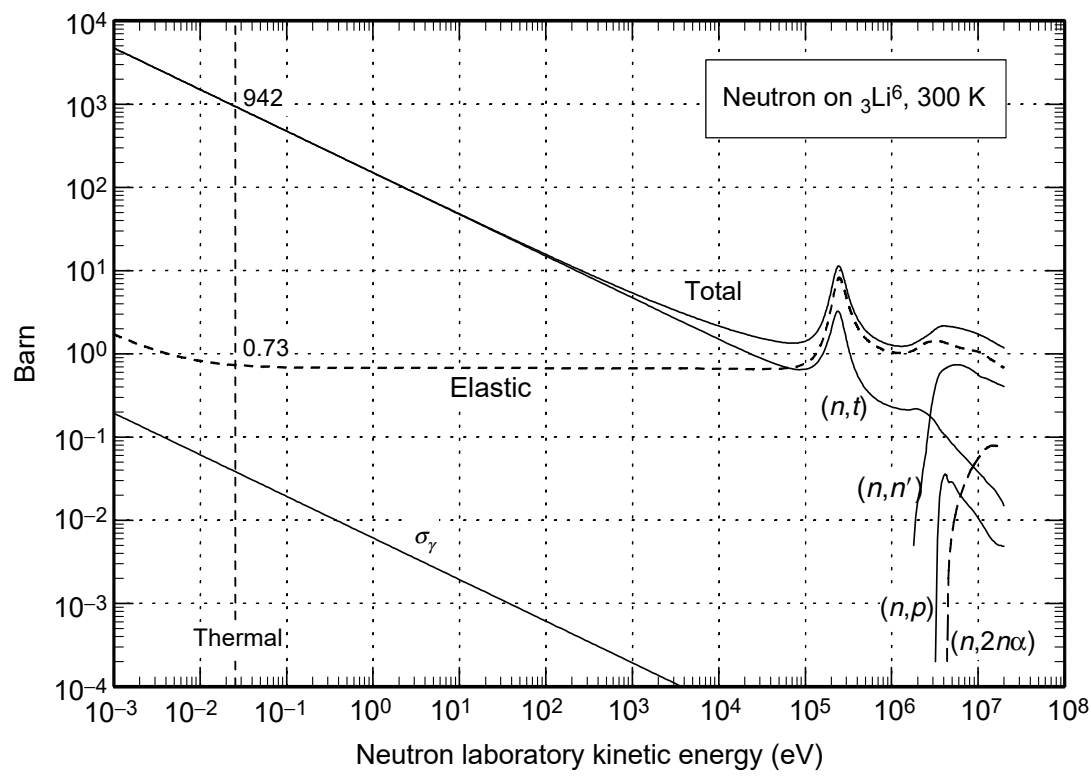

FIGURE 5.13 Principal neutron- $\mathrm{Li}^{6}$ cross sections at $300 \mathrm{~K}$. Threshold for inelastic scattering $\left(n, n^{\prime}\right)$ is about $1.76 \mathrm{MeV} . \sigma_{\gamma}$ is the capture cross section that results in gammas; the gamma production cross section may be larger. The $(n, p)$ reaction is $n+\mathrm{Li}^{6} \rightarrow p+\mathrm{He}^{6} . \mathrm{He}^{6}$ half-life is $0.8 \mathrm{sec}$; it $\beta$-decays back to $\mathrm{Li}^{6}$. The $(n, 2 n \alpha)$ process is $n+\mathrm{Li}^{6} \rightarrow 2 n+\alpha+p$. (Data from Point2004 (ENDF/B-6).)

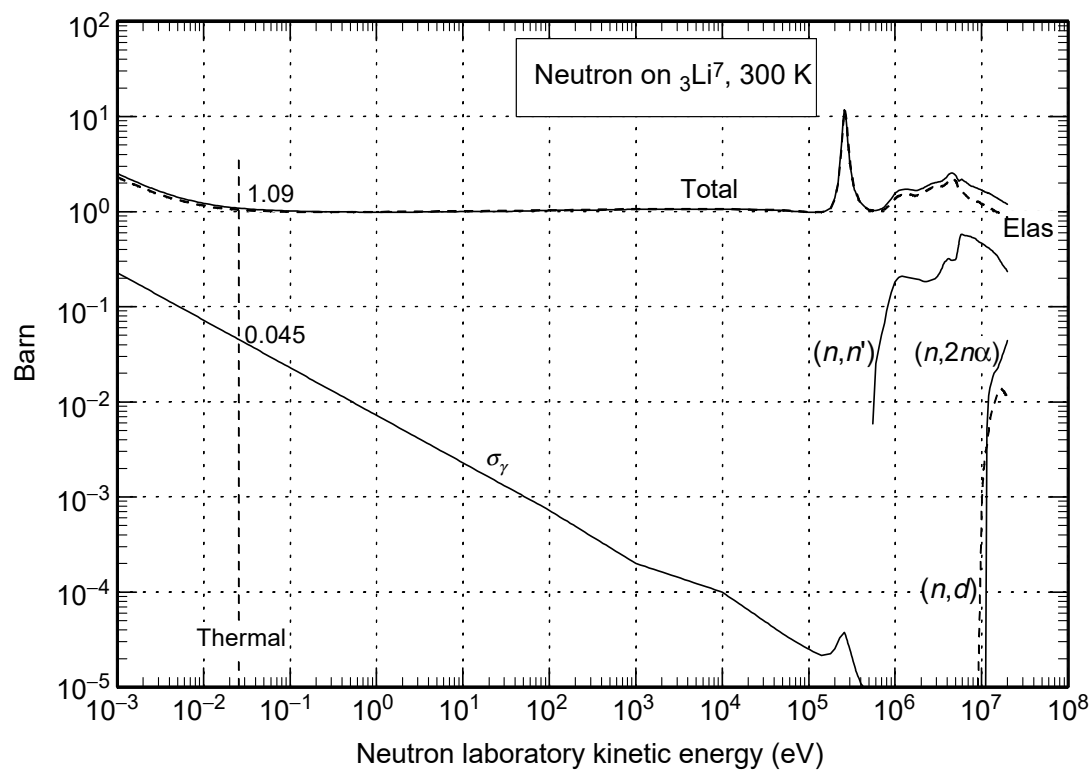

FIGURE 5.14 Principal neutron- $\mathrm{Li}^{7}$ cross sections at $300 \mathrm{~K} . \sigma_{\gamma}$ is the capture cross section; the gamma production cross section may be larger. Threshold for inelastic scattering $\left(n, n^{\prime}\right)$ is about $547 \mathrm{keV}$. The $(n, 2 \mathrm{n} \alpha)$ process is $n+\mathrm{Li}^{7} \rightarrow 2 n+\alpha+d$. $(n, d)$ is $n+\mathrm{Li}^{7} \rightarrow \mathrm{He}^{6}+d$. (Data from Point2004 (ENDF/B-6).) 


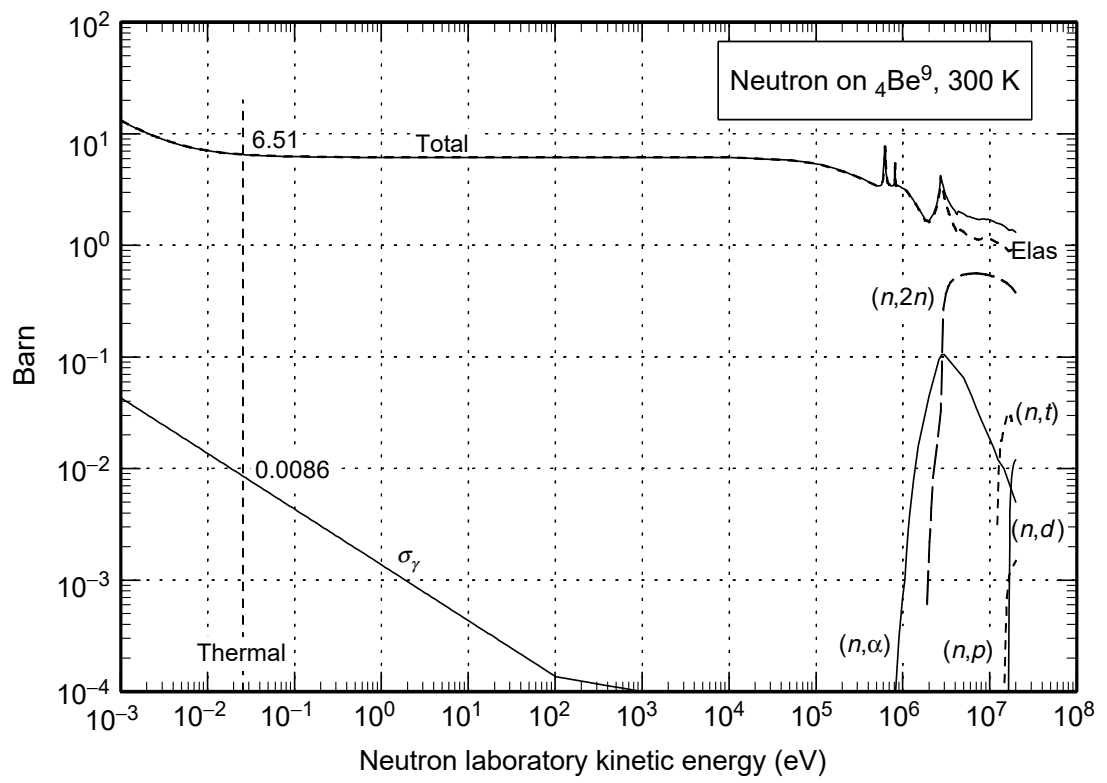

FIGURE 5.15 Principal neutron- $\mathrm{Be}^{9}$ cross sections at $300 \mathrm{~K}$. Elastic cross section (dashed) is almost equal to total cross section. $\sigma_{\gamma}$ is the radiative capture cross section; the gamma production cross section is larger. $\sigma_{\gamma}$ creates $\mathrm{Be}^{10}$ with half-life $2.6 \mathrm{Myr}$. Thermal cross section values are indicated. (Data from Point2004 (ENDF/B-6).)

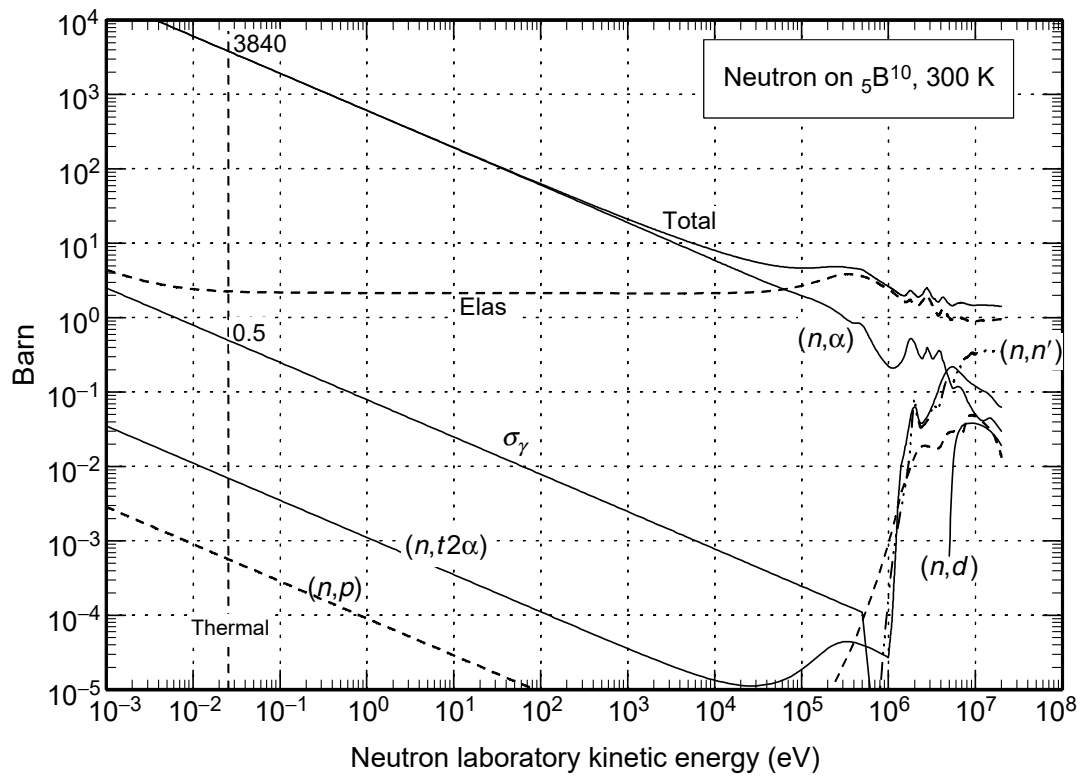

FIGURE 5.16 Principal neutron- $\mathrm{B}^{10}$ cross sections at $300 \mathrm{~K}$. Below $1 \mathrm{keV},(n, \alpha)$ cross section is almost equal to total cross section. $\sigma_{\gamma}$ is the radiative capture cross section; the gamma production cross section will be larger. Inelastic scattering, $\left(n, n^{\prime}\right)$, starts at $0.8 \mathrm{MeV}$. $(n, p)$ and $(n, t 2 \alpha)$ both have no threshold, but are small until above $1 \mathrm{MeV}$. (Data from Point2004 (ENDF/B-6).) 


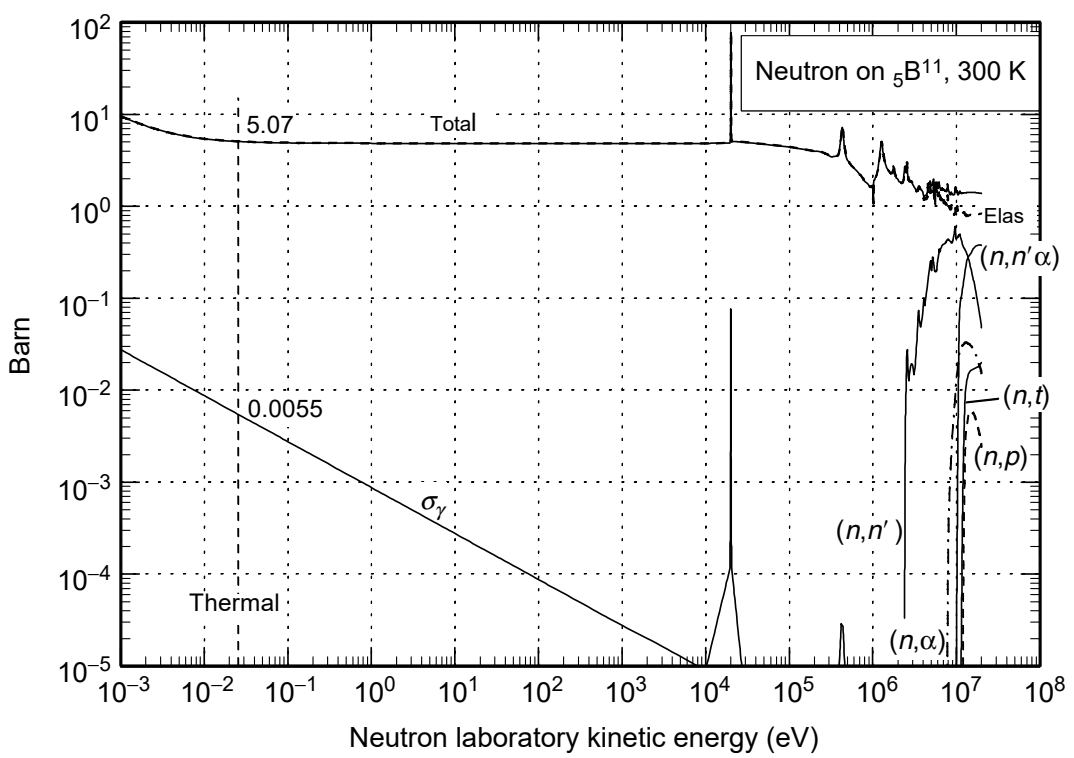

FIGURE 5.17 Some neutron-B ${ }^{11}$ cross sections at $300 \mathrm{~K} . \sigma_{\gamma}$ is the capture cross section; the gamma production cross section is larger. Below $6 \mathrm{MeV}$ scattering is mostly elastic. Inelastic scattering $\left(n, n^{\prime}\right)$, starts at $2.4 \mathrm{MeV}$. Particle production, via $(n, \alpha)$, starts near 7.7 MeV. (Data from Point2004 (ENDF/B-6).)

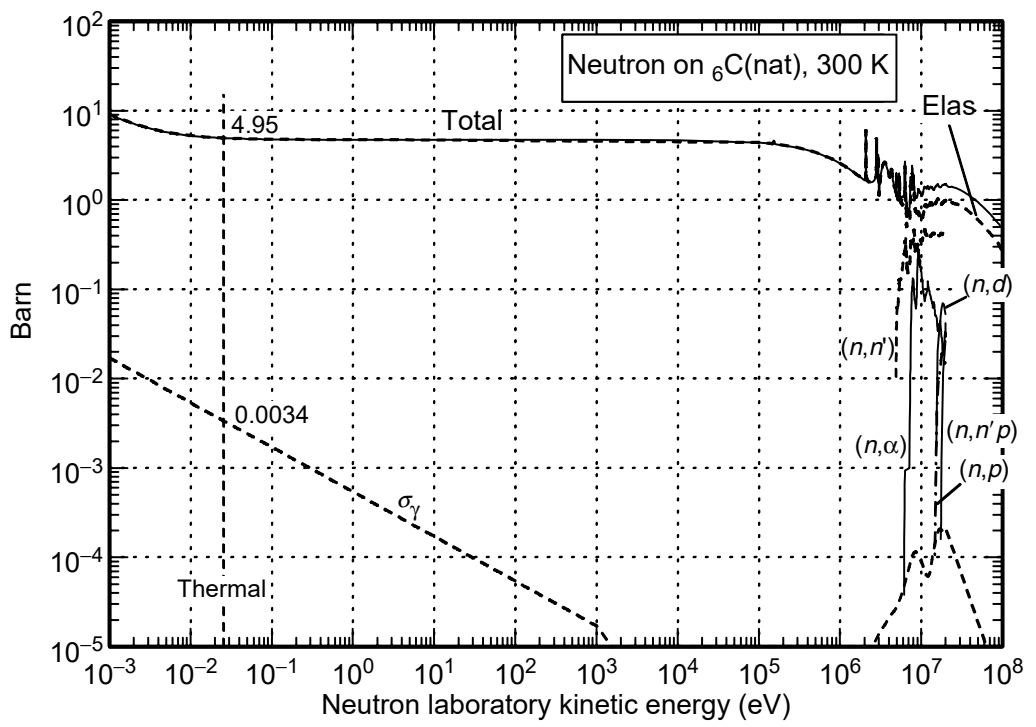

FIGURE 5.18 Principal neutron-C(natural)cross sections at $300 \mathrm{~K} . \sigma_{\gamma}$ is the capture cross section; the gamma production cross section is larger. Below $6 \mathrm{MeV}$ scattering is mostly elastic. Inelastic scattering, $\left(n, n^{\prime}\right)$, the sum of $\left(n, n_{1}^{\prime}\right)+\left(n, n_{2}^{\prime}\right)+\cdots$ starts at 4.85 MeV. Particle production, via $(n, \alpha)$, starts near 6.2 MeV. (Data from Point2004 (ENDF/B-6).) 

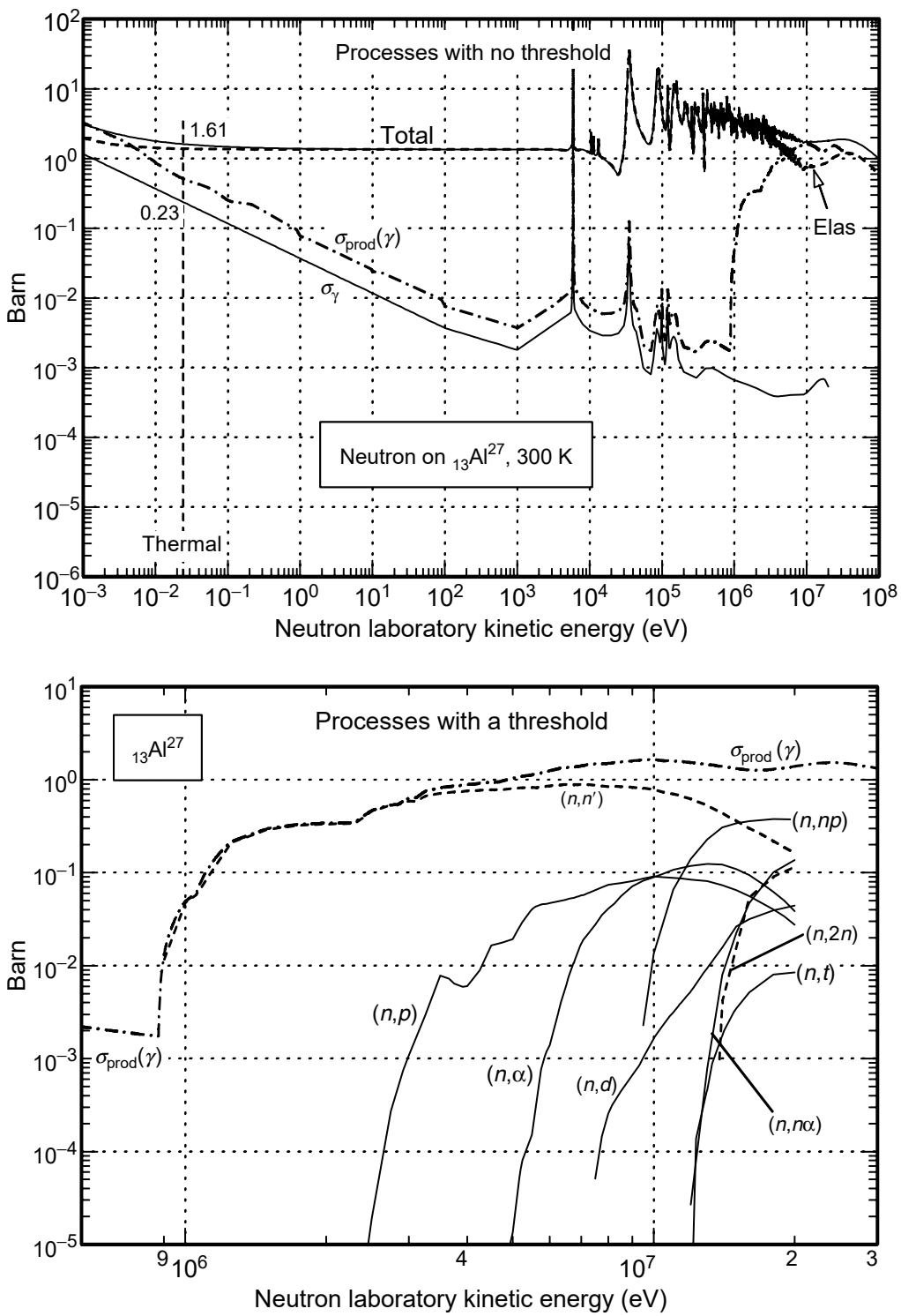

FIGURE 5.19 Principal neutron- $\mathrm{Al}^{27}$ cross sections at $300 \mathrm{~K} . \sigma_{\gamma}$ is radiative capture; $\sigma_{\text {prod }}(\gamma)$ is the gamma production cross section. The first threshold process is inelastic scattering $\left(n, n^{\prime}\right)$ at about $900 \mathrm{keV}$. (Data from Point2004 (ENDF/B-6); $\sigma_{\text {prod }}(\gamma)$ from ACE library.) 

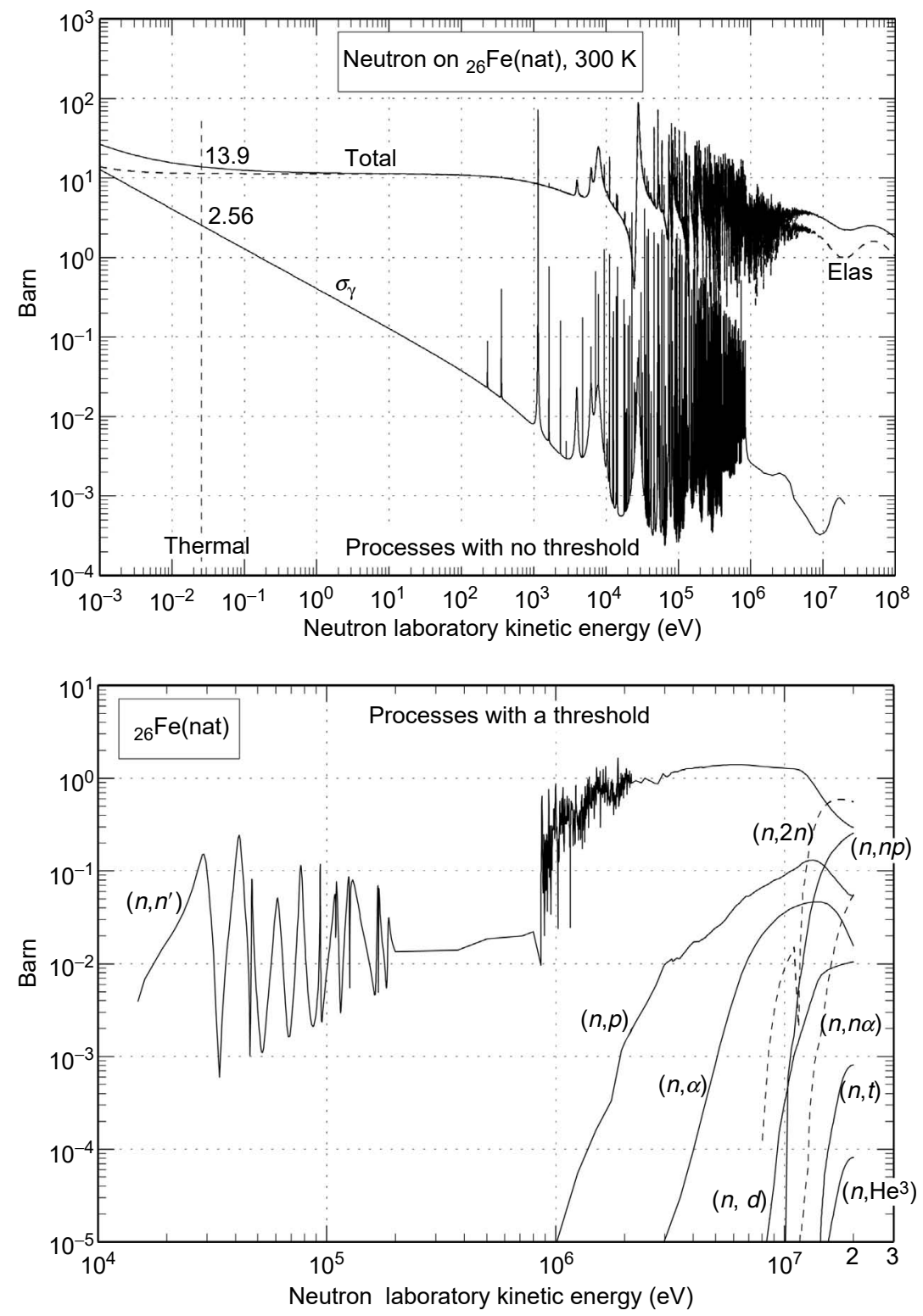

FIGURE 5.20 Principal neutron-Fe(natural) cross sections at $300 \mathrm{~K}$. The first threshold process is inelastic scattering $\left(n, n^{\prime}\right)$ at about $15 \mathrm{keV} . \sigma_{\gamma}$ is radiative capture; the gamma production cross section is larger. (Data from Point2004 (ENDF/B-6).) 

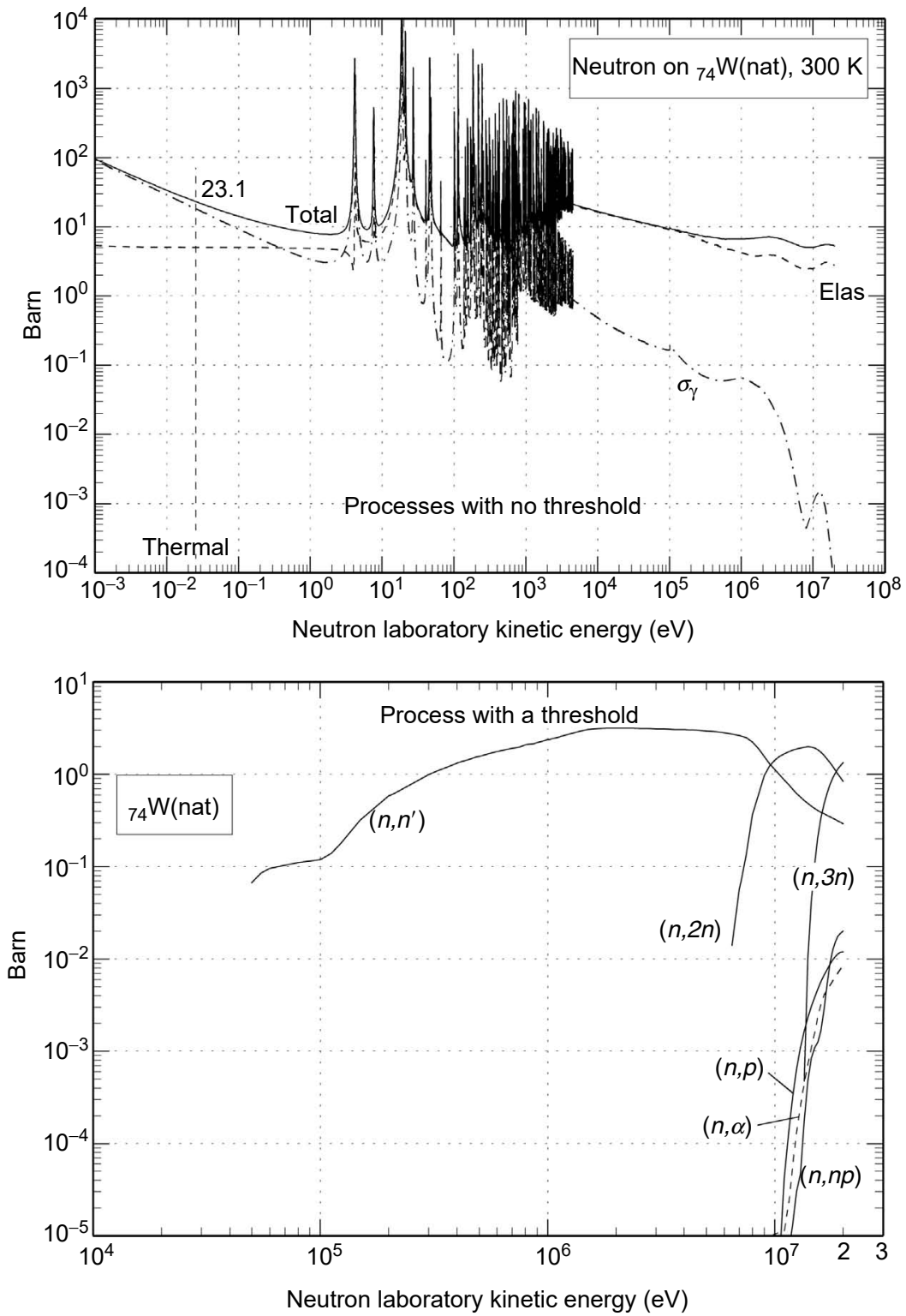

FIGURE 5.21 Principal neutron-W(natural) cross sections at $300 \mathrm{~K} . \sigma_{\gamma}$ is radiative capture; the gamma production cross section is larger. The first threshold process is inelastic scattering $\left(n, n^{\prime}\right)$ at about $50 \mathrm{keV}$. (Data from Point2004 (ENDF/B-6).) 

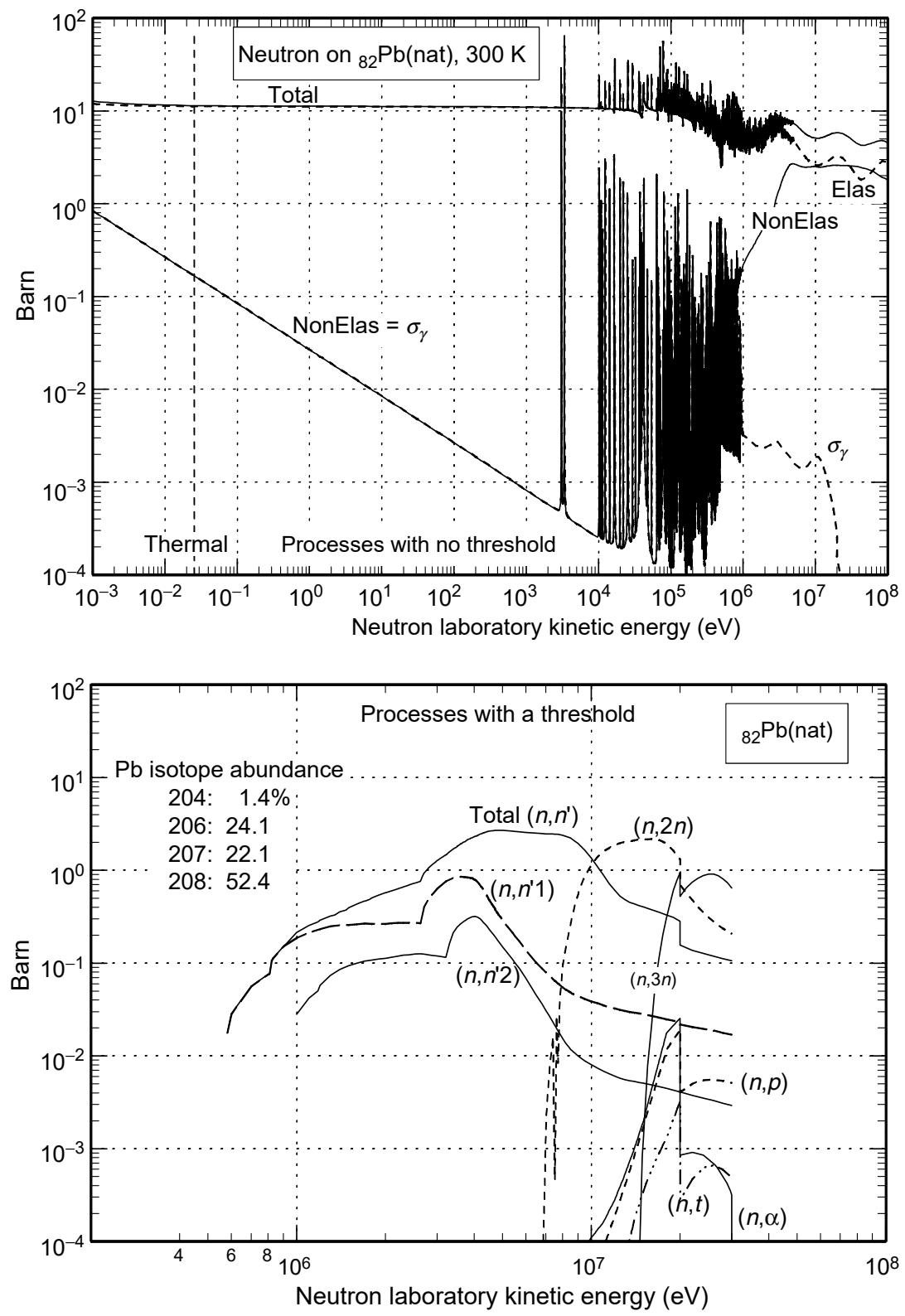

FIGURE 5.22 Principal neutron cross sections on natural $\mathrm{Pb}$ at $300 \mathrm{~K}$. The first threshold process is inelastic scattering, $\left(n, n^{\prime} 1\right)$, at $580 \mathrm{keV}$. Processes not shown occur only in $\mathrm{Pb}^{208}$ and have thresholds above $10 \mathrm{MeV}$; they contribute more than 0.01 barn to natural $\mathrm{Pb}$ only above $25 \mathrm{MeV}$. Constructed from ENDF/B-6 cross sections for individual isotopes $(206,207,208) . \mathrm{Pb}^{204}$ was ignored. 


\section{REFERENCES}

[Aj86] Ajzenberg-Selove, F., Energy levels of light nuclei $A=13-15$, Nucl. Phys. A. $449,1,1986$.

[Am59] Amaldi, E., The production and slowing down of neutrons, in Flügge, S., Ed., Handbuch der Physik, Vol. XXXVIII/2, Springer-Verlag, Berlin, 1959, pp. $1-659$.

[Am84] Amaldi, E., From the discovery of the neutron to the discovery of nuclear fission, Phys. Rep. 111(1-4): 1, 1984.

[Be70] Bell, G.I. and Glasstone, S., Nuclear Reactor Theory, Van Nostrand Reinhold, 1970.

[By94] Byrne, J., Neutrons, Nuclei, and Matter, Institute of Physics Publishing, Bristol, 1994.

[Ch32] Chadwick, J., Nature 129; 312, 1932.

[Fe50] Fermi, E., Nuclear Physics, Revised Edn. University of Chicago Press, 1950.

[Fi89] Fisher, H.M., A Nuclear Cross Section Data Handbook, Los Alamos National Laboratory report LA-11711-M, UC-705, December, 1989.

[Fi96] Firestone, R.B. and Shirley, V.S., Eds., Table of Isotopes, 8th edn., John Wiley, 1996.

[Go76] Gorbachev, V.M., Zamyatin, Y.S., and Lbov, A.A., Interaction of Radiations with Nuclei of Heavy Elements and Nuclear Fission, Atomizdat, Moscow, 1976. In Russian.

[Gr97] Grigoriev, I.S. and Meilikhov, E.Z., Handbook of Physical Quantities, CRC Press, 1997.

[Gr05] Griffin P.J., Energy-dependent neutron response of silicon sensor, Sandia National Laboratory, Draft Report SAND2005-7854P, November, 2005.

[He96] Helmer, R.G., Nuclear data sheets for $A=158$, Nuclear Data Sheets, 77(3): 471-630, March 1996.

[Ho02] Holmes-Siedle, A. and Adams, L., Handbook of Radiation Effects, 2nd edn., Oxford Univ. Press, 2002.

[La91] http://www.nis5.lanl.gov/panda_manual.htm.

[Li63a] Lindhard, J., Nielsen, V., Scharff, M., and Thompsen, P.V., Integral equations governing radiation effects, Kgl. Danske Videnskab. Selskab, Mat. Fys. Medd. 33(10): 1-42, 1963.

[Li63b] Lindhard, J., Scharff, M., and Schiøtt, H.E., Range concepts and heavy ion ranges, Kgl. Danske Videnskab. Selskab, Mat. -Fys. Medd. 33(14): 1, 1963.

[Ma60] Marion, J.B. and Fowler, J.L., Fast Neutron Physics, Wiley Interscience, 1960.

[Ma99] MacFarlane, R., LANL. Private communication, September, 3, 1999.

[McL88] McLane, V., Dunford, C.L., and Rose, P.F., Neutron Cross Sections, Vol. 2, Neutron Cross Section Curves, Academic Press, 1988.

[Me92a] Messenger, G.C., IEEE Trans. Nucl. Sci. 39(3): 468, 1992.

[Me92b] Messenger, G.C., Ash, M.S., The Effects of Radiation on Electronic Systems, 2nd edn., Van Nostrand, Reimhold, New York, 1992.

[Mu81] Mughabghab, S.F., Divadeenam, M., and Holden, N.E., Neutron cross sections, Vol. 1, Neutron Cross Section Curves, Part A: Z=1-60, Academic Press, 1981.

[Mu84] Mughabghab, S.F., Neutron Cross Sections, Vol. 1, Neutron Cross Section Curves, Part B: $Z=61-100$, Academic Press, 1984.

[PDG04] Particle Data Group, Review of particle properties, Phys. Lett. B. 592: 1, July, 2004. 
[Sa66] Sattler, A.R., Vook, F.L., and Palms, J.M., Phys. Rev. A. 143: 588, 1966.

[Sr03] Srour, J., Marshall, C.J., and Marshall, P.W., IEEE Trans. Nucl. Sci. 50(3): 653, June, 2003.

[VL80] Van Lint, V.A.J., Flanagan, T.M., Leadon, R.E., Naber, J.A., Rogers, V.C., Mechanisms of Radiation Effects in Electronic Materials, John Wiley, New York, 1980.

[We58] Weinberg, A.M. and Wigner, E.P., The Physical Theory of Neutron Chain Reactors, University of Chicago Press, 1958. 



\section{Appendix A: Some Fundamental Constants and Defined Units}

For reference we list some useful constants, taken from Peter J. Mohr and Barry N. Taylor, "CODATA recommended values of the fundamental physical constants: 2002," Rev. Mod. Phys. 77(1), 1 (January, 2005). This reference gives the constants to more significant figures than presented here. An online collection is kept by NIST at http://physics.nist. gov/cuu/constants.

In the list below, for combined constants, if the quantity in square brackets is not included, the formula reads in electrostatic units (esu). If it is included, the formula reads in SI units. For example, in esu $e^{2}=2.307 \times 10^{-19} \mathrm{erg} \mathrm{cm}$, but in SI it is $e^{2} / 4 \pi \varepsilon_{\mathrm{o}}$ that equals $2.307 \times 10^{-19} \mathrm{erg} \mathrm{cm}\left(=2.307 \times 10^{-28} \mathrm{~J} \mathrm{~m}\right)$. The fine structure constant $\alpha \approx 1 / 137$ reads as $e^{2} / \hbar c$ in esu, but in SI it is $e^{2} / 4 \pi \varepsilon_{\mathrm{o}} \hbar c$. The electromagnetic vacuum quantities $\varepsilon_{\mathrm{o}}$ and $\mu_{\mathrm{o}}$ are employed only in SI units.

Although nuclear masses are commonly expressed in terms of it, the atomic mass unit $m_{\mathrm{u}}$ is $1 / 12$ the mass of one free, neutral $\mathrm{C}^{12}$ atom with the nucleus and atom in their ground states.

\section{FUNDAMENTAL}

Magnetic permeability of space $\mu_{\mathrm{o}} \equiv 4 \pi \times 10^{-7} \mathrm{H} / \mathrm{m}$ Electric permittivity of space $\varepsilon_{\mathrm{o}}=1 / \mu_{\mathrm{o}} c^{2}=8.85419 \times 10^{-12} \mathrm{~F} / \mathrm{m}$ Speed of light $c \equiv 2.99792458 \times 10^{8} \mathrm{~m} / \mathrm{sec}$

Planck constant $h=6.62607 \times 10^{-27} \mathrm{erg} \mathrm{sec}=4.135667 \times 10^{-15} \mathrm{eV}$ sec $\hbar=h / 2 \pi=1.05457 \times 10^{-27} \mathrm{erg} \mathrm{sec}=6.58212 \times 10^{-16} \mathrm{eV} \mathrm{sec}$ $h c=1.23984 \times 10^{-4} \mathrm{eV} \mathrm{cm}=12.3984 \mathrm{keV} \AA$ $\hbar c=1.97327 \times 10^{-5} \mathrm{eV} \mathrm{cm}=1.97327 \mathrm{keV} \AA$ 


\section{ELECTRON}

Charge $e=1.602177 \times 10^{-19} \mathrm{Coul}=4.803204 \times 10^{-10} \mathrm{esu}$ $e^{2}\left[/ 4 \pi \varepsilon_{\mathrm{o}}\right]=2.30708 \times 10^{-19} \mathrm{erg} \mathrm{cm}=14.399641 \mathrm{eVA}$ $e^{2} c\left[/ 4 \pi \varepsilon_{\mathrm{o}}\right]=4.316904 \mathrm{keV} \mathrm{cm}^{2} / \mathrm{sec}$

Mass $\quad m_{\mathrm{e}}=0.910938 \times 10^{-27} \mathrm{~g}$ $m_{\mathrm{e}} c^{2}=0.5109989 \mathrm{MeV}=8.18710 \times 10^{-7} \mathrm{erg}$

Classical radius $r_{\mathrm{o}}=e^{2} /\left[4 \pi \varepsilon_{\mathrm{o}}\right] m_{\mathrm{e}} c^{2}=\alpha^{2} a_{\mathrm{o}}=2.81794 \times 10^{-13} \mathrm{~cm}$ $4 \pi r_{\mathrm{o}}^{2}=0.997869 \times 10^{-24} \mathrm{~cm}^{2} \approx 1$ barn

Thomson cross section $\sigma_{\mathrm{T}}=(8 \pi / 3) r_{\mathrm{o}}^{2}=6.65246 \times 10^{-25} \mathrm{~cm}^{2} \approx 2 / 3$ barn

Compton wavelength $\quad \lambda_{\mathrm{C}}=h / m_{\mathrm{e}} c=2.42631 \times 10^{-10} \mathrm{~cm}=2 \pi \alpha a_{\mathrm{o}}$

Velocity of $1 \mathrm{eV} \quad v(1 \mathrm{eV})=5.93097 \times 10^{7} \mathrm{~cm} / \mathrm{sec}$

Velocity of $10 \mathrm{keV} \quad v(10 \mathrm{keV})=0.194986 c$

Velocity of $79.0518 \mathrm{keV} \quad=0.5 c$

\section{ATOMIC, ETC.}

Fine structure constant $\quad \alpha=e^{2} /\left[4 \pi \varepsilon_{\mathrm{o}}\right] \hbar c=7.29735 \times 10^{-3}=1 / 137.036$

Velocity in first Bohr orbit $\alpha c=e^{2} /\left[4 \pi \varepsilon_{0}\right] \hbar=2.18769 \times 10^{8} \mathrm{~cm} / \mathrm{sec}$

Bohr radius $a_{\mathrm{o}}=\left[4 \pi \varepsilon_{\mathrm{o}}\right] \hbar^{2} / m_{\mathrm{e}} e^{2}=\hbar / m_{\mathrm{e}} c \alpha=\lambda_{\mathrm{C}} / 2 \pi \alpha=5.29177 \times 10^{-9} \mathrm{~cm}$ Atomic mass unit $m_{\mathrm{u}}=(1 / 12)$ mass of $\mathrm{C}^{12}$ atom $=1.66054 \times 10^{-24} \mathrm{~g}=$ $931.494 \mathrm{MeV}$

Avogadro number $\quad N_{\mathrm{A}}=6.022142 \times 10^{23}$ particles per mole $=1 / \mathrm{m}_{\mathrm{u}}(\mathrm{g})$

Boltzmann constant $k_{\mathrm{B}}=1.38065 \times 10^{-16} \mathrm{erg} / \mathrm{K}=8.617342 \times 10^{-5} \mathrm{eV} / \mathrm{K}=$ $1 \mathrm{eV} / 11,605 \mathrm{~K}$

Proton mass $m_{\mathrm{p}}=1.67262 \times 10^{-24} \mathrm{~g}=938.272 \mathrm{MeV}$

Neutron mass $m_{\mathrm{n}}=1.67493 \times 10^{-24} \mathrm{~g}=939.565 \mathrm{MeV}=1.001378 m_{\mathrm{p}}=$ $m_{\mathrm{p}}+1.293 \mathrm{MeV}$

\section{DEFINED UNITS}

1 fermi $\equiv 1 \mathrm{fm} \equiv 10^{-13} \mathrm{~cm}$

1 Ångstrom unit $\equiv 1 \AA \equiv 10^{-8} \mathrm{~cm}=0.1 \mathrm{~nm}$

1 barn $\equiv 1 \mathrm{~b} \equiv 10^{-24} \mathrm{~cm}^{2}$

$1 \mathrm{cal} \equiv 4.184 \mathrm{~J}$

$1 \mathrm{eV}=1.602177 \times 10^{-19} \mathrm{~J}$

$1 \mathrm{rad} \equiv 100 \mathrm{erg} / \mathrm{g}=1 \mathrm{cGy}=6.241509 \times 10^{7} \mathrm{MeV} / \mathrm{g}$

1 Gray $\equiv 1 \mathrm{~Gy} \equiv 1 \mathrm{~J} / \mathrm{kg} \equiv 10^{4} \mathrm{erg} / \mathrm{g} \equiv 100 \mathrm{rad}$ 


\section{Appendix B: Useful Web Sites}

\section{CROSS-SECTION DATA}

\section{The Four Main World Data Centers}

Data and their documentation are available at:

http://www-nds.iaea.org/-International Atomic Energy Agency, Nuclear Data Services, Vienna, Austria. ENDF (nuclear, photoatomic, and electroatomic) and other international data available here.

http://www.nndc.bnl.gov/-U.S. National Nuclear Data Center at Brookhaven National Laboratory, Upton, New York. ENDF and other data available here.

http://www3.nndc.bnl.gov/point2004/-Neutron cross sections from ENDF/B-6 in a "pointwise" form at selected temperatures. Resonance parameters have been expanded and consistency checks made on raw ENDF/B-6 data files. More useful than raw ENDF data.

http://www.nea.fr/-Nuclear Energy Agency, Issy-les-Moulineaux, and Paris, France. ENDF and other data available at http://www.nea.fr/html/dbdata/

http://www.ippe.obninsk.ru/ and http://www.rssi.ru/IPPE/-Russian Nuclear Data Center (CJD), Institute of Physics and Power Engineering, Obninsk, Russia. Most data can be obtained at: http://www.ippe.obninsk. $\mathrm{ru} / \mathrm{podr} / \mathrm{cjd} /$ index.html

\section{Other Sites for Cross Sections and Related Quantities}

http://t2.lanl.gov/—Los Alamos National Laboratory collection of neutron, photon, and electron cross sections. ENDF data and compilation from other countries are available here.

http://t2.lanl.gov/njoy/ and http://t2.lanl.gov/codes/-Documentation of the ENDF postprocessing code NJOY.

http://www.Innl.gov/cullen1/—DermottE. (Red) Cullen's site is a portal to all ENDF data, utility codes, cross-section display software, documentation, and Monte Carlo codes, specifically TART for neutron-photon transport. 
http://physics.nist.gov/PhysRefData/—General physics reference data. Electron, proton, and alpha particle stopping powers and ranges, and photon cross sections.

http://www.srim.org/—Download the SRIM code from this site. Stopping powers, ranges, and Monte Carlo transport and damage calculation of any ion in almost any material. Documentation.

http://iaeand.iaea.or.at/photonuclear/—Photonuclear cross sections from five countries, with recommended values.

\section{Other Sites of Interest}

http://www.jlab.org/ -semenov/rlinks/soft.html-Very complete list of links to physics-related software, analysis tools, graphics utilities, and so on.

http://pdg.lbl.gov-The Particle Data Group at Lawrence Berkeley National Laboratory. Reviews of particle properties, summaries of theories, and useful formulae.

http://pdg.lbl.gov/AtomicNuclearProperties/—Atomic and nuclear properties of materials (elements, compounds, mixtures, and biological materials), including muon stopping powers and ranges in them.

http://www-phys.llnl.gov/Research/scattering/_-LLNL site on photonatom elastic scattering.

http://ie.lbl.gov/toi.htm-“Isotope Explorer.” Lawrence Berkeley National Laboratory Isotopes Project. Nuclear structure and levels. Table of radioactive nuclides. LLNL/UCSC Nuclear Astrophysics Data. Thermal neutron capture data.

http://www-cxro.lbl.gov/-Center for X-ray Optics at Lawrence Berkeley National Laboratory. All aspects of EUV and x-rays. Form factors, scattering functions, and $\mathrm{x}$-ray data booklet.

http://www-rsicc.ornl.gov/—Radiation Safety Information Computational Center. Codes and data for radiation transport, dose, and damage.

http://www.ans.org/—American Nuclear Society. Technical publications and journals of interest in nuclear physics, largely in nuclear reactor applications.

http://www.inl.gov/—Idaho National Laboratory (INL) (Union of the former Idaho National Engineering and Environmental Laboratory and Argonne National Laboratory-West).

http://www.inl.gov/gammaray/catalogs/catalogs.shtml-Radioactive decay gamma spectroscopy data at INL (formerly INEEL).

http://www3.ocn.ne.jp/ tttabata/, select "emid (Database)"Tabata's Institute for Data Evaluation and Analysis. Electron-stopping powers, ranges, dose, and multiple scattering. 
http://www.napchan.com/-The low-energy electron transport code $m c$-set (Monte Carlo Simulation of Electron Transport).

http://www.nucleide.org/—Laboratoire National Henri Becquerel. Data on dosimetry and radioactivity.

http://web.utk.edu/ srcutk/htm/interact.htm-D.C. Joy, “A Database on Electron-Solid Interactions." Backscattered and secondary electron yields.

http://www.ncrponline.org/—National Council on Radiation Protection and Measurements. Standards publications, safety guidelines.

http://www.icru.org/-International Commission on Radiation Units and Measurements. Standards publications, tutorials and proper definitions, measurement procedures, and journal of the ICRU. 

(a) $\square \underset{\square}{ } \mathrm{Xe}^{135}\left(t_{1 / 2}=9.1 \mathrm{~h}\right)$

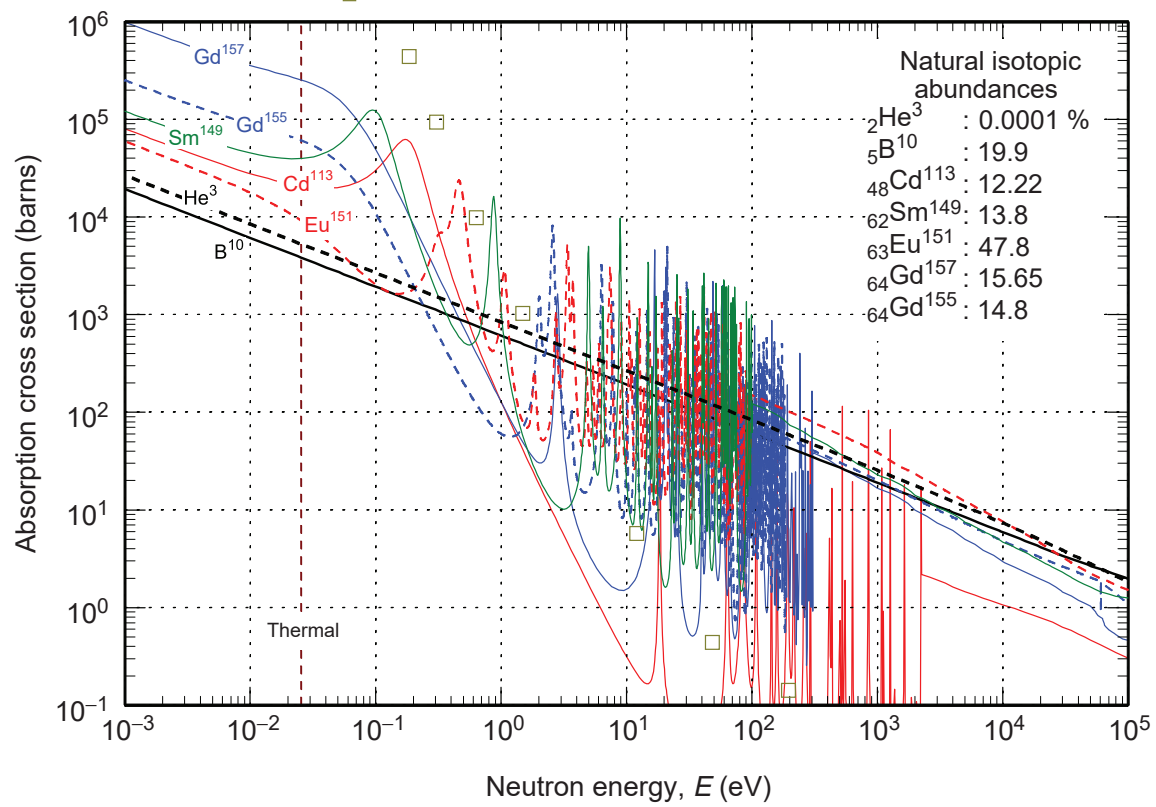

(b)

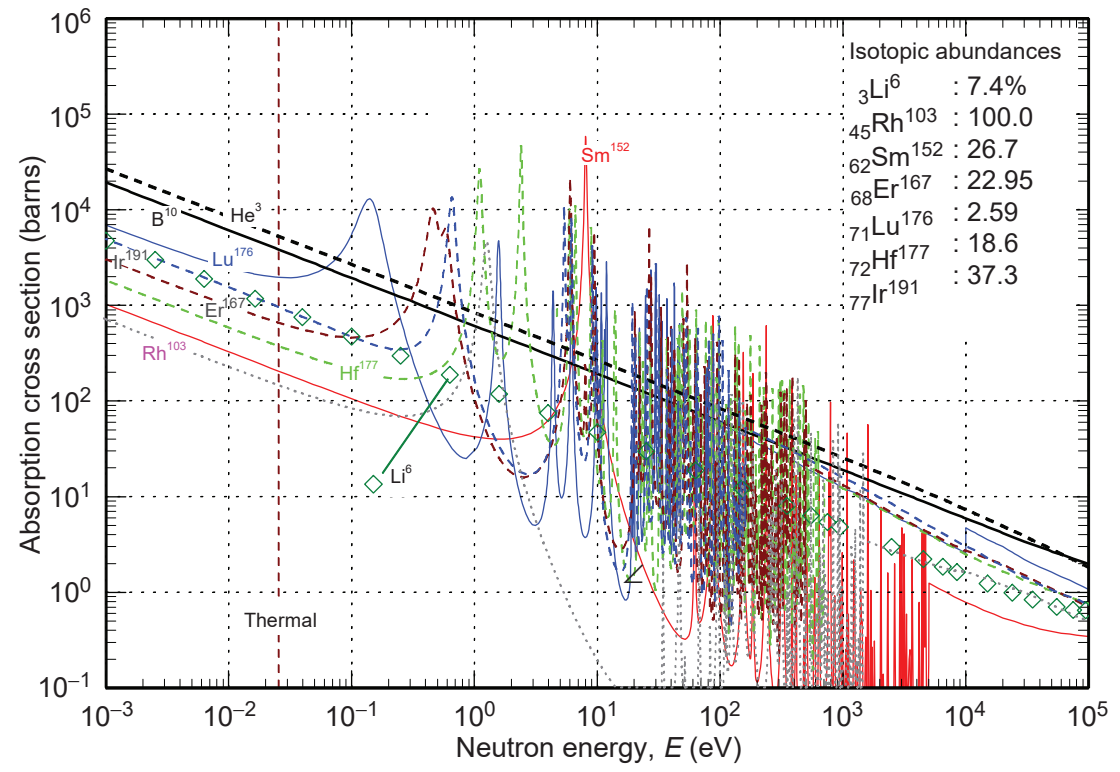

FIGURE 5.2 Cross sections at $300 \mathrm{~K}$ of good absorbers of low-energy neutrons. (a) Stable isotopes with thermal cross section greater than that of $\mathrm{B}^{10}$. All reactions are $(n, \gamma)$ or $(n, \mathrm{ce})$ except $\mathrm{He}^{3}(n, p)$ and $\mathrm{B}^{10}(n, \alpha) . \mathrm{Xe}^{135}$ (half-life $9.1 \mathrm{~h}$ ), with thermal cross section $2.5 \mathrm{Mbarn}$, is shown in squares for comparison. (b) Stable isotopes with thermal cross section less than that of $\mathrm{B}^{10}$. All reactions are $(n, \gamma)$ or $(n, c e)$ except $\mathrm{He}^{3}(n, p), \mathrm{B}^{10}(n, \alpha)$, and $\mathrm{Li}^{6}(n, t)$. 


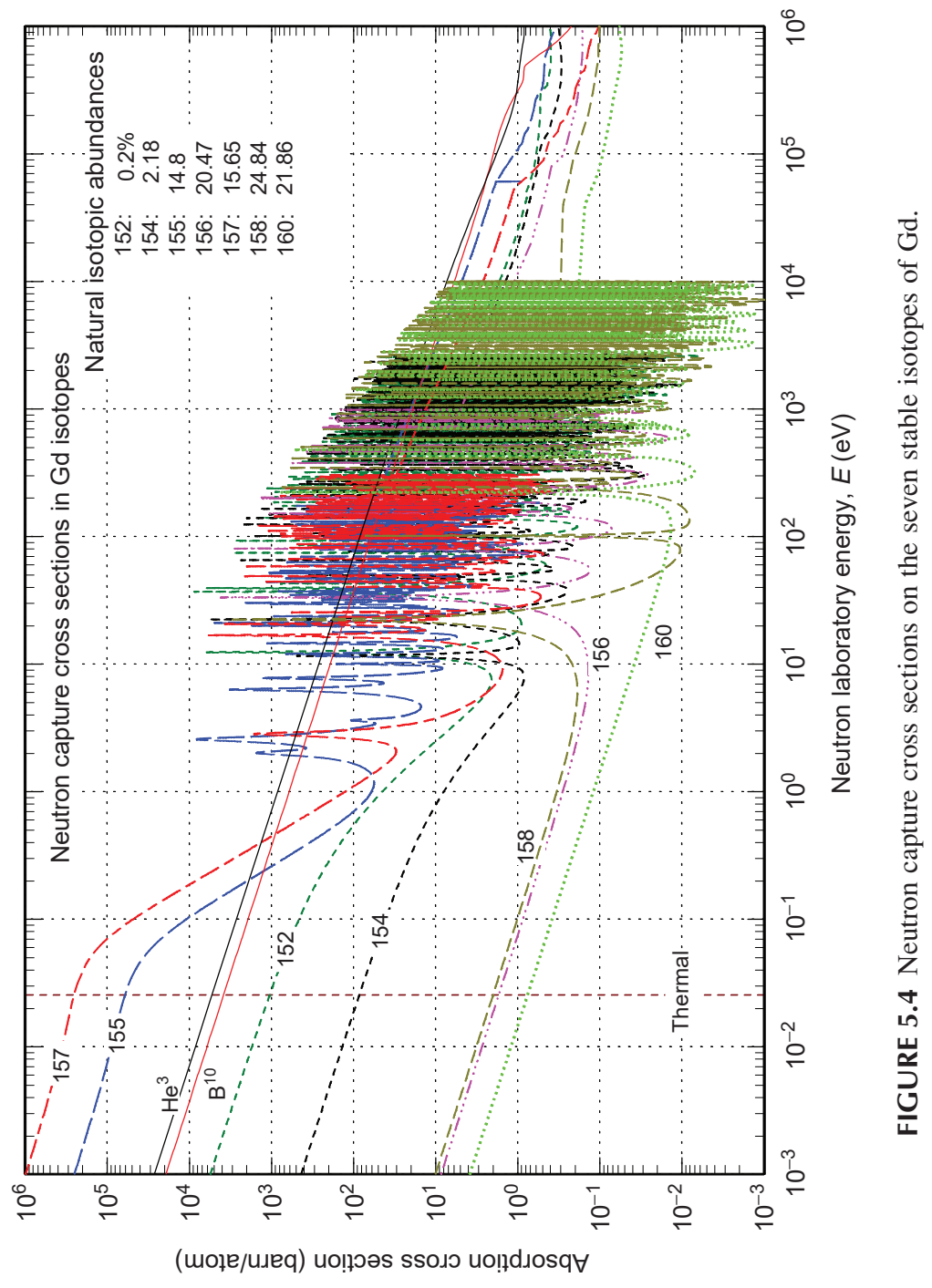




\section{An Introduction to the \\ 24 \\ Passage of Energetic Particles through Matter}

Identifying where to access data, extracting a needed subset from available resources, and knowing how to interpret the format in which data are presented can be time-consuming tasks for scientists and engineers. By collecting all of this information and providing a background in physics, An Introduction to the Passage of Energetic Particles through Matter enables specialists and nonspecialists alike to understand and apply the data.

Making modern data more accessible, this book explores the interactions with matter of energetic particles, including photons, electrons, protons, alpha particles, and neutrons. It presents quantities of interest in many applications, such as photon and neutron cross sections, charged particle stopping powers, electron mean ranges, and angular distributions. The book also discusses electron multiple scattering and models for electron mean range against both stopping power and scattering. The author uses numerous graphs throughout the book to illustrate the material and describes the basic physics underlying all processes. The accompanying CD-ROM includes full datasets and large color contour graphs of cross sections, stopping powers, and ranges in all elements at all interesting energies.

An Introduction to the Passage of Energetic Particles through Matter provides a comprehensive foundation of partiele interactions and supplies an introduction to the massive, invaluable Evaluated Nuclear Data File (ENDF) tibrary.

\section{Features}

- Presents modern data on basic particle-matter interactions

- Discusses the fundamental physics to make numerical values understandable both for specialists and nonspecialists

- Describes the physical processes that control photon, electron, and ion behavior in matter

- Provides useful graphs that show dependencies over a wide range of the independent yariable(s) and allow quick approximations of a quantity

- Incorporates data extractión tools, large scale high resolution contour plots in color, and numerical data, including photon cross sections and electron stopping powers, on the accompanying CD-ROM

This book was supported by the U.S. Air Force Office of Scientific Research (AFOSR).

IP> 34

(1) Taylor \& Francis Taylor \& Francis Group an informa business

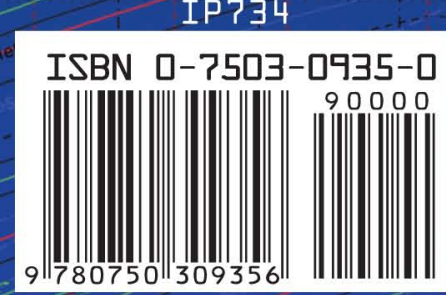

